RENATO MAIA MATARAZZO ORSINO

\title{
A CONTRIBUTION ON MODELING METHODOLOGIES FOR MULTIBODY SYSTEMS
}

Tese apresentada à Escola Politécnica da Universidade de São Paulo para obtenção do Título de Doutor em Engenharia Mecânica. 


\section{A CONTRIBUTION ON MODELING METHODOLOGIES FOR MULTIBODY SYSTEMS}

Tese apresentada à Escola Politécnica da Universidade de São Paulo para obtenção do Título de Doutor em Engenharia Mecânica.

Área de concentração: Engenharia Mecânica

Orientador:

Prof. Dr. Tarcisio Antonio Hess Coelho 
Este exemplar foi revisado e corrigido em relação à versão original, sob responsabilidade única do autor e com a anuência de seu orientador.

São Paulo de de

Assinatura do autor:

Assinatura do orientador:

Catalogação-na-publicação

Orsino, Renato Maia Matarazzo

A contribution on modeling methodologies for multibody systems / R. M.

M. Orsino -- versão corr. -- São Paulo, 2016.

$213 \mathrm{p}$.

Tese (Doutorado) - Escola Politécnica da Universidade de São Paulo. Departamento de Engenharia Mecânica.

1.Dinâmica 2.Cinemática 3.Mecanismos I.Universidade de São Paulo. Escola Politécnica. Departamento de Engenharia Mecânica II.t. 


\section{Acknowledgements}

I would like to acknowledge everyone who supported, advised and encouraged me during the last 4 years, since I begun this long journey towards my PhD degree. Professors, family, friends, colleagues, staff (both from University of São Paulo and TU Delft), thank you all!

A second acknowledgment to CNPq - Conselho Nacional de Desenvolvimento Científico e Tecnológico, of the Ministry of Science, Technology and Innovation of Brazil for sponsoring this research (processes numbers 163026/2012-2 and 203112/2014-8).

I would like to dedicate the remaining of this section for some special acknowledgements.

First of all, I would like to thank my parents, Liliane and Francisco. Without your unconditional support I would never be able to dedicate the time and effort I wanted to in this project.

A special acknowledgement to my advisor, Professor Tarcisio Antonio Hess Coelho. Thank you for your patience and dedication but, mainly, for our fertile meetings, from which I could make important decisions in the course of my research.

Another special acknowledgement to Professor Arend Leendert Schwab, who generously gave me the opportunity of working along with his team at TU Delft, where I could make substantial progress in my research.

I would also like to thank professors Celso Pupo Pesce, Raul Gonzales Lima and Roberto Spinola Barbosa, members of my qualifying exam board, for their advices on the preliminar versions of this text.

Finally, among all the PhD candidates I had the privilege of working on along the last 4 years, I would like to specially acknowledge my colleague André Garnier Coutinho, a great enthusiast of the novel methodology presented in this thesis, who gave me valuable feedback related to actual implementations and developed specialized versions that made it accessible to undergraduate students. 


\section{Resumo}

A Dinâmica de Sistemas Multicorpos tem sido responsável por revolucionar projetos de Engenharia Mecânica pela utilização de modelos matemáticos para simulação e otimização do comportamento dinâmico de uma ampla gama de sistemas mecânicos. Estes modelos matemáticos não somente podem fornecer valiosas informações acerca de um sistema que caso contrário poderiam ser obtidas somente através de experimentos com protótipos, como também têm sido responsável pelo desenvolvimento de diversos sistemas de controle baseados em modelo.

Este trabalho representa uma contribuição para a modelagem dinâmica de sistemas mecânicos multicorpos por meio do desenvolvimento de uma nova metodologia modular e recursiva que unifica as principais contribuições de diversos formalismos da Mecânica Clássica. A razão para propor tal metodologia é motivar a implementação de rotinas computacionais para a modelagem de sistemas mecânicos multicorpos complexos sem depender de pacotes de software de código fechado e, consequentemente, contribuir para o ensino de Dinâmica de Sistemas Multicorpos nos níveis de graduação e pós-graduação.

Todos os desenvolvimentos teóricos são baseados em e motivados por uma revisão crítica da literatura, conduzindo a uma forma matricial geral das equações dinâmicas de movimento de um sistema mecânico multicorpos (que podem ser expressas em termos de qualquer conjunto de variáveis adotado para a descrição dos movimentos realizados pelo sistema, ainda que tal conjunto inclua variáveis redundantes) e a uma metodologia recursiva geral para a obtenção de modelos matemáticos de sistemas complexos, dado um conjunto de equações descrevendo a dinâmica de cada um de seus subsistemas desacoplados e outro descrevendo os vínculos entre estes subsistemas (no sistema) quando acoplado. Este trabalho também inclui algumas discussões acerca da descrição de movimentos (utilizando qualquer conjunto admissível de variáveis de movimento e admitindo qualquer tipo de vínculo que seja passível de descrição por invariantes), e das condições para a solução dos problemas de dinâmica direta e inversa dado um modelo matemático de um sistema multicorpos. Finalmente, alguns exemplos de pacotes computationais baseados na nova metodologia, juntamente com alguns estudos de caso, são apresentados, ressaltando as contribuições que podem ser alcançadas por meio do uso da metodologia proposta.

Palavras chave: Sistemas Multicorpos. Mecânica Analítica. Mecânica Computacional. Dinâmica. Modelagem Matemática. 


\section{Abstract}

Multibody System Dynamics has been responsible for revolutionizing Mechanical Engineering Design by using mathematical models to simulate and optimize the dynamic behavior of a wide range of mechanical systems. These mathematical models not only can provide valuable informations about a system that could otherwise be obtained only by experiments with prototypes, but also have been responsible for the development of many model-based control systems.

This work represents a contribution for dynamic modeling of multibody mechanical systems by developing a novel recursive modular methodology that unifies the main contributions of several Classical Mechanics formalisms. The reason for proposing such a methodology is to motivate the implementation of computational routines for modeling complex multibody mechanical systems without being dependent on closed source software and, consequently, to contribute for the teaching of Multibody System Dynamics in undergraduate and graduate levels.

All the theoretical developments are based on and motivated by a critical literature review, leading to a general matrix form of the dynamic equations of motion of a multibody mechanical system (that can be expressed in terms of any set of variables adopted for the description of motions performed by the system, even if such a set includes redundant variables) and to a general recursive methodology for obtaining mathematical models of complex systems given a set of equations describing the dynamics of each of its uncoupled subsystems and another set describing the constraints among these subsystems in the assembled system. This work also includes some discussions on the description of motion (using any possible set of motion variables and admitting any kind of constraint that can be expressed by an invariant), and on the conditions for solving forward and inverse dynamics problems given a mathematical model of a multibody system. Finally, some examples of computational packages based on the novel methodology, along with some case studies, are presented, highlighting the contributions that can be achieved by using the proposed methodology.

Keywords: Multibody Systems. Analytical Mechanics. Computational Mechanics. Dynamics. Mathematical modeling. 


\section{Contents}

List of Figures iv

Symbol conventions v v

1 Introduction 1

1.1 Background and objetives . . . . . . . . . . . . . 1

1.2 Research topics and publications . . . . . . . . . . . . 4

1.3 Outline of the main topics . . . . . . . . . . . . . 8

1.4 Notation . . . . . . . . . . . . . . . . . 9

2 On the description of motion of multibody systems 12

2.1 Sufficient conditions for describing the motion of a multibody system ............................ 14

2.2 Generalized variables . . . . . . . . . . . . . . . 19

2.3 Generalized constraint invariants . . . . . . . . . . . . . 23

2.4 Variations of motion variables . . . . . . . . . . . . 28

2.5 Descriptions of motion in modular multibody formulations . . . 38

3 Multibody System Dynamics - a comparative literature review $\quad \mathbf{5 0}$

3.1 Newton-Euler formalism . . . . . . . . . . . . . . . 50

3.1.1 Newton's laws of motion . . . . . . . . . . . . . . 52

3.1 .2 Newton-Euler equations . . . . . . . . . . . . . 54

3.1.3 Applications of the Screw Theory . . . . . . . . . . . 58

3.1.4 Recursive algorithms based on Newton-Euler formalism . 62

3.2 Analytical Mechanics: fundamental principles and Lagrangian formalism . . . . . . . . . . . . . . . . . . . . . 64 
3.2.1 Principle of Virtual Work and D'Alembert's Principle . . . 64

3.2.2 Differential Variational Principles . . . . . . . . . . . 67

3.2.3 Modeling methodologies based on the Principle of Virtual Work and its variants . . . . . . . . . . . . . . . 69

3.2.4 Extended Hamilton's Principle . . . . . . . . . . . . . . . 69

3.2.5 Lagrangian equations of motion . . . . . . . . . 71

3.2.6 Lagrangian multipliers and canonical equations of motion 73

3.2.7 Modeling methodologies based on LagrangianHamiltonian formalism . . . . . . . . . . . . . 76

3.3 Gibbs-Appell, Maggi's and Boltzmann-Hamel formalisms . . . 78

3.3.1 Gibbs-Appell equations . . . . . . . . . . . . 78

3.3.2 Maggi's equations . . . . . . . . . . . . . . 83

3.3.3 Boltzmann-Hamel equations . . . . . . . . . . . . 85

3.4 Kane's and Udwadia-Kalaba methodologies . . . . . . . . . . . 86

3.4.1 Kane's equations . . . . . . . . . . . . . . 86

3.4.2 Udwadia-Kalaba methodology . . . . . . . . . . . 89

4 Modular modeling methodology for multibody systems 94

4.1 General form of equations of motion of a multibody system . . . 95

4.2 Recursive modular modeling algorithm . . . . . . . . . . . . 101

4.3 Sufficient conditions for the forward dynamics . . . . . . . . . 111

4.4 Sufficient conditions for the inverse dynamics . . . . . . . . . 118

4.5 Linearization of equations of motion . . . . . . . . . . . 133

4.6 Qualitative comparison between modeling methodologies . . . . 145

5 Computational implementations of modular modeling algorithms for multibody systems

5.1 MoSsPack - recursive modular modeling package for Mathematica $10.2 \ldots \ldots$. . . . . . . . . . . . . . . . . . . . . . . . . . . . . .

5.2 Case study: linearized tadpole tricycle model . . . . . . . . . . 154 
5.2 .1 Overview . . . . . . . . . . . . . . . . . . . . 154

5.2.2 Rider's body and frame assembly . . . . . . . . . 156

5.2 .3 Wheels and suspension . . . . . . . . . . . . . 158

5.2.4 Linearized equations of motion . . . . . . . . . . . 164

5.2 .5 Stability analysis . . . . . . . . . . . . 168

5.3 Mo2DPack - modeling and simulation of planar mechanisms . 175

5.3.1 Modeling and inverse dynamics simulation of a Whitworth quick-return mechanism . . . . . . . . . . . . . 181

5.3.2 Modeling and inverse dynamics simulation of a $3 \underline{R R R}$ mechanism . . . . . . . . . . . . . 186

6 Conclusion 193

$\begin{array}{ll}\text { References } & 195\end{array}$

Appendix A - Use of energy-like functions in the modeling of multibody systems 203

A.1 Generalized inertia forces and energy-like functions . . . . . 203

A.2 Kinetic energy and Gibbs-Appell function of a rigid body . . . . 208

A.3 Generalized forces in a multi-rigid-body system . . . . . . . 211 


\section{List of Figures}

1.1 Comparison between conventional modeling approaches and the proposed modular modeling methodology. Reproduced from Orsino e Hess-Coelho (2015). . . . . . . . . . . . . . . 4

1.22 RSS+PPaP mechanism. Reproduced from Orsino and HessCoelho (2013). . . . . . . . . . . . . . . . . . . 5

1.3 Modeling methodology for a parallel mechanism based on Kane's method. Reproduced from Orsino and HessCoelho (2013). . . . . . . . . . . . . . . . . . . . . . 6

1.4 Delta parallel mechanism. Reproduced from Orsino, HessCoelho and Pesce (2015). . . . . . . . . . . . . . . . . . . . 6

2.1 Representation of a 4-bar mechanism. Adapted from Orsino and Hess-Coelho (2015). . . . . . . . . . . . . . . . . . . 16

2.2 Representation of a knife-edge disc rolling in a plane. Adapted from Orsino and Hess-Coelho (2015). . . . . . . . . . . . 17

2.3 Representation of system $\mathscr{P} . \ldots \ldots \ldots$

2.4 Singular generalized configurations of the variant of $\mathscr{P} . \quad \ldots \quad 27$

2.5 Representation of a $2 \mathrm{D}$ rigid body element. . . . . . . . . 37

2.6 Representation of the model of a bicycle, $\mathscr{W}$. Adapted from Orsino and Hess-Coelho (2015). . . . . . . . . . . . . . . . . . 39

2.7 Representation of the model of a 5-bar mechanism, $\mathscr{P} . \quad \ldots .39$

2.8 Representation of the model of a 3RRR mechanism, Q . . . . . 39

2.9 Extra generalized coordinates defined in the model of the 5-bar mechanism. . . . . . . . . . . . . . . . 47

4.1 System $\delta \ldots \ldots \ldots \ldots \ldots$. . . . . . . . . . . . . . . . . . . .

4.2 Time history of the coordinate $x$ of the contact point of the sphere $\mathcal{S}$ with the surface $U \ldots \ldots \ldots \ldots$ 
4.3 Normalized time histories of the coordinate $x$ of the contact point of the sphere $\mathcal{S}$ with the surface $U$. . . . . . . . . . . . . 117

4.4 Time history of the quaternion parameters defining the orientation of the sphere $\delta . \ldots \ldots$. . . . . . . . . . . 117

4.5 Time history of the quaternion norm inspection. . . . . . . . 118

4.6 Representation of a 3D slider-crank mechanism in its reference configuration . . . . . . . . . . . . . . . . . . 122

4.7 Numerical error evaluation in the inverse kinematics simulation $\# 1$. . . . . . . . . . . . . . . . . . . . . . . . . . . . . . 129

4.8 Numerical error evaluation in the inverse kinematics simulation \#2. . . . . . . . . . . . . . . . . . . . . . . . . . . 129

4.9 Numerical error evaluation in the inverse dynamics simulation. . 130

4.10 Time history of the position of the slider. . . . . . . . . . . 130

4.11 Time history of the angular position of the crank. . . . . . . 131

4.12 Time history of the angular velocity of the crank. . . . . . . 131

4.13 Time history of the torque applied by the actuator. . . . . . . 132

4.14 Time history of the normal components of the contact force acting in the slider. . . . . . . . . . . . . . . . . . . . . 132

4.15 Stable value of $\theta$ as a function of the inclination angle $\bar{\xi}$ for several values of the ratio $\bar{m}_{2} / \bar{m}_{1} \ldots \ldots \ldots \ldots \ldots \ldots 14 \ldots \ldots \ldots$

4.16 Natural frequency $\bar{\omega}$ as a function of the inclination angle $\bar{\xi}$ for several values of the ratio $\bar{m}_{2} / \bar{m}_{1} \ldots \ldots \ldots \ldots \ldots 14 \ldots \ldots \ldots$

4.17 Time histories of the angle $\theta$ obtained by the non-linear $(\mathrm{NL})$ and linearized $(L)$ models compared with the reference value $(R)$ of the angle. . . . . . . . . . . . . . . . . . . . . . 142

4.18 Time histories of $\omega_{1}-{ }^{\mathrm{R}} \omega_{1}$ obtained by the non-linear $(\mathrm{NL})$ and linearized $(\mathrm{L})$ models. . . . . . . . . . . . . . . . . . . . . 143

4.19 Time histories of $\omega_{2}-{ }^{\mathrm{R}} \omega_{2}$ obtained by the non-linear $(\mathrm{NL})$ and linearized $(\mathrm{L})$ models. . . . . . . . . . . . . . . . . . . . . 143

4.20 Time histories of $\dot{\theta}-{ }^{\mathrm{R}} \dot{\theta}$ obtained by the non-linear (NL) and linearized $(\mathrm{L})$ models. . . . . . . . . . . . . . . . . . . . . . . 144 
4.21 Numerical error evaluation in the forward dynamics simulation of the non-linear model. . . . . . . . . . . . . . . . . . . . . . 144

5.1 Modelling of a spherical pendulum using MoSsPack. Adapted from the documentation available at https://github.com/ renatoorsino/MoSsPack/blob/master/doc/MoSsPack.pdf. . . . 153

5.2 Representation of system $\mathscr{T}$ and its subsystems $\ldots \ldots \ldots$

5.3 Feetz $^{\circledR}$ tricycle . . . . . . . . . . . . . . . . . . . . 166

5.4 Eigenvalues versus speed chart for the linearized tadpole tricycle model using reference parameters. . . . . . . . . . . . 170

5.5 Steer-to-lean ratio versus speed chart for the linearized tadpole tricycle model using reference parameters. . . . . . . . . . . 170

5.6 Eigenvectors tables for different speeds of the tadpole tricycle using reference parameters. . . . . . . . . . . . . . . 171

5.7 Eigenvalues versus speed charts for variant scenarios . . . . . 172

5.8 Steer-to-lean ratio versus speed charts for variant scenarios . . . 173

5.9 Algorithm for modeling and simulation of system $\delta \ldots \ldots$. . 180

5.10 Representation of a Whitworth quick-return mechanism. . . . . 182

5.11 Time history of the quasi-velocity $q_{P, 0,1}^{\langle 1\rangle}$ in the simulation "SI". . . 184

5.12 Time history of the quasi-velocity $q_{P, 3,3}^{\langle 1\rangle}$ in the simulation "SI". . . 184

5.13 Time history of the input torque $u_{1}$ in the simulation "SI". . . . . 185

5.14 Inspection of the error in the numerical solution of the constraint equations in the simulation "SI". . . . . . . . . . . . . . . . 185

5.15 Representation of a 3 RRR parallel mechanism. . . . . . . . . 187

5.16 Time history of the generalized coordinate $q_{\mathrm{N}, 1,3}^{\langle 0\rangle}$ in the simulation "SI". . . . . . . . . . . . . . . . . . . . . . . 189

5.17 Time history of the generalized coordinate $q_{\mathrm{N}, 1,4}^{\langle 0\rangle}$ in the simulation "SI". . . . . . . . . . . . . . . . . . . . . . . . 189

5.18 Time history of the quasi-velocity $q_{R, 0,7}^{\langle 1\rangle}$ in the simulation "SI". . . 190

5.19 Time history of the input torque $u_{1}$ in the simulation "SI". . . . 190

5.20 Time history of the input torque $u_{2}$ in the simulation "SI". . . . . 191 
5.21 Time history of the input torque $u_{3}$ in the simulation "SI". . . . . 191

5.22 Inspection of the error in the numerical solution of the constraint equations in the simulation "SI". . . . . . . . . . . . . . . . 192

5.23 Representation of the initial configuration of the mechanism in the simulation "SI". . . . . . . . . . . . . . . . . . . . . . . 192 


\section{Symbol conventions}

$a, b, \ldots \quad$ Scalars, elements of tuples/column-matrices or indexes

$A, B, \ldots \quad$ Scalars or elements of matrices

$\boldsymbol{a}, \boldsymbol{b}, \ldots \quad$ Tuples/column-matrices

$A, B, \ldots \quad$ Matrices (two dimensional arrays)

$\mathbf{a}, \mathbf{b}, \ldots \quad$ Vectors (first-order tensors)

A, B,$\ldots \quad$ Dyadics and other second-or-higher-order tensors

$a, b, \ldots \quad$ Points

$\mathrm{A}, \mathrm{B}, \ldots \quad$ Coordinate systems

$\mathbb{A}, \mathbb{B}, \ldots \quad$ Sets

$\mathcal{A}, \mathcal{B}, \ldots \quad$ Rigid bodies or reference frames

$\mathscr{A}, \mathscr{B}, \ldots \quad$ Multibody systems or classes

$a, A, b, B, \ldots$ Operators

$\mathrm{a}, \mathrm{A}, \mathrm{b}, \mathrm{B}, \ldots$ Labels 


\section{Introduction}

\subsection{Background and objetives}

A brief analysis on the evolution of Engineering since the second half of the twentieth century reveals that the increasing application of computational tools in design and manufacturing is directly associated to almost every major advance observed. Computational modeling has been responsible for the overcoming of several technological obstacles, expanding the frontiers of knowledge, leading to increasingly complex projects and to the reconception of many design paradigms.

One area of Mechanical Engineering in which the introduction of computational tools has been responsible for a major evolution is Multibody System Dynamics. Involving Rigid Body Dynamics, Structural Mechanics and Continuum Mechanics, this area finds applications for machinery, mechanisms and robotic systems, biomechanical systems, land, aerospace and water vehicles design, including parametric identification and optimization, simulation and experimental validation, virtual reality design, control systems design, and studies of problems of impact, contact, flexibility and multi-physical interaction (SCHIEHLEN, 2007). For all these applications, several software packages have been developed, allowing the computational implementation of the methodologies already developed for the study of Multibody System Dynamics.

However, it is noticeable that there is still a high dependence on proprietary software for applications to Multibody System Dynamics which lead to "blackbox" or "gray-box" computational models in which the user can only control the input data provided but not the modeling and simulation procedures. An evident disadvantage of this fact is that these packages do not allow the user to clearly understand how the modeling and simulation algorithms actually work. This makes it more difficult not only to interpret the obtained models, but also to identify errors in the model. It is also often that some software packages have some limitations that do not allow the choice of definition of some variables, 
parameters, constraints and constitutive equations that might be relevant for obtaing a particular model. Nevertheless, the main disadvantage of this dependence on proprietary software resides in the fact that it is a extremely difficult task to integrate different software packages (from different developers) within a given project. Once a family of software packages is chosen for the preprocessing, modeling, simulation and post-processing, it is sometimes not feasible to use input and output data from these models in other software packages (SCHIEHLEN, 1997). This represents a great obstacle to advances in Multibody System Dynamics, not only to validating results but also to using them in collaborative researches in which different software packages are adopted. A third obstacle, however, occurs in the modeling of complex multibody systems. In general, these systems can be conceived as a set of constrained subsystems, which can be quite different in terms of topology. At least in theory, various software packages and modeling methodologies could be applied to obtain the models of the subsystems, which could be used in the derivation of the equations of motion of the system. This scenario, in which the modeling of each subsystem could be optimized by choosing the most appropriate methodology for each case, seems not to be feasible due to this dependence on proprietary software.

On the other hand, it can be noticed that these software packages are based on implementations of modeling methodologies based either on NewtonEuler or Analytical Mechanics formalisms. As will be discussed latter in this text, each of these formalisms may be the optimal one for a particular application. Thus, it might be possible to make a contribution to Multibody System Dynamics that simultaneously reduce the dependence on proprietary software and explore the modular modeling of multibody systems, bringing a new perspective to this area of knowledge.

The main objective of this work is to unify the contributions of the conventional Classical Mechanics formalisms, developing a modeling methodology, applicable to any multibody systems with a finite number of degrees of freedom, that must:

(1) Aid the systematization and algorithmization of the modeling procedures, leading to accessible computational implementations (that can be performed using general-purpose open source software and programming languages, preferably).

(2) Work modularly, taking as input data the already known mathematical 
models of the subsystems and a description of the constraints among these subsystems.

(3) Be recursive, allowing its application to systems that can be conceived as a hierarchical structure of subsystems (in which the complexity increases from one level to the following).

Basically, the recursive modular methodology presented in this thesis proposes a more straightforward conception of the modeling approach as illustrated in Figure 1.1. In order to reach this goal, it is necessary that the novel methodology does not present any restriction concerning the choice of model variables and the nature of forces and motion constraints involved. Therefore, before proposing contributions to the formulation of equations of motion, it is first necessary to generalize the procedures for describing motions and constraints of multibody systems, independently of the number or type of variables chosen and of the nature of the constraints involved ${ }^{1}$. After that, a critical literature review on the main contributions of each Classical Mechanics formalism is performed and from it a general matrix form for the equations of motion of a multibody system is proposed and the novel modeling methodology is developed.

This text also intends to promote a discussion on the possibilities of using this novel methodology for the development of new modeling algorithm for multibody systems, using general-purpose software, programming languages and its libraries. The intention is that future applications of this thesis could be developed as open-source packages. However, it is not in the scope of this text the development of computational packages that explore the full potential of the novel methodology. All the packages and algorithms introduced in this text are merely illustrative.

Another important objective of this thesis is to ensure that the novel methodology can somehow be used for an improvement of teaching of Multibody System Dynamics at undergraduate and graduate levels. To achieve this goal, it is necessary to systematize the methodology in such a way that it can be presented as a step by step procedure, without restricting its scope of application, however. The wide range of areas of knowledge to which this novel methodology is applicable, along with the fact that it unifies the main contributions of

\footnotetext{
${ }^{1}$ Actually it is assumed that any constraint in a given system must be describable in terms of invariants expressed in terms of motion variables. This fact might impede the application of the methodology proposed in this text, in its current state, to some problems involving impact and contact.
} 


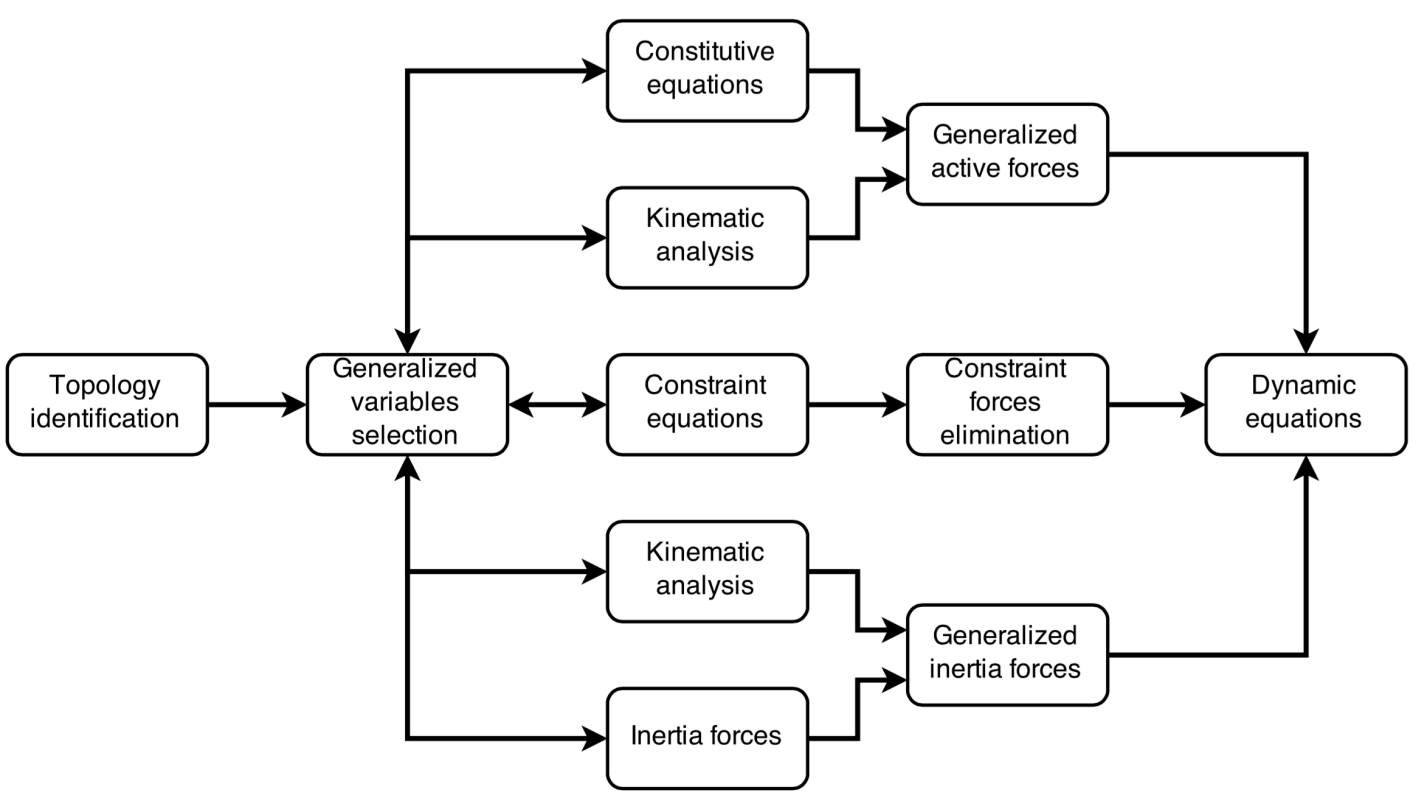

(a) Conventional modeling approaches.

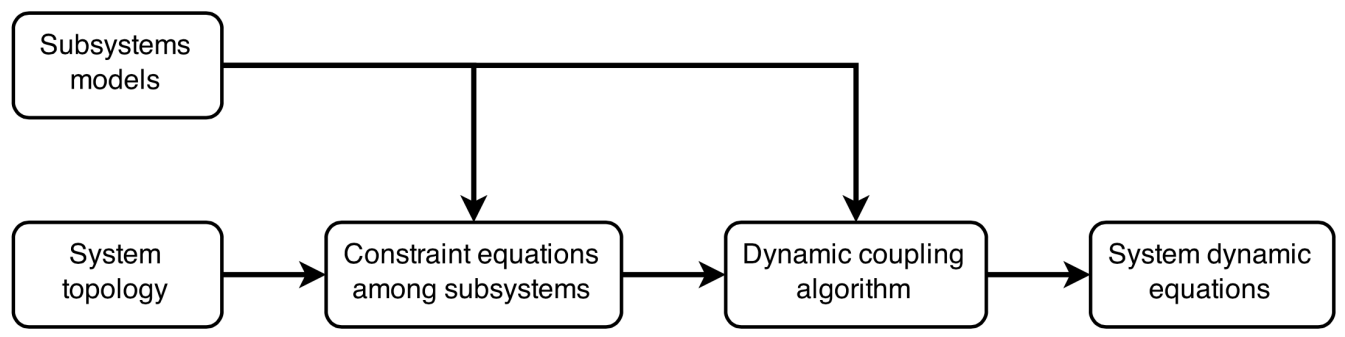

(b) Novel modular modeling methodology.

Figure 1.1: Comparison between conventional modeling approaches and the proposed modular modeling methodology. Reproduced from Orsino e Hess-Coelho (2015).

the main Classical Mechanics formalisms, makes it possible to explore its use in both theoretical and applied courses on Multibody System Dynamics.

\subsection{Research topics and publications}

The candidate's research on Multibody System Dynamics focused on two main areas of application: the study of parallel mechanisms and of wheeled single-track vehicles.

The candidate's studies on the former area started during his undergraduate research, whose objective was the formulation of a mathematical model for the study of the effects of friction and flexibility in the dynamics of a parallel asymmetric mechanism with a novel architecture. The techniques developed for the modeling and simulation of the $2 \underline{R S S}+\underline{P P a P}$ (see Figure 1.2) mecha- 
nism led to the development of some new algorithms that are applicable to the study of generic parallel mechanism. A discussion on how these techniques might be applied for deriving mathematical models of these systems led to the publication of the paper "A contribution for developing more efficient dynamic modeling algorithms of parallel robots" published in the International Journal of Mechanisms and Robotic Systems (ORSINO; HESS-COELHO, 2013), in which a modeling methodology for parallel mechanisms based on Kane's method is presented (see Figure 1.3) and applied to the $2 \underline{R S S+P P a P}$ mechanism.
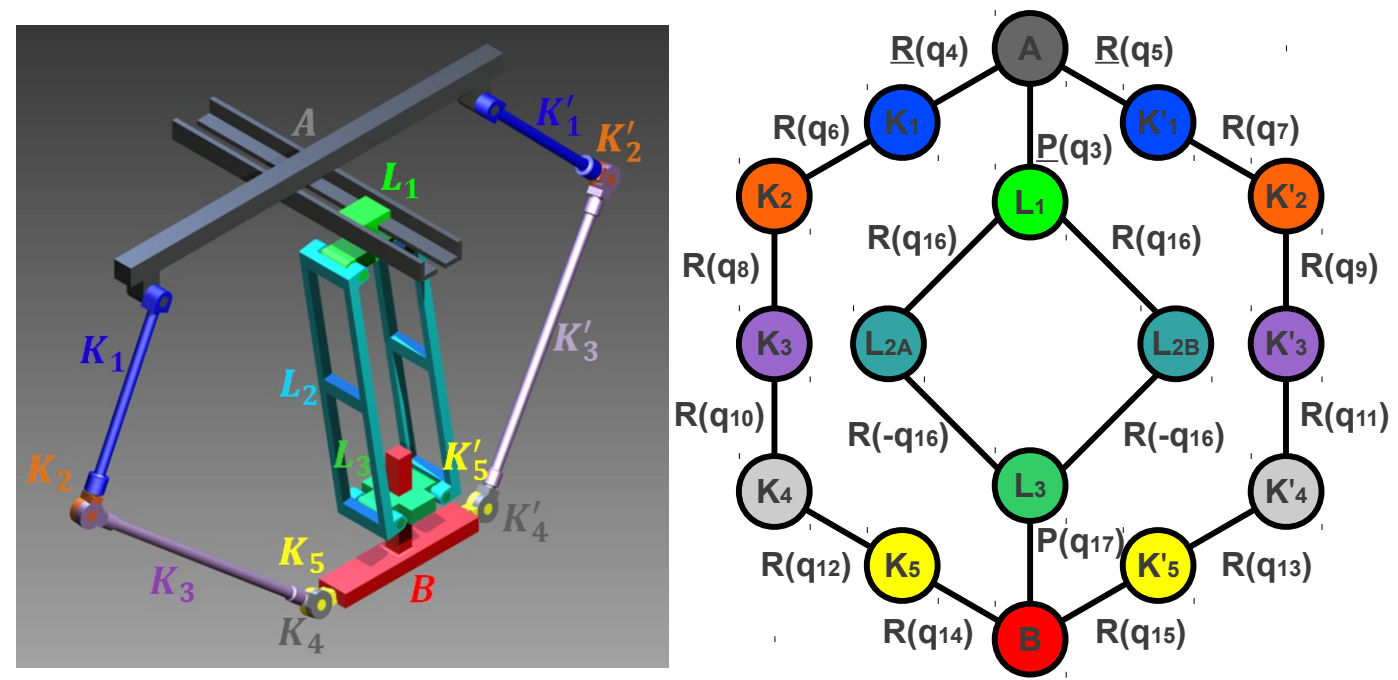

Figure 1.2: 2 RSS+PPaP mechanism. Reproduced from Orsino and Hess-Coelho (2013).

Continuing this research on parallel mechanisms, alternative modeling and simulation methodologies based on other conventional approaches (NewtonEuler, Principle of Virtual Work, Lagrange's and Maggi's equations) were developed. Inevitably, the development of such a variety of methodologies led to the necessity to draw a comparison among them. The paper entitled "Analytical mechanics approaches in the dynamic modeling of Delta mechanism", published in Robotica (ORSINO; HESS-COELHO; PESCE, 2015), takes the Delta parallel mechanism (see Figure 1.4) as a benchmark for comparing four modeling methodologies, based on the Principle Virtual Work, Lagrange's, Maggi's and Kane's equations.

After some implementations of these methodologies for different types of mechanisms and for some similar mechanical systems, three conclusions could be drawn:

(1) With minor modifications the methodologies could be adapted for applications to almost any type of multibody system. 


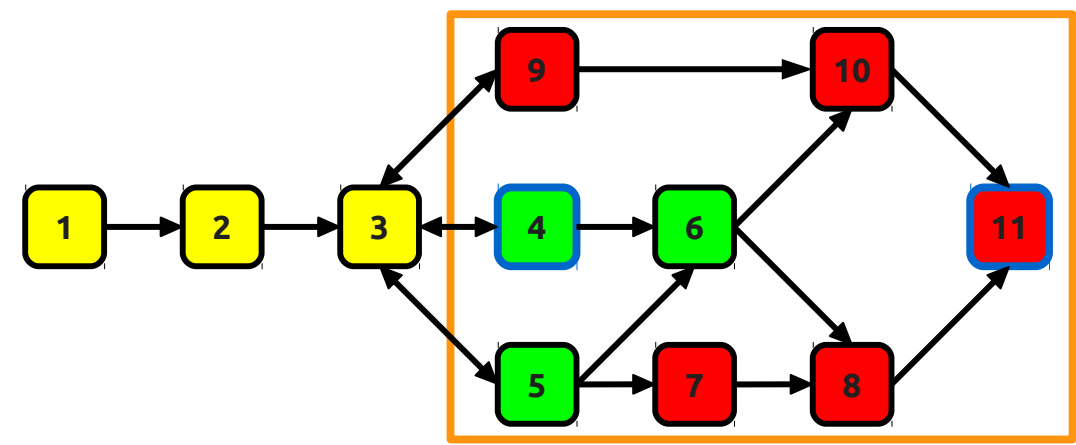

Computer aided stages

\begin{tabular}{cll}
\hline Step & Description & Group \\
\hline 1 & Chains and links identification & Topology \\
2 & Mobility analysis & \\
3 & Coordinates and generalized speeds definition & \\
\hline 4 & Constraint equations & Kinematics \\
5 & Velocities, angular velocities and its derivatives & \\
6 & Partial velocities and partial angular velocities & \\
\hline 7 & Inertia forces and inertia torques & Dynamics \\
8 & Generalized inertia forces & \\
9 & External forces/torques and additional effects & \\
& (gravity, friction, elasticity) & \\
10 & Generalized active forces & \\
\hline
\end{tabular}

Figure 1.3: Modeling methodology for a parallel mechanism based on Kane's method. Reproduced from Orsino and Hess-Coelho (2013).

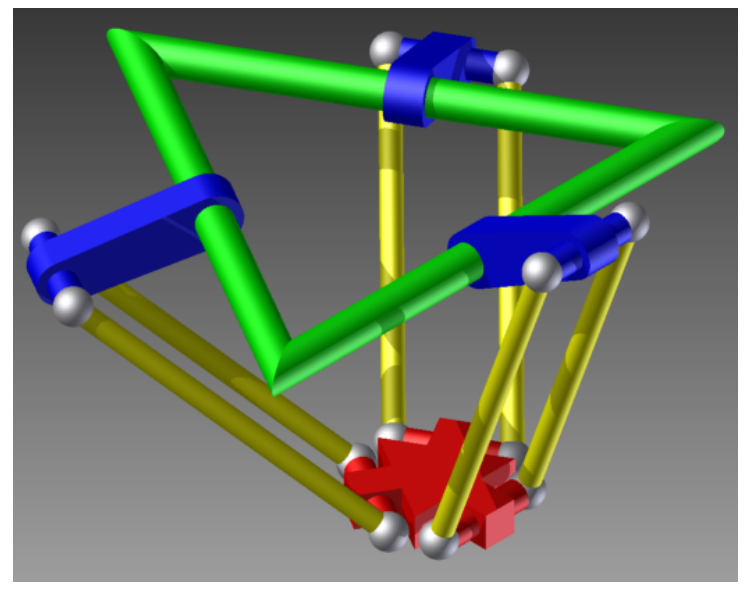

Figure 1.4: Delta parallel mechanism. Reproduced from Orsino, Hess-Coelho and Pesce (2015).

(2) For different types of systems, different methodologies have better performance for the derivations of the mathematical models. 
(3) Even in different multibody systems, some steps of modeling might be identical once these systems might have similar subsystems.

Taking these facts in considerations, the following research stage was to develop a unifying modular modeling methodology that could be applicable to generic multibody systems. The first development of such a methodology was presented in the paper entitled "A contribution on the modular modeling of multibody systems", published in Proceedings of the Royal Society A (ORSINO; HESSCOELHO, 2015). In this text an extended and recursive version of this methodology is developed.

Still on the area of parallel mechanisms, the candidate explored the use of the novel modular modeling methodology for the problem of mechanism (adaptive) balancing. This led to the publication of a book chapter entitled "Dynamic Modelling and Control of Balanced Parallel Mechanisms" (ORSINO; COUTINHO; HESS-COELHO, 2016) in the book "Dynamic Balancing of Mechanisms and Synthesizing of Parallel Robots", published by Springer.

The research on the area of wheeled single-track vehicles was mainly developed during a Split Fellowship Program, was developed at the BioMechanical Engineering Department of Delft University of Technology ${ }^{2}$, under the supervision of Professor Arend L. Schwab, Two case studies were explored during this research. The main one, was the development of an extended linearized Whipple bicycle models that can be applied to study the influence of design parameters on the wobble modes of this vehicle. In these extended models the effects of the flexibility of the bicycle frame and of the rider's body were considered along with linear side-slip-tyre models. The comparison of these models with the conventional Whipple model led to several stability analysis that could determine which of the main design parameters have more influence on the dynamic behavior of the wobble modes. This case study might lead to the publication of another paper soon. The other one, was the development of the linearized model of a tadpole tricycle in order to compare its dynamic behavior with a conventional bicycle. In order to derive this model, a package of functions for Wolfram Mathamatica 10.2, called MoSsPack, that implements the recursive modular modeling methodology presented in this text was developed. This case study along with a brief introduction to MoSsPack are presented in Chapter 5.

\footnotetext{
2 Delft, South Holland, The Netherlands.
} 


\subsection{Outline of the main topics}

This thesis is organized in six chapters, the first being the introductory one and the last reserved for the final conclusions and future work perspectives, and one appendix. The content of this text can be divided in three main topics: description of motion (Chapter 2), methodologies for deriving of equations of motion (Chapters 3 and 4 and Appendix A) and implementation of computational packages for modeling (Chapter 5 ) of multibody systems.

Chapter 2 is devoted to kinematics of multibody systems. It starts with a literature review in which a new unifying notation is introduced, in order to comprise all possible alternatives for the description of motion of multibody systems. Even unusual constraints whose order are higher than 1, like nonmaterial programmed constraints (JARZEBOWSKA, 2002; JARZEBOWSKA, 2008; JARZEBOWSKA, 2012) and unconventional parametrizations of motion variables in which quasi-accelerations, quasi-jerks, etc. are not trivially defined (i.e. they can be other variables than merely time derivatives of the quasi-velocities) are included. The only restriction that must be assumed for the statements and results presented in Chapter 2 is that they only apply to systems with a finite number of degrees of freedom. In the two last sections of this chapter, one of the most important contributions of this thesis is presented: a modular and recursive algorithm for obtaining an orthogonal complement of the Jacobian of the invariants required to describe the constraints in a multibody system. This algorithm is the basis for the novel recursive modular modeling methodology presented in this thesis.

Chapter 3 is a brief but comprehensive literature review on the conventional formalisms and techniques for deriving equations of motion for multibody systems. The notation in this chapter do not always coincide with the conventions usually adopted for the formalisms described, once it is adapted to coincide with the new notation adopted in Chapter 2.

In Chapter 4 the main contribution of this thesis is presented: the recursive modular modeling methodology for multibody systems. The first two sections of the chapter are devoted to the demonstration and description of the methodology (detailing all possible cases). Two other sections discuss sufficient conditions for the solution of forward and inverse dynamics problems associated to a system using models derived according to the procedures described in the chapter. Another section discusses the modifications that must be performed 
in the recursive modular modeling methodology in order to be able to derive linearized equations of motion (without performing the complete derivation of the non-linear ones). Along this five sections some didactic examples are discussed in order to illustrate, in detail, for the reader the step by step application of the proposed methodology. The last section of Chapter 4 is devoted to a qualitative comparison among the conventional modeling methodologies and the one proposed in this text. It aims to express an assessment of these methods based on the personal experience of the author in applying them to his own research topics (briefly introduced in the previous section). The content of Chapter 4 is complemented by Appendix $A$, in which some techniques for using energy-like functions in the derivation of the equations of motion of a multibody system are presented.

Finally, Chapter 5 is dedicated to illustrate the wide possibilities of using the novel methodology for the development of both general-purpose and specialized computational algorithms for the modeling of multibody systems. Two packages for Wolfram Mathematica 10.2 developed during the candidate's research are introduced and some case studies are used to illustrate the applications of these packages, including the tadpole tricycle mentioned in the previous section.

\subsection{Notation}

In this text, several mathematical alphabets (font styles) are used. Each of them has an specific application, as shown in the "Symbol conventions". It is worth noting that:

- In this text, it is assumed that tuples and column-matrices are equivalent representations of a multidimensional real coordinate space element. Denoting by $\mathbb{R}^{n}$ the real coordinate space of $n$ dimensions, if $x \in \mathbb{R}^{n}$, then it can be represented as a tuple of $n$ real entries or by a column-matrix with $n$ rows. Whenever matrix operations are involved in a particular context, the column-matrix representation of these elements must be assumed.

-When meaningful, column-matrices might have its rows labeled as well as matrices might have both rows and columns labeled. A label might 
refer to a sigle row (column) or to a set of them. For example:

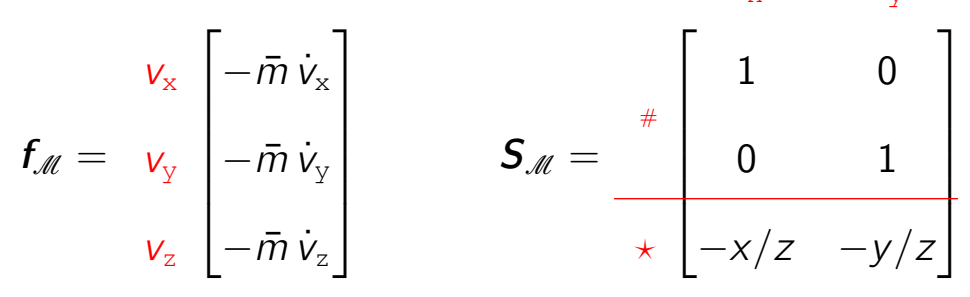

The labels can also be used to define submatrices (subtuples) and to denote specific elements of matrices (tuples). For instance:

(a) $S_{\mathscr{M} \text {.\# }}$ is the submatrix defined by the rows of $S_{\mathscr{M}}$ labeled by the symbol \#, which is an identity matrix.

(b) $S_{\mathscr{M} \ldots v_{x}}$ is the submatrix defined by the column of $S_{\mathscr{M}}$ labeled by $v_{\mathrm{x}}$.

(c) $S_{M . \# . v_{x}}$ is the submatrix defined by the rows of $S_{M}$ labeled by the symbol \# and by the column of labeled by $v_{x}$.

(d) $S_{M . * . v_{y}}=-y / z$.

(e) $f_{\mu_{1} \cdot v_{z}}=-\bar{m} \dot{v}_{z}$.

- Tuples of tuples are equivalent to a single tuple constituted by the respective elements in the same sequence. For instance, $\left(t,\left(p_{1}, p_{2}, p_{3}\right),\left(q_{1}, q_{2}\right)\right)=\left(t, p_{1}, p_{2}, p_{3}, q_{1}, q_{2}\right)$. Tuples of matrices must be understood as block-matrices assembled according to its row and column labels (see Definition 2.9).

- Overbar notation (e.g. $\overline{\boldsymbol{q}}_{\mathscr{U}}, \bar{a}$ ) is used to indicate that the corresponding symbol represents a constant or an invariant.

- Underbar notation (e.g. $\underline{\mathbf{r}}, \underline{\dot{q}}$ ) is used to indicate that the corresponding symbol denotes a function, not a variable.

- Overhat notation (e.g. $\hat{\gamma}_{K}$ ) is used to indicate that the corresponding symbol is an nondimensional quantity. In the case of vector quantities, it also indicates that the vector is a unity vector (e.g. $\hat{\mathbf{n}}_{\mathrm{x}}$ ).

- Superscripts used inside angle brackets $\langle\cdot\rangle$ or double angle brackets $\langle\langle\cdot\rangle$. refer the so-called order of the corresponding variables. Order 0 variables are related to the description of the configurations of a multibody system, order 1 to the first time derivatives of these configuration quantities, order 2 to the second time derivatives and so on. Basically, the 
angle brackets $\langle\cdot\rangle$ notation is used to denote a set of variables of a particular order. For example, $\boldsymbol{q}^{\langle\alpha\rangle}$ denotes a set of $\alpha$-th order variables. The double angle brackets $\langle\cdot\rangle\rangle$ corresponds to a shorthand notation that refers to a tuple constituted by all variables of a certain kind from 0th order up to the order indicated inside these brackets. For example, $\boldsymbol{q}^{\langle\beta\rangle}=\left(\boldsymbol{q}^{\langle 0\rangle}, \ldots, \boldsymbol{q}^{\langle\alpha\rangle}, \ldots, \boldsymbol{q}^{\langle\beta\rangle}\right), 0 \leq \alpha \leq \beta$. For further details, see Section 2.2.

- $r_{p \mid o}$ denotes the position vector of point $p$ with respect to point o (i.e. the vector defined by the directed line segment from $\circ$ to $p$ ).

- $\mathrm{v}_{\mathrm{p} \mid \varepsilon}$ denotes the velocity vector of a point $\mathrm{p}$ measured with respect to the reference frame $\varepsilon$.

- $\omega_{\mathcal{B} \mid \mathcal{E}}$ denotes the angular velocity vector of the rigid body (or reference frame) $\mathcal{B}$ with respect to the reference frame $\mathcal{E}$.

- $[\mathrm{x}]_{\mathrm{S}}$ denotes the column-matrix representing the coordinates of the vector $\mathrm{x}$ with respect to the coordinate system $\mathrm{S}$.

- $[T]_{S \mid R}$ denotes the matrix representing the dyadic $T$ with respect to the coordinate systems $R$ and $S$ (i.e. if $\mathbf{y}=\mathbf{T} \cdot \mathbf{x}$, then $[\mathrm{y}]_{S}=[\mathbf{T}]_{S \mid R}[\mathbf{x}]_{R}$ ).

- 1 denotes the unit dyadic, i.e. if $\mathrm{x}$ is an arbitrary vector, then $1 \cdot \mathrm{x}=\mathrm{x}$. $[1]_{\mathrm{S} \mid \mathrm{R}}$ represents the transformation matrix describing the change of basis between the coordinate systems $\mathrm{R}$ and $\mathrm{S}$, i.e. if $\mathrm{x}$ is an arbitrary vector, then $[\mathrm{x}]_{\mathrm{S}}=[1]_{\mathrm{S} \mid \mathrm{R}}[\mathrm{x}]_{\mathrm{R}}$. Note that $[1]_{\mathrm{R} \mid \mathrm{R}}=[1]_{\mathrm{S} \mid \mathrm{S}}=1$, with 1 denoting the identity matrix.

- $c_{\theta}$ and $s_{\theta}$ are used as shorthand notations for $\cos (\theta)$ a and $\sin (\theta)$, respectively. 


\section{On the description of motion of multibody systems}

To pursue the objective of proposing a new unifying modeling methodology that is able to use already known models of subsystems to derive the equations of motion of multibody systems, not being relevant the formalisms that led to these models, it is necessary to be able to describe the corresponding motions as generally as possible. It must be stated, however, that the concept of motion is underlain by even more fundamental notions:

... classical physics implies a certain basic descriptive order and measure. This may be characterized as the use of certain Cartesian coordinates and by the notion of universal and absolute order of time, independent of that of space. This further implies the absolute character of what may be called Euclidean order and measure (i.e., that characteristic of Euclidean geometry). With this order and measure, certain structures are possible. In essence, these are based on the quasi-rigid body, considered as a constituent element. The general characteristic of classical structure is just the analysability of everything into separate parts, which are either small, quasi-rigid bodies, or their ultimate idealization as extensionless particles. As pointed out earlier, these parts are considered to be working together in interaction (as in a machine). (BOHM, 2005, p. 153)

According to the Classical Physics point of view, any motion in a multibody system can be ultimatelly described as the motion of interacting rigid bodies and particles in an Euclidean space. The concept of rigid body plays a fundamental role in both Classical and Analytical Mechanics once it not only can be conceived as the basic constituent element of a mechanical system, but also because it can be used to define the concept of reference frame:

A reference frame will be defined here as a set of non-colinear points, with distances from each other invariant with time. A rigid 
body can be taken as a reference frame, since it fully meets the conditions that define the latter. In practice, the concepts of rigid body and reference frame will be used alternatively. A plane can be adopted as a reference frame, for the same reason. A line or point, however, does not constitute a reference frame, since it does not fulfill the conditions established in the above definition. (TENENBAUM, 2004, p. 92)

The objective of this chapter is to propose a general approach for interpreting all types of descriptions of motion normally used in the modeling of multibody systems and to discuss how to obtain, from those descriptions, relevant information for the modular modeling of such systems. The results presented in this chapter apply to any mechanical system for which the ability to track the motions of a finite number of points is enough to provide all the information needed about all possible motions it is able to perform. In other words, all the statements in the following sections apply to any multibody system with a finite number of degrees of freedom.

This chapter involves both a literature review on the main concepts of kinematics of multibody systems and some novel contributions, one of them being a new notation introduced for the development of the proposed methodology. Section 2.1 discusses the sufficient conditions for describing the motion of a multibody system, including the concepts of generalized coordinates and constraints (along with their classifications). Section 2.2 introduces the concept of generalized variables along with the corresponding notations to be used along this text. Also, there is a brief discussion on the sufficient conditions for defining new sets of generalized variables, in replacement of the trivially defined ones. Section 2.3 introduces the concepts of generalized constraint invariants and of constraint order of a multibody system. Based on them the definitions of generalized configurations, singular configurations and of number of degrees of freedom of a multibody system are revisited and reformulated in order to be compatible both with the conventional concepts and with the novel approach. Section 2.4 establishes a fundamental theorem for the variations of motion variables concerning their compatility with the constraints of a multibody system. Finally, Section 2.5 presents the central contribution of this chapter, which is a modular and recursive algorithm for applying the result presented in the previous section for a multibody system that can be conceived as a hierarchical structure of subsystems representable by a tree diagram. This algorithm is the basis of the modular modelling methodology developed in this thesis. 


\subsection{Sufficient conditions for describing the mo- tion of a multibody system}

Let $\mathscr{M}$ denote a multibody system for which tracking the motion of a finite number of points is enough to describe any possible motion that it is able to perform. Let $\varepsilon$ be a reference frame and $\mathrm{E}$ be a coordinate system rigidly attached to $\mathcal{E}$. Any motion of a point of $\mathscr{M}$ with respect to $\mathcal{E}$ can be analytically described by time histories of its Cartesian, homogeneous, cylindrical, spherical, etc. coordinates in the system E. Such kind of coordinates will generally be referred in this text as spatial coordinates, in order to avoid nomenclature conflicts.

Obtaining such time histories, however, normally correspond to the final objective of a typical multibody system dynamics analysis. Much more relevant for the modeling of such systems is the so called instantaneous kinematics approach in which the values of the spatial coordinates and its time derivatives up to $\rho$-th order $(\rho \geq 1$ ) are known (or at least can be determined from the available data) at a given time instant $t^{*}$. In this case, it is implicitly assumed that the corresponding time histories are class $\mathscr{C}^{\rho}$ at $t^{*}$. Moreover, it can be stated that in the time interval $\left[t^{*}, t^{*}+\epsilon\right.$ [ the error of the approximation of the actual time history by a $\rho$-th order Taylor polynomial is an infinitesimal ${ }^{1}$ with respect to $\epsilon^{\rho}$.

Although a finite number of spatial coordinates might be enough for the description of the motion of a multibody system, such a choice of variables will hardly ever be the most appropriate to derive the corresponding mathematical model. Indeed, it is always possible to define a finite number of variables to parametrize the descriptions of the spatial coordinates. Such variables, if sufficient to uniquely determine the positions of any point ${ }^{2}$ in a multibody system, are called generalized coordinates. In this text, the tuple (which may be interpreted as a column-matrix when convenient) representing the generalized coordinates of the multibody system $\mathscr{M}$ will be denoted by $\boldsymbol{q}_{\mathscr{M}}^{\langle 0\rangle}$. A particular generalized coordinate in this tuple will be represented by the symbol $q_{\mathscr{M} \text {.i }}^{\langle 0\rangle}$, with $i$ being a generic index. Moreover, when there is no other multibody system

\footnotetext{
${ }^{1}$ Let $x(t)$ and $y(t)$ be two real valued functions of a real variable $t$, both defined in a neighborhood of $t=0: y(t)$ is an infinitesimal with respect to $x(t)$ or, in symbolic notation, $y=o(x)$, if and only if $\lim _{t \rightarrow 0}(y(t) / x(t))=0$. For example, $t^{5 / 2}$ is an infinitesimal with respect to $t^{2}$ at $t=0$ once $t^{5 / 2} / t^{2}=t^{1 / 2}$ and $\lim _{t \rightarrow 0} t^{1 / 2}=0$.

2 Actually, local uniqueness is enough, that is, if there is more than one configuration corresponding to the same values of generalized coordinates, there must be a finite (noninfinitesimal) distance between such configurations.
} 
(than $\mathscr{M}$ ) in a particular context, the subscript $\mathscr{M}$ can be omitted for the sake of simplicity, i.e. the generalized coordinates tuple can simply be denoted by $\boldsymbol{q}^{\langle 0\rangle}$ and a particular coordinate in this tuple by $q_{. i}^{\langle 0\rangle}$.

Let $x$ denote any spatial coordinate of some point in $\mathscr{M}$. If $\boldsymbol{q}^{\langle 0\rangle}$ represents a tuple of generalized coordinates for $\mathscr{M}$, then there must be a function $\underline{x}$ parametrizing $x$ in terms of the time variable $t$ and of the generalized variables $\boldsymbol{q}^{\langle 0\rangle}$, i.e.:

$$
x=\underline{x}\left(t, \boldsymbol{q}^{\langle 0\rangle}\right)
$$

Thus, if the time history of the generalized variables of $\mathscr{M}$ is obtained for some time interval, then, the corresponding time history of $x$ can be given by the expression: $x(t)=\underline{x}\left(t, \boldsymbol{q}^{\langle 0\rangle}(t)\right)$. Moreover, applying the chain rule it can be stated that, if $\underline{x}$ is a class $\mathscr{C}^{\rho}$ function, than there must be a function $\underline{x}^{(\rho)}$ such that the $\rho$-th time derivative of $x$ can be parametrized as a function of time, of the generalized coordinates and its time derivatives up to $\rho$-th order, i.e.:

$$
\frac{\mathrm{d}^{\rho} x}{\mathrm{~d} t^{\rho}}=\underline{x}^{(\rho)}\left(t, \boldsymbol{q}^{\langle 0\rangle}, \ldots, \frac{\mathrm{d}^{\alpha} \boldsymbol{q}^{\langle 0\rangle}}{\mathrm{d} t^{\alpha}}, \ldots, \frac{\mathrm{d}^{\rho} \boldsymbol{q}^{\langle 0\rangle}}{\mathrm{d} t^{\rho}}\right)
$$

Typically, the criteria used for choosing the generalized coordinates of a system take into consideration the existing motion constraints, which often lead to the selection of a minimal set of variables for a parametric description of its possible motions. For example, in a simple pendulum, which is a system consisting of a single particle (extensionless body) constrained to move in a circumference, the specification of a single angular coordinate $\theta$ measured along such circumference is sufficient to describe any possible configuration of this system uniquely. Moreover, such a coordinate (as well as its time derivatives) can assume any value without violating any constraint of the pendulum, corresponding to a minimal parametric description for it. Consider now a spherical pendulum, which is a system consisting of a single particle constrained to move in a spherical surface. In this case, two angular coordinates, e.g. latitude and longitude, are sufficient to describe any possible configuration of this system uniquely, corresponding to a minimal parametric description for it.

However, it is not always practical to adopt a minimal description for the configurations of a multibody system in terms of generalized coordinates. Consider, for instance, the 4-bar mechanism shown in Figure 2.1. This system is constituted by 4 rigid planar bars linked by ideal revolute joints at their edges. Let $\mathcal{E}$ be a reference frame and suppose that the bar ab remains fixed with 
respect to $\mathcal{E}$.

a

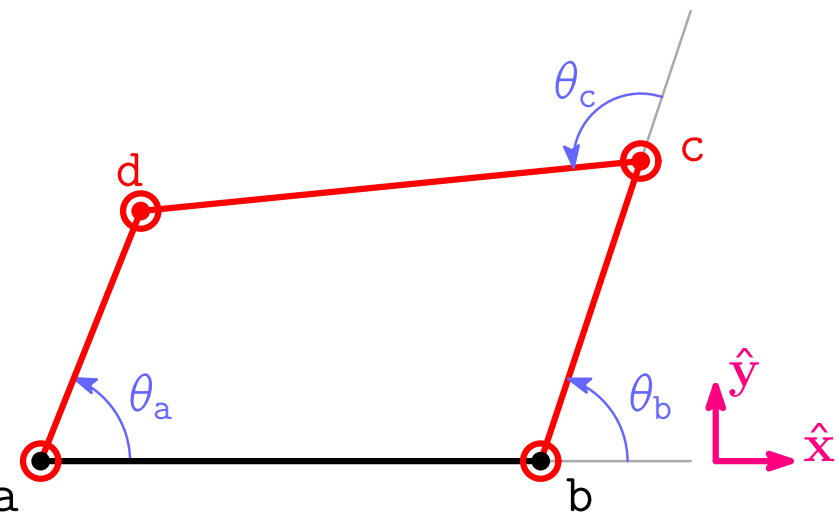

Figure 2.1: Representation of a 4-bar mechanism. Adapted from Orsino and Hess-Coelho (2015).

Noting that abcd is a quadrilateral in which the lengths of the sides remain constant, in order to uniquely determine any configuration of this mechanism with respect to the reference frame $\mathcal{E}$, it is sufficient to specify a single internal or external angle. Choosing only one angle among $\theta_{\mathrm{a}}, \theta_{\mathrm{b}}$ and $\theta_{\mathrm{c}}$, for example (see Figure 2.1), would be enough. Yet, if one tries to obtain the Cartesian coordinates of $c$ as a function of $\theta_{\mathrm{a}}$ only, for instance, it will lead to cumbersome expressions. Thus, although it is possible to obtain a mathematical model of the 4-bar mechanism using a single generalized coordinate, this is not convenient. It would be much more practical to use $\theta_{\mathrm{a}}, \theta_{\mathrm{b}}$ and $\theta_{\mathrm{c}}$ simultaneously (ORSINO; HESS-COELHO, 2015). Such a scenario, however, corresponds to the use of redundant (generalized) coordinates, i.e. coordinates whose values can not be set arbitrarily without leading to some configurations that are not compatible with the constraints of the system. In this particular example, once the value of one of the angles is given, the values of the other two can be uniquely determined. Thus, whenever redundant coordinates are used in the modeling of a multibody system, its motion constraints will lead to invariants of the form:

$$
\bar{h}_{. k}^{\langle 0\rangle}\left(t, \boldsymbol{q}^{\langle 0\rangle}\right)=0
$$

Such invariants do not only apply only to a particular configuration of a system but for all the possible configurations it might have. Any time history $\boldsymbol{q}^{\langle 0\rangle}(t)$ compatible with the motion constraints of a system must identically satisfy these invariants. This result also extends to the instantaneous kinematics approach, i.e. equations (2.3) does not only impose restrictions to the possible values of the generalized coordinates but also to its time derivatives.

However, not all constraints in a multibody system will be satisfied if minimal sets of generalized coordinates are chosen or if invariants of the form (2.3) 


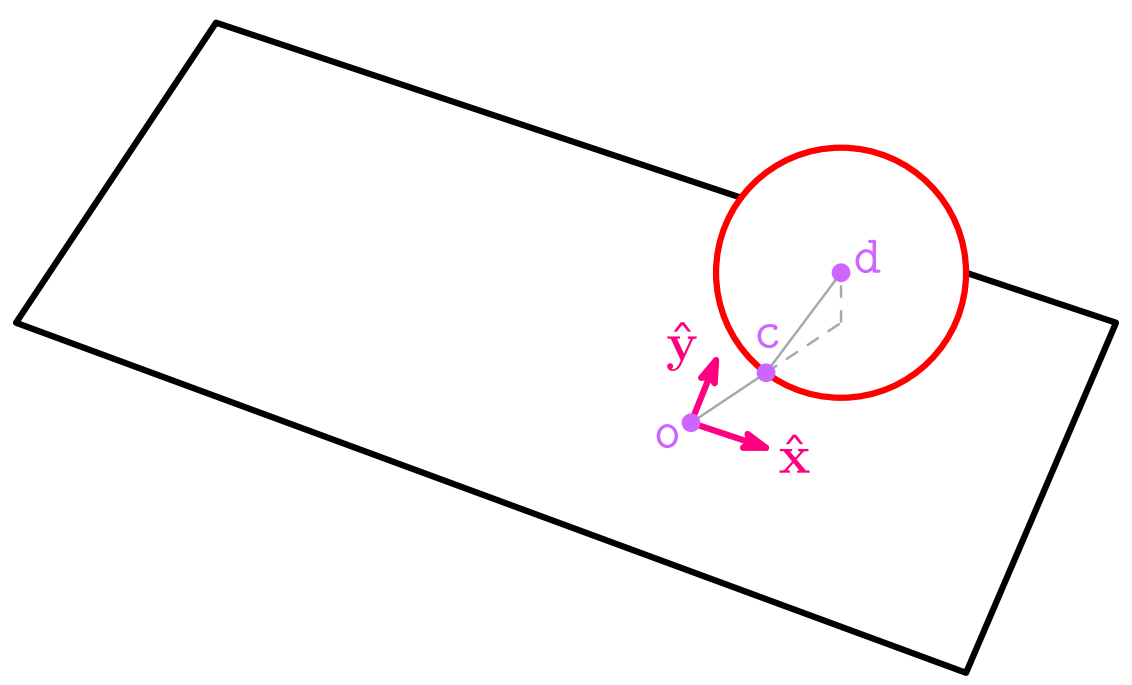

Figure 2.2: Representation of a knife-edge disc rolling in a plane. Adapted from Orsino and Hess-Coelho (2015).

are enforced. Consider, for example, the system represented in Figure 2.2, consisting of an ideal knife-edge disc rolling in a plane. Suppose that this system satisfies the no-slip constraint: points of two distinct bodies must have the same velocity (zero relative velocity) when in contact. Let $\mathcal{E}$ be a reference frame rigidly attached to the plane and $E=(0, \hat{\mathbf{x}}, \hat{\mathbf{y}}, \hat{\mathbf{z}})$ be a coordinate system in $\mathcal{E}$ such that $o$ is a point in the plane and $\hat{x}$ and $\hat{\mathbf{y}}$ are parallel to this plane. Once there always must be a point $c$ of the disc in instantaneous contact with the plane, the velocity of this point with respect to $\mathcal{E}$ must be zero at this correponding instant. Let the coordinates of $c$ in $E$ be $[c]_{E}=\left(x_{c}, y_{c}, z_{c}\right)$. Due to the contact between the disc and the plane, it can be stated that $z_{\mathrm{c}}=0$. The coordinates $x_{c}$ and $y_{c}$ can assume any value without violating any constraint of the system. Their rates, however, must satisfy some conditions in order not to violate the no-slip constraint. Thus, invariants expressing the no-slip constraint can not be expressed as functions of the generalized coordinates and time only; they must involve some of the time derivatives of the generalized constraints.

In general, enforcing kinematic constraints in a multibody system will involve the satisfaction of equations or inequalities of the following forms:

$$
\begin{aligned}
& \bar{h}_{. k}^{\langle\rho\rangle}\left(t, \boldsymbol{q}^{\langle 0\rangle}, \ldots, \frac{\mathrm{d}^{\alpha} \boldsymbol{q}^{\langle 0\rangle}}{\mathrm{d} t^{\alpha}}, \ldots, \frac{\mathrm{d}^{\rho} \boldsymbol{q}^{\langle 0\rangle}}{\mathrm{d} t^{\rho}}\right)=0 \\
& \check{h}_{. k}^{\langle\rho\rangle}\left(t, \boldsymbol{q}^{\langle 0\rangle}, \ldots, \frac{\mathrm{d}^{\alpha} \boldsymbol{q}^{\langle 0\rangle}}{\mathrm{d} t^{\alpha}}, \ldots, \frac{\mathrm{d}^{\rho} \boldsymbol{q}^{\langle 0\rangle}}{\mathrm{d} t^{\rho}}\right) \geq 0
\end{aligned}
$$

Constraints that can not be represented by equations, i.e. that do not correspond to invariants, are out of the scope of this text. 
Definition 2.1. A constraint is classified as holonomic either when it is automatically satisfied by an adequate choice of generalized coordinates or when it corresponds to invariants that can be expressed in the form presented in equation (2.3). When a holonomic constraint does not involve time $(t)$ as an explicit variable, it is further classified as scleronomic; whenever it does, the constraint is said to be rheonomic (GOLDSTEIN; POOLE JR.; SAFKO, 2002).

A holonomic system is a multibody system in which all the constraints are holonomic being the denomination nonholonomic system reserved for any system in which at least one constraint is not holonomic.

Among the nonholonomic constraints, the so called simple nonholonomic constraints (KANE; LEVINSON, 1985) stand out: such constraints can be expressed by invariants which are affine functions with respect to the time derivatives of the generalized coordinates. A simple nonholonomic system is any system in which all the constraints are either holonomic or simple nonholonomic (existing at least one simple nonholonomic constraint).

Proposition 2.2. An isolated tridimensional rigid body is a holonomic system. The positions of three noncollinear points of this body or six generalized coordinates are sufficient to describe any configuration of a rigid body with respect to any reference frame.

Proof. Consider that, without any loss in generality, at a given time instant the configuration of an isolated rigid body $\mathcal{B}$ with respect to an arbitrary reference frame $\mathcal{E}$ is known. At any other time instant, the distance between two points in this body must be exactly the same as it was in that reference configuration. Let $b_{0}$ be a point of $\mathcal{B}$ whose position is tracked. In general, three coordinates are sufficient to describe the position of $b_{0}$ with respect to its reference configuration. In any configuration, any other point $b_{1}$ of $\mathcal{B}$ must lie in a spherical surface centered in $b_{0}$ whose radius is equal to the already known distance between $b_{0}$ and $b_{1}$. Thus, tracking the position of $b_{1}$ too would require only two extra coordinates (to uniquely specify the position of a point in a spherical surface). Suppose that both $b_{0}$ and $b_{1}$ are tracked. Any point in the straight line $b_{0} b_{1}$ can simply be located by the already known distances to $b_{0}$ and $b_{1}$ without requiring any extra coordinate. Let $b_{2}$ be noncollinear to $b_{0}$ and $b_{1}$ : it must remain in the intersection of two spherical surfaces, one centered in $b_{0}$ and the other centered in $b_{1}$, whose radii are the already known distances between $b_{2}$ and each of these points. Such an intersection corresponds to a circumference. Therefore, a single extra coordinate is sufficient to locate $b_{2}$ in this intersection. 
When $b_{0}, b_{1}$ and $b_{2}$ are tracked, $a$ fourth point $b$ will remain in the intersection of three spherical surfaces with known radii centered at each of these three points. Such an intersection corresponds to two points lying in different subspaces between those defined by the plane $b_{0} b_{1} b_{2}$, which means that once the position of $b$ in the reference configuration is known, it is possible to uniquely determine which of these two points is $\mathrm{b}$ in a new configuration, without requiring the definition of any extra coordinate. Finally, once all the constraints of $\mathcal{B}$ can be automatically satisfied by an appropriate choice of a minimal set of generalized coordinates, according to Definition 2.1, it can be classified as a holonomic system.

\subsection{Generalized variables}

As discussed in the previous section, any variable used to describe a configuration of a multibody system can be parametrically described in terms of time and of a set of generalized coordinates adequately chosen to model such system. Consequently, any variable related to the motion of a system (which involves configurations and related rates) can be parametrized in terms of time, generalized coordinates and its time derivatives up to some order. For some systems, however, it might be convenient to adopt reparametrizations, in which the $\alpha$-th time derivatives of the generalized coordinates are replaced by a set of $\alpha$-th order generalized variables. In the literature, first order generalized variables are commonly called quasi-velocities (NEJMARK; FUFAEV, 1972; CAMERON; BOOK, 1997; JARZEBOWSKA, 2009; PAPASTAVRIDIS, 1988), second order generalized variables are called quasi-accelerations (MARUSKIN; BLOCH, 2007; PAPASTAVRIDIS, 1988; UDWADIA; PHOHOMSIRI, 2007a) and third order, are called quasi-jerk (MARUSKIN; BLOCH, 2007). Higher order generalized variables do not receive any special denomination. Note that generalized coordinates can also be referred as 0 -th order generalized variables.

In this text, the tuple (or column-matrix) representing the $\alpha$-th order generalized variables of a multibody system $\mathscr{M}$ will be denoted by $\boldsymbol{q}_{\mathscr{M}}^{\langle\alpha\rangle}$. A particular generalized variable in this tuple will be represented by the symbol $q_{\mathscr{M} . i}^{\langle\alpha\rangle}$, being $i$ a generic index. Again, when there is no other multibody system (than $\mathscr{M}$ ) in a particular context, the subscript $\mathscr{M}$ can be omitted, i.e. the $\alpha$-th order generalized variables tuple can simply be denoted by $\boldsymbol{q}^{\langle\alpha\rangle}$ and a particular variable in this tuple by $q_{. i}^{\langle\alpha\rangle}$. Moreover, the following notations will be adopted: 
- $\boldsymbol{q}^{\langle\beta\rangle}$ represents the tuple (or column-matrix) constituted by all generalized variables up to $\beta$-th order, i.e.:

$$
\boldsymbol{q}^{\langle\langle\beta\rangle}=\left(\boldsymbol{q}^{\langle 0\rangle}, \ldots, \boldsymbol{q}^{\langle\alpha\rangle}, \ldots, \boldsymbol{q}^{\langle\beta\rangle}\right)
$$

- $\boldsymbol{q}^{\langle\beta \mid \alpha\rangle}, \beta>\alpha$, represents an alternative set of $\beta$-th order generalized variables defined as the $(\beta-\alpha)$-th time derivative of $\boldsymbol{q}^{\langle\alpha\rangle}$, i.e. ${ }^{3}$ :

$$
\boldsymbol{q}^{\langle\beta \mid \alpha\rangle}=\frac{\mathrm{d}^{\beta-\alpha} \boldsymbol{q}^{\langle\alpha\rangle}}{\mathrm{d} t^{\beta-\alpha}}
$$

- $\boldsymbol{q}^{\langle\beta \beta+\kappa \mid \beta\rangle}, \kappa>0$, represents the tuple (or column-matrix) constituted by $\boldsymbol{q}^{\langle\beta \beta\rangle}$ and its time derivatives up to $\kappa$-th order, i.e.:

$$
\boldsymbol{q}^{\langle\langle\beta+\kappa \mid \beta\rangle\rangle}=\left(\boldsymbol{q}^{\langle\beta \beta\rangle}, \ldots, \frac{\mathrm{d}^{\kappa} \boldsymbol{q}^{\langle\beta \beta\rangle}}{\mathrm{d} t^{\kappa}}\right)
$$

Let $\nu\left(\boldsymbol{q}_{\mathscr{M}}^{\langle\alpha\rangle}\right)$ denote the number of $\alpha$-th order generalized variables for modeling a multibody system $\mathscr{M}$. Once the $(\beta+1)$-th order generalized variables are defined to replace the time derivatives of the $\beta$-th order variables, such transformation of variables will be properly defined provided a tuple (or column-matrix) $\bar{c}_{\mathscr{M}}^{\langle\mathcal{\beta}+1\rangle}$ constituted by $\nu\left(\overline{\boldsymbol{c}}_{\mathcal{M}}^{\langle\mathcal{\beta}+1\rangle}\right)=\max \left\{\nu\left(\boldsymbol{q}_{\mathscr{M}}^{\langle\mathcal{\beta}+1\rangle}\right), \nu\left(\boldsymbol{q}_{\mathcal{M}}^{\langle\mathcal{\beta}\rangle}\right)\right\}$ invariants as follows:

$$
\bar{c}_{\mathscr{M} \cdot k}^{\langle\mathcal{\beta}+1\rangle}\left(t, \boldsymbol{q}_{\mathcal{M}}^{\langle\beta+1\rangle}, \dot{\boldsymbol{q}}_{\mathcal{M}}^{\langle\beta\rangle}\right)=0
$$

It must be noticed, however, that above some order, it is not convenient to define other generalized variables than the time derivatives of the previous order ones. Indeed, if $\boldsymbol{q}_{\mathscr{M}}^{\langle\beta\rangle}=\boldsymbol{q}_{\mathscr{M}}^{\langle\beta \mid \alpha\rangle}$ for all $\beta>\alpha \geq \sigma$, it can be stated that the model of system $\mathscr{M}$ has trivial generalized variables above $\sigma$-th order. Moreover, if $\sigma=0$, i.e., the generalized coordinates are given and all other generalized variables are defined as $\boldsymbol{q}^{\langle\alpha\rangle}=\boldsymbol{q}^{\langle\alpha \mid 0\rangle}$, then it can be said that the model of system $\mathscr{M}$ has trivial generalized variables.

Example 2.1. Let $\mathscr{B}$ be a mechanical system constituted by a single free rigid body $\mathcal{B}$ whose motion is described with respect to an arbitrary reference frame $\mathcal{E}$. Consider that $\mathrm{E}=\left(\mathrm{e}_{0}, \hat{\mathbf{e}}_{\mathrm{x}}, \hat{\mathbf{e}}_{\mathrm{y}}, \hat{\mathbf{e}}_{z}\right)$ is a coordinate system fixed to $\mathcal{E}$ and that $B=\left(b_{\circ}, \hat{\mathbf{b}}_{x}, \hat{\mathbf{b}}_{y}, \hat{\mathbf{b}}_{z}\right)$ is a coordinate system that moves along with $\mathcal{B}$. Let the transformation between the coordinate systems $\mathrm{B}$ and $\mathrm{E}$ be defined by a unit quaternion $\left(q_{t}, \mathbf{q}\right)$. Define the following generalized coordinates for modeling

\footnotetext{
${ }^{3}$ Whenever useful, it can also be adopted the following convention: $\boldsymbol{q}^{\langle\alpha \mid \alpha\rangle}=\boldsymbol{q}^{\langle\alpha\rangle}$.
} 
$\mathscr{B}:$

$$
\begin{cases}q_{.1}^{\langle 0\rangle}=q_{\mathrm{x}}=\mathbf{q} \cdot \hat{\mathbf{e}}_{\mathrm{x}} & q_{.5}^{\langle 0\rangle}=x=\mathbf{r}_{\mathrm{b}_{\circ} \mid \mathbf{e}_{\circ}} \cdot \hat{\mathbf{e}}_{\mathrm{x}} \\ q_{.2}^{\langle 0\rangle}=q_{\mathrm{y}}=\mathbf{q} \cdot \hat{\mathbf{e}}_{\mathrm{y}} & q_{.6}^{\langle 0\rangle}=y=\mathbf{r}_{\mathrm{b}_{\circ} \mid \mathbf{e}_{\circ}} \cdot \hat{\mathbf{e}}_{\mathrm{y}} \\ q_{.3}^{\langle 0\rangle}=q_{\mathrm{x}}=\mathbf{q} \cdot \hat{\mathbf{e}}_{\mathrm{z}} & q_{.7}^{\langle 0\rangle}=z=\mathbf{r}_{\mathrm{b}_{\mathrm{o}} \mid \mathbf{e}_{\circ}} \cdot \hat{\mathbf{e}}_{\mathrm{z}} \\ q_{.4}^{\langle 0\rangle}=q_{\mathrm{t}} & \end{cases}
$$

Note that once $\left(q_{t}, \mathbf{q}\right)$ is a unit quaternion, the following relation must be satisfied:

$$
q_{\mathrm{t}}^{2}+q_{\mathrm{x}}^{2}+q_{\mathrm{y}}^{2}+q_{\mathrm{z}}^{2}-1=0
$$

Moreover, the matrix defining the transformation of coordinates between $\mathrm{E}$ and $\mathrm{B}$ is given by (BOTTEMA; ROTH, 1979, p. 11):

$$
[\mathbf{1}]_{\mathrm{E} \mid \mathrm{B}}=\left[\begin{array}{ccc}
q_{\mathrm{t}}^{2}+q_{\mathrm{x}}^{2}-q_{\mathrm{y}}^{2}-q_{\mathrm{z}}^{2} & 2\left(q_{\mathrm{x}} q_{\mathrm{y}}-q_{\mathrm{t}} q_{\mathrm{z}}\right) & 2\left(q_{\mathrm{x}} q_{\mathrm{z}}+q_{\mathrm{t}} q_{\mathrm{y}}\right) \\
2\left(q_{\mathrm{x}} q_{\mathrm{y}}+q_{\mathrm{t}} q_{\mathrm{z}}\right) & q_{\mathrm{t}}^{2}-q_{\mathrm{x}}^{2}+q_{\mathrm{y}}^{2}-q_{\mathrm{z}}^{2} & 2\left(q_{\mathrm{y}} q_{\mathrm{z}}-q_{\mathrm{t}} q_{\mathrm{x}}\right) \\
2\left(q_{\mathrm{x}} q_{\mathrm{z}}-q_{\mathrm{t}} q_{\mathrm{y}}\right) & 2\left(q_{\mathrm{y}} q_{\mathrm{z}}+q_{\mathrm{t}} q_{\mathrm{x}}\right) & q_{\mathrm{t}}^{2}-q_{\mathrm{x}}^{2}-q_{\mathrm{y}}^{2}+q_{\mathrm{z}}^{2}
\end{array}\right]
$$

Let $\omega_{\mathcal{B} \mid \mathcal{E}}$ denote the angular velocity of $\mathcal{B}$ with respect to $\mathcal{E}, \mathbf{v}_{\mathrm{b}_{\circ} \mid \mathcal{E}}=\dot{\mathbf{r}}_{\mathrm{b}_{\circ} \mid \mathrm{e}_{\circ}}$ be the velocity of $b_{\circ}$ with respect to $\mathcal{E}$ and define the following quasi-velocities:

$$
\left\{\begin{aligned}
q_{.1}^{\langle 1\rangle}=\omega_{\mathrm{x}}=\omega_{\mathcal{B} \mid \mathcal{E}} \cdot \hat{\mathbf{b}}_{\mathrm{x}} & q_{.4}^{\langle 1\rangle}=v_{\mathrm{x}}=\mathbf{v}_{\mathrm{b}_{\circ} \mid \mathcal{E}} \cdot \hat{\mathbf{e}}_{\mathrm{x}} \\
q_{.2}^{\langle 1\rangle}=\omega_{\mathrm{y}}=\omega_{\mathcal{B} \mid \mathcal{E}} \cdot \hat{\mathbf{b}}_{\mathrm{y}} & q_{.5}^{\langle 1\rangle}=v_{\mathrm{y}}=\mathbf{v}_{\mathrm{b}_{\circ} \mid \mathcal{E}} \cdot \hat{\mathbf{e}}_{\mathrm{y}} \\
q_{.3}^{\langle 1\rangle}=\omega_{\mathrm{z}}=\omega_{\mathcal{B} \mid \mathcal{E}} \cdot \hat{\mathbf{b}}_{\mathrm{z}} & q_{.6}^{\langle 1\rangle}=v_{\mathrm{z}}=\mathbf{v}_{\mathrm{b}_{\circ} \mid \mathcal{E}} \cdot \hat{\mathbf{e}}_{\mathrm{z}}
\end{aligned}\right.
$$

It can be proved that (BOTTEMA; ROTH, 1979, Secs. 2.3 and 2.4):

$$
[\mathbf{1}]_{\mathrm{E} \mid \mathrm{B}}^{\top}[\dot{\mathbf{1}}]_{\mathrm{E} \mid \mathrm{B}}=\left[\begin{array}{ccc}
0 & -\omega_{\mathrm{z}} & \omega_{\mathrm{y}} \\
\omega_{\mathrm{z}} & 0 & -\omega_{\mathrm{x}} \\
-\omega_{\mathrm{y}} & \omega_{\mathrm{x}} & 0
\end{array}\right]
$$

which leads to the following identities:

$$
\left\{\begin{array}{l}
\omega_{\mathrm{x}}=2\left(q_{\mathrm{t}} \dot{q}_{\mathrm{x}}+q_{\mathrm{z}} \dot{q}_{\mathrm{y}}-q_{\mathrm{y}} \dot{q}_{\mathrm{z}}-q_{\mathrm{x}} \dot{q}_{\mathrm{t}}\right) \\
\omega_{\mathrm{y}}=2\left(-q_{\mathrm{z}} \dot{q}_{\mathrm{x}}+q_{\mathrm{t}} \dot{q}_{\mathrm{y}}+q_{\mathrm{x}} \dot{q}_{\mathrm{z}}-q_{\mathrm{y}} \dot{q}_{\mathrm{t}}\right) \\
\omega_{\mathrm{z}}=2\left(q_{\mathrm{y}} \dot{q}_{\mathrm{x}}-q_{\mathrm{x}} \dot{q}_{\mathrm{y}}+q_{\mathrm{t}} \dot{q}_{\mathrm{z}}-q_{\mathrm{z}} \dot{q}_{\mathrm{t}}\right)
\end{array}\right.
$$

In this model seven generalized coordinates and six quasi-velocities are de- 
fined, i.e., $\nu\left(\boldsymbol{q}_{\mathscr{B}}^{\langle 0\rangle}\right)=7$ and $\nu\left(\boldsymbol{q}_{\mathscr{B}}^{\langle 1\rangle}\right)=6$. Suppose that the generalized variables are trivial above first order. There must be $\nu\left(\bar{c}_{\mathscr{B}}^{\langle 1\rangle}\right)=7$ invariants defining the transformation of variables between the time derivatives of the generalized coordinates and the quasi-velocities. Taking the time derivative of equation (b) along with the identities (f), it can be stated that the following invariants define this transformation of variables:

$$
\left\{\begin{array}{l}
\bar{c}_{.1}^{\langle 1\rangle}=q_{\mathrm{x}} \dot{q}_{\mathrm{x}}+q_{\mathrm{y}} \dot{q}_{\mathrm{y}}+q_{\mathrm{z}} \dot{q}_{\mathrm{z}}+q_{\mathrm{t}} \dot{q}_{\mathrm{t}}=0 \\
\bar{c}_{.2}^{\langle 1\rangle}=\omega_{\mathrm{x}}-2\left(q_{\mathrm{t}} \dot{q}_{\mathrm{x}}+q_{\mathrm{z}} \dot{q}_{\mathrm{y}}-q_{\mathrm{y}} \dot{q}_{\mathrm{z}}-q_{\mathrm{x}} \dot{q}_{\mathrm{t}}\right)=0 \\
\bar{c}_{.3}^{\langle 1\rangle}=\omega_{\mathrm{y}}-2\left(-q_{\mathrm{z}} \dot{q}_{\mathrm{x}}+q_{\mathrm{t}} \dot{q}_{\mathrm{y}}+q_{\mathrm{x}} \dot{q}_{\mathrm{z}}-q_{\mathrm{y}} \dot{q}_{\mathrm{t}}\right)=0 \\
\bar{c}_{.4}^{\langle 1\rangle}=\omega_{\mathrm{z}}-2\left(q_{\mathrm{y}} \dot{q}_{\mathrm{x}}-q_{\mathrm{x}} \dot{q}_{\mathrm{y}}+q_{\mathrm{t}} \dot{q}_{\mathrm{z}}-q_{\mathrm{z}} \dot{q}_{\mathrm{t}}\right)=0 \\
\bar{c}_{.5}^{\langle 1\rangle}=v_{\mathrm{x}}-\dot{x}=0 \\
\bar{c}_{.6}^{\langle 1\rangle}=v_{\mathrm{y}}-\dot{y}=0 \\
\bar{c}_{.7}^{\langle 1\rangle}=v_{\mathrm{z}}-\dot{z}=0
\end{array}\right.
$$

After some algebraic manipulations, such equations lead to the following identities:

$$
\left\{\begin{array}{l}
\dot{q}_{\mathrm{x}}=\frac{1}{2} q_{\mathrm{t}} \omega_{\mathrm{x}}-\frac{1}{2} q_{\mathrm{z}} \omega_{\mathrm{y}}+\frac{1}{2} q_{\mathrm{y}} \omega_{\mathrm{z}} \\
\dot{q}_{\mathrm{y}}=\frac{1}{2} q_{\mathrm{z}} \omega_{\mathrm{x}}+\frac{1}{2} q_{\mathrm{t}} \omega_{\mathrm{y}}-\frac{1}{2} q_{\mathrm{x}} \omega_{\mathrm{z}} \\
\dot{q}_{\mathrm{z}}=-\frac{1}{2} q_{\mathrm{y}} \omega_{\mathrm{x}}+\frac{1}{2} q_{\mathrm{x}} \omega_{\mathrm{y}}+\frac{1}{2} q_{\mathrm{t}} \omega_{\mathrm{z}} \\
\dot{q}_{\mathrm{t}}=-\frac{1}{2} q_{\mathrm{x}} \omega_{\mathrm{x}}-\frac{1}{2} q_{\mathrm{y}} \omega_{\mathrm{y}}-\frac{1}{2} q_{\mathrm{z}} \omega_{\mathrm{z}} \\
\dot{x}=v_{\mathrm{x}} \\
\dot{y}=v_{\mathrm{y}} \\
\dot{z}=v_{\mathrm{z}}
\end{array}\right.
$$

Consider, for instance, the motion in which $\omega_{\mathrm{y}}=\bar{\Omega}$ (constant) and all the other quasi-velocities are zero. Adopting as initial conditions all generalized coordinates being zero but $q_{t}=1$, it can be noticed that the solution of the system of 
ordinary differential equations $(h)$ is:

$$
\left\{\begin{array}{l}
x=y=z=0 \\
q_{\mathrm{x}}=q_{\mathrm{z}}=0 \\
q_{\mathrm{y}}=\sin \left(\frac{1}{2} \Omega t\right) \\
q_{\mathrm{t}}=\cos \left(\frac{1}{2} \Omega t\right)
\end{array}\right.
$$

From the definition of quaternion parameters, it can be noticed that these expressions correspond to a pure rotation around the axis through $b_{\circ}$ defined by the unit vector $\hat{\mathbf{e}}_{\mathrm{y}}$. The angle of rotation is given by $\theta=\bar{\Omega} t$, i.e., as expected, the corresponding motion is a rotation with constant angular velocity.

In this example, it could be noticed that it is possible to express $\dot{\boldsymbol{q}}^{\langle 0\rangle}$ explicitly as a function of $\boldsymbol{q}^{\langle 0\rangle}$ and $\boldsymbol{q}^{\langle 1\rangle}$. Orsino and Hess-Coelho (2015) prove (in Lemma 2.1) a generalization of this result. Let $\mathscr{M}$ be a multibody system and let $\bar{c}_{\mathscr{M}}^{\langle\alpha+1\rangle}$ denote tuples (or column-matrices) constituted by invariants describing the transformations of variables between $\dot{\boldsymbol{q}}_{\mathscr{M}}^{\langle\alpha\rangle}$ and $\boldsymbol{q}_{\mathscr{M}}^{\langle\alpha+1\rangle}$. Suppose that all $\bar{c}_{\mathscr{M}}^{\langle\alpha+1\rangle}$ belong to class $\mathscr{C}^{\beta-\alpha}$ for all $\alpha$ such that $0 \leq \alpha \leq \beta$. If all the Jacobians $\left[\partial c_{\mathscr{M}}^{\langle\alpha+1\rangle} / \partial \dot{\boldsymbol{q}}_{\mathscr{M}}^{\langle\alpha\rangle}\right]$, for $0 \leq \alpha \leq \beta$, are full rank matrices for the given values of $\left(t, \boldsymbol{q}_{\mathscr{M}}^{\langle\alpha+1\rangle}, \dot{\boldsymbol{q}}_{\mathscr{M}}^{\langle\alpha\rangle}\right)$, then it is possible to express $\boldsymbol{q}_{\mathscr{M}}^{\langle\beta \mid \alpha\rangle}(\beta \geq \alpha)$ as an explicit function of time and generalized variables of $\mathscr{M}$ up to $\beta$-th order, i.e.:

$$
\boldsymbol{q}_{\mathscr{M}}^{\langle\beta \mid \alpha\rangle}=\underline{\boldsymbol{q}}_{\mathscr{M}}^{\langle\beta \mid \alpha\rangle}\left(t, \boldsymbol{q}_{\mathscr{M}}^{\langle\beta\rangle}\right)
$$

\subsection{Generalized constraint invariants}

Let $\mathscr{M}$ be a mechanical system whose motion can be parametrically described by a finite number of variables. Suppose that the motion constraints of this system either are automatically satisfied by some proper selection of generalized coordinates or can be represented by a finite number of invariants that can be expressed in the form of equation (2.4). Also, consider that non-trivial $(\alpha+1)$-th order generalized variables are related to the time derivatives of the $\alpha$-th order ones by a transformation of variables described by invariants that can be expressed in the form of equation (2.6). For each $\sigma \geq 0$ obtain the 
following time derivatives, for all $\rho$ such that $0 \leq \rho \leq \sigma$ :

$$
\begin{aligned}
& \frac{\mathrm{d}^{\sigma-\rho}}{\mathrm{d} t^{\sigma-\rho}}\left(\bar{h}_{\mathscr{M} . k}^{\langle\rho\rangle}\left(t, \boldsymbol{q}_{\mathscr{M}}^{\langle\rho \mid 0\rangle}\right)\right)=0 \\
& \frac{\mathrm{d}^{\sigma-\rho}}{\mathrm{d} t^{\sigma-\rho}}\left(\bar{c}_{\mathscr{M} . k}^{\langle\rho\rangle}\left(t, \boldsymbol{q}_{\mathcal{M}}^{\langle\rho\rangle}, \boldsymbol{q}_{\mathscr{M}}^{\langle\rho \mid \rho-1\rangle}\right)\right)=0
\end{aligned}
$$

Replace each $q_{\mathcal{M} . i}^{\langle\beta \mid \alpha\rangle}$ in equations $(2.8,2.9)$ by their corresponding expressions in terms of $t$ and $\boldsymbol{q}_{\mathcal{M}}^{\langle\langle\beta\rangle}$ given by equations (2.7). After such replacements, all the left-hand sides of the equations $(2.8,2.9)$ that remain non-degenerate, i.e that are not identically zero by any values of $t$ and $\boldsymbol{q}_{\mathscr{M}}^{\langle\sigma\rangle}$, are denominated $\sigma$-th order generalized constraint invariants of $\mathscr{M}$ and can be generically denoted by a tuple (or column-matrix) as follows:

$$
\overline{\boldsymbol{q}}_{M}^{\langle\sigma\rangle}\left(t, \boldsymbol{q}_{M}^{\langle\sigma\rangle}\right)=0
$$

Definition 2.3. The constraint order of $\mathscr{M}$, denoted by $\nu_{\mathscr{M}}^{\circ}$, is defined as the least integer $\sigma$ for which the following conditions are simultaneously satisfied:

(1) All of the constraints of $\mathscr{M}$ either are automatically satisfied by some proper selection of generalized coordinates or can be represented by a finite number of generalized constraint invariants that can be expressed in the form of equation (2.10).

(2) The model of system $\mathscr{M}$ has trivial generalized variables above $\sigma$-th order.

(3) $\overline{\boldsymbol{q}}_{\mathscr{M}}^{\langle\sigma\rangle}$ (when present) is an affine function with respect to $\boldsymbol{q}_{\mathscr{M}}^{\langle\sigma\rangle}$.

For the sake of brevity, the subscripts $\mathscr{M}$ will be omitted in the remainder of this section. For every $\sigma \geq \nu^{\circ}$, it can be stated that there is a matrix $\boldsymbol{A}=$ $\underline{\boldsymbol{A}}\left(t, \boldsymbol{q}^{\left\langle\nu^{\circ}-1\right\rangle}\right)$ and a column-matrix $\boldsymbol{b}^{\langle\sigma\rangle}=\underline{\boldsymbol{b}}^{\langle\sigma\rangle}\left(t, \boldsymbol{q}^{\langle\sigma-1\rangle}\right)$ such that the columnmatrix $\overline{\boldsymbol{q}}^{\langle\sigma\rangle}$ can be expressed as follows:

$$
\overline{\boldsymbol{q}}^{\langle\sigma\rangle}=\boldsymbol{A} \boldsymbol{q}^{\langle\sigma\rangle}+\boldsymbol{b}^{\langle\sigma-1\rangle}=\mathbf{0}
$$

Note that $\boldsymbol{A}$ is the Jacobian matrix of the $\sigma$-th order generalized constraint invariants with respect to the $\sigma$-th order generalized variables for every $\sigma \geq \nu^{\circ}$. Whenever $\sigma$-th order generalized constraint invariants are not present in the model for $\sigma \geq \nu^{\circ}, \overline{\boldsymbol{q}}^{\langle\sigma\rangle}$ can be supposed to be an empty tuple and $\boldsymbol{A}$ can be supposed to be a $0 \times \nu\left(\boldsymbol{q}^{\left\langle\nu^{\circ}\right\rangle}\right)$ empty matrix.

If $A$ is not a full rank matrix for any values of $t$ and $\boldsymbol{q}^{\left\langle\left\langle\nu^{\circ}-1\right\rangle\right.}$, then some of the invariants in the column-matrix $\overline{\boldsymbol{q}}^{\langle\sigma\rangle}$ can be expressed as linear combina- 
tions of the remaining ones ${ }^{4}$. Thus, such invariants are redundant and can be removed, being the remaining ones referred as independent generalized constraint invariants. In this case, the new matrix $A$ has full rank.

Definition 2.4. Let $\mathscr{M}$ be a multibody system whose constraint order is equal to $\nu^{\circ}$. A generalized configuration of $\mathscr{M}$ can be defined as being the tuple $\left(t, \boldsymbol{q}^{\left\langle\left\langle\nu^{\circ}-1\right\rangle\right.}\right)$. Denote by $\boldsymbol{A}$ the Jacobian matrix of the $\sigma$-th order independent generalized constraint invariants with respect to the $\sigma$-th order generalized variables for $\sigma \geq \nu^{\circ}$. A singular (generalized) configuration of $\mathscr{M}$ is any eventual tuple of values $\left(t, \boldsymbol{q}^{\left\langle\nu^{\circ}-1\right\rangle}\right)$ for which $A$ is not a full rank matrix.

Definition 2.5. The number of degrees of freedom of $\mathscr{M}$, denoted by $\nu^{\#}$ (or $\nu_{\mathscr{M}}^{\#}$ ), is defined as the nullity ${ }^{5}$ of the matrix $A$ in any non-singular configuration of $\mathscr{M}$. Also, $\mathscr{M}$ can be classified as a $\nu^{\#-D O F}$ system. It is worth noting that this definition is also valid when $\boldsymbol{A}$ is an empty matrix: in these cases, $\nu^{\#}=\nu\left(q^{\left\langle\nu^{\circ}\right\rangle}\right)$.

Example 2.2. Consider the system $\mathscr{P}$, represented in Figure 2.3, and constituted by four rigid bodies: $\mathcal{B}_{0}$ (base), $\mathcal{B}_{1}, \mathcal{B}_{2}$ (rigid rods whose transverse sections have negligible dimensions) and $\mathcal{B}_{3}$ (slider). The kinematic pairs $\left(\mathcal{B}_{0}, \mathcal{B}_{1}\right)$, $\left(\mathcal{B}_{1}, \mathcal{B}_{2}\right)$ e $\left(\mathcal{B}_{2}, \mathcal{B}_{3}\right)$ are ideal spherical joints and the kinematic pair $\left(\mathcal{B}_{0}, \mathcal{B}_{3}\right)$ is a prismatic joint.

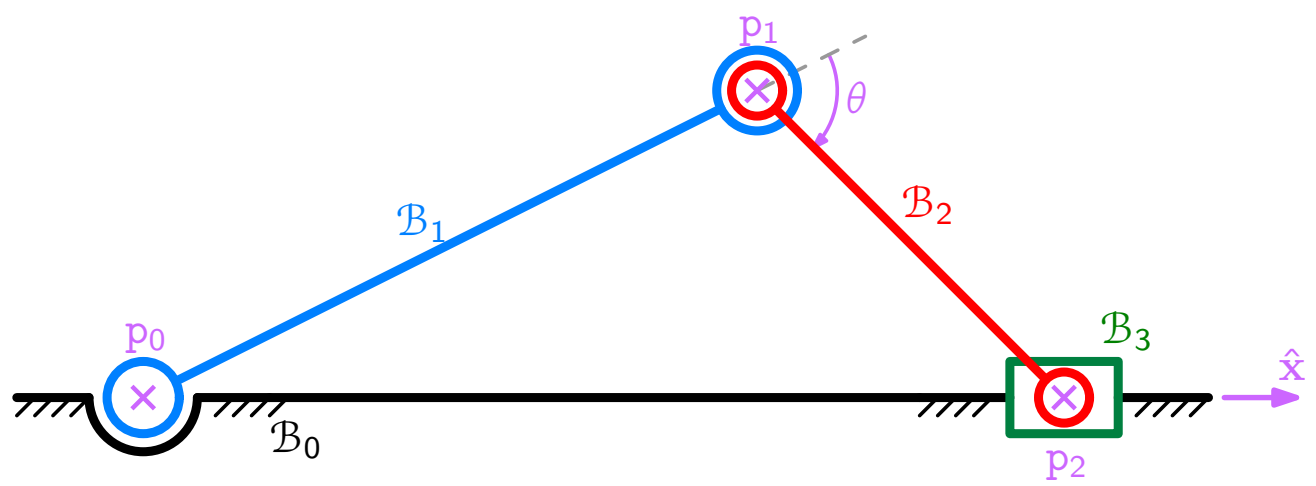

Figure 2.3: Representation of system $\mathscr{P}$.

Define $\mathrm{p}_{0}, \mathrm{p}_{1}$ and $\mathrm{p}_{2}$ as the geometric centers of the spherical joints $\left(\mathcal{B}_{0}, \mathcal{B}_{1}\right)$, $\left(\mathcal{B}_{1}, \mathcal{B}_{2}\right)$ and $\left(\mathcal{B}_{2}, \mathcal{B}_{3}\right)$, respectively, and let $\ell_{1}$ and $\ell_{2}$ denote the lengths of the rods $\mathcal{B}_{1}$ and $\mathcal{B}_{2}$, i.e., $\ell_{1}=\left\|\mathrm{r}_{\mathrm{p}_{1} \mid \mathrm{p}_{0}}\right\|$ and $\ell_{2}=\left\|\mathrm{r}_{\mathrm{p}_{2} \mid \mathrm{p}_{1}}\right\|$. Suppose that the axis of the prismatic joint $\left(\mathcal{B}_{0}, \mathcal{B}_{3}\right)$ is parallel to an unit vector $\hat{x}$ and passes through $\mathrm{p}_{0}$. Define the coordinate system $\mathrm{E}=\left(\mathrm{p}_{0}, \hat{\mathbf{x}}, \hat{\mathbf{y}}, \hat{\mathbf{z}}\right)$ which remains fixed with respect to $\mathcal{B}_{0}$. To describe the motion of this system with respect to $\mathcal{B}_{0}$ define

\footnotetext{
${ }^{4}$ Note that the coefficients of such linear combinations can be functions of $t$ and $\boldsymbol{q}^{\left\langle\nu^{\circ}-1\right\rangle}$.

${ }^{5}$ The nullity of a matrix is defined as the dimension of the kernel of the linear transformation defined by this matrix.
} 
the following generalized coordinates:

$$
\boldsymbol{q}_{\mathscr{P}}^{\langle 0\rangle}=\left(x_{1}, y_{1}, z_{1}, x_{2}, y_{2}, z_{2}, \theta\right)
$$

with $\left(x_{1}, y_{1}, z_{1}\right)$ and $\left(x_{2}, y_{2}, z_{2}\right)$ being the Cartesian coordinates of $p_{1}$ and $p_{2}$ in $E$ and $\theta$ denoting the angle between the lines $p_{0} p_{1}$ and $p_{1} p_{2}$. Consider that the model of $\mathscr{P}$ has trivial generalized variables. Due to the constraints of this system, the following invariants must be established:

$$
\left\{\begin{array}{l}
\bar{q}_{\mathscr{P} .1}^{\langle 0\rangle}=x_{1}^{2}+y_{1}^{2}+z_{1}^{2}-\ell_{1}^{2}=0 \\
\bar{q}_{\mathscr{P} .2}^{\langle 0\rangle}=\left(x_{1}-x_{2}\right)^{2}+\left(y_{1}-y_{2}\right)^{2}+\left(z_{1}-z_{2}\right)^{2}-\ell_{2}^{2}=0 \\
\bar{q}_{\mathscr{P} .3}^{\langle 0\rangle}=y_{2}=0 \\
\bar{q}_{\mathscr{P} .4}^{\langle 0\rangle}=z_{2}=0 \\
\bar{q}_{\mathscr{P} .5}^{\langle 0\rangle}=x_{2}^{2}+y_{2}^{2}+z_{2}^{2}-\ell_{1}^{2}-\ell_{2}^{2}-2 \ell_{1} \ell_{2} \mathrm{c}_{\theta}=0
\end{array}\right.
$$

All the constraints in $\mathscr{P}$ either are automatically satisfied by the selected generalized coordinates or can be represented by some generalized constraint invariants of order 0 or higher. Taking the first time derivative of equations (b), it can be noticed that according to Definition 2.3, the constraint order of $\mathscr{P}$ is $\nu_{P}^{\circ}=1$ and the corresponding matrix $\boldsymbol{A}_{\mathscr{P}}$ is given by:

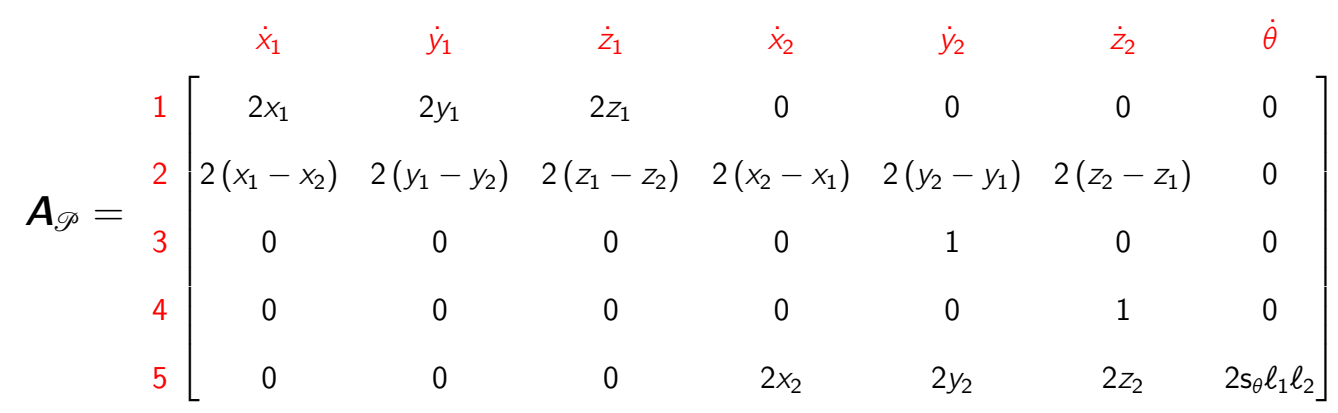

Whenever $\mathrm{s}_{\theta}=0$, the system of equations (b) has only four solutions:

$$
\left\{\begin{array}{lllll}
x_{1}=\ell_{1} & y_{1}=z_{1}=0 & x_{2}=\ell_{1}+\ell_{2} & y_{2}=z_{2}=0 & \theta=0 \\
x_{1}=-\ell_{1} & y_{1}=z_{1}=0 & x_{2}=-\ell_{1}-\ell_{2} & y_{2}=z_{2}=0 & \theta=0 \\
x_{1}=\ell_{1} & y_{1}=z_{1}=0 & x_{2}=\ell_{1}-\ell_{2} & y_{2}=z_{2}=0 & \theta=\pi \\
x_{1}=-\ell_{1} & y_{1}=z_{1}=0 & x_{2}=-\ell_{1}+\ell_{2} & y_{2}=z_{2}=0 & \theta=\pi
\end{array}\right.
$$

In any of these generalized configurations, the columns labeled by $\dot{y}_{1}, \dot{z}_{1}$ and $\dot{\theta}$ are identically zero and $\boldsymbol{A}_{\mathscr{P}}$ has rank 4 ; in any other generalized configurations its rank is equal to 5 . According to Definition 2.4, all of the generalized configu- 
rations (d) are singular. Moreover, following definition 2.5, it can be stated that $\nu_{\mathscr{P}}^{\#}=2$, i.e., $\mathscr{P}$ is a 2-DOF system.

Consider now that $\boldsymbol{q}_{\mathscr{P}}^{\langle 0\rangle}=\left(x_{1}, y_{1}, z_{1}, x_{2}, y_{2}, z_{2}\right)$, i.e. $\theta$ is not used as a generalized coordinate anymore. In this case the first 4 invariants of equation (b) are sufficient to completely describe the constraints of $\mathscr{P}$. In this case, matrix $\boldsymbol{A}_{\mathscr{P}}$ is given by:

$$
\left.\boldsymbol{A}_{\mathscr{P}}=\frac{1}{3}=\begin{array}{cccccc}
\dot{x}_{1} & \dot{y}_{1} & \dot{z}_{1} & \dot{x}_{2} & \dot{y}_{2} & \dot{z}_{2} \\
2 x_{1} & 2 y_{1} & 2 z_{1} & 0 & 0 & 0 \\
2\left(x_{1}-x_{2}\right) & 2\left(y_{1}-y_{2}\right) & 2\left(z_{1}-z_{2}\right) & 2\left(x_{2}-x_{1}\right) & 2\left(y_{2}-y_{1}\right) & 2\left(z_{2}-z_{1}\right) \\
0 & 0 & 0 & 0 & 1 & 0 \\
0 & 0 & 0 & 0 & 0 & 1
\end{array}\right]
$$

Note that matrix $\boldsymbol{A}_{\mathscr{P}}$ has full rank (equal to 4) at every generalized configuration, including the ones described by (d). Thus, there will be no singular generalized configurations in this case.

This example illustrates that some singularities in the description of motion of multibody systems are due to the kind of generalized variables chosen.
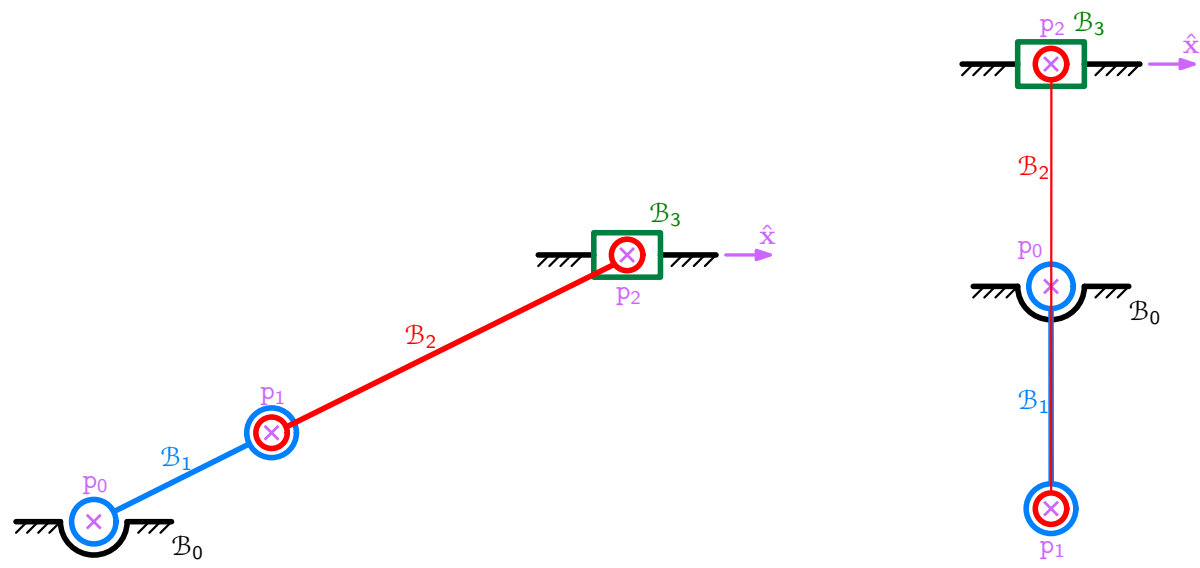

Figure 2.4: Singular generalized configurations of the variant of $\mathscr{P}$.

Example 2.3. Consider now the following variant of system $\mathscr{P}$, discussed in the previous example: adopt $\ell_{1}=1, \ell_{2}=2$ and suppose that the axis of the prismatic joint is defined by $y_{2}=1$ and $z_{2}=0$. Take $\boldsymbol{q}_{\mathscr{P}}^{\langle 0\rangle}=\left(x_{1}, y_{1}, z_{1}, x_{2}, y_{2}, z_{2}, \theta\right)$ again: whenever $s_{\theta}=0$, the corresponding system of generalized constraint equations of order 0 leads to three different solutions:

$$
\left\{\begin{array}{lllllll}
x_{1}=-\frac{2 \sqrt{2}}{3} & y_{1}=\frac{1}{3} & z_{1}=0 & x_{2}=-2 \sqrt{2} & y_{2}=1 & z_{2}=0 & \theta=0 \\
x_{1}=+\frac{2 \sqrt{2}}{3} & y_{1}=\frac{1}{3} & z_{1}=0 & x_{2}=+2 \sqrt{2} & y_{2}=1 & z_{2}=0 & \theta=0 \\
x_{1}=0 & y_{1}=-1 & z_{1}=0 & x_{2}=0 & y_{2}=1 & z_{2}=0 & \theta=\pi
\end{array}\right.
$$


In the first two of these generalized configurations (which are symmetric with respect to the $\mathrm{yz}$ plane of $\mathrm{E}$ ), the rank of the corresponiding matrix $A_{\mathscr{P}}$ is equal to 4 , and in the last one, it has rank 3 . Thus, these are the generalized singular configurations of this variant of $\mathscr{P}$ (outlined Figure 2.4).

\subsection{Variations of motion variables}

The application of Functional Analysis to the development of modeling methodologies for multibody systems is useful to apply the descriptons of motions and constraints (kinematics) to the derivation of the equations of motion (dynamics) of these systems.

Let $\mathscr{C}^{\nu}(\Omega)$ denote the linear space (over $\mathbb{R}$ ) of class $\mathscr{C}^{\nu}$ real-valued functions defined in a closed interval $\Omega \subset \mathbb{R}$. The addition and scalar multiplication in this linear space are defined respectively by: $\left(w_{1}+w_{2}\right)(t)=w_{1}(t)+w_{2}(t)$ and $(\lambda w)(t)=\lambda w(t)$ for all $w, w_{1}, w_{2} \in \mathscr{C}^{\nu}(\Omega), t \in \Omega$ and $\lambda \in \mathbb{R}$. In addition, define a norm for $\mathscr{C}^{\nu}(\Omega)$ as follows (GELFAND; FOMIN; SILVERMAN, 2000):

$$
\|w\|=\sum_{\kappa=0}^{\nu} \max _{t \in \Omega}\left|\frac{\mathrm{d}^{\kappa} w}{\mathrm{~d} t^{\kappa}}(t)\right|
$$

Also, if $w$ is a tuple (or column-matrix) of class $\mathscr{C}^{\nu}(\Omega)$ functions, define $\|w\|=$ $\max _{i}\left\|w_{. i}\right\|$, being the norms in the right-hand side of this equation obtained by (2.12).

Let $\mathscr{M}$ be a $\nu^{\#-D O F}$ multibody system whose constraint order is equal to $\nu^{\circ}$. Take an integer $\sigma \geq \nu^{\circ}$, and suppose that a generic motion of $\mathscr{M}$ during a time interval $\Omega$ is described by tuples $\boldsymbol{q}^{\langle\alpha\rangle}(t)$ of class $\mathscr{C}^{\sigma-\alpha}(\Omega)$ functions, for $0 \leq \alpha \leq \sigma$, with $\boldsymbol{q}^{\langle\alpha\rangle}$ representing the $\alpha$-th order generalized variables of the model of $\mathscr{M}$. Also, define for $0 \leq \alpha \leq \sigma$ the functions $\delta \boldsymbol{q}^{\langle\alpha\rangle}$, which will be called variations of the $\alpha$-th order generalized variables, satisfying the condition $\max _{\alpha}\left\|\delta \boldsymbol{q}^{\langle\alpha\rangle}\right\|=\epsilon$ for some $\epsilon>0$.

Let $\boldsymbol{v}^{\langle\rho\rangle}$ be a tuple constituted of arbitrary $\rho$-th order motion variables which can be defined as any variables that can be expressed as explicit functions of time and generalized variables up to $\rho$-th order, i.e., there must exist a function $\underline{\boldsymbol{v}}^{\langle\rho\rangle}$ such that $\boldsymbol{v}^{\langle\rho\rangle}=\underline{\boldsymbol{v}}^{\langle\rho\rangle}(t, \boldsymbol{q}\langle\rho \rho\rangle)$. Suppose that $\underline{\boldsymbol{v}}^{\langle\rho\rangle}$ is a class $\mathscr{C}^{1}$ function and 
consider the following first order Taylor series expansion ${ }^{6}$ :

$$
\begin{aligned}
& \underline{\boldsymbol{v}}^{\langle\rho\rangle}\left(t, \boldsymbol{q}^{\langle\rho \rho\rangle}(t)+\delta \boldsymbol{q}^{\langle\rho \rho\rangle}(t)\right)= \\
& \quad \underline{\boldsymbol{v}}^{\langle\rho\rangle}\left(t, \boldsymbol{q}^{\langle\rho \rho\rangle}(t)\right)+\delta \underline{\boldsymbol{v}}^{\langle\rho\rangle}\left(t, \boldsymbol{q}^{\langle\rho \rho\rangle}(t) ; \delta \boldsymbol{q}^{\langle\rho\rangle\rangle}(t)\right)+\boldsymbol{o}(\epsilon)
\end{aligned}
$$

with $\delta \underline{\boldsymbol{v}}^{\langle\rho\rangle}\left(t, \boldsymbol{q}^{\langle\rho \rho\rangle}(t) ; \delta \boldsymbol{q}^{\langle\rho \rho\rangle}(t)\right)$ given by:

$$
\delta \underline{\boldsymbol{v}}^{\langle\rho\rangle}\left(t, \boldsymbol{q}^{\langle\rho\rangle\rangle}(t) ; \delta \boldsymbol{q}^{\langle\rho\rangle\rangle}(t)\right)=\frac{\partial \underline{\boldsymbol{v}}^{\langle\rho\rangle}}{\partial \boldsymbol{q}\langle\rho\rangle\rangle} \delta \boldsymbol{q}^{\langle\rho\rangle\rangle}(t)=\sum_{\kappa=0}^{\rho} \frac{\partial \underline{\boldsymbol{v}}^{\langle\rho\rangle}}{\partial \boldsymbol{q}^{\langle\kappa\rangle}} \delta \boldsymbol{q}^{\langle\kappa\rangle}(t)
$$

The variation of $\boldsymbol{v}^{\langle\rho\rangle}$ is the function $\delta \boldsymbol{v}_{j}^{\langle\rho\rangle}(t)$ defined by:

$$
\delta \boldsymbol{v}^{\langle\rho\rangle}(t)=\delta \underline{\boldsymbol{v}}^{\langle\rho\rangle}\left(t, \boldsymbol{q}^{\langle\rho \rho\rangle}(t) ; \delta \boldsymbol{q}^{\langle\rho \rho\rangle}(t)\right)
$$

Let $\sigma \geq \max \left\{\rho, \nu^{\circ}\right\}$, and suppose that in a given time instant $t^{*}$ all the values of $\boldsymbol{q}^{\langle\sigma-1\rangle}\left(t^{*}\right)$ are known. Adopt $\delta \boldsymbol{q}^{\langle\sigma \sigma-1\rangle}\left(t^{*}\right)=0$ and consider that each $\delta \boldsymbol{q}^{\langle\alpha\rangle}$ is a tuple of class $\mathscr{C}^{\sigma-\alpha}(\Omega)$ functions, for $0 \leq \alpha \leq \sigma$, with $\Omega$ denoting a closed interval, $t^{*} \in \Omega$ and $\left(t^{*}+\varepsilon_{t}\right) \in \Omega$ for some $\varepsilon_{t}>0$. The following Taylor series expansions are valid in this case $^{7}$ :

$$
\delta \boldsymbol{q}^{\langle\rho\rangle}\left(t^{*}+\varepsilon_{t}\right)=\sum_{\kappa=0}^{\sigma-\rho} \frac{\varepsilon_{t}^{\kappa}}{\kappa !} \delta \boldsymbol{q}^{\langle\rho+\kappa \mid \rho\rangle}\left(t^{*}\right)+\boldsymbol{o}\left(\varepsilon_{t}^{\sigma-\rho}\right)
$$

Moreover, using equation (2.7), it can be stated that:

$$
\delta \boldsymbol{q}^{\langle\rho+\kappa \mid \rho\rangle}\left(t^{*}\right)=\sum_{\alpha=0}^{\rho+\kappa} \frac{\partial \underline{\boldsymbol{q}}^{\langle\rho+\kappa \mid \rho\rangle}}{\partial \boldsymbol{q}^{\langle\alpha\rangle}} \delta \boldsymbol{q}^{\langle\alpha\rangle}\left(t^{*}\right)
$$

However, by hypothesis, all $\delta \boldsymbol{q}^{\langle\alpha\rangle}\left(t^{*}\right)=0$ for $0 \leq \alpha \leq(\sigma-1)$; thus, if $(\rho+\kappa)<\sigma$, i.e. $\kappa<(\sigma-\rho)$, then, according to equation (2.17), $\delta \boldsymbol{q}^{\langle\rho+\kappa \mid \rho\rangle}\left(t^{*}\right)=0$. Also, from this same equation, it can be stated that:

$$
\delta \boldsymbol{q}^{\langle\sigma \mid \rho\rangle}\left(t^{*}\right)=\frac{\partial \underline{\boldsymbol{q}}^{\langle\sigma \mid \rho\rangle}}{\partial \boldsymbol{q}^{\langle\sigma\rangle}} \delta \boldsymbol{q}^{\langle\sigma\rangle}\left(t^{*}\right)
$$

Therefore, equation (2.16) can be rewritten as follows:

$$
\delta \boldsymbol{q}^{\langle\rho\rangle}\left(t^{*}+\varepsilon_{t}\right)=\frac{\varepsilon_{t}^{\sigma-\rho}}{(\sigma-\rho) !} \frac{\partial \underline{\boldsymbol{q}}^{\langle\sigma \mid \rho\rangle}}{\partial \boldsymbol{q}^{\langle\sigma\rangle}} \delta \boldsymbol{q}^{\langle\sigma\rangle}\left(t^{*}\right)+\boldsymbol{o}\left(\varepsilon_{t}^{\sigma-\rho}\right)
$$

${ }^{6}$ Denote by $o(\epsilon)$ any terms or sum of terms in a Taylor series expansion satisfying the following property:

$$
\lim _{\epsilon \rightarrow 0} \frac{\|\boldsymbol{o}(\epsilon)\|}{\epsilon}=0
$$

7 In this expansion, the following property is assumed: "the derivative of the variation is equal to the variation of the derivative" (LANCZOS, 1986, Sec. II-9). 
Furthermore, according to equations (2.14) and (2.19), it can be stated that:

$$
\delta \boldsymbol{v}^{\langle\rho\rangle}\left(t^{*}+\varepsilon_{t}\right)=\frac{\varepsilon_{t}^{\sigma-\rho}}{(\sigma-\rho) !} \boldsymbol{V}^{\langle\rho, \sigma\rangle} \delta \boldsymbol{q}^{\langle\sigma\rangle}\left(t^{*}\right)+\boldsymbol{o}\left(\varepsilon_{t}^{\sigma-\rho}\right)
$$

with $V^{\langle\rho, \sigma\rangle}$ defined as follows:

$$
\boldsymbol{V}^{\langle\rho, \sigma\rangle}=\frac{\partial \underline{\underline{v}}^{\langle\rho\rangle}}{\partial \boldsymbol{q}^{\langle\rho\rangle}} \frac{\partial \underline{\boldsymbol{q}}^{\langle\sigma \mid \rho\rangle}}{\partial \boldsymbol{q}^{\langle\sigma\rangle}}
$$

Definition 2.6. Let $\mathscr{M}$ be a multibody system whose constraint order is equal to $\nu^{\circ}$. Adopt $\sigma \geq \nu^{\circ}$, and consider that at a time instant $t^{*}$ the values of all generalized variables $\boldsymbol{q}^{\langle\sigma\rangle}\left(t^{*}\right)$ are known and satisfy the constraints of the system, i.e., if there are any independent generalized constraint invariants then $\overline{\boldsymbol{q}}^{\langle\sigma\rangle}\left(t^{*}, \boldsymbol{q}^{\langle\sigma\rangle}\left(t^{*}\right)\right)=\boldsymbol{0}$. In this case, the corresponding variations of the generalized variables at $t^{*}$ are said to be compatible with the constraints of $\mathscr{M}$ if and only if $\overline{\boldsymbol{q}}^{\langle\sigma\rangle}\left(t^{*}, \boldsymbol{q}^{\langle\sigma \sigma\rangle}\left(t^{*}\right)+\delta \boldsymbol{q}^{\langle\sigma\rangle\rangle}\left(t^{*}\right)\right)=\mathbf{0}$.

Proposition 2.7. Adopt the same hypothesis and conventions presented in Definition 2.6. If $\overline{\boldsymbol{q}}^{\langle\sigma\rangle}\left(t^{*}, \boldsymbol{q}^{\langle\sigma\rangle\rangle}\left(t^{*}\right)\right)=0$ then the values of $\delta \boldsymbol{q}^{\langle\sigma\rangle}\left(t^{*}\right)$ are compatible with the constraints of $\mathscr{M}$ if and only if:

$$
\delta \overline{\boldsymbol{q}}^{\langle\sigma\rangle}\left(t^{*}, \boldsymbol{q}\langle\sigma\rangle\left(t^{*}\right) ; \delta \boldsymbol{q}^{\langle\sigma \sigma\rangle}\left(t^{*}\right)\right)=0
$$

Proof. According to Definition 2.6, the values of $\delta \boldsymbol{q}^{\langle\sigma\rangle}\left(t^{*}\right)$ are compatible the constraints of $\mathscr{M}$ if and only if:

$$
\overline{\boldsymbol{q}}^{\langle\sigma\rangle}\left(t^{*}, \boldsymbol{q}^{\langle\sigma \sigma\rangle}\left(t^{*}\right)+\delta \boldsymbol{q}^{\langle\sigma\rangle\rangle}\left(t^{*}\right)\right)=0
$$

Using a Taylor series expansion similar to the one in equation (2.13), then:

$$
\begin{aligned}
& \overline{\boldsymbol{q}}^{\langle\sigma\rangle}\left(t^{*}, \boldsymbol{q}^{\langle\sigma \sigma\rangle}\left(t^{*}\right)+\delta \boldsymbol{q}^{\langle\sigma\rangle}\left(t^{*}\right)\right)= \\
& \overline{\boldsymbol{q}}^{\langle\sigma\rangle}\left(t^{*}, \boldsymbol{q}^{\langle\langle\sigma\rangle}\left(t^{*}\right)\right)+\delta \overline{\boldsymbol{q}}^{\langle\sigma\rangle}\left(t^{*}, \boldsymbol{q}^{\langle\langle\sigma\rangle}\left(t^{*}\right) ; \delta \boldsymbol{q}^{\langle\sigma\rangle}\left(t^{*}\right)\right)+\boldsymbol{o}(\epsilon)=\mathbf{0}
\end{aligned}
$$

However, by hypothesis, $\overline{\boldsymbol{q}}^{\langle\sigma\rangle}\left(t^{*}, \boldsymbol{q}^{\langle\sigma\rangle}\left(t^{*}\right)\right)=0$. Thus:

$$
\delta \overline{\boldsymbol{q}}^{\langle\sigma\rangle}\left(t^{*}, \boldsymbol{q}^{\langle\langle\sigma\rangle}\left(t^{*}\right) ; \delta \boldsymbol{q}^{\langle\sigma\rangle}\left(t^{*}\right)\right)=\boldsymbol{o}(\epsilon)
$$

Once $\delta \overline{\boldsymbol{q}}^{\langle\sigma\rangle}$ is a linear function of the $\delta \boldsymbol{q}^{\langle\alpha\rangle}$, with $0 \leq \alpha \leq \sigma$, and considering that $\epsilon=\max _{\alpha}\left\|\delta \boldsymbol{q}^{\langle\alpha\rangle}\right\|$, equation (c) implies that $\delta \overline{\boldsymbol{q}}^{\langle\sigma\rangle}$ must be identically zero (GELFAND; FOMIN; SILVERMAN, 2000, p. 12).

Theorem 2.8. Let $\mathscr{M}$ be a $\nu^{\#-D O F ~ m u l t i b o d y ~ s y s t e m ~ w h o s e ~ c o n s t r a i n t ~ o r d e r ~ i s ~}$ equal to $\nu^{\circ}$. Choose an integer $\sigma \geq \nu^{\circ}$ and consider that in a given time instant $t^{*}$ the values of $\boldsymbol{q}^{\langle\sigma-1\rangle}\left(t^{*}\right)$ are known and do not violate any constraint of $\mathscr{M}$. 
Adopt $\delta \boldsymbol{q}^{\langle\sigma-1\rangle}\left(t^{*}\right)=0$. It can be stated that, unless $\left(t^{*}, \boldsymbol{q}^{\langle\sigma-1\rangle}\left(t^{*}\right)\right)$ corresponds to a singular generalized configuration, there is a rank $\nu^{\#}$ matrix $S$ such that $A S=0$. Thus, for every variation $\delta \boldsymbol{q}^{\langle\sigma\rangle}$ compatible with the constraints of $\mathscr{M}$ there must be a column-matrix $\tilde{\boldsymbol{q}}^{\langle\sigma\rangle} \in \mathbb{R}^{\nu *}$ such that:

$$
\delta \boldsymbol{q}^{\langle\sigma\rangle}\left(t^{*}\right)=\boldsymbol{S} \tilde{\boldsymbol{q}}^{\langle\sigma\rangle}\left(t^{*}\right)
$$

Consequently, if $\boldsymbol{v}^{\langle\rho\rangle}$ denotes any column-matrix (or tuple) of $\rho$-th order motion variables $(\rho \leq \sigma)$, considering the definition of $\boldsymbol{V}^{\langle\rho, \sigma\rangle}$ given in equation (2.21), it can be stated that, for any variation $\delta v^{\langle\rho\rangle}\left(t^{*}+\varepsilon_{t}\right)$ compatible with the constraints of $\mathscr{M}$ there is a column-matrix $\tilde{\boldsymbol{q}}^{\langle\sigma\rangle} \in \mathbb{R}^{\nu^{*}}$ such that:

$$
\delta \boldsymbol{v}^{\langle\rho\rangle}\left(t^{*}+\varepsilon_{t}\right)=\frac{\varepsilon_{t}^{\sigma-\rho}}{(\sigma-\rho) !} \boldsymbol{V}^{\langle\rho, \sigma\rangle} \boldsymbol{S} \tilde{\boldsymbol{q}}^{\langle\sigma\rangle}\left(t^{*}\right)+\boldsymbol{o}\left(\varepsilon_{t}^{\sigma-\rho}\right)
$$

Proof. Assume that $\delta \boldsymbol{q}^{\langle\sigma-1\rangle}\left(t^{*}\right)=0$. Equation (2.22) can be written as follows:

$$
\boldsymbol{A} \delta \boldsymbol{q}^{\langle\sigma\rangle}\left(t^{*}\right)=0
$$

From this equation, it can be stated that any variation $\delta \boldsymbol{q}^{\langle\sigma\rangle}\left(t^{*}\right)$ compatible with the constraints of $\mathscr{M}$ must lie in the kernel of the linear transformation defined by matrix $A$. According to Definition 2.5, the dimension of the kernel of this linear transformation is equal to the number of degrees of freedom of $\mathscr{M}\left(\nu^{\#}\right)$. Thus, there must exist a full rank matrix $S$ with $\nu^{\#}$ columns such that $A S=0$. Such a matrix is called an orthogonal complement of $A$. Moreover, it there is any other matrix $S^{\prime}$ such that $A S^{\prime}=0$, it can be stated that the rank of $S^{\prime}$ must be less than or equal to $\nu^{*}$. Therefore, any variation $\delta \boldsymbol{q}^{\langle\sigma\rangle}\left(t^{*}\right)$ compatible with the constraints of $\mathscr{M}$ can be expressed as a linear combination of the columns of $\boldsymbol{S}$. It means that, there must exist a column-matrix $\tilde{\boldsymbol{q}}^{\langle\sigma\rangle}\left(t^{*}\right) \in \mathbb{R}^{\nu *}$ such that $\delta \boldsymbol{q}^{\langle\sigma\rangle}\left(t^{*}\right)$ can be given by equation (2.23). Finally, equation (2.24) can be obtained by applying equation (2.23) to (2.20).

From Theorem 2.8 it can be stated that any (compatible) variations of variables of motion in a $\nu^{\#-D O F}$ multibody system $\mathscr{M}$ can be expressed as a linear combination of $\nu^{*}$ independent parameters. The coefficients of these linear combinations can be expressed as functions of the generalized configuration of $\mathscr{M}$. The most intuitive choice for a matrix $S$ satisfying the conditions of Theorem 2.8 is trying to choose some independent variables among the elements of the column-matrix $\delta \boldsymbol{q}^{\langle\sigma\rangle}\left(t^{*}\right)$ and, by some algebraic manipulations in the $\sigma$-th order constraint invariants, express explicitly all the remaining elements of this column-matrix as linear combinations of the independent ones. 
Assume, without loss of generality ${ }^{8}$, that the column-matrix $\delta \boldsymbol{q}^{\langle\sigma\rangle}\left(t^{*}\right)$ and the matrix $A$ can be partitioned in the following form:

$$
\boldsymbol{A}=\left[\begin{array}{ll}
\boldsymbol{q}_{. \#}^{\langle\sigma\rangle} & \boldsymbol{q}_{\cdot \star}^{\langle\sigma\rangle} \\
\boldsymbol{A}_{\cdot \#} & \boldsymbol{A}_{\cdot \star}
\end{array}\right] \quad \delta \boldsymbol{q}^{\langle\sigma\rangle}\left(t^{*}\right)=\left[\begin{array}{c}
\delta \boldsymbol{q}_{\cdot \#}^{\langle\sigma\rangle}\left(t^{*}\right) \\
\delta \boldsymbol{q}_{\cdot \star}^{\langle\sigma\rangle}\left(t^{*}\right)
\end{array}\right]
$$

In this case, equation (2.25) can be written as follows:

$$
\boldsymbol{A}_{\cdot \#} \delta \boldsymbol{q}_{. \#}^{\langle\sigma\rangle}\left(t^{*}\right)+\boldsymbol{A}_{\cdot \star} \delta \boldsymbol{q}_{\cdot \star}^{\langle\sigma\rangle}\left(t^{*}\right)=0
$$

It can be ensured that $\delta \boldsymbol{q}_{\text {.\# }}^{\langle\sigma\rangle}\left(t^{*}\right)$ represents an independent set of $\nu^{\#}$ variations of generalized variables if $\boldsymbol{A}_{\text {.太 }}$ is an invertible matrix ${ }^{9}$. Thus, denoting by $\boldsymbol{1}_{\nu \#}$ an identity matrix of order $\nu^{\#}$, it can be stated that:

$$
\delta \boldsymbol{q}^{\langle\sigma\rangle}\left(t^{*}\right)=\left[\begin{array}{c}
\delta \boldsymbol{q}_{. \#}^{\langle\sigma\rangle}\left(t^{*}\right) \\
\delta \boldsymbol{q}_{. \star}^{\langle\sigma\rangle}\left(t^{*}\right)
\end{array}\right]=\left[\begin{array}{c}
\boldsymbol{1}_{\nu \#} \\
-\boldsymbol{A}_{\cdot \star}^{-1} \boldsymbol{A}_{. \#}
\end{array}\right] \delta \boldsymbol{q}_{. \#}^{\langle\sigma\rangle}\left(t^{*}\right)
$$

Finally, from this last equation, it can be identified that:

$$
\boldsymbol{S}=\underset{\boldsymbol{q}_{\cdot \star \star}^{\langle\sigma\rangle}}{\boldsymbol{q}_{\cdot \#}^{\langle\sigma\rangle}}\left[\begin{array}{c}
\boldsymbol{q}_{\cdot \#}^{\langle\sigma\rangle} \\
\boldsymbol{1}_{\nu \#} \\
-\boldsymbol{A}_{\cdot \star}^{-1} \boldsymbol{A}_{\cdot \#}
\end{array}\right] \quad \tilde{\boldsymbol{q}}^{\langle\sigma\rangle}\left(t^{*}\right)=\delta \boldsymbol{q}_{\cdot \#}^{\langle\sigma\rangle}\left(t^{*}\right)
$$

Even though the method illustrated in equations $(2.26-2.29)$ seems to be the most intuitive one to obtain an orthogonal complement of $A$, it must be highlighted that any algorithm that is able to derive an expression for a rank $\nu^{\#}$ matrix $S$ such that $A S=0$ shall also apply.

Example 2.4. Back to system $\mathscr{P}$ presented in Example 2.2, it can be noticed that unless $\ell_{1}=\ell_{2}, x_{2}$ will never be zero. Moreover, if $\ell_{1}=\ell_{2}$, then $x_{2}=0$ only in a singular generalized configuration of this system. The same can be stated about configurations in which $s_{\theta}=0$ (see Figures 2.3 and 2.4). Furthermore, $y_{1}$ and $z_{1}$ will only be simultaneously zero in singular configurations too.

Thus, whenever $y_{1} \neq 0$, the variations of $\delta \dot{z}_{1}$ and $\delta \dot{x}_{2}$ can be adopted as being independent, and the method presented in equations $(2.26-2.29)$ leads

\footnotetext{
${ }^{8}$ Once it is always possible to reorder the columns of $A$ based on any ordering assumed for $\boldsymbol{q}^{\langle\sigma\rangle}$.

${ }^{9}$ Note that, once $\boldsymbol{A}$ is a full rank matrix, there must be $\left(\nu\left(\overline{\boldsymbol{q}}_{\mathscr{M}}^{\langle\sigma\rangle}\right)-\nu^{\#}\right)$ columns of $\boldsymbol{A}$ constituting an invertible square matrix.
} 
to:

$$
\left[\begin{array}{c}
\delta \dot{x}_{1} \\
\delta \dot{y}_{1} \\
\delta \dot{z}_{1} \\
\delta \dot{x}_{2} \\
\delta \dot{y}_{2} \\
\delta \dot{z}_{2} \\
\delta \dot{\theta}
\end{array}\right]=\left[\begin{array}{cc}
\frac{z_{2}}{x_{2}} & \frac{x_{1}}{x_{2}}-1 \\
\frac{z_{1} x_{2}-x_{1} z_{2}}{y_{1} x_{2}} & \frac{x_{1}\left(x_{2}-x_{1}\right)}{y_{1} x_{2}} \\
1 & 0 \\
0 & 1 \\
0 & 0 \\
0 & 0 \\
0 & \frac{x_{2}}{l_{1} l_{2} \mathrm{~s}_{\theta}}
\end{array}\right]\left[\begin{array}{c}
\delta \dot{z}_{1} \\
\delta \dot{x}_{2}
\end{array}\right]
$$

On the other hand, when $z_{1} \neq 0$, the variations of $\delta \dot{y}_{1}$ and $\delta \dot{x}_{2}$ can be assumed as independent, leading to:

$$
\left[\begin{array}{c}
\delta \dot{x}_{1} \\
\delta \dot{y}_{1} \\
\delta \dot{z}_{1} \\
\delta \dot{x}_{2} \\
\delta \dot{y}_{2} \\
\delta \dot{z}_{2} \\
\delta \dot{\theta}
\end{array}\right]=\left[\begin{array}{cc}
\frac{y_{2}}{x_{2}} & \frac{x_{1}}{x_{2}}-1 \\
1 & 0 \\
\frac{y_{1} x_{2}-x_{1} y_{2}}{z_{1} x_{2}} & \frac{x_{1}\left(x_{2}-x_{1}\right)}{z_{1} x_{2}} \\
0 & 1 \\
0 & 0 \\
0 & 0 \\
0 & \frac{x_{2}}{\ell_{1} \ell_{2} s_{\theta}}
\end{array}\right]\left[\begin{array}{c}
\delta \dot{y}_{1} \\
\delta \dot{x}_{2}
\end{array}\right]
$$

This example illustrates the statement of Theorem 2.8.

Example 2.5. Let $\delta$ denote a mechanical system consisting of an ideal rigid sphere $\mathcal{S}$ rolling without slipping in a rigid surface $\mathcal{U}$. Suppose that the sphere always touches the surface in a single point. Denote by $U=\left(\mathbf{u}_{\circ}, \hat{\mathbf{u}}_{\mathrm{x}}, \hat{\mathbf{u}}_{\mathrm{y}}, \hat{\mathbf{u}}_{z}\right) \mathbf{a}$ coordinate system rigidly attached to $\mathcal{U}$ and consider that the Cartesian coordinates of the instantaneous contact point between the sphere and the surface in $\mathrm{U}$ are denoted by $(x, y, z)$. Moreover, consider that $\mathrm{S}=\left(\mathrm{s}, \hat{\mathrm{S}}_{\mathrm{x}}, \hat{\mathrm{S}}_{\mathrm{y}}, \hat{\mathrm{S}}_{\mathrm{z}}\right)$ is a coordinate system rigidly attached to $\mathcal{S}$, that $\mathrm{s}$ is the geometric center of the sphere and that the transformation between $\mathrm{S}$ and $\mathrm{U}$ is defined by a unit quaternion $\left(q_{\mathrm{t}}, \mathbf{q}\right)=\left(q_{\mathrm{t}}, q_{\mathrm{x}} \hat{\mathbf{u}}_{\mathrm{x}}+q_{\mathrm{y}} \hat{\mathbf{u}}_{\mathrm{y}}+q_{\mathrm{z}} \hat{\mathbf{u}}_{\mathrm{z}}\right)$. Finally, let $\left(v_{\mathrm{x}}, v_{\mathrm{y}}, v_{\mathrm{z}}\right)$ denote the components of $\mathbf{v}_{\mathbf{s} \mid u}$ in $\mathrm{U}$ and $\left(\omega_{\mathrm{x}}, \omega_{\mathrm{y}}, \omega_{\mathrm{z}}\right)$ denote the components of $\omega_{\mathcal{S} \mid u}$ in $\mathrm{U}$ too. Define the following generalized variables to describe the motion of $\mathcal{S}$ with respect to 
U:

$$
\begin{aligned}
& \boldsymbol{q}_{\mathcal{S}}^{\langle 0\rangle}=\left(q_{\mathrm{x}}, q_{\mathrm{y}}, q_{\mathrm{z}}, q_{\mathrm{t}}, x, y, z\right) \\
& \boldsymbol{q}_{\mathcal{S}}^{\langle 1\rangle}=\left(\omega_{\mathrm{x}}, \omega_{\mathrm{y}}, \omega_{\mathrm{z}}, v_{\mathrm{x}}, v_{\mathrm{y}}, v_{\mathrm{z}}\right)
\end{aligned}
$$

Let $\mathcal{U}$ be defined by an equation of the form $\underline{\gamma}(x, y, z)=0$. and suppose, without any loss in generality, $\nabla \underline{\gamma}(x, y, z)$ points to the half-space in which $\mathcal{S}$ moves. For the sake of brevity, define, with all the partial derivatives being evaluated at the contact point coordinates $(x, y, z)$ :

$$
\begin{aligned}
& \gamma_{\mathrm{n}}=\sqrt{\left(\frac{\partial \underline{\gamma}}{\partial x}\right)^{2}+\left(\frac{\partial \underline{\gamma}}{\partial y}\right)^{2}+\left(\frac{\partial \underline{\gamma}}{\partial z}\right)^{2}} \\
& \gamma_{\mathrm{x}}=\frac{1}{\gamma_{\mathrm{n}}} \frac{\partial \underline{\gamma}}{\partial x} \quad \gamma_{\mathrm{y}}=\frac{1}{\gamma_{\mathrm{n}}} \frac{\partial \underline{\gamma}}{\partial y} \quad \gamma_{\mathrm{z}}=\frac{1}{\gamma_{\mathrm{n}}} \frac{\partial \underline{\gamma}}{\partial z}
\end{aligned}
$$

Denoting by $\bar{r}$ the radius of this sphere, it can be stated that the coordinates of $\mathrm{s}$ in $\mathrm{U}$ are given by $[\mathrm{s}]_{\mathrm{U}}=(x, y, z)+\bar{r}\left(\gamma_{\mathrm{x}}, \gamma_{\mathrm{y}}, \gamma_{\mathrm{z}}\right)$. Considering that $\left(q_{\mathrm{t}}, \mathbf{q}\right)$ is a unit quaternion, that $\left(v_{\mathrm{x}}, v_{\mathrm{y}}, v_{\mathrm{z}}\right)=\frac{\mathrm{d}}{\mathrm{d} t}[\mathrm{~s}]_{\mathrm{U}}$ and that the skew-symmetric representation of $\left(\omega_{\mathrm{x}}, \omega_{\mathrm{y}}, \omega_{\mathrm{z}}\right)$ is given by $[1]_{\mathrm{U} \mid \mathrm{S}}[1]_{\mathrm{U} \mid \mathrm{S}}^{\top}$, the following invariants define the transformations between the quasi-velocities and the time derivatives of the generalized coordinates in this model:

$$
\left\{\begin{array}{l}
\bar{c}_{\delta .1}^{\langle 1\rangle}=q_{\mathrm{x}} \dot{q}_{\mathrm{x}}+q_{\mathrm{y}} \dot{q}_{\mathrm{y}}+q_{\mathrm{z}} \dot{q}_{\mathrm{z}}+q_{\mathrm{t}} \dot{q}_{\mathrm{t}}=0 \\
\bar{c}_{\mathcal{S} .2}^{\langle 1\rangle}=\omega_{\mathrm{x}}-2\left(q_{\mathrm{t}} \dot{q}_{\mathrm{x}}-q_{\mathrm{z}} \dot{q}_{\mathrm{y}}+q_{\mathrm{y}} \dot{q}_{\mathrm{z}}-q_{\mathrm{x}} \dot{q}_{\mathrm{t}}\right)=0 \\
\bar{c}_{\delta .3}^{\langle 1\rangle}=\omega_{\mathrm{y}}-2\left(q_{\mathrm{z}} \dot{q}_{\mathrm{x}}+q_{\mathrm{t}} \dot{q}_{\mathrm{y}}-q_{\mathrm{x}} \dot{q}_{\mathrm{z}}-q_{\mathrm{y}} \dot{q}_{\mathrm{t}}\right)=0 \\
\bar{c}_{\delta .4}^{\langle 1\rangle}=\omega_{\mathrm{z}}-2\left(-q_{\mathrm{y}} \dot{q}_{\mathrm{x}}+q_{\mathrm{x}} \dot{q}_{\mathrm{y}}+q_{\mathrm{t}} \dot{q}_{\mathrm{z}}-q_{\mathrm{z}} \dot{q}_{\mathrm{t}}\right)=0 \\
\bar{c}_{\delta .5}^{\langle 1\rangle}=v_{\mathrm{x}}-\dot{x}-\frac{\mathrm{d}}{\mathrm{d} t}\left(\bar{r} \gamma_{\mathrm{x}}\right)=0 \\
\bar{c}_{\delta .6}^{\langle 1\rangle}=v_{\mathrm{y}}-\dot{y}-\frac{\mathrm{d}}{\mathrm{d} t}\left(\bar{r} \gamma_{\mathrm{y}}\right)=0 \\
\bar{c}_{\delta .7}^{\langle 1\rangle}=v_{\mathrm{z}}-\dot{z}-\frac{\mathrm{d}}{\mathrm{d} t}\left(\bar{r} \gamma_{\mathrm{z}}\right)=0
\end{array}\right.
$$

Due to the no-slip constraints present in this system, the instantaneous contact point between $\mathcal{S}$ and $\mathcal{U}$ must have zero velocity with respect to $\mathcal{U}$. This fact 
leads to the following identity:

$$
\left[\begin{array}{c}
v_{\mathrm{x}} \\
v_{\mathrm{y}} \\
v_{\mathrm{z}}
\end{array}\right]=\bar{r}\left[\begin{array}{ccc}
0 & -\omega_{\mathrm{z}} & \omega_{\mathrm{y}} \\
\omega_{\mathrm{z}} & 0 & -\omega_{\mathrm{x}} \\
-\omega_{\mathrm{y}} & \omega_{\mathrm{x}} & 0
\end{array}\right]\left[\begin{array}{c}
\gamma_{\mathrm{x}} \\
\gamma_{\mathrm{y}} \\
\gamma_{\mathrm{z}}
\end{array}\right]
$$

Thus, the following invariants are obtained:

$$
\left\{\begin{array}{l}
\bar{q}_{S .1}^{\langle 1\rangle}=v_{\mathrm{x}}-\bar{r}\left(\omega_{\mathrm{y}} \gamma_{\mathrm{z}}-\omega_{\mathrm{z}} \gamma_{\mathrm{y}}\right)=0 \\
\bar{q}_{\delta .2}^{\langle 1\rangle}=v_{\mathrm{y}}-\bar{r}\left(\omega_{\mathrm{z}} \gamma_{\mathrm{x}}-\omega_{\mathrm{x}} \gamma_{\mathrm{z}}\right)=0 \\
\bar{q}_{S .3}^{\langle 1\rangle}=v_{\mathrm{z}}-\bar{r}\left(\omega_{\mathrm{x}} \gamma_{\mathrm{y}}-\omega_{\mathrm{y}} \gamma_{\mathrm{x}}\right)=0
\end{array}\right.
$$

A possible matrix $S_{\mathcal{S}}$ for this system can be given by the following expression:

$$
S_{S}=\begin{gathered}
\omega_{x} \\
\omega_{y} \\
v_{x} \\
v_{y}
\end{gathered}\left[\begin{array}{ccc}
1 & \omega_{y} & \omega_{z} \\
0 & 1 & 0 \\
0 & 0 & 1 \\
0 & r \gamma_{z} & -r \gamma_{y} \\
-r \gamma_{z} & 0 & r \gamma_{x} \\
r \gamma_{y} & -r \gamma_{x} & 0
\end{array}\right]
$$

Consider, for instance, that $\mathcal{U}$ is defined by the equation $\underline{\gamma}(x, y, z)=$ $z-\mu y^{2}=0$. In this case, $\gamma_{x}=0, \gamma_{y}=-2 \mu y / \sqrt{1+4 \mu^{2} y^{2}}$ and $\gamma_{z}=$ $1 / \sqrt{1+4 \mu^{2} y^{2}}$. Let $\mathrm{b}$ be a point in the surface of $\mathcal{S}$, whose local coordinates are given by $[\mathrm{b}]_{\mathrm{S}}=(0,0,-\bar{r})$. Define $\boldsymbol{v}^{\langle 0\rangle}$ as follows:

$$
\boldsymbol{v}^{\langle 0\rangle}=[\mathrm{b}]_{\mathrm{U}}=[\mathrm{s}]_{\mathrm{U}}+[1]_{\mathrm{U} \mid \mathrm{S}}[\mathrm{b}]_{\mathrm{S}}
$$

It can be stated that:

$$
\boldsymbol{V}^{\langle 0,1\rangle}=v_{\cdot \mathrm{x}}^{v_{\cdot \mathrm{y}}^{\langle 0\rangle}}\left[\begin{array}{cccccc}
\omega_{\mathrm{x}} & \omega_{\mathrm{y}} & \omega_{\mathrm{z}} & v_{\mathrm{x}} & v_{\mathrm{y}} & v_{\mathrm{z}} \\
0 & -\bar{r}\left(1-2 q_{\mathrm{x}}^{2}-2 q_{\mathrm{y}}^{2}\right) & 2 \bar{r}\left(q_{\mathrm{y}} q_{\mathrm{z}}-q_{\mathrm{x}} q_{\mathrm{t}}\right) & 1 & 0 & 0 \\
v_{\cdot \mathrm{c}}^{\langle 0\rangle} & 0 & -2 \bar{r}\left(q_{\mathrm{x}} q_{\mathrm{z}}+q_{\mathrm{y}} q_{\mathrm{t}}\right) & 0 & 1 & 0 \\
\bar{r}\left(1-2 q_{\mathrm{x}}^{2}-2 q_{\mathrm{y}}^{2}\right) & 0 & 0 & 0 & 0 & 1
\end{array}\right]
$$


Moreover:

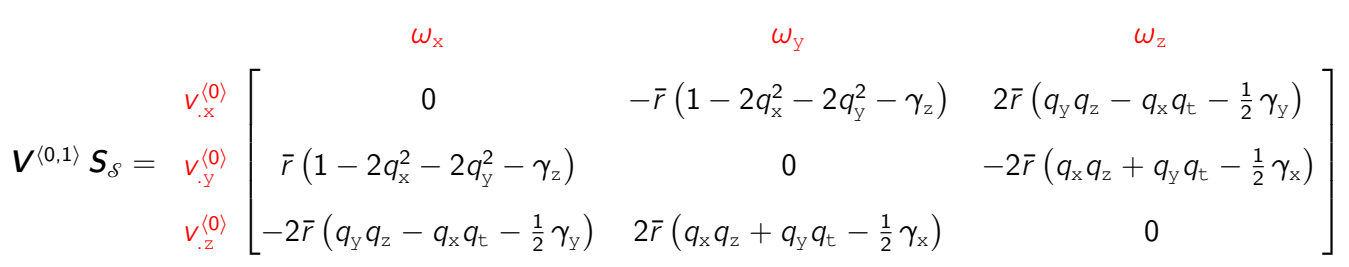

Consider that, at a time instant $t^{*}, y\left(t^{*}\right)=0$ (and, consequently, $\gamma_{\mathrm{x}}=0, \gamma_{\mathrm{y}}=0$, $\gamma_{\mathrm{z}}=1$ and $\left.\gamma_{\mathrm{n}}=1\right), q_{\mathrm{x}}\left(t^{*}\right)=q_{\mathrm{y}}\left(t^{*}\right)=q_{\mathrm{z}}\left(t^{*}\right)=0$ and $q_{\mathrm{t}}\left(t^{*}\right)=1$. In this configuration, $\mathrm{b}$ is instantaneously in contact with $\mathcal{S}$, and, as expected, $\boldsymbol{V}^{\langle 0,1\rangle} \boldsymbol{S}_{\mathcal{S}}=\boldsymbol{0}$. Indeed, by Theorem 2.8, $\delta \boldsymbol{v}^{\langle 0\rangle}\left(t^{*}+\varepsilon_{t}\right)=o\left(\varepsilon_{t}\right)$, i.e., the variation of the position of $\mathrm{b}$ in a time interval $\left[t^{*}, t^{*}+\varepsilon_{t}\right.$ [ is negligible comparatively to $\varepsilon_{t}$, for $\varepsilon_{t}$ sufficiently small.

Example 2.6 (2D rigid body element). Let $\mathscr{B}$ denote a system constituted by a single rigid body $\mathcal{B}$ that is constrained to perform a plane motions only, i.e., given any two points of $\mathcal{B}$ their trajectories must be plane curves and the corresponding planes must be parallel. Such a system can be conceived as a $2 D$ rigid body element. Let $\mathcal{N}$ denote the reference frame with respect to the motion of $\mathcal{B}$ is described and define a coordinate system $N=(0, \hat{\mathbf{x}}, \hat{\mathbf{y}}, \hat{\mathbf{z}})$ such that $\hat{\mathbf{x}}$ and $\hat{\mathbf{y}}$ are unit vectors which are parallel to the planes of motion and $\hat{z}$ is a unit vector orthogonal to these planes. Thus, if $\mathrm{p}$ is any point of $\mathcal{B}$, then $\mathbf{v}_{\mathrm{p} \mid \mathcal{N}} \cdot \hat{\mathbf{z}}=0$. Due to this condition, it can also be stated that $\omega_{\mathcal{B} \mid \mathcal{N}} \cdot \hat{\mathbf{x}}=\omega_{\mathcal{B} \mid \mathcal{N}} \cdot \hat{\mathbf{y}}=0$. Thus choosing a particular point $\mathrm{b}^{\star}$ of $\mathcal{B}$ and denoting $\mathbf{v}_{\mathrm{b}^{\star} \mid \mathcal{N}}=v_{\mathcal{B}, \mathrm{x}} \hat{\mathbf{x}}+v_{\mathcal{B}, Y} \hat{\mathbf{y}}$ and $\omega_{\mathcal{B} \mid \mathcal{N}}=\omega_{\mathcal{B}, z} \hat{\mathbf{z}}$, the following quasi-velocities can be defined for the modeling of $\mathscr{B}: \boldsymbol{q}_{\mathscr{B}}^{\langle 1\rangle}=\left(v_{\mathcal{B}, \mathrm{x}}, v_{\mathcal{B}, \mathrm{Y}}, \omega_{\mathcal{B}, \mathrm{z}}\right)$.

Moreover, once $\mathscr{B}$ is a holonomic system, to describe its configurations, it is enough to specify the position of a one point of $\mathcal{B}$ along with the orientation of a line orthogonal to $\hat{z}$ that moves along with this rigid body. For instance, if $p_{\mathcal{B}, \mathrm{x}}$ and $p_{\mathcal{B}, y}$ denote the $\mathrm{x}$ and $\mathrm{y}$ coordinates of $\mathrm{b}^{\star}$ in $\mathrm{N}$ and $\theta_{\mathcal{B}}$ represents the angle between a line orthogonal to $\hat{z}$ that moves along with $\mathcal{B}$ and the direction of $\hat{\mathbf{x}}$, the following generalized coordinates can be defined for the modeling of $\mathscr{B}: \boldsymbol{q}_{\mathscr{B}}^{\langle 0\rangle}=\left(p_{\mathcal{B}, \mathrm{x}}, p_{\mathcal{B}, \mathrm{Y}}, \theta_{\mathcal{B}}\right)$. In this case, $\boldsymbol{q}_{\mathscr{B}}^{\langle 1\rangle}=\dot{\boldsymbol{q}}_{\mathscr{B}}^{\langle 0\rangle}$.

Another alternative for describing a generic configuration of $\mathscr{B}$ is by selecting a finite number of points of $\mathcal{B}$ as shown in Figure 2.5. Once the position of a point and the orientation of a line are enough for it, the positions of at least two points of $\mathcal{B}$ are sufficient for specifying the position of any other point of this body. Referring to these tracked points of $\mathcal{B}$ as nodes and denoting them by $b_{k}$ and their corresponding Cartesian coordinates by $\left(p_{\mathcal{B}, k, x}, p_{\mathcal{B}, k, y}\right)$, it can 


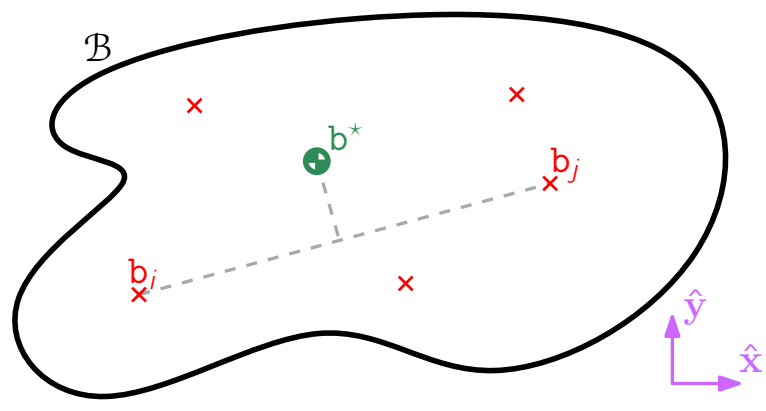

Figure 2.5: Representation of a 2D rigid body element.

be stated that a possible set of generalized coordinates for this system can be defined as the collection of the Cartesian coordinates of all these nodes (with the number of nodes begin equal to or greater than 2). Also, in this case, let $\boldsymbol{q}_{\mathscr{B}}^{\langle 1\rangle}=\left(v_{\mathcal{B}, \mathrm{x}}, v_{\mathcal{B}, \mathrm{y}}, \omega_{\mathcal{B}, \mathrm{z}}, \dot{\boldsymbol{q}}_{\mathscr{B}}^{\langle 0\rangle}\right)$. Thus, the 0 -th order generalized constraint invariants of this system can be given by equations of the form (with $\bar{\ell}_{\mathcal{B}, i, j}$ representing the distance between $\mathrm{b}_{i}$ and $\mathrm{b}_{j}, i<j$ ):

$$
\left(p_{\mathcal{B}, i, \mathrm{x}}-p_{\mathcal{B}, j, \mathrm{x}}\right)^{2}+\left(p_{\mathcal{B}, i, Y}-p_{\mathcal{B}, j, Y}\right)^{2}-\bar{\ell}_{\mathcal{B}, i, j}^{2}=0
$$

Sometimes $b^{\star}$ will not not be one of the nodes of the model. In such cases, suppose that its position is specified in terms of two nodes $b_{i}$ and $b_{j}$. There must exist two adimensional constants $\hat{\gamma}_{\mathcal{B}}$ and $\hat{\rho}_{\mathcal{B}}$ such that:

$$
\mathbf{r}_{\mathrm{b}^{\star} \mid \mathrm{b}_{i}}=\hat{\gamma}_{\mathcal{B}} \mathbf{r}_{\mathrm{b}_{j} \mid \mathrm{b}_{i}}+\hat{\rho}_{\mathcal{B}} \hat{\mathbf{z}} \times \mathbf{r}_{\mathrm{b}_{j} \mid \mathrm{b}_{i}}
$$

The time derivative of this equation leads to the following identity:

$$
\mathbf{v}_{\mathrm{b}_{i} \mid \mathcal{N}}=\mathbf{v}_{\mathrm{b}^{\star} \mid \mathcal{N}}+\omega_{\mathcal{B}, \mathrm{z}}\left(\hat{\rho}_{\mathcal{B}} \mathbf{r}_{\mathrm{b}_{j} \mid \mathrm{b}_{i}}-\hat{\gamma}_{\mathcal{B}} \hat{\mathbf{z}} \times \mathbf{r}_{\mathrm{b}_{j} \mid \mathrm{b}_{i}}\right)
$$

In general, for any node $\mathrm{b}_{k}$ :

$$
\mathbf{v}_{\mathrm{b}_{k} \mid \mathcal{N}}=\left(v_{\mathcal{B}, \mathrm{x}} \hat{\mathbf{x}}+v_{\mathcal{B}, \mathrm{Y}} \hat{\mathbf{y}}\right)+\omega_{\mathcal{B}, \mathrm{z}}\left(\hat{\rho}_{\mathcal{B}} \mathbf{r}_{\mathrm{b}_{j} \mid \mathrm{b}_{i}}-\hat{\gamma}_{\mathcal{B}} \hat{\mathbf{z}} \times \mathbf{r}_{\mathrm{b}_{j} \mid \mathrm{b}_{i}}+\hat{\mathbf{z}} \times \mathbf{r}_{\mathrm{b}_{k} \mid \mathrm{b}_{i}}\right)
$$

Using equation (c), any quasi-velocity of the model of $\mathscr{B}$ can be expressed as a function of $\left(v_{\mathcal{B}, \mathrm{x}}, v_{\mathcal{B}, \mathrm{y}}, \omega_{\mathcal{B}, \mathrm{z}}\right)$. Thus, $\boldsymbol{S}_{\mathscr{B}}$ can be expressed as follows:

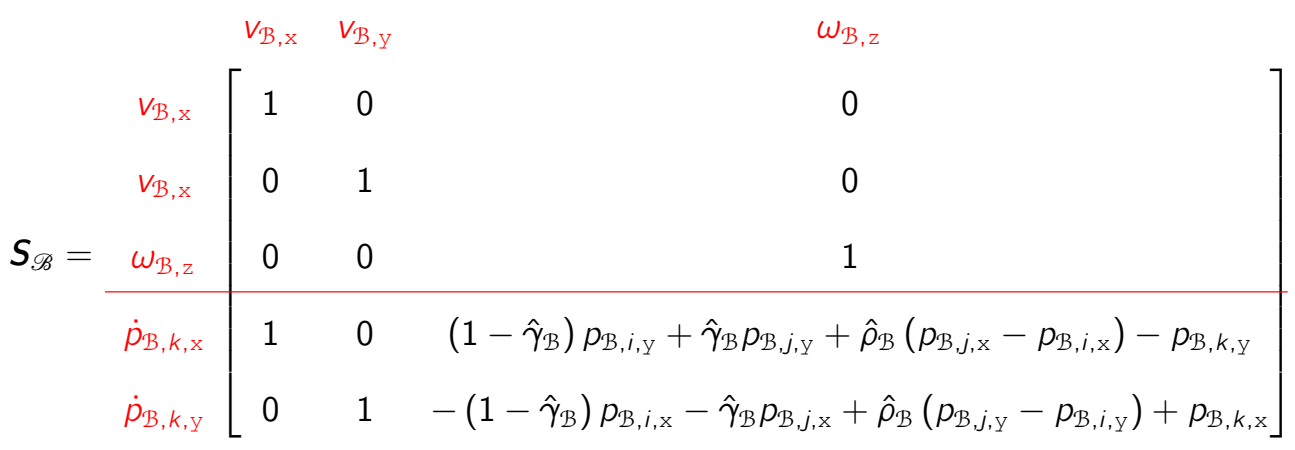


The last 2 rows shown in matrix $S_{\mathscr{B}}$ actually represent the general expression of the rows that correspond to a generic node $b_{k}$, i.e., there must be a pair of rows like these for each node used in the model of $\mathscr{B}$.

\subsection{Descriptions of motion in modular multibody formulations}

The definitions and results presented along this chapter are valid for any finite degree-of-freedom multibody system. However, in order to propose modular formulations which are able to use already known mathematical models of subsystems to derive the equations of a multibody system, some extra statements and techniques must be discussed.

First of all, it must be noticed that whenever the modular approach is used, a multibody system can be conceived as a hierarchical structure of subsystems that can be represented by a tree diagram. Consider for instance the models of a bicycle $(\mathscr{W})$, a 5-bar mechanism $(\mathscr{P})$ and a 3 RRR mechanism $(\mathcal{Q})$ presented in Figures 2.6, 2.7 and 2.8, respectively. The bicycle model is conceived as a tree structure with 3 levels: the root (level 0) corresponds to the bicycle model $\mathscr{W}$ itself; the first level consists of the subsystems of $\mathscr{W}$ which are the rear wheel $\mathscr{R}$, the front wheel $\mathscr{F}$ and the assembly $\mathscr{A}$ constituted by the body of the rider and by the frame, handlebar and front fork of the bicycle; the second level, in this example, consists of the subsystems of $\mathscr{A}$, which are the system $\mathscr{B}$ constituted by the body of the rider and the frame of the bicycle and the system $\mathscr{H}$ constituted by the handlebar and front fork of it. The 5-bar mechanism model $\mathscr{P}$ is conceived as a tree structure with 4 levels: the root corresponds to the system itself, the first level consists of its subsystems, which are the left and right actuators, $\mathscr{A}_{\mathrm{L}}$ and $\mathscr{A}_{\mathrm{R}}$ respectively, and the mechanism $\mathscr{R}$; the second level, is constituted by the subsystems of $\mathscr{R}$, which are the left and right kinematic chains, $\mathscr{H}_{\mathrm{L}}$ and $\mathscr{H}_{\mathrm{R}}$ and the payload $\mathscr{L}$; finally, the third level is constituted by the bars $\mathscr{U}_{\mathrm{L}}, \mathscr{B}_{\mathrm{L}}, \mathscr{U}_{\mathrm{R}}$ and $\mathscr{B}_{\mathrm{R}}$ which are subsystems of the chains $\mathscr{H}_{\mathrm{L}}$ and $\mathscr{H}_{\mathrm{R}}$. A similar hierarchical structure is presented to model the $3 \underline{R} R R$ mechanism $Q$, with the diference that in this case the actuators are supposed to be subsystems of the corresponding kinematic chains.

Let $\mathscr{M}_{n}$ denote a generic system at the $n$-th level of a tree structure. For instance, for the bicycle model, $\mathscr{M}_{0}=\mathscr{W}, \mathscr{M}_{1} \in\{\mathscr{A}, \mathscr{R}, \mathscr{F}\}$ and $\mathscr{M}_{2} \in\{\mathscr{B}, \mathscr{H}\} ;$ for the 5-bar mechanism model, $\mathscr{M}_{0}=\mathscr{P}, \mathscr{M}_{1} \in\left\{\mathscr{A}_{\mathrm{L}}, \mathscr{A}_{\mathrm{R}}, \mathscr{R}\right\}, \mathscr{M}_{2} \in\left\{\mathscr{H}_{\mathrm{L}}, \mathscr{H}_{\mathrm{R}}, \mathscr{L}\right\}$ 


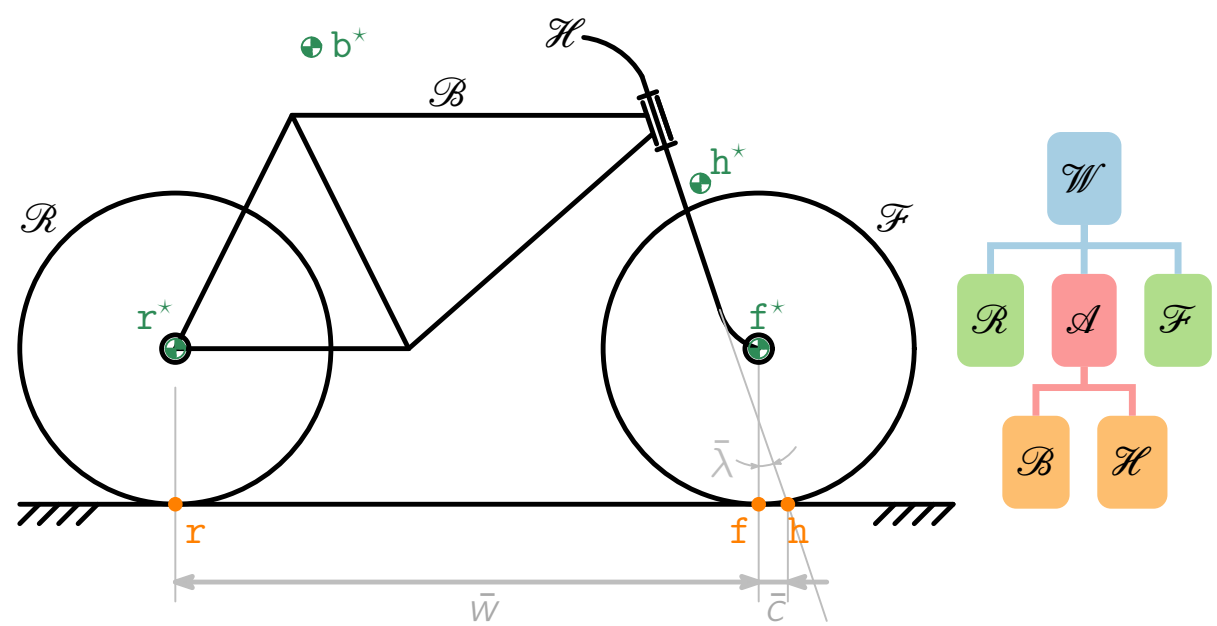

Figure 2.6: Representation of the model of a bicycle, $\mathscr{W}$. Adapted from Orsino and Hess-Coelho (2015).
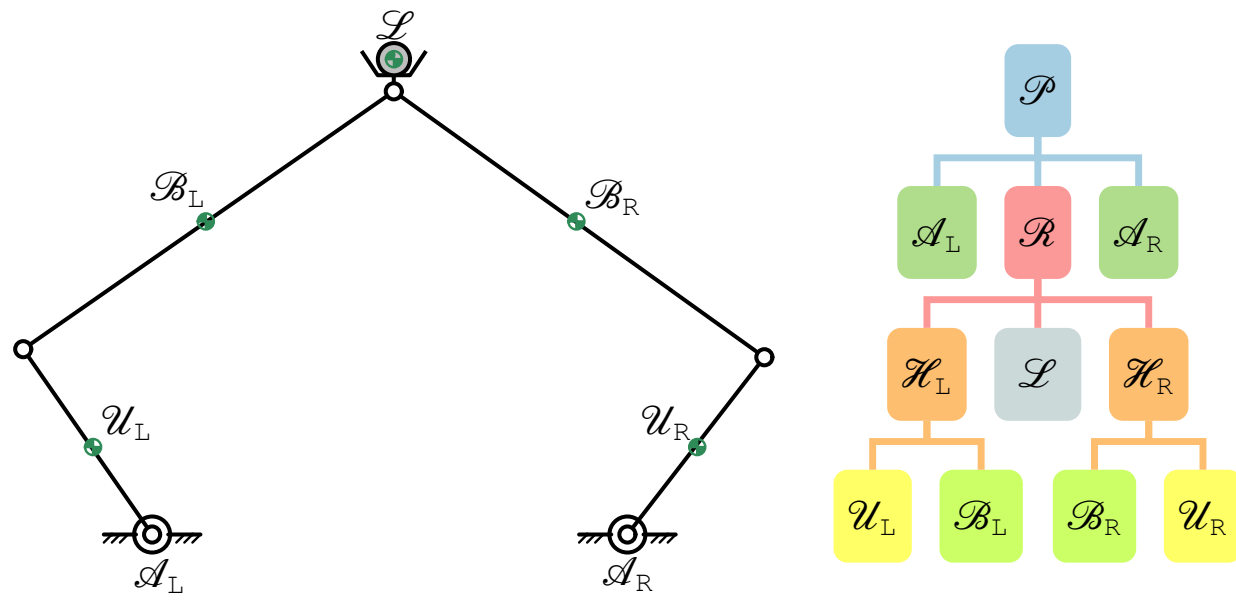

Figure 2.7: Representation of the model of a 5-bar mechanism, $\mathscr{P}$.

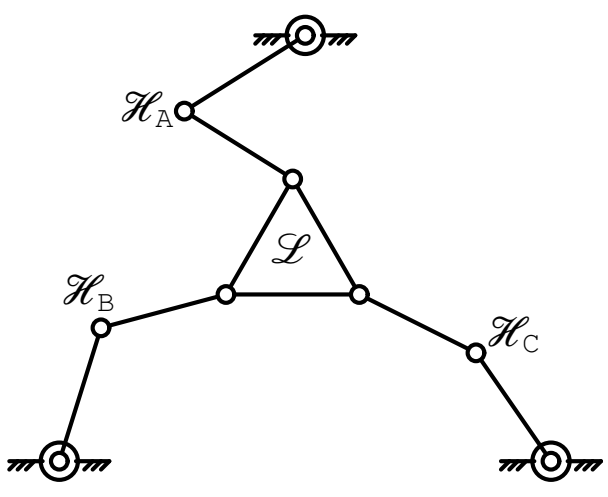

Q

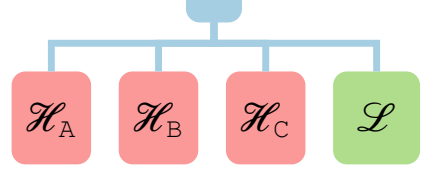

$\mathscr{H}_{\mathrm{K}}$

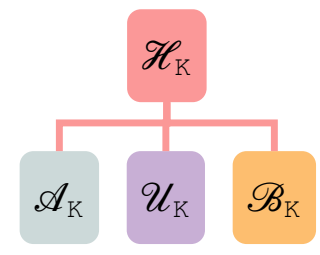

Figure 2.8: Representation of the model of a 3RRR mechanism, $Q$.

and $\mathscr{M}_{3} \in\left\{\mathscr{U}_{\mathrm{L}}, \mathscr{B}_{\mathrm{L}}, \mathscr{U}_{\mathrm{R}}, \mathscr{B}_{\mathrm{R}}\right\}$; finally, for the $3 \underline{R} \mathrm{RR}$ mechanism model, $\mathscr{M}_{0}=\mathscr{Q}$, $\mathscr{M}_{1} \in\left\{\mathscr{H}_{\mathrm{A}}, \mathscr{H}_{\mathrm{B}}, \mathscr{H}_{\mathrm{C}}, \mathscr{L}\right\}$ and $\mathscr{M}_{2} \in\left\{\mathscr{A}_{\mathrm{K}}, \mathscr{U}_{\mathrm{K}}, \mathscr{B}_{\mathrm{K}} \mid \mathrm{K} \in\{\mathrm{A}, \mathrm{B}, \mathrm{C}\}\right\}$. Also, denote by $\mathbb{S}\left(\mathscr{M}_{n}\right)$ the set of all subsystems of $\mathscr{M}_{n}$, i.e. $\mathscr{M}_{n+1} \in \mathbb{S}\left(\mathscr{M}_{n}\right)$ if and only if $\mathscr{M}_{n+1}$ is a subsystem of $\mathscr{M}_{n}$. For example, in the 5-bar mechanism model, $\mathbb{S}(\mathscr{R})=\left\{\mathscr{H}_{\mathrm{L}}, \mathscr{H}_{\mathrm{R}}, \mathscr{L}\right\}$ and $\mathbb{S}\left(\mathscr{H}_{\mathrm{R}}\right)=\left\{\mathscr{U}_{\mathrm{R}}, \mathscr{B}_{\mathrm{R}}\right\}$, whereas in the 3 RRR mechanism 
model, $\mathbb{S}\left(\mathscr{H}_{\mathrm{K}}\right)=\left\{\mathscr{A}_{\mathrm{K}}, \mathscr{U}_{\mathrm{K}}, \mathscr{B}_{\mathrm{K}}\right\}$ for all $\mathrm{K} \in\{\mathrm{A}, \mathrm{B}, \mathrm{C}\}$.

Let $\boldsymbol{q}_{\mathscr{M}_{n}}^{\langle\alpha\rangle}$ denote the tuple of $\alpha$-th order generalized variables used in the description of motion of $\mathscr{M}_{n}$. Such variables either come from the model of a subsystem of $\mathscr{M}_{n}$ or are extra variables, defined exclusively for modeling this system. Denote by $\boldsymbol{q}_{\mathscr{M}_{n} . \mathscr{M}_{n+1}}^{\left\langle\left\langle{ }_{n}\right.\right.}$ the subtuple of $\boldsymbol{q}_{\mathscr{M}_{n}}^{\langle\alpha\rangle}$ constituted by the $\alpha$-th order generalized variables of the model $\mathscr{M}_{n}$ that come from the subsystem $\mathscr{M}_{n+1}$, i.e. $\boldsymbol{q}_{\mathscr{M}_{n} . \mathscr{M}_{n+1}}^{\langle\alpha\rangle}=\boldsymbol{q}_{\mathscr{M}_{n+1}}^{\langle\alpha\rangle}$ for all $\mathscr{M}_{n+1} \in \mathbb{S}\left(\mathscr{M}_{n}\right)$. Also, let $\boldsymbol{q}_{\mathscr{M}_{n} \oplus \oplus}^{\langle\alpha\rangle}$ denote the subtuple corresponding to extra $\alpha$-th order generalized variables. Moreover, consider that $\overline{\boldsymbol{q}}_{\mathscr{M}_{n}}^{\langle\alpha\rangle}$ denote the tuple constituted by $\alpha$-th order generalized constraint invariants of $\mathscr{M}_{n}$. These invariants describe either internal constraints of some subsystem of $\mathscr{M}_{n}$ or constraints among these subsystems, which might be referred to as external constraints. Thus, analogously, the subtuples $\overline{\boldsymbol{q}}_{\mathscr{M}_{n} . \mathscr{M}_{n+1}}^{\langle\alpha\rangle}$ are be defined by $\overline{\boldsymbol{q}}_{\mathscr{M}_{n} . \mathscr{M}_{n+1}}^{\langle\alpha\rangle}=\overline{\boldsymbol{q}}_{\mathscr{M}_{n+1}}^{\langle\alpha\rangle}$ for all $\mathscr{M}_{n+1} \in \mathbb{S}\left(\mathscr{M}_{n}\right)$, whereas the subtuple $\overline{\boldsymbol{q}}_{\mathscr{M}_{n, \oplus}}^{\langle\alpha\rangle}$ is constituted by the external constraints of $\mathscr{M}_{n}$. Summing up, it can be stated that:

$$
\begin{array}{ll}
\boldsymbol{q}_{\mathscr{M}_{n}}^{\langle\alpha\rangle}=\left(\boldsymbol{q}_{\mathscr{M}_{n} \oplus}^{\langle\alpha\rangle},\left(\ldots, \boldsymbol{q}_{\mathscr{M}_{n+1}}^{\langle\alpha\rangle}, \ldots\right)\right) & \forall \mathscr{M}_{n+1} \in \mathbb{S}\left(\mathscr{M}_{n}\right) \\
\overline{\boldsymbol{q}}_{\mathscr{M}_{n}}^{\langle\alpha\rangle}=\left(\overline{\boldsymbol{q}}_{\mathscr{M}_{n} \oplus}^{\langle\alpha\rangle},\left(\ldots, \overline{\boldsymbol{q}}_{\mathscr{M}_{n+1}}^{\langle\alpha\rangle}, \ldots\right)\right) & \forall \mathscr{M}_{n+1} \in \mathbb{S}\left(\mathscr{M}_{n}\right)
\end{array}
$$

Suppose that the constraint order of $\mathscr{M}_{n}$ is equal to $\nu_{\mathscr{M}_{n}}^{\circ}$ and choose any integer $\sigma \geq \nu_{\mathscr{M}_{n}}^{\circ}$. It is worth noting that the Jacobian matrix of the $\sigma$-th order generalized constraint invariants with respect to the $\sigma$-th order generalized variables can be written, apart from the rows corresponding to the external constraints, as a block-diagonal matrix. Indeed, consider for instance the model of the 5-bar mechanism presented in Figure 2.7; the Jacobian $\boldsymbol{A}_{\mathscr{R}}$ can be ex- 
pressed as follows ${ }^{10}$ :

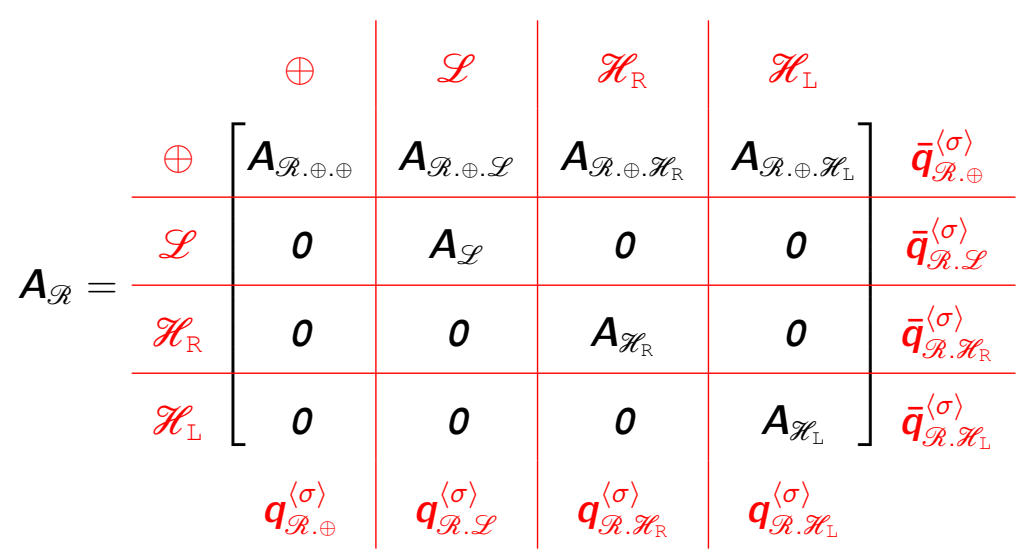

Moreover, $\boldsymbol{A}_{\mathscr{H}_{\mathrm{K}}}$ for $\mathrm{K} \in\{\mathrm{R}, \mathrm{L}\}$ can be expressed as follows:

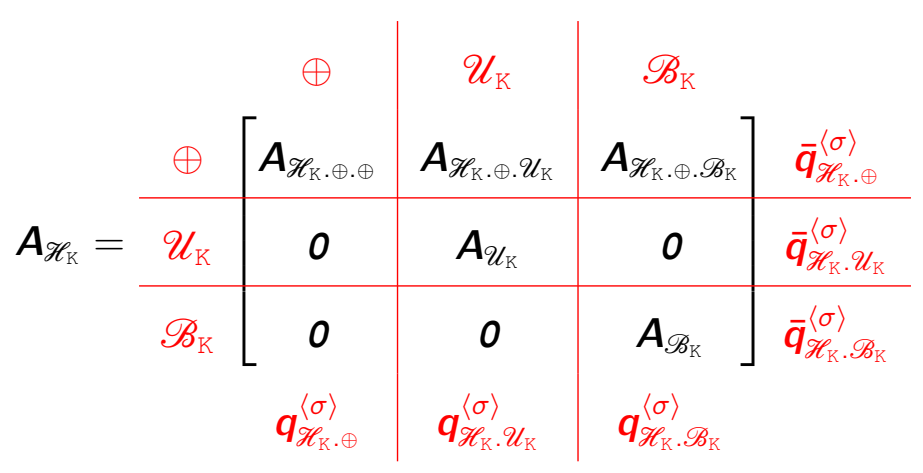

Definition 2.9. Let $X, Y$ and $Z$ denote arbitrary matrices for which it is meaningful to label the rows and columns. Such matrices will be shortly referred as labeled matrices. Define $W=(X, Y)$ as the labeled matrix defined according to the following rules:

(1) The sets of labels identifying the rows and columns of $W$ are the union of the respective sets of labels of $X$ and $Y$.

(2) Let $i$ denote a row label and $j$ a column label of $W$. The entry of $W$ corresponding to these labels is denoted by $w_{. i . j}$. Assume that if either $i$ is not a row label or $j$ is not a column label of $X$, then $X_{. i . j}=0$. Do an analogous assumption for $Y$.

(3) Define $\boldsymbol{W}_{. i . j}=\boldsymbol{X}_{. i . j}+\boldsymbol{Y}_{. i . j}$.

It can be noticed that the "tuples of labeled matrices" can be interpreted as an operator with the following properties:

\footnotetext{
${ }^{10}$ In this example the row labels are presented both in the left and right sides of the matrix: the left ones corresponds to the "shorthand forms", while the right ones are the "full forms", which shown in this example only to clarify what the labels stand for. A similar consideration can be done about the column labels which are shown above in "shorthand form" and below in "full form".
} 
- $(X, Y)=(Y, X)$

$\cdot(X, Y, Z)=((X, Y), Z)=(X,(Y, Z))$

Theorem 2.10. Let $\mathscr{M}_{n}$ be a $\nu_{\mathscr{M}_{n}}^{\#}$-DOF system in the $n$-th level of a hierarchical structure representing the model of a multibody system whose constraint order is equal to $\nu^{\circ}$. Choose an integer $\sigma \geq \nu^{\circ}$ and consider that in a given time instant $t^{*}$ the values of $\boldsymbol{q}_{\mathscr{M}_{n}}^{\langle\sigma-1\rangle}\left(t^{*}\right)$ are known, do not violate any constraint of $\mathscr{M}_{n}$ and do not correspond to a singular configuration of $\mathscr{M}_{n}$. Adopt $\delta \boldsymbol{q}_{\mathscr{U}_{n}}^{\langle\sigma-1\rangle}\left(t^{*}\right)=$ o. Suppose that, if $\mathscr{M}_{n}$ has any subsystem, for every $\mathscr{M}_{n+1} \in \mathbb{S}\left(\mathscr{M}_{n}\right)$ both the Jacobian matrix $\boldsymbol{A}_{\mathscr{U}_{n+1}}$ of the $\sigma$-th order generalized constraint invariants of $\mathscr{M}_{n+1}$ with respect to its $\sigma$-th order generalized variables and an orthogonal complement $S_{\mathscr{U}_{n+1}}$ of this Jacobian are known. Define matrix $R_{\mathscr{M}_{n}}$ as follows:

$$
R_{\mathscr{U}_{n}}=\left(R_{\mathscr{U}_{k} \oplus \oplus \oplus}\left(\ldots, S_{\mathscr{U}_{k+1}}, \ldots\right)\right) \text { with } R_{\mathscr{M}_{k} \oplus \oplus \oplus}=1, \forall \mathscr{M}_{n+1} \in \mathbb{S}\left(\mathscr{M}_{n}\right)
$$

Let $C_{\mathscr{U}_{n}}$ be an orthogonal complement of $B_{\mathscr{M}_{n}}=\boldsymbol{A}_{\mathscr{U}_{n} \oplus} \boldsymbol{R}_{\mathscr{U}_{n}}$ with $\boldsymbol{A}_{\mathscr{U}_{n} \oplus}$ representing the Jacobian matrix of the $\sigma$-th order external generalized constraint invariants of $\mathscr{M}_{n}, \overline{\boldsymbol{q}}_{\left.\mathcal{M}_{n \cdot \oplus}\right\rangle}^{\langle\sigma\rangle}$, with respect to its $\sigma$-th order generalized variables, $\boldsymbol{q}_{\mathscr{M}_{n}}^{\langle\sigma\rangle}$. It can be stated that a matrix $S_{\mathscr{M}_{n}}$ satisfying the conditions of Theorem 2.8 can be obtained by the following expression:

$$
S_{\mathscr{M}_{n}}=R_{\mathscr{M}_{n}} C_{\mathscr{M}_{n}}
$$

Proof. It must be proved that the nullity of $B_{\mathcal{M}_{n}}$ is equal to $\nu_{\mathscr{M}_{n}}^{\#}$ and consequently, the ranks of both $C_{\mathscr{M}_{n}}$ and $S_{\mathscr{M}_{n}}$ will be equal to $\nu_{\mathscr{M}_{n}}^{\#}$ too. Consider, without loss of generality, that $\mathscr{M}_{n}$ has $\kappa$ subsystems denoted by $\mathscr{M}_{n+1}^{[\iota]}$, with $\iota$ being an integer index and $1 \leq \iota \leq \kappa$. In this case, $\boldsymbol{A}_{\mathscr{M}_{n}}$ and $\boldsymbol{R}_{\mathscr{M}_{n}}$ can be expressed by:

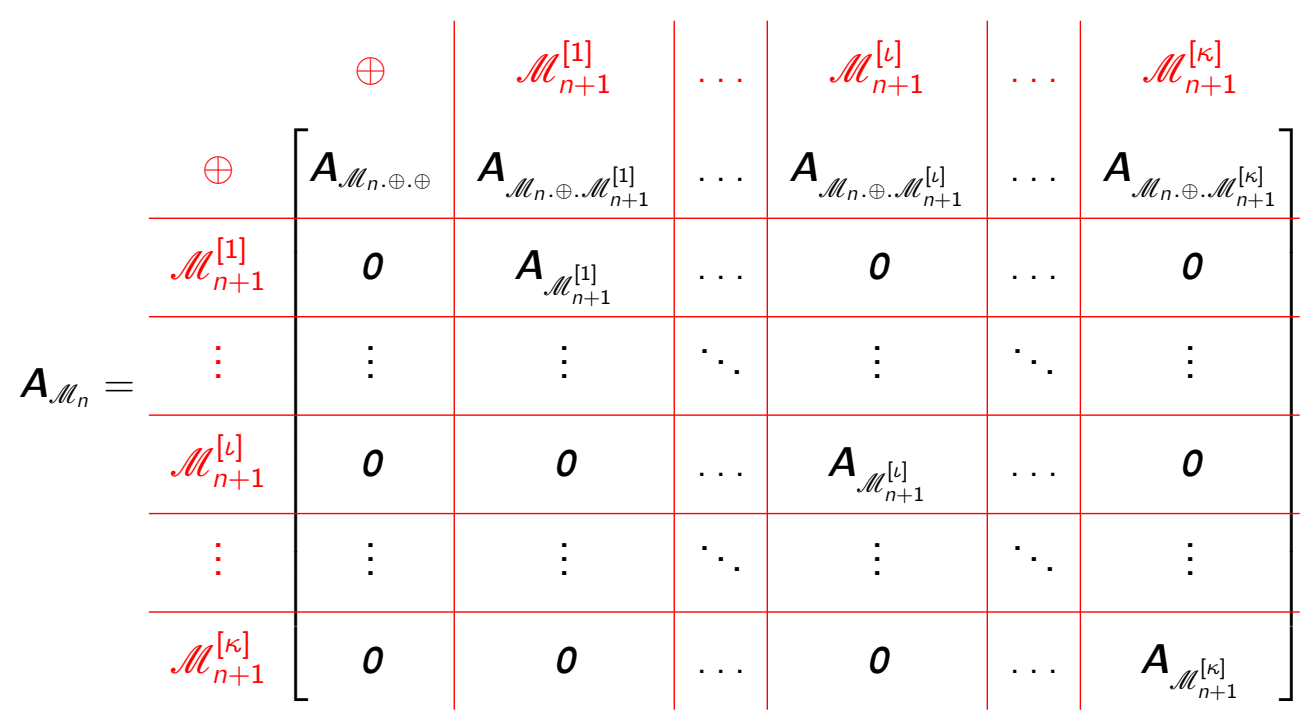




$$
\left.\begin{array}{c|c|c|c|c|c|c}
\oplus & \mathscr{M}_{n+1}^{[1]} & \ldots & \mathscr{M}_{n+1}^{[\iota]} & \ldots & \mathscr{M}_{n+1}^{[\kappa]} \\
\hline \boldsymbol{R}_{\mathscr{M}_{n}}= & \mathbf{1} & 0 & \ldots & 0 & \ldots & 0 \\
\hline \mathscr{M}_{n+1}^{[1]} & 0 & S_{\mathscr{M}_{n+1}^{[1]}} & \ldots & 0 & \ldots & 0 \\
\hline \vdots & \vdots & \vdots & \ddots & \vdots & \ddots & \vdots \\
\hline \mathscr{M}_{n+1}^{[\iota]} & 0 & 0 & \ldots & S_{\mathscr{M}_{n+1}^{[l]}} & \ldots & 0 \\
\hline \vdots & \vdots & \vdots & \ddots & \vdots & \ddots & \vdots \\
\hline \mathscr{M}_{n+1}^{[\kappa]} & 0 & 0 & \ldots & 0 & \ldots & S_{\mathscr{M}_{n+1}^{[\kappa]}}
\end{array}\right]
$$

Considering that, by hypothesis $S_{\mathscr{M}_{n+1}^{[l]}}$ is an orthogonal complement of $A_{\mathscr{M}_{n+1}^{[l]}}$, then $A_{\mathscr{M}_{n+1}^{[l]}} S_{\mathscr{M}_{n+1}^{[l]}}=0$. Thus:

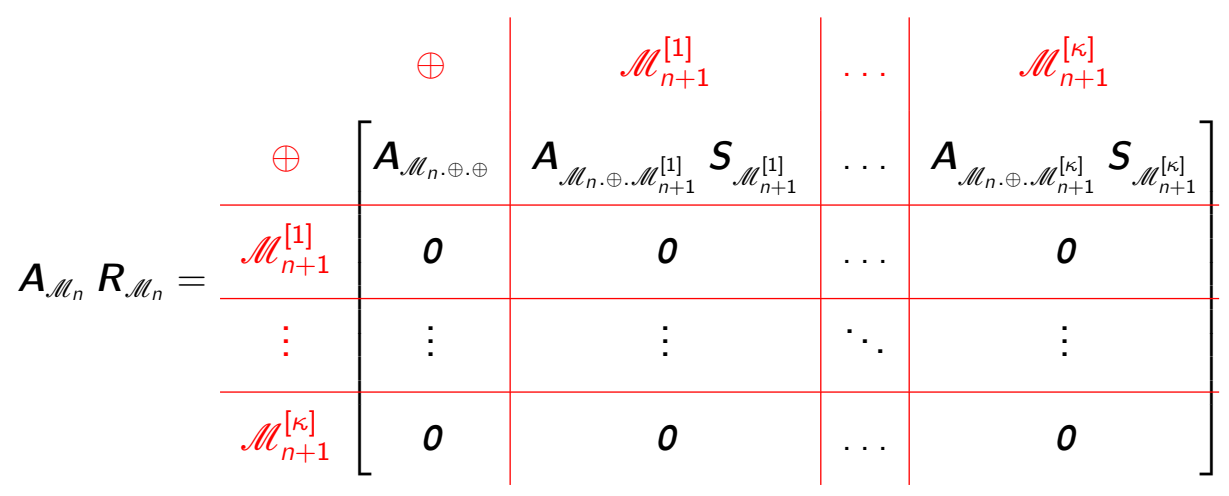

Therefore it can be stated that:

i. Every variation $\delta \boldsymbol{q}_{\mathscr{M}_{n}}^{\langle\sigma\rangle}$ lying in the image of the linear transformation defined by the matrix $R_{\mathscr{M}_{n}}$ is compatible with all the internal constraints of $\mathscr{M}_{n}$ (once all the rows corresponding to internal constraint invariants in $\boldsymbol{A}_{\mathscr{M}_{n}} \boldsymbol{R}_{\mathscr{M}_{n}}$ are identically zero).

ii. Moreover, considering that $R_{\mathscr{M}_{n}}$ is a block-diagonal matrix and analyzing the statement of Theorem 2.8, it can be noticed that $R_{\mathscr{M}_{n}}$ is a maximal rank matrix with such property. Thus, every variation $\delta \boldsymbol{q}_{\mathscr{M}_{n}}^{\langle\sigma\rangle}$ compatible with all the constraints of $\mathscr{M}_{n}$ (both internal and external) must lie in the image of $R_{\mathscr{M}_{n}}$.

iii. If $x$ lies in the kernel of $A_{\mathscr{M}_{n}} R_{\mathscr{M}_{n}}$ then $y=R_{\mathscr{M}_{n}} x$ will lie in the kernel of $\boldsymbol{A}_{\mathscr{M}_{n}}$. By the previous statement, the converse is also true, i.e., if $y$ lies in the kernel of $\boldsymbol{A}_{\mathscr{M}_{n}}$ then there must exist a column-matrix $x$ such that $y=R_{\mathscr{M}_{n}} x$. Thus, the dimension of the kernel of $\boldsymbol{A}_{\mathscr{M}_{n}}$ (which, according to Definition 2.5, is $\nu_{\mathscr{M}_{n}}^{\#}$ ) is equal to the dimension of the kernel of $\boldsymbol{A}_{\mathscr{M}_{n}} \boldsymbol{R}_{\mathscr{M}_{n}}$. 
iv. As it can be noticed in equation (c), apart from the rows corresponding to the external constraint invariants, all the remaing rows of $A_{\mathscr{M}_{n}} \boldsymbol{R}_{\mathscr{M}_{n}}$ are identically zero. Then, the kernel of $A_{\mathscr{M}_{n}} R_{\mathscr{M}_{n}}$ is identical to the kernel of $B_{\mathscr{M}_{n}}=\boldsymbol{A}_{\mathscr{M}_{n} \oplus} \boldsymbol{R}_{\mathscr{M}_{n}}$.

Finally, if $C_{\mathscr{M}_{n}}$ is an orthogonal complement of $B_{\mathscr{M}_{n}}$, its rank is equal to $\nu_{\mathscr{M}_{n}}^{\#}$ and, according to statement iii, $S_{\mathscr{M}_{n}}=R_{\mathscr{M}_{n}} C_{\mathscr{M}_{n}}$ will be a rank $\nu_{\mathscr{M}_{n}}^{\#}$ matrix satisfying the conditions of Theorem 2.8 for the multibody system $\mathscr{M}_{n}$.

In order to be able to use the same notation for multibody systems $\mathscr{M}_{n}$ that have no subsystems (which are the leaves in the tree structures), it is enough to define $R_{\mathscr{M}_{n}}=1$. In this case $C_{\mathscr{M}_{n}}$ will be defined as an orthogonal complement of $B_{\mathscr{M}_{n}}=A_{\mathscr{M}_{n} \oplus} R_{\mathscr{M}_{n}}=A_{\mathscr{M}_{n} \oplus}=A_{\mathscr{M}_{n}}$, and $S_{\mathscr{M}_{n}}=R_{\mathscr{M}_{n}} C_{\mathscr{M}_{n}}=C_{\mathscr{M}_{n}}$. It will be shown in Chapter 4 that matrices $C_{\mathscr{M}_{n}}$ have a key role in the derivation of the equations of motion of a multibody system when the proposed modular modeling methodology is used.

Example 2.7. Consider the 5-bar mechanism $(\mathscr{P})$ presented in Figure 2.7. In order to model it according to the hierarchical structure shown in the tree diagram, it is first of all necessary to describe the motions of the subsystems $\mathscr{A}_{\mathrm{L}}$, $\mathscr{A}_{\mathrm{R}}, \mathscr{L}, \mathscr{U}_{\mathrm{L}}, \mathscr{U}_{\mathrm{R}}, \mathscr{B}_{\mathrm{L}}$ and $\mathscr{B}_{\mathrm{R}}$, which are the leaves of the tree. For this system, a left-right symmetry is supposed, i.e. subsystems $\mathscr{A}_{\mathrm{L}}$ and $\mathscr{A}_{\mathrm{R}}$, for instance, have identical mathematical models, which will simply be denoted by $\mathscr{A}_{\mathrm{K}}$, the same happening to the pair of subsystems $\mathscr{U}_{\mathrm{L}}$ and $\mathscr{U}_{\mathrm{R}}$ (model $\mathscr{U}_{\mathrm{K}}$ ), as well as to $\mathscr{B}_{\mathrm{L}}$ and $\mathscr{B}_{\mathrm{R}}\left(\right.$ model $\left.\mathscr{B}_{\mathrm{K}}\right)$.

Actuators $\mathscr{A}_{\mathrm{L}}$ and $\mathscr{A}_{\mathrm{R}}$. Let $\omega_{\mathrm{K}}$ denote the angular speed of the rotation motion of the axis of the actuator $\mathscr{A}_{\mathrm{K}}$ and $i_{\mathrm{K}}$ denote the electrical current in the armature circuit of this actuator. Adopt $\boldsymbol{q}_{\mathscr{A}_{\mathrm{K}}}^{\langle 1\rangle}=\left(\omega_{\mathrm{K}}, i_{\mathrm{K}}\right)$. No generalized constraint invariants are needed for this selection of variables.

Bars $\mathscr{U}_{\mathrm{L}}$ and $\mathscr{U}_{\mathrm{R}}$. Following the analysis shown in Example 2.6, the positions of the centers of the two revolute joints defined by $\mathcal{U}_{\mathrm{K}}$ and $\mathscr{A}_{\mathrm{K}}$ and by $\mathscr{U}_{\mathrm{K}}$ and $\mathscr{B}_{\mathrm{K}}$ are enough to fully specify any of its configurations. However, since the former center remains fixed with respect to the mechanism base, only the Cartesian coordinates of the latter are enough as generalized coordinates of $\mathcal{U}_{\mathrm{K}}$, i.e. $\boldsymbol{q}_{\mathcal{U}_{\mathrm{K}}}^{\langle 0\rangle}=\left(p_{\mathcal{U}_{\mathrm{K}}, 2, \mathrm{x}}, p_{\mathcal{U}_{\mathrm{K}}, 2, \mathrm{Y}}\right)$. Also, following Example 2.6, it 
can be stated that if $\boldsymbol{q}_{\mathcal{U}_{\mathrm{K}}}^{\langle 1\rangle}=\left(v_{\mathcal{U}_{\mathrm{K}}, \mathrm{x}}, v_{\mathcal{U}_{\mathrm{K}, \mathrm{Y}}}, \omega_{\mathcal{U}_{\mathrm{K}, \mathrm{z}}}, \dot{p}_{\mathcal{U}_{\mathrm{K}, 2, \mathrm{x}}}, \dot{p}_{\mathcal{U}_{\mathrm{K}, 2, \mathrm{Y}}}\right)$, then:

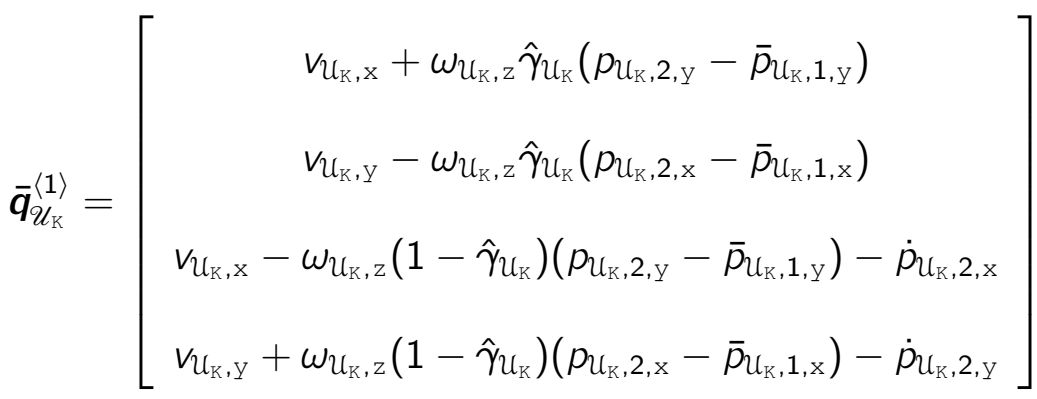

$$
\begin{aligned}
& \omega_{\mathcal{U}_{K}, Z}
\end{aligned}
$$

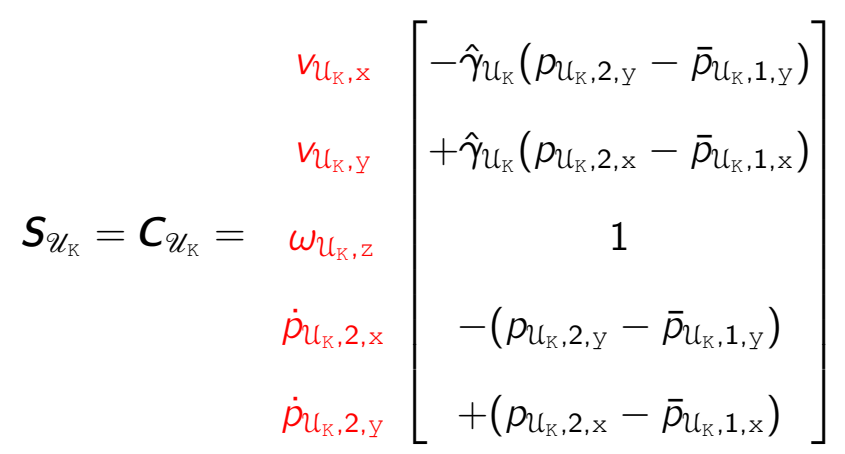

Bars $\mathscr{B}_{\mathrm{L}}$ and $\mathscr{B}_{\mathrm{R}}$. Again, the models for these subsystems are based on Example 2.6. Two nodes, coinciding with the corresponding centres of the revolute joints, are chosen. Thus, $\boldsymbol{q}_{\mathscr{B}_{\mathrm{K}}}^{\langle 0\rangle}=\left(p_{\mathcal{B}_{\mathrm{K}}, 1, \mathrm{x}}, p_{\mathcal{B}_{\mathrm{K}}, 1, \mathrm{y}}, p_{\mathcal{B}_{\mathrm{K}}, 2, \mathrm{x}}, p_{\mathcal{B}_{\mathrm{K}}, 2, \mathrm{Y}}\right)$, $\boldsymbol{q}_{\mathscr{B}_{\mathrm{K}}}^{\langle 1\rangle}=\left(v_{\mathcal{B}_{K}, \mathrm{x}}, v_{\mathcal{B}_{K}, Y}, \omega_{\mathcal{B}_{K}, \mathrm{z}}, \dot{p}_{\mathcal{B}_{K}, 1, x}, \dot{p}_{\mathcal{B}_{K}, 1, Y}, \dot{p}_{\mathcal{B}_{K}, 2, \mathrm{x}}, \dot{p}_{\mathcal{B}_{K}, 2, Y}\right)$ and:

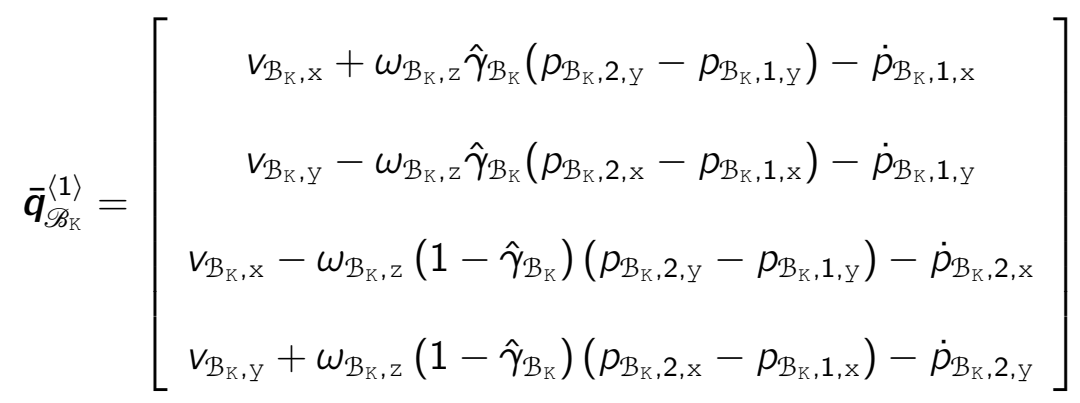

$$
\begin{aligned}
& v_{\mathcal{B}_{\mathrm{K}}, \mathrm{X}} \quad v_{\mathcal{B}_{\mathrm{K}, \mathrm{Y}}} \quad \omega_{\mathcal{B}_{\mathrm{K}}, \mathrm{z}}
\end{aligned}
$$

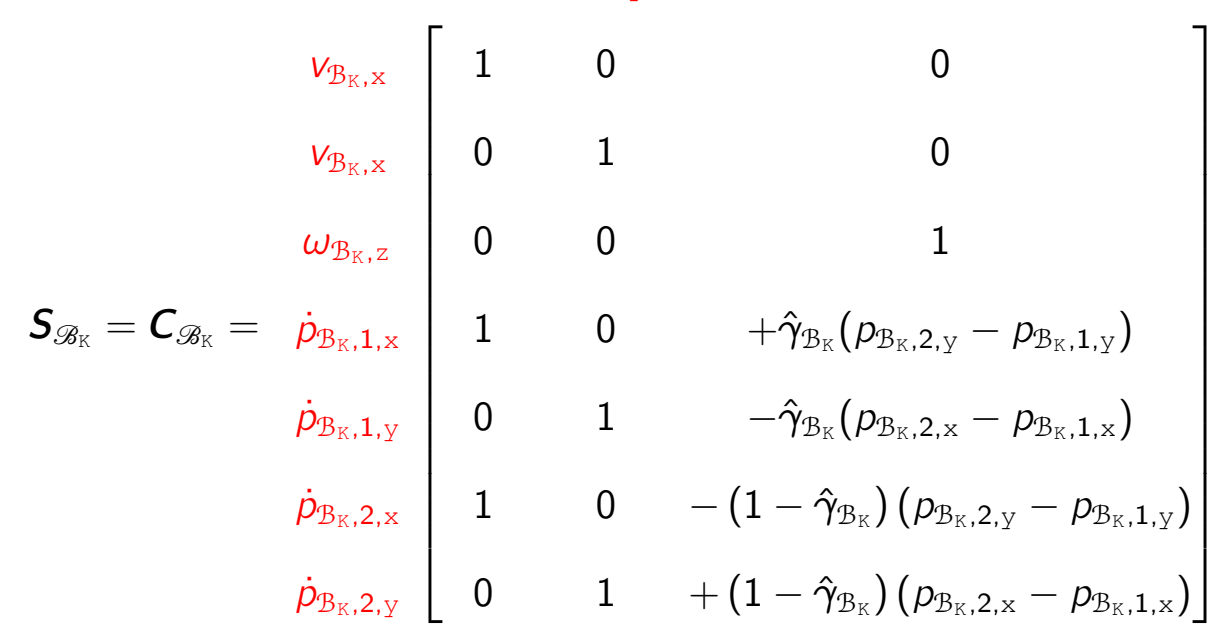


Payload $\mathscr{L}$. The payload will be modelled as a single particle, being two Cartesian coordinates sufficient to describe its configuration. Thus, adopt: $\boldsymbol{q}_{\mathscr{L}}^{\langle 0\rangle}=(x, y)$ and $\boldsymbol{q}_{\mathscr{L}}^{\langle 1\rangle}=(\dot{x}, \dot{y})$. No generalized constraint invariants are needed for this selection of variables.

Kinematic chains $\mathscr{H}_{\mathrm{L}}$ and $\mathscr{H}_{\mathrm{R}}$. A kinematic chain $\mathscr{H}_{\mathrm{K}}$ is constituted by two subsystems, $\mathscr{U}_{\mathrm{K}}$ and $\mathscr{B}_{\mathrm{K}}$. In the modeling of this system, it is convenient to define two extra generalized coordinates: $\theta_{\mathrm{K}}$ representing the angle between the longitudinal direction of the bar $\mathcal{U}_{\mathrm{K}}$ and the horizontal line of the base of the mechanism and $\phi_{\mathrm{K}}$ representing the angle between the longitudinal directions of the bars $\mathcal{B}_{\mathrm{K}}$ and this same horizontal, both measured in the counterclockwise direction (see Figure 2.9). Thus, adopt $\boldsymbol{q}_{\mathscr{H}_{\mathrm{K}} \oplus \oplus}^{\langle 0\rangle}=\left(\theta_{\mathrm{K}}, \phi_{\mathrm{K}}\right)$ and $\boldsymbol{q}_{\mathscr{H}_{\mathrm{K} \cdot \oplus}^{\langle 1\rangle}}^{\langle 1}=\left(\dot{\theta}_{\mathrm{K}}, \dot{\phi}_{\mathrm{K}}\right)$. The invariants associated to the external constraints in this system are given by:

$$
\overline{\boldsymbol{q}}_{\mathscr{\mathcal { H }}_{\mathrm{K}} \oplus}^{\langle 1\rangle}=\left[\begin{array}{c}
\dot{p}_{\mathcal{B}_{\mathrm{K}}, 1, \mathrm{x}}-\dot{p}_{\mathcal{U}_{\mathrm{K}}, 2, \mathrm{x}} \\
\dot{p}_{\mathcal{B}_{\mathrm{K}}, 1, \mathrm{Y}}-\dot{p}_{\mathcal{U}_{\mathrm{K}, 2, Y}} \\
\dot{\theta}_{\mathrm{K}}-\omega_{\mathcal{U}_{\mathrm{K}, \mathrm{Z}}} \\
\dot{\phi}_{\mathrm{K}}-\omega_{\mathcal{B}_{\mathrm{K}}, \mathrm{z}}
\end{array}\right]
$$

Denoting by $\bar{a}_{\mathcal{U}_{K}}$ and $\bar{a}_{\mathcal{B}_{K}}$ the lengths of bars $\mathcal{U}_{\mathrm{K}}$ and $\mathcal{B}_{\mathrm{K}}$, it can be stated that, using the new coordinates defined:

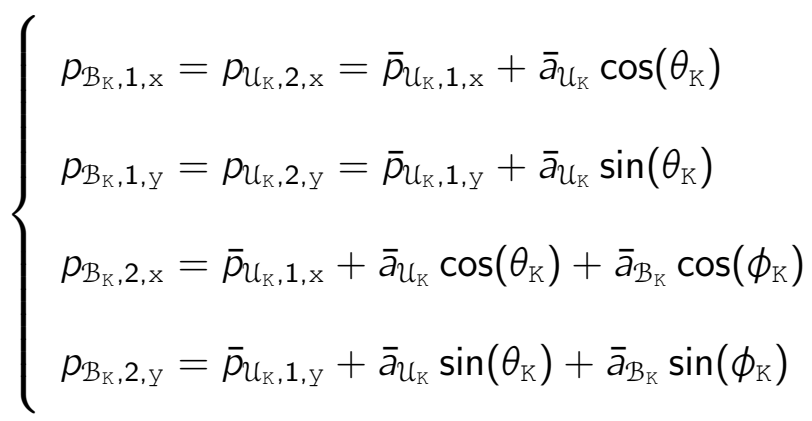


Thus, expressions for $A_{\mathscr{H}_{\mathrm{K} . \oplus}} \boldsymbol{R}_{\mathscr{H}_{\mathrm{K}}}$ and and $\boldsymbol{C}_{\mathscr{H}_{\mathrm{K}}}$ are given by:

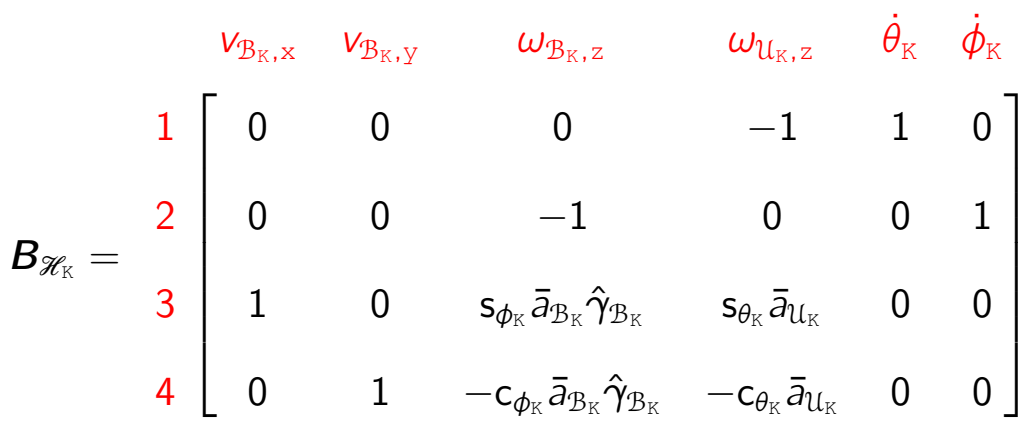

$$
\begin{aligned}
& C_{\mathscr{H}_{\mathrm{K}}}=\begin{array}{cc}
\dot{\theta}_{\mathrm{K}} & \dot{\phi}_{\mathrm{K}} \\
v_{\mathcal{B}_{\mathrm{K}}, \mathrm{x}} \\
v_{\mathcal{B}_{\mathrm{K}}, \mathrm{Y}} \\
\omega_{\mathcal{B}_{\mathrm{K}}, \mathrm{z}} \\
\omega_{\mathcal{U}_{\mathrm{K}, \mathrm{Z}}} \\
\dot{\theta}_{\mathrm{K}} \\
\dot{\phi}_{\mathrm{K}}
\end{array}\left[\begin{array}{cc}
-\mathrm{s}_{\theta_{\mathcal{K}}} \bar{a}_{\mathcal{U}_{\mathrm{K}}} & -\mathrm{s}_{\phi_{\mathcal{K}}} \bar{a}_{\mathcal{B}_{\mathrm{K}}} \hat{\gamma}_{\mathcal{B}_{\mathrm{K}}} \\
\mathrm{c}_{\theta_{\mathcal{K}}} \bar{a}_{\mathfrak{U}_{\mathrm{K}}} & \mathrm{c}_{\phi_{\mathcal{K}}} \overline{\mathrm{a}}_{\mathcal{B}_{\mathrm{K}}} \hat{\gamma}_{\mathcal{B}_{\mathrm{K}}} \\
0 & 1 \\
1 & 0 \\
1 & 0 \\
0 & 1
\end{array}\right]
\end{aligned}
$$

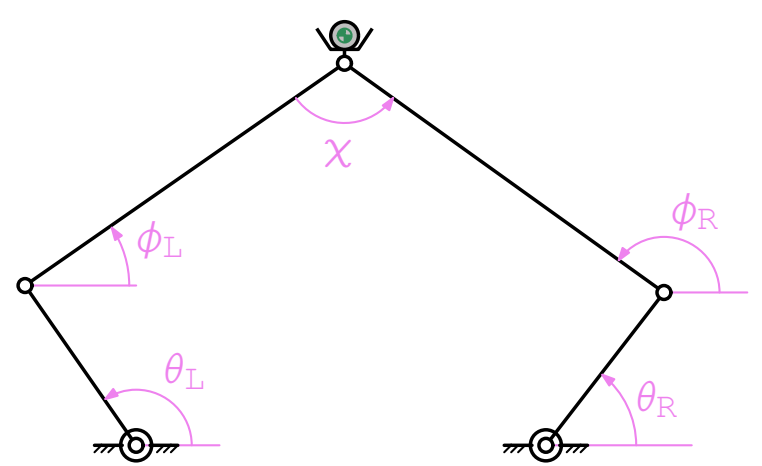

Figure 2.9: Extra generalized coordinates defined in the model of the 5-bar mechanism.

Unactuated 5-bar mechanism $\mathscr{R}$. The unactuated 5-bar mechanism $\mathscr{R}$ is constituted by the subsystems $\mathscr{H}_{\mathrm{L}}, \mathscr{H}_{\mathrm{R}}$ and $\mathscr{L}$. It is convenient to define an extra generalized coordinate $\chi$ representing the angle between the longitudinal directions of $\mathcal{B}_{\mathrm{L}}$ and $\mathcal{B}_{\mathrm{R}}$, measured in the counterclockwise direction (ORSINO; COUTINHO; HESS-COELHO, 2016). Thus, adopt $\boldsymbol{q}_{\mathscr{R} . \oplus}^{\langle 0\rangle}=(\chi)$ and $\boldsymbol{q}_{\mathscr{R} . \oplus}^{\langle 1\rangle}=(\dot{\chi})$. The invariants associated to the external constraints in 
this system are given by:

$$
\overline{\boldsymbol{q}}_{\mathscr{R} . \oplus}^{\langle 1\rangle}=\left[\begin{array}{c}
\dot{p}_{\mathcal{B}_{\mathrm{L}}, 2, \mathrm{x}}-\dot{x} \\
\dot{p}_{\mathcal{B}_{\mathrm{L}}, 2, \mathrm{Y}}-\dot{y} \\
\dot{p}_{\mathcal{B}_{\mathrm{R}}, 2, \mathrm{x}}-\dot{x} \\
\dot{p}_{\mathcal{B}_{\mathrm{R}}, 2, \mathrm{Y}}-\dot{y} \\
\dot{\chi}-\left(\omega_{\mathcal{B}_{\mathrm{R}}, \mathrm{z}}-\omega_{\mathcal{B}_{\mathrm{L}}, \mathrm{z}}\right)
\end{array}\right]
$$

Noting that $\chi=\phi_{\mathrm{R}}-\phi_{\mathrm{L}}$, it can be stated that:

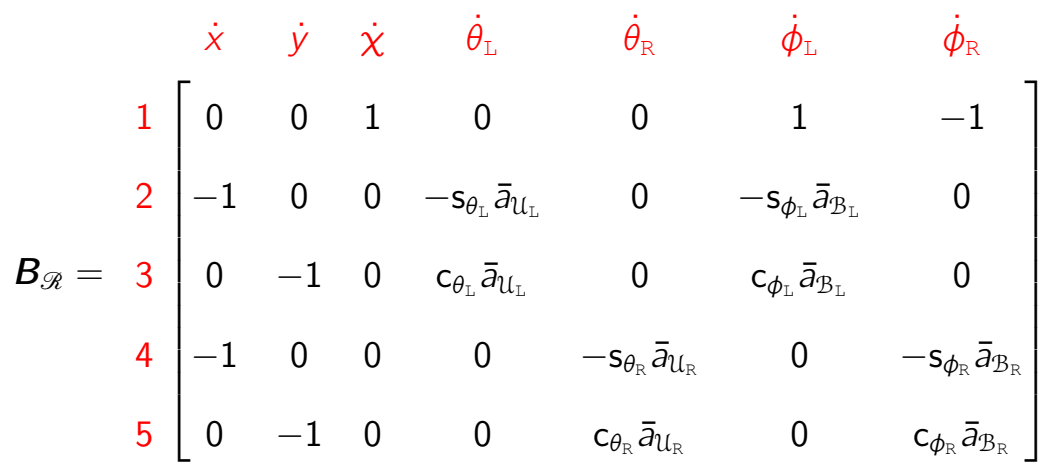

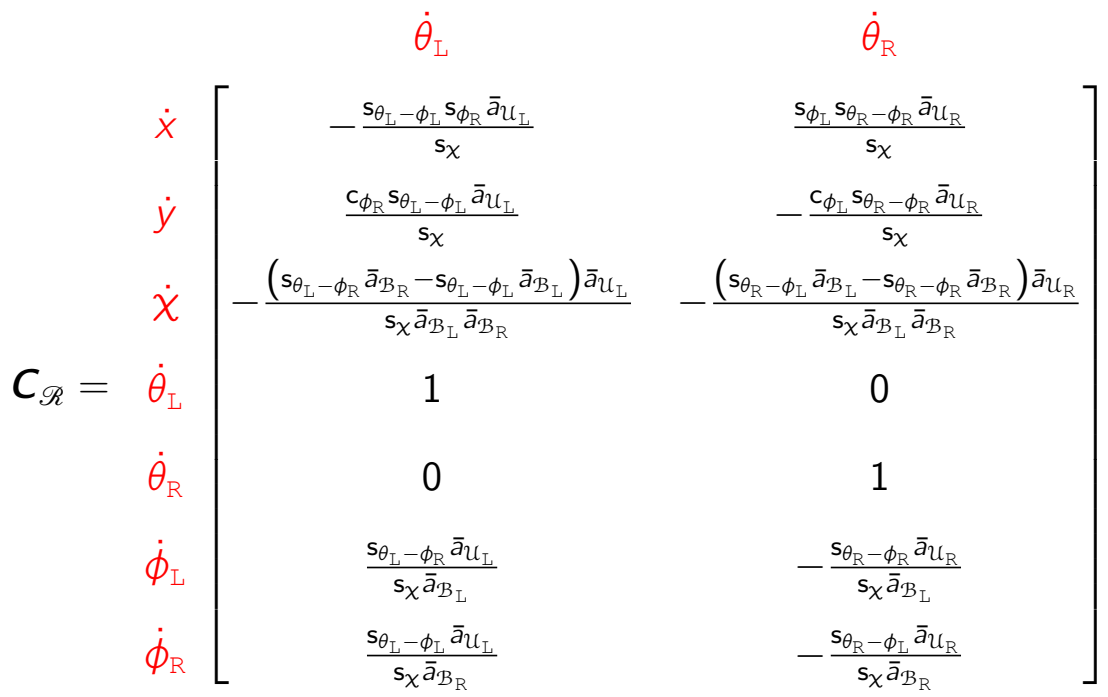

Actuated 5-bar mechanism $\mathscr{P}$. System $\mathscr{P}$ is constituted by the subsystems $\mathscr{A}_{\mathrm{L}}, \mathscr{A}_{\mathrm{R}}$ and $\mathscr{R}$. No extra generalized variables are needed in this final step. Moreover, the invariants associated to the external constraints in this system are given by:

$$
\overline{\boldsymbol{q}}_{\mathscr{P} . \oplus}^{\langle 1\rangle}=\left[\begin{array}{c}
\dot{\theta}_{\mathrm{R}}-\omega_{\mathrm{R}} / \bar{\eta}_{\mathrm{R}} \\
\dot{\theta}_{\mathrm{L}}-\omega_{\mathrm{L}} / \bar{\eta}_{\mathrm{L}}
\end{array}\right]
$$


Therefore, it can be stated that:

$$
\boldsymbol{C}_{\mathscr{P}}=\begin{gathered}
i_{\mathrm{L}} \\
i_{\mathrm{R}} \\
\omega_{\mathrm{L}} \\
\omega_{\mathrm{R}} \\
\dot{\theta}_{\mathrm{L}} \\
\dot{\theta}_{\mathrm{R}}
\end{gathered}\left[\begin{array}{llll}
1 & 0 & 0 & 0 \\
0 & 1 & 0 & 0 \\
0 & 0 & \bar{\theta}_{\mathrm{L}} & 0 \\
0 & 0 & 0 & \bar{\theta}_{\mathrm{R}} \\
0 & 0 & 1 & 0 \\
0 & 0 & 0 & 1
\end{array}\right]
$$

In Chapter 4, it will be shown how the matrices $C$ can be used in a recursive algorithm for obtaining the equations of motion of this system. 


\section{Multibody System Dynamics - a comparative literature review}

A general comparison among methodologies for obtaining the equations of motion of multibody systems points out that they can differ in two fundamental points:

- The approach for including constraint effects in the derivations.

- The techniques used to describe the action of external forces and inertial effects.

The principles underlying such methodologies, however, are just two: they are based either on the principles of Newtonian Mechanics (Newton's laws of motion) or on the fundamental principles of Analytical Mechanics.

The essential difference between the Newtonian and Analytical Mechanics approaches is the concept of force. In the former, forces have a key role on describing the interaction between material bodies (even the constraint effects are modeled as forces), while in the latter, the generalized forces are supposed to be methodological intermediate in the relation between the motion of a body and its interaction with the physical environment as a function of the instantaneous state of the system (JAMMER, 2012; MAUGIN, 2013). In Analytical Mechanics, concepts of work and energy are more important than the concept of force, not being necessary (although sometimes convenient) to use forces for describing the effects of constraints on the dynamics of a multibody system.

\subsection{Newton-Euler formalism}

Newton-Euler formalism is one of the most traditional approaches used in the derivation of equations of motion of multi-rigid-body systems. The so called Newton-Euler equations are based on Newton's laws of motion and on the descriptions of translation and rotations of a rigid body originally developed by 
Euler. The first fields of Multibody System Dynamics on which this formalism was applied were on mechanism theory, gyroscopes and biomechanics (SCHIEHLEN, 1997).

The main advantage of Newton-Euler equations is that, independently of the geometry, inertia or constraints of motion of a rigid body, its equations of motion will always have the same form. Even the choice of variables of motion will hardly be influenced by any of these factors. Indeed, the configuration of a rigid body is either described by the coordinates of a finite number of points of the body ${ }^{1}$ or by the coordinates of a single point along with three Euler Angles or four quaternion parameters. To complete the description of the state of a rigid body it is enough to specify the components of the velocity of one of its points along with the components of the angular velocity of the body ${ }^{2}$. Therefore, in order to obtain the mathematical model of a rigid body, the following it is necessary to derive:

- Kinematic equations expressing time derivatives of the generalized coordinates in terms of the chosen quasi-velocities.

- Six dynamic equations of motion, three of them relating the resultant of the force system applied to the body to its instantaneous translation and the other three relating the resultant torque of this force system to the instantaneous rotation of the body.

In order to describe motion constraints in Newton-Euler formalism, it is necessary to introduce one, a priori unknown, force or torque component for each independent first order generalized constraint invariant. Whenever the described constraint is related to a pair of rigid bodies within the studied system, these unknown constraint forces or torques must constitute pairs of action and reaction between the involved bodies. No modifications on the form of the dynamic equations of motion due to constraints are performed, however, i.e. the topology of motion does not require further than including associated constraint forces and torques when necessary.

Such features of Newton-Euler formalism explain its relative success in the development of several algorithms for deriving the equations of motion of

\footnotetext{
${ }^{1}$ At least two distinct points are required in the case of a plane motion and at least three non-collinear points are needed in the case of a tridimensional motion.

2 In the case of a plane motion, two components of velocity (parallel to the plane) and a single component of angular velocity (orthogonal to the plane) are enough; for a general tridimensional motion, three components of velocity and three of angular velocity are required. Once the Newton's laws of motion are applied, velocities and angular velocities must be measured with respect to an inertial reference frame.
} 
multi-rigid-body systems, including the ones used including many free and proprietary software implementations (PAUL, 1975). Among the several applications of Newton-Euler methodology to modeling and simulation of multibody systems, the recursive algorithms stand out.

This Section starts with a brief discussion on Newton's laws of motion in Subsection 3.1.1. Newton-Euler equations of motion for a rigid body are presented in Subsection 3.1.2. Applications of the Screw Theory to Newton-Euler formalism are discussed in Subsection 3.1.3 and, finally, some comments on the main recursive algorithms based on this formalism are presented in Subsection 3.1.4.

\subsubsection{Newton's laws of motion}

Newton's laws of motion can be understood as the first formalization of Classical Mechanics providing general relations between the motion of a body and the set of forces acting on it.

Before stating the three fundamental laws of motion it is important to mention two definitions presented in "Philosophiae Naturalis Principia Mathematica" (JAMMER, 2012, p. $119-121$ ):

- The vis insita, or the innate force of matter is a power of resisting by which every body, as much in it lies, continues in its present state, whether it be of rest, or of moving uniformly forwards in a right line.

- The vis impressa, or the impressed force is an action exerted upon a body, in order to change its state, either of rest, or of uniform motion in a right line.

From these definitions, along with some others, Newton set out three postulates, known as Newton's laws of motion, which were originally stated as follows (JAMMER, 2012, p. 123):

Law I Every body continues in its state of rest, or of uniform motion in a right line, unless it is compelled to change that state by force impressed upon it.

Law II The change of motion is proportional to the motive force impressed; and is made in the direction of the right line in which that force is impressed. 
Law III To every action there is always opposed an equal reaction; or, the mutual actions of two bodies upon each other are always equal, and directed to contrary parts.

In order to correctly interpret these laws of motion two notions are fundamental: the concept of particle and the concept of inertial reference frame. Following the remarks in the beginning of Chapter 2, a particle can be conceived as an material body of infinitesimal volume and finite mass that can be represented by a single point in space. Whenever Newton mentions "body", it should be understood as a particle. It does mean any restriction to the application of the laws of motion, on condition that Integral Calculus is applied to generalize their application to extensive bodies. This, however, requires the adoption of the concept of density, which is a continuous function defined within the domain of the material body and represents, in each point, the limit of the mass to volume ratio in open neighborhoods of this point. In this case, it is possible to consider that in infinitesimal portions of an extensive body, the mass is proportional to the volume; moreover, if these portions are sufficiently small, they can be conceived as particles, being possible to apply the Newton's laws of motion to them.

Concerning the reference frames adopted in descriptions of motion, an special class named inertial reference frames is characterized by Law I: if in a given reference frame a particle, without any force impressed upon it, remains in rest or describes an uniform motion along a straight line, this reference frame is inertial. Moreover, due to Galilean relativity, it can be stated that any reference frame performing a rectilinear translation with rectilinear translation with respect to an inertial reference frame is also a reference frame. Law II is only true whenever the "change of motion" (which should be understood as the time derivative of linear momentum) of a "body" (particle with constant mass) is measured with respect to an inertial reference frame.

The linear momentum of a particle with constant mass is defined by the scalar multiplication of the velocity vector of a particle by its mass. Denoting by $\mathbf{f}$ the impressed force acting in a particle, by $\bar{m}$ its mass (constant) and by $\mathbf{v}$ the velocity of this particle with respect to an inertial reference frame $\mathcal{N}$, Law II can be expressed by the following equation:

$$
\mathbf{f}=\frac{\mathrm{d}(\bar{m} \mathbf{v})}{\mathrm{d} t}=\bar{m} \dot{\mathbf{v}}
$$

According to Law I, if there is no force impressed upon a particle it remain in 
a state of rest or move along a straight line with constant velocity with respect to an inertial reference frame. Consider, however, a particle constrained to move in a curvilinear trajectory (measured with respect to an inertial reference frame). In order not to violate neither the laws of motion nor the constraints imposed to the particle, the corresponding motion constraints must be associated with forces, generally called constraint forces, that must be compatible with the observed motion. In general, whenever the modeling methodology is directly based on Newton's laws of motion, constraint forces corresponding to each motion constraint must be introduced, which does not necessarily happen in Analytical Mechanics based approaches.

\subsubsection{Newton-Euler equations}

Newton-Euler equations represent the most classical formulation to derive equations of motion of multibody systems constituted by ideal rigid bodies. The derivation of such equations come from the application of Integral Calculus to Newton's laws of motion.

Let $\mathcal{N}$ denote an inertial reference frame and $\mathcal{B}$ an ideal rigid body with finite extension and constant mass. Let $\mathbf{v}$ denote the velocity of an arbitrary point of $\mathcal{B}$ with respect to $\mathcal{N}$. It can be stated that the application of Newton's laws of motion to body $\mathcal{B}$ leads to the following equations, the first representing an integral form of Law II and the second an integral of the moments (with respect to the center of mass of $\mathcal{B}$ ) of the respective physical quantities ${ }^{3}$ :

$$
\begin{aligned}
& \int_{\mathcal{B}} \mu \dot{\mathbf{v}} \mathrm{d} \mathcal{B}=\int_{\mathcal{B}} \boldsymbol{\gamma} \mathrm{d} \mathcal{B}+\int_{\widetilde{\partial}_{\mathcal{B}}} \boldsymbol{\sigma} \mathrm{d}_{\mathcal{B}}+\sum_{k} \mathbf{f}_{k} \\
& \int_{\mathcal{B}} \mathbf{r} \times \mu \dot{\mathbf{v}} \mathrm{d} \mathcal{B}=\int_{\mathcal{B}} \mathbf{r} \times \boldsymbol{\gamma} \mathrm{d} \mathcal{B}+\int_{\coprod_{\mathcal{B}}} \mathbf{r} \times \boldsymbol{\sigma} \mathrm{d} \coprod_{\mathcal{B}}+\sum_{k} \mathbf{r}_{k} \times \mathbf{f}_{k}
\end{aligned}
$$

In these equations the following notation is used:

- $\mu$ represents the density function defined within the domain of body $\mathcal{B}$;

- $\gamma$ represents the field forces (forces per unity of volume) of $\mathcal{B}$, which must be an integrable function defined within the domain of $\mathcal{B}$.

${ }^{3}$ Actually, the first equation involves the use of both Law II and the weak form of Law III (once internal forces within the rigid body form pairs of action and reaction that cancel when added up), while in the second one, Law II is used along with the strong form of Law III which additionally imposes that the pairs of action and reaction act along the straight line defined by the points in which these forces act (GOLDSTEIN; POOLE JR.; SAFKO, 2002). 
- $\sigma$ represents the surface forces (forces per unity of area) of $\mathcal{B}$, which must be an integral function defined within the the boundary of $\mathcal{B}$, which is denoted by $ð_{\mathcal{B}}$;

- $\mathbf{f}_{k}$ represent a finite number of concentrated forces applied in some points of $\mathcal{B}$;

- $\mathbf{r}$ is a vector that represents the position of an arbitrary point of $\mathcal{B}$ with respect to the center of mass of this body;

- $\mathbf{r}_{k}$ is defined analogously for the special points where the respective forces $\mathbf{f}_{k}$ are applied.

Once $\mathrm{r}$ represents the position vector from the center of mass of $\mathcal{B}$, and considering that $\mu$ is independent of time (and consequently, $\dot{\mu}=0$ ) then, by the definition of center of mass (TENENBAUM, 2004, p. 409):

$$
\int_{\mathcal{B}} \mu \mathbf{r} \mathrm{d} \mathcal{B}=\int_{\mathcal{B}} \mu \dot{\mathbf{r}} \mathrm{d} \mathcal{B}=\int_{\mathcal{B}} \mu \ddot{\mathbf{r}} \mathrm{d} \mathcal{B}=\mathbf{0}
$$

Define also the mass of $\mathcal{B}$ as $\bar{m}=\int_{\mathcal{B}} \mu \mathrm{d} \mathcal{B}$ and the inertia tensor of $\mathcal{B}$ with respect to its own center of mass, denoted by $\overline{\mathbf{I}}^{\star}$, as the second order symmetric tensor satisfying the following identities for any vectors $\mathrm{x}$ and $\mathrm{y}$ (KANE; LEVINSON, 1985; TENENBAUM, 2004):

$$
\begin{aligned}
& \overline{\mathbf{I}}^{\star} \cdot \mathbf{x}=\int_{\mathcal{B}} \mathbf{r} \times(\mathbf{x} \times \mathbf{r}) \mu \mathrm{d} \mathcal{B}=\int_{\mathcal{B}}((\mathbf{r} \cdot \mathbf{r}) \mathbf{x}-(\mathbf{x} \cdot \mathbf{r}) \mathbf{r}) \mu \mathrm{d} \mathcal{B} \\
& \mathbf{x} \cdot\left(\overline{\mathbf{I}}^{\star} \cdot \mathbf{y}\right)=\int_{\mathcal{B}}((\mathbf{x} \cdot \mathbf{y})(\mathbf{r} \cdot \mathbf{r})-(\mathbf{x} \cdot \mathbf{r})(\mathbf{y} \cdot \mathbf{r})) \mu \mathrm{d} \mathcal{B}=\mathbf{y} \cdot\left(\overline{\mathbf{I}}^{\star} \cdot \mathbf{x}\right)
\end{aligned}
$$

Moreover, let $\mathbf{v}^{\star}$ denote the velocity of the center of mass of $\mathcal{B}$ with respect to $\mathcal{N}$. Then, clearly, $\mathbf{v}=\mathbf{v}^{\star}+\dot{\mathbf{r}}$. Furthermore, if $\omega$ denotes the angular velocity of $\mathcal{B}$ with respect to $\mathcal{N}$, then $\dot{\mathbf{r}}=\omega \times \mathbf{r}$ and $\ddot{\mathbf{r}}=\dot{\omega} \times \mathbf{r}+\omega \times(\omega \times \mathbf{r})$. Thus, it can be stated that:

$$
\begin{aligned}
\int_{\mathcal{B}} \mu \dot{\mathbf{v}} \mathrm{d} \mathcal{B}=\int_{\mathcal{B}} \mu\left(\ddot{\mathbf{r}}+\dot{\mathbf{v}}^{\star}\right) \mathrm{d} \mathcal{B}=\int_{\mathcal{B}} \mu \ddot{\mathbf{r}} \mathrm{d} \mathcal{B}+\dot{\mathbf{v}}^{\star} \int_{\mathcal{B}} \mu \mathrm{dB}=\bar{m} \dot{\mathbf{v}}^{\star} \\
\int_{\mathcal{B}} \mathbf{r} \times \mu \dot{\mathbf{v}} \mathrm{d} \mathcal{B}=\int_{\mathcal{B}} \mathbf{r} \times \mu\left(\ddot{\mathbf{r}}+\dot{\mathbf{v}}^{\star}\right) \mathrm{d} \mathcal{B} \\
=\int_{\mathcal{B}} \mathbf{r} \times \mu \ddot{\mathbf{r}} \mathrm{d} \mathcal{B}-\dot{\mathbf{v}}^{\star} \times \int_{\mathcal{B}} \mu \mathbf{r} \mathrm{d} \mathcal{B} \\
=\int_{\mathcal{B}} \mathbf{r} \times(\mu \omega \times(\omega \times \mathbf{r})) \mathrm{d} \mathcal{B}+\int_{\mathcal{B}} \mathbf{r} \times(\mu \dot{\omega} \times \mathbf{r}) \mathrm{d} \mathcal{B} \\
=\omega \times\left(\int_{\mathcal{B}} \mathbf{r} \times(\omega \times \mathbf{r}) \mu \mathrm{d} \mathcal{B}\right)+\int_{\mathcal{B}} \mathbf{r} \times(\dot{\omega} \times \mathbf{r}) \mu \mathrm{dB} \\
=\omega \times\left(\overline{\mathbf{I}}^{\star} \cdot \omega\right)+\overline{\mathbf{I}}^{\star} \cdot \dot{\omega}
\end{aligned}
$$


Finally, define the resultant force $\mathbf{f}$ and the resultant torque $\mathbf{t}^{\star}$ acting in $\mathcal{B}$ as follows:

$$
\begin{aligned}
& \mathbf{f}=\int_{\mathcal{B}} \boldsymbol{\gamma} \mathrm{d} \mathcal{B}+\int_{\widetilde{\partial}_{\mathcal{B}}} \boldsymbol{\sigma} \mathrm{d}_{\mathcal{B}}+\sum_{k} \mathbf{f}_{k} \\
& \mathbf{t}^{\star}=\int_{\mathcal{B}} \mathbf{r} \times \gamma \mathrm{d} \mathcal{B}+\int_{\widetilde{\partial}_{\mathcal{B}}} \mathbf{r} \times \boldsymbol{\sigma} \mathrm{d} \widetilde{\partial}_{\mathcal{B}}+\sum_{k} \mathbf{r}_{k} \times \mathbf{f}_{k}
\end{aligned}
$$

In this case, equations $(3.2,3.3)$ can be rewritten as follows:

$$
\begin{aligned}
& \bar{m} \dot{\mathbf{v}}^{\star}=\mathbf{f} \\
& \overline{\mathbf{I}}^{\star} \cdot \dot{\boldsymbol{\omega}}=\mathbf{t}^{\star}-\boldsymbol{\omega} \times\left(\overline{\mathbf{I}}^{\star} \cdot \boldsymbol{\omega}\right)
\end{aligned}
$$

It is common to express equations $(3.11,3.12)$ in terms of the linear and angular momenta of $\mathcal{B}$. The (linear) momentum of $\mathcal{B}$ can be defined by:

$$
\mathbf{p}=\int_{\mathcal{B}} \mu \mathbf{v} \mathrm{d} \mathcal{B}=\bar{m} \mathbf{v}^{\star}
$$

Also, let $\mathbf{r}_{\mid \mathrm{p}}$ denote the position vector of an arbitrary point of $\mathcal{B}$ with respect to a given point $\mathrm{p}$. The angular momentum of $\mathcal{B}$ with respect to the point $\mathrm{p}$ is given by (TENENBAUM, 2004):

$$
\mathbf{h}_{\mid \mathrm{p}}=\int_{\mathcal{B}} \mathbf{r}_{\mid \mathrm{p}} \times \mu \mathbf{v} \mathrm{dB}=\overline{\mathbf{I}}^{\star} \cdot \omega+\mathbf{p} \times \mathbf{r}_{\mathrm{p} \mid \mathbf{b}^{\star}}
$$

It can be stated that, equations $(3.11,3.12)$ are equivalent to the following ones, with $v_{p}$ denoting the velocity of point $p$ with respect to the inertial reference frame $\mathcal{N}$ and with $t_{\mid p}$ denoting the resultant torque with respect to the point $\mathrm{p}$ :

$$
\begin{aligned}
& \dot{\mathbf{p}}=\mathbf{f} \\
& \dot{\mathbf{h}}_{\mid \mathrm{p}}=\mathbf{t}_{\mid \mathrm{p}}+\mathbf{p} \times \mathbf{v}_{\mathrm{p}}
\end{aligned}
$$

Actually, equations $(3.15,3.16)$ can be understood as generalizations of $(3.11,3.12)$. Note also that $p$ is a fixed point or if $v_{p}$ is parallel to $p$, equation (3.16) simplifies to the following form: $\dot{\mathbf{h}}_{\mid \mathrm{p}}=\mathbf{t}_{\mid \mathrm{p}}$. The latter condition includes the case in which $\mathrm{p}$ coincides with the center of mass of $\mathcal{B}$; denoting the corresponding angular momentum by $\mathbf{h}^{\star}$, it can be stated that $\mathbf{h}^{\star}=\overline{\mathbf{I}}^{\star} \cdot \omega$ and $\dot{\mathbf{h}}^{\star}=\mathbf{t}^{\star}$.

In order to obtain the so called Newton-Euler equations, it is necessary to choose coordinate systems to obtain components of the vectors involved in equations $(3.11,3.12)$. Let $E^{\prime}$ and $E^{\prime \prime}$ denote the coordinate systems chosen to decompose the vectors in these equations, respectively. The Newton-Euler 
equations of motion of $\mathcal{B}$ are given by:

$$
\left[\begin{array}{cc}
\bar{m} \mathbf{1} & 0 \\
0 & {\left[\overline{\mathbf{I}}^{\star}\right]_{\mathrm{E}^{\prime \prime} \mid \mathrm{E}^{\prime \prime}}}
\end{array}\right]\left[\begin{array}{c}
{\left[\dot{\mathbf{v}}^{\star}\right]_{E^{\prime}}} \\
{[\dot{\omega}]_{E^{\prime \prime}}}
\end{array}\right]=\left[\begin{array}{c}
{[\mathbf{f}]_{E^{\prime}}} \\
{\left[\mathbf{t}^{\star}\right]_{E^{\prime \prime}}-[\omega]_{E^{\prime \prime} \mid E^{\prime \prime}}^{\times}\left[\overline{\mathbf{I}}^{\star}\right]_{E^{\prime \prime} \mid E^{\prime \prime}}[\boldsymbol{\omega}]_{E^{\prime \prime}}}
\end{array}\right]
$$

In this equation, $[\omega]_{E^{\prime \prime} \mid E^{\prime \prime}}^{\times}$stands for the skew-symmetric matrix representation of the matrix-column $[\omega]_{E^{\prime \prime}}$, i.e.:

$$
[\omega]_{\mathrm{E}^{\prime \prime}}^{\times}=\left[\begin{array}{ccc}
0 & -\omega_{\mathrm{z}} & \omega_{\mathrm{y}} \\
\omega_{\mathrm{z}} & 0 & -\omega_{\mathrm{x}} \\
-\omega_{\mathrm{y}} & \omega_{\mathrm{x}} & 0
\end{array}\right] \Leftrightarrow \quad[\omega]_{\mathrm{E}^{\prime \prime} \mid \mathrm{E}^{\prime \prime}}=\left[\begin{array}{c}
\omega_{\mathrm{x}} \\
\omega_{\mathrm{y}} \\
\omega_{\mathrm{z}}
\end{array}\right]
$$

When an arbitrary coordinate system is adopted, the components of the time derivative of a vector are generally not equal to the time derivatives of the components of this vector. Let $\mathrm{N}$ and $\mathrm{B}$ denote a coordinate systems rigidly attached to $\mathcal{N}$ and $\mathcal{B}$, respectively, and consider that the origin of the latter one coincides with the center of mass of $\mathcal{B}$. It can be stated that $\left[\dot{\mathbf{v}}^{\star}\right]_{\mathrm{N}}$ and $[\dot{\omega}]_{\mathrm{N}}$ are equal to the time derivatives of $\left[\mathbf{v}^{\star}\right]_{N}$ and $[\omega]_{N}$, respectively ${ }^{4}$. Thus, it is useful to adopt $\mathrm{E}^{\prime}=\mathrm{N}$. Moreover, $[\dot{\omega}]_{\mathrm{B}}$ is equal to the time derivative of $[\omega]_{\mathrm{B}}$. Once the components of the inertia tensor of $\mathcal{B}$ with respect to a coordinate system fixed to the body are invariants, it is usual do adopt $E^{\prime \prime}=B$. Therefore, the typical form of Newton-Euler equations for an arbitrary rigid body $\mathcal{B}$ is the following:

$$
\left[\begin{array}{cc}
\bar{m} 1 & 0 \\
0 & {\left[\overline{\mathbf{I}}^{\star}\right]_{\mathrm{B} \mid \mathrm{B}}}
\end{array}\right]\left[\begin{array}{c}
{\left[\dot{\mathbf{v}}^{\star}\right]_{\mathrm{N}}} \\
{[\dot{\boldsymbol{\omega}}]_{\mathrm{B}}}
\end{array}\right]=\left[\begin{array}{c}
{[\mathbf{f}]_{\mathrm{N}}} \\
{\left[\mathbf{t}^{\star}\right]_{\mathrm{B}}-[\boldsymbol{\omega}]_{\mathrm{B} \mid \mathrm{B}}^{\times}\left[\overline{\mathbf{I}}^{\star}\right]_{\mathrm{B} \mid \mathrm{B}}[\boldsymbol{\omega}]_{\mathrm{B}}}
\end{array}\right]
$$

A relevant particular case of equation (3.19) occurs when the axis of the coordinate system B coincide with the so called principal axes of inertia of $\mathcal{B}$, i.e., when $\left[\overline{\mathbf{I}}^{\star}\right]_{\mathrm{B} \mid \mathrm{B}}$ corresponds to a diagonal matrix. In this case, the Newton-Euler

${ }^{4}$ The following identity stand for other coordinate systems than $\mathrm{N}$, when $\mathbf{z}=\mathbf{v}$ or $\mathbf{z}=\omega$, with $\mathcal{E}$ denoting the reference frame to which the coordinate system $\mathrm{E}$ is attached:

$$
\left[\frac{\mathrm{d} \mathbf{z}}{\mathrm{d} t}\right]_{\mathrm{E}}=\frac{\mathrm{d}}{\mathrm{d} t}[\mathbf{z}]_{\mathrm{E}}+\left[\omega_{\varepsilon \mid \mathcal{N}}\right]_{\mathrm{E} \mid \mathrm{E}}^{\times}[\mathbf{z}]_{\mathrm{E}}
$$


equations can be expressed in the following form ${ }^{5}$ :

$$
\begin{array}{ll}
\dot{v}_{\mathrm{x}}^{\star}=\frac{f_{\mathrm{x}}}{\bar{m}} & \dot{\omega}_{\mathrm{x}}=\frac{t_{\mathrm{x}}^{\star}+\left(\bar{l}_{\mathrm{y}}-\bar{l}_{\mathrm{z}}\right) \omega_{\mathrm{y}} \omega_{\mathrm{z}}}{\bar{l}_{\mathrm{x}}} \\
\dot{v}_{\mathrm{y}}^{\star}=\frac{f_{\mathrm{y}}}{\bar{m}} & \dot{\omega}_{\mathrm{y}}=\frac{t_{\mathrm{y}}^{\star}+\left(\bar{l}_{\mathrm{z}}-\bar{l}_{\mathrm{x}}\right) \omega_{\mathrm{z}} \omega_{\mathrm{x}}}{\bar{l}_{\mathrm{y}}} \\
\dot{v}_{\mathrm{z}}^{\star}=\frac{f_{\mathrm{z}}}{\bar{m}} & \dot{\omega}_{\mathrm{z}}=\frac{t_{\mathrm{z}}^{\star}+\left(\bar{I}_{\mathrm{x}}-\bar{l}_{\mathrm{y}}\right) \omega_{\mathrm{x}} \omega_{\mathrm{y}}}{\bar{l}_{\mathrm{z}}}
\end{array}
$$

\subsubsection{Applications of the Screw Theory}

Screw theory, initially formalized by Sir Robert Stawell Ball at the end of the nineteenth century, comprises several mathematical tools for the description of instantaneous kinematics of rigid bodies and force systems (in which the forces can be conceived as sliding vectors) by geometrical entities called screws. A screw is an axis associated to a (real) scalar called pitch (BALL, 1998). An infinite pitch screw is an element that represents a direction in space and is not bound to any particular line.

In order to describe the instantaneous kinematics of rigid body motion it is sufficient a vector $\omega$ representing the angular velocity of the rigid body (with respect to a reference frame $\mathcal{E}$ ), a point p moving along with this body and the velocity of this point $\mathbf{v}$ (with respect to $\mathcal{E}$ ). Thus, a 3 -tuple $(\omega, \mathbf{v}, \mathrm{p})$ is sufficient to describe the instantaneous kinematics of any rigid body motion. Another $3-$ tuple $\left(\omega^{\prime}, \mathbf{v}^{\prime}, p^{\prime}\right)$ describes the same rigid body motion described by $(\omega, \mathbf{v}, p)$ if and only if:

$$
\left\{\begin{array}{l}
\omega^{\prime}=\omega \\
\mathbf{v}^{\prime}=\mathbf{v}+\omega \times \mathbf{r}_{\mathrm{p}^{\prime} \mid \mathrm{p}}
\end{array}\right.
$$

The equivalence class of 3-tuples that describe the same rigid body motion is called twist. The twist associated to the equivalence class defined by $(\omega, \mathbf{v}, \mathrm{p})$ is denoted by $\vee=\llbracket(\omega, \mathbf{v}, \mathrm{p}) \rrbracket$. It can be proved that, unless $\omega$ is a zero vector, there exists a point $h$ (moving along with the rigid body) and a scalar $\eta$ such that $\llbracket(\omega, \mathrm{v}, \mathrm{p}) \rrbracket=\llbracket(\omega, \eta \omega, \mathrm{h}) \rrbracket$. Moreover, if $\mathrm{h}^{\prime}$ is any point such that $\mathbf{r}_{\mathrm{h}^{\prime} \mid \mathrm{h}}$ is parallel to $\omega$, then $\llbracket(\omega, \eta \omega, \mathrm{h}) \rrbracket=\llbracket\left(\omega, \eta \omega, \mathrm{h}^{\prime}\right) \rrbracket$. Therefore, any twist with non-zero $\omega$ can be represented by a screw passing through $h$, parallel to $\omega$ and whose pitch is equal to $\eta$. On the other hand, $\llbracket(0, v, p) \rrbracket=\llbracket\left(0, v, p^{\prime}\right) \rrbracket$ for any points $p$ and $\mathrm{p}^{\prime}$.

\footnotetext{
${ }^{5}$ The overbar notation is used just to reinforce that the corresponding parameters are constants.
} 
Thus, any twist with $\omega=0$ can be represented by an infinite pitch screw whose direction is defined by $\mathbf{v}$.

Analogously, in order to fully describe a force system in which forces can be represented by sliding vectors (i.e., forces are bounded to a line of action but not to a specific point of this line), it is enough to specify the resultant force $f$, to choose a point $\mathrm{p}$ and to obtain the resultant torque $\mathrm{t}$ with respect to the point p. A 3-tuple (f, t, p) fully describes a force system. Moreover, the 3-tuples $(\mathbf{f}, \mathbf{t}, \mathrm{p})$ and $\left(\mathbf{f}^{\prime}, \mathbf{t}^{\prime}, \mathrm{p}^{\prime}\right)$ describe the same force system if and only if:

$$
\left\{\begin{array}{l}
\mathbf{f}^{\prime}=\mathbf{f} \\
\mathbf{t}^{\prime}=\mathbf{t}+\mathbf{f} \times \mathbf{r}_{\mathrm{p}^{\prime} \mid \mathrm{p}}
\end{array}\right.
$$

The equivalence class of 3-tuples that describe the same force system is called wrench. The wrench associated to the equivalence class defined by $(f, t, p)$ is denoted by $\mathbb{a}^{*}=\llbracket(f, t, p) \rrbracket$. Analogously to the cases presented above for twists, any wrench with non-zero resultant force can be represented by a finite pitch screw and any wrench with zero resultant force can be represented by an infinite pitch screw whose direction is defined by $t$.

It can be stated that twists and wrenches constitute linear spaces denoted respectively by $\mathbb{M}^{6}$ and $\mathbb{F}^{6}$ (FEATHERSTONE, 2008). Let $E=\left(e, \hat{\mathbf{e}}_{1}, \hat{\mathbf{e}}_{2}, \hat{\mathbf{e}}_{3}\right)$ be a coordinate system rigidly attached to a reference frame $\mathcal{E}$ and let $\mathcal{B}$ denote a rigid body. Denote by $\omega$ the angular velocity of $\mathcal{B}$ with respect to $\mathcal{E}$ and by $\mathbf{v}$ the velocity of a point that moves along with $\mathcal{B}$ and instantly coincides with e. The instantaneous kinematics of $\mathcal{B}$ can be described by the twist $\vee=\llbracket(\omega, \mathbf{v}, e) \rrbracket$. Also, denote by $f$ the resultant of a force system acting in $\mathcal{B}$ and by $t$ the resultant torque of this system with respect to point e. This force system can be described by the wrench $\mathbb{F}^{*}=\llbracket(\mathbf{f}, \mathbf{t}, \mathrm{e}) \rrbracket$. Define the following unit screws:

$$
\begin{array}{ll}
\hat{\mathbb{E}}_{1}=\hat{\mathbb{E}}_{4}^{*}=\llbracket\left(\hat{\mathbf{e}}_{1}, 0, \mathrm{e}\right) \rrbracket & \hat{\mathbb{E}}_{4}=\hat{\mathbb{E}}_{1}^{*}=\llbracket\left(0, \hat{\mathbf{e}}_{1}, \mathrm{e}\right) \rrbracket \\
\hat{\mathbb{E}}_{2}=\hat{\mathbb{E}}_{5}^{*}=\llbracket\left(\hat{\mathbf{e}}_{2}, 0, \mathrm{e}\right) \rrbracket & \hat{\mathbb{E}}_{5}=\hat{\mathbb{e}}_{2}^{*}=\llbracket\left(0, \hat{\mathbf{e}}_{2}, \mathrm{e}\right) \rrbracket \\
\hat{\mathbb{E}}_{3}=\hat{\mathbb{E}}_{6}^{*}=\llbracket\left(\hat{\mathbf{e}}_{3}, 0, \mathrm{e}\right) \rrbracket & \hat{\mathbb{E}}_{6}=\hat{\mathbb{E}}_{3}^{*}=\llbracket\left(0, \hat{\mathbf{e}}_{3}, \mathrm{e}\right) \rrbracket
\end{array}
$$

Considering that $\omega=\omega_{\mathrm{x}} \hat{\mathbf{e}}_{1}+\omega_{\mathrm{y}} \hat{\mathbf{e}}_{2}+\omega_{\mathrm{z}} \hat{\mathbf{e}}_{3}, \mathbf{v}=v_{\mathrm{x}} \hat{\mathbf{e}}_{1}+v_{\mathrm{y}} \hat{\mathbf{e}}_{2}+v_{\mathrm{z}} \hat{\mathbf{e}}_{3}, \mathbf{f}=f_{\mathrm{x}} \hat{\mathbf{e}}_{1}+$ $f_{\mathrm{y}} \hat{\mathbf{e}}_{2}+f_{\mathrm{z}} \hat{\mathbf{e}}_{3}, \mathbf{t}=t_{\mathrm{x}} \hat{\mathbf{e}}_{1}+t_{\mathrm{y}} \hat{\mathbf{e}}_{2}+t_{\mathrm{z}} \hat{\mathbf{e}}_{3}$, it can be stated that:

$$
\begin{aligned}
& \nabla=\omega_{\mathrm{x}} \hat{\mathbb{e}}_{1}+\omega_{\mathrm{y}} \hat{\mathbb{e}}_{2}+\omega_{\mathrm{z}} \hat{\mathbb{E}}_{3}+v_{\mathrm{x}} \hat{\mathbb{E}}_{4}+v_{\mathrm{y}} \hat{\mathbb{E}}_{5}+v_{\mathrm{z}} \hat{\mathbb{E}}_{6} \\
& \mathbb{\mathbb { F }}^{*}=t_{\mathrm{x}} \hat{\mathbb{E}}_{1}^{*}+t_{\mathrm{y}} \hat{\mathbb{E}}_{2}^{*}+t_{\mathrm{z}} \hat{\mathbb{E}}_{3}^{*}+f_{\mathrm{x}} \hat{\mathbb{E}}_{4}^{*}+f_{\mathrm{y}} \hat{\mathbb{E}}_{5}^{*}+f_{\mathrm{z}} \hat{\mathbb{E}}_{6}^{*}
\end{aligned}
$$


Thus, the so called Plücker coordinates of $\vee$ and $\mathbb{E}^{*}$ in $\mathrm{E}$ are given by:

$$
\begin{aligned}
& {[\mathrm{v}]_{\mathrm{E}}=\left(\omega_{\mathrm{x}}, \omega_{\mathrm{y}}, \omega_{\mathrm{z}}, v_{\mathrm{x}}, v_{\mathrm{y}}, v_{\mathrm{z}}\right)=\left([\omega]_{\mathrm{E}},[\mathbf{v}]_{\mathrm{E}}\right)} \\
& {\left[\mathbb{F}^{*}\right]_{\mathrm{E}}=\left(t_{\mathrm{x}}, t_{\mathrm{y}}, t_{\mathrm{z}}, f_{\mathrm{x}}, f_{\mathrm{y}}, f_{\mathrm{z}}\right)=\left([\mathbf{t}]_{\mathrm{E}},[\mathbf{f}]_{\mathrm{E}}\right)}
\end{aligned}
$$

Moreover, $\mathbb{M}^{6}$ and $\mathbb{F}^{6}$ are can be conceived as dual spaces, with $\hat{\mathbb{e}}_{i} \cdot \hat{\mathbb{e}}_{j}^{*}=\hat{\mathbb{e}}_{j}^{*} \cdot \hat{\mathbb{e}}_{i}=$ $\underline{\delta}_{i j}\left(\underline{\delta}_{i j}\right.$ representing the Kronecker delta, i.e., its value is equal to 1 if and only if $i=j$, otherwise its value is 0 ). Thus,

$$
\mathbb{T}^{*} \cdot \nabla=\mathbf{t} \cdot \mathbf{\omega}+\mathbf{f} \cdot \mathbf{v}
$$

Note that $\mathbb{Q}^{*} \cdot \vee$ represents the power delivered by the force system represented by $\mathbb{~}^{*}$ when it is applied to a rigid body whose instantaneous motion is given by v.

Finally, let $\mathbb{1}$ denote the identity tensor of $\mathbb{M}^{6} \times \mathbb{M}^{6}$ and $\mathbb{1}^{*}$ denote the identity tensor of $\mathbb{F}^{6} \times \mathbb{F}^{6}$.

Let $\mathrm{E}=\left(\mathrm{e}, \hat{\mathrm{e}}_{1}, \hat{\mathrm{e}}_{2}, \hat{\mathrm{e}}_{3}\right)$ and $\mathrm{E}^{\prime}=\left(\mathrm{e}^{\prime}, \hat{\mathrm{e}}_{1}^{\prime}, \hat{\mathbf{e}}_{2}^{\prime}, \hat{\mathrm{e}}_{3}^{\prime}\right)$ be two coordinate systems rigidly attached to a reference frame $\mathcal{E}$. It can be stated that (FEATHERSTONE, 2008; SELIG, 2005):

$$
\begin{aligned}
& {[\mathbb{1}]_{E \mid E^{\prime}}=\left[\begin{array}{cc}
{[1]_{E \mid E^{\prime}}} & 0 \\
{\left[r_{e^{\prime} \mid e}\right]_{E}^{\times}[1]_{E \mid E^{\prime}}} & {[1]_{E \mid E^{\prime}}}
\end{array}\right]} \\
& {\left[\mathbb{1}^{*}\right]_{E \mid E^{\prime}}=\left[\begin{array}{cc}
{[1]_{E \mid E^{\prime}}} & {\left[r_{e^{\prime} \mid e}\right]_{E}^{\times}[1]_{E \mid E^{\prime}}} \\
0 & {[1]_{E \mid E^{\prime}}}
\end{array}\right]}
\end{aligned}
$$

It can also be noticed that $\left[\mathbb{1}^{*}\right]_{E \mid E^{\prime}}^{\top}[\mathbb{1}]_{E \mid E^{\prime}}=1$.

Let $\mathcal{N}$ be an inertial reference frame and $\mathrm{N}=\left(\mathrm{o}, \hat{\mathbf{n}}_{x}, \hat{\mathbf{n}}_{y}, \hat{\mathbf{n}}_{z}\right)$ be a coordinate system rigidly attached to it. Consider that $\mathcal{B}$ is a rigid body and that $B$ and $B^{\star}$ are two coordinate systems moving along with $\mathcal{B}$ such that the former instantly coincides with $N$ and the latter has its origin in $b^{\star}$, the centre of mass of $\mathcal{B}$. Suppose that the instantaneous kinematics of $\mathcal{B}$ is described by the twist $\vee=$ $\llbracket\left(\omega, \mathbf{v}^{\star}, \mathrm{b}^{\star}\right) \rrbracket$ and the force system acting on $\mathcal{B}$ is described by the wrench $\mathbb{f}=$ $\llbracket\left(\mathbf{f}, \mathbf{t}^{\star}, \mathrm{b}^{\star}\right) \rrbracket$. In this case, the Newton-Euler equations for $\mathcal{B}$ can be expressed 
as follows, see equation (3.19):

$$
\begin{aligned}
& {\left[\begin{array}{cc}
{[\omega]_{\mathrm{B}^{\star}}^{\times}} & {\left[\mathbf{v}^{\star}\right]_{\mathrm{B}^{\star}}^{\times}} \\
0 & {[\omega]_{\mathrm{B}^{\star}}^{\times}}
\end{array}\right]\left[\begin{array}{cc}
{\left[\overline{\mathbf{I}}^{\star}\right]_{\mathrm{B}^{\star}}} & 0 \\
0 & \bar{m} \mathbf{1}
\end{array}\right]\left[\begin{array}{c}
{[\omega]_{\mathrm{B}^{\star}}} \\
{\left[\mathbf{v}^{\star}\right]_{\mathrm{B}^{\star}}}
\end{array}\right]} \\
& +\left[\begin{array}{cc}
{\left[\overline{\mathbf{I}}^{\star}\right]_{\mathrm{B}^{\star} \mid \mathrm{B}^{\star}}} & 0 \\
0 & \bar{m} \mathbf{1}
\end{array}\right]\left[\begin{array}{c}
{[\dot{\omega}]_{\mathrm{B}^{\star}}} \\
{\left[\dot{\mathbf{v}}^{\star}\right]_{\mathrm{B}^{\star}}-[\omega]_{\mathrm{B}^{\star}}^{\times}\left[\mathbf{v}^{\star}\right]_{\mathrm{B}^{\star}}}
\end{array}\right]=\left[\begin{array}{c}
{\left[\mathbf{t}^{\star}\right]_{\mathrm{B}^{\star}}} \\
{[\mathbf{f}]_{\mathrm{B}^{\star}}}
\end{array}\right]
\end{aligned}
$$

Adopt the following notations:

$$
\begin{aligned}
& {[\mathrm{v}]_{\mathrm{B}^{\star}}=\left[\begin{array}{c}
{[\omega]_{\mathrm{B}^{\star}}} \\
{\left[\mathbf{v}^{\star}\right]_{\mathrm{B}^{\star}}}
\end{array}\right]} \\
& {[\dot{\mathrm{v}}]_{\mathrm{B}^{\star}}=\frac{\mathrm{d}}{\mathrm{d} t}[\mathrm{v}]_{\mathrm{B}^{\star}}=\left[\begin{array}{c}
{[\dot{\omega}]_{\mathrm{B}^{\star}}} \\
{\left[\dot{\mathbf{v}}^{\star}\right]_{\mathrm{B}^{\star}}-[\omega]_{\mathrm{B}^{\star}}^{\times}\left[\mathbf{v}^{\star}\right]_{\mathrm{B}^{\star}}}
\end{array}\right]} \\
& {[\mathrm{v}]_{\mathrm{B}^{\star} \mid \mathrm{B}^{\star}}^{\times *}=\left[\begin{array}{cc}
{[\omega]_{\mathrm{B}^{\star}}^{\times}} & {\left[\mathbf{v}^{\star}\right]_{\mathrm{B}^{\star}}^{\times}} \\
0 & {[\omega]_{\mathrm{B}^{\star}}^{\times}}
\end{array}\right]} \\
& {\left[\mathbb{G}^{*}\right]_{\mathrm{B}^{\star}}=\left[\begin{array}{cc}
{\left[\mathbf{t}^{\star}\right]_{\mathrm{B}^{\star}}} \\
{[\mathbf{f}]_{\mathrm{B}^{\star}}}
\end{array}\right]} \\
& {[\overline{\mathbb{I}}]_{\mathrm{B}^{\star} \mid \mathrm{B}^{\star}}=\left[\begin{array}{cc}
{\left[\overline{\mathbf{I}}^{\star}\right]_{\mathrm{B}^{\star} \mid \mathrm{B}^{\star}}} & 0 \\
0 & \bar{m} \mathbf{1}
\end{array}\right]}
\end{aligned}
$$

With this new notation, equation (3.31) can be rewritten in the following form:

$$
[\overline{\mathbb{I}}]_{\mathrm{B}^{\star} \mid \mathrm{B}^{\star}}[\dot{\mathrm{v}}]_{\mathrm{B}^{\star}}+[\mathbb{\mathrm { v }}]_{\mathrm{B}^{\star} \mid \mathrm{B}^{\star}}^{\times *}[\overline{\mathbb{I}}]_{\mathrm{B}^{\star} \mid \mathrm{B}^{\star}}[\mathbb{\mathrm { v }}]_{\mathrm{B}^{\star}}=\left[\mathbb{F}^{*}\right]_{\mathrm{B}^{\star}}
$$

It can be proved that the following identities are valid (FEATHERSTONE, 2008):

$$
\begin{aligned}
& {[\mathrm{\vee}]_{\mathrm{B}^{\star}}=[\mathbb{1}]_{\mathrm{B}^{\star} \mid \mathrm{B}}[\mathrm{v}]_{\mathrm{B}}} \\
& {[\dot{\mathrm{v}}]_{\mathrm{B}^{\star}}=[\mathbb{1}]_{\mathrm{B}^{\star} \mid \mathrm{B}}[\dot{\mathrm{v}}]_{\mathrm{B}}} \\
& {[\mathrm{\vee}]_{\mathrm{B}^{\star} \mid \mathrm{B}^{\star}}^{\times *}=\left[\mathbb{1}^{*}\right]_{\mathrm{B}^{\star} \mid \mathrm{B}}[\mathrm{v}]_{\mathrm{B} \mid \mathrm{B}}^{\times *}[\mathbb{1}]_{\mathrm{B}^{\mathrm{B}} \mathrm{B}^{\star}}^{\top}=\left[\mathbb{1}^{*}\right]_{\mathrm{B}^{\star} \mid \mathrm{B}}[\mathrm{v}]_{\mathrm{B} \mid \mathrm{B}}^{\times *}\left[\mathbb{1}^{*}\right]_{\mathrm{B} \mid \mathrm{B}^{\star}}}
\end{aligned}
$$

Applying these identities to equation (3.37), it can be stated that:

$$
\begin{aligned}
& {[\overline{\mathbb{I}}]_{\mathrm{B}^{\star}}[\mathbb{1}]_{\mathrm{B}^{\star} \mid \mathrm{B}}[\dot{\mathbb{v}}]_{\mathrm{B}}+\left[\mathbb{1}^{*}\right]_{\mathrm{B}^{\star} \mid \mathrm{B}}[\mathbb{\nabla}]_{\mathrm{B} \mid \mathrm{B}}^{\times *}\left[\mathbb{1}^{*}\right]_{\mathrm{B}^{\mathrm{B}^{*}}}[\overline{\mathbb{I}}]_{\mathrm{B}^{\star}}[\mathbb{1}]_{\mathrm{B}^{\star} \mid \mathrm{B}}[\mathbb{\nabla}]_{\mathrm{B}}=\left[\mathbb{1}^{*}\right]_{\mathrm{B}^{\star} \mid \mathrm{B}}\left[\mathbb{F}^{*}\right]_{\mathrm{B}}} \\
& \left(\left[\mathbb{1}^{*}\right]_{\mathrm{B}^{\mathrm{B}^{*}}}[\overline{\mathbb{I}}]_{\mathrm{B}^{\star}}[\mathbb{1}]_{\mathrm{B}^{\star} \mid \mathrm{B}}\right)[\dot{\mathbb{v}}]_{\mathrm{B}}+[\mathbb{\nabla}]_{\mathrm{B} \mid \mathrm{B}}^{\times *}\left(\left[\mathbb{1}^{*}\right]_{\mathrm{B}^{\mathrm{B}^{*}}}[\overline{\mathbb{I}}]_{\mathrm{B}^{\star}}[\mathbb{1}]_{\mathrm{B}^{\star} \mid \mathrm{B}}\right)[\mathbb{\nabla}]_{\mathrm{B}}=\left[\mathbb{F}^{*}\right]_{\mathrm{B}}
\end{aligned}
$$

Finally, taking $[\mathbb{I}]_{\mathrm{B}}=\left[\mathbb{1}^{*}\right]_{\mathrm{B}^{\mathrm{B}^{\star}}}[\overline{\mathbb{I}}]_{\mathrm{B}^{\star}}[\mathbb{1}]_{\mathrm{B}^{\star} \mid \mathrm{B}}$, it can be stated that the Newton-Euler equations of motion of $\mathcal{B}$ expressed in terms of twists and wrenches are given 
by the following expression:

$$
[\overline{\mathbb{I}}]_{\mathrm{B}}[\dot{\mathbb{v}}]_{\mathrm{B}}+[\mathbb{\nabla}]_{\mathrm{B} \mid \mathrm{B}}^{\times *}[\overline{\mathbb{I}}]_{\mathrm{B}}[\mathbb{v}]_{\mathrm{B}}=\left[\mathbb{F}^{*}\right]_{\mathrm{B}}
$$

By hypothesis, B coincides instantaneously with $N$; then, $[\omega]_{\mathrm{B}}^{\times}=[\omega]_{\mathrm{N}}^{\times}=[1]_{N \mid B}$. Therefore, it can be stated that:

$$
\begin{aligned}
& {[\mathrm{v}]_{\mathrm{B}}=[\mathrm{v}]_{\mathrm{N}}=\left(\omega_{\mathrm{x}}, \omega_{\mathrm{y}}, \omega_{\mathrm{z}}, v_{\mathrm{x}}, v_{\mathrm{y}}, v_{\mathrm{z}}\right)} \\
& {[\dot{\mathrm{v}}]_{\mathrm{B}}=[\dot{\mathrm{v}}]_{\mathrm{N}}=\left(\dot{\omega}_{\mathrm{x}}, \dot{\omega}_{\mathrm{y}}, \dot{\omega}_{\mathrm{z}}, \dot{v}_{\mathrm{x}}, \dot{v}_{\mathrm{y}}, \dot{v}_{\mathrm{z}}\right)} \\
& {\left[\mathbb{F}^{*}\right]_{\mathrm{B}}=\left[\mathbb{F}^{*}\right]_{\mathrm{N}}=\left(t_{\mathrm{x}}, t_{\mathrm{y}}, t_{\mathrm{z}}, f_{\mathrm{x}}, f_{\mathrm{y}}, f_{\mathrm{z}}\right)}
\end{aligned}
$$

Taking these identities into consideration, equation (3.43) can be understood as a system of first order ordinary differential equations whose variables are the quasi-velocities defined as the Plücker coordinates of the twists that describe the instantaneous kinematics of the motion of the rigid body $\mathcal{B}$ with respect to an inertial reference frame $\mathcal{N}$.

\subsubsection{Recursive algorithms based on Newton-Euler formal- ism}

The main recursive algorithm for inverse dynamics problems (in which it is desired to determine the forces and torques imposed by actuators for a multibody system to perform some specific motion), is the Recursive Newton-Euler Algorithm, RNEA (FEATHERSTONE, 2008; FEATHERSTONE; ORIN, 2000; KHAN et al., 2005a; CRAIG, 2005; TSAI, 1999). In this algorithm multi-rigid-body systems are conceived as kinematic chains that can be represented by graphs in which each node corresponds to a body and each edge stands for a joint. The nodes are enumerated such that node 0 represents the inertial reference frame chosen for the modeling and that each of the remaining nodes has an index number greater than its parents in the extension tree. For systems constituted by open loop kinematic chains only, the velocities and accelerations of relevant points and the angular velocities and angular accelerations can be obtained recursively in ascending order of the nodal indexes. From Newton-Euler equations, these informations can be used to obtain the force systems that must be applied to the leaves of the extension tree. Then, recursively, in descending order of nodal indexes, the corresponding force systems can be obtained for the corresponding parental bodies using the corresponding Newton-Euler equations. The projections of these force systems in the directions of the axes of the actuators determine the corresponding torque and force components. The 
remaining unknown components calculated by this algorithm correspond to the constraint forces and torques. On the other hand, whenever closed loop kinematic chains are involved, some of the edges of the graph can not be included in an extension tree of it, being necessary to include a loop-closing algorithm involving both the corresponding constraint invariants and the respective forces and torques associated to these edges (FEATHERSTONE, 2008).

For the forward dynamics problems, in which the objective is to describe the motion of a system when some external force systems are applied to it (including forces and torques from actuators and due to disturbances), the main algorithms based on Newton-Euler formalism are the following:

- Composite Rigid Body Algorithm (CRBA): follows an approach similar to RNEA to obtain the forces and torques that are independent from the time derivatives of components of velocity and angular velocity and derives, recursively, an expression for the generalized inertia matrix associated to the system (FEATHERSTONE, 2008; FEATHERSTONE; ORIN, 2000; KHAN et al., 2005a).

- Articulated-body Algorithm ( $A B A$ ): obtains recursively, for each rigid body in the system, from the leaves to the root of the extension tree (propagating external, constraint and inertia forces and torques), the associated "articulated-body inertia matrix" and "bias force", which describe an affine transformation between the components of acceleration and angular acceleration of a body and the force system acting on it (FEATHERSTONE, 2008; RODRIGUEZ, 1987; FEATHERSTONE; ORIN, 2000; KHAN et al., 2005a).

- Constraint Force Algorithm (CFA), Hybrid Direct/lterative Algorithm (HDIA), Divide-and-Conquer Algorithm (DCA) and Total Force Algorithm (TFA): variants of Articulated-body Algorithm that use parallel computation to decrease the complexity of the necessary operations to perform the numerical simulations of multi-rigid-body systems with several bodies involved (FEATHERSTONE, 1999a; FEATHERSTONE, 1999b; MITIGUY; BANERJEE, 2002; FIJANY; FEATHERSTONE, 2012).

It is worth noting that, frequently, these algorithms are implemented using some form of Newton-Euler equations expressed in terms of twists and wrenches, like the one presented in equation (3.43). 


\subsection{Analytical Mechanics: fundamental princi- ples and Lagrangian formalism}

Analytical Mechanics is a collection of formalisms based on the same fundamental principles, which can ultimately be conceived as two (PAPASTAVRIDIS, 1998): the first relating the active forces (i.e., forces caused by physical effects not exclusively related to the motion of a system and described by constitutive equations) and inertial effects (if relevant) to any possible motion of a system and the second establishing analogous relations between the constraint forces (which are strictly to the enforcement of the motion constraints of a system) to any possible motion of a system. The first fundamental principle has several variants, the most well-known being the Principle of Virtual Work and its counterpart that takes inertial effects in consideration, which is typically called D'Alembert's Principle or Lagrange's Principle. Other commonly used formulations of this first principle are: Principle of Virtual Power or Principle of Jourdain, Gauss' Principle of Least Restraint, Principle of Hamilton, etc. Under some circumstances the statements of different versions of the first principle are equivalent Papastavridis (1987). The second principle is normally referred as Principle of Relaxation of Constraints.

In order to derive the equations of motion of a mechanical system, the use of an appropriate form of the first principle must be sufficient. In some cases, however, it might be preferable to use formulations that are based on both principles, like the ones that use the method of Lagrange multipliers.

This section presents the statements of the Principle of Virtual Work and D'Alembert's Principle, along with the corresponding differential forms, and discusses some of the modeling algorithms in the literature based on these principles. Also, the extended version of Hamilton's Principle is presented and from it, the expressions for Lagrangian and canonical equations of motion of a mechanical system are derived. Finally, the use of these formalisms in the development of modeling algorithms for multibody systems is briefly discussed.

\subsubsection{Principle of Virtual Work and D'Alembert's Principle}

Let $\mathcal{S}$ denote a mechanical system for whose motion can be described by tracking a finite number of points, which will be generally denoted by $\mathrm{p}_{k}$. Let $\mathcal{N}$ be an inertial reference frame, denote by $\mathrm{N}$ a coordinate system rigidly attached to $\mathcal{N}$, by $\mathbf{r}_{k}$ the position vector of $\mathrm{p}_{k}$ with respect to $\mathrm{N}$ and by $\delta \mathbf{r}_{k}$ a variation of $\mathbf{r}_{k}$ 
compatible with the constraints of $\mathcal{N}$, called virtual displacement of $\mathrm{p}_{k}$. Suppose that the force system acting in $\mathcal{S}$ is equivalent to applying two forces ${ }^{\mathrm{A}} \mathbf{f}_{k}$ and ${ }^{\mathrm{C}} \mathbf{f}_{k}$ to each point $\mathrm{p}_{k}$, the former representing active forces and the latter, constraint forces. The virtual work of the force system acting on $\delta$ is defined by:

$$
\delta W=\delta W_{\mathrm{A}}+\delta W_{\mathrm{C}} \text { with }\left\{\begin{array}{l}
\delta W_{\mathrm{A}}=\sum_{k}{ }^{\mathrm{A}} \mathbf{f}_{k} \cdot \delta \mathbf{r}_{k} \\
\delta W_{\mathrm{C}}=\sum_{k}{ }^{\mathrm{C}} \mathbf{f}_{k} \cdot \delta \mathbf{r}_{k}
\end{array}\right.
$$

If $\mathcal{S}$ is a system of particles in which each $\mathrm{p}_{k}$ represents one particle, then, according to Newton's laws of motion, $\mathcal{S}$ is in a static equilibrium state if and only if all of the $\mathbf{f}_{k}=0$, which leads to $\delta W=0$. In general, the Principle of Virtual Work states that (LANCZOS, 1986, p. 75):

A mechanical system will be in equilibrium if, and only if, the total virtual work of all the impressed forces vanishes, i.e. $\delta W=0$.

It is more desirable to have an alternative statement of this fundamental principle involving the active forces acting on a mechanical system only. To this end, a single postulate is necessary (LEECH, 1971, p. 13):

Postulate A. For every virtual displacement $\delta \mathbf{r}_{k}$ compatible with the constraints of a mechanical system, it can be stated that:

$$
{ }^{\mathrm{C}} \mathbf{f}_{k} \cdot \delta \mathbf{r}_{k} \geq 0
$$

From this postulate, it is evident that $\delta W_{C} \geq 0$. Therefore, if $\delta W=0$, then $\delta W_{\mathrm{A}}=-\delta W_{\mathrm{C}} \leq 0$. Moreover:

This postulate is not restricted to the realm of statics. It applies equally to dynamics, when the principle of virtual work is suitably generalized by means of d'Alembert's principle. Since all the fundamental variational principles of mechanics, the principles of Euler, Lagrange, Jacobi, Hamilton, are but alternative formulations of d'Alembert's principle, Postulate A is actually the only postulate of analytical mechanics, and thus of fundamental importance.

Those scientists who claim that analytical mechanics is nothing but a mathematically different formulation of the laws of Newton must assume that Postulate $A$ is deducible from the Newtonian laws of motion. The author is unable to see how this can be done. Certainly 
the third law of motion, "action equals reaction," is not wide enough to replace Postulate A (LANCZOS, 1986, p. 77).

Particularly, consider that $\delta$ is in a configuration in which if $\delta \mathbf{r}_{k}$ is a virtual displacement compatible with the constraints of the system, so will be $-\delta \mathbf{r}_{k}$. In this case, the virtual displacements of $\delta$ are reversible and it can be stated that:

$$
\sum_{k}{ }^{A} \mathbf{f}_{k} \cdot \delta \mathbf{r}_{k} \leq 0 \quad \text { and } \quad \sum_{k}{ }^{A} \mathbf{f}_{k} \cdot\left(-\delta \mathbf{r}_{k}\right) \leq 0 \Rightarrow \sum_{k}{ }^{A} \mathbf{f}_{k} \cdot \delta \mathbf{r}_{k}=0
$$

Thus, the Principle of Virtual Work can be stated as follows:

A mechanical system will be in equilibrium if, and only if, the virtual work of the impressed active forces is equal to zero for every reversible virtual displacements compatible with the constraints of the system. If, in a particular configuration, some virtual displacement is not reversible, then the system is in equilibrium if the virtual work of the impressed active forces is equal to or less than zero.

In order to make a distinction between the conventional statement of the Principle of Virtual Work for equilibrium states of mechanical systems and its general version for arbitrary states, the latter one is called D'Alembert's Principle (LANCZOS, 1986), Principle of D'Alembert in Lagrange's form or Lagrange's Principle (PAPASTAVRIDIS, 1998). This nomenclature is due to the concept of inertia force introduced by Jean le Rond d'Alembert, that enables a straightforward extension of the Principle of Virtual Work to non-equilibrium states. Under the same hypothesis consider that the force system acting in $\delta$ is equivalent to applying not only the forces ${ }^{\mathrm{A}} \mathbf{f}_{k}$ and ${ }^{\mathrm{C}} \mathbf{f}_{k}$ to each point $\mathrm{p}_{k}$ but also an inertia force ${ }^{\mathrm{I}} \mathbf{f}_{k}$. For example, if $\mathcal{S}$ is a system of particles and each $\mathrm{p}_{k}$ represents a particle whose mass is denoted by $\bar{m}_{k}$ and whose velocity with respect to $\mathcal{N}$ is denoted by $\mathbf{v}_{k}$, then ${ }^{\mathrm{I}} \mathbf{f}_{k}=-\bar{m}_{k} \dot{\mathbf{v}}_{k}$. Thus, considering Postulate $\mathrm{A}$, it can be stated that:

$$
\sum_{k}\left({ }^{\mathrm{A}} \mathbf{f}_{k}+{ }^{\mathrm{I}} \mathbf{f}_{k}\right) \cdot \delta \mathbf{r}_{k} \leq 0
$$

The sum $\left({ }^{\mathrm{A}} \mathbf{f}_{k}+{ }^{\mathrm{I}} \mathbf{f}_{k}\right)$ is sometimes called effective force and D'Alembert's Principle can be stated as follows (LANCZOS, 1986; LEECH, 1971):

The virtual work of the effective forces in a mechanical system is zero for every reversible virtual displacements compatible with the 
constraints of the system. If any virtual displacement is not reversible, than the corresponding virtual work is equal to or less than zero.

\subsubsection{Differential Variational Principles}

The first fundamental principle of Analytical Mechanics can also have alternative statements which are generally referred as Differential Variational Principles (PAPASTAVRIDIS, 1998). The most well known forms of this category are the Principle of Virtual Power or Jourdain's Principle (PAPASTAVRIDIS, 1992) and the Gauss' Principle. The former is particularly useful in problems of impulsive motion, while the latter is a genuine minimum principle, also known as Gauss' Principle of Least Constraint (LANCZOS, 1986), which underlie the UdwadiaKalaba methodology (see Section 3.4) and can be extended to applications in the study of singular configurations of mechanical systems (PAPASTAVRIDIS, 1998).

Let $\delta$ be a mechanical system satisfying the same conditions listed in the previous subsection. Define $\mathbf{r}_{k}^{(0)}=\mathbf{r}_{k}, \mathbf{r}_{k}^{(\beta)}=\mathrm{d}^{\beta} \mathbf{r}_{k} / \mathrm{d} t^{\beta}$, and consider that in a given time instant $t^{*}$, all $\delta \mathbf{r}_{k}^{(\alpha)}\left(t^{*}\right)=0$ for every $\alpha$ such that $0 \leq \alpha \leq \rho-1$. Assuming that $\mathbf{r}_{k}(t)$ is a class $\mathscr{C}^{\rho}$ function in a neighborhood of $t^{*}$, it can be stated that for an infinitesimal $\varepsilon$ :

$$
\delta \mathbf{r}_{k}\left(t^{*}+\varepsilon\right)=\frac{\varepsilon^{\rho}}{\rho !} \delta \mathbf{r}_{k}^{(\rho)}\left(t^{*}\right)+\mathbf{o}\left(\varepsilon^{\rho}\right)
$$

Thus, applying the Principle of Virtual Work for $\delta$ in the time instant $(t+\varepsilon)$, it can be stated that, for $\varepsilon>0$ :

$$
\begin{aligned}
& \sum_{k}\left({ }^{\mathrm{A}} \mathbf{f}_{k}\left(t^{*}+\varepsilon\right)+{ }^{\mathrm{I}} \mathbf{f}_{k}\left(t^{*}+\varepsilon\right)\right) \cdot \delta \mathbf{r}_{k}\left(t^{*}+\varepsilon\right) \leq 0 \\
& \sum_{k}\left({ }^{\mathrm{A}} \mathbf{f}_{k}\left(t^{*}\right)+{ }^{\mathrm{I}} \mathbf{f}_{k}\left(t^{*}\right)+\mathbf{o}(\varepsilon)\right) \cdot\left(\frac{\varepsilon^{\rho}}{\rho !} \delta \mathbf{r}_{k}^{(\rho)}\left(t^{*}\right)+\mathbf{o}\left(\varepsilon^{\rho}\right)\right) \leq 0 \\
& \frac{\varepsilon^{\rho}}{\rho !} \sum_{k}\left({ }^{\mathrm{A}} \mathbf{f}_{k}\left(t^{*}\right)+{ }^{\mathrm{I}} \mathbf{f}_{k}\left(t^{*}\right)\right) \cdot \delta \mathbf{r}_{k}^{(\rho)}\left(t^{*}\right)+\mathbf{o}\left(\varepsilon^{\rho}\right) \leq 0 \\
& \sum_{k}\left({ }^{\mathrm{A}} \mathbf{f}_{k}\left(t^{*}\right)+{ }^{\mathrm{I}} \mathbf{f}_{k}\left(t^{*}\right)\right) \cdot \delta \mathbf{r}_{k}^{(\rho)}\left(t^{*}\right)+\rho ! \frac{\mathbf{o}\left(\varepsilon^{\rho}\right)}{\varepsilon^{\rho}} \leq 0
\end{aligned}
$$

Finally, taking the limit $\varepsilon \rightarrow 0$ :

$$
\sum_{k}\left({ }^{A} \mathbf{f}_{k}\left(t^{*}\right)+{ }^{\mathrm{I}} \mathbf{f}_{k}\left(t^{*}\right)\right) \cdot \delta \mathbf{r}_{k}^{(\rho)}\left(t^{*}\right) \leq 0
$$


Thus, if $\rho>0$, whenever it is possible to consider that all $\delta \mathbf{r}_{k}^{(\alpha)}=0$ for every $\alpha$ such that $0 \leq \alpha \leq \rho-1$, the corresponding $\rho$-th order variational principle can be stated:

$$
\sum_{k}\left({ }^{\mathrm{A}} \mathbf{f}_{k}+{ }^{\mathrm{I}} \mathbf{f}_{k}\right) \cdot \delta \mathbf{r}_{k}^{(\rho)} \leq 0
$$

Moreover, if all the variations $\delta \mathbf{r}_{k}^{(\rho)}$ can be supposed reversible, this principle can be stated as follows (JARZEBOWSKA, 2012; MANGERON; DELEANU, 1962; PAPASTAVRIDIS, 1998):

$$
\sum_{k}\left({ }^{\mathrm{A}} \mathbf{f}_{k}+{ }^{\mathrm{I}} \mathbf{f}_{k}\right) \cdot \delta \mathbf{r}_{k}^{(\rho)}=0
$$

When $\rho=0$, equations (3.53) and (3.54) correspond to D'Alembert's Principle and when $\rho=1$ to Jourdain's Principle.

For $\rho=2$, equation (3.54) represents the statement of Gauss' Principle of Least Constraint. Indeed, consider without loss of generality, that $\delta$ is a system of particles in which each $\mathrm{p}_{k}$ represents a particle whose mass is denoted by $\bar{m}_{k}$, and define $Z$, the Gaussian deviation (PAPASTAVRIDIS, 1998) or "constraint" (LANCZOS, 1986) of system $\mathcal{S}$ as follows:

$$
Z=\sum_{k} \frac{\bar{m}_{k}}{2}\left(\ddot{\mathbf{r}}_{k}-\frac{{ }^{\mathrm{A}} \mathbf{f}_{k}}{\bar{m}_{k}}\right)^{2}=\sum_{k} \frac{1}{2 \bar{m}_{k}}\left({ }^{\mathrm{A}} \mathbf{f}_{k}-\bar{m}_{k} \ddot{\mathbf{r}}_{k}\right)^{2}
$$

Assume that all $\delta \mathbf{r}_{k}=\mathbf{0}$ and all $\delta \dot{\mathbf{r}}_{k}=\mathbf{0}$. The variation of $Z$ is given by:

$$
\delta Z=-\sum_{k}\left({ }^{A} \mathbf{f}_{k}-\bar{m}_{k} \ddot{\mathbf{r}}_{k}\right) \cdot \delta \ddot{\mathbf{r}}_{k}
$$

Thus, if all the variations $\delta \ddot{\mathbf{r}}_{k}$ can be supposed reversible, then the expression for Gauss' Principle for $\mathcal{S}$ is:

$$
\delta Z=0
$$

Once $Z$ is a sum of non-negative terms, it must have a minimum. It can be proved that the actual motion of a system not only corresponds to a stationary Gaussian deviation (i.e., $\delta Z=0$ ), but also to a minimum of $Z$ (PAPASTAVRIDIS, 1998). 


\subsubsection{Modeling methodologies based on the Principle of Vir- tual Work and its variants}

Through the use of adequate formulations, applications of the Principle of Virtual Work and of its variant forms can be found in almost every area of Multibody System Dynamics, including continuous systems (SCHWAB; MEIJAARD, 2003; MAUGIN, 2013). For instance, the "weak formulations" of the statics and dynamics problems of continuous systems are based on the integral form of the equation obtained by the application of the Principle of Virtual Work to them. Due to the freedom to choose variables and to the lack of restrictions to the nature of the motion constraints involved, it can be found in the literature several modeling methodologies derived from this first fundamental principle of Analytical Mechanics. Some of these methodologies, however, are so specialized to some kinds of problems that sometimes the modeling algorithms impose restrictions for the choice of variables and use of some mathematical tools. Such peculiarities can become deterrent if someone wants to use more than one of these methodologies in the same problem. Even though they derive from the same principle, the integration between the corresponding modeling algorithms might not be straightforward, requiring effort and skill from users.

One area in Multibody System Dynamics in which specialized methodologies have been successful is the modeling of closed-loop kinematic chains, particularly parallel mechanisms (TSAI, 1999), for which were even developed recursive matrix algorithms based on Principle of Virtual Work (STAICU; ZHANG, 2008; LI; STAICU, 2011; STAICU, 2013). Other relevant applications are related to modeling methodologies based on Graph Theory. Shi and McPhee (2000), McPhee, Schmitke and Redmond (2004) and Schmitke and McPhee (2005), for instance, show that the integration between graphs and algorithms based on the Principle of Virtual Work led to the development of a package for Maple called DynaFlex, that can be used in the symbolic or numerical derivation of the equations of motion of multibody systems composed of both rigid and flexible bodies.

\subsubsection{Extended Hamilton's Principle}

Hamilton's Principle can be understood as a variant form of the fundamental principle of Analytical Mechanics, being equivalent to D'Alembert's Principle for the study of generic mechanical systems. It must be highlighted, however, that 
the applications of Hamilton's Principle are followed by several mathematical tools from Calculus of Variations (GELFAND; FOMIN; SILVERMAN, 2000), which are helpful in deriving of equations of motion, in finding solutions for them and in obtaining conservation laws for the modeled systems.

From this principle, Lagrangian equations of motion and Hamilton's canonical equations of motion can be derived from "energy functions" called Lagrangian and Hamiltonian, expressed in terms of the generalized coordinates, of its time derivatives or equivalently, of the associated generalized momenta and of some physical and geometrical parameters of the model. In case of nonconservative force systems, techniques based on any variant of the Principle of Virtual Work can be used to obtain the corresponding generalized forces.

Let $\delta$ be a mechanical system, and suppose, for the sake of simplicity, that it is constituted by a finite number of particles, each of them being represented by a point $\mathrm{p}_{k}$. Let $\mathcal{N}$ be an inertial reference frame and $\mathrm{N}$ be a coordinate system rigidly attached to $\mathcal{N}$. Denote by $m_{k}$ the mass of the particle represented by $\mathrm{p}_{k}$, by $\mathbf{r}_{k}$ the position vector of $\mathrm{p}_{k}$ with respect to $\mathrm{N}$ and by $\mathbf{v}_{k}=\dot{\mathbf{r}}_{k}$ the velocity of the corresponding particle with respect to $\mathcal{N}$. The kinetic energy of $\delta$ is defined as follows:

$$
T=\sum_{k} \frac{1}{2} m_{k} \mathbf{v}_{k} \cdot \mathbf{v}_{k}
$$

It can be stated that the virtual work of the inertia forces of $\mathcal{\delta}$ satisfies the following identity:

$$
\begin{aligned}
\sum_{k}{ }^{\mathrm{I}} \mathbf{f}_{k} \cdot \delta \mathbf{r}_{k} & =-\sum_{k} m_{k} \ddot{\mathbf{r}}_{k} \cdot \delta \mathbf{r}_{k} \\
& =\sum_{k} m_{k} \dot{\mathbf{r}}_{k} \cdot \delta \dot{\mathbf{r}}_{k}-\sum_{k} m_{k} \frac{\mathrm{d}}{\mathrm{d} t}\left(\dot{\mathbf{r}}_{k} \cdot \delta \mathbf{r}_{k}\right) \\
& =\delta T-\sum_{k} m_{k} \frac{\mathrm{d}}{\mathrm{d} t}\left(\dot{\mathbf{r}}_{k} \cdot \delta \mathbf{r}_{k}\right)
\end{aligned}
$$

Consider that $\delta$ is in a configuration in which all the virtual displacements are reversible. Due to D'Alembert's Principle, it can be stated that:

$$
\delta T+\sum_{k}{ }^{A} \mathbf{f}_{k} \cdot \delta \mathbf{r}_{k}=\sum_{k} m_{k} \frac{\mathrm{d}}{\mathrm{d} t}\left(\dot{\mathbf{r}}_{k} \cdot \delta \mathbf{r}_{k}\right)
$$

Suppose additionally that there are two distinct time instants $t_{0}$ and $t_{1}, t_{1}>t_{0}$, for which the positions of all particles of the system are known. Thus, adopt $\delta \mathbf{r}_{k}\left(t_{0}\right)=\delta \mathbf{r}_{k}\left(t_{1}\right)=\mathbf{0}, \forall k$. Taking the integral of equation (3.60) in the interval 
$t_{0}<t<t_{1}$, it can be stated that:

$$
\begin{aligned}
\int_{t_{0}}^{t_{1}}(\delta T+ & \left.\sum_{k}{ }^{A} \mathbf{f}_{k} \cdot \delta \mathbf{r}_{k}\right) d t \\
& =\sum_{k} m_{k}\left(\dot{\mathbf{r}}_{k}\left(t_{1}\right) \cdot \delta \mathbf{r}_{k}\left(t_{1}\right)-\dot{\mathbf{r}}_{k}\left(t_{0}\right) \cdot \delta \mathbf{r}_{k}\left(t_{0}\right)\right)=0
\end{aligned}
$$

Assuming that "the variation of a definite integral is equal to the definite integral of the variation" (LANCZOS, 1986, Sec. II-9), Extended Hamilton's Principle can be expressed as follows:

$$
\delta \int_{t_{0}}^{t_{1}} T \mathrm{~d} t+\int_{t_{0}}^{t_{1}} \delta W_{\mathrm{A}} \mathrm{d} t=0
$$

The classical form of Hamilton's Principle is valid whenever the force system acting in $\delta$ is conservative, i.e. if there is a single scalar differentiable function $V$ called potential energy of $\delta$ such that for every $\mathrm{p}_{k}$ :

$$
{ }^{\mathrm{A}} \mathbf{f}_{k}=-\frac{\partial V}{\partial \mathbf{r}_{k}}
$$

In this case, system $\delta$ is classified as conservative and:

$$
\int_{t_{0}}^{t_{1}} \delta W_{\mathrm{A}} \mathrm{d} t=-\int_{t_{0}}^{t_{1}} \sum_{k} \frac{\partial V}{\partial \mathbf{r}_{k}} \cdot \delta \mathbf{r}_{k} \mathrm{~d} t=-\delta \int_{t_{0}}^{t_{1}} V \mathrm{~d} t
$$

Defining the Lagrangian of $\mathcal{S}$ as the function $L=T-V$, it can be stated that equation (3.62) can be rewritten as follows:

$$
\delta \int_{t_{0}}^{t_{1}} L \mathrm{~d} t=0
$$

This is the classical form of Hamilton's Principle, valid for conservative systems only.

\subsubsection{Lagrangian equations of motion}

Let $\mathcal{S}$ be a conservative mechanical system whose Lagrangian function is given by $L=L(t, \boldsymbol{q}, \dot{\boldsymbol{q}})$, with $\boldsymbol{q}$ standing for the generalized coordinates of the model. It can be stated that:

$$
\begin{aligned}
& \delta L=\left(\delta \boldsymbol{q}^{\top} \frac{\partial \underline{\underline{L}}}{\partial \boldsymbol{q}}+\delta \dot{\boldsymbol{q}}^{\top} \frac{\partial \underline{\underline{L}}}{\partial \dot{\boldsymbol{q}}}\right)=\delta \boldsymbol{q}^{\top}\left(\frac{\partial \underline{\underline{L}}}{\partial \boldsymbol{q}}-\frac{\mathrm{d}}{\mathrm{d} t}\left(\frac{\partial \underline{\underline{L}}}{\partial \dot{\boldsymbol{q}}}\right)\right)+\frac{\mathrm{d}}{\mathrm{d} t}\left(\delta \boldsymbol{q}^{\top} \frac{\partial \underline{\underline{L}}}{\partial \dot{\boldsymbol{q}}}\right) \\
& \delta \int_{t_{0}}^{t_{1}} L \mathrm{~d} t=\int_{t_{0}}^{t_{1}} \delta \boldsymbol{q}^{\top}\left(\frac{\partial \underline{\underline{L}}}{\partial \boldsymbol{q}}-\frac{\mathrm{d}}{\mathrm{d} t}\left(\frac{\partial \underline{\underline{L}}}{\partial \dot{\boldsymbol{q}}}\right)\right) \mathrm{d} t+\left.\delta \boldsymbol{q}^{\top} \frac{\partial \underline{L}}{\partial \dot{\boldsymbol{q}}}\right|_{t_{0}} ^{t_{1}}
\end{aligned}
$$


Consider that there are two time instants $t_{0}$ and $t_{1}$ in which the configurations of $\mathcal{S}$ are known. Thus, it is possible to adopt $\delta \boldsymbol{q}\left(t_{0}\right)=\delta \boldsymbol{q}\left(t_{1}\right)=0$, which cancels the second term in the right-hand side of equation (3.67). If the number of generalized coordinates adopted in the modeling of $\delta$ is equal to the number of degrees of freedom of the system (which is only possible if $\mathcal{S}$ is a holonomic system), it can be stated that all the components of $\delta q$ are independent, and to ensure that Hamilton's principle, given by equation (3.65) is satisfied, it is necessary that:

$$
\frac{\partial \underline{L}}{\partial \boldsymbol{q}}-\frac{\mathrm{d}}{\mathrm{d} t}\left(\frac{\partial \underline{L}}{\partial \dot{\boldsymbol{q}}}\right)=0
$$

The system of equations (3.68) are the Lagrangian equations of motion for a holonomic conservative mechanical system for which a minimal set of generalized coordinates is adopted.

For a non-conservative system, the extended form of Hamilton's principle, given by equation (3.62) must be used. Analogously to equation (3.67), it can be stated that:

$$
\delta \int_{t_{0}}^{t_{1}} T \mathrm{~d} t=\int_{t_{0}}^{t_{1}} \delta \boldsymbol{q}^{\top}\left(\frac{\partial \underline{T}}{\partial \boldsymbol{q}}-\frac{\mathrm{d}}{\mathrm{d} t}\left(\frac{\partial \underline{T}}{\partial \dot{\boldsymbol{q}}}\right)\right) \mathrm{d} t
$$

Moreover, once any $\mathbf{r}_{k}$ can be expressed in the form $\mathbf{r}_{k}=\underline{\mathbf{r}}_{k}(t, \boldsymbol{q})$, then:

$$
\delta \mathbf{r}_{k}=\frac{\partial \mathbf{r}_{k}}{\partial \boldsymbol{q}} \delta \boldsymbol{q}
$$

Thus:

$$
\delta W_{\mathrm{A}}=\sum_{k} \delta \mathbf{r}_{k} \cdot{ }^{\mathrm{A}} \mathbf{f}_{k}=\delta \boldsymbol{q}^{\top}\left(\sum_{k} \frac{\partial \mathbf{r}_{k}}{\partial \boldsymbol{q}} \cdot{ }^{\mathrm{A}} \mathbf{f}_{k}\right)=\delta \boldsymbol{q}^{\top \mathrm{A}} \boldsymbol{f}
$$

Finally:

$$
\delta \int_{t_{0}}^{t_{1}} T \mathrm{~d} t+\int_{t_{0}}^{t_{1}} \delta W_{\mathrm{A}} \mathrm{d} t=\int_{t_{0}}^{t_{1}} \delta \boldsymbol{q}^{\top}\left(\frac{\partial \underline{T}}{\partial \boldsymbol{q}}-\frac{\mathrm{d}}{\mathrm{d} t}\left(\frac{\partial \underline{T}}{\partial \dot{\boldsymbol{q}}}\right)+{ }^{\mathrm{A}} \boldsymbol{f}\right) \mathrm{d} t=0
$$

Again, if $\delta$ is a holonomic system and the number of generalized coordinates of its model is equal to the number of degrees of freedom of the system, it can be stated that the satisfaction of Extended Hamilton's Principle requires that:

$$
\frac{\partial \underline{T}}{\partial \boldsymbol{q}}-\frac{\mathrm{d}}{\mathrm{d} t}\left(\frac{\partial \underline{T}}{\partial \dot{\boldsymbol{q}}}\right)+{ }^{\mathrm{A}} \boldsymbol{f}=0
$$

This form of Lagrangian equations of motion is valid for any holonomic system in which a minimal set of generalized coordinates is defined.

If some of the forces acting in $\delta$ are conservative, then there must exist a 
single differentiable scalar function $V$ such that the corresponding components of these forces are given by the gradient of $-V$, i.e.:

$$
{ }^{\mathrm{A}} \boldsymbol{f}=-\frac{\partial V}{\partial \boldsymbol{q}}+{ }^{\mathrm{A}} \boldsymbol{f}^{\prime}
$$

with ${ }^{\mathrm{A}} \boldsymbol{f}^{\prime}$ denoting the generalized forces associated to the non-conservative force systems acting in $\mathcal{S}$. Therefore, equation (3.73) can be written in the following forms:

$$
\begin{aligned}
& \frac{\partial \underline{T}}{\partial \boldsymbol{q}}-\frac{\partial V}{\partial \boldsymbol{q}}-\frac{\mathrm{d}}{\mathrm{d} t}\left(\frac{\partial \underline{T}}{\partial \dot{\boldsymbol{q}}}\right)+{ }^{\mathrm{A}} \boldsymbol{f}^{\prime}=\mathbf{0} \\
& \frac{\partial \underline{\underline{L}}}{\partial \boldsymbol{q}}-\frac{\mathrm{d}}{\mathrm{d} t}\left(\frac{\partial \underline{L}}{\partial \dot{\boldsymbol{q}}}\right)+{ }^{\mathrm{A}} \boldsymbol{f}^{\prime}=\mathbf{0}
\end{aligned}
$$

Note that the $V$ is supposed to be a function of time and of the generalized coordinates of $\mathcal{S}$ only, i.e. $V=\underline{V}(t, \boldsymbol{q})$.

\subsubsection{Lagrangian multipliers and canonical equations of motion}

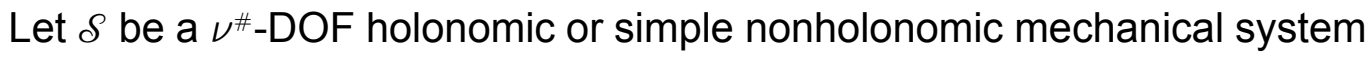
whose constraint order is equal to 1 . Consider that are defined $\nu(q)$ generalized coordinates and $\nu(\boldsymbol{q})$ quasi-velocities for the modeling of $\mathcal{S}$, such that $\boldsymbol{q}^{\langle 0\rangle}=\boldsymbol{q}$ and $\boldsymbol{q}^{\langle 1\rangle}=\dot{\boldsymbol{q}}$. The variations of the independent generalized constraint invariants of $\mathcal{S}$ (if any) can be expressed in the following form, with $A=\underline{A}(t, q)$ :

$$
A \delta q=0
$$

Thus, for any time history $(t, \boldsymbol{q}(t), \dot{\boldsymbol{q}}(t))$ compatible with the constraints of $\mathcal{\delta}$, $\delta L$ can be replaced by $\delta L+\delta \boldsymbol{q}^{\top} \boldsymbol{A}^{\top} \boldsymbol{\lambda}$, with $\boldsymbol{\lambda} \in \mathbb{R}^{\nu(q)-\nu^{\#}}$ representing a columnmatrix of the so called Lagrangian multipliers. The corresponding expression of Extended Hamilton's Principle for $\mathcal{S}$ is:

$$
\int_{t_{0}}^{t_{1}} \delta \boldsymbol{q}^{\top}\left(\frac{\partial \underline{\underline{L}}}{\partial \boldsymbol{q}}-\frac{\mathrm{d}}{\mathrm{d} t}\left(\frac{\partial \underline{\underline{L}}}{\partial \dot{\boldsymbol{q}}}\right)+{ }^{\mathrm{A}} \boldsymbol{f}^{\prime}+\boldsymbol{A}^{\top} \boldsymbol{\lambda}\right) \mathrm{d} t=0
$$

Once the $\nu(\boldsymbol{q})-\nu^{\#}$ elements of $\lambda$ are a priori undetermined, they can be chosen to cancel $\nu(\boldsymbol{q})-\nu^{\#}$ of the elements of the column-matrix left-multiplied by $\delta \boldsymbol{q}^{\top}$. There will still remain $\nu^{\#}$ non-zero elements in this column-matrix. However, now these elements represent the coefficients of $\nu^{\#}$ variations of generalized coordinates in equation (3.78). Considering that $\nu^{\#}$ variations of generalized coordinates of $\delta$ can be assumed as independent, then the only form of ensuring the satisfaction of the Extended Hamilton's Principle (3.78) is to impose that 
the corresponding coefficients of these variations must be zero too. Therefore, the Lagrangian equations of motion of $\mathcal{S}$ are given by:

$$
\frac{\partial \underline{L}}{\partial \boldsymbol{q}}-\frac{\mathrm{d}}{\mathrm{d} t}\left(\frac{\partial \underline{L}}{\partial \dot{\boldsymbol{q}}}\right)+{ }^{\mathrm{A}} \boldsymbol{f}^{\prime}+\boldsymbol{A}^{\top} \boldsymbol{\lambda}=\mathbf{0}
$$

The system of equations (3.79) represents the most general form of Lagrange's equation of motion for systems with constant mass, either holonomic or simple nonholonomic constraints and trivial generalized variables. Pesce (2003) presentes an even more general version of Lagrange's equations of motion, applicable to variable-mass systems.

In order to obtain Hamilton's canonical equations of motion of $\mathcal{S}$, let $L^{*}$ be defined as follows (LANCZOS, 1986):

$$
L^{*}=\underline{L}^{*}(t, \boldsymbol{q}, \dot{\boldsymbol{q}}, \boldsymbol{p}, \boldsymbol{v})=\underline{L}(t, \boldsymbol{q}, \boldsymbol{v})+\boldsymbol{p}^{\top}(\dot{\boldsymbol{q}}-\boldsymbol{v})
$$

It can be stated that:

$$
\delta L^{*}=\delta \boldsymbol{q}^{\top} \frac{\partial \underline{L}^{*}}{\partial \boldsymbol{q}}+\delta \dot{\boldsymbol{q}}^{\top} \frac{\partial \underline{L}^{*}}{\partial \dot{\boldsymbol{q}}}+\delta \boldsymbol{p}^{\top} \frac{\partial \underline{L}^{*}}{\partial \boldsymbol{p}}+\delta \boldsymbol{v}^{\top} \frac{\partial \underline{L}^{*}}{\partial \boldsymbol{v}}
$$

with:

$$
\begin{array}{ll}
\frac{\partial \underline{L}^{*}}{\partial \boldsymbol{q}}=\frac{\partial \underline{L}}{\partial \boldsymbol{q}} & \frac{\partial \underline{L}^{*}}{\partial \dot{\boldsymbol{q}}}=\boldsymbol{p} \\
\frac{\partial \underline{L}^{*}}{\partial \boldsymbol{p}}=\dot{\boldsymbol{q}}-\boldsymbol{v} & \frac{\partial \underline{L}^{*}}{\partial \boldsymbol{v}}=\frac{\partial \underline{L}}{\partial \boldsymbol{v}}-\boldsymbol{p}
\end{array}
$$

Applying Extended Hamilton's Principle with $L^{*}$ replacing $L$, it can be stated that:

$$
\int_{t_{0}}^{t_{1}}\left(\delta \boldsymbol{q}^{\top}\left(\frac{\partial \underline{L}^{*}}{\partial \boldsymbol{q}}-\frac{\mathrm{d}}{\mathrm{d} t}\left(\frac{\partial \underline{L}^{*}}{\partial \dot{\boldsymbol{q}}}\right)+{ }^{\mathrm{A}} \boldsymbol{f}^{\prime}\right)+\delta \boldsymbol{p}^{\top} \frac{\partial \underline{\underline{L}}^{*}}{\partial \boldsymbol{p}}+\delta \boldsymbol{v}^{\top} \frac{\partial \underline{L}^{*}}{\partial \boldsymbol{v}}\right) \mathrm{d} t=0
$$

Assuming that $\delta p$ and $\delta v$ are column-matrices of independent variations, whose values can be arbitrarily chosen, it can be stated that two necessary conditions for the satisfaction of equation (3.83) are the following:

$$
\begin{aligned}
& \frac{\partial \underline{L}^{*}}{\partial \boldsymbol{p}}=0 \quad \Rightarrow \quad \boldsymbol{v}=\dot{\boldsymbol{q}} \\
& \frac{\partial \underline{L}^{*}}{\partial \boldsymbol{v}}=0 \quad \Rightarrow \quad \boldsymbol{p}=\frac{\partial \underline{\underline{L}}}{\partial \boldsymbol{v}}
\end{aligned}
$$

From (3.80), it can be noted that if $\boldsymbol{v}=\dot{\boldsymbol{q}}$, then $L^{*}=L$, which proves that equation (3.83) is indeed the expression of the Extended Hamilton's Principle for system $\mathcal{S}$.

Suppose that there is a function $H=\underline{H}(t, \boldsymbol{q}, \boldsymbol{p})$, called the Hamiltonian of $\delta$ 
such that:

$$
L^{*}=\boldsymbol{p}^{\top} \dot{\boldsymbol{q}}-H
$$

Calculating the corresponding partial derivatives of $L^{*}$ by equation (3.86), it can be stated that:

$$
\begin{array}{ll}
\frac{\partial \underline{L}^{*}}{\partial \boldsymbol{q}}=-\frac{\partial \underline{H}}{\partial \boldsymbol{q}} & \frac{\partial \underline{L}^{*}}{\partial \dot{\boldsymbol{q}}}=\boldsymbol{p} \\
\frac{\partial \underline{L}^{*}}{\partial \boldsymbol{p}}=\dot{\boldsymbol{q}}-\frac{\partial \underline{H}}{\partial \boldsymbol{p}} & \frac{\partial \underline{L}^{*}}{\partial \boldsymbol{v}}=\boldsymbol{0}
\end{array}
$$

The compatibility between (3.82) and (3.87) requires that:

$$
\frac{\partial \underline{L}}{\partial \boldsymbol{q}}=-\frac{\partial \underline{H}}{\partial \boldsymbol{q}} \quad \text { and } \quad \dot{\boldsymbol{q}}=\frac{\partial \underline{H}}{\partial \boldsymbol{p}}
$$

Finally, from equations (3.87) and (3.88), it can be stated that (3.83) leads to:

$$
\int_{t_{0}}^{t_{1}} \delta \boldsymbol{q}^{\top}\left(-\frac{\partial \underline{H}}{\partial \boldsymbol{q}}-\dot{\boldsymbol{p}}+{ }^{\mathrm{A}} \boldsymbol{f}^{\prime}\right) \mathrm{d} t=0
$$

Therefore, if $\mathcal{S}$ is a holonomic system with a minimal set of generalized coordinates, (3.89) leads to the following Hamilton's canonical equations of motion of $\delta$ :

$$
\begin{aligned}
& \dot{\boldsymbol{q}}=\frac{\partial \underline{H}}{\partial \boldsymbol{p}} \\
& \dot{\boldsymbol{p}}=-\frac{\partial \underline{H}}{\partial \boldsymbol{q}}+{ }^{\mathrm{A}} \boldsymbol{f}^{\prime}
\end{aligned}
$$

Otherwise, if $\mathcal{S}$ is any holonomic or simple nonholonomic system whose constraints demand the satisfaction of an equation like (3.77), the strategy of using the Lagrange multipliers method can be applied, leading to the a system of canonical equations of motion similar to $(3.90,3.91)$, with (3.91) being replaced by:

$$
\dot{\boldsymbol{p}}=-\frac{\partial \underline{H}}{\partial \boldsymbol{q}}+{ }^{\mathrm{A}} \boldsymbol{f}^{\prime}+\boldsymbol{A}^{\top} \boldsymbol{\lambda}
$$

It is important to highlight that the column-matrix $v$ is constituted of auxiliary variables introduced only for the derivations. Taking the conventional expression of the Lagrangian of $\mathcal{S}, L=\underline{L}(t, \boldsymbol{q}, \dot{\boldsymbol{q}})$, the generalized momenta $\boldsymbol{p}$ can be defined as follows:

$$
\boldsymbol{p}=\frac{\partial \underline{\underline{L}}}{\partial \dot{\boldsymbol{q}}}
$$


Moreover, the Hamiltonian of $\mathcal{S}$ can be obtained by following expression:

$$
H=\underline{H}(t, \boldsymbol{q}, \boldsymbol{p})=\boldsymbol{p}^{\top} \dot{\boldsymbol{q}}-\underline{L}(t, \boldsymbol{q}, \underline{\dot{q}}(t, \boldsymbol{q}, \boldsymbol{p}))
$$

with $\dot{\boldsymbol{q}}=\dot{\boldsymbol{q}}(t, \boldsymbol{q}, \boldsymbol{p})$ representing a solution of the system of equations (3.93) in terms of $\dot{\boldsymbol{q}}$.

\subsubsection{Modeling methodologies based on Lagrangian- Hamiltonian formalism}

Lagrangian-Hamiltonian formalism has been responsible for the development of some of the most used methodologies for modeling multibody systems, allying the fundamental principles of Analytical Mechanics to mathematical techniques from Calculus of Variations. Lagrangian or canonical equations of motion combine the versatility of applications to a great variety of mechanical systems ${ }^{6}$ with descriptions of configurations based on the use of generalized coordinates and, eventually, with the use of the method of Lagrange multipliers. Integrating all these techniques, Lagrangian-Hamiltonian formalism systematize typical modeling procedures, enabling the development of algorithms, which explains the great variety of software packages for Multibody System Dynamics based on this formalism (PAUL, 1975).

The method of Lagrange multipliers also enable several extensions to the application of Lagrangian-Hamiltonian formalism. For instance, Seliger and Whitham (1968) use the undetermined multipliers to generalize the variational principles of mechanics to problems of continuous mechanics that originally adopt an Eulerian description of motion. In applications of Graph Theory to the modeling of mechanical systems, Lagrangian multipliers are important to interpret the internal conexions among the subsystems involved, which might lead to some simplifications in the modeling procedure (KARNOPP, 1997; RICHARD; BOUAZARA, 2012). Other relevant application of the multipliers is in the develpent of algorithms for obtaining approximate solutions of equations of motion (HE, 1999).

On the other hand, the use of undetermined multipliers can lead to some disadvantages in the use of mathematical models based on Lagrangian or canonical equations. Particularly, whenever the elimination of the multipliers

\footnotetext{
${ }^{6}$ An adequate rederivation can lead to versions of Lagrangian equations of motion that are applicable to the modeling of continuous and general nonholonomic systems (BLOCH, 2003; TEODORESCU, 2009).
} 
from the equations of motion is not trivial, they are held in the system of equations, which increase the number of variables in the model and may reduce the efficiency of numerical simulation algorithms. In the case of inverse dynamics simulations, a high number of multipliers might lead to sparse matrix that must be inverted at some point of the algorithm, which may generate some numerical instabilities (ORSINO; HESS-COELHO; PESCE, 2015). In the case of forward dynamics simulations, the Lagrangian equations of motion are generally represented as follows:

$$
\left[\begin{array}{cc}
M & A^{\top} \\
\boldsymbol{A} & 0
\end{array}\right]\left[\begin{array}{l}
\ddot{\boldsymbol{q}} \\
\boldsymbol{\lambda}
\end{array}\right]=\left[\begin{array}{c}
{ }^{\mathrm{A}} \boldsymbol{f}+{ }^{\mathrm{G}} \boldsymbol{f} \\
-\boldsymbol{b}
\end{array}\right]
$$

with $M$ representing the generalized inertia matrix, $q$ the column-matrix of generalized coordinates of the model, $\boldsymbol{A}$ the Jacobian of the constraint invariants, $\boldsymbol{\lambda}$ the column-matrix of Lagrangian multipliers ${ }^{\mathrm{A}} \boldsymbol{f}$ the generalized active forces, ${ }^{\mathrm{G}} \boldsymbol{f}$ the generalized gyroscopic inertia forces (i.e., components of inertia forces that are independent of $\ddot{\boldsymbol{q}})$ and $\boldsymbol{b}=\underline{\boldsymbol{b}}(t, \boldsymbol{q}, \dot{\boldsymbol{q}})=\overline{\boldsymbol{q}}^{\langle 2\rangle}(t, \boldsymbol{q}, \dot{\boldsymbol{q}}, \ddot{\boldsymbol{q}})-\boldsymbol{A} \ddot{\boldsymbol{q}}$. Equation (3.95) clearly shows that if $\nu(\boldsymbol{\lambda})$ undetermined multipliers are used, then a $\nu(\boldsymbol{\lambda}) \times \nu(\boldsymbol{\lambda})$ block of zeros must be included in the "extended generalized inertia matrix". Thus, for each undetermined multiplier in the model, up to two zero eigenvalues are included in the system of equations of motion, which might lead to numerical instabilities unless some stabilization algorithm is used (SCHIEHLEN; GUSE; SEIFRIED, 2006; BAUCHAU; BOTTASSO; TRAINELLI, 2003).

An alternative to the use of undetermined multipliers in Lagragian formulation was proposed by Bayo, Jalón and Serna (1988). In this methodology, fictitious terms are added to the expressions of kinetic energy, potential energy and dissipation function in the expression of Extended Hamilton's Principle, in order to represent the motion constraints. These terms are multiplied by previously chosen penalty factors that enforce the constraints without the need of using Lagrangian multipliers. In order to improve the numerical performance of this methodology, an iterative procedure can be included to make it less sensitive to the choice of the penalty factors (DOPICO et al., 2014). 


\subsection{Gibbs-Appell, Maggi's and Boltzmann-Hamel formalisms}

In this section, it is presented a brief discussion on the main advances in Analytical Mechanics in the period between the end of 19th century and the first decades of the 20th. The formalisms from this period were characterized by remarkable developments in the study of nonholonomic systems (including non-linear nonholonomic constraints) and by the introduction of the concept of quasi-velocities (nonholonomic variables). These advances led to formulations in which the use of undetermined multipliers was not required anymore, and the number of equations of motion can be reduced to the number of degrees of freedom of the system independently of the nature of the constraint involved.

\subsubsection{Gibbs-Appell equations}

Gibbs-Appell equations, also known as Appell's equations, were independently developed by the American scientist Josiah Willard Gibbs and the French mathematician Paul Émile Appell.

Gibbs (1879) discusses the generalization of the concept of virtual displacment along with its application in the modeling of systems in which the force systems are continuous or impulsive. For systems subject to generic constraints (either holonomic or nonholonomic, either unilateral or bilateral) it is possible to apply some form of Gauss' Principle along with the concept of quasi-velocity to obtain a minimal system of the equations of motion ${ }^{7}$. Gibbs also shows that the expressions for the generalized inertia forces can be obtained from time derivatives of the Gibbs-Appell function, also known as "energy of accelerations".

Indeed, consider that $\delta$ is a mechanical system whose Gaussian constraint is given by equation (3.55). Note that:

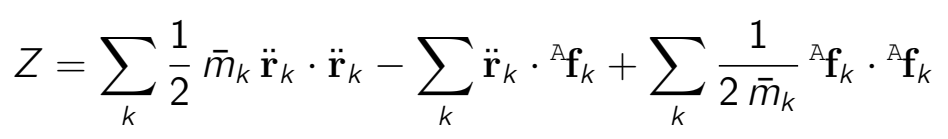

The Gibbs-Appell function of $\mathcal{\delta}$, can be defined as follows:

$$
S=\sum_{k} \frac{1}{2} \bar{m}_{k} \ddot{\mathbf{r}}_{k} \cdot \ddot{\mathbf{r}}_{k}
$$

Note that the nomenclature "energy of accelerations" comes from the similarity

\footnotetext{
${ }^{7}$ Altough developing a general formulation, Gibbs (1879) fails to highlight that it can also be applicable to the modeling of nonholonomic systems (PAPASTAVRIDIS, 1988).
} 
between (3.97) and the equation (3.58) that defines the kinetic energy of $\delta$. Assuming that all $\delta \mathbf{r}_{k}=\mathbf{0}$ and all $\delta \dot{\mathbf{r}}_{k}=\mathbf{0}$, it can be stated that all $\delta^{\mathrm{A}} \mathbf{f}_{k}=\mathbf{0}$. Indeed, in Classical Mechanics it is assumed that force systems can be functions of the state of the mechanical system to which they are applied. This means that these force systems can depend on the positions and velocities of points in a mechanical system; however, they can not depend on any acceleration or higher order derivatives of the position vectors. Thus, the application of Gauss' Principle to $\mathcal{S}$ leads to:

$$
\delta Z=\delta S-\sum_{k} \delta \ddot{\mathbf{r}}_{k} \cdot{ }^{\mathrm{A}} \mathbf{f}_{k}=0
$$

Appell (1900) not only reproduced independently the results already obtained by Gibbs (1879), but also started a wide discussion on the use of this new formalism for modeling nonholonomic system, which led to an extensive monograph on this subject (APPELL, 1925). Due to the greater significance of Appell's constributions, some authors opt to refer to this formalism as Appell's equations (PAPASTAVRIDIS, 1988).

The following derivation of Gibbs-Appell equations is based in the one presented in Papastavridis (1988), which highlights that the same formulation is valid for any bilateral constraints (holonomic, linear nonholonomic and nonlinear nonholonomic). Let $\delta$ be a $\nu^{\#-D O F ~ m e c h a n i c a l ~ s y s t e m, ~ s u b j e c t ~ t o ~ b i-~}$ lateral constraints only. Let $\boldsymbol{q}^{\langle 0\rangle}$ denote the column-matrix of generalized coordinates of the model of $\mathcal{\delta}$. If the number of generalized coordinates $\nu\left(\boldsymbol{q}^{\langle 0\rangle}\right)$ is greater than the number of degrees of freedom $\nu^{\#}$, than there must be $\nu\left(\boldsymbol{q}^{\langle 0\rangle}\right)-\nu^{\#}$ independent invariants constituting a column-matrix $\overline{\boldsymbol{h}}^{\langle 1\rangle}$ such that:

$$
\overline{\boldsymbol{h}}_{\cdot \star}^{\langle 1\rangle}\left(t, \boldsymbol{q}^{\langle 0\rangle}, \dot{\boldsymbol{q}}^{\langle 0\rangle}\right)=0
$$

Define the quasi-velocities of $\delta$ as follows:

$$
\boldsymbol{q}^{\langle 1\rangle}=\left[\begin{array}{c}
\boldsymbol{q}_{\cdot \#}^{\langle 1\rangle} \\
\boldsymbol{q}_{. \star}^{\langle 1\rangle}
\end{array}\right]=\left[\begin{array}{c}
\underline{\boldsymbol{h}}_{. \#}^{\langle 1\rangle}\left(t, \boldsymbol{q}^{\langle 0\rangle}, \dot{\boldsymbol{q}}^{\langle 0\rangle}\right) \\
\overline{\boldsymbol{h}}_{\cdot \star}^{\langle 1\rangle}\left(t, \boldsymbol{q}^{\langle 0\rangle}, \dot{\boldsymbol{q}}^{\langle 0\rangle}\right)
\end{array}\right]=\underline{\boldsymbol{q}}^{\langle 1\rangle}\left(t, \boldsymbol{q}^{\langle 0\rangle}, \dot{\boldsymbol{q}}^{\langle 0\rangle}\right)
$$

with $\underline{\boldsymbol{h}}_{\text {.\# }}^{\langle 1\rangle}\left(t, \boldsymbol{q}^{\langle 0\rangle}, \dot{\boldsymbol{q}}^{\langle 0\rangle}\right)$ representing a column-matrix constituted by $\nu^{\#}$ real-valued functions defined such that the Jacobian $\partial \underline{q}^{\langle 1\rangle} / \partial \dot{\boldsymbol{q}}^{\langle 0\rangle}$ is an invertible squarematrix in any non-singular configuration od $\mathcal{S}$. Due the Implicit Function Theorem (HAASER; SULLIVAN, 1991), it can be stated that there is a column-matrix $\dot{\boldsymbol{q}}^{\langle 0\rangle}\left(t, \boldsymbol{q}^{\langle 0\rangle}, \boldsymbol{q}^{\langle 1\rangle}\right)$ constituted by $\nu\left(\boldsymbol{q}^{\langle 0\rangle}\right)$ real-valued functions such that if $\dot{\boldsymbol{q}}^{\langle 0\rangle}=$ 
$\dot{\dot{\boldsymbol{q}}}^{\langle 0\rangle}\left(t, \boldsymbol{q}^{\langle 0\rangle}, \boldsymbol{q}^{\langle 1\rangle}\right)$, then:

$$
\left\{\begin{array}{l}
\underline{\boldsymbol{h}}_{. \#}^{\langle 1\rangle}\left(t, \boldsymbol{q}^{\langle 0\rangle}, \underline{\dot{\boldsymbol{q}}}^{\langle 0\rangle}\left(t, \boldsymbol{q}^{\langle 0\rangle}, \boldsymbol{q}^{\langle 1\rangle}\right)\right)=\boldsymbol{q}_{. \#}^{\langle 1\rangle} \\
\overline{\boldsymbol{h}}_{\cdot \star}^{\langle 1\rangle}\left(t, \boldsymbol{q}^{\langle 0\rangle}, \underline{\dot{\boldsymbol{q}}}^{\langle 0\rangle}\left(t, \boldsymbol{q}^{\langle 0\rangle}, \boldsymbol{q}^{\langle 1\rangle}\right)\right)=\mathbf{0}
\end{array}\right.
$$

Moreover, for any values of $\left(t, \boldsymbol{q}^{\langle 0\rangle}, \dot{\boldsymbol{q}}^{\langle 0\rangle}\right)$ compatible with the constraints of the system:

$$
\dot{\dot{\boldsymbol{q}}}^{\langle 0\rangle}\left(t, \boldsymbol{q}^{\langle 0\rangle}, \underline{\boldsymbol{q}}^{\langle 1\rangle}\left(t, \boldsymbol{q}^{\langle 0\rangle}, \dot{\boldsymbol{q}}^{\langle 0\rangle}\right)\right)=\dot{\boldsymbol{q}}^{\langle 0\rangle}
$$

Thus, it can be stated that:

$$
\frac{\partial \underline{\dot{q}}^{\langle 0\rangle}}{\partial \boldsymbol{q}^{\langle 1\rangle}} \frac{\partial \underline{\boldsymbol{q}}^{\langle 1\rangle}}{\partial \dot{\boldsymbol{q}}^{\langle 0\rangle}}=\mathbf{1} \quad \text { and } \quad \frac{\partial \underline{\boldsymbol{q}}^{\langle 1\rangle}}{\partial \dot{\boldsymbol{q}}^{\langle 0\rangle}} \frac{\partial \underline{\dot{q}}^{\langle 0\rangle}}{\partial \boldsymbol{q}^{\langle 1\rangle}}=\mathbf{1}
$$

Using equation (3.103) and noting that, by the definition given by equation (3.100), $\delta \boldsymbol{q}_{\cdot \star \star}^{\langle 1\rangle}=0$, it can also be stated that:

$$
\delta \boldsymbol{q}^{\langle 1\rangle}=\frac{\partial \underline{\boldsymbol{q}}^{\langle 1\rangle}}{\partial \dot{\boldsymbol{q}}^{\langle 0\rangle}} \delta \dot{\boldsymbol{q}}^{\langle 0\rangle} \quad \Rightarrow \quad \delta \dot{\boldsymbol{q}}^{\langle 0\rangle}=\frac{\partial \dot{\boldsymbol{q}}^{\langle 0\rangle}}{\partial \boldsymbol{q}^{\langle 1\rangle}} \delta \boldsymbol{q}^{\langle 1\rangle}=\frac{\partial \dot{\boldsymbol{q}}^{\langle 0\rangle}}{\partial \boldsymbol{q}_{\text {.\# }}^{\langle 1\rangle}} \delta \boldsymbol{q}_{\text {.\# }}^{\langle 1\rangle}
$$

Moreover, for any integer $\alpha \geq 1$ :

$$
\delta \boldsymbol{q}^{\langle\alpha \mid 0\rangle}=\frac{\partial \dot{\boldsymbol{q}}^{\langle 0\rangle}}{\partial \boldsymbol{q}_{\text {.\# }}^{\langle 1\rangle}} \delta \boldsymbol{q}_{\text {.\# }}^{\langle\alpha \mid 1\rangle}
$$

Furthermore, it is convenient to define:

$$
\delta \boldsymbol{q}^{\langle 0\rangle}=\frac{\partial \underline{\dot{\boldsymbol{q}}}^{\langle 0\rangle}}{\partial \boldsymbol{q}_{. \#}^{\langle 1\rangle}} \delta \boldsymbol{q}_{. \#}^{\langle 0 \mid 1\rangle}
$$

with $\delta \boldsymbol{q}_{. \#}^{\langle 0 \mid 1\rangle}$ representing variations of independent quasi-coordinates ${ }^{8}$.

Therefore, considering that $\delta$ is in a non-singular configuration in which all the variations are reversible, adopting an integer $\rho \geq 0$ and applying the $\rho$-th order variational principle for $\mathcal{S}$, given by equation (3.54), it can be stated that:

$$
\sum_{k} \delta \mathbf{r}_{k}^{(\rho)} \cdot\left({ }^{{ }^{A}} \mathbf{f}_{k}+{ }^{\mathrm{I}} \mathbf{f}_{k}\right)=\left(\delta \boldsymbol{q}_{. \#}^{\langle\rho \mid 1\rangle}\right)^{\top} \sum_{k} \mathbf{V}_{k \cdot \#} \cdot\left({ }^{\mathrm{A}} \mathbf{f}_{k}+{ }^{\mathrm{I}} \mathbf{f}_{k}\right)=0
$$

\footnotetext{
${ }^{8}$ It is important to highlight that, in general, it is not possible to interpret quasi-coordinates as an alternative set of generalized coordinates, once some of the time integrals of quasivelocities might not be related to the description of configurations of the system (for instance, the time integrals of components of angular velocity of a rigid body do not have any relation with its orientation); thus, only the variations of quasi-coordinates, but not quasi-coordinates themselves, are defined.
} 
with the second order tensor $\mathbf{V}_{k \text {.\# }}$ defined as follows:

$$
\mathbf{V}_{k . \#}=\frac{\partial \underline{\mathbf{r}}_{k}^{(\rho)}}{\partial \boldsymbol{q}^{\langle\rho \mid 0\rangle}} \frac{\partial \dot{\dot{q}}^{\langle 0\rangle}}{\partial \boldsymbol{q}_{. \#}^{\langle 1\rangle}}
$$

Once the components of $\delta \boldsymbol{q}_{. \#}^{\langle\rho \mid 1\rangle}$ are independent, it can be stated that to ensure the condition imposed by equation (3.107), it is necessary that:

$$
{ }^{\mathrm{I}} \boldsymbol{f}_{\text {.\# }}+{ }^{\mathrm{A}} \boldsymbol{f}_{\text {.\# }}=0
$$

with ${ }^{\mathrm{I}} \boldsymbol{f}_{\text {.\# }}$ and ${ }^{\mathrm{A}} \boldsymbol{f}_{\text {.\# }}$ defined by:

$$
\begin{aligned}
& { }^{\mathrm{I}} \boldsymbol{f}_{\cdot \#}=\sum_{k} \mathbf{V}_{k \cdot \#} \cdot{ }^{\mathrm{I}} \mathbf{f}_{k} \\
& { }^{\mathrm{A}} \boldsymbol{f}_{\text {.\# }}=\sum_{k} \mathbf{V}_{k \cdot \#} \cdot{ }^{\mathrm{A}} \mathbf{f}_{k}
\end{aligned}
$$

The components of ${ }^{\mathrm{I}} \boldsymbol{f}_{\text {.\# }}$ and are called generalized inertia forces and generalized active forces, respectively. Finally, consider that the Gibbs-Appell function is expressed as a function of the generalized variables of the model of $\mathcal{S}$ up to second order, i.e. $S=\underline{S}\left(t, \boldsymbol{q}^{\langle 0\rangle}, \boldsymbol{q}^{\langle 1\rangle}, \dot{\boldsymbol{q}}^{\langle 1\rangle}\right)$. Let, for instance, $S$ be given by equation (3.97) and assume that it is possible to express each acceleration as a function of the generalized coordinates and its time derivatives up to second order, i.e. $\ddot{\mathbf{r}}_{k}=\underline{\ddot{r}}_{k}\left(t, \boldsymbol{q}^{\langle 0\rangle}, \dot{\boldsymbol{q}}^{\langle 0\rangle}, \ddot{\boldsymbol{q}}^{\langle 0\rangle}\right)$. It can be stated that:

$$
\frac{\partial \underline{S}}{\partial \dot{\boldsymbol{q}}_{. \#}^{\langle 1\rangle}}=\sum_{k} \bar{m}_{k} \ddot{\underline{\mathbf{r}}}_{k} \cdot \frac{\partial \underline{\ddot{\mathbf{r}}}_{k}}{\partial \ddot{\boldsymbol{q}}^{\langle 0\rangle}} \frac{\partial \underline{\ddot{\boldsymbol{q}}}^{\langle 0\rangle}}{\partial \dot{\boldsymbol{q}}_{. \#}^{\langle 1\rangle}}=\sum_{k} \bar{m}_{k} \ddot{\ddot{r}}_{k} \cdot \frac{\partial \underline{\ddot{\mathbf{r}}}_{k}}{\partial \ddot{\boldsymbol{q}}^{\langle 0\rangle}} \frac{\partial \dot{\boldsymbol{q}}^{\langle 0\rangle}}{\partial \boldsymbol{q}_{\text {.\# }}^{\langle 1\rangle}}=-{ }^{\mathrm{I}} \boldsymbol{f}_{\text {.\# }}
$$

Therefore, equation (3.109) can be rewritten as follows:

$$
\frac{\partial \underline{S}}{\partial \dot{\boldsymbol{q}} . \#}={ }^{A} \boldsymbol{f}_{\text {.\# }}
$$

This is the matrix form of the Gibbs-Appell equations for system $\mathcal{S}$ (GIBBS, 1879; APPELL, 1925; PAPASTAVRIDIS, 1988; BARUH, 1999).

Papastavridis (1988) also presents a method for obtaining the expressions of the generalized constraint forces based on Gibbs-Appell formulation and on the Principle of Relaxation of Constraints:

(1) Obtain a description of the motion of $\delta$ in terms of all the generalized variables considering, a priori, that the variables $\boldsymbol{q}_{\star \star}^{\langle 1\rangle}$ are independent, i.e. without setting $q_{\cdot \star \star}^{\langle 1\rangle}=0$.

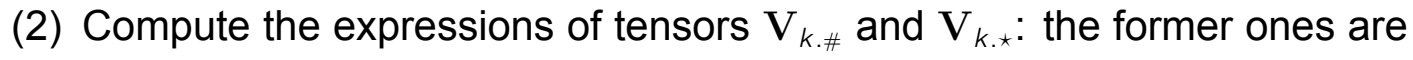
given by equation (3.108), while the latter ones are given by analogous 
expressions in which the partial derivatives with respect to $\boldsymbol{q}_{\text {.\# }}^{\langle 1\rangle}$ are replaced by partial derivatives with respect to $\boldsymbol{q}_{. \star}^{\langle 1\rangle}$.

(3) Obtain the expressions of ${ }^{\mathrm{I}} \boldsymbol{f}_{\text {.\# }}$ and ${ }^{\mathrm{A}} \boldsymbol{f}_{\text {.\# }}$ using equations (3.110) and (3.111).

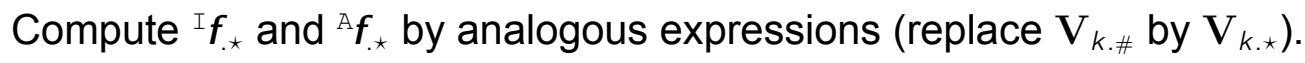

(4) Set $q_{\cdot \star}^{\langle 1\rangle}=0$ in the expressions derived in the previous step.

The equations of motion of $\mathcal{S}$ are given by (3.109) and the generalized constraint forces of $\mathcal{S}$ (components of ${ }^{\mathrm{C}} \boldsymbol{f}_{. \star}$ ) are obtained as follows:

$$
{ }^{\mathrm{C}} \boldsymbol{f}_{\cdot \star}=-{ }^{\mathrm{I}} \boldsymbol{f}_{\cdot \star}-{ }^{\mathrm{A}} \boldsymbol{f}_{\cdot \star \star}
$$

It is worth noting that:

- The equations of motion of a system (3.109) are totally decoupled from the equations to compute the generalized constraint forces (3.114). This is a significant advantage with respect to the Lagrangian-Hamiltonian formulation, particularly in the case of nonholonomic systems or models in which redundant generalized coordinates are defined, once no undetermined multipliers are required in these cases.

- To derive the equations of motion only, variables $\boldsymbol{q}_{\cdot \star}^{\langle 1\rangle}$ are not necessary apart from their use to choose the independent quasi-velocities $\boldsymbol{q}_{\text {.\# }}^{\langle 1\rangle}$ and to obtain the expressions $\underline{\dot{q}}^{\langle 0\rangle}\left(t, \boldsymbol{q}^{\langle 0\rangle}, \boldsymbol{q}^{\langle 1\rangle}\right)$. The number of equations of motion is equal to the number of degrees of freedom of the system.

- Inverse dynamics problems can be interpreted as mechanical system to which extra constraints, representing the desired motion of the system, are imposed. Thus, in Gibbs-Appell formulation, these problems can be reduced to computing the values of ${ }^{C} \boldsymbol{f}_{. \star}$ that correspond to these extra constraints.

Gibbs-Appell equations are still widely studied, being found in several textbooks on Multibody System Dynamics (TEODORESCU, 2009; BARUH, 1999; BARUH, 2000). This formalism also finds applications in non-linear control (KIRGETOV, $1964)$ and in the formulation of recursive modeling algorithms for mechanisms (MATA et al., 2002a; MATA et al., 2002b; KORAYEM; SHAFEI, 2009). 


\subsubsection{Maggi's equations}

Maggi's equations, introduced by the Italian mathematician and physicist Gian Antonio Maggi in 1896 (LAULUSA; BAUCHAU, 2008), can be understood as an extension of Lagrangian-Hamiltonian formalism for simple nonholonomic systems (and holonomic systems with redundant generalized coordinates) that allows the elimination of the undetermined multipliers from the equations of motion of a mechanical system by a matrix algorithm.

Basically, consider that the constraint invariants of equations (3.99) are affine functions with respect to $\dot{\boldsymbol{q}}^{\langle 0\rangle}$, i.e.:

$$
\overline{\boldsymbol{h}}_{. \star}^{\langle 1\rangle}\left(t, \boldsymbol{q}^{\langle 0\rangle}, \dot{\boldsymbol{q}}^{\langle 0\rangle}\right)=\underline{\boldsymbol{A}}_{. \star}\left(t, \boldsymbol{q}^{\langle 0\rangle}\right) \dot{\boldsymbol{q}}^{\langle 0\rangle}+\underline{\boldsymbol{b}}_{. \star}\left(t, \boldsymbol{q}^{\langle 0\rangle}\right)=\mathbf{0}
$$

Assume also that the function $\underline{\boldsymbol{h}}_{. \#}^{\langle 1\rangle}\left(t, \boldsymbol{q}^{\langle 0\rangle}, \dot{\boldsymbol{q}}^{\langle 0\rangle}\right)$ is affine with respect to $\dot{\boldsymbol{q}}^{\langle 0\rangle}$ so that, equation (3.100) can be rewritten as follows:

$$
\boldsymbol{q}^{\langle 1\rangle}=\left[\begin{array}{c}
\boldsymbol{q}_{. \#}^{\langle 1\rangle} \\
\boldsymbol{q}_{. \star}^{\langle 1\rangle}
\end{array}\right]=\left[\begin{array}{c}
\underline{\boldsymbol{A}}_{. \#}\left(t, \boldsymbol{q}^{\langle 0\rangle}\right) \dot{\boldsymbol{q}}^{\langle 0\rangle}+\underline{\boldsymbol{b}}_{. \#}^{\langle 0\rangle}\left(t, \boldsymbol{q}^{\langle 0\rangle}\right) \\
\underline{\boldsymbol{A}}_{. \star}\left(t, \boldsymbol{q}^{\langle 0\rangle}\right) \dot{\boldsymbol{q}}^{\langle 0\rangle}+\underline{\boldsymbol{b}}_{. \star}^{\langle 0\rangle}\left(t, \boldsymbol{q}^{\langle 0\rangle}\right)
\end{array}\right]=\underline{\boldsymbol{q}}^{\langle 1\rangle}\left(t, \boldsymbol{q}^{\langle 0\rangle}, \dot{\boldsymbol{q}}^{\langle 0\rangle}\right)
$$

Define $\boldsymbol{A}=\partial \underline{\boldsymbol{q}}^{\langle 1\rangle} / \partial \dot{\boldsymbol{q}}^{\langle 0\rangle}$, and adopt the following notation:

$$
\boldsymbol{A}=\left[\begin{array}{c}
A_{\cdot \#} \\
A_{\cdot \star}
\end{array}\right] \quad A^{-1}=\left[\begin{array}{ll}
C_{. \star} & C_{. \#}
\end{array}\right]
$$

Considering that $\boldsymbol{A A ^ { - 1 }}=\boldsymbol{A}^{-1} \boldsymbol{A}=\mathbf{1}$, the following identities can be verified:

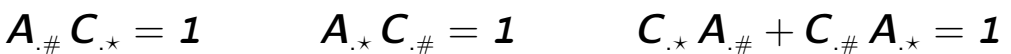

$$
\begin{aligned}
& A_{\text {. }} C_{\text {.太 }}=0 \quad A_{\text {.\# }} C_{\text {.\# }}=0
\end{aligned}
$$

It can be noticed that $C_{\text {.* }}$ is an orthogonal complement of $A_{\text {.* }}$ and that $C_{\text {.\# }}$ is an

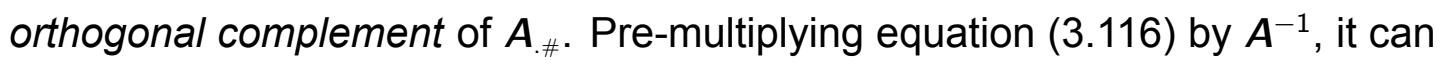
be stated that:

$$
\dot{\boldsymbol{q}}=\boldsymbol{C}_{. \star} \boldsymbol{q}_{\cdot \#}^{\langle 1\rangle}-\left(\boldsymbol{C}_{. \#} \boldsymbol{b}_{. \star}^{\langle 0\rangle}+\boldsymbol{C}_{. \star} \boldsymbol{b}_{\cdot \#}^{\langle 0\rangle}\right)
$$

By equation (3.106), $\delta \boldsymbol{q}^{\langle 0\rangle}$ satisfies the following expression:

$$
\delta \boldsymbol{q}^{\langle 0\rangle}=C_{\cdot \star \star} \delta \boldsymbol{q}_{. \#}^{\langle 0 \mid 1\rangle}
$$

Let the kinetic energy of $\mathcal{S}$ be expressed as a function of time, generalized 
coordinates and its time derivatives, i.e. $T=\underline{T}(t, \boldsymbol{q}, \dot{\boldsymbol{q}})$, with $\boldsymbol{q}=\boldsymbol{q}^{\langle 0\rangle}$. Applying the Extended Hamilton's Principle, equation (3.72), it can be stated that:

$$
\int_{t_{0}}^{t_{1}}\left(\delta \boldsymbol{q}_{\cdot \#}^{\langle 0 \mid 1\rangle}\right)^{\top} C_{. \star}^{\top}\left(\frac{\partial \underline{T}}{\partial q_{i}}-\frac{\mathrm{d}}{\mathrm{d} t}\left(\frac{\partial \underline{T}}{\partial \dot{\boldsymbol{q}}}\right)+{ }^{\mathrm{A}} \boldsymbol{f}\right) \mathrm{d} t=0
$$

Once $\mathcal{S}$ is a $\nu^{\#-D O F}$ system, then the $\nu^{\#}$ components of $\delta \boldsymbol{q}_{\text {.\# }}^{\langle 0 \mid 1\rangle}$ are independent variables. Thus, to ensure the satisfaction of equation (3.121) for values of $\left(t, \boldsymbol{q}^{\langle 0\rangle}, \boldsymbol{q}^{\langle 1\rangle}\right)$ compatible with the constraints of the system, it is necessary and sufficient that:

$$
C_{. \star}^{\top}\left(\frac{\partial \underline{T}}{\partial q_{i}}-\frac{\mathrm{d}}{\mathrm{d} t}\left(\frac{\partial \underline{T}}{\partial \dot{\boldsymbol{q}}}\right)+{ }^{\mathrm{A}} \boldsymbol{f}\right)=0
$$

These are the Maggi's equations for system $\mathcal{S}$ (BYACHKOV; SUSLONOV, 2002; BARUH, 2000).

Moreover, suppose that the Lagrangian equations of motion with undetermined multipliers for this same system are expressed as follows:

$$
\left[\begin{array}{cc}
M & A_{\cdot \star}^{\top} \\
\boldsymbol{A}_{\cdot \star} & 0
\end{array}\right]\left[\begin{array}{l}
\ddot{\boldsymbol{q}} \\
\boldsymbol{\lambda}
\end{array}\right]=\left[\begin{array}{c}
{ }^{\mathrm{A}} \boldsymbol{f}+{ }^{\mathrm{G}} \boldsymbol{f} \\
-\boldsymbol{b}^{\langle 1\rangle}
\end{array}\right]
$$

Considering that $C_{\cdot \star}^{\top} A_{\cdot \star}^{\top}=0$, it is possible to reduce the system of equations (3.123) to the following form:

$$
\left[\begin{array}{c}
C_{. \star}^{\top} M \\
A_{\cdot \star}
\end{array}\right] \ddot{\boldsymbol{q}}=\left[\begin{array}{c}
C_{. \star}^{\top}\left({ }^{\mathrm{A}} \boldsymbol{f}+{ }^{\mathrm{G}} \boldsymbol{f}\right) \\
-\boldsymbol{b}^{\langle 1\rangle}
\end{array}\right]
$$

The system of equations (3.124) correspond to the matrix form of Maggi's equations of motion of $\mathcal{S}$. Futhermore, once $C_{\text {.\# }}^{\top} A_{\text {. }}^{\top}=1$, pre-multiplying equation (3.123) by $C_{\text {.\# }}^{\top}$ is can be stated that (LAULUSA; BAUCHAU, 2008):

$$
\lambda=C_{. \#}^{\top}\left({ }^{\mathrm{A}} \boldsymbol{f}+{ }^{\mathrm{G}} \boldsymbol{f}-M \ddot{q}\right)
$$

As well as in Gibbs-Appell formalism, in Maggi's approach the equations of motion are decoupled from the equations for computing the generalized constraint forces, represented, in this case by the undetermined multipliers.

A brief analysis in the literature shows that several modeling methodologies are based on Maggi's formalism, applying orthogonal complement techniques for the formulation of equations of motion without need of including generalized constraint forced or undetermined multipliers. Once the matrix $C_{\text {.` }}$ satis-

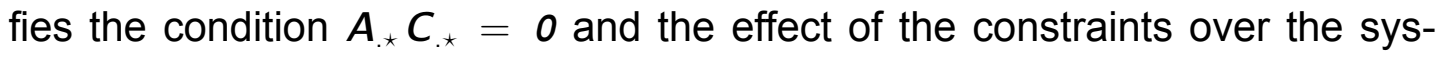


tem can be expressed as a linear combination of the columns of $A_{\star \star}^{\top}$ (rows of $\boldsymbol{A}_{\text {. }}$ ), pre-multiplying the equations of motion by $C_{\text {.* }}^{\top}$, automatically cancels the terms related to constraint forces and torques (BLOCH; MARSDEN; ZENKOV, 2009; LAULUSA; BAUCHAU, 2008; KHAN et al., 2005a). This fact enables the extension of orthogonal complement based techniques for every classical modeling formalism, not only the ones based on the fundamental principles of Analytical Mechanics, but also the ones based on Newton's laws of motion.

One of these applications is the Natural Orthogonal Complement Method (NOC) and its variant, called Decoupled Natural Orthogonal Complement Method (DeNOC), which use an approach based on Screw Theory for obtaining orthogonal complement matrices to cancel the constraint forces and torques from Newton-Euler equations expressed in terms of twists and wrenches (KHAN et al., 2005b; SAHA; ANGELES, 1991). Shah, Saha and Dutt (2012) propose recursive modular algorithms based on DeNOC, which under an adequate interpretation, correspond to particular forms of the modeling methodology proposed in this thesis. Due to specific criteria for defining the generalized variables (which are essentially joint variables and twists components), the NOC matrices in the intra-modular level (considering that the modules are always serial kinematic chains) and the so called module-DeNOC matrices, for the inter-modular level, always have the same block-matrix structure.

Another similar application is associated to Graph Theory based modeling algorithms, in which the derivation of equations of motion can be interpreted analogously to the application fo Kirchhoff laws for electrical circuits. In these cases, the generalized constraint force terms are also cancelled by the projection of the equations of motion by orthogonal complement matrices (MCPHEE; SCHMITKE; REDMOND, 2004; MCPHEE; REDMOND, 2006). It is important to highlight that these methodologies are applicable both to holonomic and nonholonomic systems.

\subsubsection{Boltzmann-Hamel equations}

Boltzmann-Hamel equations can be understood as a further generalization of Lagrangian-Hamiltonian formalism, applicable to any mechanical system with bilateral constraints (including non-linear nonholonomic constraints), and using a description of motion completely based on generalized coordinates and quasi-velocities, defined according to the convention presented in equation (3.100). Instead of expressing the kinetic energy of $\delta$ as a function of 
time, generalized coordinates and its time derivatives, the latter variables are replaced by the quasi-velocities, i.e. $T=\underline{T}\left(t, \boldsymbol{q}^{\langle 0\rangle}, \boldsymbol{q}^{\langle 1\rangle}\right)$. In this case, the Extended Hamilton's principle for $\mathcal{S}$ can be expressed as follows (see Section A.1, Appendix A):

$$
\begin{aligned}
\int_{t_{0}}^{t_{1}} & \left(\delta \boldsymbol{q}^{\langle 1\rangle}\right)^{\top}\left(\left(\frac{\partial \underline{\dot{q}}^{\langle 0\rangle}}{\partial \boldsymbol{q}^{\langle 1\rangle}}\right)^{\top} \frac{\partial \underline{T}}{\partial \boldsymbol{q}^{\langle 0\rangle}}-\frac{\mathrm{d}}{\mathrm{d} t}\left(\frac{\partial \underline{T}}{\partial \boldsymbol{q}^{\langle 1\rangle}}\right)\right. \\
& \left.-\left(\frac{\partial \underline{\boldsymbol{q}}^{\langle 0\rangle}}{\partial \boldsymbol{q}^{\langle 1\rangle}}\right)^{\top}\left(\frac{\mathrm{d}}{\mathrm{d} t} \frac{\partial \underline{\boldsymbol{q}}^{\langle 1\rangle}}{\partial \dot{\boldsymbol{q}}^{\langle 0\rangle}}-\frac{\partial \underline{\boldsymbol{q}}^{\langle 1\rangle}}{\partial \boldsymbol{q}^{\langle 0\rangle}}\right)^{\top} \frac{\partial \underline{T}}{\partial \boldsymbol{q}^{\langle 1\rangle}}+\left(\frac{\partial \dot{\boldsymbol{q}}^{\langle 0\rangle}}{\partial \boldsymbol{q}^{\langle 1\rangle}}\right)^{\top}{ }^{\mathrm{A}} \boldsymbol{f}^{\prime}\right) \mathrm{d} t=0
\end{aligned}
$$

Once all the $\nu^{\#}$ components of $\delta \boldsymbol{q}_{\text {.\# }}^{\langle 1\rangle}$ are independent, and the values of $\boldsymbol{q}_{\text {. }}^{\langle 1\rangle}$ and $\delta \boldsymbol{q}_{. \star}^{\langle 1\rangle}$ must be identically zero to satisfy the conditions imposed by the motion constraints. Thus, to ensure the condition imposed by equation (3.126) it is necessary and sufficient that:

$$
\begin{aligned}
\frac{\mathrm{d}}{\mathrm{d} t}\left(\frac{\partial \underline{T}}{\partial \boldsymbol{q}_{. \#}^{\langle 1\rangle}}\right)+\left(\frac{\partial \underline{\dot{q}}^{\langle 0\rangle}}{\partial \boldsymbol{q}_{. \#}^{\langle 1\rangle}}\right)^{\top}\left(\frac{\mathrm{d}}{\mathrm{d} t} \frac{\partial \underline{\boldsymbol{q}}_{. \#}^{\langle 1\rangle}}{\partial \dot{\boldsymbol{q}}^{\langle 0\rangle}}-\frac{\partial \underline{\boldsymbol{q}}_{. \#}^{\langle 1\rangle}}{\partial \boldsymbol{q}^{\langle 0\rangle}}\right)^{\top} \frac{\partial \underline{T}}{\partial \boldsymbol{q}_{. \#}^{\langle 1\rangle}} \\
-\left(\frac{\partial \underline{\dot{\boldsymbol{q}}}^{\langle 0\rangle}}{\partial \boldsymbol{q}_{. \#}^{\langle 1\rangle}}\right)^{\top} \frac{\partial \underline{T}}{\partial \boldsymbol{q}^{\langle 0\rangle}}=\left(\frac{\partial \underline{\dot{q}}^{\langle 0\rangle}}{\partial \boldsymbol{q}_{. \#}^{\langle 1\rangle}}\right)^{\mathrm{A}} \boldsymbol{f}^{\prime}
\end{aligned}
$$

These are the Boltzmann-Hamel equations of system $\mathcal{S}$ (JARZEBOWSKA, 2009; MARUSKIN; BLOCH, 2007). It is important to highlight that the derivations of these equations were also presented by Poincare (1901), at the same time of the first publications of Hamel (BREMER, 2014) ${ }^{9}$.

Boltzmann-Hamel equations are successfully applied in the study of nonholonomic systems, including the analysis of some mechanisms (CAMERON; BOOK, 1997) and applications for the development of non-linear optimal control this kind of system, including strategies that involve the enforcement of extra constraints of higher order than first (JARZEBOWSKA, 2012; JARZEBOWSKA, 2009; MARUSKIN; BLOCH, 2007).

\subsection{Kane's and Udwadia-Kalaba methodologies}

\subsubsection{Kane's equations}

Kane's equations, originally presented in Kane (1961) and Kane and Wang (1965), represent one of the most popular methodologies for modeling honolomic and simples nonholonomic multibody systems. Originally developed

\footnotetext{
9 Poincaré, however, did not not do further discussions on the use of these equations for modeling nonholonomic systems.
} 
at the same time of the first computational implementations of Multibody System Dynamics packages, Kane's methodology is already an algorithm that describes, step by step, a procedure for obtaining expressions for generalized active and inertia forces of a system, considering the effects of the motion constraints. This possibility of interpreting Kane's method as an algorithm is explored by several authors (SANDINO; BEJAR; OLLERO, 2011; BUFFINTON, 2005; GILLESPIE; ARBOR, 2003). Moreover, being a contemporaneous of the first researchers to apply computational tools to the modeling and simulation of multibody systems, gave Kane the opportunity to identify some of the major difficulties found by them in these implementations, allowing to make his own methodology adequate to their needs (KANE; LEVINSON, 1983a).

In order to do introduce the use of this methodology to derive the equations of motion of a mechanical system, the procedure described in Kane and Levinson (1985) is presented below with some adaptations in the notation. Assume that the constraint invariants of $\delta$ are given by equation (3.115). Define as much quasi-velocities as the number of generalized coordinates used, such that there will exist an invertible square matrix $A^{\bullet}$ and a column-matrix $b^{\bullet}$ such that:

$$
\dot{\boldsymbol{q}}^{\langle 0\rangle}=\underline{\boldsymbol{A}}^{\bullet}\left(t, \boldsymbol{q}^{\langle 0\rangle}\right) \boldsymbol{q}^{\langle 1\rangle}+\underline{\boldsymbol{b}}^{\bullet}\left(t, \boldsymbol{q}^{\langle 0\rangle}\right)
$$

Thus, equation (3.115) can be rewritten as follows:

$$
A_{\cdot \star} A^{\bullet} \boldsymbol{q}^{\langle 1\rangle}+\left(\boldsymbol{A}_{\cdot \star \star} \boldsymbol{b}^{\bullet}+\boldsymbol{b}_{\cdot \star}\right)=0
$$

Therefore, $\nu^{\#}$ variables can be chosen among the quasi-velocities of the model to constitute a column-matrix $\boldsymbol{q}_{\text {.\# }}^{\langle 1\rangle}$ and there will be a rank $\nu^{\#}$ matrix $C^{\bullet}$ and a column-matrix $d^{\bullet}$ such that:

$$
\boldsymbol{q}^{\langle 1\rangle}=\underline{\boldsymbol{C}}^{\bullet}\left(t, \boldsymbol{q}^{\langle 0\rangle}\right) \boldsymbol{q}_{. \#}^{\langle 1\rangle}+\underline{\boldsymbol{d}}^{\bullet}\left(t, \boldsymbol{q}^{\langle 0\rangle}\right)
$$

In order to satisfy the constraint equations (3.129) the following relations must be valid:

$$
\left\{\begin{array}{l}
A_{\star \star} A^{\bullet} C^{\bullet}=0 \\
A_{\star \star}\left(A^{\bullet} d^{\bullet}+b^{\bullet}\right)+b_{. \star}=0
\end{array}\right.
$$

Consider that the expressions of positions and velocities of relevant points $\mathrm{p}_{k}$ of $\mathcal{S}$, measured with respect to an inertial reference frame $\mathcal{N}$, are expressed as follows: $\mathbf{r}_{k}=\underline{\mathbf{r}}_{k}\left(t, \boldsymbol{q}^{\langle 0\rangle}\right)$ and $\mathbf{v}_{k}=\underline{\mathbf{v}}_{k}\left(t, \boldsymbol{q}^{\langle 0\rangle}, \boldsymbol{q}^{\langle 1\rangle}\right)$. Denote by $c_{j}^{\bullet}$ the $j$-th 
column of matrix $C^{\bullet}$. Kane e Levinson (1985) defines the $j$-th nonholonomic partial velocity of $\mathrm{p}_{k}$ in $\mathcal{N}$ as follows:

$$
\mathbf{v}_{k . j}^{\bullet}=\frac{\partial \underline{\mathbf{r}}_{k}}{\partial \boldsymbol{q}^{\langle 0\rangle}} \boldsymbol{A}^{\bullet} \boldsymbol{c}_{j}^{\bullet}=\frac{\partial \underline{\mathbf{v}}_{k}}{\partial \boldsymbol{q}^{\langle 1\rangle}} \boldsymbol{c}_{j}^{\bullet}
$$

From this, Kane e Levinson (1985) defines the $j$-th nonholonomic generalized inertia force for $\mathcal{S}$ in $\mathcal{N},{ }^{\mathrm{I}} f_{j}^{\bullet}$, and the $j$-th nonholonomic generalized active force for $\mathcal{S}$ in $\mathcal{N},{ }^{\mathrm{A}} f_{j}^{\bullet}$, as follows:

$$
\begin{aligned}
& { }^{\mathrm{I}} f_{j}^{\bullet}=\sum_{k} \mathbf{v}_{k \cdot j}^{\bullet} \cdot{ }^{\mathrm{I}} \mathbf{f}_{k} \\
& { }^{\mathrm{A}} f_{j}^{\bullet}=\sum_{k} \mathbf{v}_{k \cdot j}^{\bullet} \cdot{ }^{\mathrm{A}} \mathbf{f}_{k}
\end{aligned}
$$

Finally, Kane's equations for $\mathcal{S}$ are:

$$
{ }^{\mathrm{I}} f_{j}^{\bullet}+{ }^{\mathrm{A}} f_{j}^{\bullet}=0 \quad \text { for all } \quad j \in\left\{1, \ldots, \nu^{\#}\right\}
$$

Kane's methodology is widely applied in different areas of Mechanical Engineering including, for example, the modeling of terrestrial and aerial vehicles (SANDINO; BEJAR; OLLERO, 2011; BOLENDER, 2009), robotic mechanisms (BUFFINTON, 2005; KANE; LEVINSON, 1983b) and systems with flexible bodies (HU; JIA; XU, 2012; BUFFINTON, 2005).

Besides its huge success in several applications to Multibody System Dynamics problems, Kane's approach can not be considered a new theoretical formalism once this methodology, developed in the 1960s, is based on the formalisms of Gibbs-Appell, Maggi and others which date back to the early twentieth century. It is evident, for instance, the similarity between Kane's (3.135) and Gibbs-Appell equations (3.109). Some authors claim their equivalence (BARUH, 1999), while others state that Kane's equations are a particular form of GibbsAppell equations applicable to holonomic or simple nonholonomic systems, being less general than the latter ones which are applicable to any nonholonomic system whose constraint order is equal to 1 (DESLOGE, 1987; PAPASTAVRIDIS, 1988). Desloge's paper (1987) was harshly criticized by some supporters of Kane's methodology, like his co-author Levinson (1987), who claims that in Gibbs-Appell formalism it is necessary to explicitly obtain the expression of the function $S$ to derive the equations of motion, which is a cumbersome procedure not required in Kane's method. This argument is invalid, once it is possible to obtain Gibbs-Appell equations from expressions (3.109-3.111), without needing to compute the expression of Gibbs-Appell function S. Papastavridis 
(1988) not only invalidates this argumentation, but also points out some incompatible terminologies adopted by Kane and Levinson (1985). According to him, referring to the $\mathbf{v}_{k . j}^{\cdot}$ as "partial velocities" prevents the user from a correct interpretation of the meaning of these vectors, not enabling further generalizations to systems with non-linear nonholonomic constraints, for example. Borri, Bottasso and Mantegazza (1990) adopt a slightly different interpretation to show that Kane's equations are equivalent to Maggi's. Piedboeuf (1993), in turn, discusses the equivalence between Kane's equations and Jourdain's principle (whose statement dates from 1909).

\subsubsection{Udwadia-Kalaba methodology}

Among the conventional methodologies in Analytical Mechanics, two techniques for the inclusion of the effect of constraints in the equations of motion of a mechanical system stand out (LAULUSA; BAUCHAU, 2008): the inclusion of generalized constraint forces (using the method of Lagrange multipliers and its variants) or the use of orthogonal complement methods (which are used to find the projections of active and inertial forces and torques in directions which are orthogonal to the ones defined by the action of the constraints).

Udwadia-Kalaba methodology brings back the generalized constraint forces to the equations of motion of a mechanical system, providing explicit expressions for their computation in terms of inertial and active forces along with terms coming from the differential form of the constraint invariants. The method for obtaining these explicit expressions involve the use of MoorePenrose pseudo-inverse matrices and was derived for the first time in Udwadia and Kalaba (1992) from Gauss' Principle. The solution to the minimization problem associated to this principle leads to a condition in which, in any state, the difference between the real accelerations of a constrained mechanical system and the accelerations it would have in the absence of these constraints is proportional to the extent to which these latter accelerations violate the corresponding constraints.

The following derivation of Udwadia-Kalaba methodology is adapted from Udwadia and Kalaba (2002) and Udwadia and Phohomsiri (2007a). Let $\delta$ be a mechanical system for which are defined $\nu_{0}$ generalized coordinates, represented by the column-matrix $\boldsymbol{q}^{\langle 0\rangle}$, and $\nu_{1}$ quasi-velocities, represented by the column-matrix $q^{\langle 1\rangle}$. Assume that in the absence of some constraints the equations of motion of $\mathcal{S}$ can be expressed by ${ }^{\mathrm{A}} \boldsymbol{f}+{ }^{\mathrm{I}} \boldsymbol{f}=\boldsymbol{0}$, with ${ }^{\mathrm{A}} \boldsymbol{f}$ and ${ }^{\mathrm{I}} \boldsymbol{f}$ being 
column-matrices representing, respectively, the generalized active forces and the generalized active forces associated to the quasi-velocities $q^{\langle 1\rangle}$. Consider that ${ }^{\mathrm{I}} \boldsymbol{f}=-\underline{\boldsymbol{M}}\left(t, \boldsymbol{q}^{\langle 0\rangle}, \boldsymbol{q}^{\langle 1\rangle}\right) \dot{\boldsymbol{q}}^{\langle 1\rangle}+{ }^{\mathrm{G}} \boldsymbol{f}\left(t, \boldsymbol{q}^{\langle 0\rangle}, \boldsymbol{q}^{\langle 1\rangle}\right)$ and suppose that there exists a column-matrix ${ }^{\mathrm{C}} \boldsymbol{f}$ constituted by generalized constraint forces, such that when no constraints are neglected, the equations of motion of $\delta$ are given by:

$$
M \dot{\boldsymbol{q}}^{\langle 1\rangle}={ }^{\mathrm{A}} \boldsymbol{f}+{ }^{\mathrm{G}} \boldsymbol{f}+{ }^{\mathrm{C}} \boldsymbol{f}
$$

Assume that all $\delta \mathbf{r}_{k}=0$ and all $\delta \dot{r}_{k}=0$. In this case, Postulate A, see equation (3.48), can be stated as follows: for every variation $\delta \mathbf{r}_{k}$ compatible with the constraints of $\mathcal{S}$, it can be stated that $\delta \ddot{\mathbf{r}}_{k} \cdot{ }^{C} \mathbf{f}_{k} \geq 0$, with $\mathbf{r}_{k}$ representing the position vector of a point $\mathrm{p}_{k}$ of $\delta$ with respect to an inertial reference frame and ${ }^{\mathrm{C}} \mathbf{f}_{k}$ representing the constraint force acting in this point. Analogously to equations $(3.110,3.111)$, define:

$$
{ }^{\mathrm{C}} \boldsymbol{f}=\sum_{k} \mathbf{V}_{k} \cdot{ }^{{ }^{C}} \mathbf{f}_{k} \quad \text { with } \quad \mathbf{V}_{k}=\frac{\partial \underline{\mathbf{r}}_{k}}{\partial \boldsymbol{q}^{\langle 0\rangle}} \frac{\partial \dot{\dot{\boldsymbol{q}}}^{\langle 0\rangle}}{\partial \boldsymbol{q}^{\langle 1\rangle}}
$$

Once, $\delta \ddot{\mathbf{r}}_{k}=\mathrm{V}_{k} \delta \dot{\boldsymbol{q}}^{\langle 1\rangle}$, then, using equation (3.137), it can be stated that Postulate $\mathrm{A}$ for system $\delta$ can be stated as follows:

$$
\left(\delta \dot{\boldsymbol{q}}^{\langle 1\rangle}\right)^{\top}\left({ }^{\mathrm{C}} \boldsymbol{f}\right) \geq 0
$$

Unlike usual formulations, Udwadia-Kalaba methodology enables a straightforward derivation for the equations of motion of mechanical systems in which the total work done by all the constraint forces may not be zero. For this, it is assumed that there exists a function ${ }^{\mathrm{C}} \boldsymbol{f}^{*}$, empirically determined, such that for every variation $\delta \dot{\boldsymbol{q}}^{\langle 1\rangle}$ compatible with the constraints of $\mathcal{\delta}$ :

$$
\left(\delta \dot{\boldsymbol{q}}^{\langle 1\rangle}\right)^{\top}\left({ }^{\mathrm{C}} \boldsymbol{f}\right)=\left(\delta \dot{\boldsymbol{q}}^{\langle 1\rangle}\right)^{\top}\left({ }^{\mathrm{C}} \boldsymbol{f}^{*}\right)
$$

Thus, even if ${ }^{\mathrm{C}} \boldsymbol{f}$ is a priori unknown (note that it is not necessary that ${ }^{\mathrm{C}} \boldsymbol{f}={ }^{\mathrm{C}} \boldsymbol{f}^{*}$ ), it is possible to obtain the virtual power associated with the constraint forces of $\mathcal{S}$. When the total work of the constraint forces is equal to zero, it can be simply assumed that ${ }^{\mathrm{C}} \boldsymbol{f}^{*}=0$.

Consider that the independent generalized constraint invariants of $\mathcal{S}$ can be expressed as follows:

$$
\boldsymbol{A} \dot{\boldsymbol{q}}^{\langle 1\rangle}+\boldsymbol{b}^{\langle 1\rangle}=0
$$

Assume that $M$ is an invertible matrix and define $\check{A}=A M^{-1 / 2}$. The general 
solution of equation (3.140) is given by (UDWADIA; PHOHOMSIRI, 2007a):

$$
M^{1 / 2} \dot{\boldsymbol{q}}^{\langle 1\rangle}=-\check{\boldsymbol{A}}^{\mathrm{P}} \boldsymbol{b}^{\langle 1\rangle}+\left(1-\check{\boldsymbol{A}}^{\mathrm{P}} \check{\boldsymbol{A}}\right) \boldsymbol{w}
$$

with $w \in \mathbb{R}^{\nu_{1}}$ representing an arbitrary column-matrix and $\check{A}^{\mathrm{P}}$ denoting the Moore-Penrose pseudo-inverse of matriz $\check{A}$. It can also be stated that:

$$
\check{A} M^{1 / 2} \delta \dot{\boldsymbol{q}}^{\langle 1\rangle}=0 \quad \Rightarrow \quad M^{1 / 2} \delta \dot{\boldsymbol{q}}^{\langle 1\rangle}=\left(1-\check{A}^{\mathrm{P}} \check{A}\right) z
$$

with $z \in \mathbb{R}^{\nu_{1}}$ representing an arbitrary column-matrix. From equations (3.136), (3.141) and (3.142), it can be stated that:

$$
\begin{aligned}
& \left(\delta \dot{\boldsymbol{q}}^{\langle 1\rangle}\right)^{\top}\left({ }^{\mathrm{C}} \boldsymbol{f}\right)=\left(\boldsymbol{M}^{1 / 2} \delta \dot{\boldsymbol{q}}^{\langle 1\rangle}\right)^{\top} \boldsymbol{M}^{-1 / 2}\left({ }^{\mathrm{C}} \boldsymbol{f}\right) \\
& =z^{\top}\left(1-\check{A}^{\mathrm{P}} \check{A}\right)^{\top}\left(M^{1 / 2} \dot{\boldsymbol{q}}^{\langle 1\rangle}-M^{-1 / 2}\left({ }^{\mathrm{G}} \boldsymbol{f}+{ }^{\mathrm{A}} \boldsymbol{f}\right)\right) \\
& =z^{\top}\left(\boldsymbol{1}-\check{\boldsymbol{A}}^{\mathrm{P}} \check{\boldsymbol{A}}\right)^{\mathrm{T}}\left(-\check{\boldsymbol{A}}^{\mathrm{P}} \boldsymbol{b}^{\langle 1\rangle}+\left(\boldsymbol{1}-\check{\boldsymbol{A}}^{\mathrm{P}} \check{\boldsymbol{A}}\right) \boldsymbol{w}-\boldsymbol{M}^{-1 / 2}\left({ }^{\mathrm{G}} \boldsymbol{f}+{ }^{\mathrm{A}} \boldsymbol{f}\right)\right)
\end{aligned}
$$

For further simplifications of this expression, the following identities must be considered (UDWADIA; PHOHOMSIRI, 2007a):

$$
\left\{\begin{array}{l}
\left(1-\check{A}^{\mathrm{P}} \check{A}\right)^{T}=\left(1-\check{A}^{\mathrm{P}} \check{A}\right) \\
\left(1-\check{A}^{\mathrm{P}} \check{A}\right)\left(1-\check{A}^{\mathrm{P}} \check{A}\right)=\left(1-\check{A}^{\mathrm{P}} \check{A}\right) \\
\left(1-\check{A}^{\mathrm{P}} \check{A}\right) \check{A}^{\mathrm{P}}=0
\end{array}\right.
$$

Applying these identities to equation (3.143), it can be stated that:

$$
\left(\delta \dot{\boldsymbol{q}}^{\langle 1\rangle}\right)^{\top}\left({ }^{\mathrm{C}} \boldsymbol{f}\right)=z^{\mathrm{T}}\left(\left(\boldsymbol{1}-\check{\boldsymbol{A}}^{\mathrm{P}} \check{\boldsymbol{A}}\right) \boldsymbol{w}-\left(\boldsymbol{1}-\check{\boldsymbol{A}}^{\mathrm{P}} \check{\boldsymbol{A}}\right) \boldsymbol{M}^{-1 / 2}\left({ }^{\mathrm{G}} \boldsymbol{f}+{ }^{\mathrm{A}} \boldsymbol{f}\right)\right)
$$

On the other hand:

$$
\begin{gathered}
\left(\delta \dot{\boldsymbol{q}}^{\langle 1\rangle}\right)^{\top}\left({ }^{\mathrm{C}} \boldsymbol{f}\right)=\left(\delta \dot{\boldsymbol{q}}^{\langle 1\rangle}\right)^{\top}\left({ }^{\mathrm{C}} \boldsymbol{f}^{*}\right)=\left(M^{1 / 2} \delta \dot{\boldsymbol{q}}^{\langle 1\rangle}\right)^{\top} M^{-1 / 2}\left({ }^{\mathrm{C}} \boldsymbol{f}^{*}\right)= \\
z^{\top}\left(\boldsymbol{1}-\check{\boldsymbol{A}}^{\mathrm{P}} \check{\boldsymbol{A}}\right) M^{-1 / 2}\left({ }^{\mathrm{C}} \boldsymbol{f}^{*}\right)
\end{gathered}
$$

Combining expressions (3.145) and (3.146), it can be stated that:

$$
z^{\top}\left(\left(1-\check{A}^{\mathrm{P}} \check{A}\right) w-\left(1-\check{A}^{\mathrm{P}} \check{A}\right) M^{-1 / 2}\left({ }^{\mathrm{G}} \boldsymbol{f}+{ }^{\mathrm{A}} \boldsymbol{f}+{ }^{\mathrm{C}} \boldsymbol{f}^{*}\right)\right)=0
$$

Once this identity must be true for any values chosen for the components of $z$, then it is necessary and sufficient that:

$$
\left(1-\check{A}^{\mathrm{P}} \check{\boldsymbol{A}}\right) \boldsymbol{w}=\left(1-\check{A}^{\mathrm{P}} \check{A}\right) M^{-1 / 2}\left({ }^{\mathrm{G}} \boldsymbol{f}+{ }^{\mathrm{A}} \boldsymbol{f}\right)+\left(1-\check{A}^{\mathrm{P}} \check{\boldsymbol{A}}\right) M^{-1 / 2}\left({ }^{\mathrm{C}} \boldsymbol{f}^{*}\right)
$$

Noting that, in the absence of the constraints represented by equation (3.140), the generalized accelerations are given by $\dot{q}_{\bullet}^{\langle 1\rangle}=M^{-1}\left({ }^{G} f+f\right)$, it can be stated 
that, replacing equation (3.148) in (3.141), leads, after factorization, to the following equation (UDWADIA; PHOHOMSIRI, 2007a):

$$
\begin{aligned}
\dot{\boldsymbol{q}}^{\langle 1\rangle}-\dot{\boldsymbol{q}}_{\bullet}^{\langle 1\rangle}= & -M^{-1 / 2} \check{\boldsymbol{A}}^{\mathrm{P}}\left(\boldsymbol{A} \dot{\boldsymbol{q}}_{\bullet}^{\langle 1\rangle}+\boldsymbol{b}^{\langle 1\rangle}\right) \\
& +M^{-1 / 2}\left(1-\check{\boldsymbol{A}}^{\mathrm{P}} \check{\boldsymbol{A}}\right) M^{-1 / 2 \mathrm{C}} \boldsymbol{f}^{*}
\end{aligned}
$$

Pre-multiplying this last equation by $M$ and using equation (3.136), it can be stated that:

$$
{ }^{\mathrm{C}} \boldsymbol{f}=-\boldsymbol{M}^{1 / 2} \check{A}^{\mathrm{P}}\left(\boldsymbol{A} \dot{\boldsymbol{q}}_{0}^{\langle 1\rangle}+\boldsymbol{b}^{\langle 1\rangle}\right)+\boldsymbol{M}^{1 / 2}\left(1-\check{A}^{\mathrm{P}} \check{\boldsymbol{A}}\right) M^{-1 / 2}\left({ }^{\mathrm{C}} \boldsymbol{f}^{*}\right)
$$

An alternative approach presented by Udwadia and Phohomsiri (2006) allows the derivation of similar equations of motion even when $M$ is a singular matrix. First of all, consider that:

$$
\boldsymbol{A} \delta \dot{\boldsymbol{q}}^{\langle 1\rangle}=0 \quad \Rightarrow \quad \delta \dot{\boldsymbol{q}}^{\langle 1\rangle}=\left(1-\boldsymbol{A}^{\mathrm{P}} \boldsymbol{A}\right) \check{z}
$$

with $z \in \mathbb{R}^{\nu_{1}}$ representing an arbitrary column-matrix. Replacing this identity in equation (3.139), leads to:

$$
\check{z}^{\top}\left(1-\boldsymbol{A}^{\mathrm{P}} \boldsymbol{A}\right)^{\top}\left({ }^{\mathrm{C}} \boldsymbol{f}\right)=\check{z}^{\top}\left(1-\boldsymbol{A}^{\mathrm{P}} \boldsymbol{A}\right)^{\top}\left({ }^{\mathrm{C}} \boldsymbol{f}^{*}\right)
$$

Using equation (3.136) along with the identities (3.144) and, considering that $z$ is arbitrary, it can be stated that:

$$
\left(\boldsymbol{1}-\boldsymbol{A}^{\mathrm{P}} \boldsymbol{A}\right) \boldsymbol{M} \dot{\boldsymbol{q}}^{\langle 1\rangle}=\left(\boldsymbol{1}-\boldsymbol{A}^{\mathrm{P}} \boldsymbol{A}\right)\left({ }^{\mathrm{G}} \boldsymbol{f}+{ }^{\mathrm{A}} \boldsymbol{f}+{ }^{\mathrm{C}} \boldsymbol{f}^{*}\right)
$$

Equations (3.140) and (3.153) can be represented in the following matrix form:

$$
\left[\begin{array}{c}
\left(1-A^{\mathrm{P}} \boldsymbol{A}\right) \boldsymbol{M} \\
\boldsymbol{A}
\end{array}\right] \dot{\boldsymbol{q}}^{\langle 1\rangle}=\left[\begin{array}{c}
\left(\boldsymbol{1}-\boldsymbol{A}^{\mathrm{P}} \boldsymbol{A}\right)\left({ }^{\mathrm{G}} \boldsymbol{f}+{ }^{\mathrm{A}} \boldsymbol{f}+{ }^{\mathrm{C}} \boldsymbol{f}^{*}\right) \\
-\boldsymbol{b}^{\langle 1\rangle}
\end{array}\right]
$$

If $\left[\begin{array}{ll}M^{\top} & A^{\top}\end{array}\right]^{\top}$ is a full rank matrix ${ }^{10}$, it can be stated that (UDWADIA; PHOHOMSIRI, 2006):

$$
\dot{\boldsymbol{q}}^{\langle 1\rangle}=\left[\begin{array}{c}
\left(\boldsymbol{1}-\boldsymbol{A}^{\mathrm{P}} \boldsymbol{A}\right) \boldsymbol{M} \\
\boldsymbol{A}
\end{array}\right]^{\mathrm{P}}\left[\begin{array}{c}
\left({ }^{\mathrm{G}} \boldsymbol{f}+{ }^{\mathrm{A}} \boldsymbol{f}+{ }^{\mathrm{C}} \boldsymbol{f}^{*}\right) \\
-\boldsymbol{b}^{\langle 1\rangle}
\end{array}\right]
$$

Udwadia and Schutte (2010) present a third variant of this methodology,

\footnotetext{
10 This condition might be satisfied even in cases in which $M$ is a singular matrix, being a less restrictive condition than the one necessary to be able to use equation (3.149).
} 
that uses inertia matrices of the form $M+\alpha^{2} A^{\mathrm{P}} A$, which are positive-definite matrices (and, therefore, non-singular) for every $\alpha \neq 0$, whenever $M$ is at least semi-positive-definite. Udwadia and Wanichanon (2010) do an extensive discussion on the modeling of nonholonomic systems and on the so-called Hamel's paradox: unlike in the modeling of holonomic systems, in which some coordinate transformations might lead to identically satisfied constraint equations, for nonholonomic systems, similar variable eliminations might lead to wrong equations of motion.

Falco, Pennestrì and Vita (2005) and Pennestrì and Valentini (2004) compare the efficiency of different strategies for implementing numerical simulation algorithms based in Udwadia-Kalaba methodology, discussing their use in education and other applications in Multibody System Dynamics in comparison to conventional methodologies. Pennestrì, Valentini and Falco (2010) discuss the use of Udwadia-Kalada methodology in the formulation of equations of motion of flexible multibody systems.

Finally, it is worth mentioning that non-linear tracking control, particularly inverse dynamics problems can be conceived as determining the generalized constraint forces related to a set of extra constraints that represent a desired trajectory. Thus, Udwadia-Kalaba methodology becomes an alternative to solve such problems, once explicit expressions as the one presented in equation (3.150) can be used to find the expressions of these extra generalized constraint forces that represent the effect to the actuators in the motion of the system (UDWADIA; PHOHOMSIRI, 2007b; UDWADIA, 2006; UDWADIA, 2003). 


\section{Modular modeling methodology for multibody systems}

In his extensive literature review "Multibody System Dynamics: Roots and Perspectives", Schiehlen (1997) points out the necessity of introducing a standardized, formalism-independent description format that "would permit the alternate use of validated multibody system models with different simulation systems":

The goal of such a standardization process is the unification and standardization of the neutral data format, independent of any formalism. Only by means of such a standardization it will be possible in future to achieve a situation in which a single, unique model description is sufficient for describing a mechanical system with precision to make it accessible for analysis using any program package or formalism. The standardized model description is then used as the basis for the input. In a first step the input format required by the relevant formalism is generated from the standardized model description and evaluated using a preprocessor or converter. In a subsequent step, the formalism generates the mathematical model equations. (SCHIEHLEN, 1997, p. 15)

This chapter presents a novel modeling methodology to accomplish this goal by deriving the equations of motion of a multibody system from already known models of its subsystems. This novel methodology is not only modular and recursive, allowing to express the mathematical model of a multibody system in a standardized format, but also formalism-independent, once the formalisms used in the derivations of the models of the subsystems, ultimately, do not have any influence in the overall modeling procedure.

In the first section, a general and standardized form of the equations of motion of a multibody system is presented along with an illustrative and didactic example in which a spherical pendulum is modeled using different sets 
of generalized coordinates. Section 4.2 presents the novel recursive modular modeling algorithm, which is the central contribution of this thesis. Two didactic examples are discussed to illustrate the generality of this modeling procedure, one of them being the continuation of the modeling of a planar 5-bar mechanism (started in Section 2.5). Sections 4.3 and 4.4 present sufficient conditions for the existence of solutions for the forward and inverse dynamics problems, respectively, whenever a standard mathematical model derived according to the novel methodology presented in this text is provided. The examples discussed in these sections come with numerical simulations performed in Wolfram Mathematica 10.2. Section 4.5 extends the modeling methodology for the derivation of linearized equations of motion, providing a procedure that does not require the full derivation of a non-linear model prior to the linearization. Finally, Section 4.6 is devoted to a qualitative comparison between modeling methodologies, in which the conventional ones, discussed in Chapter 3 , are assessed along with the novel methodology presented in this text.

Complementing the content of this chapter, Appendix A discusses the use of energy-like functions in the modeling of multibody systems. It is divided in three sections: Section A.1 presents some results that allow the derivation of the generalized inertia forces associated to a system from the corresponding expressions of kinetic energy or Gibbs-Appell function; Section A.2 shows the derivations of the expressions for the kinetic energy and Gibbs-Appell function of a rigid body, and Section A.3 uses these results to derive a general expression for the generalized inertia forces associated to a multi-rigid-body system.

\subsection{General form of equations of motion of a multibody system}

Let $\mathscr{M}$ denote a $\nu^{\#-D O F}$ system whose constraint order is equal to $\nu^{\circ}$. Assume that $\nu^{\#}$ is a finite integer such that the motion of $\mathscr{M}$ can be described by tracking the trajectories of a finite number of points $\mathrm{p}_{k}$ of $\mathscr{M}$. Consider also that the force system representing both active and inertia forces acting acting in $\mathscr{M}$ is represented by an effective force $\mathrm{f}_{k}$ applied to each point $\mathrm{p}_{k}$. Let $\mathcal{N}$ denote an inertial reference frame and $\mathbf{r}_{k}$ denote the position vector of $\mathrm{p}_{k}$ with respect to a point fixed to $\mathcal{N}$. Adopt the following shorthand notation $\mathbf{r}_{k}^{(\rho)}=\mathrm{d}^{\rho} \mathbf{r}_{k} / \mathrm{d} t^{\rho}$.

The $\rho$-th order variational principle ${ }^{1}$ for $\mathscr{M}$ can be stated as follows (see

\footnotetext{
${ }^{1}$ If $\rho>0$ it is assumed that all the $\delta \mathbf{r}_{k}^{(\alpha)}=0$ for all $\alpha$ such that $0 \leq \alpha \leq \rho-1$.
} 
Section 3.2.2):

$$
\sum_{k} \delta \mathbf{r}_{k}^{(\rho)} \cdot \mathbf{f}_{k} \leq 0
$$

In order to obtain a more general version of principle (4.1), let $\boldsymbol{v}^{\langle\rho\rangle}$ denote a tuple (or column-vector) of $\rho$-th order motion variables and $\mathbf{f}^{\prime}$ denote a tuple (or column-vector) of generalized effective forces associated to these motion variables. Assume that the set of motion variables constituting $v^{\langle\rho\rangle}$ is sufficient to describe the instantaneous kinematics of $\mathscr{M}$ up to $\rho$-th order whenever the description up to $(\rho-1)$-th order is already known. Moreover, if $x$ and $y$ represent two tuples (or column-matrices) of the same vector space $\mathbb{R}^{\nu}$, define:

$$
x \cdot y=x^{\top} y=y^{\top} x=y \cdot x
$$

Adopting this notation and assuming that, if $\rho>0$ then the variation of any motion variable of a lower order than $\rho$-th is equal to zero (in shorthand notation, $\delta \boldsymbol{v}\langle\rho-1\rangle=0)$, it can be stated that the $\rho$-th order variational principle for system $\mathscr{M}$, can be expressed as follows:

$$
\delta \boldsymbol{v}^{\langle\rho\rangle} \cdot \boldsymbol{f}^{\prime} \leq 0
$$

Note that if both equations (4.1) and (4.3) represent two versions of the $\rho$-th order variational principle for the same system $\mathscr{M}$, then it is necessary that $\delta \boldsymbol{v}^{\langle\rho\rangle} \cdot \boldsymbol{f}^{\prime}=\sum_{k} \delta \mathbf{r}_{k}^{(\rho)} \cdot \mathbf{f}_{k}$.

Particularly, consider that $\boldsymbol{v}^{\langle\rho\rangle}$ can be expressed as a function of time and generalized variables of the model of $\mathscr{M}$ up to $\rho$-th order, i.e. $\left.\boldsymbol{v}^{\langle\rho\rangle}=\underline{\boldsymbol{v}}^{\langle\rho\rangle}(t, \boldsymbol{q}\langle\rho\rangle\rangle\right)$. Choose an integer $\sigma$ such that $\sigma \geq \rho$ and $\sigma \geq \nu^{\circ}$ and assume that at a time instant $t^{*}$ the values of $\boldsymbol{q}^{\langle\sigma \sigma-1\rangle}\left(t^{*}\right)$ are known and do not correspond to any singular configuration of $\mathscr{M}$. Adopt $\delta \boldsymbol{q}^{\langle\sigma \sigma-1\rangle}\left(t^{*}\right)=0$. Also, suppose that there is a time interval $\Omega$ in the neighborhood of $t^{*}$ such that $f^{\prime}$ is a class $\mathscr{C}^{0}(\Omega)$ function (i.e. a continuous function in $\Omega$ ). According to Theorem 2.8, it can be stated that, in a time instant $\left(t^{*}+\varepsilon_{t}\right) \in \Omega$, with $\varepsilon_{t} \geq 0$ :

$$
\begin{aligned}
& \left(\frac{\varepsilon_{t}^{\sigma-\rho}}{(\sigma-\rho) !} \boldsymbol{V S} \tilde{\boldsymbol{q}}^{\langle\sigma\rangle}\left(t^{*}\right)+\boldsymbol{o}\left(\varepsilon_{t}^{\sigma-\rho}\right)\right) \cdot\left(\boldsymbol{f}^{\prime}\left(t^{*}\right)+o(1)\right) \leq 0 \\
& \left(\boldsymbol{V} \boldsymbol{S} \tilde{\boldsymbol{q}}^{\langle\sigma\rangle}\left(t^{*}\right)\right) \cdot \boldsymbol{f}^{\prime}\left(t^{*}\right)+\frac{(\sigma-\rho) ! o\left(\varepsilon_{t}^{\sigma-\rho}\right)}{\varepsilon_{t}^{\sigma-\rho}} \leq 0 \\
& \tilde{\boldsymbol{q}}^{\langle\sigma\rangle}\left(t^{*}\right) \cdot\left(\boldsymbol{S}^{\top} \boldsymbol{V}^{\top} \boldsymbol{f}^{\prime}\left(t^{*}\right)\right)+\frac{(\sigma-\rho) ! o\left(\varepsilon_{t}^{\sigma-\rho}\right)}{\varepsilon_{t}^{\sigma-\rho}} \leq 0
\end{aligned}
$$


Taking the limit $\varepsilon_{t} \rightarrow 0$, it can be stated that:

$$
\tilde{\boldsymbol{q}}^{\langle\sigma\rangle}\left(t^{*}\right) \cdot\left(\boldsymbol{S}^{\top} \boldsymbol{V}^{\top} \boldsymbol{f}^{\prime}\left(t^{*}\right)\right) \leq 0
$$

Values of $\tilde{\boldsymbol{q}}^{\langle\sigma\rangle}\left(t^{*}\right)$ are infinitesimals that can be freely chosen, being the satisfaction of the motion constraints ensured, unless the generalized configuration $\boldsymbol{q}^{\langle\sigma-1\rangle}\left(t^{*}\right)$ is singular. Thus, the inequality (4.5) must remain the same if $\tilde{\boldsymbol{q}}^{\langle\sigma\rangle}\left(t^{*}\right)$ is replaced by $-\tilde{\boldsymbol{q}}^{\langle\sigma\rangle}\left(t^{*}\right)$, which means that:

$$
S^{\top} V^{\top} f^{\prime}=0
$$

Thus, if $\boldsymbol{q}^{\langle\sigma-1\rangle}\left(t^{*}\right)$ is known, equation (4.6) can be used to determine the evolution of the motion of this system, leading ultimately, along with the constraint invariants of $\mathscr{M}$, to values of $\dot{\boldsymbol{q}}^{\langle\sigma-1\rangle}\left(t^{*}\right)$. Thus, interpreting this as an initial conditions problem, shows that is not just an expression for an invariant valid at some time instant $t^{*}$ but a general matrix form of the dynamic equations of motion of $\mathscr{M}$.

Example 4.1 (Modeling of a spherical pendulum). Denote by $\mathcal{N}$ an inertial reference frame and let $\mathrm{N}=(0, \hat{\mathbf{x}}, \hat{\mathbf{y}}, \hat{\mathbf{z}})$ be a coordinate system rigidly attached to $\mathcal{N}$. Assume that the unit vector $\hat{x}$ points in the same direction as the local acceleration of gravity. Let $\mathcal{P}$ denote a material particle whose mass is equal to $m$ constrained to move in a spherical surface of radius $\bar{r}$ and centered at $o$. The system constituted by $\mathcal{N}$ and $\mathcal{P}$ will be referred as a spherical pendulum $\mathscr{P}$. Two mathematical models are derived for $\mathscr{P}$ :

Model in spherical coordinates Once $\mathcal{P}$ is constrained to move in a spherical surface, a description by spherical coordinates is an immediate choice. Once the radial coordinate is constant and equal to $\bar{r}$, only the zenith angle $(\theta)$ and azimuth angle $(\phi)$ are sufficient as generalized coordinates of $\mathscr{P}$. Assume that this model has trivial generalized variables. Denoting by $\mathbf{r}$ the position vector of the center of $\mathcal{P}$ with respect to $\mathrm{N}$, it can be stated that:

$$
\mathbf{r}=\bar{r}\left(\mathrm{c}_{\theta} \hat{\mathbf{x}}+\mathrm{c}_{\phi} \mathrm{s}_{\theta} \hat{\mathbf{y}}+\mathrm{s}_{\phi} \mathrm{s}_{\theta} \hat{\mathbf{z}}\right)
$$


Let $\boldsymbol{v}^{\langle 0\rangle}=[\mathbf{r}]_{\mathrm{N}}=(x, y, z)$. Once $\mathbf{f}=-m \ddot{\mathbf{r}}+m g \hat{\mathbf{x}}$, then:

$$
f^{\prime}=y\left[\begin{array}{c}
m g+m \bar{r}\left(\mathrm{c}_{\theta} \dot{\theta}^{2}+\mathrm{s}_{\theta} \ddot{\theta}\right) \\
-m \bar{r}\left(\mathrm{c}_{\theta}\left(\mathrm{c}_{\phi} \ddot{\theta}-2 \mathrm{~s}_{\phi} \dot{\phi} \dot{\theta}\right)-\mathrm{s}_{\theta}\left(\mathrm{c}_{\phi}\left(\dot{\phi}^{2}+\dot{\theta}^{2}\right)+\mathrm{s}_{\phi} \ddot{\phi}\right)\right) \\
-m \bar{r}\left(\mathrm{~s}_{\theta}\left(\mathrm{c}_{\phi} \ddot{\phi}-\mathrm{s}_{\phi}\left(\dot{\phi}^{2}+\dot{\theta}^{2}\right)\right)+\mathrm{c}_{\theta}\left(2 \mathrm{c}_{\phi} \dot{\phi} \dot{\theta}+\mathrm{s}_{\phi} \ddot{\theta}\right)\right)
\end{array}\right]
$$

Also, from equation (a):

$$
\boldsymbol{V}=y\left[\begin{array}{cc}
\phi & \theta \\
0 & -\bar{r} \mathrm{~s}_{\theta} \\
-\bar{r} \mathrm{~s}_{\phi} \mathrm{s}_{\theta} & \bar{r} \mathrm{c}_{\phi} \mathrm{s}_{\theta} \\
\bar{r} \mathrm{c}_{\phi} \mathrm{s}_{\theta} & \bar{r} \mathrm{~s}_{\phi} \mathrm{c}_{\theta}
\end{array}\right]
$$

Finally, once no generalized constraint invariants are needed to describe the constraints of $\mathscr{M}$, it can be assumed that $S=1$. The dynamic equations of motion of $\mathscr{P}$ are given by:

$$
\theta\left[\begin{array}{c}
-m \bar{r}^{2} \mathrm{~s}_{\theta}\left(2 \mathrm{c}_{\theta} \dot{\phi} \dot{\theta}+\mathrm{s}_{\theta} \ddot{\phi}\right) \\
-m g \bar{r} \mathrm{~s}_{\theta}+m \bar{r}^{2}\left(\mathrm{~s}_{\theta} \mathrm{c}_{\theta} \dot{\phi}^{2}-\ddot{\theta}\right)
\end{array}\right]=0
$$

After some algebraic simplification, these equations of motion can be written as follows:

$$
\left\{\begin{array}{l}
\ddot{\phi}=-\frac{2 \mathrm{c}_{\theta} \dot{\phi} \dot{\theta}}{\mathrm{s}_{\theta}} \\
\ddot{\theta}=\mathrm{s}_{\theta}\left(\mathrm{c}_{\theta} \dot{\phi}^{2}-\frac{g}{\bar{r}}\right)
\end{array}\right.
$$

The singularity $s_{\theta}=0$ in equation (e) is inherent from a description by spherical coordinates. Indeed, when $\mathrm{s}_{\theta}=0$, any variation in the coordinate $\phi$ will not correspond to any variation in the configuration of the system, as can be noticed in equation (a).

Model in Cartesian coordinates (first version) Consider, on the other hand, that the Cartesian coordinates of the center of $\mathcal{P}$ are adopted as generalized coordinates, i.e. $\boldsymbol{q}^{\langle 0\rangle}=(x, y, z)$. Assume again that the model has trivial generalized variables. In this case the position vector $r$ can be 
written as follows:

$$
\mathbf{r}=x \hat{\mathbf{x}}+y \hat{\mathbf{y}}+z \hat{\mathbf{z}}
$$

with the coordinates $x, y$ e $z$ satisfying the following invariant:

$$
\bar{q}^{\langle 0\rangle}=x^{2}+y^{2}+z^{2}-\bar{r}^{2}=0
$$

Note that, in this case the constraint order of $\mathscr{P}$ is $\nu^{\circ}=1$ Adopting, for instance $\sigma=2$ and $\boldsymbol{v}^{\langle 2\rangle}=\ddot{\boldsymbol{q}}^{\langle 0\rangle}$, it can be stated that:

$$
\boldsymbol{f}^{\prime}=\ddot{\ddot{x}}\left[\begin{array}{c}
m g-m \ddot{x} \\
-m \ddot{y} \\
-m \ddot{z}
\end{array}\right]
$$

Note also that $V=1$. Moreover,

$$
\boldsymbol{A}=\left[\begin{array}{ccc}
\ddot{x} & \ddot{y} & \ddot{z} \\
2 x & 2 y & 2 z
\end{array}\right]
$$

A possible orthogonal complement for $A$, in this case, is:

$$
\boldsymbol{S}=\ddot{\ddot{x}}\left[\begin{array}{cc}
\tilde{q}_{1} & \tilde{q}_{2} \\
\ddot{z} & -z \\
x & 0 \\
0 & x
\end{array}\right]
$$

The equations of motion of $\mathscr{P}$ can be given by the second time derivative of equation ( $\mathrm{g}$ ) along with the dynamic equations of the form (4.6). These equations of motion can be reorganized in matrix form as follows:

$$
\left[\begin{array}{ccc}
2 x & 2 y & 2 z \\
-y & 0 & y \\
-z & x & 0
\end{array}\right]\left[\begin{array}{c}
\ddot{x} \\
\ddot{y} \\
\ddot{z}
\end{array}\right]=\left[\begin{array}{c}
-2\left(\dot{x}^{2}+\dot{y}^{2}+\dot{z}^{2}\right) \\
-g y \\
-g z
\end{array}\right]
$$

After some algebraic manipulation, these equations can be written in an 
explicit form:

$$
\left\{\begin{array}{l}
\ddot{x}=\frac{g}{\bar{r}^{2}}\left(y^{2}+z^{2}\right)-\frac{1}{\bar{r}^{2}} \times\left(\dot{x}^{2}+\dot{y}^{2}+\dot{z}^{2}\right) \\
\ddot{y}=-\frac{g}{\bar{r}^{2}} x y-\frac{1}{\bar{r}^{2}} y\left(\dot{x}^{2}+\dot{y}^{2}+\dot{z}^{2}\right) \\
\ddot{z}=-\frac{g}{\bar{r}^{2}} x z-\frac{1}{\bar{r}^{2}} z\left(\dot{x}^{2}+\dot{y}^{2}+\dot{z}^{2}\right)
\end{array}\right.
$$

The description in Cartesian coordinates does not have any intrinsic singularity and no trigonometric functions are involved in the equations of motion, which are polinomial (this might be an advantage in terms of numerical simulations).

Model in Cartesian coordinates (second version) A brief analysis at equations (I) shows that it might be useful to define a new quasi-velocity in order to simplify the mathematical model of $\mathscr{P}$. From now on assume that $\boldsymbol{q}^{\langle 0\rangle}=(x, y, z)$ and $\boldsymbol{q}^{\langle 1\rangle}=(\dot{x}, \dot{y}, \dot{z}, v)$, with trivial generalized variables above first order. Two generalized constraint invariants are required in this case, for instance:

$$
\overline{\boldsymbol{q}}^{\langle 1\rangle}=\left[\begin{array}{c}
2 x \dot{x}+2 y \dot{y}+2 z \dot{z} \\
\dot{x}^{2}+\dot{y}^{2}+\dot{z}^{2}-v
\end{array}\right]=0
$$

Now the constraint order of the model is $\nu^{\circ}=2$ and:

$$
\boldsymbol{A}=\left[\begin{array}{cccc}
\ddot{x} & \ddot{y} & \ddot{z} & \dot{v} \\
2 x & 2 y & 2 z & 0 \\
2 \dot{x} & 2 \dot{y} & 2 \dot{z} & -1
\end{array}\right]
$$

Noting that the generalized effective forces associated to $\dot{v}$ are zero, it can be stated that following the same procedure which led to the previous models of $\mathscr{P}$, the following explicit equations of motion are obtained in this case:

$$
\left\{\begin{array}{l}
\ddot{x}=g-\frac{x(v+g x)}{\bar{r}^{2}} \\
\ddot{y}=-\frac{y(v+g x)}{\bar{r}^{2}} \\
\ddot{z}=-\frac{z(v+g x)}{\bar{r}^{2}} \\
\dot{v}=2 g \dot{x}
\end{array}\right.
$$


It is evident that the last equation of the system (o) comes from the time derivative of the mechanical energy of $\mathscr{P}$. Indeed, the mechanical energy of the spherical pendulum can be expressed as follows:

$$
E=\frac{m}{2}(v-2 g x)
$$

Once $\mathscr{P}$ is a conservative system, then $\dot{E}=0$, which leads to the last equation of system (o).

\subsection{Recursive modular modeling algorithm}

In the previous section it was shown that the following three steps are sufficient for obtaining the dynamic equations of motion of a multibody system:

(1) Obtain expressions of generalized effective forces (i.e. the sum of generalized active and inertia forces) associated to a generic set of $\rho$-th order motion variables.

(2) Determine a transformation matrix associated to the conversion between variations of these generic $\rho$-th order motion variables and variations of $\sigma$-th order generalized variables for some $\sigma \geq \max \left\{\rho, \nu^{\circ}\right\}$.

(3) Compute an orthogonal complement of the Jacobian of the $\sigma$-th order independent generalized constraint invariants.

Consider that a multibody system is divided in subsystems. The first and second steps can be performed within the scope of each subsystem ${ }^{2}$, being only necessary to consider the motion constraints between the subsystems in the final step. Thus, the techniques presented in Section 2.5 can be used to develop a recursive modular modeling algorithm for multibody systems.

Consider a generic multibody system which can be conceived as a hierarchical structure of subsystems representable by a tree diagram. Following the convention presented in Section 2.5, let $\mathscr{M}_{n}$ denote a generic system at the $n$-th level of a tree structure (with $\mathscr{M}_{0}$ denoting the whole system itself). and denote by $\mathbb{S}\left(\mathscr{M}_{n}\right)$ the set of all subsystems of $\mathscr{M}_{n}$. Moreover, let $\boldsymbol{q}_{\mathscr{M}_{n}}^{\langle\alpha\rangle}$ denote the tuple (or column-matrix) of $\alpha$-th order generalized variables used in the description of motion of $\mathscr{M}_{n}$. If $\mathbb{S}\left(\mathscr{M}_{n}\right)$ is not an empty set, then it can be stated

\footnotetext{
${ }^{2}$ Even though some constitutive equations might lead to expressions of generalized effective forces that involve variables associated to more than one subsystem, each generalized force is associated to a single subsystem.
} 
that $\boldsymbol{q}_{\mathcal{M}_{n}}^{\langle\alpha\rangle}$ is constituted both by variables coming from the models of the subsystems of $\mathscr{M}_{n}$, and by extra variables specially chosen for modeling $\mathscr{M}_{n}$, if any, i.e. $\boldsymbol{q}_{\mathscr{M}_{n}}^{\langle\alpha\rangle}=\left(\boldsymbol{q}_{\mathscr{M}_{n \oplus \oplus}}^{\langle\alpha\rangle}\left(\ldots, \boldsymbol{q}_{\mathscr{M}_{n+1}}^{\langle\alpha\rangle}, \ldots\right)\right)$ for all $\mathscr{M}_{n+1} \in \mathbb{S}\left(\mathscr{M}_{n}\right)$. Analogously, let $\overline{\boldsymbol{q}}_{\mathscr{M}_{n}}^{\langle\alpha\rangle}$ denote the tuple (or column-matrix) constituted by the $\alpha$-th order generalized constraint invariants of $\mathscr{M}_{n}$. If $\mathbb{S}\left(\mathscr{M}_{n}\right)$ is not an empty set, then it can be stated that $\overline{\boldsymbol{q}}_{\mathcal{M}_{n}}^{\langle\alpha\rangle}$ is constituted both by invariants describing the internal constraints of the subsystem of $\mathscr{M}_{n}$, if any, and by the invariants describing the (external) constraints among these subsystems, if any, i.e. $\left.\overline{\boldsymbol{q}}_{\mathcal{M}_{n}}^{\langle\alpha\rangle}=\left(\overline{\boldsymbol{q}}_{\mathcal{M}_{n} \oplus}^{\langle\alpha}, \ldots, \overline{\boldsymbol{q}}_{\mathcal{M}_{n+1}}^{\langle\alpha\rangle}, \ldots\right)\right)$ for all $\mathscr{M}_{n+1} \in \mathbb{S}\left(\mathscr{M}_{n}\right)$.

Focus the attention to the cases in which $\mathbb{S}\left(\mathscr{M}_{n}\right)=\varnothing$, i.e. $\mathscr{M}_{n}$ has no subsystems, being a leaf in the tree structure representing system $\mathscr{M}_{0}$. Define the unconstrained generalized effective forces of $\mathscr{M}_{n}, f_{\mathscr{M}_{n}}$, and the constrained generalized effective forces of $\mathscr{M}_{n} \bar{f}_{\mathcal{M}_{n}}$, as follows:

$$
\begin{aligned}
& f_{\mathscr{U}_{n}}=V_{\mathscr{U}_{n}} \cdot f_{\mathscr{U}_{n}}^{\prime}=V_{\mathscr{U}_{n}}^{\top} f_{\mathscr{U}_{n}}^{\prime} \\
& \bar{f}_{\mathcal{U}_{n}}=C_{\mathscr{M}_{n}} \cdot f_{\mathscr{U}_{n}}=C_{\mathscr{U}_{n}}^{\top} \boldsymbol{f}_{\mathscr{U}_{n}}
\end{aligned}
$$

Noting that if $\mathbb{S}\left(\mathscr{M}_{n}\right)=\varnothing$ then $C_{\mathscr{M}_{n}}=S_{\mathscr{M}_{n}}$ (see Section 2.5), it can be stated that, according to equation (4.6), the dynamic equations of motion of $\mathscr{M}_{n}$ when it is not constrained to any other system are given by $\bar{f}_{\mathcal{M}_{n}}=0$.

Consider now that $\mathbb{S}\left(\mathscr{M}_{n}\right)$ is not empty, i.e. $\mathscr{M}_{n}$ has its own subsystems. Once $\boldsymbol{q}_{\mathscr{M}_{n}}^{\langle\alpha\rangle}=\left(\boldsymbol{q}_{\mathscr{M}_{n \rightarrow \oplus}}^{\langle\alpha\rangle}\left(\ldots, \boldsymbol{q}_{\mathscr{M}_{n+1}}^{\langle\alpha\rangle}, \ldots\right)\right)$ for all $\mathscr{M}_{n+1} \in \mathbb{S}\left(\mathscr{M}_{n}\right)$, let $\delta \boldsymbol{v}_{\mathscr{M}_{n}}^{\langle\rho\rangle}=$ $\left(\delta \boldsymbol{q}_{\mathscr{M}_{n, \oplus}}^{\langle\rho \mid \sigma\rangle},\left(\ldots, \delta \boldsymbol{v}_{\mathscr{M}_{n+1}}^{\langle\rho\rangle}, \ldots\right)\right)$ for all $\mathscr{M}_{n+1} \in \mathbb{S}\left(\mathscr{M}_{n}\right)$. Thus,

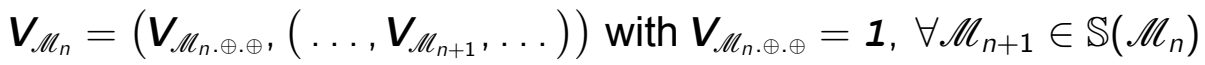

$$
\begin{aligned}
& f_{\mathscr{M}_{n}}^{\prime}=\left(f_{\mathscr{M}_{n} \oplus},\left(\ldots, f_{\mathscr{M}_{n+1}}^{\prime}, \ldots\right)\right), \forall \mathscr{M}_{n+1} \in \mathbb{S}\left(\mathscr{M}_{n}\right)
\end{aligned}
$$

with $f_{\|_{n} \oplus}$ denoting the generalized effective forces associated with the extra generalized variables of $\mathscr{M}_{n}$.

From Theorem 2.10, it can be stated that equation (4.6) for $\mathscr{M}_{n}$ can be written as follows:

$$
\begin{aligned}
& \bar{f}_{\mathscr{U}_{n}}=S_{\mathscr{M}_{n}}^{\top} V_{\mathscr{M}_{n}}^{\top} f_{\mathscr{M}_{n}}^{\prime}=0 \\
& \bar{f}_{\mathscr{U}_{n}}=C_{\mathscr{M}_{n}}^{\top} \boldsymbol{R}_{\mathscr{M}_{n}}^{\top} \boldsymbol{V}_{\mathscr{M}_{n}}^{\top} \boldsymbol{f}_{\mathscr{M}_{n}}^{\prime}=0 \\
& \bar{f}_{\mathscr{U}_{n}}=C_{\mathscr{M}_{n}}^{\top}\left(f_{\mathscr{M}_{n} \oplus},\left(\ldots, S_{\mathscr{M}_{n+1}}^{\top} \boldsymbol{V}_{\mathscr{M}_{n+1}}^{\top} \boldsymbol{f}_{\mathscr{M}_{n+1}}^{\prime}, \ldots\right)\right)=0, \forall \mathscr{M}_{n+1} \in \mathbb{S}\left(\mathscr{M}_{n}\right) \\
& \bar{f}_{\mathscr{M}_{n}}=C_{\mathscr{M}_{n}}^{\top}\left(f_{\mathscr{M}_{n} \oplus},\left(\ldots, \bar{f}_{\mathscr{M}_{n+1}}, \ldots\right)\right)=0, \forall \mathscr{M}_{n+1} \in \mathbb{S}\left(\mathscr{M}_{n}\right)
\end{aligned}
$$


Defining the unconstrained generalized effective forces of $\mathscr{M}_{n}, f_{\mathscr{M}_{n}}$, by:

$$
\boldsymbol{f}_{\mathscr{M}_{n}}=\left(\boldsymbol{f}_{\mathscr{M}_{n} \oplus},\left(\ldots, \overline{\boldsymbol{f}}_{\mathscr{M}_{n+1}}, \ldots\right)\right)
$$

it can be stated that, according to equation (4.11), the constrained generalized effective forces of $\mathscr{M}_{n}, \bar{f}_{\mathscr{M}_{n}}$, are given by equation (4.8), i.e.:

$$
\bar{f}_{\mathscr{M}_{n}}=C_{\mathscr{M}_{n}} \cdot f_{\mathscr{M}_{n}}=C_{\mathscr{M}_{n}}^{\top} \boldsymbol{f}_{\mathscr{M}_{n}}
$$

Therefore, a recursive algorithm can be proposed for the modeling of multibody systems which can be represented by a hierarchy of subsystems. All the computations must begin at the deepest levels of the tree structure, followed by the corresponding parents, representing a "leaves-to-root" algorithm (i.e. the algorithm can only be applied to a node in the tree if it was already applied to all its descendants). The algorithm must be defined for two cases based on whether or not $\mathscr{M}_{n}$ has subsystems.

Case 1A: $\mathscr{M}_{n}$ has no subsystems, $\mathbb{S}\left(\mathscr{M}_{n}\right)=\varnothing$, and its model is already known

(1) Identify the constraint order $\nu_{\mathscr{M}_{n}}^{\circ}$ and the $\alpha$-th order generalized variables of $\mathscr{M}_{n}, \boldsymbol{q}_{\mathscr{M}_{n}}^{\langle\alpha\rangle}$, for all $\alpha$ such that $0 \leq \alpha \leq \nu_{\mathscr{M}_{n}}^{\circ}$.

(2) Identify the expressions, if any, of the $\alpha$-th generalized constraint invariants of $\mathscr{M}_{n}, \boldsymbol{q}_{\mathscr{M}_{n}}^{\langle\alpha\rangle}$, for all $\alpha$ such that $0 \leq \alpha \leq \nu_{\mathscr{M}_{n}}^{\circ}$.

(3) If there are any non-degenerate $\nu_{\mathscr{M}_{n}}^{\circ}$-th order generalized constraint invariants of $\mathscr{M}_{n}$, identify the expression of $\boldsymbol{A}_{\mathscr{M}_{n}}$, let $B_{\mathscr{M}_{n}}=\boldsymbol{A}_{\mathscr{M}_{n}}$ and obtain an expression (if not already known) for an orthogonal complement $C_{\mathscr{M}_{n}}$; else, adopt $C_{\mathscr{M}_{n}}=1$.

(4) If the already known dynamic equations of motion of $\mathscr{M}_{n}$ involve undetermined multipliers, pre-multiply them by $C_{\mathscr{M}_{n}}^{\top}$ in order to eliminate these auxiliary variables. Obtain the expressions for $\bar{f}_{\mathscr{M}_{n}}$.

(5) Adopt $S_{\mathscr{M}_{n}}=C_{\mathscr{M}_{n}}$.

Case 1: $\mathscr{M}_{n}$ has no subsystems, $\mathbb{S}\left(\mathscr{M}_{n}\right)=\varnothing$

(1) Choose a set of generalized variables for $\mathscr{M}_{n}$ and identify its constraint order $\nu_{\mathscr{M}_{n}}^{\circ}$.

(2) Write down the non-trivial expressions of the invariants $\overline{\boldsymbol{c}}_{\mathscr{M}_{n}}^{\langle\alpha+1\rangle}\left(t, \boldsymbol{q}_{\mathscr{M}_{n}}^{\langle\langle\alpha+1\rangle}, \dot{\boldsymbol{q}}_{\mathscr{M}_{n}}^{\langle\alpha\rangle}\right)=0$ defining the transformations of variables between the $\dot{\boldsymbol{q}}_{\mathscr{M}_{n}}^{\langle\alpha\rangle}$ and the $\boldsymbol{q}_{\mathscr{M}_{n}}^{\langle\alpha+1\rangle}$. 
(3) Obtain, if necessary, expressions for the $\alpha$-th generalized constraint invariants of $\mathscr{M}_{n}, \overline{\boldsymbol{q}}_{\mathcal{M}_{n}}^{\langle\alpha\rangle}\left(t, \boldsymbol{q}_{\mathcal{M}_{n}}^{\langle\alpha\rangle}\right)=0$, for all $\alpha$ such that $0 \leq \alpha \leq \nu_{\mathscr{M}_{n}}^{\circ}$.

(4) If there are any non-degenerate $\nu_{M_{n}}^{\circ}$-th order generalized constraint invariants of $\mathscr{M}_{n}$, identify the expression of $\boldsymbol{A}_{\mathscr{M}_{n}}$, let $B_{\mathscr{M}_{n}}=\boldsymbol{A}_{\mathscr{M}_{n}}$ and obtain an expression for an orthogonal complement $C_{\mathscr{M}_{n}}$; else, adopt $C_{\mathscr{M}_{n}}=1$.

(5) Consider that it is possible to completely describe the effective force system acting in $\mathscr{M}_{n}$ by a tuple (or column-matrix) $f_{\mathscr{M}_{n}}^{\prime}$ of generalized effective forces associated to a set of generic $\rho$-th order motion variables $v_{\mathscr{M}_{n}}^{\langle\rho\rangle}$. Obtain the expressions for these motion variables in terms of time and of the generalized variables of the model of $\mathscr{M}_{n}$, i.e., $\boldsymbol{v}_{\mathscr{M}_{n}}^{\langle\rho\rangle}=\underline{\boldsymbol{v}}_{\mathscr{M}_{n}}^{\langle\rho\rangle}\left(t, \boldsymbol{q}_{\mathcal{M}_{n}}^{\langle\rho\rangle\rangle}\right)$. Choose some $\sigma \geq \max \left\{\nu_{\mathcal{M}_{n}}^{\circ}, \rho\right\}$ and compute $V_{\mathscr{M}_{n}}$ by equation (2.21):

$$
\boldsymbol{V}_{\mathscr{M}_{n}}=\frac{\partial \underline{\underline{v}}_{\mathcal{M}_{n}}^{\langle\rho\rangle}}{\partial \boldsymbol{q}_{\mathcal{M}_{n}}^{\langle\rho}} \frac{\partial \underline{\boldsymbol{q}}_{\mathcal{M}_{n}}^{\langle\rho \mid \rho\rangle}}{\partial \boldsymbol{q}_{\mathcal{M}_{n}}^{\langle\sigma\rangle}}
$$

(6) Obtain $f_{\mathscr{M}_{n}}=V_{\mathscr{M}_{n}}^{\top} f_{\mathcal{M n}_{n}}^{\prime}$.

(7) Obtain $\bar{f}_{\mathcal{M}_{n}}=C_{\mathcal{M}_{n}}^{\top} f_{\mathcal{M}_{n}}$.

(8) Adopt $S_{\mathscr{M}_{n}}=C_{\mathscr{M}_{n}}$.

Case 2: $\mathscr{M}_{n}$ has subsystems, $\mathbb{S}\left(\mathscr{M}_{n}\right) \neq \varnothing$

(1) Define extra generalized variables if convenient, and let $\boldsymbol{q}_{\mathscr{M}_{n}}^{\langle\alpha\rangle}=$ $\left(\boldsymbol{q}_{\mathscr{M}_{n \rightarrow \oplus}}^{\langle\alpha\rangle}\left(\ldots, \boldsymbol{q}_{\mathscr{M}_{n+1}}^{\langle\alpha\rangle}, \ldots\right)\right)$ for all $\mathscr{M}_{n+1} \in \mathbb{S}\left(\mathscr{M}_{n}\right)$. Identify the constrain order $\nu_{\mathscr{M}_{n}}^{\circ}$ of the system noting that $\nu_{\mathscr{M}_{n}}^{\circ} \geq \nu_{\mathscr{U}_{n+1}}^{\circ}$ for all $\mathscr{M}_{n+1} \in \mathbb{S}\left(\mathscr{M}_{n}\right)$.

(2) Obtain, if necessary, expressions for the external generalized constraint invariants of $\mathscr{M}_{n}, \overline{\boldsymbol{q}}_{\mathscr{M}_{n}}^{\langle\alpha\rangle}$ for all $\alpha$ such that $0 \leq \alpha \leq \nu_{\mathscr{M}_{n}}^{\circ}$.

(3) Choose an integer $\sigma \geq \nu_{\mu_{n}}^{\circ}$ and write down all the $\sigma$-th order generalized constraints of $\mathscr{M}_{n}$ as follows: $\overline{\boldsymbol{q}}_{\mathscr{M}_{n}}^{\langle\sigma\rangle}=\boldsymbol{A}_{\mathscr{M}_{n}} \boldsymbol{q}_{\mathcal{M}_{n}}^{\langle\sigma\rangle}+\boldsymbol{b}_{\mathscr{M}_{n}}^{\langle\sigma-1\rangle}$.

(4) Following the statement of Theorem 2.10 define $R_{\mathscr{U}_{n}}$ by equation (2.34):

$$
R_{\mathscr{U}_{n}}=\left(R_{\mathscr{U}_{n \cdot \oplus \oplus},}\left(\ldots, S_{\mathscr{M}_{n+1}}, \ldots\right)\right) \text { with } R_{\mathscr{U}_{n} \oplus \oplus \oplus}=1, \forall \mathscr{M}_{n+1} \in \mathbb{S}\left(\mathscr{M}_{n}\right)
$$

(5) If $A_{\mathscr{U}_{n \cdot \oplus}}$ is an empty matrix, adopt $C_{\mathscr{M}_{n}}=1$, else obtain $C_{\mathscr{U}_{n}}$ as an orthogonal complement of $B_{\mathscr{U}_{n}}=A_{\mathscr{U}_{n} \oplus} \boldsymbol{R}_{\mathscr{U}_{n}}$.

(6) If necessary, include extra generalized effective force associated to the extra $\rho$-th order generalized variables of $\mathscr{M}_{n}$, and let $f_{\mathscr{M}_{n}}=$ 


$$
\left(f_{\mathscr{M}_{n \cdot \oplus}},\left(\ldots, \bar{f}_{\mathscr{M}_{n+1}}, \ldots\right)\right) \text { for all } \mathscr{M}_{n+1} \in \mathbb{S}\left(\mathscr{M}_{n}\right) .
$$

(7) Obtain $\bar{f}_{\mathscr{M}_{n}}=C_{M_{n}}^{\top} f_{\mathscr{M}_{n}}$.

(8) Compute, by equation (2.35), $\boldsymbol{S}_{\mathscr{U}_{n}}=\boldsymbol{R}_{\mathscr{U}_{n}} \boldsymbol{C}_{\mathscr{U}_{n}}$ (this last step is optional for the root level, i.e. for $\mathscr{M}_{0}$ ).

A simplified comparison between "Case 1" and "Case 2" algorithms is shown in Table 4.1. The first three rows in this table are constituted by steps of the algorithm which can not be completely automated, requiring some input from the user. The following rows, however, represent steps of the algorithms that can be fully automated in both cases.

Table 4.1: Comparison between "Case 1" and "Case 2" algorithms

\begin{tabular}{|c|c|c|}
\hline & Case 1: $\mathbb{S}\left(\mathscr{M}_{n}\right)=\varnothing$ & Case 2: $\mathbb{S}\left(\mathscr{M}_{n}\right) \neq \varnothing$ \\
\hline$q_{\mathscr{M}_{n}}^{\langle\alpha\rangle}$ & Input & $\left(\boldsymbol{q}_{\mathcal{M}_{n \rightarrow \oplus},}^{\langle\alpha\rangle}\left(\ldots, \boldsymbol{q}_{\mathcal{M}_{n+1}}^{\langle\alpha\rangle}, \ldots\right)\right)$ \\
\hline$\overline{\boldsymbol{q}}_{\mathscr{M}_{n}}^{\langle\sigma\rangle}$ & $\boldsymbol{A}_{\mathscr{M}_{n}} \boldsymbol{q}_{\mathscr{M}_{n}}^{\langle\sigma\rangle}+\boldsymbol{b}_{\mathscr{M}_{n}}^{\langle\sigma-1\rangle}$ & $\boldsymbol{A}_{\mathscr{M}_{n}} \boldsymbol{q}_{\mathscr{M}_{n}}^{\langle\sigma\rangle}+\boldsymbol{b}_{\mathscr{M}_{n}}^{\langle\sigma-1\rangle}$ \\
\hline$f_{\mathscr{M}_{n}}$ & 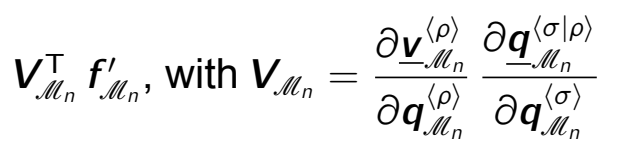 & $\left(f_{\mathcal{M}_{n \cdot \oplus}}\left(\ldots, \bar{f}_{\mathcal{M}_{n+1}}, \ldots\right)\right)$ \\
\hline$R_{\mathscr{M}_{n}}$ & 1 & $\begin{array}{l}\left(R_{\mathscr{M}_{n, \oplus . \oplus}}\left(\ldots, S_{\mathscr{M}_{n+1}}, \ldots\right)\right) \\
\quad \text { with } R_{\mathscr{M}_{n} \oplus \oplus \oplus}=\mathbf{1}\end{array}$ \\
\hline$B_{\mathscr{M}_{n}}$ & $\boldsymbol{A}_{\mathscr{M}_{n}}$ & $\boldsymbol{A}_{\mathscr{M}_{n} \oplus \oplus} \boldsymbol{R}_{\mathscr{M}_{n}}$ \\
\hline$C_{\mathscr{M}_{n}}$ & An orthogonal compl. of $B_{\mathscr{M}_{n}}$ & An orthogonal compl. of $B_{\mathscr{M}_{n}}$ \\
\hline$S_{\mathscr{M}_{n}}$ & $C_{\mathscr{M}_{n}}$ & $R_{\mathscr{U}_{n}} C_{\mathscr{U}_{n}}$ \\
\hline $\bar{f}_{\mathscr{M}_{n}}$ & $C_{\mathscr{M}_{n}}^{\top} f_{\mathscr{U}_{n}}$ & $C_{\mathscr{M}_{n}}^{\top} f_{\mathscr{U}_{n}}$ \\
\hline
\end{tabular}

Example 4.2. Consider the system $\delta$ shown in Figure 4.1 and suppose that it is composed by two subsystems, $\mathscr{R}$ and $\mathscr{D}$, the former constituted only by a rigid thin homogeneous ring $\mathcal{B}_{1}$ whose center is $b_{1}^{\star}$ and the latter constituted only by a rigid massive homogeneous disc $\mathcal{B}_{2}$ whose center is $\mathrm{b}_{2}^{\star}$. Assume that $\mathcal{B}_{1}$ and $\mathcal{B}_{2}$ can only perform plane motions in a vertical plane. Let $\mathrm{N}=$ $(o, \hat{\mathbf{x}}, \hat{\mathbf{y}}, \hat{\mathbf{z}})$ be a coordinate system rigidly attached to an inertial reference frame $\mathcal{N}$. Assume that there is an inclined plane which makes an angle $\bar{\xi}$ with respect to the horizontal and remains fixed with respect to $\mathcal{N}$. Let $\hat{y}$ be orthogonal to this inclined plane and $\hat{z}$ be orthogonal to the planes defined by the trajectories of the points of $\mathcal{B}_{1}$ and $\mathcal{B}_{2}$. Define $\boldsymbol{q}_{\mathscr{R}}^{\langle 1\rangle}=\left(u_{1}, v_{1}, \omega_{1}\right)$ and $\boldsymbol{q}_{\mathscr{D}}^{\langle 1\rangle}=\left(u_{2}, v_{2}, \omega_{2}\right)$ with $u_{k}$ and $v_{k}$ denoting the $\mathrm{x}$ and $\mathrm{y}$ components in $\mathrm{N}$ of the velocity of $\mathrm{b}_{k}^{\star}$ with respect 


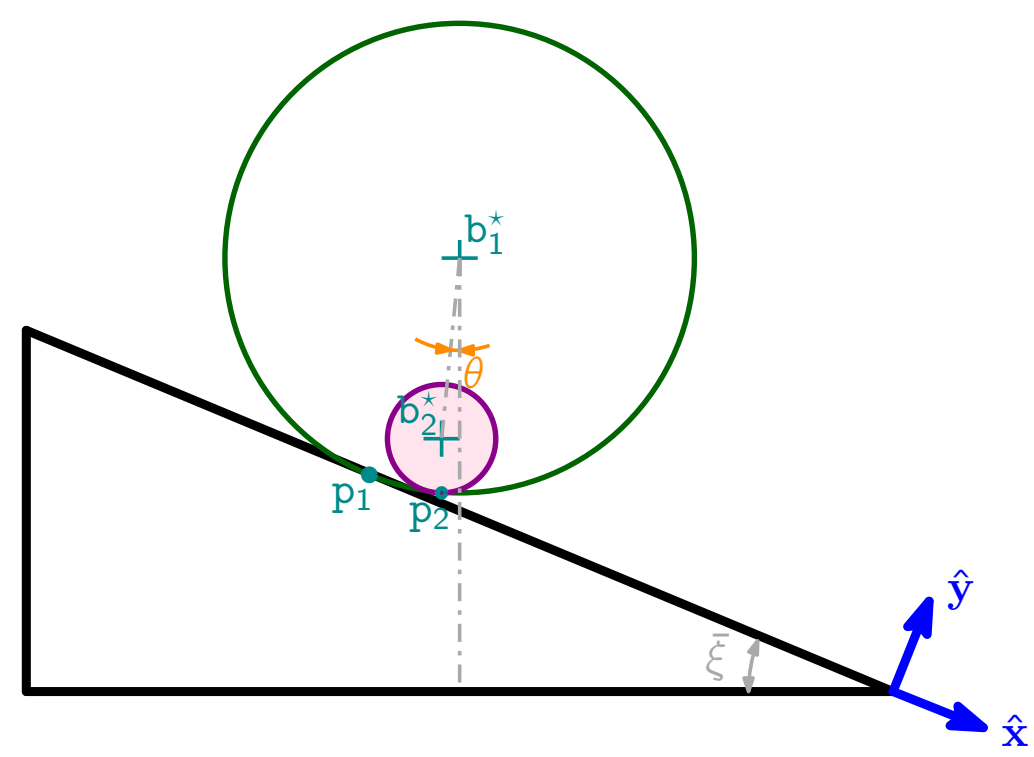

Figure 4.1: System $\delta$.

to $\mathcal{N}$ and $\omega_{k}$ denoting the $z$ component in $\mathrm{N}$ of the angular velocity of $\mathcal{B}_{k}$ with respect to $\mathcal{N}, k \in\{1,2\}$. Denote by $\bar{m}_{k}$ the mass of $\mathcal{B}_{k}$, by $\bar{T}_{k}$ its moment of inertia with respect to $b_{k}^{\star}$ and by $\bar{r}_{k}$ its corresponding radius, $k \in\{1,2\}$. Due to the homogeneous mass distribution of the rigid bodies, it can be stated that $\bar{l}_{1}=\bar{m}_{1} \bar{r}_{1}^{2}$ and $\bar{l}_{2}=\bar{m}_{2} \bar{r}_{2}^{2} / 2$. Also, let $\overline{\mathbf{g}}=\bar{g}(\sin \bar{\xi} \hat{\mathbf{x}}-\cos \bar{\xi} \hat{\mathbf{y}})$, be the local acceleration of gravity. It can be stated that:

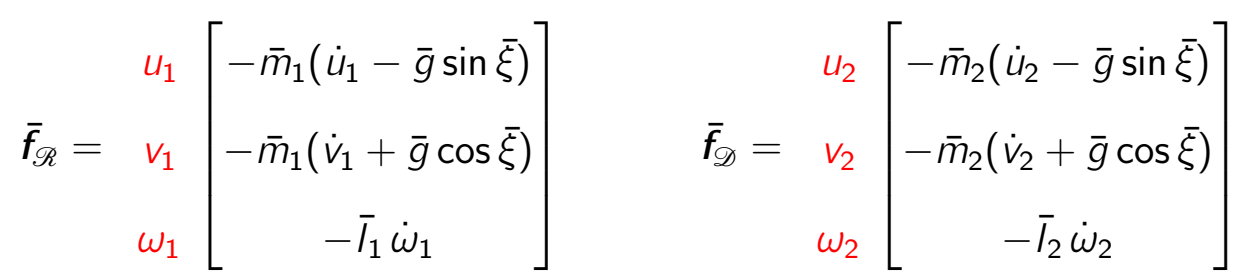

It can also be assumed that $S_{\mathscr{R}}=C_{\mathscr{R}}=1$ and $S_{\mathscr{D}}=C_{\mathscr{D}}=1$.

Assume that in $\mathcal{S}$, the ring $\mathcal{B}_{1}$ rolls without slipping down the inclined plane and the disc $\mathcal{B}_{2}$ rolls without slipping on the internal surface of the ring. Suppose that in a particular motion the angle between the line $b_{1}^{\star} b_{2}^{\star}$ and the vertical is constant and equal to $\bar{\theta}$. Holics(2011) proposes to find a relation between the ratio $\bar{m}_{2} / \bar{m}_{1}$ and the angles $\bar{\theta}$ and $\bar{\xi}$. For this, adopt an extra generalized coordinate for $\mathcal{S}, \theta$, denoting the clockwise angle between the line $\mathrm{b}_{1}^{\star} \mathrm{b}_{2}^{\star}$ and the vertical, let $\boldsymbol{q}_{\delta}^{\langle 0\rangle}=(\theta)$ and $\boldsymbol{q}_{\delta}^{\langle 1\rangle}=\left(\dot{\theta}, u_{1}, v_{1}, \omega_{1}, u_{2}, v_{2}, \omega_{2}\right)$, and assume that the generalized variables of $\mathcal{S}$ are trivial above first order.

Let $p_{1}$ and $p_{2}$ denote the contact points between $\mathcal{B}_{1}$ and the inclined plane and between $\mathcal{B}_{2}$ and $\mathcal{B}_{1}$, respectively. Denote by $\mathrm{p}_{1}(\mathcal{N})$ and $\mathrm{p}_{1}\left(\mathcal{B}_{1}\right)$ points that instantaneously coincide with $\mathrm{p}_{1}$ and move along with $\mathcal{N}$ and $\mathcal{B}_{1}$, respectively. Analogously, define $\mathrm{p}_{2}\left(\mathcal{B}_{1}\right)$ and $\mathrm{p}_{2}\left(\mathcal{B}_{2}\right)$. Due to the "roll without slipping" con- 
straint, it is necessary that:

$$
\begin{aligned}
& \mathbf{v}_{\mathrm{p}_{1}(\mathcal{N}) \mid \mathcal{N}}=\mathbf{v}_{\mathrm{p}_{1}\left(\mathcal{B}_{1}\right) \mid \mathcal{N}} \quad \Rightarrow \quad 0=\mathbf{v}_{\mathrm{b}_{1}^{\star} \mid \mathcal{N}}+\omega_{\mathcal{B}_{1} \mid \mathcal{N}} \times \mathbf{r}_{\mathrm{p}_{1} \mid \mathrm{b}_{1}^{\star}} \\
& \mathbf{v}_{\mathrm{p}_{2}\left(\mathcal{B}_{1}\right) \mid \mathcal{N}}=\mathbf{v}_{\mathrm{p}_{2}\left(\mathcal{B}_{2}\right) \mid \mathcal{N}} \Rightarrow \mathbf{v}_{\mathrm{b}_{1}^{\star} \mid \mathcal{N}}+\omega_{\mathcal{B}_{1} \mid \mathcal{N}} \times \mathbf{r}_{\mathrm{p}_{2} \mid \mathrm{b}_{1}^{\star}}=\mathbf{v}_{\mathrm{b}_{2}^{\star} \mid \mathcal{N}}+\omega_{\mathcal{B}_{2} \mid \mathcal{N}} \times \mathbf{r}_{\mathrm{p}_{2} \mid \mathrm{b}_{2}^{\star}}
\end{aligned}
$$

It can also be stated that:

$$
u_{2}-u_{1}=\frac{\mathrm{d}}{\mathrm{d} t}\left(\left(\bar{r}_{1}-\bar{r}_{2}\right) \sin (\bar{\xi}-\theta)\right)
$$

Thus, due to equations (b-d), it can be stated that the external constraint invariants of $\mathcal{S}$ are the following:

$$
\overline{\boldsymbol{q}}_{\mathcal{S}}^{\langle 1\rangle}=\left[\begin{array}{c}
u_{1}+\omega_{1} \bar{r}_{1} \\
v_{1} \\
u_{1}-u_{2}+\cos (\bar{\xi}-\theta) \omega_{1} \bar{r}_{1}-\cos (\bar{\xi}-\theta) \omega_{2} \bar{r}_{2} \\
v_{1}-v_{2}+\sin (\bar{\xi}-\theta) \omega_{1} \bar{r}_{1}-\sin (\bar{\xi}-\theta) \omega_{2} \bar{r}_{2} \\
-u_{1}+u_{2}+\cos (\bar{\xi}-\theta) \dot{\theta}\left(\bar{r}_{1}-\bar{r}_{2}\right)
\end{array}\right]
$$

Adopting the notation $\mathrm{c}_{\theta}^{\bullet}=\cos (\bar{\xi}-\theta)$ and $\mathrm{s}_{\theta}^{\bullet}=\sin (\bar{\xi}-\theta)$, it can be stated that:

$$
\boldsymbol{A}_{\mathcal{S}}=\left[\begin{array}{ccccccc}
\dot{\theta} & u_{1} & v_{1} & \omega_{1} & u_{2} & v_{2} & \omega_{2} \\
0 & 1 & 0 & \bar{r}_{1} & 0 & 0 & 0 \\
0 & 0 & 1 & 0 & 0 & 0 & 0 \\
0 & 1 & 0 & \mathrm{c}_{\theta}^{\bullet} \bar{r}_{1} & -1 & 0 & -\mathrm{c}_{\theta}^{\bullet} \bar{r}_{2} \\
0 & 0 & 1 & \mathrm{~s}_{\theta}^{\bullet} \bar{r}_{1} & 0 & -1 & -\mathrm{s}_{\theta}^{\bullet} \bar{r}_{2} \\
\mathrm{c}_{\theta}^{\bullet}\left(\bar{r}_{1}-\bar{r}_{2}\right) & -1 & 0 & 0 & 1 & 0 & 0
\end{array}\right]
$$


A possible expression for matrix $C_{\mathcal{S}}$ is the following:

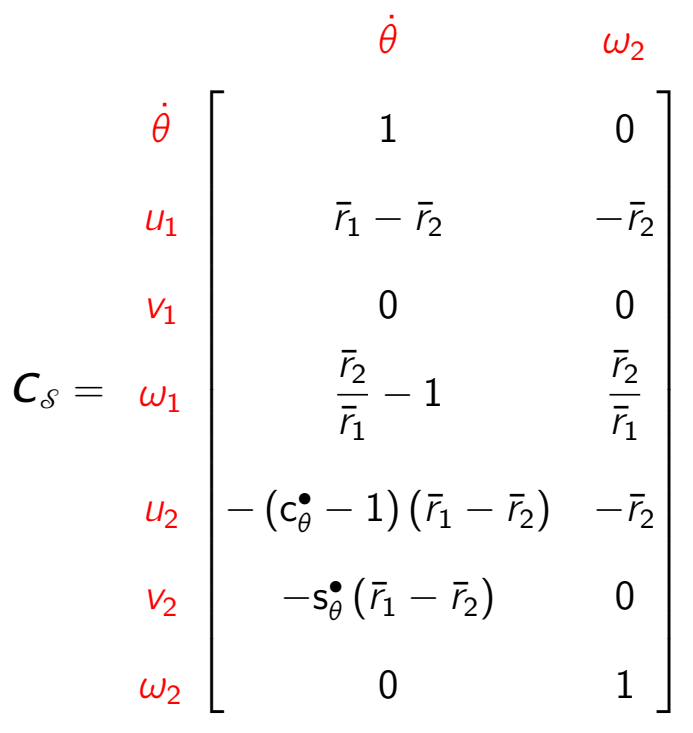

Using the modular modeling algorithm, the equations of motion of $\delta$ can be obtained. Solving these equations for the motion in which $\theta=\bar{\theta}, \dot{\theta}=0$ and $\ddot{\theta}=0$ leads to the following identities:

$$
\left\{\begin{array}{l}
\dot{u}_{1}=\dot{u}_{2}=\frac{2 \bar{g} \sin (\bar{\theta})}{2 \cos (\bar{\theta}-\bar{\xi})+1} \\
\dot{v}_{1}=\dot{v}_{2}=0 \\
\dot{\omega}_{1}=-\frac{\dot{u}_{1}}{\bar{r}_{1}} \\
\dot{\omega}_{2}=-\frac{\dot{u}_{2}}{\bar{r}_{2}} \\
\frac{\bar{m}_{2}}{\bar{m}_{1}}=\frac{\sin (\bar{\theta})}{2 \sin (\bar{\xi}) \cos (\bar{\theta}-\bar{\xi})-3 \sin (\bar{\theta})+\sin (\bar{\xi})}-1
\end{array}\right.
$$

Thus, it can be noted that the stable value $\bar{\theta}$ of the angle $\theta$ between the line joining the centers of the ring and the disc and the vertical depends on the slope of the inclined plane, $\bar{\xi}$, and on the ratio between the masses of the disc and the ring, $\bar{m}_{2} / \bar{m}_{1}$.

Example 4.3. Consider the 5-bar mechanism (system $\mathscr{P}$ ) discussed in Example 2.7. Assume that the models of the subsystems which are the leaves in the tree structure are already known. The derivation of a model for the actuators $\mathscr{A}_{\mathrm{L}}$ and $\mathscr{A}_{\mathrm{R}}$, for instance, can be found in Craig (2005), corresponding to the 
following dynamic invariants:

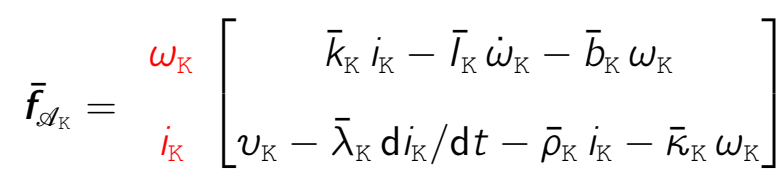

In these invariants the following constants show up:

- $\bar{k}_{\mathrm{K}}$ and $\bar{\kappa}_{\mathrm{K}}$ are respectively the motor torque constant and the back emf constant of the actuator $\mathscr{A}_{\mathrm{K}}$.

- $\bar{l}_{\mathrm{K}}$ and $\bar{b}_{\mathrm{K}}$ are the inertia and the viscous damping coefficient for the rotor of the actuator $\mathscr{A}_{\mathrm{K}}$.

- $\bar{\lambda}_{\mathrm{K}}$ and $\bar{\rho}_{\mathrm{K}}$ are the inductance of armature windings and the associated resistance of the actuator $\mathscr{A}_{\mathrm{K}}$.

Also, $v_{\mathrm{K}}$ denotes the voltage source of the actuator $\mathscr{A}_{\mathrm{K}}$, which is an input variable. Analogously, for the models of $\mathscr{U}_{\mathrm{L}}, \mathscr{U}_{\mathrm{R}}, \mathscr{B}_{\mathrm{L}}, \mathscr{B}_{\mathrm{R}}$ and $\mathscr{L}$ it can be stated that:

$$
\begin{aligned}
& \boldsymbol{f}_{\mathcal{U}_{\mathrm{K}}}=v_{v_{\mathfrak{U}_{\mathrm{K}}, \mathrm{Y}}}\left[\begin{array}{c}
-\bar{m}_{\mathfrak{U}_{\mathrm{K}}, \mathrm{x}} \dot{v}_{\mathfrak{U}_{\mathrm{K}}, \mathrm{x}} \\
\omega_{\mathcal{U}_{\mathrm{K}}, \mathrm{z}}
\end{array}\right]
\end{aligned}
$$

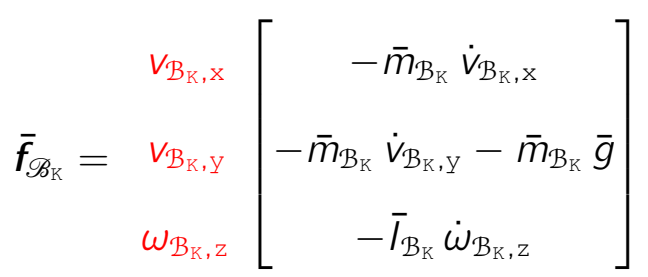

$$
\begin{aligned}
& \bar{f}_{\mathscr{L}}=\begin{array}{c}
\dot{x} \\
\dot{y}
\end{array}\left[\begin{array}{c}
-\bar{m}_{\mathcal{L}} \ddot{x} \\
-\bar{m}_{\mathcal{L}} \ddot{y}-\bar{m}_{\mathcal{L}} \bar{g}
\end{array}\right]
\end{aligned}
$$

with the $\bar{m}$ symbols denoting the masses of the respective rigid bodies, the $\bar{l}$ symbols their moment of inertia with respect to their own centres of mass and with $\bar{g}$ denoting the magnitude of the local acceleration of gravity.

From the application of the modular modeling algorithm, it can be stated that the dynamic equations of motion for subsystems $\mathscr{H}_{\mathrm{L}}$ and $\mathscr{H}_{\mathrm{R}}$ are given by 
the following invariants:

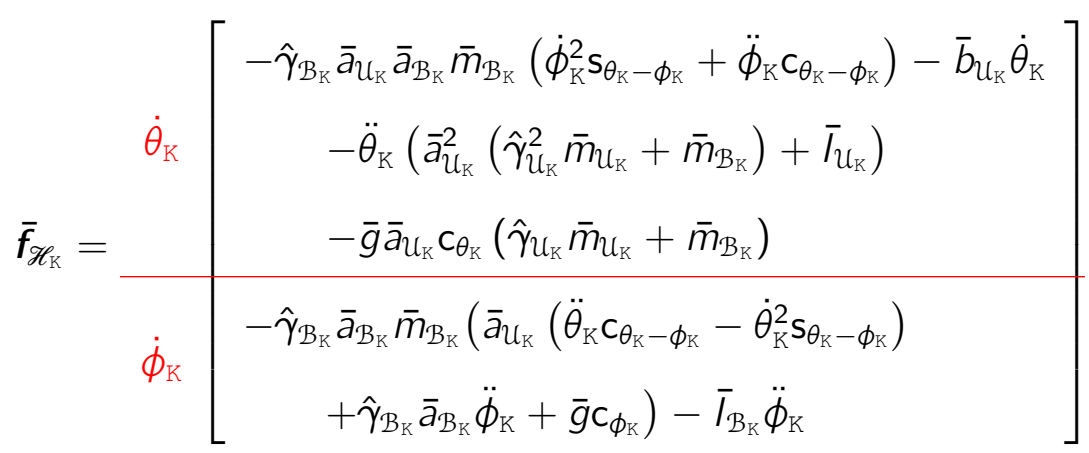

Applying the recursive algorithm two other times, the models of $\mathscr{R}$ and $\mathscr{P}$ are obtained. Particularly, for the latter one, it can be stated that:

$$
\bar{f}_{\mathscr{P}}=\begin{gathered}
i_{\mathrm{I}} \\
i_{\mathrm{R}} \\
\dot{\theta}_{\mathrm{L}} \\
\dot{\theta}_{\mathrm{R}}
\end{gathered}\left[\begin{array}{c}
\bar{f}_{\mathscr{P} . i_{\mathrm{L}}} \\
\bar{f}_{\mathscr{P} . i_{\mathrm{R}}} \\
\bar{f}_{\mathscr{P} . \dot{\theta}_{\mathrm{L}}} \\
\bar{f}_{\mathscr{P} . \dot{\theta}_{\mathrm{R}}}
\end{array}\right]=0
$$

with the expressions for the $\bar{f}_{\mathscr{P} . i_{x}}$ and the $\bar{f}_{\mathscr{P} . \dot{\theta}_{x}}$ given by (consider that when $\mathrm{X}=\mathrm{L}$ then $\mathrm{Y}=\mathrm{R}$ and vice versa):

$$
\begin{aligned}
& \bar{f}_{\mathscr{P} . i_{\mathrm{X}}}=v_{\mathrm{X}}-\bar{\lambda}_{\mathrm{X}} \mathrm{d} i_{\mathrm{X}} / \mathrm{d} t-\bar{\rho}_{\mathrm{X}} i_{\mathrm{X}}-\bar{\kappa}_{\mathrm{X}} \bar{\eta}_{\mathrm{X}} \dot{\theta}_{\mathrm{X}} \\
& \bar{f}_{\mathscr{P} . \dot{\theta}_{X}}=\frac{\hat{\gamma}_{\mathcal{B}_{Y}} \bar{a}_{\mathcal{U}_{Y}} \bar{a}_{\mathcal{U}_{X}} \bar{m}_{\mathcal{B}_{Y}} \dot{\theta}_{Y}^{2} s_{\theta_{Y}-\phi_{Y}} s_{\theta_{X}-\phi_{X}}}{\mathrm{~s}_{\phi_{Y}-\phi_{X}}}+\frac{\hat{\gamma}_{\mathcal{B}_{X}} \bar{a}_{\mathcal{U}_{X}}^{2} \bar{m}_{\mathcal{B}_{X}} \dot{\theta}_{X}^{2} \mathrm{~s}_{\theta_{X}-\phi_{X}} \mathrm{~s}_{\theta_{X}-\phi_{Y}}}{\mathrm{~s}_{\phi_{Y}}-\phi_{X}}
\end{aligned}
$$

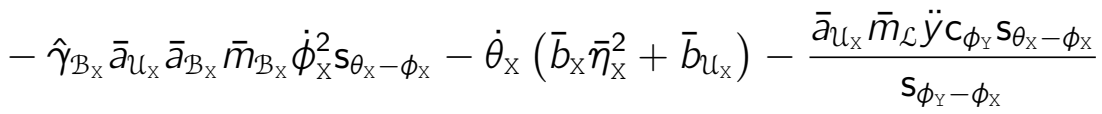

$$
\begin{aligned}
& -\frac{\hat{\gamma}_{\mathcal{B}_{Y}} \bar{a}_{\mathcal{U}_{Y}} \bar{a}_{\mathcal{U}_{X}} \bar{m}_{\mathcal{B}_{Y}} \ddot{\theta}_{Y} c_{\theta_{Y}-\phi_{Y}} s_{\theta_{X}-\phi_{X}}}{s_{\phi_{Y}-\phi_{X}}}+\frac{\bar{a}_{U_{X}} \bar{m}_{\mathrm{L}} \ddot{x} s_{\phi_{Y}} s_{\theta_{X}-\phi_{X}}}{\mathrm{~s}_{\phi_{Y}-\phi_{X}}}
\end{aligned}
$$

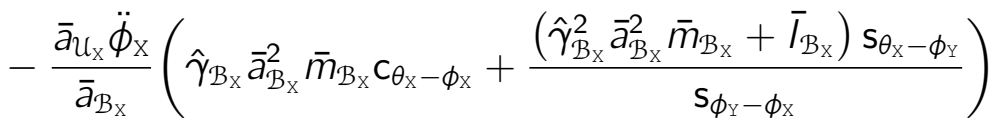

$$
\begin{aligned}
& +\frac{1}{2} \ddot{\theta}_{\mathrm{X}}\left(\bar{a}_{\mathfrak{U}_{\mathrm{X}}}^{2}\left(\bar{m}_{\mathcal{B}_{\mathrm{X}}}\left(\hat{\gamma}_{\mathcal{B}_{\mathrm{X}}}\left(1-\frac{\mathrm{s}_{-\phi_{\mathrm{Y}}+2 \theta_{\mathrm{X}}-\phi_{\mathrm{X}}}}{\mathrm{s}_{\phi_{\mathrm{Y}}-\phi_{\mathrm{X}}}}\right)-2\right)-2 \hat{\gamma}_{\mathfrak{U}_{\mathrm{x}}}^{2} \bar{m}_{\mathfrak{U}_{\mathrm{X}}}\right)\right. \\
& \left.-2\left(\bar{I}_{\mathrm{X}} \bar{\eta}_{\mathrm{X}}^{2}+\bar{l}_{\mathcal{U}_{\mathrm{X}}}\right)\right)-\frac{\bar{a}_{\mathcal{U}_{\mathrm{X}}} \ddot{\phi}_{\mathrm{Y}} \mathrm{s}_{\theta_{\mathrm{X}}-\phi_{\mathrm{X}}}\left(\hat{\gamma}_{\mathcal{B}_{\mathrm{Y}}}^{2} \bar{a}_{\mathcal{B}_{Y}}^{2} \bar{m}_{\mathcal{B}_{\mathrm{Y}}}+\bar{I}_{\mathcal{B}_{\mathrm{Y}}}\right)}{\bar{a}_{\mathcal{B}_{\mathrm{Y}}} \mathrm{s}_{\phi_{\mathrm{Y}}-\phi_{\mathrm{X}}}}+\bar{k}_{\mathrm{X}} \bar{\eta}_{\mathrm{X}} i_{\mathrm{X}} \\
& -\bar{g} \bar{a}_{\mathfrak{u}_{\mathrm{x}}}\left(\frac{\hat{\gamma}_{\mathcal{B}_{Y}} \bar{m}_{\mathcal{B}_{Y}} c_{\phi_{Y}} \mathrm{~s}_{\theta_{\mathrm{X}}-\phi_{\mathrm{X}}}}{\mathrm{s}_{\phi_{\mathrm{Y}}-\phi_{\mathrm{X}}}}+\bar{m}_{\mathcal{B}_{\mathrm{X}}}\left(\frac{\hat{\gamma}_{\mathcal{B}_{\mathrm{X}}} \mathrm{c}_{\phi_{\mathrm{X}}} \mathrm{s}_{\theta_{\mathrm{X}}-\phi_{\mathrm{Y}}}}{\mathrm{s}_{\phi_{\mathrm{Y}}-\phi_{\mathrm{X}}}}+\mathrm{c}_{\theta_{\mathrm{X}}}\right)+\hat{\gamma}_{\mathfrak{U}_{\mathrm{x}}} \bar{m}_{\mathfrak{U}_{\mathrm{X}}} \mathrm{c}_{\theta_{\mathrm{X}}}\right)
\end{aligned}
$$

This example illustrates the use of the recursive modular modeling algorithm to derive the equations of motion of a closed loop kinematic chain system by exploring the existing symmetries, taking as a starting point the models of its basal subsystems, which are much less complex. 


\subsection{Sufficient conditions for the forward dynam- ics}

This section establishes a theorem providing sufficient conditions for existence and uniqueness of solutions for a system of equations of motion of a multibody system derived according to the modular modeling algorithm presented in the previous section. Basically, the sufficient conditions listed must guarantee the application of Picard's Existence Theorem to the associated system of ordinary differential equations (ODEs).

Let $\mathscr{M}_{n}$ be a $\nu_{\mathscr{M}_{n}}^{\#}$-DOF multibody system whose constraint order is equal to $\nu_{\mathscr{M}_{n}}^{\circ}$. Adopt an integer $\sigma$ such that $\sigma \geq \nu_{\mathscr{M}_{n}}^{\circ}$ and that no equation of motion of $\mathscr{M}$ depend on any generalized variable above $\sigma$-th order. It can be stated that the system of differential-algebraic equations (DAEs) of motion of $\mathscr{M}_{n}$ can be written as follows:

$$
\left\{\begin{array}{l}
\dot{\boldsymbol{q}}_{\mathscr{M}_{n}}^{\langle\langle\beta}=\dot{\boldsymbol{q}}_{\mathscr{M}_{n}}^{\langle\langle\beta}\left(t, \boldsymbol{q}_{\mathscr{M}_{n}}^{\langle\langle\beta+1\rangle\rangle}\right) \quad \text { for } \quad 0 \leq \beta \leq(\sigma-1) \\
\overline{\boldsymbol{q}}_{\mathscr{M}_{n}}^{\langle\sigma\rangle}=\boldsymbol{A}_{\mathscr{M}_{n}} \boldsymbol{q}_{\mathscr{M}_{n}}^{\langle\sigma\rangle}+\boldsymbol{b}_{\mathscr{M}_{n}}^{\langle\sigma-1\rangle}=0 \\
\overline{\boldsymbol{f}}_{\mathscr{M}_{n}}=C_{\mathscr{M}_{n}}^{\top} \boldsymbol{f}_{\mathscr{M}_{n}}=0
\end{array}\right.
$$

The necessary initial conditions at a time instant $t^{*}$ to find a solution for the system of equations of motion (4.13) are the values of $\boldsymbol{q}_{\mathscr{M}_{n}}^{\langle\langle\sigma-1\rangle}\left(t^{*}\right)$. These values, however, must be compatible with all the the constraints of the system, i.e. if some constraints of $\mathscr{M}_{n}$ are associated to invariants that can be expressed as functions of generalized variables up to $(\sigma-1)$-th order, the values $\boldsymbol{q}_{\mathscr{M}_{n}}^{\langle\sigma \sigma-1\rangle}\left(t^{*}\right)$ must identically satisfy these invariants at instant $t^{*}$. Moreover, the values $\boldsymbol{q}_{\mathscr{M}_{n}}^{\langle\sigma-1\rangle}\left(t^{*}\right)$ must not lead to any algebraic singularity in the equations of motion (like, for instance, a zero denominator).

Theorem 4.1 (Existence and uniqueness of solutions of equations of motion of a multibody system). Let $\mathscr{M}_{n}$ be a $\nu_{\mathscr{M}_{n}}^{\#}$-DOF multibody system whose constraint order is equal to $\nu_{\mathscr{M}_{n}}^{\circ}$. Adopt an integer $\sigma \geq 2$ according to the criteria listed above. Assume that the corresponding initial conditions satisfy the conditions previously mentioned. Suppose also that:

- The invariants $\overline{\boldsymbol{c}}_{\mathscr{M}_{n}}^{\langle\beta+1\rangle}\left(t, \boldsymbol{q}_{\mathscr{M}_{n}}^{\langle\beta+1\rangle}, \dot{\boldsymbol{q}}_{\mathscr{M}_{n}}^{\langle\beta\rangle}\right)=0$ that define the transformations of variables between $\dot{\boldsymbol{q}}_{\mathscr{M}_{n}}^{\langle\beta\rangle}$ and $\boldsymbol{q}_{\mathscr{M}_{n}}^{\langle\beta+1\rangle}, 0 \leq \beta \leq(\sigma-1)$, are class $\mathscr{C}^{2}$ functions.

- $\boldsymbol{A}_{\mathscr{M}_{n}}, \boldsymbol{b}_{\mathscr{M}_{n}}^{\langle\sigma-1\rangle}$ can be expressed as functions of $\left(t, \boldsymbol{q}_{\mathscr{M}_{n}}^{\langle\langle\sigma-1\rangle)}\right.$ that are continuous with respect to time $t$ in some interval in the neighborhood of $t^{*}$ 
and satisfy Lipschitz condition for every $\boldsymbol{q}_{\mathcal{M n}_{n}}^{\langle\sigma-1\rangle}$ in some neighborhood of $\boldsymbol{q}_{\mathscr{M}_{n}}^{\langle\sigma \sigma-1\rangle}\left(t^{*}\right)$.

- $\boldsymbol{A}_{\mathscr{U}_{n}}$ is a full rank matrix for the given values of $\boldsymbol{q}_{\mathscr{U}_{n}}^{\langle\sigma-1\rangle}\left(t^{*}\right)$.

- Any unconstrained generalized effective force associated to $\mathscr{M}_{n}, f_{\mathscr{U}_{n}}$, can be expressed as functions of $\left(t, \boldsymbol{q}_{\mathcal{M}_{n}}^{\langle\langle\rangle}\right)$which must be affine with respect to $\boldsymbol{q}_{\mathcal{M}_{n}}^{\langle\sigma\rangle}$, continuous with respect to time $t$ in some interval in the neighborhood of $t^{*}$ and must satisfy Lipschitz condition for every $\boldsymbol{q}_{\mathcal{M}_{n}}^{\langle\sigma-1\rangle}$ in some neighborhood of $\boldsymbol{q}_{\mathcal{M}_{n}}^{\| \sigma-1\rangle}\left(t^{*}\right)$.

In this case, it can be stated that the system of differential-algebraic equations (4.13) has a unique solution in some open interval around $t^{*}$ that satisfy simultaneously all the constraint invariants and dynamic invariants of $\mathscr{M}_{n}$.

Proof. First of all, due to the hypotheses of this theorem, it can be stated that:

- According to Lemma 2.1 of Orsino and Hess-Coelho (2015), all the $\underline{\dot{q}}_{\mathcal{M}_{n}}^{\langle\beta\rangle}\left(t, \boldsymbol{q}_{\mathscr{M}_{n}}^{\langle\langle\beta+1\rangle}\right)$ are class $\mathscr{C}^{1}$ functions, for $0 \leq \beta \leq(\sigma-1)$.

- According to Theorems 2.8 and 2.10, it can be stated that $C_{\mathscr{M}_{n}}$ must be a continuous function with respect to time $t$ in some open interval around $t^{*}$ and must satisfy the Lipschitz condition for every $\boldsymbol{q}_{\mathscr{M}_{n}} \| \sigma{ }$ in some neighborhood of $\boldsymbol{q}_{\mathscr{M}_{n}}^{\left\langle\left\langle t^{*}\right)\right.}$.

Thus, suppose that it is possible to isolate algebraically $\boldsymbol{q}_{\mathcal{M}_{n}}^{\langle\sigma\rangle}$ in the system of equations (4.13), obtaining for such variables explicit and non-singular expressions in some neighborhood of the initial conditions $\left(t^{*}, \boldsymbol{q}_{\mathcal{M}_{n}}^{\| \sigma-1\rangle}\left(t^{*}\right)\right)$. Due to the previous two remarks it is possible to apply Picard's Existence Theorem (SOTOMAYOR-TELLO, 2011) to the ordinary differential equations in (4.13) and it can be stated that there is a unique solution defined in some open interval around $t^{*}$ that satisfy all constraint and dynamic invariants of $\mathscr{M}_{n}$.

Thus, the remainder of this proof will show that, if a system is in a non-singular generalized configuration, then there is a neighborhood of $\left(t^{*}, \boldsymbol{q}_{\mathcal{M}_{n}}^{\langle\sigma-1\rangle}\left(t^{*}\right)\right)$ for which it is possible to obtain explicit and non-singular expressions for the $\boldsymbol{q}_{\mathcal{M}_{n}}^{\langle\sigma\rangle}$ in the system of equations (4.13).

Consider that the general solution of $\boldsymbol{A}_{\mathscr{M}_{n}} \boldsymbol{q}_{\mathscr{M}_{n}}^{\langle\sigma\rangle}+\boldsymbol{b}_{\mathscr{M}_{n}}^{\langle\sigma-1\rangle}=0$ must have the following form:

$$
\boldsymbol{q}_{\mathscr{M}_{n}}^{\langle\sigma\rangle}=S_{\mathscr{M}_{n}} z_{\mathscr{U}_{n}}^{\langle\sigma\rangle}+e_{\mathscr{M}_{n}}^{\langle\sigma-1\rangle}=R_{\mathscr{M}_{n}} C_{\mathscr{M}_{n}} z_{\mathscr{M}_{n}}^{\langle\sigma\rangle}+e_{\mathscr{M}_{n}}^{\langle\sigma-1\rangle}
$$


Also, once it is possible to derive an expression for $f_{\mathcal{M}_{n}}$ from the algorithm presented in Section 4.2, it can be assumed that there is a positive-definite matrix $M_{\mathscr{U}_{n}}$ a matrix $V_{\mathscr{U}_{n}}$ and a column-matrix $g_{\mathscr{U}_{n}}^{\langle\sigma-1\rangle}$ such that:

$$
\boldsymbol{f}_{\mathscr{U}_{n}}=-\boldsymbol{R}_{\mathscr{M}_{n}}^{\top} \boldsymbol{V}_{\mathscr{M}_{n}}^{\top} \boldsymbol{M}_{\mathscr{M}_{n}} \boldsymbol{V}_{\mathscr{U}_{n}} \boldsymbol{q}_{\mathscr{M}_{n}}^{\langle\sigma\rangle}+\boldsymbol{g}_{\mathscr{M}_{n}}^{\langle\sigma-1\rangle}
$$

Indeed, consider for instance a multi-rigid-body system for which it can be adopted $\sigma=2$. Once the expression for the effective forces in this system can be obtained using the expressions (A.40 - A.45), from Section A.3 of Appendix $A$, it can be stated that:

$$
\frac{\partial \boldsymbol{f}_{\mathscr{U}_{n}}}{\partial \dot{\boldsymbol{q}}_{\mathscr{M}_{n}}^{(2\rangle}}=-\sum_{r}\left(\mathbf{V}_{r}^{\star} \cdot\left(m_{r} \mathbf{V}_{r}^{\star}\right)+\boldsymbol{\Omega}_{r} \cdot\left(\mathbf{I}_{r}^{\star} \cdot \boldsymbol{\Omega}_{r}\right)\right)
$$

In this case $V_{\mathscr{U}_{n}}$ is a block diagonal matrix in which each block correspond to the matrix representation of a second order tensor among the $\mathrm{V}_{r}^{\star}$ and $\Omega_{r}^{\star}$. Moreover, $M_{\mathscr{M}_{n}}$ is also block diagonal, being each block given by:

$$
\left[\begin{array}{cc}
m_{r} 1 & 0 \\
0 & {\left[\mathbf{I}_{r}^{ \pm}\right]_{E_{r} \mid E_{r}}}
\end{array}\right]
$$

with $\left[\mathbf{I}_{r}^{\star}\right]_{\mathrm{E}_{r} \mid \mathrm{E}_{r}}$ being the matrix representation of the inertia tensor $\mathbf{I}_{r}^{\star}$ in a particular coordinate system $\mathrm{E}_{r}$. Once each inertia tensor must be symmetric and positive-definite, so it must be $M_{\mathscr{M}_{n}}$. Finally, once this multi-rigid-body system does not have any subsystem, then $R_{\mathscr{U}_{n}}=1$. Thus, the expression for the column-matrix of unconstrained generalized effective forces associated to this system can be expressed by equation (b).

Adopting $W_{\mathscr{M}_{n}}=V_{\mathscr{M}_{n}} S_{\mathscr{M}_{n}}=V_{\mathscr{M}_{n}} R_{\mathscr{M}_{n}} C_{\mathscr{M}_{n}}$ and using equations (a) and (b), it can be stated that:

$$
\bar{f}_{\mathscr{M}_{n}}=-W_{\mathscr{M}_{n}}^{\top} M_{\mathscr{M}_{n}} W_{\mathscr{M}_{n}} z_{\mathscr{M}_{n}}^{\langle\sigma\rangle}+\boldsymbol{h}_{\mathscr{M}_{n}}^{\langle\sigma-1\rangle}
$$

Theorem 2.8 proves that $W_{\mathscr{M}_{n}}$ is a rank $\nu_{\mathscr{M}_{n}}^{\#}$ matrix. Indeed, if the rank of this matrix was less than $\nu_{\mathscr{M}_{n}}^{\#}$, it would be possible to express arbitrary variations of a sufficient set of motion variables ${ }^{3}$ in a time instant immediately after $t^{*}$ as a function of less than $\nu_{\mathscr{M}_{n}}^{\#}$ independent parameters, which contradicts either the initial assumption that $\mathscr{U}_{n}$ is a $\nu_{\mathscr{U}_{n}}^{\#}$-DOF system or the fact that the system is not in a singular generalized configuration at $t^{*}$.

\footnotetext{
${ }^{3}$ That is, a set of variables that is sufficient to describe the instantaneous kinematics of any possible motion of the system up to some order, provided that information up to the previous order is already known, as discussed in Section 4.1.
} 
Once $M_{\mathscr{M}_{n}}$ is symmetric and positive-definite and $W_{\mathscr{M}_{n}}$ is a full rank matrix, it can be shown that $W_{\mathscr{M}_{n}}^{\top} M_{\mathscr{M}_{n}} W_{\mathscr{M}_{n}}$ is invertible. Indeed, let $z \in \mathbb{R}^{\nu_{\mathscr{M}_{n}}^{*}}$ and assume that $z \neq 0$. Once $W_{\mathscr{M}_{n}}$ is full rank, then it can be stated that $W_{\mathscr{M}_{n}} z \neq 0$ and:

$$
z^{\top} W_{\mathscr{M}_{n}}^{\top} M_{\mathscr{M}_{n}} W_{\mathscr{M}_{n}} z=\left(W_{\mathscr{M}_{n}} z\right)^{\top} M_{\mathscr{M}_{n}}\left(W_{\mathscr{M}_{n}} z\right)>0
$$

This proves that $W_{\mathscr{M}_{n}}^{\top} M_{\mathscr{M}_{n}} W_{\mathscr{M}_{n}}$ is also positive-definite and, therefore, invertible. Finally, it can be stated that:

$$
z_{\mathscr{M}_{n}}^{\langle\sigma\rangle}=\left(W_{\mathscr{M}_{n}}^{\top} M_{\mathscr{M}_{n}} W_{\mathscr{M}_{n}}\right)^{-1} \boldsymbol{h}_{\mathscr{M}_{n}}^{\langle\sigma-1\rangle}
$$

The replacement of this last equation in (a) concludes the proof.

It is worth doing the following remarks about the result presented in Theorem 4.1:

- The numerical simulation of the system of equations (4.13) given compatible initial conditions and providing explicit expressions for the constitutive equations, allowing to express all effective forces acting in a system as functions of time and of the generalized variables of the model, constitutes the solution of a forward dynamics problem associated to this system.

- The maximal solutions of the system of equations (4.13) will not be defined for an infinite time interval whenever the generalized configuration of the system indefinitely approaches a singularity.

- Throughout the proof of the theorem, it is shown that it is possible to obtain from (4.13) a system of explicit ordinary differential equations whenever the system is not in a singular configuration. These transformations are done exclusively to be able to apply the conventional statement of Picard's Existence Theorem and might not necessarily relate to the integration algorithms applied in an actual numerical simulation.

- The assumption that $M_{\mathscr{M}_{n}}$ is a positive-definite matrix will only be assuredly true when conventional non-degenerate mechanical systems are modeled (hypothesis concerning some negligible inertia parameter might lead to degenerate models, for instance). There are cases in which the statement of this theorem is applicable even when $M_{\mathscr{M}_{n}}$ is not a positivedefinite matrix. The proof for these cases, however, is out of the scope of this text. 
Example 4.4. Back to the system $\mathcal{S}$ of Example 2.5 (Section 2.4), consider that the equation of surface $\mathcal{U}$ is defined by $\gamma(x, y, z)=0$. Let $\mathrm{b}$ be a point in the surface of $\mathcal{S}$, whose local coordinates are given by $[\mathrm{b}]_{\mathrm{S}}=(0,0,-\bar{r})$ and consider that there is a point electric charge of magnitude $\bar{\kappa}$ that remains fixed with respect to the sphere and has negligible inertia. Consider that the local acceleration of gravity is given by $\overline{\mathrm{g}}=-\bar{g} \hat{\mathbf{z}}$ and that there is a local electric field $\mathbf{e}=e \hat{\mathbf{x}}$. Denote by $\bar{m}$ the mass of the sphere $\mathcal{S}$ and assume that it has an homogeneous mass distribution such that the moment of inertia about any axis passing through its center is equal to $\bar{J}$. For this system it can be stated that:

$$
\boldsymbol{f}_{\mathcal{S}}=\boldsymbol{f}_{\mathcal{S}}^{\prime}+\left(\boldsymbol{V}^{\langle 0,1\rangle}\right)^{\top} \boldsymbol{f}_{\mathcal{S}}^{\prime \prime}
$$

with $V^{\langle 0,1\rangle}$ denoting the matrix given by equation (i) of Example 2.5 and with $\boldsymbol{f}_{\mathcal{S}}^{\prime}$ and $f_{\delta}^{\prime \prime}$ defined as follows:

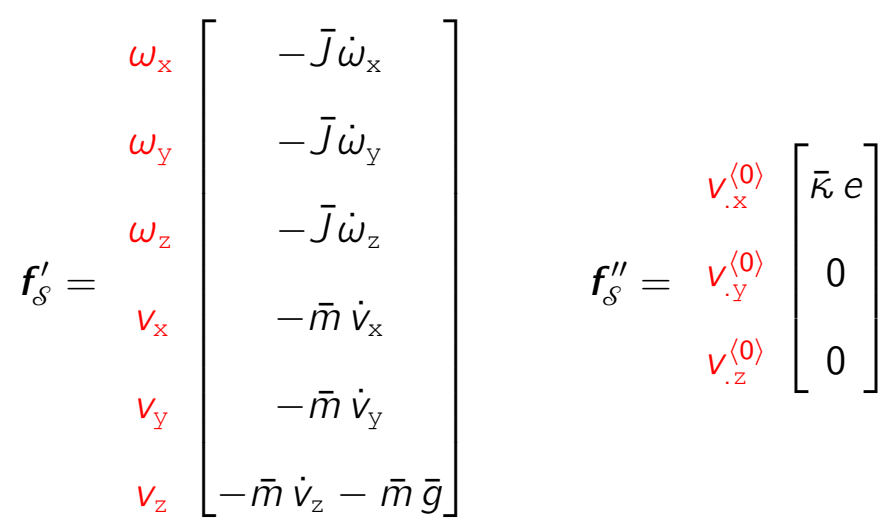

Once $\delta$ has no subsystems it can be stated that $C_{\mathcal{S}}=S_{\mathcal{S}}$, with $S_{\mathcal{\delta}}$ given by equation equation (g) of Example 2.5, for example. Thus, $\bar{f}_{\mathcal{\delta}}=C_{\mathcal{S}}^{\top} f_{\mathcal{S}}=0$, i.e.:

$$
\left\{\begin{array}{l}
\bar{r} \gamma_{\mathrm{y}}\left(-\bar{m} \dot{v}_{\mathrm{z}}-\bar{m} \bar{g}\right)-\bar{J} \dot{\omega}_{\mathrm{x}}+\bar{m} \bar{r} \gamma_{\mathrm{z}} \dot{v}_{\mathrm{y}}=0 \\
\bar{r} \gamma_{\mathrm{z}}\left(e \bar{\kappa}-\bar{m} \dot{v}_{\mathrm{x}}\right)+\bar{r} \gamma_{\mathrm{x}}\left(\bar{m} \dot{v}_{\mathrm{z}}+\bar{m} \bar{g}\right)-\bar{J} \dot{\omega}_{\mathrm{y}}+e \bar{\kappa} \bar{r}\left(2 q_{\mathrm{x}}^{2}+2 q_{\mathrm{y}}^{2}-1\right)=0 \\
-\bar{r} \gamma_{\mathrm{y}}\left(e \bar{\kappa}-\bar{m} \dot{v}_{\mathrm{x}}\right)-\bar{J} \dot{\omega}_{\mathrm{z}}-\bar{m} \bar{r} \gamma_{\mathrm{x}} \dot{v}_{\mathrm{y}}+e \bar{\kappa} \bar{r}\left(2 q_{\mathrm{y}} q_{\mathrm{z}}-2 q_{\mathrm{t}} q_{\mathrm{x}}\right)=0
\end{array}\right.
$$

Equations (c) along with equations $(\mathrm{d}, \mathrm{f})$ from example 2.5, constitute the system of differential-algebraic equations of motion for system $\delta$.

In order to illustrate the dynamic behavior of this system, consider the following values for the physical parameters of $\delta$ :

$$
\begin{array}{lll}
\bar{r}=0.01 \mathrm{~m} & \bar{g}=9.8 \mathrm{~m} / \mathrm{s}^{2} & \bar{m}=0.05 \mathrm{~kg} \\
\bar{J}=\frac{2}{5} \bar{m} \bar{r}^{2} & e=\left(1 \times 10^{4} \mathrm{~N} / \mathrm{C}\right) \sin \left(\frac{1}{2} \pi t\right) & \bar{\kappa}=5 \times 10^{-6} \mathrm{C}
\end{array}
$$


Also, let $\underline{\gamma}(x, y, z)=z-\frac{1}{2} y^{2}=0$ and assume that the initial conditions are given by:

$$
\begin{array}{lll}
x(0)=0 & y(0)=0.1 \mathrm{~m} & z(0)=0.005 \mathrm{~m} \\
q_{1}(0)=q_{2}(0)=q_{3}(0)=0 & q_{4}(0)=1 & \\
v_{\mathrm{x}}(0)=v_{\mathrm{y}}(0)=v_{\mathrm{z}}(0)=0 & \omega_{\mathrm{x}}(0)=\omega_{\mathrm{y}}(0)=\omega_{\mathrm{z}}(0)=0 &
\end{array}
$$

Some of the results of the numerical simulation performed in Wolfram Mathematica 10.2 for the first $50 \mathrm{~s}$ of motion are shown in Figures $4.2-4.5$.

The most interesting result to be noticed is due to the effect of the no-slip constraint in the time history of coordinate $x$. The electric force is the only active force acting in the direction $\hat{x}$ and, even though the electric field is oscillating harmonically with a period of $4 \mathrm{~s}$, the translation motion in this direction is not. Another remarkable result is that, due to the geometry of surface $U$ (parabolic trough) the coordinate $z$ oscillates at twice the frequency of coordinate $y$.

Finally, it is worth mentioning that, in this example, one way to control the quality of the results obtained in the numerical simulation is by inspecting the norm of the quaternion (that must be unitary) as shown in Figure 4.5.

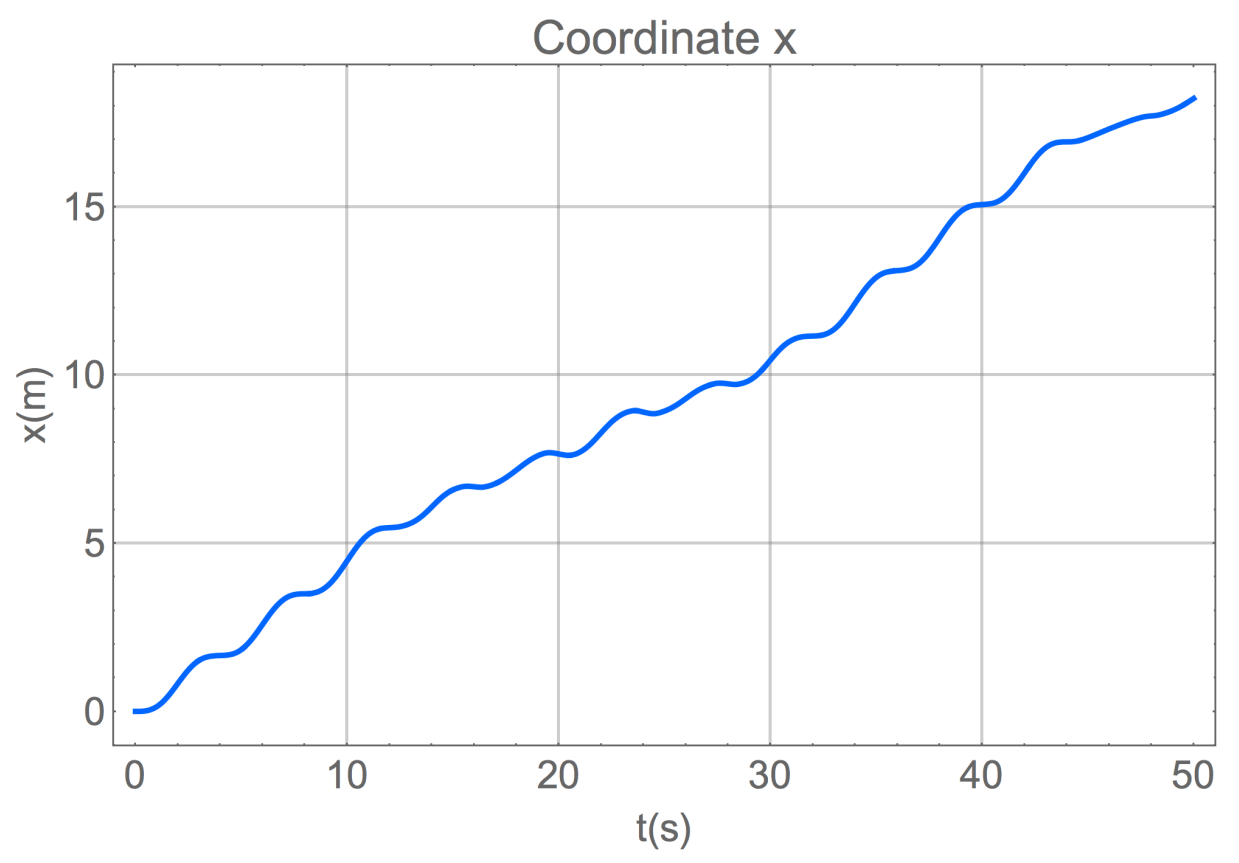

Figure 4.2: Time history of the coordinate $x$ of the contact point of the sphere $\mathcal{S}$ with the surface $\mathcal{U}$. 


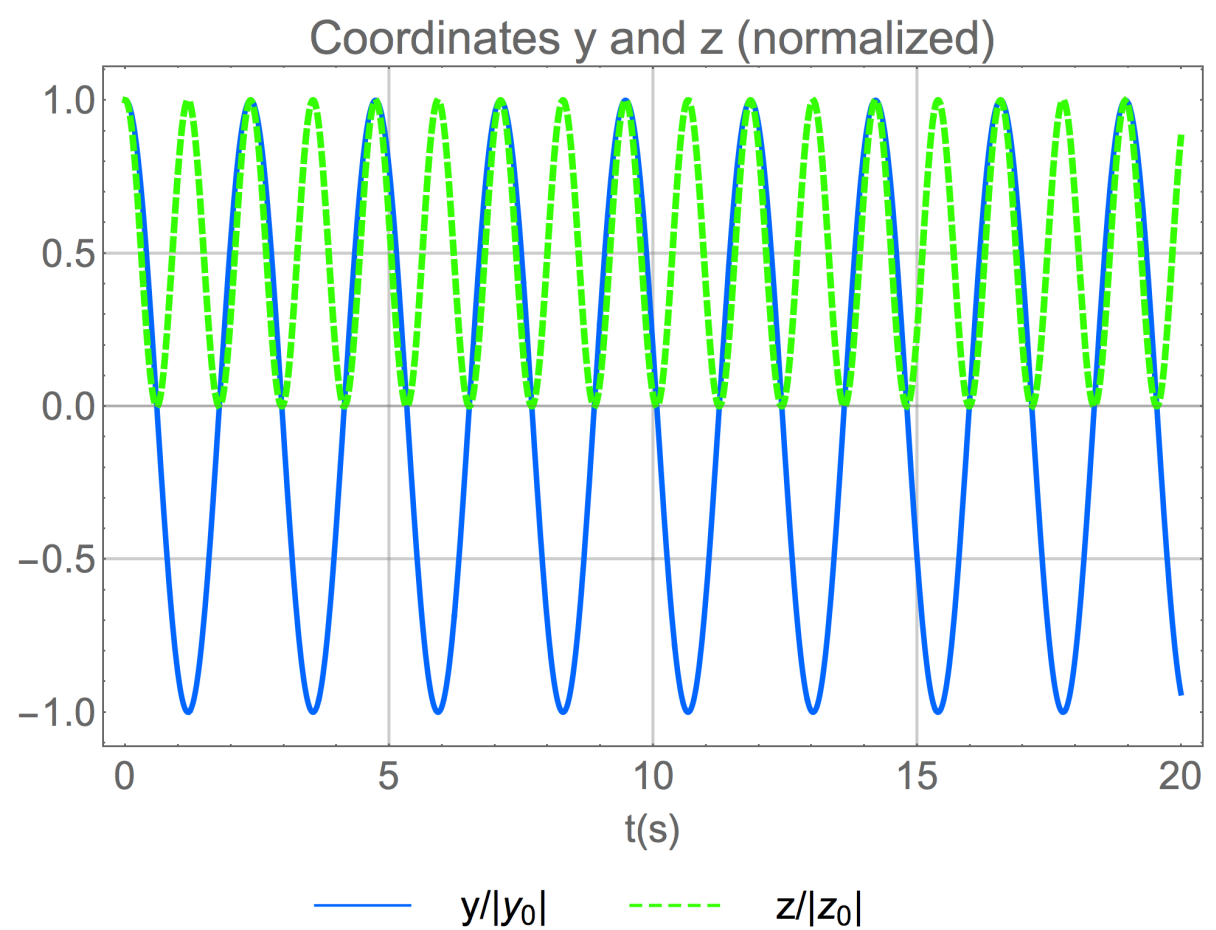

Figure 4.3: Normalized time histories of the coordinate $x$ of the contact point of the sphere $\mathcal{S}$ with the surface $\mathcal{U}$.
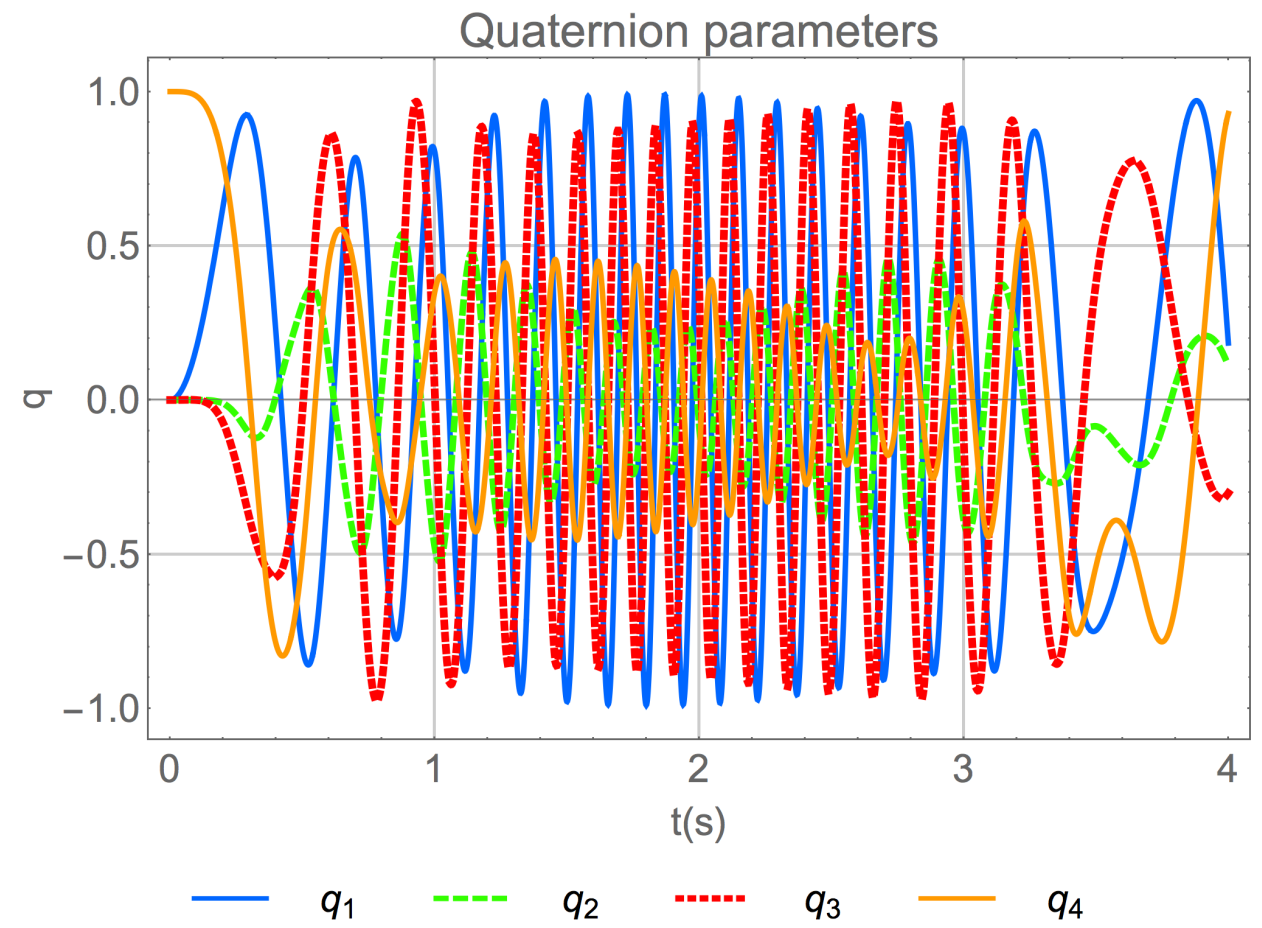

Figure 4.4: Time history of the quaternion parameters defining the orientation of the sphere $\mathcal{S}$. 


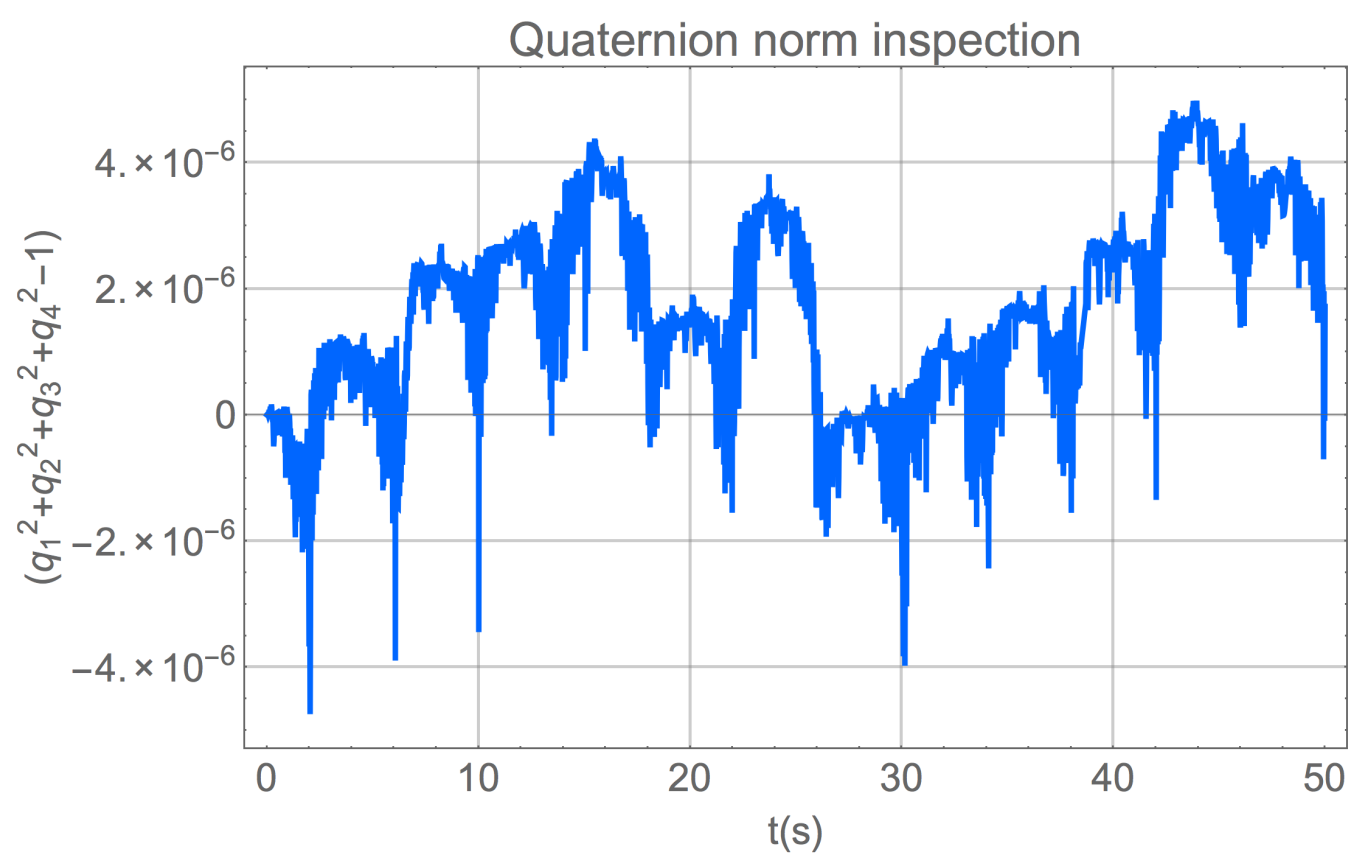

Figure 4.5: Time history of the quaternion norm inspection.

\subsection{Sufficient conditions for the inverse dynam- ics}

In an inverse dynamics problem, it is considered that some of the forces acting in a multibody system $\mathscr{M}$ can be expressed as functions of time, of the generalized variables of the model of $\mathscr{M}$ and of a finite number of input parameters. In this text, the tuple (or column-matrix) constituted by the the inputs of system $\mathscr{M}$ is denoted by $\boldsymbol{u}_{\mathscr{U}}$.

The inputs of $\mathscr{M}$ constitute a set of variables that can somehow be manipulated in order to try to modify its "natural" response. Let $\nu^{\circ}$ denote the constraint order of $\mathscr{M}$, choose an integer $\sigma \geq \nu^{\circ}$ such that in a given time instant $t^{*}, \boldsymbol{q}^{\langle\sigma \sigma-1\rangle}\left(t^{*}\right)$ is known and assume that $\delta \boldsymbol{q}^{\langle\sigma-1\rangle}\left(t^{*}\right)=0$. Denote by $\boldsymbol{q}_{\mathcal{M} . u}^{\langle\sigma\rangle}$ a subtuple (or the corresponding column-matrix) constituted by some of the $\sigma$-th order generalized variables of the model of $\mathscr{M}$. These generalized variables are said to be associated with the inputs $u_{\mathscr{M}}$ if and only if the virtual work associated to these inputs at a time instant $\left(t^{*}+\varepsilon\right)$, with $\varepsilon$ denoting an infinitesimal, is proportional to $\left(\delta \boldsymbol{q}_{\mathscr{M} . u}^{\langle\sigma\rangle} \cdot \boldsymbol{u}_{\mathscr{U}}\right)$.

For instance, consider a multi-rigid-body system whose constraint order is 
equal to $\nu^{\circ}=1$. Adopting $\sigma=1$ it can be stated that:

- If $u_{\mathscr{M} . k}$ represents a component of force, the associated quasi-velocity $q_{M . M . k}^{\langle 1\rangle}$ must represent the corresponding component (i.e. the component about the same axis) of the velocity of the point of application of this force (measured with respect to an inertial reference frame).

- If $u_{\mathscr{M} . k}$ represents a component of torque, the associated quasi-velocity $q_{M l k . k}^{\langle 1\rangle}$ must represent the corresponding component (i.e. the component about the same axis) of the angular velocity of the rigid body to which this torque is applied (measured with respect to an inertial reference frame).

Proposition 4.2. Let $\boldsymbol{q}_{\mathcal{M} \text {.u }}^{\langle\sigma\rangle}$ be a $\sigma$-th order generalized variable from the model of $\mathscr{M}$ associated to the input $u_{\mathscr{M}}$. It can be stated that the unconstrained generalized effective forces associated to $\boldsymbol{q}_{\mu . u}^{\langle\sigma\rangle}$ can be expressed as follows:

$$
\boldsymbol{f}_{\mathscr{M} . u}=\boldsymbol{u}_{\mathscr{M}}+\underline{\boldsymbol{f}}_{\mathscr{M} . u}^{*}\left(t, \boldsymbol{q}_{\mathscr{M}}^{\langle\sigma\rangle}\right)
$$

Proof. Considering that the variables $\boldsymbol{q}_{\mathscr{M} . u}^{\langle\sigma\rangle}$ are associated to the inputs $u_{\mathscr{M}}$, it can be stated that the $\sigma$-th order variational principle for system $\mathscr{M}$, can be expressed as follows:

$$
\delta \boldsymbol{v}_{\mathscr{M}}^{\langle\sigma\rangle} \cdot \underline{\boldsymbol{f}}_{\mathscr{M}}^{\prime}\left(t, \boldsymbol{q}_{\mathscr{M}}^{\langle\sigma\rangle}\right)+\delta \boldsymbol{q}_{\mathscr{M} . u}^{\langle\sigma\rangle} \cdot \boldsymbol{u}_{\mathscr{M}} \leq 0
$$

Note that it is possible to rewrite the previous inequality as follows:

$$
\left(\delta \boldsymbol{v}_{\mathscr{M}}^{\langle\sigma\rangle}, \delta \boldsymbol{q}_{\mathcal{M} . u}^{\langle\sigma\rangle}\right) \cdot\left(\underline{f}_{\mathscr{M}}^{\prime}\left(t, \boldsymbol{q}_{\mathcal{M}}^{\langle\sigma\rangle}\right), \boldsymbol{u}_{\mathscr{M}}\right) \leq 0
$$

Thus, the same derivation applied in Section 4.1 can also be used for the previous inequality leading to the following expression for $f_{\mu l}$ :

$$
f_{\mathscr{M}}=\boldsymbol{V}_{\mathscr{M}}^{\top} \underline{\underline{f}}_{\mathscr{M}}^{\prime}\left(t, \boldsymbol{q}_{M}^{\langle\sigma\rangle}\right)+{ }_{\star}^{u}\left[\begin{array}{l}
1 \\
0
\end{array}\right] \boldsymbol{u}_{\mathscr{M}}
$$

This completes the proof.

Theorem 4.3 (Sufficient conditions for a solution of an inverse dynamics problem). Let $\mathscr{M}$ be a $\nu^{\#-D O F}$ multibody system as described earlier in this section. Assume that the number of inputs in $\mathscr{M}$ is equal to the number of degrees of freedom of it, $\nu^{\#}$. Suppose also that for each input there is an associated $\sigma$-th order generalized variable, with $\sigma \geq \nu^{\circ}$. If, in a given generalized configuration of the system $\delta \boldsymbol{q}_{\mathcal{M}, u}^{\langle\sigma\rangle}$ is an independent set of variations (i.e. none of them can 
be expressed as a linear combination of the others), it can be stated that the dynamic equations of motion of $\mathscr{M}$ can be expressed as follows:

$$
u_{\mathscr{M}}=-C_{\mathscr{M}}^{\top} f_{\mathscr{M}}^{*}
$$

with $u_{\mathscr{M}}$ representing the column-matrix constituted by the inputs and $\boldsymbol{f}_{\mathscr{M}}^{*}$ corresponding to the effective forces associated to $\mathscr{M}$ when $u_{\mathscr{M}}=0$.

Equation (4.15) can be interpreted as an open-loop control law for $\mathscr{M}$, leading to the general solution for the inverse dynamics problem associated to this system.

Proof. If the variations $\delta \boldsymbol{q}_{\mathscr{M} . u}^{\langle\sigma\rangle}$ are independent, then according to the results of Theorems 2.8 and 2.10 , using the procedure described by equations (2.26 - 2.29), it is possible to obtain a matrix $C_{\mathscr{M}}$ for $\mathscr{M}$ such that the rows of this matrix that correspond to the $\sigma$-th order variables associated to the inputs of $\mathscr{M}$ constitute an identity matrix, i.e. $C_{\mathscr{M} . u}=1$. This fact, along with the result presented in Proposition 4.2 leads to:

$$
\bar{f}_{\mathscr{M}}=C_{\mathscr{M}}^{\top} f_{\mathscr{M}}=u_{\mathscr{M}}+C_{\mathscr{M}}^{\top} f_{\mathscr{M}}^{*}
$$

This leads to equation (4.15).

Note also that, being explicit, equation (4.15) can be used to determine for each $\left(t, \boldsymbol{q}^{\langle\sigma\rangle}(t)\right)$ that corresponds to a non-singular configuration of $\mathscr{M}$ and is compatible with the constraints of thus system, a unique set of values for the inputs $u_{\mathscr{M}}$. On the other hand, from the result of Theorem 4.1, it can be stated that each set of values for the inputs of the system will correspond to a unique set of values for $\dot{\boldsymbol{q}}^{\langle\sigma \sigma-1\rangle}$, which concludes the proof that equation (4.15) indeed represents a solution for the inverse dynamics problem associated to $\mathscr{M}$.

The application of Theorem 4.3 is not restricted to the solution of the inverse dynamics problem associated to a given multibody system. Some active forces in a mechanical system might be described as functions of components of constraint forces. This is the case of Coulomb friction, in which the tangential component of the contact force between two surfaces in relative motion (which corresponds to the effect of the friction) is modeled as being proportional to the normal component of this same force (which is a constraint force). This tangential component is parallel and opposite to the relative velocity between the contact points, corresponding to an active force. As discussed in Chapter 3, constraint forces do not normally appear in equations of motion derived by An- 
alytical Mechanics approaches. The same happens when the methodology described in Sections 4.1 and 4.2 is applied. In these cases, adopting a correct interpretation of the Principle of Relaxation of Constraints, active forces due to control inputs can be interpreted as constraint forces and vice versa, once their primary function is to enforce the system to follow an specific trajectory in the state space, constraining the the system to it (UDWADIA, 2006). Such an analogy allows the development of an approach, based on Theorem 4.3, that leads to expressions to compute some of the constraint forces acting in a multibody system when convenient.

The technique proposed here is adapted from Kane e Levinson (1985) and consists on the following steps:

1. Define $\sigma$-th order generalized variabels associated to the required constraint forces or torques. Such variables will describe motions that either are not allowed or prescribed according to the existing motion constraints.

2. Derive the equations of motion of the system supposing that all these extra generalized variables are independent.

3. Replace the values of these extra variables in the equations of motion by zero, if the corresponding motion is not allowed in the real system or by their expressions as functions of time if the motion is prescribed. Do any other replacement of variables that might be associated with these fictitious or imposed motions.

4. Use the result of Theorem 4.3 to obtain explicit expressions for these constraint forces or torques. If some active force depends on these constraint forces, it might be necessary to implement an iterative procedure for their computations.

Example 4.5 (Modeling of 3D slider-crank mechanism). Consider the 3D slidercrank mechanism $\mathscr{M}$ represented in Figure 4.6. Suppose that is is constituted by three subsystems $\mathscr{B}_{1}, \mathscr{B}_{2}$ and $\mathscr{B}_{3}$ each constituted by a single rigid body: $\mathscr{B}_{1}$ corresponds to the crank (body $\mathcal{B}_{1}$ ), $\mathscr{B}_{2}$ to the connecting rod (body $\mathcal{B}_{2}$ ) and $\mathscr{B}_{3}$ to the slider (body $\mathcal{B}_{3}$ ). By hypothesis, all the rigid bodies are supposed to be rigid and all the existing joints are ideal, not allowing any kind of clearance 
during motion.

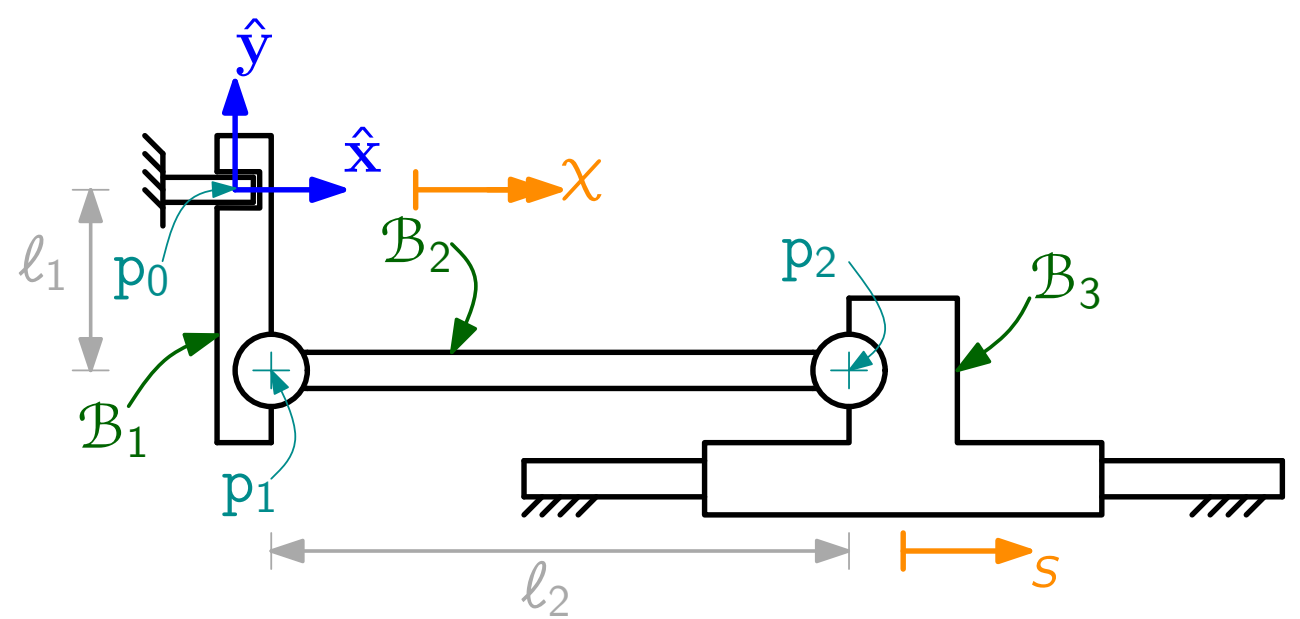

Figure 4.6: Representation of a 3D slider-crank mechanism in its reference configuration

In this system, the crank is constrained to a fixed basis (which is rigidly attached to an inertial reference frame $\mathcal{N}$ ) by a revolute joint, which allows a relative rotation motion around an axis passing through $\mathrm{p}_{0}$ which has the direction of the unit vector $\hat{\mathbf{x}}$. The slider is constrained to the same fixed basis by a prismatic joint, which allows it to translate in the direction defined by the unit vector $\hat{\mathbf{x}}$. The connecting rod is constrained to both the slider and the crank by spherical joints. Once all the constraints in this system are due to restrictions imposed by joints, it can be stated that this mechanism is a holonomic system. Considering that this system has two 1-DOF joints (one revolute and one prismatic) and two 3-DOF joints (both spherical), the application of the Chebychev-Grübler-Kutzbach criterion ${ }^{4}$ (GOGU, 2005; ANGELES, 1988) leads to $\nu_{\mathrm{M}}=6 \cdot(4-1)-(5 \cdot 2+3 \cdot 2)=2$. However, once the revolution of the connecting rod around its own longitudinal axis is irrelevant for the model, a 1-DOF model can be conceived for this mechanism.

Moreover, it will be supposed that any effect due to friction is negligible in the revolute and spherical joints. In the prismatic joint, however, dry friction will

\footnotetext{
${ }^{4}$ Chebychev-Grübler-Kutzbach criterion provides an equation to compute the mobility $\nu_{M}$ of a mechanism based on whether it is a $2 \mathrm{D}$ or $3 \mathrm{D}$ mechanism and on the number and type of joints it has. The mobility of a holonomic mechanism corresponds to the number of degrees of freedom it has. Consider that $\nu_{\# \#}$ stands for the number of degrees of freedom of a rigid body in a given multidimensional space. For a $2 \mathrm{D}$ mechanism adopt $\nu_{\#}=3$ and for a $3 \mathrm{D}$ one adopt $\nu_{\#}=6$. Denote by $\nu_{\mathrm{B}}$ the number of rigid bodies composing the mechanism (base included) and by $\nu_{J}(k)$ the number of joints that allow a $k$-DOF relative motion between a pair of rigid bodies. It can be stated that:
}

$$
\nu_{\mathrm{M}}=\nu_{\#}\left(\nu_{\mathrm{B}}-1\right)-\sum_{k}\left(\nu_{\#}-k\right) \nu_{\mathrm{J}}(k)
$$


be considered according to Coulomb's model (i.e., the tangential component is proportional to the normal component of the contact force between the surfaces). Furthermore, the motion of the crank will be supposed to be controlled by an input torque $u_{1} \hat{\mathbf{x}}$ applied to it.

In order to develop a mathematical model for this system, it is convenient to defined the following parameters:

- $\bar{\ell}_{1}$ represents the distance between the centres of the spherical joint $\left(\mathcal{B}_{1}, \mathcal{B}_{2}\right)$ and the axis of the revolute joint $\left(\mathcal{N}, \mathcal{B}_{1}\right)$, i.e. the distance between $\mathrm{p}_{1}$ and $\mathrm{p}_{0}$;

- $\bar{\ell}_{2}$ represents the distance between the centres of the spherical joints $\left(\mathcal{B}_{1}, \mathcal{B}_{2}\right), \mathrm{p}_{1}$, and $\left(\mathcal{B}_{2}, \mathcal{B}_{3}\right), \mathrm{p}_{2}$;

- $\bar{m}_{r}$ represents the mass of $\mathcal{B}_{r}(r=1,2,3)$;

- $\bar{l}_{1}$ represents the moment of inertia of the body crank $\mathcal{B}_{1}$ with respect to an axis parallel to $\hat{\mathrm{x}}$ passing through $\mathrm{p}_{0}$.

- $\bar{I}_{2}$ represents the moment of inertia of the connecting rod $\mathcal{B}_{2}$ measured with respect to any transversal axis passing through its center of mass $b_{2}^{\star}$;

- $\hat{\gamma}_{2}$ represents the ratio between the distance from $\mathrm{p}_{1}$ to $\mathrm{b}_{2}^{\star}$ and $\ell_{2}$;

- $\hat{\mu}$ is the coefficient of friction in the prismatic joint $\left(\mathcal{N}, \mathcal{B}_{3}\right)$.

Adopt the shorthand notation $\boldsymbol{q}_{n}^{\langle\sigma\rangle}=\boldsymbol{q}_{\mathscr{B}_{n}}^{\langle\sigma\rangle}$ and $\boldsymbol{q}^{\langle\sigma\rangle}=\boldsymbol{q}_{\mathscr{M}}^{\langle\sigma\rangle}$; adopt analogous conventions for subscripts of other variables. Define $\boldsymbol{q}_{1}^{\langle 0\rangle}=(\chi), \boldsymbol{q}_{2}^{\langle 0\rangle}=(\psi, \phi, \theta)$, $\boldsymbol{q}_{3}^{\langle 0\rangle}=(s)$ and $\boldsymbol{q}_{1}^{\langle 1\rangle}=\left(\omega_{1 \mathrm{x}}\right), \boldsymbol{q}_{2}^{\langle 1\rangle}=\left(v_{2 \mathrm{x}}, v_{2 \mathrm{y}}, v_{2 \mathrm{z}}, \omega_{2 \mathrm{y}}, \omega_{2 \mathrm{z}}\right), \boldsymbol{q}_{3}^{\langle 1\rangle}=\left(v_{3 \mathrm{x}}, v_{3 \mathrm{y}}, v_{3 \mathrm{z}}\right)$, considering that:

- $s$ represents the displacement, from the reference configuration shown in Figure 4.6 of the slider oriented by the unit vector $\hat{\mathbf{x}}$.

- $\chi$ represents the angle measuring the angle revolution of the crank from the reference configuration shown in Figure 4.6 (oriented according to the unit vector $\hat{\mathbf{x}}$ ).

- $\psi, \phi$ and $\theta$ are the zyx Euler angles describing the transformation between the coordinate system $N=\left(p_{0}, \hat{\mathbf{x}}, \hat{\mathbf{y}}, \hat{\mathbf{z}}\right)$ and a coordinate system $\mathrm{B}_{2}=\left(\mathrm{b}_{2}^{\star}, \hat{\mathbf{x}}_{2}, \hat{\mathbf{y}}_{2}, \hat{\mathbf{z}}_{2}\right)$ rigidly attached to $\mathcal{B}_{2}$, with $\hat{\mathbf{x}}_{2}$ being parallel to the line $\mathrm{p}_{1} \mathrm{p}_{2}$. 
- $\omega_{1 \mathrm{x}}, \omega_{2 \mathrm{y}}$ and $\omega_{2 \mathrm{z}}$ are the components, in the local bases of the angular velocities of $\mathcal{B}_{1}$ and $\mathcal{B}_{2}$ (with respect to $\mathcal{N}$ ), respectively. Consider that these local bases coincide with the one of $N$ in the reference configuration shown in Figure 4.6.

- $v_{2 x}, v_{2 y}, v_{2 z}$ and $v_{3 x}$ represent the components in the basis of $\mathrm{N}$ of the centres of mass of $\mathcal{B}_{2}$ and $\mathcal{B}_{3}$ (with respect to $\mathcal{N}$ ), respectively.

- $v_{3 y}$ and $v_{3 z}$ are fictitious components of the velocity of the center of mass of the slider in the directions defined by the unit vectors $\hat{\mathbf{y}}$ and $\hat{\mathbf{z}}$, which will be used to include the constraint forces $y_{3}^{*}$ and $z_{3}^{*}$ in the model (to obtain expressions to compute them).

It can be stated that:

$$
\begin{aligned}
& \bar{f}_{1}=\omega_{1 \mathrm{x}}\left[u_{1}-\bar{l}_{1} \dot{\omega}_{1 \mathrm{x}}\right]
\end{aligned}
$$

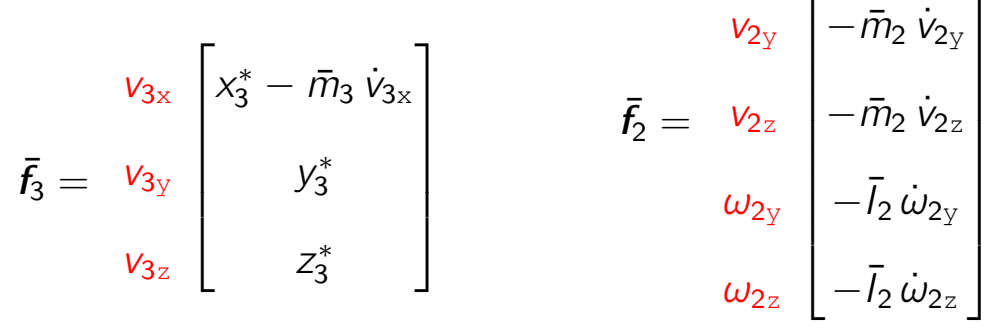

Coulomb's model for the friction force $x_{3}^{*}$, considering that $y_{3}^{*}$ and $z_{3}^{*}$ represent normal components of the contact force in the directions of $\hat{\mathbf{y}}$ and $\hat{z}$, leads to the following equation:

$$
x_{3}^{*}=-\hat{\mu} \operatorname{sign}\left(v_{3 x}\right) \sqrt{\left(y_{3}^{*}\right)^{2}+\left(z_{3}^{*}\right)^{2}}
$$

Also, matrix $[1]_{\mathrm{N} \mid \mathrm{B}_{2}}$ is given by:

$$
[1]_{\mathrm{N} \mid \mathrm{B}_{2}}=\left[\begin{array}{ccc}
\mathrm{c}_{\psi} \mathrm{c}_{\phi} & \mathrm{c}_{\psi} \mathrm{s}_{\phi} \mathrm{s}_{\theta}-\mathrm{c}_{\theta} \mathrm{s}_{\psi} & \mathrm{c}_{\psi} \mathrm{c}_{\theta} \mathrm{s}_{\phi}+\mathrm{s}_{\psi} \mathrm{s}_{\theta} \\
\mathrm{c}_{\phi} \mathrm{s}_{\psi} & \mathrm{c}_{\psi} \mathrm{c}_{\theta}+\mathrm{s}_{\psi} \mathrm{s}_{\phi} \mathrm{s}_{\theta} & \mathrm{c}_{\theta} \mathrm{s}_{\psi} \mathrm{s}_{\phi}-\mathrm{c}_{\psi} \mathrm{s}_{\theta} \\
-\mathrm{s}_{\phi} & \mathrm{c}_{\phi} \mathrm{s}_{\theta} & \mathrm{c}_{\phi} \mathrm{c}_{\theta}
\end{array}\right]
$$

Due to the configuration constraints imposed by the spherical joints, it can be stated that:

$$
\overline{\boldsymbol{q}}^{\langle 0\rangle}=\left[\begin{array}{c}
\bar{\ell}_{2}\left(1-\mathrm{c}_{\psi} \mathrm{c}_{\phi}\right)+s \\
\bar{\ell}_{1}\left(1-\mathrm{c}_{\chi}\right)+\bar{\ell}_{2} \mathrm{c}_{\phi} \mathrm{s}_{\psi} \\
\bar{\ell}_{1} \mathrm{~s}_{\chi}+\bar{\ell}_{2} \mathrm{~s}_{\phi}
\end{array}\right]=0
$$


Moreover, neglecting the revolution of the connecting rod around its own lon-

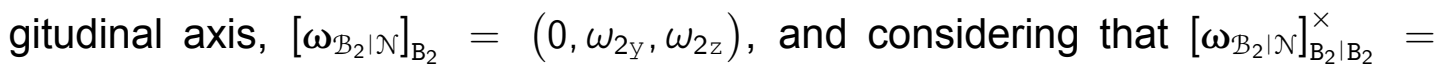
$[1]_{\mathbb{N} \mid B_{2}}^{\top}[1]_{N \mid B_{2}}$, the following identities can be stated:

$$
\left\{\begin{array}{l}
\dot{s}-v_{3 x}=0 \\
\dot{\chi}-\omega_{1 x}=0 \\
\dot{\theta}-\dot{\psi} s_{\phi}=0 \\
c_{\phi} \dot{\psi} s_{\theta}+c_{\theta} \dot{\phi}-\omega_{2 y}=0 \\
c_{\phi} c_{\theta} \dot{\psi}-\omega_{2 z}-\dot{\phi} s_{\theta}=0
\end{array}\right.
$$

Moreover, once:

$$
\begin{aligned}
& \mathbf{v}_{\mathrm{p}_{2} \mid \mathcal{N}}=\mathbf{v}_{\mathrm{p}_{1} \mid \mathcal{N}}+\omega_{\mathcal{B}_{2} \mid \mathcal{N}} \times \mathbf{r}_{\mathrm{p}_{2} \mid \mathrm{p}_{1}} \\
& \left(\mathbf{v}_{\mathrm{b}_{2}^{\star} \mid \mathcal{N}}-\mathbf{v}_{\mathrm{p}_{1} \mid \mathcal{N}}\right)=\hat{\gamma}_{2}\left(\mathbf{v}_{\mathrm{p}_{2} \mid \mathcal{N}}-\mathbf{v}_{\mathrm{p}_{1} \mid \mathcal{N}}\right)
\end{aligned}
$$

the first order constraint invariants of this system are given by:

$$
\overline{\boldsymbol{q}}^{\langle 1\rangle}=\left[\begin{array}{c}
\bar{\ell}_{2}\left(\mathrm{~s}_{\psi}\left(\mathrm{s}_{\theta} \omega_{2 \mathrm{y}}+\mathrm{c}_{\theta} \omega_{2 z}\right)+\mathrm{c}_{\psi} \mathrm{s}_{\phi}\left(\mathrm{c}_{\theta} \omega_{2 \mathrm{y}}-\mathrm{s}_{\theta} \omega_{2 z}\right)\right)+v_{3 \mathrm{x}} \\
-\mathrm{s}_{\chi} \bar{\ell}_{1} \omega_{1 \mathrm{x}}+\bar{\ell}_{2}\left(\mathrm{~s}_{\psi} \mathrm{s}_{\phi}\left(\mathrm{c}_{\theta} \omega_{2 y}-\mathrm{s}_{\theta} \omega_{2 z}\right)-\mathrm{c}_{\psi}\left(\mathrm{s}_{\theta} \omega_{2 \mathrm{y}}+\mathrm{c}_{\theta} \omega_{2 z}\right)\right)+v_{3 \mathrm{y}} \\
\mathrm{c}_{\chi} \bar{\ell}_{1} \omega_{1 \mathrm{x}}+\mathrm{c}_{\phi} \bar{\ell}_{2}\left(c_{\theta} \omega_{2 \mathrm{y}}-\mathrm{s}_{\theta} \omega_{2 z}\right)+v_{3 z} \\
v_{2 \mathrm{x}}-v_{3 \mathrm{x}} \hat{\gamma}_{2} \\
v_{2 \mathrm{y}}+\mathrm{s}_{\chi} \bar{\ell}_{1} \omega_{1 \mathrm{x}}\left(\hat{\gamma}_{2}-1\right)-v_{3 \mathrm{y}} \hat{\gamma}_{2} \\
v_{2 z}-c_{\chi} \bar{\ell}_{1} \omega_{1 \mathrm{x}}\left(\hat{\gamma}_{2}-1\right)-v_{3 z} \hat{\gamma}_{2}
\end{array}\right]
$$

Noting that for this system $\nu^{\circ}=1$, the expression for matrix $A$ can be obtained from the Jacobian of (f) with respect to $q^{(1)}$. Also, for this system $B=A$. The matrix $C$ (orthogonal complement of $B$ ) obtained according to Theorem 4.3 for the solution of the invers dynamics problem associated to this system is given 
by (with $E_{i, j}$ denoting the $i$-th row, $j$-th column element of matrix $E=[1]_{\mathbb{N} \mid B_{2}}$ ):

$$
\boldsymbol{C}=\left[\begin{array}{ccc}
1 & 0 & 0 \\
\frac{\bar{\ell}_{1} \hat{\gamma}_{2}\left(s_{\chi} E_{2,1}-c_{\chi} E_{3,1}\right)}{E_{1,1}} & -\frac{\hat{\gamma}_{2} E_{2,1}}{E_{1,1}} & -\frac{\hat{\gamma}_{2} E_{3,1}}{E_{1,1}} \\
-s_{\chi} \bar{\ell}_{1}\left(\hat{\gamma}_{2}-1\right) & \hat{\gamma}_{2} & 0 \\
c_{\chi} \bar{\ell}_{1}\left(\hat{\gamma}_{2}-1\right) & 0 & \hat{\gamma}_{2} \\
-\frac{\bar{\ell}_{1}\left(c_{\chi} E_{2,2}+s_{\chi} E_{3,2}\right)}{\bar{\ell}_{2} E_{1,1}} & \frac{E_{3,2}}{\bar{\ell}_{2} E_{1,1}} & -\frac{E_{2,2}}{\bar{\ell}_{2} E_{1,1}} \\
-\frac{\bar{\ell}_{1}\left(c_{\chi} E_{2,3}+s_{\chi} E_{3,3}\right)}{\bar{\ell}_{2} E_{1,1}} & \frac{E_{3,3}}{\bar{\ell}_{2} E_{1,1}} & -\frac{E_{2,3}}{\bar{\ell}_{2} E_{1,1}} \\
\frac{\bar{\ell}_{1}\left(s_{\chi} E_{2,1}-c_{\chi} E_{3,1}\right)}{E_{1,1}} & -\frac{E_{2,1}}{E_{1,1}} & -\frac{E_{3,1}}{E_{1,1}} \\
0 & 1 & 0 \\
0 & 0 & 1
\end{array}\right]
$$

Note that this expression for $C$ is not defined only when $E_{1,1}=0$, i.e. when the longitudinal direction of $\mathcal{B}_{2}$ is orthogonal to the direction of $\hat{x}$. In this situation, it is impossible to do any work to move the slider for any torque $u_{1}$ applied to the crank, regardless of its magnitude.

Thus, the dynamic equations of motion of this system can be written as follows:

$$
\boldsymbol{u}=\boldsymbol{C} \boldsymbol{M} \dot{\boldsymbol{q}}^{\langle 1\rangle}+x_{3}^{*} x_{3}
$$

with $\boldsymbol{u}=\left(u_{1}, y_{3}^{*}, z_{3}^{*}\right), \boldsymbol{q}^{\langle 1\rangle}=\left(\boldsymbol{q}_{1}^{\langle 1\rangle}, \boldsymbol{q}_{2}^{\langle 1\rangle}, \boldsymbol{q}_{3}^{\langle 1\rangle}\right)$ and:

$$
\begin{aligned}
& \boldsymbol{M}=\left[\begin{array}{lllllllll}
l_{1} & m_{2} & m_{2} & m_{2} & l_{2} & l_{2} & m_{3} & m_{3} & m_{3}
\end{array}\right]^{\mathrm{D}} \\
& x_{3}=\left[\begin{array}{c}
\frac{\bar{\ell}_{1}\left(c_{\chi} E_{3,1}-s_{\chi} E_{2,1}\right)}{E_{1,1}} \\
\frac{E_{2,1}}{E_{1,1}} \\
\frac{E_{3,1}}{E_{1,1}}
\end{array}\right]
\end{aligned}
$$

In order to perform a numerical inverse dynamics simulation for this system, 
consider the following values for the parameters:

$$
\begin{array}{ll}
\bar{\ell}_{1}=\frac{1}{2} \mathrm{~m} & \bar{\ell}_{2}=\sqrt{2} \mathrm{~m} \\
\hat{\gamma}_{2}=\frac{\sqrt{2}}{2} & \hat{\mu}=0.50 \\
\bar{l}_{1}=0.20 \mathrm{~kg} \cdot \mathrm{m}^{2} & \bar{l}_{2}=0.10 \mathrm{~kg} \cdot \mathrm{m}^{2} \\
\bar{m}_{2}=0.50 \mathrm{~kg} & \bar{m}_{3}=2.0 \mathrm{~kg}
\end{array}
$$

Consider that the objective of this analysis is to determine the time history of the torque $u_{1}$ which, according to the model, must be applied to the crank to ensure a given motion of the slider. For this particular simulation, consider that the position of the slider is described as follows:

$$
s(t)=-\left(1-\hat{\gamma}_{2}\right) \bar{\ell}_{2} \frac{\left(1+8 \sin ^{2}(2 \pi t)\right)}{10}
$$

Note that $v_{3 x}=\dot{s}$ and that $v_{3 y}$ and $v_{3 z}$ must be identically zero in order not to violate the constraints of the system. Thus, it is necessary, from the constraint equations of the model, to obtain time histories for the remaining generalized variables that are compatible with the motion prescribed for the slider. Then, it is enough to replace the values of these motion variables in the system of equations (h), to be able to obtain the time histories of $u_{1}, y_{3}^{*}$ and $z_{3}^{*}$.

The simulations presented in this example were performed by an algorithm written in Wolfram Language and running in Wolfram Mathematica 10.2. Basically, the built-in function NDSolve was used both for numerically solving the constraint equations (inverse kinematics simulation) and of the dynamic equations of motion in the variables $u_{1}, y_{3}^{*}$ and $z_{3}^{*}$ (inverse dynamics simulation). This function automatically chooses among a variety of built-in procedures for numerical integration, the best one for a particular system of differential-algebraic equations (DAEs).

For the inverse kinematics simulation, two algorithms were developed using the function NDSolve. In the first one, equations $(d, e, f)$ were used, and the values of $s(t), v_{3 x}(t)=\dot{s}(t), v_{3 y}(t)=0$ and $v_{3 z}(t)=0$ were prescribed. Once NDSolve can not solve systems with redundant equations, the first three equations of (f) were deleted ${ }^{5}$. The results given by these algorithms are interpolation functions that give an approximation of the time history of the involved variables. In order to inspect how satisfactory are these results, the time histories of the norms $\left\|\overline{\boldsymbol{q}}^{\langle 0\rangle}\right\|$ and $\left\|\overline{\boldsymbol{q}}^{\langle 1\rangle}\right\|$ are computed. The closer to zero these norms

\footnotetext{
${ }^{5}$ Note that these deleted equations correspond to the differential forms of equations (d) when the replacement $\dot{\boldsymbol{q}}^{\langle 0\rangle}=\dot{\boldsymbol{q}}^{\langle 0\rangle}\left(t, \boldsymbol{q}^{\langle 1\rangle\rangle}\right)$ is performed.
} 
are, the better the results are. At the initial time instant, $\left\|\overline{\boldsymbol{q}}^{\langle 0\rangle}\right\|(0)=6.245 \cdot 10^{-17}$ e $\left\|\overline{\boldsymbol{q}}^{\langle 1\rangle}\right\|(0)=0$, what can be considered satisfactory. However, as shown in Figure 4.7, the values of $\left\|\overline{\boldsymbol{q}}^{\langle 0\rangle}\right\|(t)$ and $\left\|\overline{\boldsymbol{q}}^{\langle 1\rangle}\right\|(t)$ in the remaining of the time interval in this first simulation (denoted by $\# 1$ ) are not so satisfactory (although the algorithm is quite stable and the magnitude of the norms tend to return to zero right after some growth). In the second algorithm for inverse kinematics simulation (simulation $\# 2$ ), the time derivative of the algebraic equations are taken, converting them into differential equations. Equations $\overline{\boldsymbol{q}}^{\langle 0\rangle}=0 \mathrm{e}$ $\overline{\boldsymbol{q}}^{\langle 1\rangle}=0$ are replaced, respectively, by the following ODEs:

$$
\left\{\begin{array}{l}
\frac{\mathrm{d}^{2} \overline{\boldsymbol{q}}^{\langle 0\rangle}}{\mathrm{d} t^{2}}+2 \bar{b}_{1} \bar{b}_{2} \frac{\mathrm{d} \overline{\boldsymbol{q}}^{\langle 0\rangle}}{\mathrm{d} t}+\bar{b}_{2}^{2} \overline{\boldsymbol{q}}^{\langle 0\rangle}=\mathbf{0} \\
\frac{\mathrm{d} \overline{\boldsymbol{q}}^{\langle 1\rangle}}{\mathrm{d} t}+\bar{b}_{3} \overline{\boldsymbol{q}}^{\langle 1\rangle}=0
\end{array}\right.
$$

The values of the constants $\bar{b}_{k}$ can be tunned to increase the numerical stability of the integration procedure (BAUMGARTE, 1972). Taking as initial conditions for these ODEs the results obtained in simulation $\# 1$ and setting $\bar{b}_{1}=\bar{b}_{2}=\bar{b}_{3}=1$, much more precise results were obtained as it can be noticed in the charts in Figure 4.8.

Replacing the interpolation functions obtained in simulation \#2 in the system of equations ( $h$ ), the function NDSolve is used to write an algorithm to find the corresponding time histories of $u_{1}, y_{3}^{*}$ and $z_{3}^{*}$. Moreover, the numerical error in this inverse dynamics simulation can be controlled by calculating the norm $\|\bar{f}\|(t)=\left\|\boldsymbol{u}-\boldsymbol{C} \boldsymbol{M} \dot{\boldsymbol{q}}^{\langle 1\rangle}-x_{3}^{*} x_{3}\right\|(t)$, whose time history is presented in Figure 4.9.

The results of the numerical simulation are presented in the charts of Figures $4.10-4.14$. It is relevant to note that the constitutive equation used for modeling the friction force (Coulomb's model) is responsible for a discontinuity in the results when the direction of the motion of the slider is reversed. These discontinuities will not be observed in the real system, regardless of how fast the actuator can vary the magnitude of the torque applied. Thus, in order to be able to use this model for the project of a control system for this mechanism it would be fundamental to consider the dynamics of the actuator too, which could be done by finding a model for the actuator and applying the recursive modular modeling algorithm presented in Section 4.2. Otherwise, significative errors would be observed in the real system whenever the motion of the slider is reversed. 


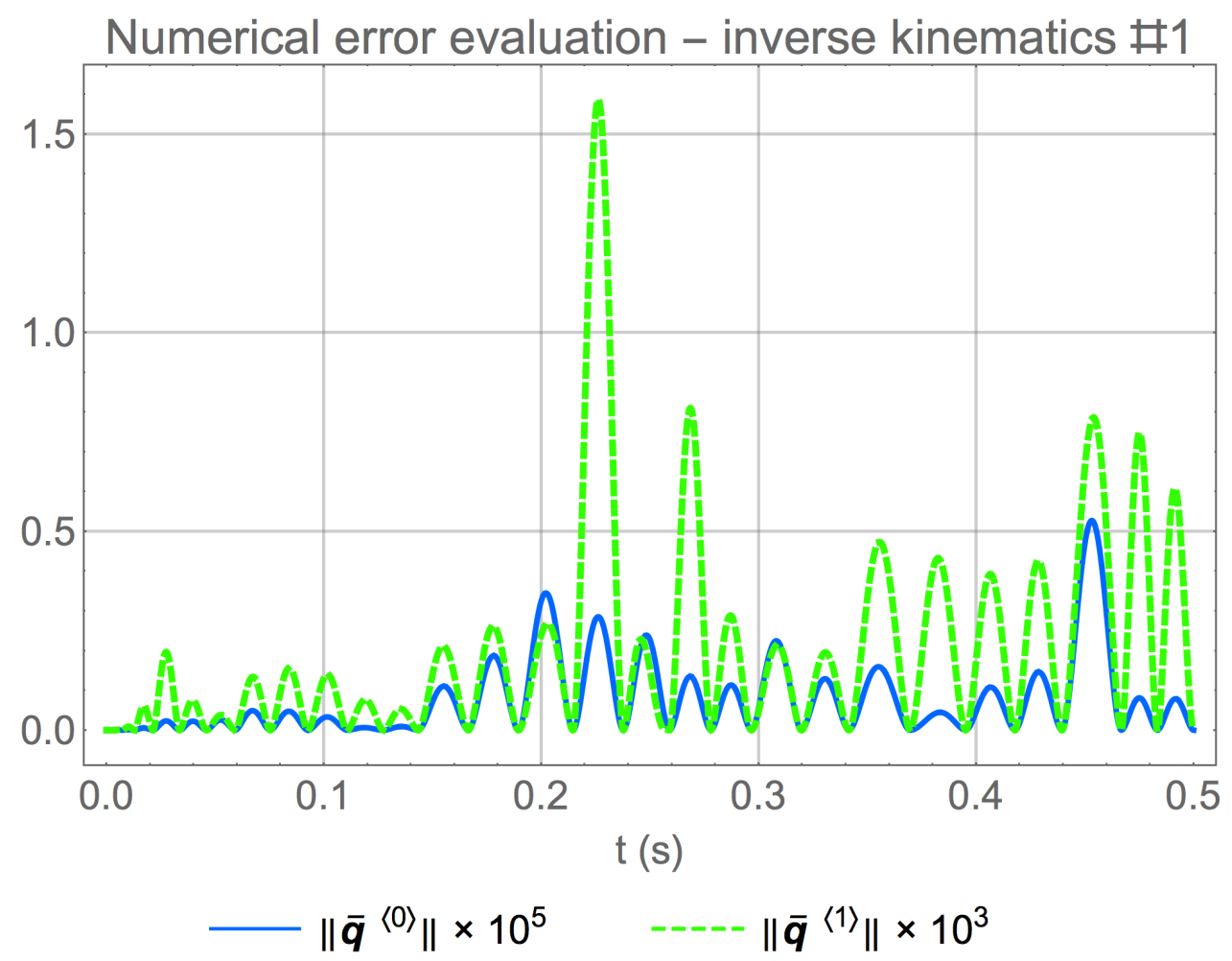

Figure 4.7: Numerical error evaluation in the inverse kinematics simulation $\# 1$.

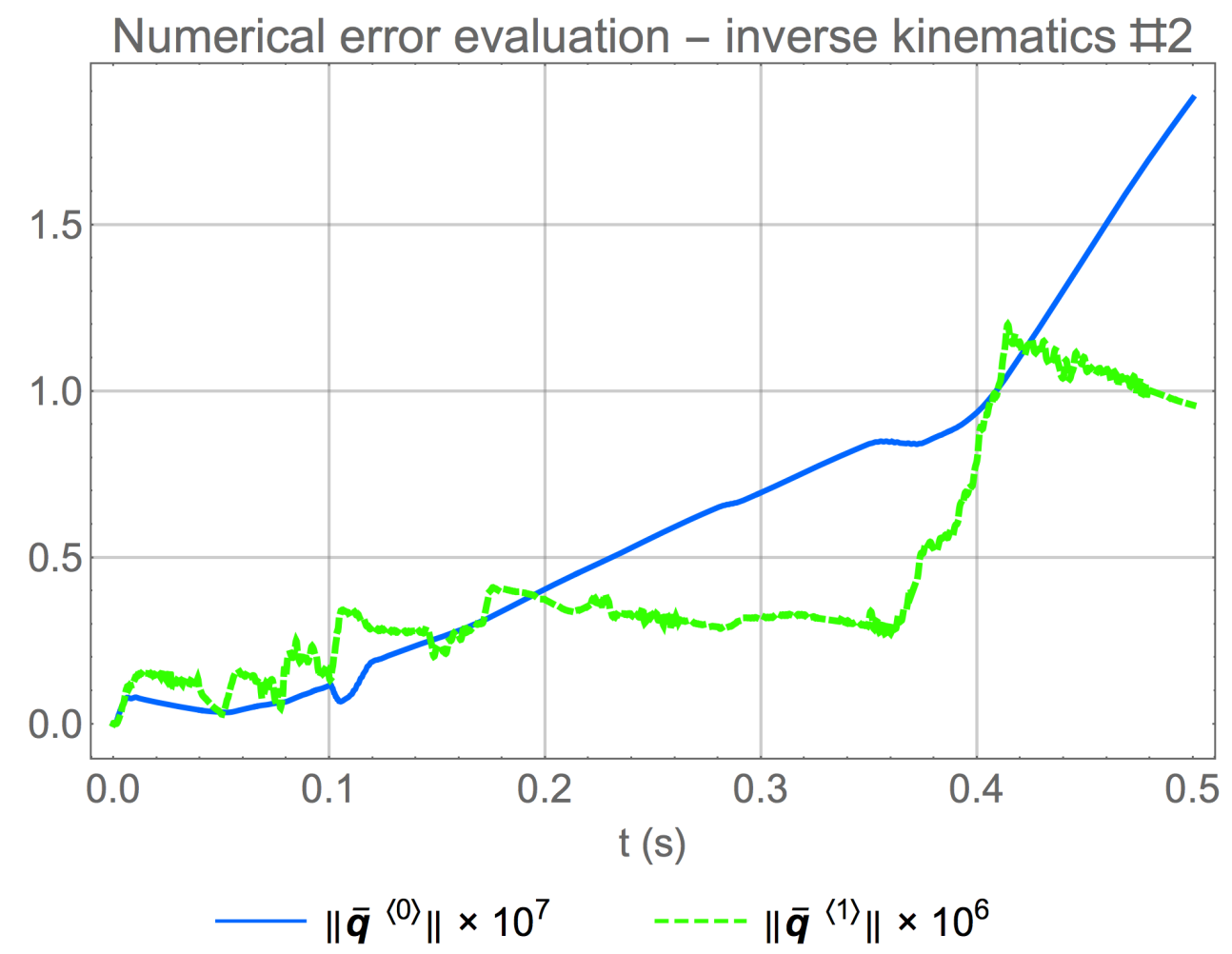

Figure 4.8: Numerical error evaluation in the inverse kinematics simulation \#2. 


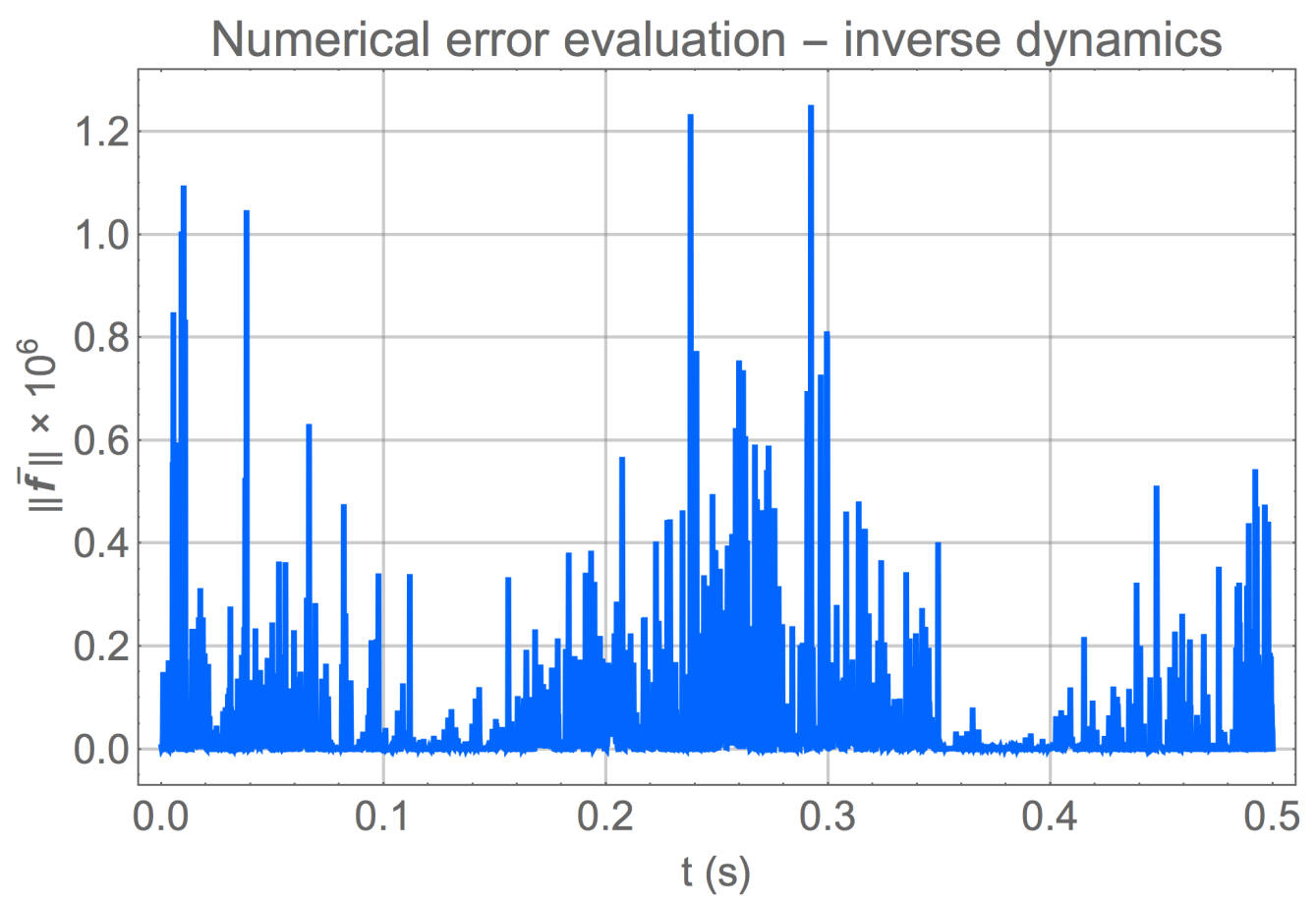

Figure 4.9: Numerical error evaluation in the inverse dynamics simulation.

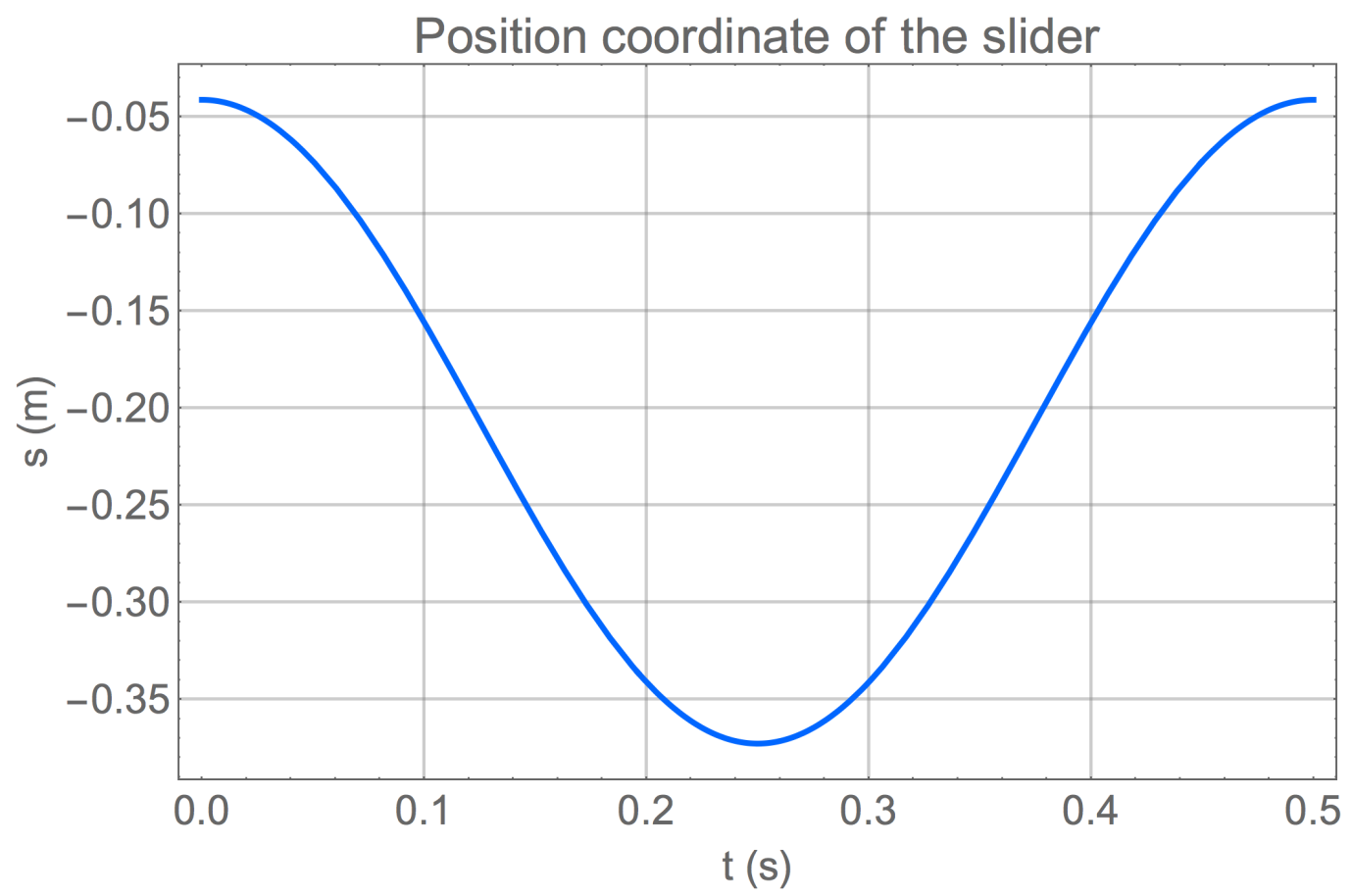

Figure 4.10: Time history of the position of the slider. 


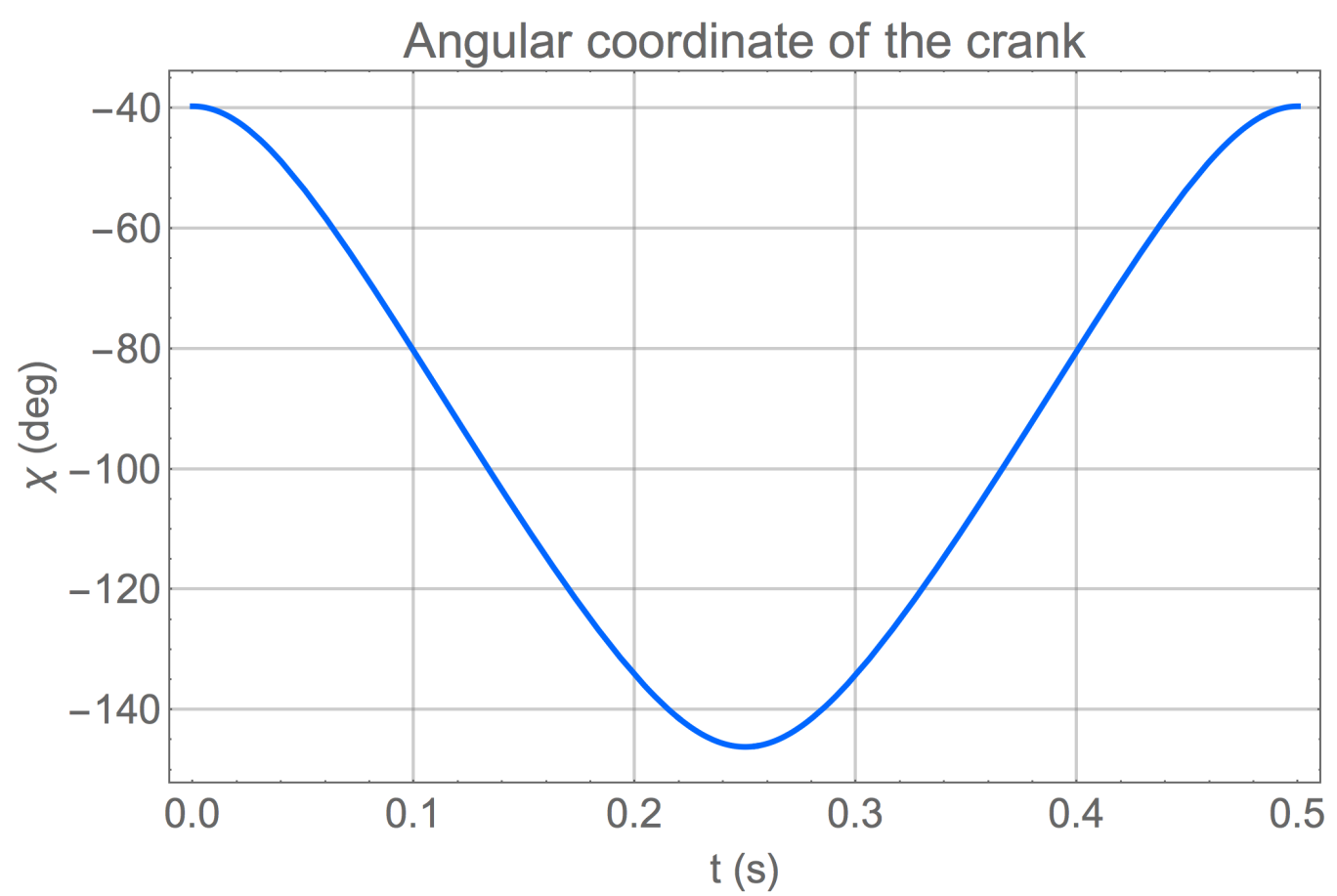

Figure 4.11: Time history of the angular position of the crank.

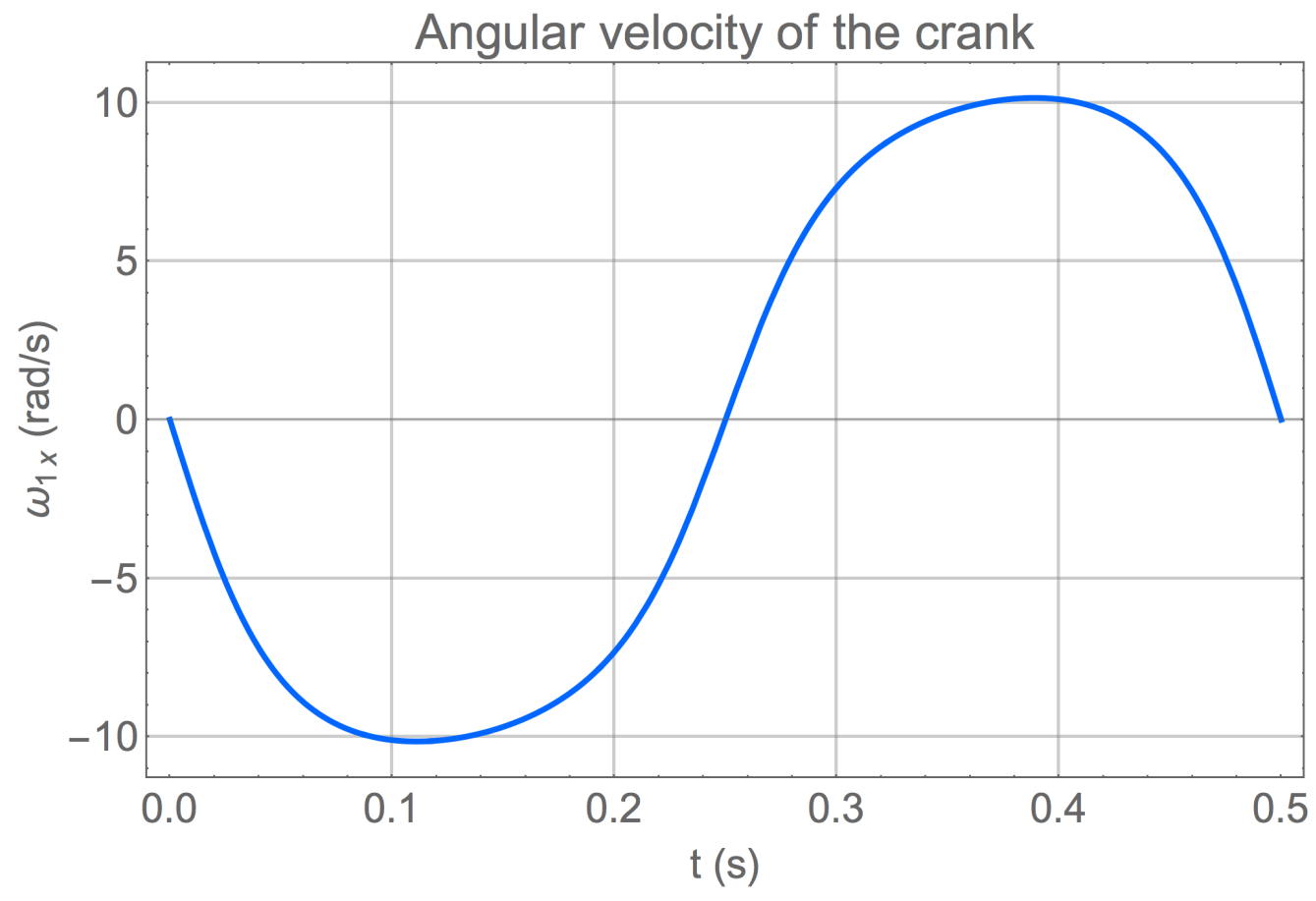

Figure 4.12: Time history of the angular velocity of the crank. 


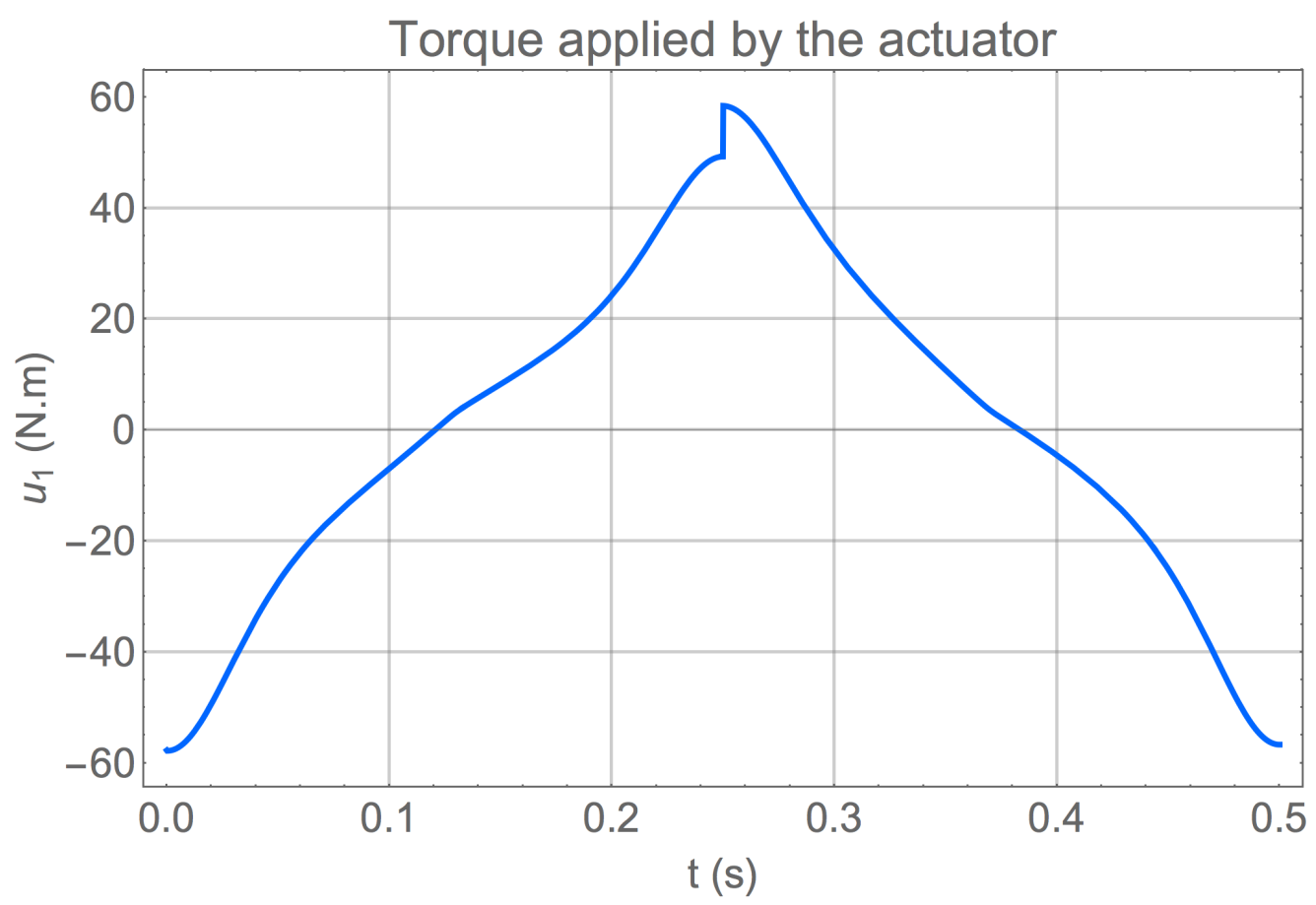

Figure 4.13: Time history of the torque applied by the actuator.

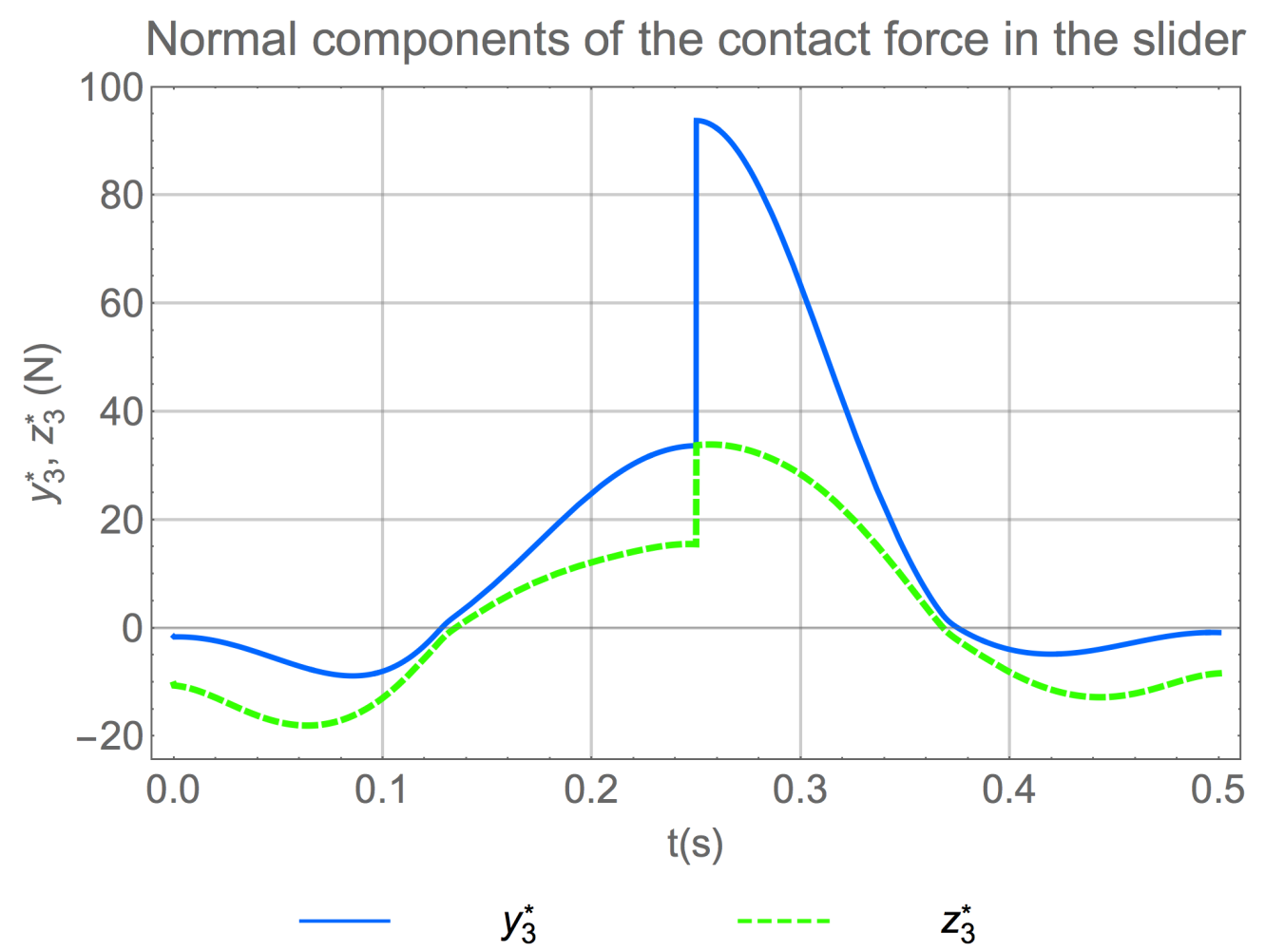

Figure 4.14: Time history of the normal components of the contact force acting in the slider. 


\subsection{Linearization of equations of motion}

Linearized mathematical models are particularly important to study the dynamic behavior of a multibody system in the neighborhood of a motion that it actually performs, particularly when stability analyses or the design of some types of control and measurement systems are involved. An evident advantage of working with linearized equations of motion is that most of the techniques for linear systems are universal (i.e., not dependent on what kind of system is being modeled) and can easily lead to precise results.

This section aims to discuss the modifications that must be performed in the recursive modular modeling methodology presented in Sections 4.1 and 4.2 in order to obtain linearized models of multibody systems, without needing to previously derive the non-linear equations of motion. Provided a reference state or a reference trajectory (which must be an a priori known solution of the original of equations of motion), a system of linearized equations of motion can be obtained for each subsystem corresponding to a leaf in the hierarchical tree structure representing a multibody system. Moreover, linearized expressions for the constraint invariants among the subsystem and for the Jacobians of the constraint invariants with respect to the highest order generalized variables in their expressions can be obtained. Therefore, the recursive modular modeling methodology proposed in this text can also be applied for the direct derivation of the linearized equations of motion. Such a procedure will result in a system of equations which is equivalent to the one obtained by a conventional approach in which the linearization is performed after the non-linear equations are derived, i.e., a system of linear ordinary differential equations whose solutions are approximations to the actual solutions of the original (non-linear) equations of motion, in which the error is an infinitesimal with respect to the first order terms.

Let $\mathscr{M}_{n}$ be a $\nu_{\mathscr{M}_{n}}^{\#}$-DOF multibody system whose constraint order is equal to $\nu_{\mathscr{U}_{n}}^{\circ}$. Adopt an integer $\sigma$ such that $\sigma \geq \nu_{\mathcal{M}_{n}}^{\circ}$ and that no equation of motion of $\mathscr{M}$ depend on any generalized variable above $\sigma$-th order. The equations of motion for $\mathscr{M}_{n}$ can be expressed by the system of differential-algebraic equa- 
tions (4.13).

$$
\left\{\begin{array}{l}
\dot{\boldsymbol{q}}_{\mathscr{M}_{n}}^{\langle\beta\rangle}=\dot{\boldsymbol{q}}_{\mathscr{M}_{n}}^{\langle\beta\rangle}\left(t, \boldsymbol{q}_{\mathscr{M}_{n}}^{\langle\beta+1\rangle}\right) \quad \text { for } \quad 0 \leq \beta \leq(\sigma-1) \\
\overline{\boldsymbol{q}}_{\mathscr{M}_{n}}^{\langle\sigma\rangle}=\boldsymbol{A}_{\mathscr{M}_{n}} \boldsymbol{q}_{\mathscr{M}_{n}}^{\langle\sigma\rangle}+\boldsymbol{b}_{\mathscr{M}_{n}}^{\langle\sigma-1\rangle}=0 \\
\overline{\boldsymbol{f}}_{\mathscr{M}_{n}}=C_{\mathscr{M}_{n}}^{\top} \boldsymbol{f}_{\mathscr{M}_{n}}=0
\end{array}\right.
$$

Let $\boldsymbol{q}_{\mathscr{M}_{n}}^{\langle\sigma\rangle}={ }^{\mathrm{R}} \boldsymbol{q}_{\mathscr{M}_{n}}^{\langle\sigma \nabla\rangle}(t)$ be a solution of (4.13), associated to a set of inputs $\boldsymbol{u}_{\mathscr{M}_{n}}={ }^{\mathrm{R}} \boldsymbol{u}_{\mathscr{M}_{n}}(t)$. Assume that this solution is taken as reference for the derivation of the linearized model, i.e. it is desired to obtain a system of linear ordinary differential equations that is able to estimate solutions of the original system that are in a neighborhood of the reference solution, $\boldsymbol{q}_{\mathscr{M}_{n}}^{\langle\sigma\rangle}={ }^{\mathrm{R}} \boldsymbol{q}_{\mathcal{M}_{n}}^{\langle\langle\sigma\rangle}(t)$, and associated to inputs in a neighborhood of the reference ones, $\boldsymbol{u}_{\mathscr{M}_{n}}={ }^{\mathrm{R}} \boldsymbol{u}_{\mathscr{M}_{n}}(t)$. Formally, defining ${ }^{\mathrm{L}} \boldsymbol{q}_{\mathscr{M}_{n}}^{\langle\langle\sigma\rangle}=\boldsymbol{q}_{\mathscr{M}_{n}}^{\langle\langle\sigma\rangle}-{ }^{\mathrm{R}} \boldsymbol{q}_{\mathscr{M}_{n}}^{\langle\langle\sigma\rangle}$ and ${ }^{\mathrm{L}} \boldsymbol{u}_{\mathscr{M}_{n}}=\boldsymbol{u}_{\mathscr{M}_{n}}-{ }^{\mathrm{R}} \boldsymbol{u}_{\mathscr{M}_{n}}$, it can be stated that it is desired a system of linear ordinary differential equations that provides an estimation of a solution whose error is an infinitesimal with respect to $\epsilon$ whenever $\|\left({ }^{\mathrm{L}} \boldsymbol{q}_{\mathscr{U}_{n}}\left\langle\left\langle{ }^{\mathrm{L}} \boldsymbol{u}_{\mathscr{M}_{n}}\right) \|<\epsilon\right.\right.$, for an adequately defined norm.

In order to linearize the functions $\underline{\dot{q}}_{\mathscr{M}_{n}}^{\langle\beta\rangle}$ and $\overline{\boldsymbol{q}}_{\mathscr{M}_{n}}^{\langle\sigma\rangle}$, it is enough to use the conventional Taylor series expansion suppressing all the terms above first order, that can be generically denoted by $o(\epsilon)$. If the non-linear expressions for the functions $\underline{\dot{q}}_{\mathscr{M}_{n}}^{\langle\beta\rangle}$ are not explicitly known, then it is possible to apply this same linearization procedure to the invariants $\overline{\boldsymbol{c}}_{\mathscr{M}_{n}}^{\langle\beta+1\rangle}\left(t, \boldsymbol{q}_{\mathscr{M}_{n}}^{\langle\beta+1\rangle}, \dot{\boldsymbol{q}}_{\mathscr{M}_{n}}^{\langle\beta\rangle}\right)=0$, that define the transformations of variables between $\dot{\boldsymbol{q}}_{\mathscr{M}_{n}}^{\langle\beta\rangle}$ and $\boldsymbol{q}_{\mathscr{M}_{n}}^{\langle\beta+1\rangle}, 0 \leq \beta \leq(\sigma-1)$, and, after that, use some symbolic linear solving method to express the ${ }^{\mathrm{I}} \dot{\boldsymbol{q}}_{\mathscr{M}_{n}}^{\langle\mathcal{\langle \beta}}$ as an explicit linear function of the remaining variables, which will correspond to the linearized form of $\underline{\dot{q}}_{\mathscr{M}_{n}}^{\langle\beta\rangle}$.

Adopt the notation ${ }^{\mathrm{L}} \boldsymbol{v}_{\mathscr{M}_{n}}$ for the linearized form of $\boldsymbol{v}_{\mathscr{M}_{n}}$. Concerning the dynamic equations of motion, it is relevant to note that the linearized expressions of the unconstrained generalized effective forces are given by:

$$
{ }^{\mathrm{L}} \boldsymbol{f}_{\mathscr{M}_{n}}=\left\{\begin{array}{l}
{ }^{\mathrm{L}}\left({ }^{\mathrm{L}} \boldsymbol{V}_{\mathscr{M}_{n}} \cdot{ }^{\mathrm{L}} \boldsymbol{f}_{\mathscr{M}_{n}}^{\prime}\right), \text { if } \mathbb{S}\left(\mathscr{M}_{n}\right)=\varnothing \\
\left({ }^{\mathrm{L}} \boldsymbol{f}_{\mathscr{M}_{n} \oplus},\left(\ldots,{ }^{\mathrm{L}} \overline{\boldsymbol{f}}_{\mathscr{M}_{n+1}}, \ldots\right)\right), \text { otherwise }
\end{array}\right.
$$

In the case of matrix $C_{\mathscr{M}_{n}}$, however, not always a non-linear expression will be available. Thus, it is necessary to develop an algorithm for obtaining a linearized matrix ${ }^{\mathrm{L}} \boldsymbol{C}_{\mathscr{M}_{n}}$ without the need of previously obtaining a non-linear matrix 
$C_{\mathscr{M}_{n}}$. Define:

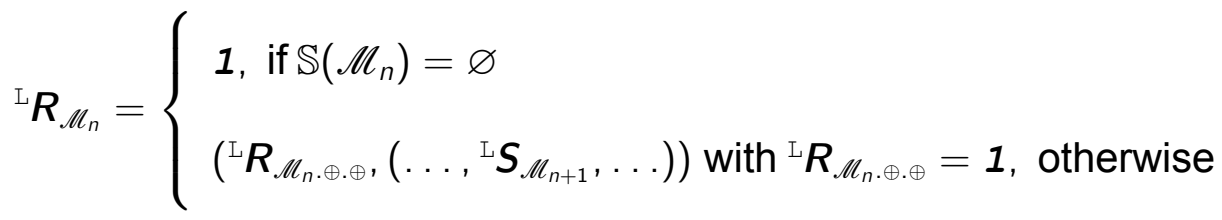

$$
\begin{aligned}
& { }^{\mathrm{L}} \boldsymbol{B}_{\mathscr{M}_{n}}=\left\{\begin{array}{l}
{ }^{\mathrm{L}} \boldsymbol{A}_{\mathscr{M}_{n}}, \text { if } \mathbb{S}\left(\mathscr{M}_{n}\right)=\varnothing \\
{ }^{\mathrm{L}}\left({ }^{\mathrm{L}} \boldsymbol{A}_{\mathscr{M}_{n} \oplus}{ }^{\mathrm{L}} \boldsymbol{R}_{\mathscr{M}_{n}}\right), \text { otherwise }
\end{array}\right.
\end{aligned}
$$

The least squares algorithm for obtaining a linearized orthogonal complement for $B_{\mathscr{M}_{n}}$ is presented in the following proposition.

Proposition 4.4. Let $B=\underline{B}(x)$ denote a matrix which can be expressed as an explicit function of $x=\left(x_{1}, \ldots, x_{\nu}\right)$. Denote by ${ }^{\mathrm{L}} B$ the linearized form of $B$, which can be expressed as follows:

$$
{ }^{\mathrm{L}} \boldsymbol{B}=\overline{\boldsymbol{B}}_{0}+\sum_{j=1}^{\nu} \overline{\boldsymbol{B}}_{j} x_{j}
$$

Let ${ }^{\mathrm{L}} \mathrm{C}$ be a matrix that can be expressed as follows:

$$
{ }^{\mathrm{I}} \boldsymbol{C}=\overline{\boldsymbol{C}}_{0}+\sum_{j=1}^{\nu} \overline{\boldsymbol{C}}_{j} x_{j}
$$

Consider that the coefficient matrices $\bar{C}_{j}$ are obtained by the following algorithm:

(1) Obtain $\bar{C}_{0}$ as an orthogonal complement of $\bar{B}_{0}$ using a least squares solver.

(2) Choose some constant $\gamma_{j}$ and define $\bar{\Gamma}_{j}^{+}$as the orthogonal complement of $\left(\bar{B}_{0}+\gamma_{j} \bar{B}_{j}\right)$ obtained by the same method as in the previous step, i.e. the orthogonal complement that should satisfy $\lim _{\gamma_{j} \rightarrow 0} \bar{\Gamma}_{j}^{+}=\bar{C}_{0}$.

(3) Analogously, obtain $\bar{\Gamma}_{j}^{-}$as the orthogonal complement of $\left(\bar{B}_{0}-\gamma_{j} \bar{B}_{j}\right)$, that should satisfy $\lim _{\gamma_{j} \rightarrow 0} \bar{\Gamma}_{j}^{-}=\bar{C}_{0}$.

(4) Obtain $\bar{C}_{j}, j \in\{1,2, \ldots, \nu\}$, by the expression:

$$
\overline{\boldsymbol{C}}_{j}=\frac{1}{\gamma_{j}}\left(\overline{\boldsymbol{\Gamma}}_{j}^{+}-\overline{\boldsymbol{\Gamma}}_{j}^{-}\right)
$$

It can be stated that ${ }^{\mathrm{I}} \mathrm{C}$ is a linear approximation for an orthogonal complement of $B$. 
Proof. First of all, note that:

$$
\boldsymbol{B}{ }^{\mathrm{I}} \boldsymbol{C}={ }^{\mathrm{I}} \boldsymbol{B}{ }^{\mathrm{I}} \boldsymbol{C}+\boldsymbol{O}\left(\|\boldsymbol{x}\|^{2}\right)=\overline{\boldsymbol{B}}_{0} \bar{C}_{0}+\sum_{i=1}^{\nu} x_{i}\left(\bar{B}_{0} \overline{\boldsymbol{C}}_{i}+\bar{B}_{i} \bar{C}_{0}\right)+\boldsymbol{O}\left(\|\boldsymbol{x}\|^{2}\right)
$$

If ${ }^{\mathrm{I}} C$ is a linear approximation for an orthogonal complement of $B$, it is necessary that $B{ }^{\mathrm{I}} C=O\left(\|x\|^{2}\right)$. Thus, it is necessary that $\bar{B}_{0} \bar{C}_{0}=0$, i.e. $\bar{C}_{0}$ must be an orthogonal complement of $\bar{B}_{0}$. Moreover, let $x_{i}=\underline{\delta}_{i j} \gamma_{j}$, with $\underline{\delta}_{i j}$ representing the Kronecker delta. In this case:

$$
\begin{aligned}
\boldsymbol{B}^{\mathrm{I}} \boldsymbol{C} & =\bar{B}_{0} \overline{\boldsymbol{C}}_{0}+\gamma_{j}\left(\bar{B}_{0} \overline{\boldsymbol{C}}_{j}+\bar{B}_{j} \overline{\boldsymbol{C}}_{0}\right)+\boldsymbol{O}\left(\|\boldsymbol{x}\|^{2}\right) \\
& =\left(\bar{B}_{0}+\gamma_{j} \bar{B}_{j}\right)\left(\bar{C}_{0}+\gamma_{j} \bar{C}_{j}\right)+\boldsymbol{O}\left(\|\boldsymbol{x}\|^{2}\right)
\end{aligned}
$$

Thus, obtaining $\bar{\Gamma}_{j}^{+}=\left(\bar{C}_{0}+\gamma_{j} \bar{C}_{j}\right)$ as an orthogonal complement of $\left(\bar{B}_{0}+\gamma_{j} \bar{B}_{j}\right)$ for sufficiently small values of $\gamma_{j}$ is enough to ensure that the linear part of equation (a) will also be identically zero, which justifies the steps 2,3 and 4 of the algorithm and completes the proof.

Obtaining a linear matrix ${ }^{\mathrm{I}} \boldsymbol{C}_{\mathscr{M}_{n}}$ from the expression of ${ }^{\mathrm{L}} \boldsymbol{B}_{\mathscr{M}_{n}}$ using the algorithm described in Proposition 4.4, it can be stated that:

$$
\begin{aligned}
& { }^{\mathrm{I}} \boldsymbol{S}_{\mathscr{M}_{n}}={ }^{\mathrm{I}}\left({ }^{\mathrm{I}} \boldsymbol{R}_{\mathscr{U}_{n}}{ }^{\mathrm{I}} \boldsymbol{C}_{\mathscr{M}_{n}}\right) \\
& { }^{\mathrm{I}} \overline{\boldsymbol{f}}_{\mathscr{U}_{n}}={ }^{\mathrm{I}}\left({ }^{\mathrm{I}} \boldsymbol{C}_{\mathscr{U}_{n}} \cdot{ }^{\mathrm{I}} \boldsymbol{f}_{\mathscr{U}_{n}}\right)
\end{aligned}
$$

Finally, the linear algebraic equations ${ }^{\mathrm{I}} \overline{\boldsymbol{q}}_{\mathcal{M}_{n}}^{\langle\sigma\rangle}=0$ and ${ }^{\mathrm{L}} \overline{\boldsymbol{f}}_{\mathcal{M}_{n}}=0$ can be solved in

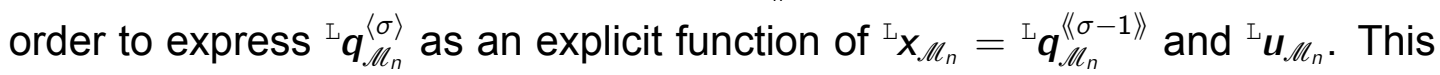
allows to replace the $\sigma$-th order generalized variables ${ }^{\mathrm{L}} \boldsymbol{q}_{\mathcal{M}_{n}}^{\langle\sigma\rangle}$ in the explicit differential equations for obtaining ${ }^{\mathrm{L}} \dot{\boldsymbol{x}}_{\mathscr{U}_{n}}={ }^{\mathrm{L}} \dot{\boldsymbol{q}}_{\mathcal{U}_{n}}^{\langle\langle\sigma-1\rangle}$, which will lead to the following linearized equations of motion for $\mathscr{M}_{n}$ :

$$
{ }^{\mathrm{I}} \dot{\boldsymbol{X}}_{\mathscr{M}_{n}}=\overline{\boldsymbol{G}}_{\mathscr{M}_{n}}{ }^{\mathrm{I}} \boldsymbol{X}_{\mathscr{U}_{n}}+\overline{\boldsymbol{H}}_{\mathscr{M}_{n}}{ }^{\mathrm{I}} \boldsymbol{u}_{\mathscr{M}_{n}}
$$

Example 4.6 (Modeling of a spherical pendulum). Back to Example 4.1, consider the second version of the model in Cartesian coordinates. It is already 
known that:

$$
\begin{aligned}
& \overline{\boldsymbol{q}}^{\langle 2\rangle}=\left[\begin{array}{c}
2 x \ddot{x}+2 y \ddot{y}+2 z \ddot{z}+2 v \\
2 \dot{x} \ddot{x}+2 \dot{y} \ddot{y}+2 \dot{z} \ddot{z}-\dot{v}
\end{array}\right]=0 \\
& \begin{array}{llll}
\ddot{x} & \ddot{y} & \ddot{z} & \dot{v}
\end{array} \\
& \boldsymbol{A}=\left[\begin{array}{cccc}
2 x & 2 y & 2 z & 0 \\
2 \dot{x} & 2 \dot{y} & 2 \dot{z} & -1
\end{array}\right]
\end{aligned}
$$

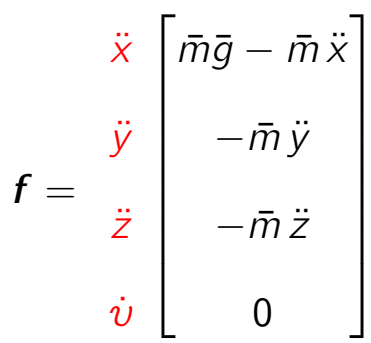

Adopting as reference state for linearization $\boldsymbol{q}^{\langle 0\rangle}=(\bar{a}, 0,0)$ and $\boldsymbol{q}^{\langle 1\rangle}=(0,0,0,0)$, for which $\boldsymbol{q}^{\langle 2\rangle}=\dot{\boldsymbol{q}}^{\langle 1\rangle}=(0,0,0,0)$, it can be stated that:

$$
\begin{aligned}
& { }^{\mathrm{L}} \overline{\boldsymbol{q}}^{\langle 2\rangle}=\left[\begin{array}{c}
2 a{ }^{\mathrm{L}} \ddot{x}+2^{\mathrm{L}} v \\
{ }^{\mathrm{L}} \dot{v}
\end{array}\right]=\mathbf{0} \\
& { }^{\mathrm{L}} \boldsymbol{A}=\left[\begin{array}{cccc}
2\left(\bar{a}+{ }^{\mathrm{L}} X\right) & 2^{\mathrm{L}} y & 2^{\mathrm{L}} z & 0 \\
2^{\mathrm{L}} \dot{x} & 2^{\mathrm{L}} \dot{y} & 2^{\mathrm{L}} \dot{z} & -1
\end{array}\right]
\end{aligned}
$$

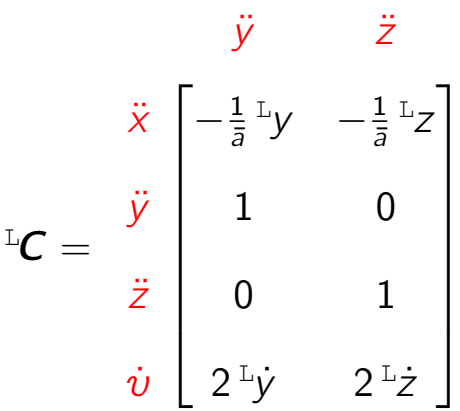

Noting that the expression of $f$ of this model is already linear, the following 
linearized equations of motion are obtained:

$$
{ }^{\mathrm{L}} \overline{\boldsymbol{f}}=\left[\begin{array}{c}
-\bar{m}\left(\frac{\bar{g}}{\bar{a}}{ }^{\mathrm{L}} y+{ }^{\mathrm{L} \ddot{y})}\right. \\
-\bar{m}\left(\frac{\bar{g}}{\bar{a}}{ }^{\mathrm{L}} z+{ }^{\mathrm{L}} \ddot{z}\right)
\end{array}\right]=0
$$

Omitting the pre-superscripts $\mathrm{L}$ for the sake of simplicity:

$$
\left\{\begin{array}{l}
\ddot{x}=-\frac{v}{\bar{a}} \\
\ddot{y}=-\frac{\bar{g}}{\bar{a}} y \\
\ddot{z}=-\frac{\bar{g}}{\bar{a}} z \\
\dot{v}=0
\end{array}\right.
$$

Note that the linearized equations correspond to a uniformly accelerated motion for coordinate $x$ (whose acceleration is proportional to the magnitude of the initial velocity of the pendulum) and to simple harmonic motions, whose angular frequencies are $\bar{\omega}=\sqrt{\bar{g} / \bar{a}}$ for the coordinates $y$ and $z$.

Consider now the reference state $\boldsymbol{q}^{\langle 0\rangle}=\left(\frac{4}{5} \bar{a}, \frac{3}{5} \bar{a}, 0\right)$ and $\boldsymbol{q}^{\langle 1\rangle}=(0,0,0,0)$, for which $\dot{\boldsymbol{q}}^{\langle 1\rangle}=\left(\frac{9}{25} \bar{g},-\frac{12}{25} \bar{g}, 0,0\right)$. Taking this state as reference for linearizing the model, it can be stated that (omitting the pre-superscripts $\mathrm{L}$ for the sake of simplicity):

$$
\begin{aligned}
& { }^{\mathrm{L}} \boldsymbol{A}=\left[\begin{array}{cccc}
\frac{8 \bar{a}}{5}+2 x & \frac{6 \bar{a}}{5}+2 y & 2 z & 0 \\
2 \dot{x} & 2 \dot{y} & 2 \dot{z} & -1
\end{array}\right]
\end{aligned}
$$

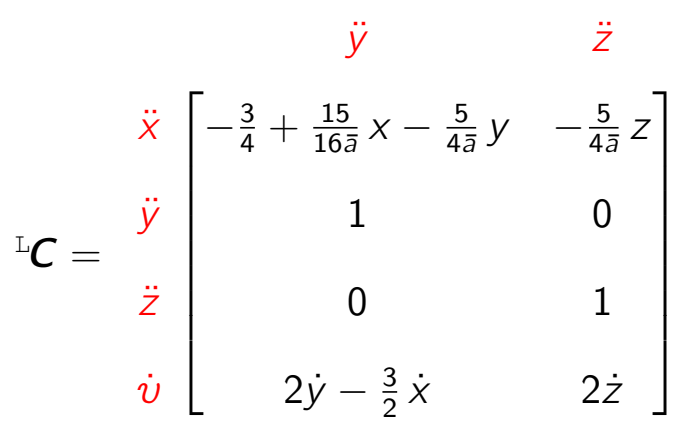


Thus, the dynamic equations of motion of the spherical pendulum are given by:

$$
\left\{\begin{array}{l}
\ddot{x}=-\frac{72 \bar{g}}{125 \bar{a}} x+\frac{96 \bar{g}}{125 \bar{a}} y-\frac{4}{5 \bar{a}} v \\
\ddot{y}=+\frac{21 \bar{g}}{125 \bar{a}} x-\frac{28 \bar{g}}{125 \bar{a}} y-\frac{3}{5 \bar{a}} v \\
\ddot{z}=-\frac{4 \bar{g}}{5 \bar{a}} z \\
\dot{v}=\frac{18 \bar{g}}{25} \dot{x}-\frac{24 \bar{g}}{25} \dot{y}
\end{array}\right.
$$

The eigenvalues of this system of linear ODEs are $\bar{\lambda}_{1}=0$ with algebraic multiplicity equal to 3 and $\bar{\lambda}_{2}=-i \bar{\omega}$ e $\bar{\lambda}_{3}=+i \bar{\omega}$, both with algebraic multiplicity equal to 2 and with $\bar{\omega}=\sqrt{4 \bar{g} / 5 \bar{a}}$.

Example 4.7. In this example the linearized equations of motion of the model studied in Example 4.2 are derived. Adopting the notation $c_{\theta}^{\bullet}=\cos (\bar{\xi}-\theta)$, $\mathbf{s}_{\theta}^{\bullet}=\sin (\bar{\xi}-\theta), c_{\bar{\xi}}=\cos (\bar{\xi})$ and $s_{\bar{\xi}}=\sin (\bar{\xi})$, it can be stated that:

$$
\begin{aligned}
& \overline{\boldsymbol{q}}^{\langle 2\rangle}=\left[\begin{array}{c}
\dot{v}_{1} \\
\dot{u}_{1}+\bar{r}_{1} \dot{\omega}_{1} \\
s_{\theta}^{\bullet} \omega_{1} \dot{\theta} \bar{r}_{1}-s_{\theta}^{\bullet} \omega_{2} \dot{\theta} \bar{r}_{2}+\dot{u}_{1}-\dot{u}_{2}+c_{\theta}^{\bullet}\left(\bar{r}_{1} \dot{\omega}_{1}-\bar{r}_{2} \ddot{z}_{2}\right) \\
-c_{\theta}^{\bullet} \omega_{1} \dot{r}_{1}+c_{\theta}^{\bullet} \omega_{2} \dot{\theta}_{2}+\dot{v}_{1}-\dot{v}_{2}+s_{\theta}^{\bullet}\left(\bar{r}_{1} \dot{\omega}_{1}-\bar{r}_{2} \ddot{z}_{2}\right) \\
-\dot{u}_{1}+\dot{u}_{2}+\left(\bar{r}_{1}-\bar{r}_{2}\right)\left(s_{\theta} \dot{\theta}^{2}+c_{\theta}^{\bullet} \ddot{\theta}\right)
\end{array}\right] \\
& \bar{f}=\left[\begin{array}{c}
\bar{m}_{2}\left(-\bar{g} s_{1}+\bar{g} s_{\bar{\xi}}+\dot{v}_{2} s_{\theta}^{\bullet}+\dot{u}_{2}\left(c_{\theta}^{\dot{\theta}}-1\right)\right)+\bar{m}_{1}\left(\bar{g} s_{\bar{\xi}}+\dot{\omega}_{1} \bar{r}_{1}-\dot{u}_{1}\right) \\
2 \bar{m}_{1}\left(\bar{g} s_{\bar{\xi}}+\dot{\omega}_{1} \bar{r}_{1}-\dot{u}_{1}\right)+\bar{m}_{2}\left(2 \bar{g} s_{\bar{\xi}}+\ddot{z}_{2} \bar{r}_{2}-2 \dot{u}_{2}\right)
\end{array}\right]
\end{aligned}
$$

The objective is to obtain the linearized equations of motion for this system in the neighborhood of a state in which the value of $\theta$ is constant and equal to $\bar{\theta}$, which is given by the equation:

$$
\frac{\bar{m}_{2}}{\bar{m}_{1}}=\frac{\sin (\bar{\theta})}{2 \sin (\bar{\xi}) \cos (\bar{\theta}-\bar{\xi})-3 \sin (\bar{\theta})+\sin (\bar{\xi})}-1
$$

Thus, assume that the reference state is given by $\boldsymbol{q}^{\langle 0\rangle}=(\bar{\theta})$ and $\boldsymbol{q}^{\langle 1\rangle}=0$, with $\bar{\theta}=\underline{\theta}\left(\bar{\xi}, \bar{m}_{2} / \bar{m}_{1}\right)$. In this state:

$$
\dot{u}_{1}=\dot{u}_{2}=-\bar{r}_{1} \dot{\omega}_{1}=-\bar{r}_{2} \dot{\omega}_{2}=\frac{2 \bar{g} \sin (\bar{\theta})}{2 \cos (\bar{\theta}-\bar{\xi})+1} \quad \dot{v}_{1}=\dot{v}_{2}=\ddot{\theta}=0
$$

Assuming these conditions, the linearized model of the system is given by (omit- 
ting the pre-superscripts $L$ for the sake of simplicity):

$$
\left[\begin{array}{c}
\dot{\theta} \\
\dot{u}_{1} \\
\dot{v}_{1} \\
\dot{\omega}_{1} \\
\dot{u}_{2} \\
\dot{v}_{2} \\
\dot{\omega}_{2} \\
\ddot{\theta}
\end{array}\right]=\left[\begin{array}{cccccccc}
0 & 0 & 0 & 0 & 0 & 0 & 0 & 1 \\
\bar{G}_{u_{1}, \theta} & 0 & 0 & 0 & 0 & 0 & 0 & 0 \\
0 & 0 & 0 & 0 & 0 & 0 & 0 & 0 \\
\bar{G}_{\omega_{1}, \theta} & 0 & 0 & 0 & 0 & 0 & 0 & 0 \\
\bar{G}_{u_{2}, \theta} & 0 & 0 & 0 & 0 & 0 & 0 & 0 \\
\bar{G}_{v_{2}, \theta} & 0 & 0 & 0 & 0 & 0 & 0 & 0 \\
\bar{G}_{\omega_{2}, \theta} & 0 & 0 & 0 & 0 & 0 & 0 & 0 \\
\bar{G}_{\dot{\theta}, \theta} & 0 & 0 & 0 & 0 & 0 & 0 & 0
\end{array}\right]\left[\begin{array}{c}
\theta \\
u_{1} \\
v_{1} \\
\omega_{1} \\
u_{2} \\
v_{2} \\
\omega_{2} \\
\dot{\theta}
\end{array}\right]
$$

with:

$$
\begin{aligned}
& \bar{G}_{u_{1}, \theta}=\frac{2 \bar{g}(\cos (\bar{\theta})+2 \cos (\bar{\xi}))(3 \sin (\bar{\theta})-\sin (\bar{\xi})+\sin (\bar{\theta}-2 \bar{\xi}))}{3 \sin (\bar{\theta})+5 \sin (2 \bar{\theta}-\bar{\xi})-2 \sin (\bar{\xi})+5 \sin (\bar{\theta}-2 \bar{\xi})+3 \sin (3 \bar{\theta}-2 \bar{\xi})+3 \sin (2 \bar{\theta}-3 \bar{\xi})+\sin (3 \bar{\theta}-4 \bar{\xi})} \\
& \bar{G}_{\omega_{1}, \theta}=-\frac{2 \bar{g}(\cos (\bar{\theta})+2 \cos (\bar{\xi}))(3 \sin (\bar{\theta})-\sin (\bar{\xi})+\sin (\bar{\theta}-2 \bar{\xi}))}{(3 \sin (\bar{\theta})+5 \sin (2 \bar{\theta}-\bar{\xi})-2 \sin (\bar{\xi})+5 \sin (\bar{\theta}-2 \bar{\xi})+3 \sin (3 \bar{\theta}-2 \bar{\xi})+3 \sin (2 \bar{\theta}-3 \bar{\xi})+\sin (3 \bar{\theta}-4 \bar{\xi})) \bar{r}_{1}} \\
& \bar{G}_{u_{2}, \theta}=\frac{\bar{g}(\cos (\bar{\theta})+2 \cos (\bar{\xi}))(3 \sin (\bar{\theta})+5(\sin (2 \bar{\theta}-\bar{\xi})+\sin (\bar{\theta}-2 \bar{\xi}))+3 \sin (2 \bar{\theta}-3 \bar{\xi}))}{(2 \cos (\bar{\theta}-\bar{\xi})+1)^{2}(2 \sin (\bar{\theta})+3 \sin (2 \bar{\theta}-\bar{\xi})-2 \sin (\bar{\xi})+2 \sin (\bar{\theta}-2 \bar{\xi})+\sin (2 \bar{\theta}-3 \bar{\xi}))} \\
& \bar{G}_{v_{2}, \theta}=\frac{\bar{g}(\cos (\bar{\theta})+2 \cos (\bar{\xi})) \sin (\bar{\xi})}{(2 \cos (\bar{\theta}-\bar{\xi})+1)(3 \cos (\bar{\theta})+2 \cos (\bar{\xi})+\cos (\bar{\theta}-2 \bar{\xi}))} \\
& \bar{G}_{\omega_{2}, \theta}=-\frac{2 \bar{g}(\cos (\bar{\theta})+2 \cos (\bar{\xi}))(\sin (\bar{\theta})+3(\sin (2 \bar{\theta}-\bar{\xi})+\sin (\bar{\theta}-2 \bar{\xi}))+\sin (2 \bar{\theta}-3 \bar{\xi}))}{(2 \cos (\bar{\theta}-\bar{\xi})+1)^{2}(2 \sin (\bar{\theta})+3 \sin (2 \bar{\theta}-\bar{\xi})-2 \sin (\bar{\xi})+2 \sin (\bar{\theta}-2 \bar{\xi})+\sin (2 \bar{\theta}-3 \bar{\xi})) \bar{r}_{2}} \\
& \bar{G}_{\dot{\theta}, \theta}=\frac{2 \bar{g}(\cos (\bar{\theta})+2 \cos (\bar{\xi})) \sin (\bar{\xi})}{(2 \cos (\bar{\theta}-\bar{\xi})+1)(2 \sin (\bar{\theta})+3 \sin (2 \bar{\theta}-\bar{\xi})-2 \sin (\bar{\xi})+2 \sin (\bar{\theta}-2 \bar{\xi})+\sin (2 \bar{\theta}-3 \bar{\xi}))\left(\bar{r}_{1}-\bar{r}_{2}\right)}
\end{aligned}
$$

When $\bar{r}_{1}>\bar{r}_{2}$ (i.e. the radius of the ring is greater that the radius of the disc), the eigenvalues of the system of linear ODEs are $\lambda_{1}=0$ with algebraic multiplicity equal to $6, \bar{\lambda}_{2}=-\mathrm{i} \bar{\omega}$ and $\bar{\lambda}_{3}=+\mathrm{i} \bar{\omega}$ both with algebraic multiplicity equal to 1 and with $\bar{\omega}=\sqrt{-\bar{G}_{\dot{\theta}, \theta}}$.

Adopt for this system the parameters proposed by Holics (2011), i.e. $\bar{r}_{1}=$ $1 \mathrm{~m}, \bar{r}_{2}=0.3 \mathrm{~m}, \bar{m}_{1}=0.670 \mathrm{~kg}$. The charts in Figures 4.15 and 4.16 show respectively the values of the reference angular coordinate $\bar{\theta}$ and of the angular frequency $\bar{\omega}$ of the system as functions of $\bar{\xi}$ for the following values of the ratio $\bar{m}_{2} / \bar{m}_{1}: 0,8 / 67,1 / 2,1,5$. 


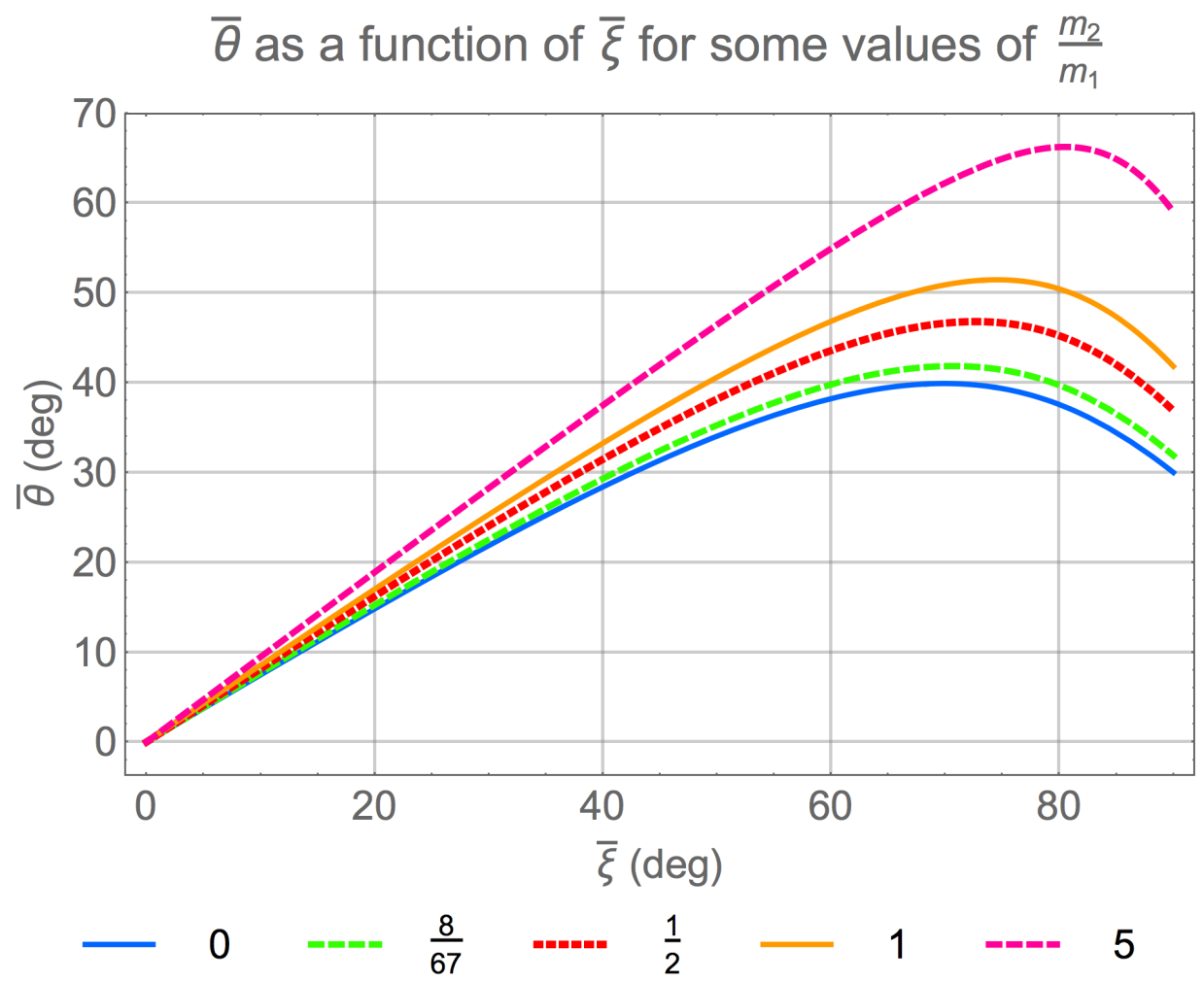

Figure 4.15: Stable value of $\theta$ as a function of the inclination angle $\bar{\xi}$ for several values of the ratio $\bar{m}_{2} / \bar{m}_{1}$.

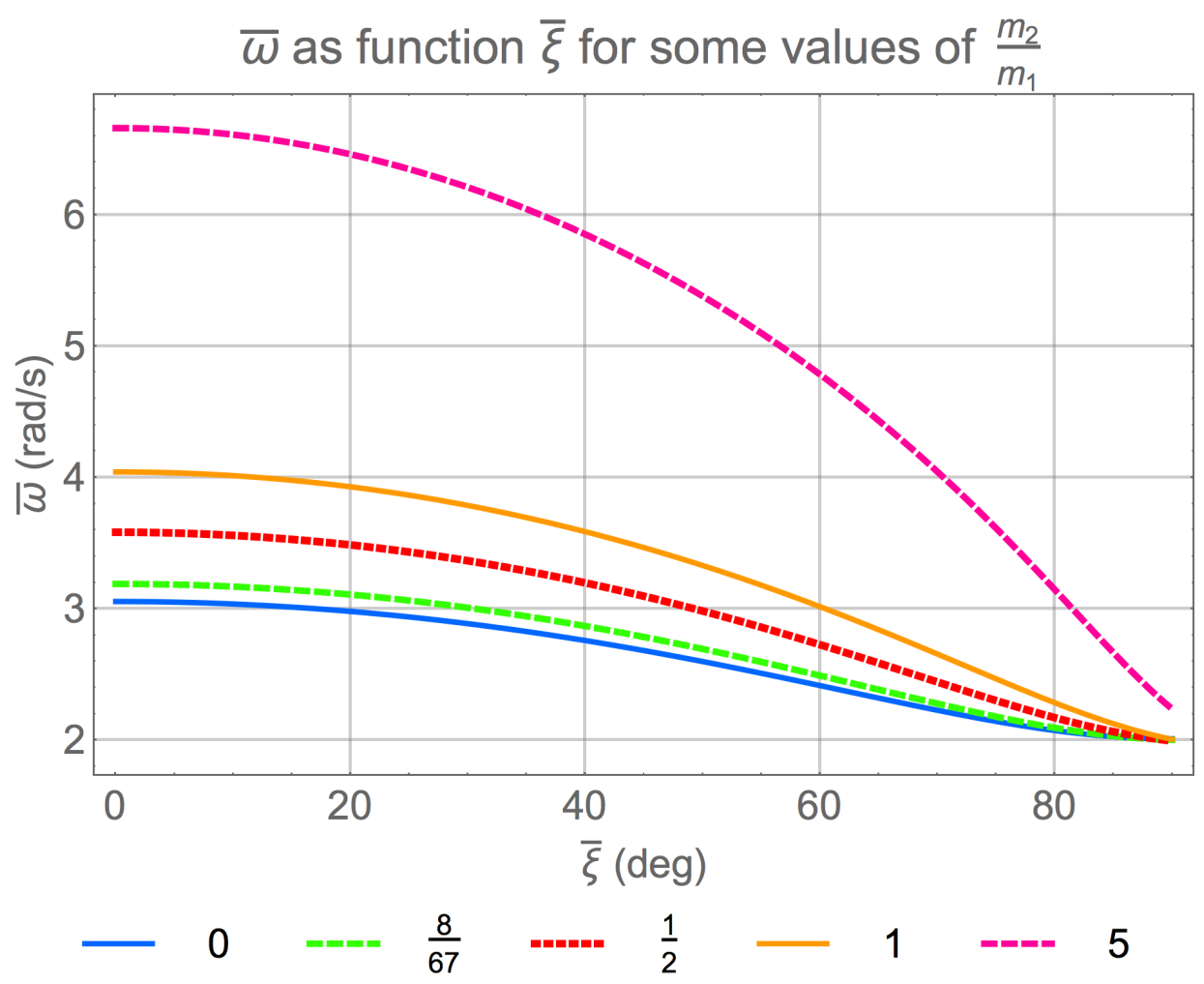

Figure 4.16: Natural frequency $\bar{\omega}$ as a function of the inclination angle $\bar{\xi}$ for several values of the ratio $\bar{m}_{2} / \bar{m}_{1}$.

In order to perform forward dynamics simulations of both non-linear and linearized models of the system, assume that the ratio $\bar{m}_{2} / \bar{m}_{1}=8 / 67$ and that 
the inclination angle $\bar{\xi}=\arcsin (4 / 5) \approx 53^{\circ} 08^{\prime}$, such that $\bar{\theta}=\arcsin (3 / 5) \approx 36^{\circ} 52^{\prime}$. Adopt as initial conditions $\boldsymbol{q}^{\langle 0\rangle}(0)=(\bar{\theta}+\pi / 6)$ and $\boldsymbol{q}^{\langle 1\rangle}(0)=0$. Denote with the pre-superscript $\mathrm{R}$ the reference solution obtained when the initial conditions are ${ }^{\mathrm{R}} \boldsymbol{q}^{\langle 0\rangle}(0)=(\bar{\theta})$ and ${ }^{\mathrm{R}} \boldsymbol{q}^{\langle 1\rangle}(0)=0$. Figures $4.17-4.20$ show the time histories of some of the generalized of the models (subtracting the reference solution values), obtained from the numerical simulations of both non-linear (NL) and linearized $(\mathrm{L})$ models.

Note that, in these simulations, the linearized model can adequately reproduce the period and the amplitude of oscillations in the neighborhood of the reference solution. The assessment on how satisfactory these approximated solutions are, depend on the desired applications for the results of the numerical simulations.

Finally, in order to evaluate the numerical error of the simulation of the nonlinear model (as in Example 4.5, the function NDSolve of Mathematica 10.2 was applied), Figure 4.21 shows the time history of the numerical error computed by the expression $\sqrt{\left\|\overline{\boldsymbol{q}}^{\langle 2\rangle}\right\|^{2}+\|\overline{\boldsymbol{f}}\|^{2}}$. Note that the algorithms used in this simulation keep the numerical error under control.

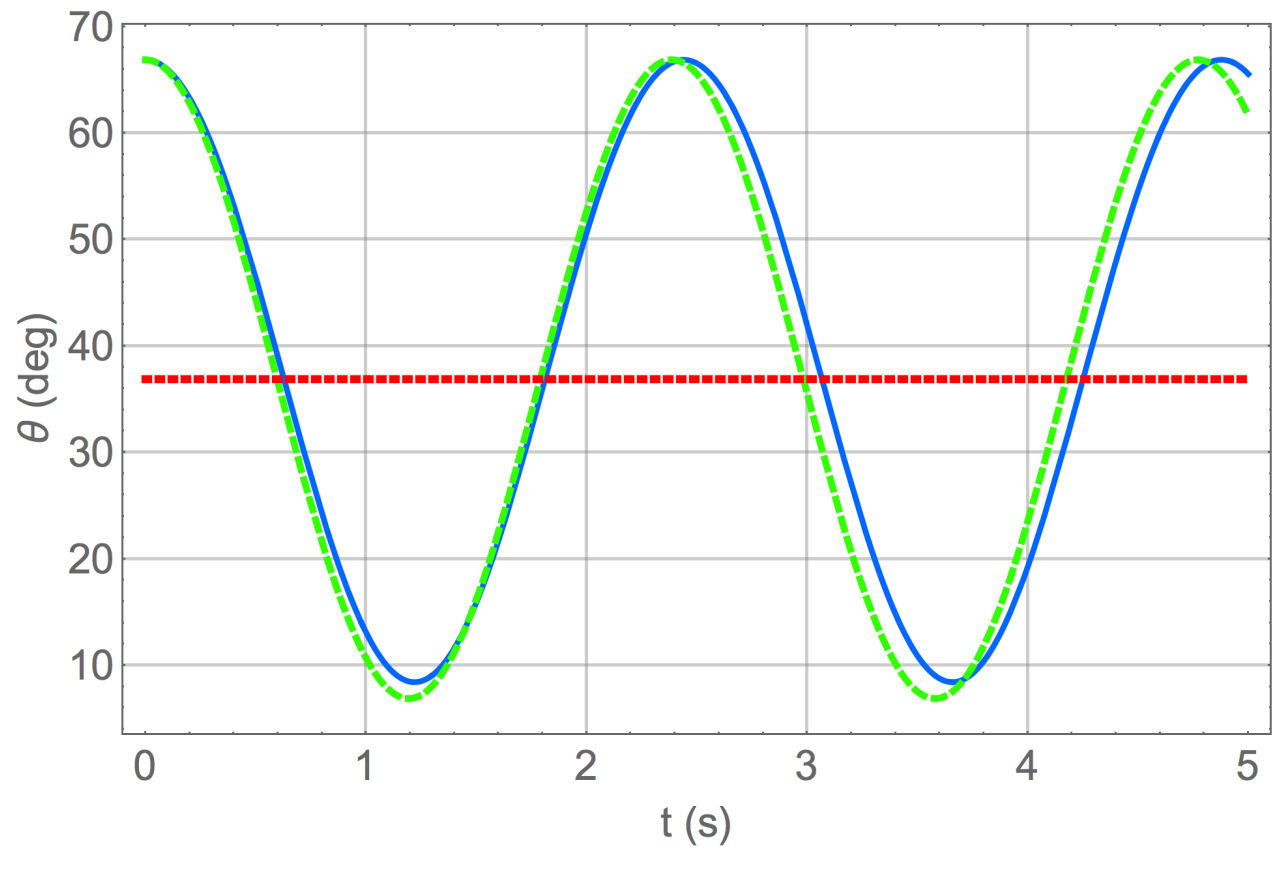

$\longrightarrow \quad \mathrm{NL} \quad----\quad \mathrm{L} \quad \cdots \cdots \quad \mathrm{R}$

Figure 4.17: Time histories of the angle $\theta$ obtained by the non-linear (NL) and linearized $(L)$ models compared with the reference value $(R)$ of the angle. 


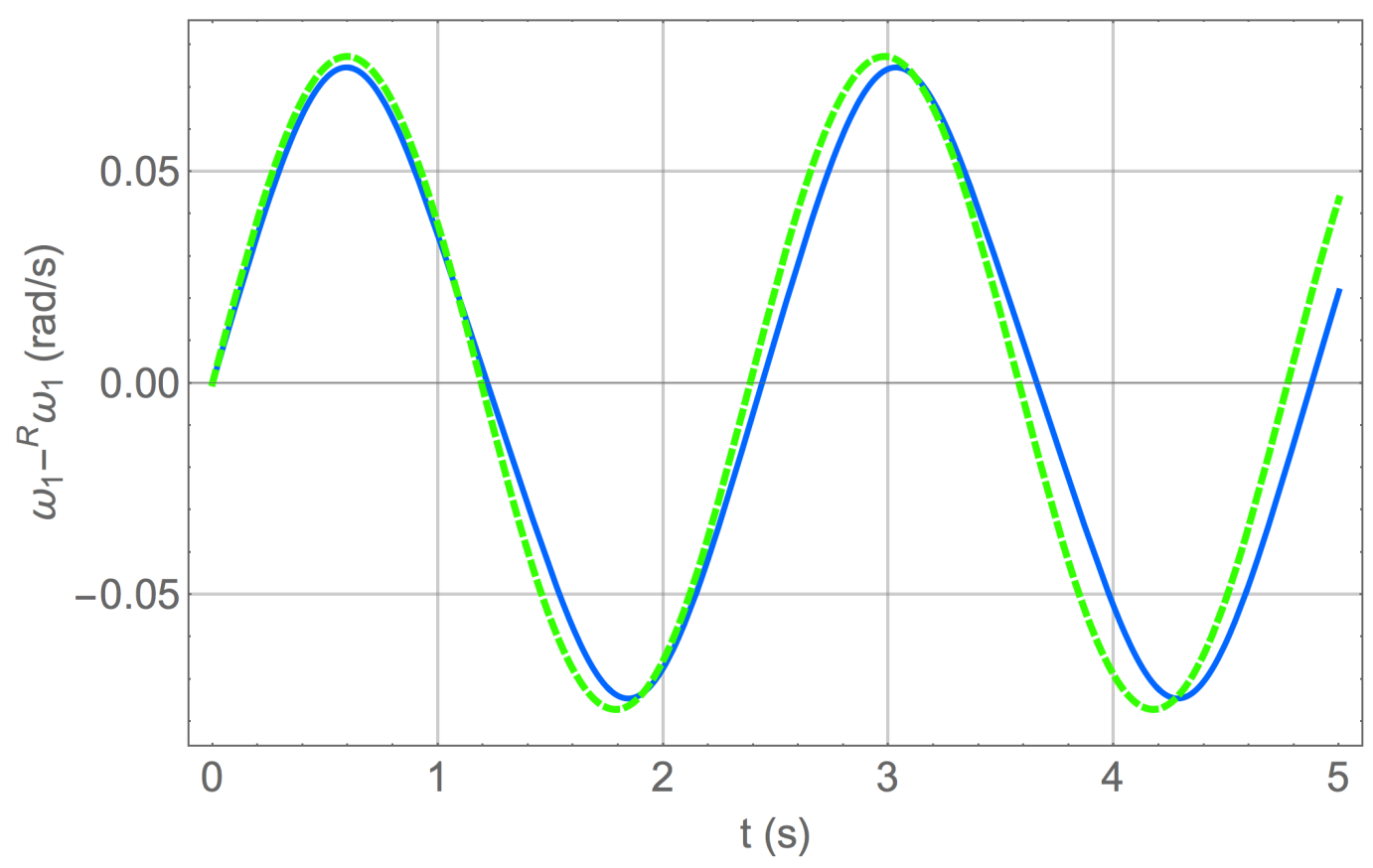

$-\quad \mathrm{NL}$

Figure 4.18: Time histories of $\omega_{1}-{ }^{R} \omega_{1}$ obtained by the non-linear (NL) and linearized (L) models.

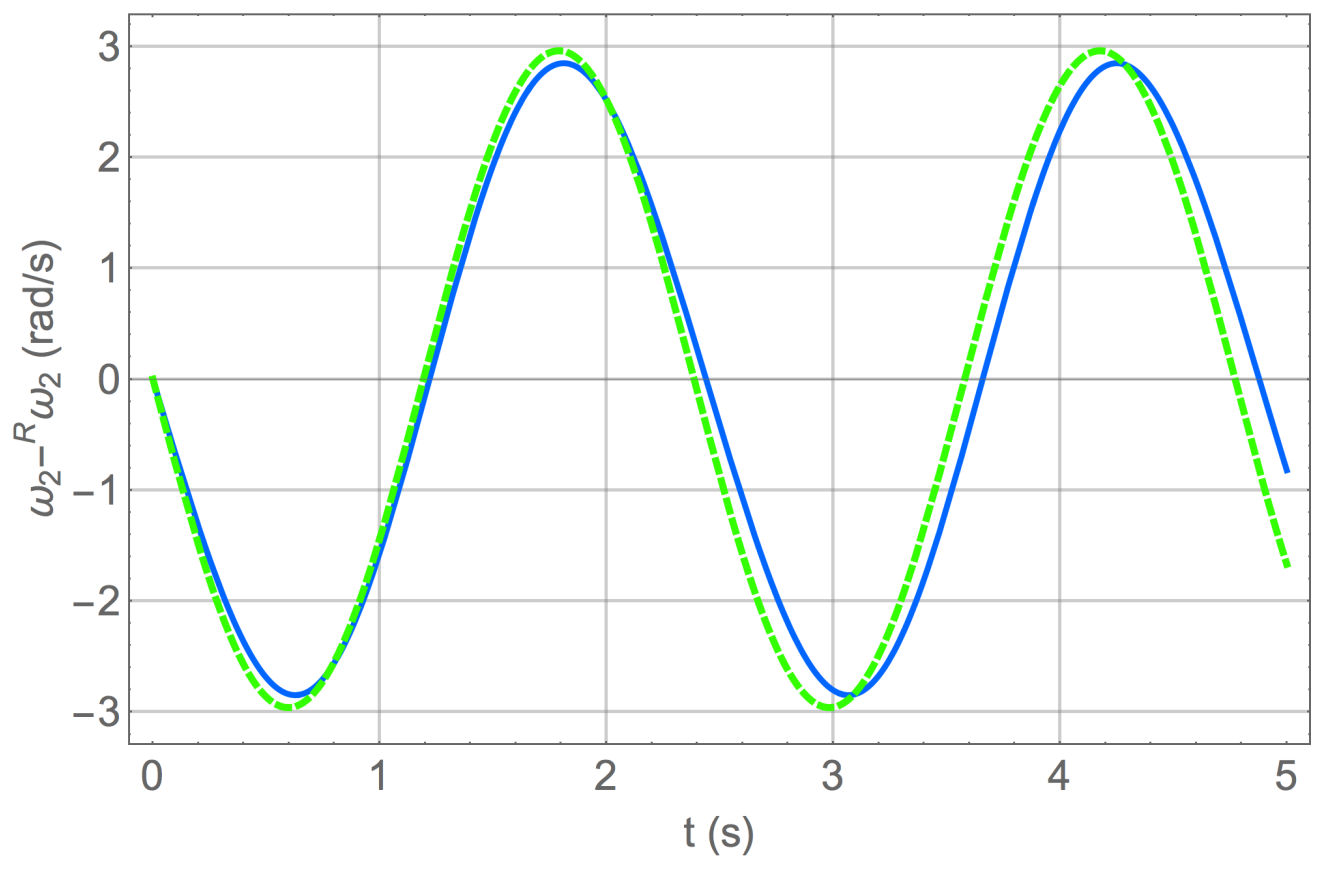

$\longrightarrow \quad \mathrm{NL} \quad \ldots-\overline{\mathrm{L}}$

Figure 4.19: Time histories of $\omega_{2}-{ }^{R} \omega_{2}$ obtained by the non-linear (NL) and linearized $(\mathrm{L})$ models. 


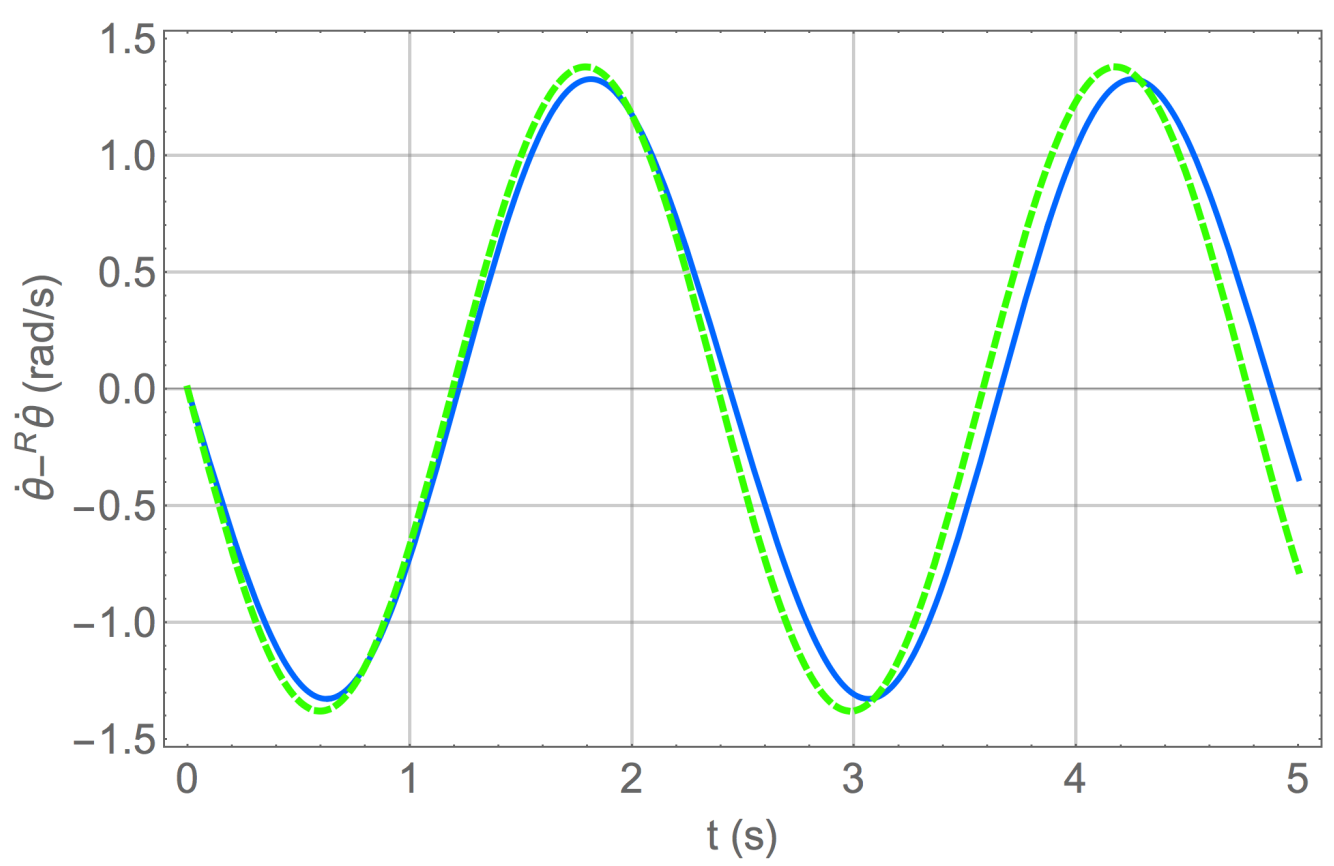

NL

Figure 4.20: Time histories of $\dot{\theta}-{ }^{\mathrm{R}} \dot{\theta}$ obtained by the non-linear (NL) and linearized $(L)$ models.

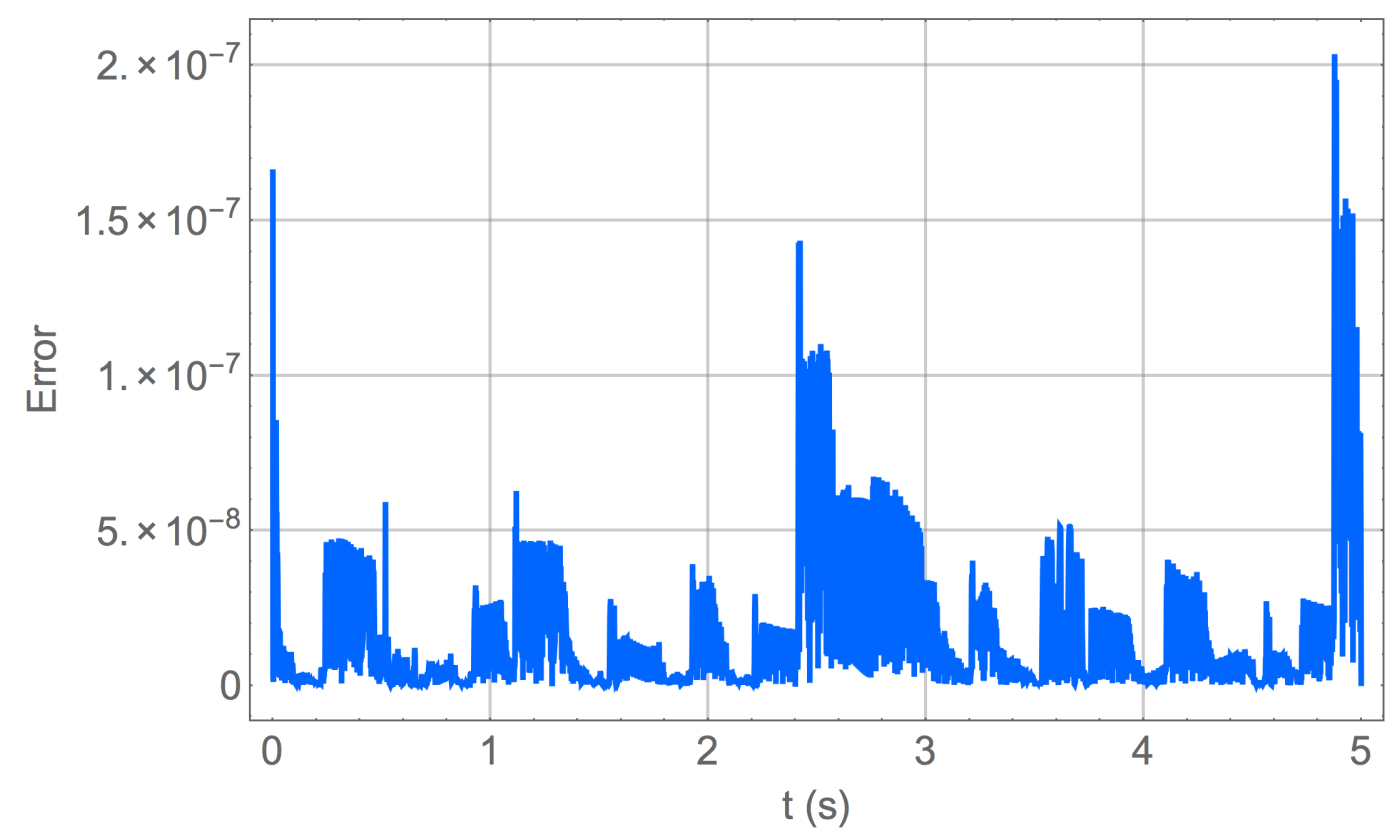

Figure 4.21: Numerical error evaluation in the forward dynamics simulation of the non-linear model. 


\subsection{Qualitative comparison between modeling methodologies}

This last section of Chapter 4 is dedicated to a qualitative comparison among the conventional modeling methodologies applied for the modeling of multibody system and the novel methodology discussed in this chapter. Based on the literature review presented in Chapter 3 and on the author's personal experience, assessments for each set of methodologies are performed, attributing a score from 0 to 5 points to each of the following eight features it might have:

- Modularity: ability to derive mathematical models of complex multibody systems from already known models of its subsystems, using a particular methodology.

- Recursivity: ability to produce recursive algorithms for the procedures of modeling and simulation of a multibody system.

- Variables: versatility for the selection of generalized variables.

- Number of EOM: how easy is to derive a minimal set of equations of motion applying a particular methodology.

- Algorithmization: how easy is to implement generic algorithms (applicable for a wide range of multibody systems) based on a particular methodology.

- Interpretability: how easy is to physically interpret the meaning of each term in the system of equations of motion derived from a particular methodology.

- Constraints: how easy is to elimine the undesired constraint forces and to find expressions for the ones that might be of interest, using a particular methodology.

- Energy: ability to use energy-like functions in the derivation of the equations of motion of a multibody system, using a particular methodology.

The score $5 / 5$ is assigned to the set of methodologies that has the best performance in a given feature; the score $1 / 5$ represents a poor performance and the score $0 / 5$ means that the particular set of methodologies does not have the corresponding feature. Table 4.2 presents the assessment performed for the following sets of modeling methodologies: 
- Newton-Euler (Section 3.1): modeling methodologies for multi-rigid-body systems based on the use of Newton-Euler equations.

- PVW (Subsections 3.2.1 - 3.2.3): modeling methodologies based on the standard statements of the Principle of Virtual Work and its differential variants (Principle of Virtual Power, Gauss' Principle, etc.)

- Lagrange (Subsections 3.2.4 - 3.2.7): modeling methodologies based on the use of Lagrangian and Hamiltonian canonical equations along with the method of Lagrangian multipliers, if necessary (see Section 3.1).

- Gibbs-Appell (Subsection 3.3.1): modeling methodologies based on the Gibbs-Appell equations.

- Maggi (Subsection 3.3.2): modeling methodologies based on Maggi's equations and the standard forms of orthogonal complement methods.

- Boltzmann-Hamel (Subsection 3.3.3): modeling methodologies based on the Boltzmann-Hamel equations.

- Kane (Subsection 3.4.1): Kane's methodology.

- Udwadia-Kalaba (Subsection 3.4.2): Udwadia-Kalaba methodology.

- Orsino (Sections 4.1 and 4.2): novel recursive modular modeling methodology presented in this chapter.

Regarding "modularity", the greatest score is assigned to the novel methodology presented in this text, once the procedure described in Section 4.2 is the most general one among the evaluated ones, being applicable for any multibody system, regardless the particular form of the models of the subsystems involved. The runner-up, scoring $4 / 5$ on "modularity" is Udwadia-Kalaba methodology, whose algorithm requires all the constraints among all the subsystems involved to be considered simultaneously. Scoring 3/5, Newton-Euler based methodologies still perform better than the other formalisms, which score $2 / 5$, once the corresponding modular modeling procedure only involves the inclusion, in the equations of motion of the subsystems, of constraint forces and torques corresponding to each motion constraint among them. For the remaning formalisms no general modular modeling procedure exists, although it is still possible to use several informations of the models of the subsystems in the corresponding derivations. 


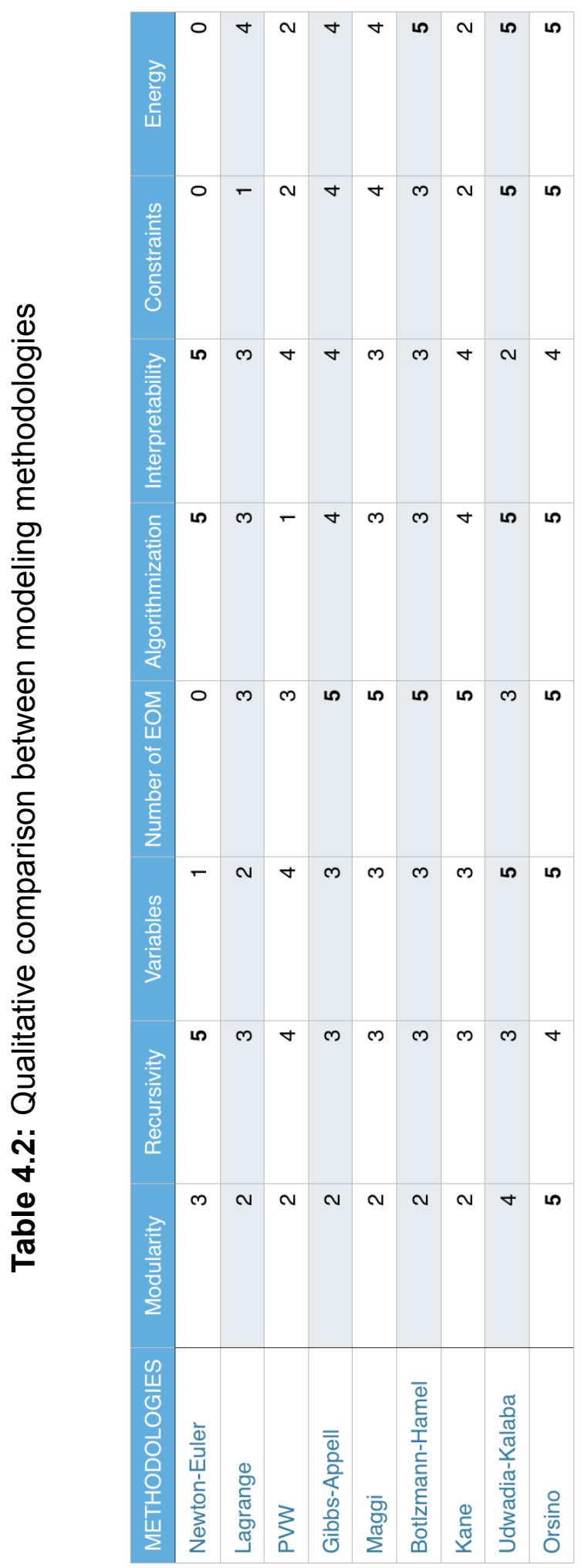


Newton-Euler based methodologies have the best performance regarding "recursivity", once they lead to some of the simpler, most generic and most widely used recursive modeling and simulation algorithms for multi-rigid-body systems. Both the novel methodology presented in this text and PVW based ones score $4 / 5$ on "recursivity" due to the great versatility of the corresponding recursive algorithms. With less versatile recursive algorithms associated, the remaining methodologies score $3 / 5$ on "Recursivity".

In terms of "selection of variables" both Udwadia-Kalaba methodology and the one developed in this chapter can be considered the best ones, once there are no restrictions on the choice of generalized variables in these methodologies. Scoring $4 / 5$, the PVW based methodologies also have no restrictions on the choice of modeling variables. These methodologies, however, lack a general procedure for expressing generic variations in these systems in terms of an independent set of variables that ensure the satisfaction of the constraints involved. Gibbs-Appell, Maggi, Boltzmann-Hamel and Kane's methodologies score $3 / 5$ on "selection of variables", because, although they allow the use both of generalized coordinates and quasi-velocities, they require the user to find a minimal set of quasi-velocities for each system (as much quasi-velocities as the number of degrees of freedom). Lagrange's formalism, that only allows the choice of generalized coordinates (being the higher order generalized variables simply time derivatives of them), scores $2 / 5$, while Newton-Euler formalism, being the less flexible in terms of choice of variables, scores $1 / 5$.

Regarding "number of EOM", all the methodologies that lead to the derivation of minimal systems of equations of motion, with as much dynamic equations as the number of degrees of freedom, score $5 / 5$. Scoring $3 / 5$ are the methods in which the number of equations of motion depends on the number of generalized variables selected. Finally, once Newton-Euler formalism does not allow to limit the number of dynamic equations of motion (which only depends on the number of rigid bodies in the system), its score is $0 / 5$ on "number of EOM".

Regarding "algorithmization", the Newton-Euler based methodologies along with Udwadia-Kalaba and the novel one presented in this text score 5/5: the former ones due to the fact that Newton-Euler equations always preserve their general form independently of the topology of the systems involved, which makes it easier to develop algorithms; the other two methodologies, due to the possibility of fully exploring the modularity of multibody systems, which even allows the use of libraries of subsystems to in the algorithms. Kane and Gibbs- 
Appell methodologies score $4 / 5$, once they are widely used in the formulation of algorithms available in the literature. Lagrange, Maggi and Boltzmann-Hamel score $3 / 5$ due to the complexity involved in formulating efficient algorithm for obtaining generalized effective forces from the expressions of energy-like functions. In the last position, scoring 1/5 comes the PVW based methodologies in which some kind of specialization must be performed (using other procedures than simply the ones based on the fundamental principles of Analytical Mechanics) to be able to implement algorithms, once these methodologies themselves can lead to a wide variety of forms for the EOMs of multibody systems.

Regarding "interpretability", again there is an evident advantage in the application of Newton-Euler based methodologies, once the identification of the physical meaning of each term in each equation is almost immediate. Scoring $4 / 5$ are the methodologies in which the terms in the dynamic equations can be interpreted as projections of forces and torques. The score $3 / 5$ is attributed to methodologies in which energy-like functions are used to derive the equations of motion, making it less simple to identify the meaning of each term. Finally, Udwadia-Kalaba methodology scores 2/5 once the methodology for including external constraints is a purely mathematical algorithm (without physical meaning).

Regarding "constraints", both Udwadia-Kalaba methodology and the novel one presented in this text score $5 / 5$, once both of them enable the inclusion of any constraint that can be expressed by invariants written in terms of generalized variables, without the need of including any undesired constraint force and providing explicit expressions for the desired ones. The same happen with Gibbs-Appell and Maggi's formalisms, with the disadvantage that it is necessary to find a minimal set of quasi-velocities, which make they score $4 / 5$ on "constraints". Scoring 3/5, Boltzmann-Hamel formalism is very similar to GibbsAppell and Maggi's but does not enable, by itself, the derivation of expressions for constraint forces. Kane's and PVW based methodologies score 2/5: the former for not allowing the inclusion of non-linear nonholonomic constraints in its original procedure, and the latter for the lack of a general method for including the effects of constraints in the equations of motion and for obtaining explicit expressões for the eventually desired constraint forces. Scoring $1 / 5$, Lagrangian formalism requires the use of undetermined multipliers whenever there are any nonholonomic constraints or redundant generalized coordinates. Finally, Newton-Euler methodologies score 0/5, once for each constraint there must be included a corresponding constraint force or torque; moreover, there 
is no general method for eliminating the undesired ones from the equations of motion.

In terms of "energy", Hamel-Boltzmann, Udwadia-Kalaba and the novel methodology presented in this text score $5 / 5$ once they enable the use of kinetic energy and other energy-like functions, expressed in terms of generalized variables, in the derivation of the equations of motion of a multibody system. Scoring 4/5, Gibbs-Appell formalism only allows the use of Gibbs-Appell function, which has a more complex expression than the kinetic energy, in the derivation of generalized inertia forces. Maggi's and Lagrangian formalisms also score $4 / 5$, because they require that the kinetic energy to be expressed in terms of the generalized coordinates and its time derivatives, instead of allowing the use of quasi-velocities. With almost no advantage in the use of energy-like functions to the derivations of equations of motion, Kane's and PVW based methodologies score 2/5. Newton-Euler formalism scores $0 / 5$ once it does not involve the use of energy-like functions on any modeling procedure. 


\section{Computational implementations of modular modeling algorithms for multibody systems}

This chapter illustrates the application of the novel modular modeling methodology presented in Chapter 4 to the development of computational algorithms, by showing two actual implementations of packages of functions for Wolfram Mathematica 10.2. The first one, MoSsPack, consists of a set of generic functions that implement all the modeling procedures shown in Table 4.1, requesting the user to provide minimal information about the models of the leaf subsystems and about the external constraints. It also implements the linearization procedures presented in Section 4.5. The second implementation presented, Mo2DPack, is a package for the symbolic derivations and numerical simulations (forward and inverse dynamics) of models of planar mechanisms, that illustrates the potential of the novel modular modeling methodology for the development of specialized computational packages.

The choice of Wolfram Mathematica, which is a proprietary software, for the development of these packages does not mean that some particular closedsource feature is necessary for the implementation of any algorithm presented here. Once the objective of the packages presented in this chapter is merely to illustrate the potential applications of the novel methodology, the use of Mathematica is justified due to the practicality of drafting functions for both symbolic and numerical computations using Wolfram Language along with functional programming. This does not mean, however, that the final version of these packages must be developed in Wolfram Mathematica. Ideally, the final version of any package based on the modular methodology can be developed in an open source programming language which is adequate for both symbolic and numerical computations. PyDy (Multibody Dynamics with Python) project ${ }^{1}$, for instance, is a successful example of the use of Python in the development of packages similar to the ones shown in this chapter.

\footnotetext{
1 Visit http: //www . pydy . org.
} 
Section 5.1 presents a brief introduction to MoSsPack. Section 5.2 shows a case study in which a linearized model of a tadpole tricycle is developed aided by MoSsPack; a brief stability analysis based on the linearized model is also presented. Finally, Section 5.3 presents a brief introduction to Mo2DPack along with some examples of application to the modeling and simulation of planar mechanisms.

\subsection{MoSsPack - recursive modular modeling package for Mathematica 10.2}

The recursive form of the modular modeling methodology presented in Section 4.2 can be implemented as a package of functions that are able to reproduce each procedure listed in Table 4.1 once the required inputs are provided by the user. The first implementation of such an algorithm resulted in MoSsPack package for Wolfram Mathematica 10.2, which can be downloaded, along with its complete documentation, at https://github. com/renatoorsino/MoSsPack. For this package to work properly, it is required another package of generalpurpose and matrix algebra functions package for Mathematica 10 developed by the autor, called MathMatrixPack (also with complete documentation) which can be downloaded at https://github. com/renatoorsino/MathMatrixPack.

Figure 5.1 illustrates the use of MoSsPack package for the modular modeling of a spherical pendulum. In this example, $\mathscr{B}$ denotes a system constituted by a single free rigid body $\mathcal{B}$ in a gravitational field. Its mathematical model is given by the corresponding Newton-Euler equations, so that $\boldsymbol{q}_{\mathscr{B}}^{\langle 0\rangle}=\left(p_{\mathcal{B}, x}, p_{\mathcal{B}, y}, p_{\mathcal{B}, z}\right)$ and $\boldsymbol{q}_{\mathscr{B}}^{\langle 1\rangle}=\left(v_{\mathcal{B}, x}, v_{\mathcal{B}, y}, v_{\mathcal{B}, z}, \omega_{\mathcal{B}, x}, \omega_{\mathcal{B}, y}, \omega_{\mathcal{B}, z}\right)$ with $p_{\mathcal{B}, x}, p_{\mathcal{B}, y}$ and $p_{B, z}$ representing the Cartesian coordinates of the center of mass of $\mathcal{B}$ in a coordinate system rigidly attached to an inertial reference frame $\mathcal{N}, v_{\mathcal{B}, x}, v_{\mathcal{B}, y}$ and $v_{\mathcal{B}, z}$ being the corresponding velocity components of this center of mass of $\mathcal{B}$ and $\omega_{\mathcal{B}, x}, \omega_{\mathcal{B}, y}$ and $\omega_{\mathcal{B}, z}$ being the local basis components of the angular velocity of $\mathcal{B}$, both measured with respect to $\mathcal{N}$. System $\mathcal{S}$ is obtained by constraining the center of mass of $\mathcal{B}$ to move in a spherical surface of radius $\bar{a}$, parametrically described by the invariants:

$$
\overline{\boldsymbol{q}}_{\delta, \oplus}^{\langle 0\rangle}=\left[\begin{array}{c}
p_{\mathcal{B}, \mathrm{x}}-\bar{a} \sin (\theta) \cos (\phi) \\
p_{\mathcal{B}, Y}-\bar{a} \sin (\theta) \sin (\phi) \\
p_{\mathcal{B}, \mathrm{z}}+\bar{a} \cos (\theta)
\end{array}\right]=0
$$




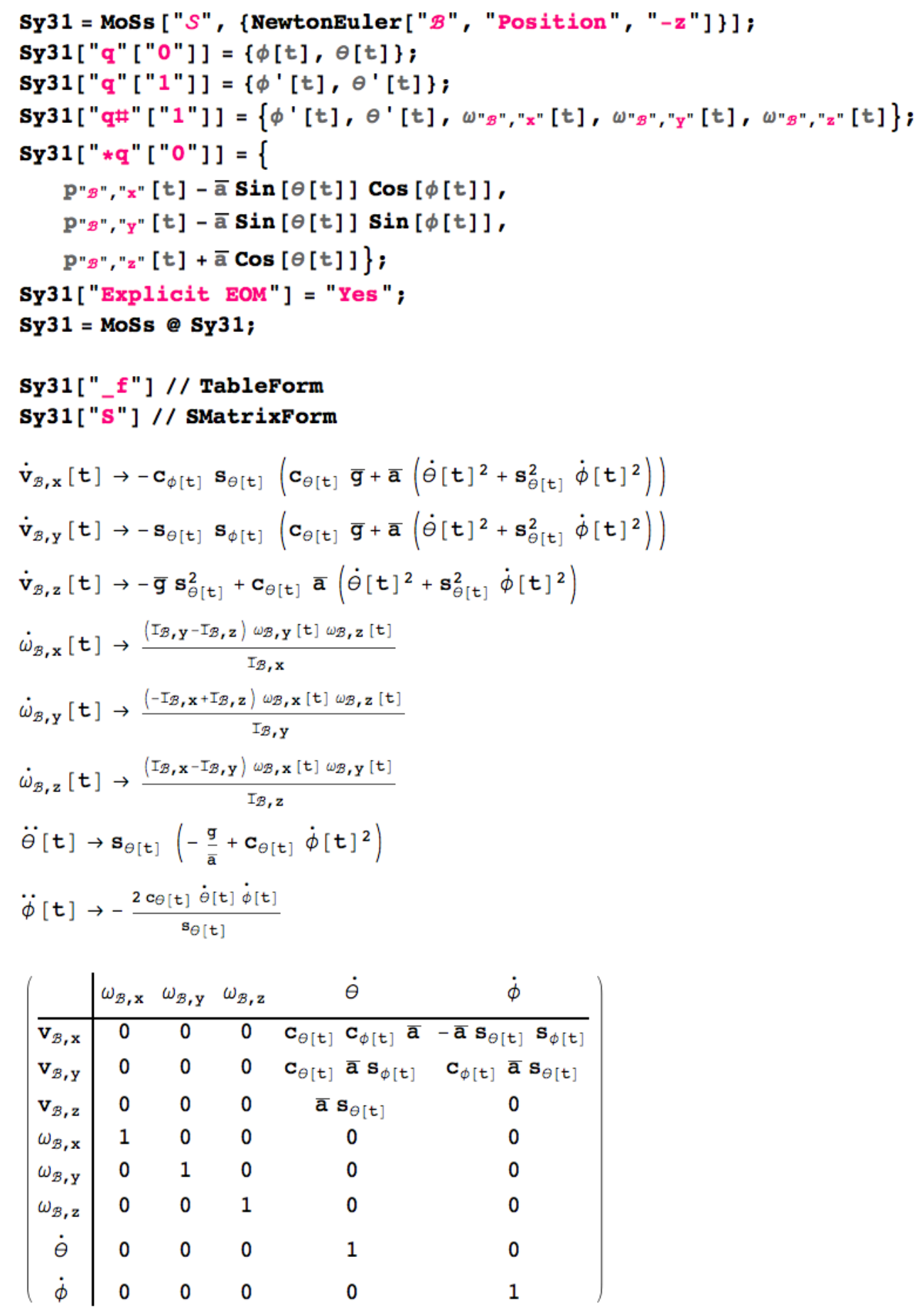

(a) Piece of a Mathematica notebook showing the derivation of the spherical pendulum model

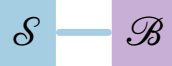

(b) Tree structure diagram of the spherical pendulum model

Figure 5.1: Modelling of a spherical pendulum using MoSsPack. Adapted from the documentation available at https:

//github.com/renatoorsino/MoSsPack/blob/master/doc/MoSsPack.pdf. 
Thus, it is convenient to define the following extra generalized variables for modeling $\delta: \boldsymbol{q}_{\delta . \oplus}^{\langle 0\rangle}=(\phi, \theta)$ and $\boldsymbol{q}_{\delta . \oplus}^{\langle 1\rangle}=(\dot{\phi}, \dot{\theta})$. Moreover, it can be stated that, for every state of $\delta$, the following subset of quasi-velocities is constituted of independent variables: $\boldsymbol{q}_{\delta . \#}^{\langle 1\rangle}=\left(\dot{\phi}, \dot{\theta}, \omega_{\mathcal{B}, x}, \omega_{\mathcal{B}, y}, \omega_{\mathcal{B}, z}\right)$. The outputs presented in Figure 5.1a are the explicit form os the equations of motion of system $\delta$ and the expression of the matrix $S_{\delta}$, respectively.

MoSsPack (used along with MathMatrixPack) not only implements the modular modeling methodology presented in Section 4.2 but also provides the procedures discussed in Section 4.5 for obtaining linearized equations of motion of multibody system based on either non-linear or linearized models of its subsystems. A remarkable attribute of this latter implementation, it that it allows to obtain linearized equations of motion for systems in which the number of constraint invariants exceed the difference between the number of $\nu^{\circ}$-th order generalized variables and the number of degrees of freedom. In these cases, the corresponding orthogonal complements are obtained by a least squares solver instead of a conventional linear solver. When the redundant invariants are compatible, i.e., whenever apart from singularities it is always possible to find in the neighborhood of a given state other states that satisfy all the invariants simultaneously, the calculated orthogonal complement will correspond to an exact solution (even when the least squares solver is used). However, when these invariants are not compatible, i.e., whenever, apart from special cases it is never possible to find in the neighborhood of a given state other states that satisfy all the invariants simultaneously, no exact orthogonal complement will exist and the obtained solution will correspond to a least squares approximation compatible with the norm adopted (in this case, the norm can be used to tune the results according to the physical interpretation of the invariants). This latter case is explored in the case study presented in the next section.

\subsection{Case study: linearized tadpole tricycle model}

\subsubsection{Overview}

In this section a linearized model of a tadpole tricycle is obtained and a brief stability analysis of this system is performed. All the procedures presented here were aided by a Wolfram Mathematica 10.2 script that uses functions from the MoSsPack package (see Section 5.1).

A tadpole tricycle $\mathscr{T}$ will be conceived as an assembly of 8 subsystems (see 
Figure 5.2):

- $\mathscr{W}, \mathscr{F}_{\mathrm{R}}$ and $\mathscr{F}_{\mathrm{L}}$, representing the rear, front right and front left wheels respectively,

- $\mathscr{A}$, representing the rider's body and frame assembly,

- $\mathcal{S}_{\mathrm{R}}$ and $\mathcal{S}_{\mathrm{L}}$, representing the left and right steering bars,

- $\mathscr{K}_{\mathrm{R}}$ and $\mathscr{K}_{\mathrm{L}}$, representing the left and right knuckles.

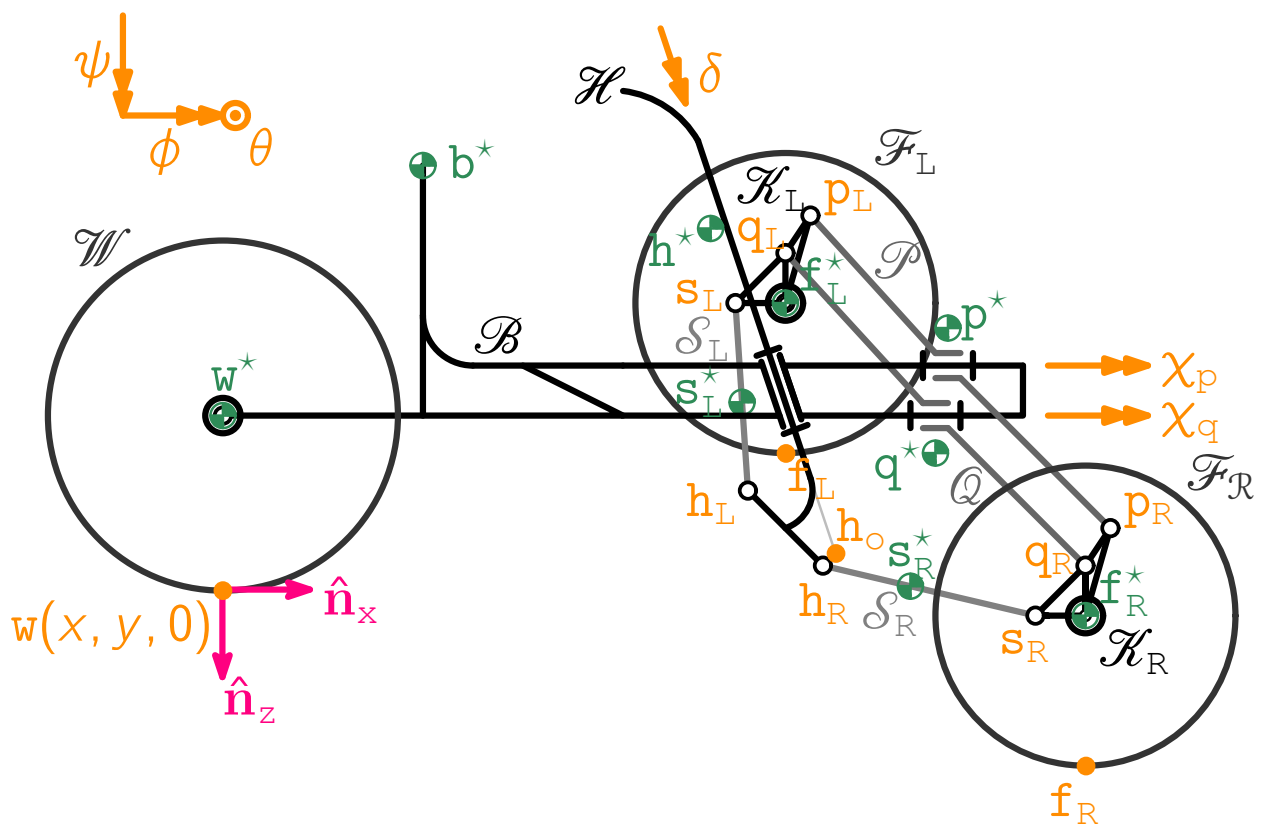

(a) Tadpole tricycle model.

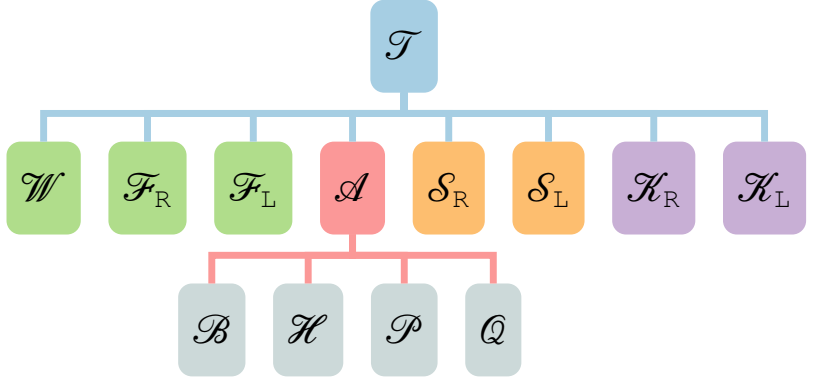

(b) Tree structure diagram of the tadpole tricycle model

Figure 5.2: Representation of system $\mathscr{T}$ and its subsystems

Subsystems $\mathscr{W}, \mathscr{F}_{\mathrm{R}}$ and $\mathscr{F}_{\mathrm{L}}$ are modeled as rigid knife-edge discs rolling in a plane; both geometry and mass distribution of these discs respect a cylindrical symmetry. Moreover, all the wheels are supposed to respect the no-slip constraint, i.e. neither longitudinal nor lateral slipping are allowed in the contact between the disc and the plane. 
Subsystem $\mathscr{A}$ is supposed to be an assembly of 4 other subsystems, each one composed of a single ideal rigid body symmetric (both in terms of geometry and mass distribution) with respect to a local plane that coincides with the (vertical) $y$-plane when the system is in the reference configuration shown in Figure 5.2a:

- $\mathscr{B}$, representing rider's body and the rear frame of the tricycle,

- $\mathscr{H}$, representing the handlebar,

- $\mathscr{P}$, representing the upper transverse rod of the suspension and

- $Q$, representing the lower transverse rod of the suspension

In this model, the rider's body will be considered to be rigidly attached to the rear frame. Moreover, the frame is linked to the handlebar and transverse suspension rods by ideal revolute joints, being negligible any clearance, as well as, any flexibility or damping effects.

Both subsystems $\delta_{\mathrm{R}}$ and $\delta_{\mathrm{L}}$ are constituted by identical rigid bodies with cylindrical symmetry and negligible axial inertia, i.e. they represent thin cylindrical bars. These subsystems are considered to be constrained both to the handlebar and to the respective knuckle by ideal spherical joints. No clearances nor flexibility and damping effects are considered.

Both subsystems $\mathscr{K}_{\mathrm{R}}$ and $\mathscr{K}_{\mathrm{L}}$ are constituted by identical rigid bodies with no special symmetry. They are constrained to the respective front wheels by ideal revolute joints and to the transverse rods of the suspension and steering bars by ideal spherical joints. Again, no clearances nor flexibility and damping effects are considered.

\subsubsection{Rider's body and frame assembly}

Consider that $\mathcal{B}, \mathcal{H}, \mathcal{P}$ and $Q$ denote the rigid bodies of subsystems $\mathscr{B}, \mathscr{H}$, $\mathscr{P}$ and $Q$, respectively. Define local coordinate systems, $B=\left(\mathrm{b}_{\circ}, \hat{\mathbf{b}}_{\mathrm{x}}, \hat{\mathbf{b}}_{\mathrm{y}}, \hat{\mathbf{b}}_{\mathrm{z}}\right)$, $\mathrm{H}=\left(\mathrm{h}_{\circ}, \hat{\mathbf{h}}_{x}, \hat{\mathbf{h}}_{y}, \hat{\mathbf{h}}_{z}\right), \mathrm{P}=\left(\mathrm{p}_{\circ}, \hat{\mathbf{p}}_{x}, \hat{\mathbf{p}}_{y}, \hat{\mathbf{p}}_{z}\right)$ and $\mathrm{Q}=\left(\mathrm{q}_{\circ}, \hat{\mathbf{q}}_{x}, \hat{\mathbf{q}}_{\mathrm{y}}, \hat{\mathbf{q}}_{z}\right)$, respectively, rigidly attached to of each of these rigid bodies. Let $\mathcal{N}$ be an inertial reference frame and denote by $\mathrm{N}=\left(\mathrm{n}_{\circ}, \hat{\mathbf{n}}_{\mathrm{x}}, \hat{\mathbf{n}}_{\mathrm{y}}, \hat{\mathbf{n}}_{z}\right)$ a coordinate system rigidly attached to $\mathcal{N}$.

Consider that the origin of $\mathrm{B}$ coincides, in the reference configuration, with the rear wheel contact point, i.e. $\mathrm{n}_{\circ}=\mathrm{w}$, the origin $\mathrm{h}_{\circ}$ of $\mathrm{H}$ coincides with the 
intersection of the local $x$-axis of $B$ and the steering axis and the origin of $P(Q)$ is defined such that:

- $p_{\circ}\left(q_{\circ}\right)$ remains in the corresponding revolute joint axis and

- the plane $p_{\circ} p_{R} p_{L}\left(q_{\circ} q_{R} q_{L}\right)$ is orthogonal to this axis.

Adopt the following notations (see Figure 5.2a):

$$
\begin{array}{lll}
{[\mathrm{w}]_{\mathrm{N}}=(x, y, 0)} & {\left[\mathrm{w}^{\star}\right]_{\mathrm{B}}=\left(0,0,-\bar{r}_{\mathcal{W}}\right)} & {\left[\mathrm{b}^{\star}\right]_{\mathrm{B}}=\left(\bar{x}_{\mathrm{b}^{\star} \mid \mathrm{B}}, 0, \bar{z}_{\mathrm{b}^{\star} \mid \mathrm{B}}\right)} \\
{\left[\mathrm{h}_{\circ}\right]_{\mathrm{B}}=(\bar{w}+\bar{c}, 0,0)} & {\left[\mathrm{p}_{\circ}\right]_{\mathrm{B}}=\left(\bar{x}_{\mathrm{p}_{\circ} \mid \mathrm{B}}, 0, \bar{z}_{\mathrm{p}_{\circ} \mid \mathrm{B}}\right)} & {\left[\mathrm{q}_{\circ}\right]_{\mathrm{B}}=\left(\bar{x}_{\mathrm{q} \mid \mathrm{B}}, 0, \bar{z}_{\mathrm{q}_{\circ} \mid \mathrm{B}}\right)} \\
{\left[\mathrm{h}^{\star}\right]_{\mathrm{H}}=\left(\bar{x}_{\mathrm{h}^{\star} \mid \mathrm{H}}, 0, \bar{z}_{\mathrm{h}^{\star} \mid \mathrm{H}}\right)} & {\left[\mathrm{p}^{\star}\right]_{\mathrm{P}}=\left(\bar{x}_{\mathrm{p}^{\star} \mid \mathrm{P}}, 0, \bar{z}_{\mathrm{p}^{\star} \mid \mathrm{P}}\right)} & {\left[\mathrm{q}^{\star}\right]_{\mathrm{Q}}=\left(\bar{x}_{\mathrm{q}^{\star} \mid \mathrm{Q}}, 0, \bar{z}_{\mathrm{q}^{\star} \mid \mathrm{Q}}\right)} \\
{\left[\mathrm{h}_{\mathrm{R}}\right]_{\mathrm{H}}=\left(\bar{x}_{\mathrm{h} \mid \mathrm{H}}, \bar{y}_{\mathrm{h} \mid \mathrm{H}}, \bar{z}_{\mathrm{h} \mid \mathrm{H}}\right)} & {\left[\mathrm{p}_{\mathrm{R}}\right]_{\mathrm{P}}=\left(0, \bar{y}_{\mathrm{p} \mid \mathrm{P}}, \bar{z}_{\mathrm{p} \mid \mathrm{P}}\right)} & {\left[\mathrm{q}_{\mathrm{R}}\right]_{\mathrm{Q}}=\left(0, \bar{y}_{\mathrm{q} \mid \mathrm{Q}}, \bar{z}_{\mathrm{q} \mid \mathrm{Q}}\right)} \\
{\left[\mathrm{h}_{\mathrm{L}}\right]_{\mathrm{H}}=\left(\bar{x}_{\mathrm{h} \mid \mathrm{H}},-\bar{y}_{\mathrm{h} \mid \mathrm{H}}, \bar{z}_{\mathrm{h} \mid \mathrm{H}}\right)} & {\left[\mathrm{p}_{\mathrm{L}}\right]_{\mathrm{P}}=\left(0,-\bar{y}_{\mathrm{p} \mid \mathrm{P}}, \bar{z}_{\mathrm{p} \mid \mathrm{P}}\right)} & {\left[\mathrm{q}_{\mathrm{L}}\right]_{\mathrm{Q}}=\left(0,-\bar{y}_{\mathrm{q} \mid \mathrm{Q}}, \bar{z}_{\mathrm{q} \mid \mathrm{Q}}\right)}
\end{array}
$$

Moreover, adopt for subsystems $\mathscr{B}, \mathscr{H}, \mathscr{P}$ and $\mathbb{Q}$ the generic Newton-Euler model, for a single rigid body symmetric with respect to the local $y$-plane (both in terms of geometry and mass distribution) subjected to a gravitational field $\overline{\mathrm{g}}=\bar{g}\left(\sin \bar{\xi} \hat{\mathbf{n}}_{\mathrm{x}}+\cos \bar{\xi} \hat{\mathbf{n}}_{\mathrm{z}}\right)$, and to an eventual torque applied in the direction of its local y-axis. In order to model $\mathscr{A}$ the following extra generalized coordinates are defined (see Figure 5.2a):

- $x$ and $y$, the $x$ and $y$ Cartesian coordinates of $w$ in $N$ (note that the $z$ coordinate of this point is always zero).

- $\psi, \phi$ and $\theta$, representing a sequence of zxy Euler angles (heading, lean and pitch angles) that are used to describe the orientation of $B$ with respect to $\mathrm{N}$.

- $\delta$, the steering angle, representing the relative revolute motion between handlebar and frame along the steering axis.

- $\chi_{\mathrm{p}}\left(\right.$ and $\chi_{\mathrm{q}}$ ) representing the relative revolute motion between the upper (lower) transverse rod of the suspension and the frame along the corresponding revolute joint axis.

Define also as extra quasi-velocities the time derivatives of these extra coordinates. Denote by $u_{\delta}$ the steering torque (assumed as a control input), by $u_{\phi}$ the lean torque (assumed as a generic disturbance). The following extra 
generalized forces must be included in the model:

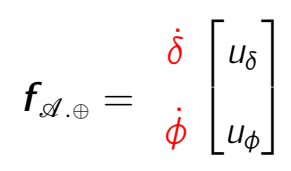

Suppose that, when the system is in the reference configuration shown in Figure 5.2a, all the unit vectors $\hat{\mathbf{b}}_{\mathrm{x}}, \hat{\mathbf{b}}_{\mathrm{y}}$ and $\hat{\mathbf{b}}_{\mathrm{z}}$ coincide with $\hat{\mathbf{n}}_{\mathrm{x}}, \hat{\mathbf{n}}_{\mathrm{y}}$ and $\hat{\mathbf{n}}_{\mathrm{z}}$. Let also $\hat{\mathbf{h}}_{z}$ have the direction of the steering axis, and $\hat{\mathbf{p}}_{x}$ and $\hat{\mathbf{q}}_{x}$ have the same direction as the respective revolute joint axes. It can be stated that:

$$
\left\{\begin{array}{l}
{[1]_{\mathrm{N} \mid \mathrm{L}}=\mathrm{R}_{z}(\psi) \mathrm{R}_{\mathrm{x}}(\phi) \mathrm{R}_{\mathrm{y}}(\theta)} \\
{[1]_{\mathrm{N} \mid \mathrm{H}}=\mathrm{R}_{\mathrm{z}}(\psi) \mathrm{R}_{\mathrm{x}}(\phi) \mathrm{R}_{\mathrm{y}}(\theta) \mathrm{R}_{\mathrm{y}}(\bar{\lambda}) \mathrm{R}_{\mathrm{z}}(\delta)} \\
{[1]_{\mathrm{N} \mid \mathrm{P}}=\mathrm{R}_{\mathrm{z}}(\psi) \mathrm{R}_{\mathrm{x}}(\phi) \mathrm{R}_{\mathrm{y}}(\theta) \mathrm{R}_{\mathrm{y}}\left(\bar{\lambda}_{\mathrm{p}}\right) \mathrm{R}_{\mathrm{x}}\left(\chi_{\mathrm{p}}\right)} \\
{[1]_{\mathrm{N} \mid \mathrm{Q}}=\mathrm{R}_{\mathrm{z}}(\psi) \mathrm{R}_{\mathrm{x}}(\phi) \mathrm{R}_{\mathrm{y}}(\theta) \mathrm{R}_{\mathrm{y}}\left(\bar{\lambda}_{\mathrm{q}}\right) \mathrm{R}_{\mathrm{x}}\left(\chi_{\mathrm{q}}\right)}
\end{array}\right.
$$

Thus, coordinates of the relevant points with respect to $\mathrm{N}$ can be obtained by:

$$
[j]_{\mathbb{N}}=\left[j_{\circ}\right]_{\mathbb{N}}+[1]_{\mathbb{N} / J}[j]_{J}
$$

For $j \in\left\{\mathrm{w}^{\star}, \mathrm{b}^{\star}, \mathrm{h}_{\circ}, \mathrm{p}_{\circ}, \mathrm{q}_{\circ}\right\}$, adopt $\mathrm{j}_{\circ}=\mathrm{w}$ and $\mathrm{J}=\mathrm{B}$; for $\mathrm{j} \in\left\{\mathrm{h}^{\star}, \mathrm{h}_{\mathrm{R}}, \mathrm{h}_{\mathrm{L}}\right\}$, adopt $j_{\circ}=h_{\circ}$ and $J=H$; for $j \in\left\{p^{\star}, p_{R}, p_{L}\right\}$, adopt $j_{\circ}=p_{\circ}$ and $J=P$ and for $j \in$ $\left\{\mathrm{q}^{\star}, \mathrm{q}_{\mathrm{R}}, \mathrm{q}_{\mathrm{L}}\right\}$, adopt $\mathrm{j}_{\circ}=\mathrm{q}_{\circ}$ and $\mathrm{J}=\mathrm{Q}$. The invariants necessary for describing the "internal" constraints of $\mathscr{A}$ can be obtained from the following equations:

$$
\left\{\begin{array}{l}
\frac{d}{d t}\left[j^{\star}\right]_{\mathbb{N}}=\left[v_{j^{\star} \mid \mathcal{N}}\right]_{\mathbb{N}}=\left(v_{\mathcal{g}, x}, v_{\mathcal{g}, y}, v_{g, z}\right) \\
{[1]_{\mathbb{N} \mid J}^{\top} \cdot \frac{d}{d t}[1]_{\mathbb{N} \mid J}=\left[\omega_{\mathcal{J} \mid \mathcal{N}}\right]_{\mathcal{J} J J}^{S}=\left(\omega_{\mathcal{J}, x}, \omega_{\mathcal{J}, y}, \omega_{\mathcal{J}, z}\right)^{S}}
\end{array}\right.
$$

for $\mathrm{j}^{\star} \in\left\{\mathrm{b}^{\star}, \mathrm{h}^{\star}, \mathrm{p}^{\star}, \mathrm{q}^{\star}\right\}$ and, correspondingly, $\mathrm{J} \in\{\mathrm{B}, \mathrm{H}, \mathrm{P}, \mathrm{Q}\}$ and $\mathcal{J} \in$ $\{\mathcal{B}, \mathcal{H}, \mathcal{P}, Q\}$.

\subsubsection{Wheels and suspension}

As previously mentioned, the wheels of the tricycle $\left(\mathscr{W}, \mathscr{F}_{\mathrm{R}}\right.$ and $\left.\mathscr{F}_{\mathrm{L}}\right)$ will be modeled as rigid no-slip knife-edge discs. Define a mechanical system $\mathscr{E}$ constituted by a single rigid body $\mathcal{E}$ rolling in the plane ground $\mathcal{N}$, representing a bicycle wheel. Consider that $\mathcal{E}$ is a flat disc with knife edge whose mass distribution respects the corresponding cylindrical symmetry. Let $\mathrm{E}=\left(\mathrm{e}_{\circ}, \hat{\mathrm{e}}_{\mathrm{x}}, \hat{\mathrm{e}}_{\mathrm{y}}, \hat{\mathrm{e}}_{z}\right)$ be a coordinate system fixed to the rigid body $\varepsilon$ : the origin $e_{\circ}$ corresponds to 
the geometrical center of the cylinder and the unit vectors $\hat{\mathbf{e}}_{\mathrm{x}}, \hat{\mathbf{e}}_{\mathrm{y}}$ and $\hat{\mathbf{e}}_{\mathrm{z}}$ constitute an orthonormal basis such that $\hat{e}_{y}$ has the direction of the axis the cylinder. Define also $\hat{\mathbf{e}}_{\mathrm{x}}^{\prime}=\left(\hat{\mathbf{e}}_{\mathrm{y}} \times \hat{\mathbf{n}}_{\mathrm{z}}\right) /\left\|\hat{\mathbf{e}}_{\mathrm{y}} \times \hat{\mathbf{n}}_{\mathrm{z}}\right\|$ as an unit vector representing the direction in the plane which is instantaneously tangent to the edge of the cylinder and $\hat{\mathbf{e}}_{\mathrm{y}}^{\prime}=\hat{\mathbf{n}}_{\mathrm{z}} \times \hat{\mathbf{e}}_{\mathrm{x}}^{\prime}$ as an unit vector representing the direction in the plane which is perpendicular to this tangent one. Let $e_{c}$ be the instantaneous contact point of the knife edge of the cylinder with the plane ground and let $\mathbf{v}_{\mathbf{e}_{\mathrm{C}} \mid \mathcal{N}}$ denote the velocity of this point with respect to the reference frame of $\mathcal{N}$. In the modeling of $\mathscr{E}$, due to the no-slip constraint, it is supposed that $\mathbf{v}_{\mathbf{e}_{\mathrm{C}} \mid \mathcal{N}}=0$.

Define the following generalized coordinates for describing the instantaneous configurations of $\mathcal{E}$ (with respect to a reference frame in $\mathcal{N}$ ):

- $p_{\varepsilon, \mathrm{x}}, p_{\mathcal{\varepsilon}, \mathrm{y}}$ and $p_{\varepsilon, \mathrm{z}}$, the coordinates of $\mathrm{e}_{\odot}$ in the coordinate system $\mathrm{N}$.

- $\psi_{\varepsilon}, \phi_{\varepsilon}$ and $\theta_{\varepsilon}$ representing a sequence of zxy Euler angles (i.e. rotations about $\hat{\mathbf{n}}_{\mathrm{z}}, \hat{\mathbf{e}}_{\mathrm{x}}^{\prime}$ and $\hat{\mathbf{e}}_{\mathrm{y}}$, respectively), that are used to describe the orientation of $\mathrm{E}$ with respect to $\mathrm{N}$ :

- $\psi_{\varepsilon}$ is the heading (yaw) angle of $\mathcal{E}$, i.e. the angle between the directions of $\hat{\mathbf{e}}_{\mathrm{x}}^{\prime}$ and $\hat{\mathbf{n}}_{\mathrm{x}}$.

- $\phi_{\mathcal{E}}$ is the lean angle of $\mathcal{E}$, i.e. the angle between the directions of $\hat{\mathbf{e}}_{\mathrm{y}}$ and $\hat{\mathbf{e}}_{\mathrm{y}}^{\prime}$.

- $\theta_{\varepsilon}$ is the angle that describes the intrinsic rotation (spin) of $\varepsilon$, i.e. the angle between the directions of $\hat{\mathbf{e}}_{\mathrm{x}}$ and $\hat{\mathbf{e}}_{\mathrm{x}}^{\prime}$.

Define also the following quasi-velocities for describing the motion of $\mathcal{E}$ with respect to $\mathcal{N}$ :

- $v_{\mathcal{E}, \mathrm{x}}, v_{\mathcal{E}, \mathrm{y}}$ and $v_{\mathcal{E}, \mathrm{z}}$, the components of the center of mass $\mathrm{e}^{\star}=\mathrm{e}_{\circ}$ of $\mathcal{E}$ (with respect to $\mathcal{N}$ ) in the coordinate system $N$, i.e. $\mathbf{v}_{\mathrm{e}^{\star} \mid \mathcal{N}}=v_{\mathcal{E}, \mathrm{x}} \hat{\mathbf{n}}_{\mathrm{x}}+v_{\mathcal{E}, \mathrm{y}} \hat{\mathbf{n}}_{\mathrm{Y}}+$ $v_{\mathcal{E}, \mathrm{z}} \hat{\mathbf{n}}_{\mathrm{z}}$.

- $\omega_{\mathcal{E}, \mathrm{x}}, \omega_{\mathcal{E}, \mathrm{y}}$ and $\omega_{\mathcal{E}, \mathrm{z}}$, the components of the angular velocity of $\mathcal{E}$ (with respect to $\mathcal{N}$ ) in the coordinate system E, i.e. $\omega_{\mathrm{e}^{\star} \mid \mathcal{N}}=\omega_{\mathcal{\varepsilon}, \mathrm{x}} \hat{\mathbf{e}}_{\mathrm{x}}+\omega_{\mathcal{E}, \mathrm{y}} \hat{\mathbf{e}}_{\mathrm{y}}+$ $\omega_{\mathcal{E}, \mathrm{z}} \hat{\mathbf{e}}_{\mathrm{z}}$.

- $\dot{\psi}_{\varepsilon}, \dot{\phi}_{\varepsilon}$ and $\dot{\theta}_{\varepsilon}$.

Consider that the mass distribution of $\mathcal{E}$ respects its cylindrical symmetry. Denote by $\bar{m}_{\mathcal{E}}$ the mass of $\mathcal{E}$, by $\bar{l}_{\mathcal{E}, a}$ its axial moment of inertia and by $\bar{l}_{\mathcal{E}, \mathrm{r}}$ its 
in-plane moment of inertia. Adopting, without loss of generality, that the local acceleration of gravity is given by $\overline{\mathbf{g}}=\bar{g}\left(\sin \bar{\xi} \hat{\mathbf{n}}_{\mathrm{x}}+\cos \bar{\xi} \hat{\mathbf{n}}_{z}\right)$, and supposing that there is a torque $-u_{\varepsilon} \hat{\mathbf{e}}_{\mathrm{y}}$ applied to $\mathcal{E}$, it can be stated that:

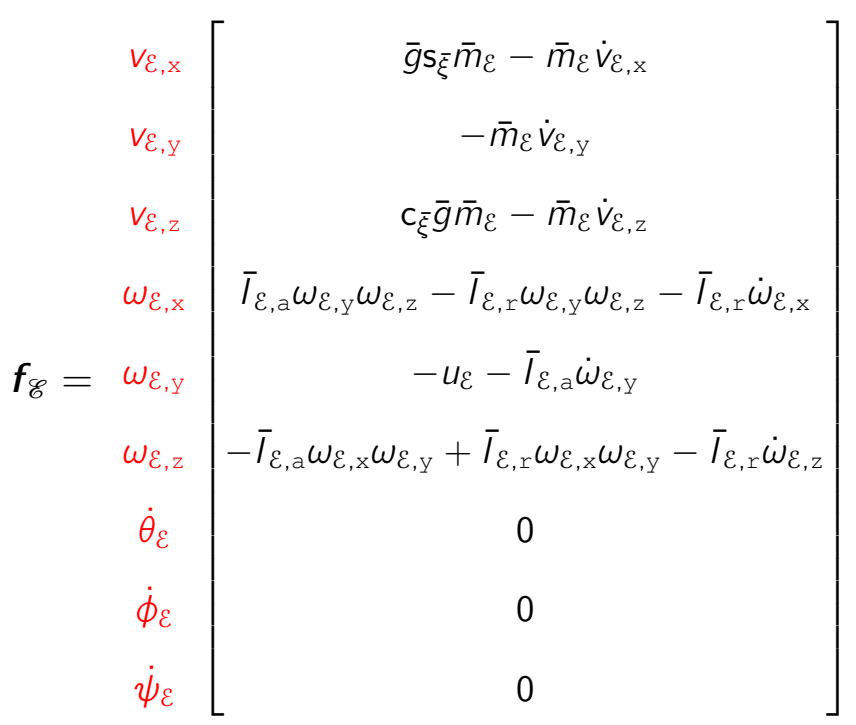

Moreover, considering that there must be three invariants expressing the relations between the three components of angular velocity $\left(\omega_{\mathcal{\varepsilon}, x}, \omega_{\mathcal{\varepsilon}, y}, \omega_{\mathcal{\varepsilon}, z}\right)$ and the three time derivatives of the Euler Angles $\left(\dot{\psi}_{\varepsilon}, \dot{\phi}_{\varepsilon}, \dot{\theta}_{\varepsilon}\right)$, as well as three invariants expressing the constraints $\mathbf{r}_{e_{c} \mid n_{\circ}} \cdot \hat{\mathbf{n}}_{z}=0$ (i.e. there must be a contact point between the wheel and the plane), $\mathbf{v}_{\mathbf{e}_{c} \mid \mathcal{N}} \cdot \hat{\mathbf{e}}_{\mathrm{x}}^{\prime}=0$ and $\mathbf{v}_{\mathrm{e}_{c} \mid \mathcal{N}} \cdot \hat{\mathbf{e}}_{\mathrm{y}}^{\prime}=v_{\varepsilon}$ (i.e. there is no longitudinal slip in this contact). All these constraints can be expressed by an invariant $\overline{\boldsymbol{q}}_{\mathscr{E}}^{\langle 1\rangle}=\boldsymbol{A}_{\mathscr{\delta}} \boldsymbol{q}_{\mathscr{E}}^{\langle 1\rangle}$, with:

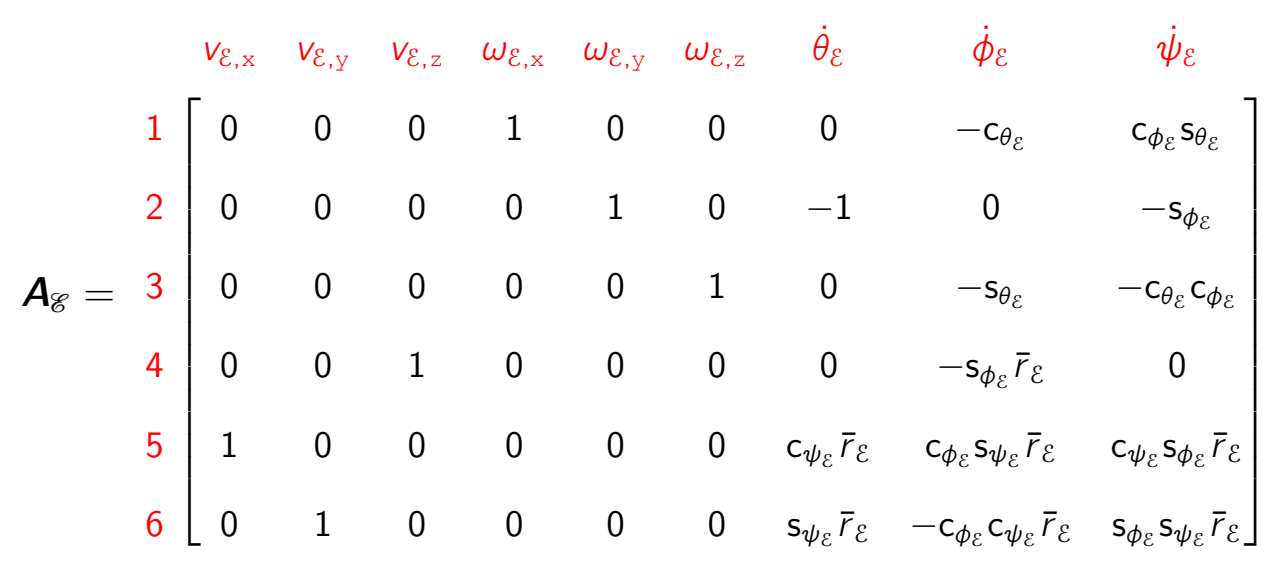


For $\mathscr{E}, B_{\mathscr{E}}=A_{\mathscr{E}}$ and a possible expression for $S_{\mathscr{E}}=C_{\mathscr{E}}$ is given by:

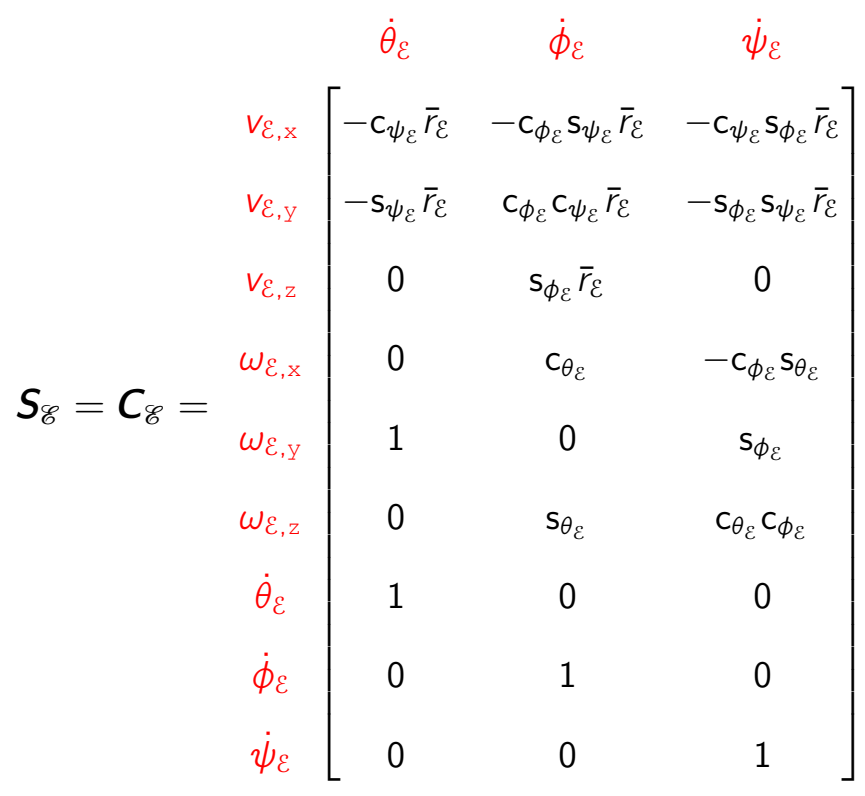

The steering bars $\delta_{\mathrm{R}}$ and $\mathcal{S}_{\mathrm{L}}$ are modeled as thin rigid cylinders $\mathcal{S}_{\mathrm{R}}$ and $\mathcal{S}_{\mathrm{L}}$ with negligible radius. The mass distribution of these bodies respects the cylindrical symmetry, thus their axial moments of inertia are also negligible. Adopt for each of these bodies local coordinate systems $S_{R}$ and $S_{L}$ with origins in their centers of mass ( $\mathrm{s}_{\mathrm{R}}^{\star}$ and $\mathrm{s}_{\mathrm{L}}^{\star}$, respectively) and with local $\mathrm{x}$-axes aligned to the cylinder axes. Define as generalized coordinates for these subsystems the Cartesian coordinates of their centers of mass with respect to a coordinate system $\mathrm{N}$ rigidly attached to an inertial reference frame $\mathcal{N}$ and zyx Euler angles representing a sequence of canonical rotations around local axes that describes the orientation of the body-attached coordinate systems with respect to $\mathrm{N}$. Define as quasi-velocities for each of these systems, the components of the velocity of the center of mass with respect to $\mathcal{N}$ in the coordinate system $\mathrm{N}$, the components of the angular velocity of the body with respect to $\mathcal{N}$ in the body-attached coordinate system and the time derivatives of the Euler angles. 
In this case, it can be stated that, for $J \in\{R, L\}$ :

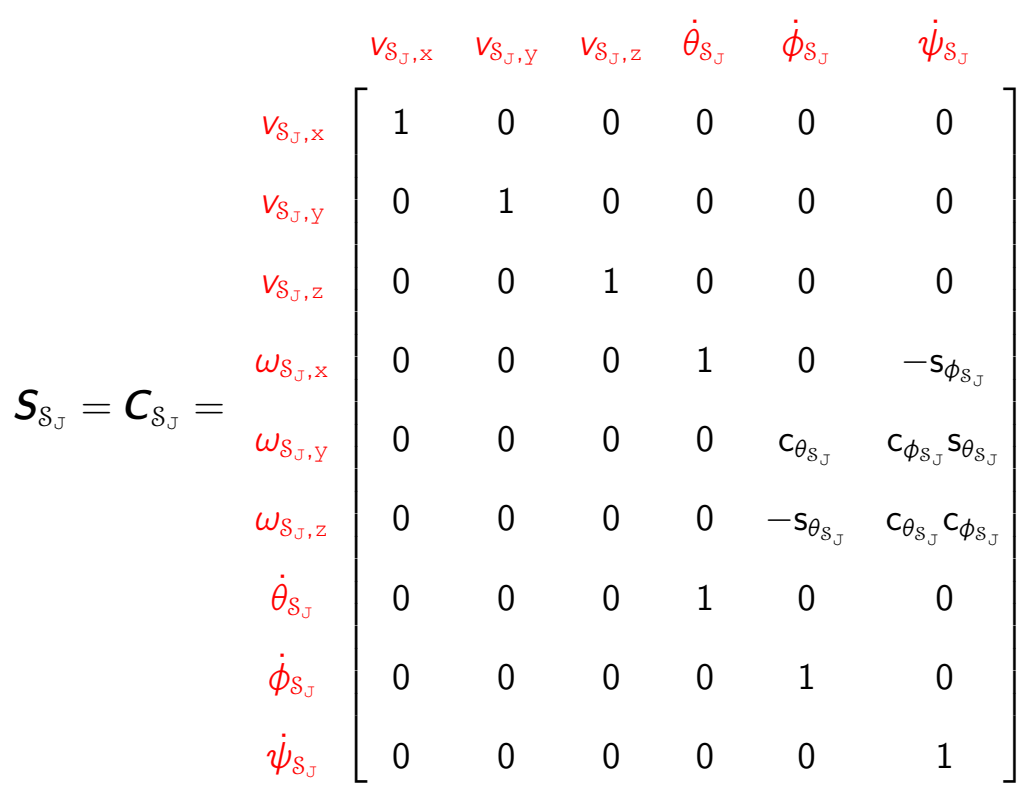

Morevover, considering that each bar is subjected to a gravitational field $\overline{\mathrm{g}}=$ $\bar{g}\left(\sin \bar{\xi} \hat{\mathbf{n}}_{\mathrm{x}}+\cos \bar{\xi} \hat{\mathbf{n}}_{\mathrm{z}}\right)$, for $\mathrm{J} \in\{\mathrm{R}, \mathrm{L}\}$ :

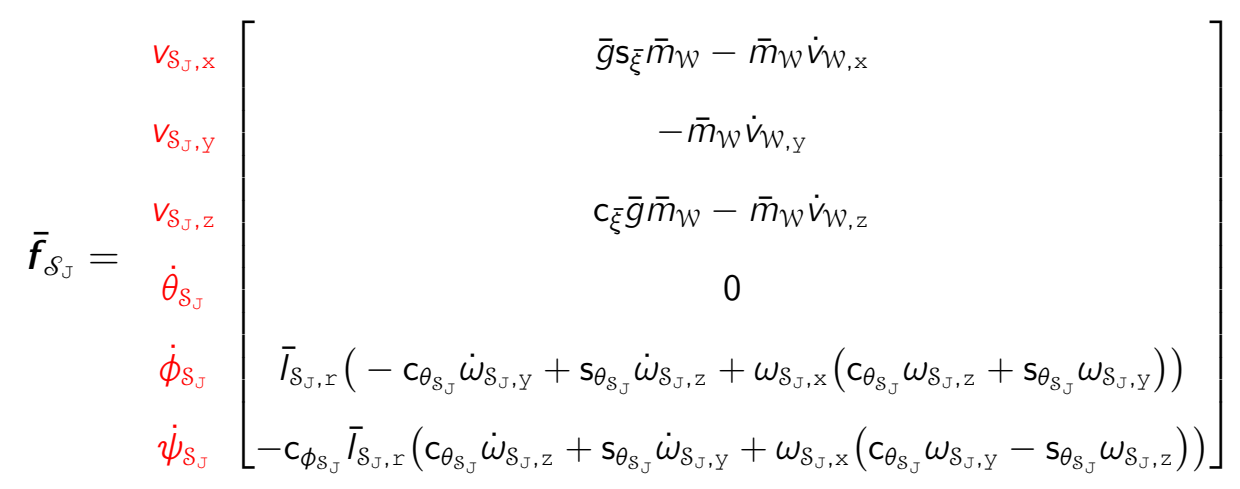

Finally, it is convenient to express the Cartesian coordinates of $h_{R}, s_{R}, h_{L}$ and $\mathrm{s}_{\mathrm{L}}$ (which are geometric centers of spherical joints, see Figure 5.2a) in the coordinate system $\mathrm{N}$ as functions of the generalized coordinates of $\mathcal{S}_{\mathrm{R}}$ and $\mathcal{S}_{\mathrm{L}}$. For this, define $\bar{\ell}_{S_{\mathrm{R}}}=\left\|\mathrm{r}_{\mathrm{S}_{\mathrm{R}} \mid \mathrm{h}_{\mathrm{R}}}\right\|$ and $\bar{\ell}_{S_{\mathrm{L}}}=\left\|\mathrm{r}_{\mathrm{s}_{\mathrm{L}} \mid \mathrm{h}_{\mathrm{L}}}\right\|$ and let $\hat{\gamma}_{S_{\mathrm{R}}}$ and $\hat{\gamma}_{S_{\mathrm{L}}}$ be adimensional quantities that satisfy the following identities: $\mathbf{r}_{s_{R}^{\star} \mid h_{R}}=\hat{\gamma}_{\delta_{R}} r_{s_{R} \mid h_{R}}$ and $\mathbf{r}_{\mathrm{s}_{\mathrm{L}}^{\star} \mid \mathrm{h}_{\mathrm{L}}}=\hat{\gamma}_{S_{\mathrm{L}}} \mathbf{r}_{\mathrm{S}_{\mathrm{L}} \mid \mathrm{h}_{\mathrm{L}}}$. It can be stated that, for $\mathrm{J} \in\{\mathrm{R}, \mathrm{L}\}$ :

$$
\left\{\begin{array}{l}
{\left[\mathrm{h}_{J}\right]_{\mathrm{N}}=\left(p_{S_{J}, \mathrm{x}}, p_{S_{J}, Y}, p_{S_{J}, \mathrm{z}}\right)+[1]_{\mathrm{N} \mid S_{J}} \cdot\left(-\hat{\gamma}_{S_{J}} \bar{\ell}_{S_{J}}, 0,0\right)} \\
{\left[\mathbf{s}_{J}\right]_{\mathrm{N}}=\left(p_{S_{J}, \mathrm{x}}, p_{S_{J}, Y}, p_{S_{J}, \mathrm{z}}\right)+[1]_{\mathrm{N} \mid S_{J}} \cdot\left(\left(1-\hat{\gamma}_{S_{J}}\right) \bar{\ell}_{S_{J}}, 0,0\right)}
\end{array}\right.
$$

The knuckles $\mathscr{K}_{\mathrm{R}}$ and $\mathscr{K}_{\mathrm{L}}$ are modeled as rigid bodies $\mathcal{K}_{\mathrm{R}}$ and $\mathcal{K}_{\mathrm{L}}$ with no special symmetry property. Define body-attached coordinate systems, $K_{R}$ and $\mathrm{K}_{\mathrm{L}}$, with origins in the corresponding centers of mass of these bodies and axes 
that coincide with the global axes (of $\mathrm{N}$ ) whenever the tricycle is in the reference configuration shown in Figure 5.2a. Consider also that this model uses the same kind of generalized coordinates and quasi-velocities defined for the modeling of the steering bars, except from the angular coordinates which are now set as zxy Euler angles. It can be stated that, for $J \in\{R, L\}$ :

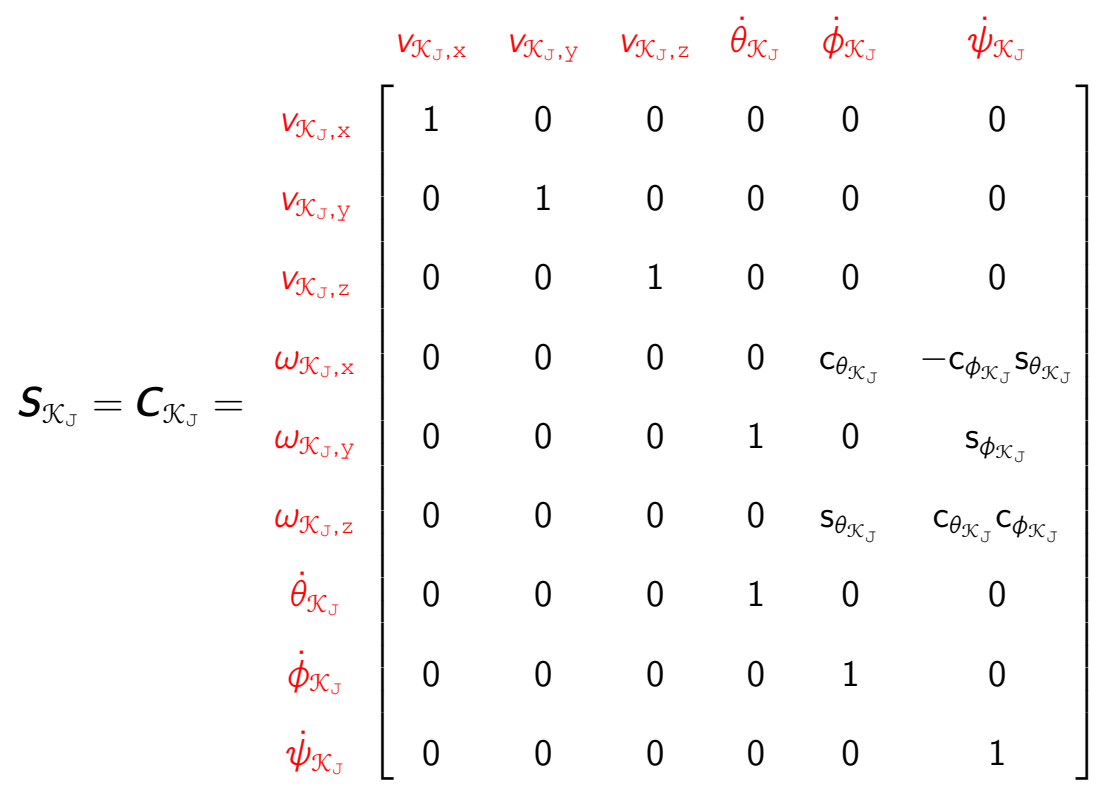

Moreover, considering that each knuckle is subjected to a gravitational field $\overline{\mathbf{g}}=\bar{g}\left(\sin \bar{\xi} \hat{\mathbf{n}}_{\mathrm{x}}+\cos \bar{\xi} \hat{\mathbf{n}}_{\mathrm{z}}\right)$, for $\mathrm{J} \in\{\mathrm{R}, \mathrm{L}\}$ :

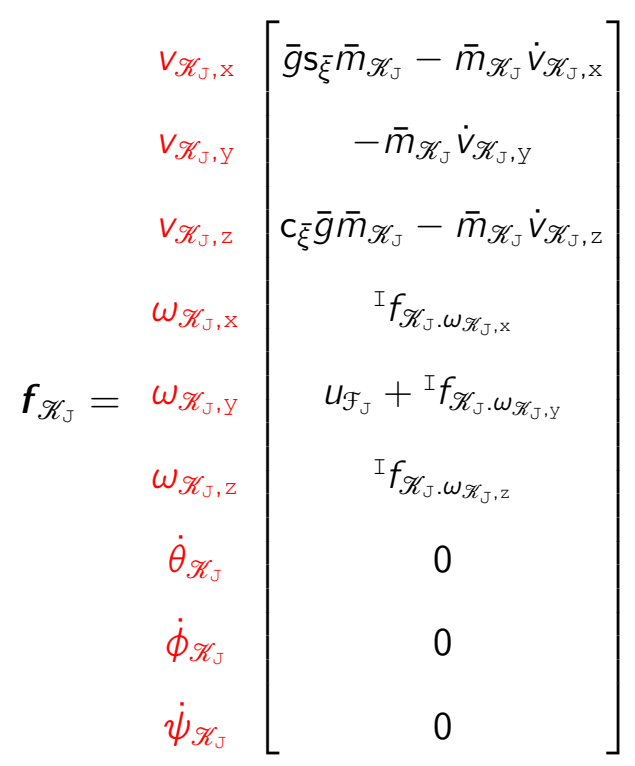

with:

$$
\begin{aligned}
& { }^{\mathrm{I}} f_{\mathscr{K}_{J}, \omega_{\mathscr{K}_{J}, \mathrm{a}}}=-\bar{I}_{\mathscr{K}_{J}, \mathrm{bc}} \omega_{\mathscr{K}_{J}, \mathrm{~b}}^{2}-\bar{I}_{\mathscr{K}_{J}, \mathrm{ac}} \omega_{\mathscr{K}_{J}, \mathrm{a}} \omega_{\mathscr{K}_{J}, \mathrm{~b}}+\bar{I}_{\mathscr{K}_{J}, \mathrm{bb}} \omega_{\mathscr{K}_{J}, \mathrm{c}} \omega_{\mathscr{K}_{J}, \mathrm{~b}} \\
& -\bar{l}_{\mathscr{K}_{J}, \mathrm{Cc}} \omega_{\mathscr{K}_{J}, \mathrm{C}} \omega_{\mathscr{K}_{J}, \mathrm{~b}}+\bar{I}_{\mathscr{K}_{\mathrm{J}, \mathrm{bc}}} \omega_{\mathscr{K}_{\mathrm{J}, \mathrm{C}}}^{2}+\bar{I}_{\mathscr{K}_{J}, \mathrm{ab}} \omega_{\mathscr{K}_{J}, \mathrm{a}} \omega_{\mathscr{K}_{J}, \mathrm{C}} \\
& -\bar{l}_{\mathscr{K}_{J}, \mathrm{aa}} \dot{\omega}_{\mathscr{K}_{J}, \mathrm{a}}-\bar{I}_{\mathscr{K}_{\mathrm{J}, \mathrm{ab}}} \dot{\omega}_{\mathscr{K}_{J}, \mathrm{~b}}-\bar{I}_{\mathscr{K}_{\mathrm{J}}, \mathrm{ac}} \dot{\omega}_{\mathscr{K}_{J}, \mathrm{c}}
\end{aligned}
$$


for all $(a, b, c) \in\{(x, y, z),(y, z, x),(z, x, y)\}$.

Then, $\overline{\boldsymbol{f}}_{\mathscr{K}_{\mathrm{R}}}=\boldsymbol{C}_{\mathcal{K}_{\mathrm{R}}}^{\top} \boldsymbol{f}_{\mathscr{K}_{\mathrm{R}}}$ and $\overline{\boldsymbol{f}}_{\mathscr{K}_{\mathrm{L}}}=\boldsymbol{C}_{\mathcal{K}_{\mathrm{L}}}^{\top} \boldsymbol{f}_{\mathscr{K}_{\mathrm{L}}}$. Finally, for $\mathrm{J} \in\{\mathrm{R}, \mathrm{L}\}$, it is convenient to express the Cartesian coordinates of $\mathrm{p}_{\mathrm{J}}, \mathrm{q}_{\mathrm{J}}, \mathrm{s}_{\mathrm{J}}$ and $\mathrm{f}_{\mathrm{J}}^{\star}$ (which are geometric centers of spherical joints, see Figure 5.2a) in the coordinate system $\mathrm{N}$ as functions of the generalized coordinates of $\mathscr{K}_{\mathrm{J}}$. These expressions can be given by (with the local coordinates, denoted with overbar, treated as known physical parameters):

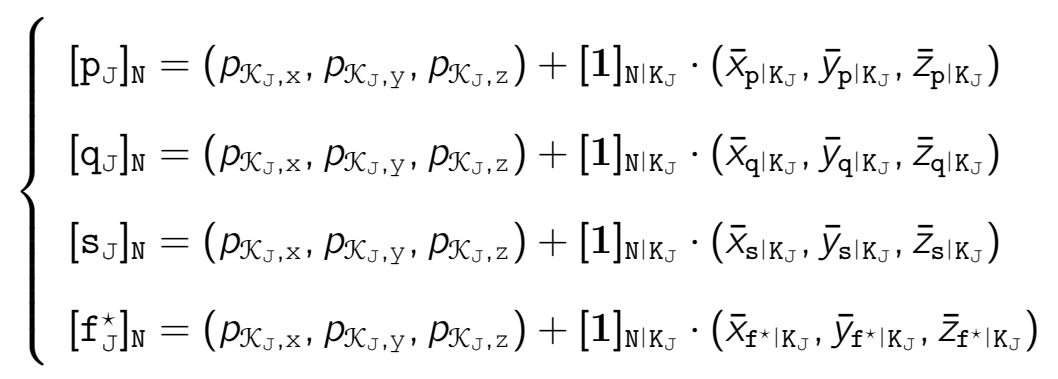

\subsubsection{Linearized equations of motion}

As discussed in Section 5.2.1, the tadpole tricycle $\mathscr{T}$ is constituted by 8 subsystems, whose models have already been derived (see Sections 5.2.2 and 5.2.3). No extra generalized variables or extra generalized forces are needed. Thus, it is necessary to obtain invariants describing the "external" constraints among these subsystems to be able to apply the modular modeling algorithm. The constraints among these subsystems are due to ideal spherical and revolute joints:

- The constraint between the rear wheel and frame can be modeled as an ideal revolute joint. The parametric descriptions of the position of $\mathrm{w}^{\star}$ using coordinates of $\mathscr{A}$ and $\mathscr{W}$ must be the same, as well as, the local y axes must be parallel, i.e.:

$$
\left\{\begin{array}{l}
{\left[\mathrm{w}^{\star}\right]_{\mathrm{N}}\left(t, \boldsymbol{q}_{\mathscr{A}}^{\langle 0\rangle}\right)=\left[\mathrm{w}^{\star}\right]_{\mathrm{N}}\left(t, \boldsymbol{q}_{\mathscr{W}}^{\langle 0\rangle}\right)=\left(p_{\mathcal{W}, \mathrm{x}}, p_{\mathcal{W}, \mathrm{y}}, p_{\mathcal{W}, \mathrm{z}}\right)} \\
{[\mathbf{1}]_{\mathrm{N} \mid \mathrm{B}} \cdot \hat{\boldsymbol{e}}_{\mathrm{Y}}=[\mathbf{1}]_{\mathrm{N} \mid \mathrm{W}} \cdot \hat{\boldsymbol{e}}_{\mathrm{y}}}
\end{array}\right.
$$

with $\hat{e}_{\mathrm{y}}=(0,1,0)$ and $\left[\mathrm{w}^{\star}\right]_{\mathrm{N}}\left(t, \boldsymbol{q}_{\mathscr{d}}^{\langle 0\rangle}\right)$ being given by equation (5.5).

- The constraints between the handlebar and the steering arms can be modeled as ideal spherical joints. In this case the parametric description of $\mathrm{h}_{\mathrm{R}}\left(\mathrm{h}_{\mathrm{L}}\right)$ in terms of coordinates of $\mathscr{A}$, given by equation (5.5), and of coordinates of $\mathcal{S}_{\mathrm{R}}\left(\mathcal{S}_{\mathrm{L}}\right)$, given by equation (5.12), must be the same. 
- The constraints between the upper and lower transverse rods of the suspension and the left and right knuckles can also be modeled as ideal spherical joints. In this case the parametric descriptions of the positions of $p_{R}, p_{L}, q_{R}$ and $q_{L}$ must be the same when described in terms of the generalized coordinates of $\mathscr{A}$, equation (5.5), and of the generalized coordinates of the respective knuckle, equation (5.16).

- The constraints between the knuckles and the steering arms can be modeled as ideal spherical joints too. Thus, it is enough to guarantee that the parametric descriptions of the positions of $\mathrm{s}_{\mathrm{R}}$ and $\mathrm{s}_{\mathrm{L}}$ coincide when expressed in terms of the generalized coordinates of the respective steering arm, equation (5.12), and of the generalized coordinates of the respective knuckle, equation (5.16).

- The constraints between the front wheels and the corresponding knuckles can be modeled as ideal revolute joints. Thus, analogously to equation (5.17), the following conditions must be satisfied, for $J \in\{R, L\}$ :

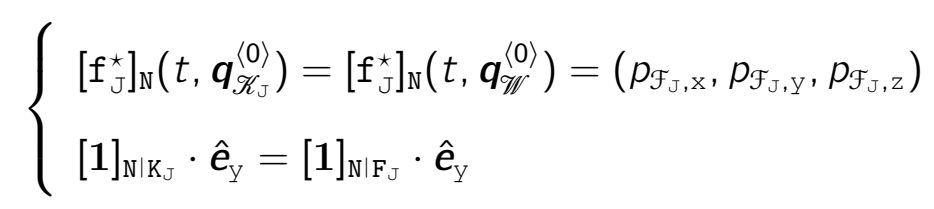

with $\left[f_{J}^{\star}\right]_{\mathbb{N}}\left(t, \boldsymbol{q}_{\mathscr{K}_{J}}^{\langle 0\rangle}\right)$ being given by equation (5.16).

- Finally, by the symmetry hypotheses considered, rotations of the steering arms around their own axes do not have any effect, nor are affected by any other motion in the system. Thus, without any loss of generality, it can be adopted that $\theta_{S_{R}}=0$ and $\theta_{S_{\perp}}=0$.

By these conditions, the corresponding invariants $\overline{\boldsymbol{q}}_{\mathscr{T} . \oplus}^{\langle 0\rangle}$ and, consequently, $\overline{\boldsymbol{q}}_{\mathscr{T} . \oplus}^{\langle 1\rangle}=\boldsymbol{A}_{\mathscr{T} . \oplus} \boldsymbol{q}_{\mathscr{T}}^{\langle 1\rangle}$ can be obtained.

However, it can be noticed that if all the corresponding invariants are considered, the model will be overconstrained, i.e., apart from the reference configuration there will be no other configuration in which all the equations can be satisfied simultaneously. Indeed, it can be noticed that this tricycle has clearances in its spherical joints (Figure 5.3) that allow the operation of the steering mechanism.

Altough the elimination of a constraint or an addition of a new joint representing an "equivalent" clearance would solve this problem, it can be noticed that such hypotheses would, in some configurations, lead to unrealistic and bi- 


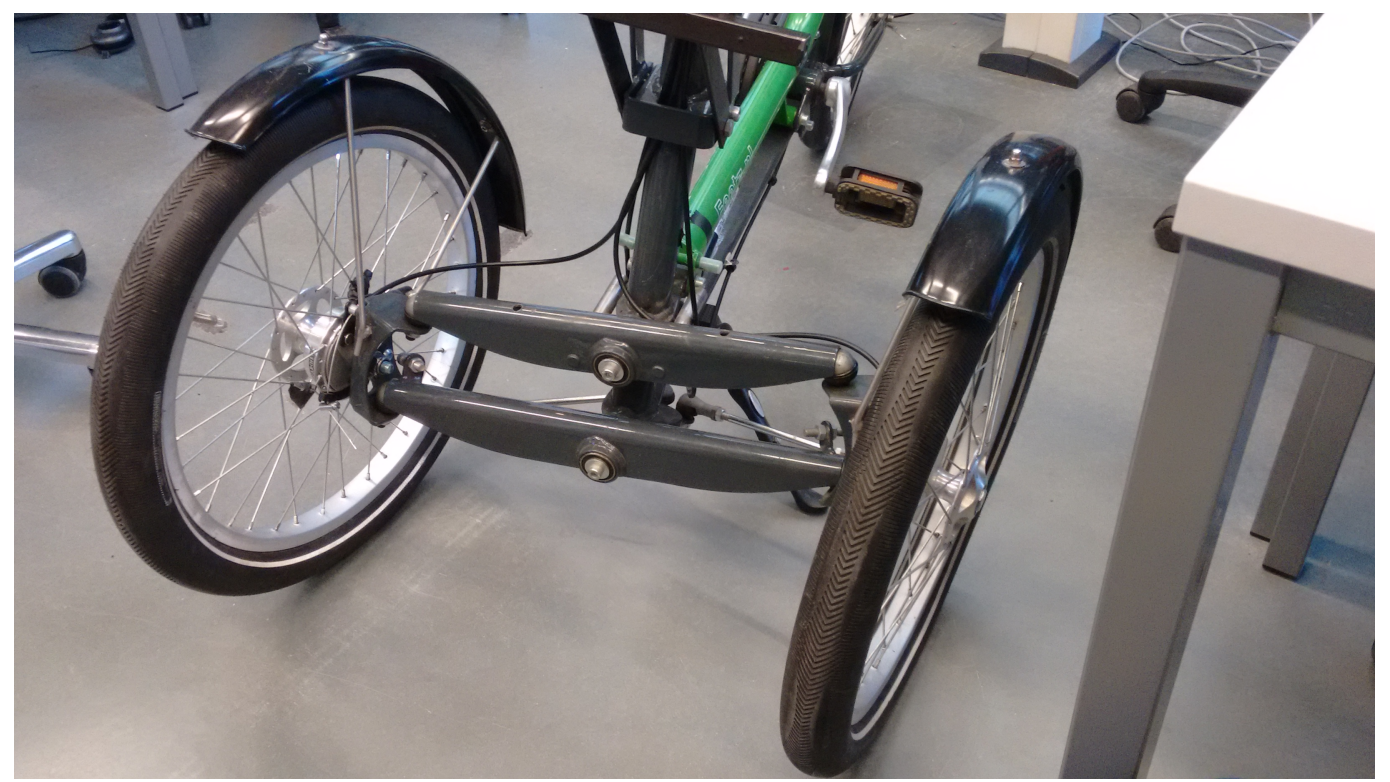

Figure 5.3: Feetz ${ }^{\circledR}$ tricycle

ased clearances. Indeed, in every configuration, the mechanism "distributes" the clearances among the joints such that no joint presents an excessive clearance and the violation of the corresponding constraint invariants is somehow minimized. Considering that the objective of this analysis is to obtain a linearized model of the tadpole tricycle, then the use of the least squares procedure described in Section 4.5 for obtaining a linearized expression for $C_{\mathscr{T}}$ based on a linearized expression of $B_{\mathscr{T}}$, would lead to a $C_{\mathscr{T}}$ matrix that effectively minimizes the violation of the constraint invariants without requiring any other additional hypothesis.

The linearizations will be performed around a reference state in which the tricycle remains in the reference configuration shown in Figure 5.2a and translates longitudinally with a constant velocity $v \hat{\mathbf{n}}_{\mathrm{x}}$ with respect to $\mathcal{N}$, which corresponds to the following reference values for the state variables: $p_{\mathcal{W}, x}-x=\bar{x}_{\mathcal{W}}$, $p_{\mathcal{W}, Y}-y=\bar{y}_{\mathcal{W}}, p_{\mathcal{W}, z}=-\bar{r}_{\mathcal{W}}, p_{\mathcal{F}_{R}, x}-x=p_{\mathcal{F}_{\mathrm{L}, \mathrm{X}}}-x=\bar{x}_{\mathcal{F}}, p_{\mathcal{F}_{R}, Y}-y=y-p_{\mathcal{F}_{\mathrm{L}, Y}}=\bar{y}_{\mathcal{F}}$, $p_{\mathcal{F}_{R}, z}=p_{\mathcal{F}_{\mathrm{L}}, \mathrm{z}}=-\bar{r}_{\mathcal{F}}, p_{\mathcal{S}_{R}, \mathrm{x}}-x=p_{\mathcal{S}_{\mathrm{L}}, \mathrm{x}}-x=\bar{x}_{S}, p_{\mathcal{S}_{R}, \mathrm{Y}}-y=y-p_{\mathcal{S}_{\mathrm{L}}, \mathrm{Y}}=\bar{y}_{S}$, $p_{\delta_{R}, z}=p_{S_{\mathrm{I}}, z}=\bar{z}_{S}, \psi_{S_{\mathrm{R}}}=-\psi_{S_{\mathrm{L}}}=\bar{\psi}_{S}, \phi_{S_{\mathrm{R}}}=\phi_{S_{\mathrm{L}}}=\bar{\phi}_{S}, p_{\mathcal{K}_{\mathrm{R}}, \mathrm{x}}-x=p_{\mathcal{K}_{\mathrm{L}}, \mathrm{x}}-x=\bar{x}_{\mathcal{K}}$, $p_{\mathcal{K}_{R}, Y}-y=y-p_{\mathcal{K}_{\mathrm{L}, Y}}=\bar{y}_{\mathcal{K}}, p_{\mathcal{K}_{R}, z}=p_{\mathcal{K}_{\mathrm{L}, Z}}=\bar{z}_{\mathcal{K}}, \dot{x}=\dot{p}_{\mathcal{W}_{, x}}=v_{\mathcal{W}, x}=$ $\dot{p}_{\mathcal{F}_{R}, x}=v_{\mathcal{F}_{R}, x}=\dot{p}_{\mathcal{F}_{\mathrm{L}}, x}=v_{\mathcal{F}_{\mathrm{L}}, x}=v_{\mathcal{B}_{,} x}=v_{\mathcal{H}, x}=v_{\mathcal{P}, x}=v_{Q, x}=\dot{p}_{\mathcal{S}_{R}, x}=v_{\mathcal{S}_{R}, x}=$ $\dot{p}_{S_{1}, x}=v_{S_{\mathrm{L}}, x}=\dot{p}_{\mathcal{K}_{\mathrm{R},}, x}=v_{\mathcal{K}_{R}, x}=\dot{p}_{\mathcal{K}_{\mathrm{L}}, x}=v_{\mathcal{K}_{\mathrm{L}}, x}=v, \dot{\theta}_{\mathcal{W}}=\omega_{\mathcal{W}, y}=-v / \bar{r}_{\mathcal{W}}$ and $\dot{\theta}_{\mathcal{F}_{R}}=\omega_{\mathcal{F}_{R, Y}}=\dot{\theta}_{\mathcal{F}_{\mathrm{L}}}=\omega_{\mathcal{F}_{\mathrm{I}, Y}}=-v / \bar{r}_{\mathcal{F}}$. Incremental generalized variables can be defined as the difference between the generalized variables and their reference values. From this point on, unless explicitly mentioned, all the generalized variables involved in mathematical expressions are incremental. 
Using the procedure described in Section 4.5 for obtaining a linearized form for $C_{\mathscr{T}}$ based on a linearized expression of $B_{\mathscr{T}}$, the following matrix is obtained:

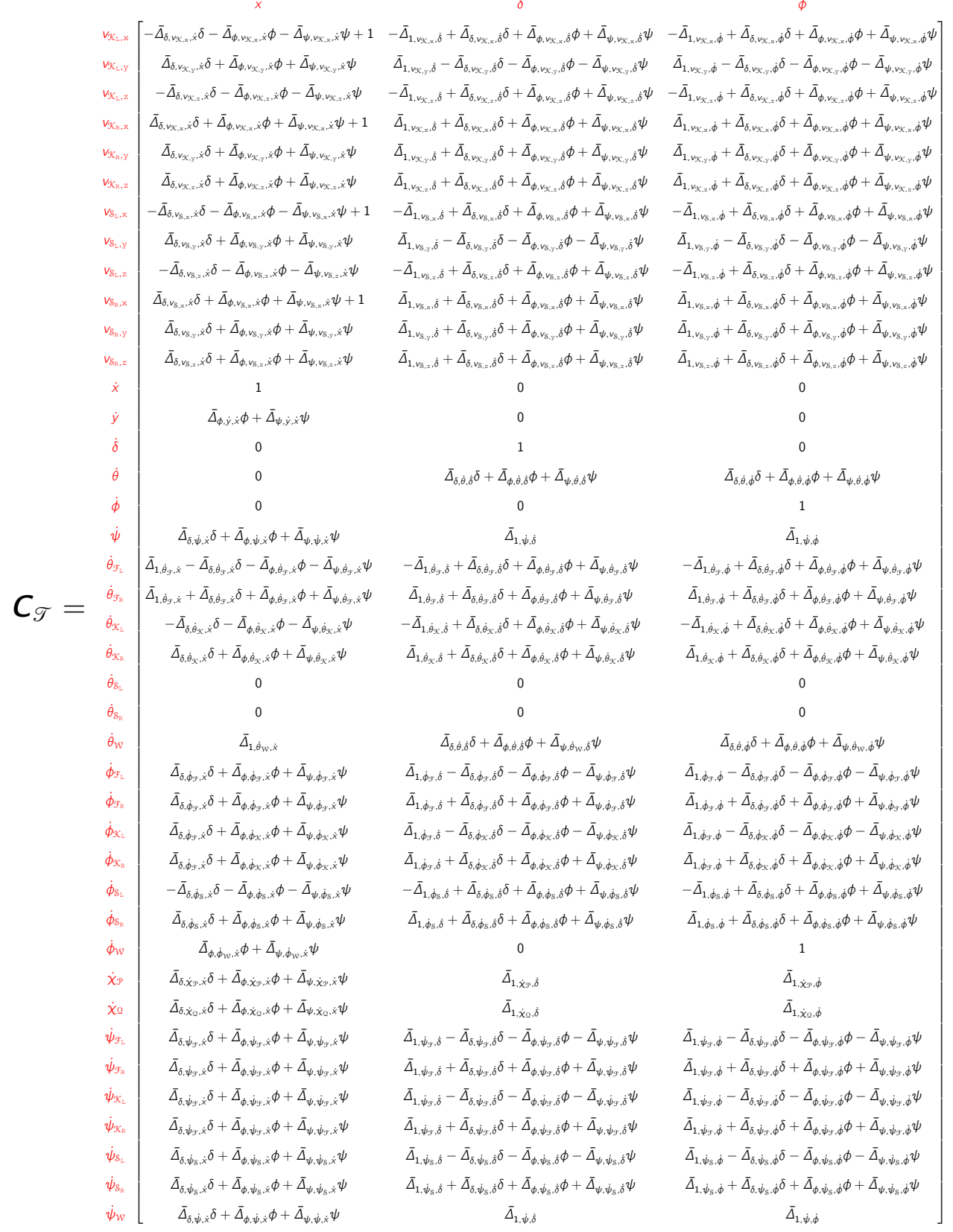

In this case, the $\bar{\Delta}$ coefficients are obtained by a least squares algorithm in which the left-right symmetry of the tricycle is enforced. That is why, in equation (5.19) rows corresponding to $R$ and $L$ variables have similar expressions in which only the signs of some terms are different.

Applying similar least squares solvers (in which left-right symmetry are enforced) to the linearized expressions of $\overline{\boldsymbol{q}}_{\mathscr{T}}^{\langle 0\rangle}$ and $\overline{\boldsymbol{q}}_{\mathscr{T}}^{\langle 1\rangle}$, all the incremental generalized coordinates $\boldsymbol{q}_{\mathscr{T}}^{\langle 0\rangle}$ can be expressed as a linear combination of a subset of "independent" incremental coordinates $\boldsymbol{q}_{\mathscr{T}}^{\langle 0\rangle}$.\# as well as all the incremental quasi-velocities $\boldsymbol{q}_{\mathscr{T}}^{\langle 1\rangle}$ can be expressed as a linear combination of $\boldsymbol{q}_{\mathscr{T} \text {.\# }}^{\langle 0\rangle}$ and a subset of "independent" incremental quasi-velocities $\boldsymbol{q}_{\mathscr{T}^{. \#}}^{\langle 1\rangle}$. A selection of vari- 
ables which is adequate for the desired stability analysis is:

$$
\left\{\begin{array}{l}
\boldsymbol{q}_{\mathscr{T}^{\prime} \#}^{\langle 0\rangle}=\left(x, y, \delta, \phi, \psi, \theta_{\mathcal{W}}, \theta_{\mathcal{F}_{\mathbb{R}}}, \theta_{\mathcal{F}_{\mathrm{L}}}\right) \\
\boldsymbol{q}_{\mathscr{T}^{\prime}, \#}^{\langle 1\rangle}=(\dot{x}, \dot{\delta}, \dot{\phi})
\end{array}\right.
$$

Finally, after linearizing $f_{\mathscr{T}}$ and applying the corresponding transformations of variables to express them in terms of $\boldsymbol{q}_{\mathscr{T}^{\text {.\# }}}^{\langle 0\rangle}, \boldsymbol{q}_{\mathscr{T}^{\text {.\# }}}^{\langle 1\rangle}$ and $\dot{\boldsymbol{q}}_{\mathscr{T} \text {.\# }}^{\langle 1\rangle}$, the linearized equations of motion of $\mathscr{T}$ can be obtained by premultiplying the linearized expression of $C_{\mathscr{T}}^{\top}$ with the linearized expression of $f_{\mathscr{T}}$, neglecting all second order terms. After collecting the terms, the equations of motion of $\mathscr{T}$ can be expressed in the following form, with $\boldsymbol{u}_{\mathscr{T}}=\left(u_{\mathcal{W}}, u_{\mathcal{F}_{R}}, u_{\mathcal{F}_{\bar{L}}}, u_{\delta}, u_{\phi}\right)$ :

$$
\bar{M} \dot{\boldsymbol{q}}_{\mathscr{T} . \#}^{\langle 1\rangle}+v \bar{C}_{1} \boldsymbol{q}_{\mathscr{T}^{\prime \#}}^{\langle 1\rangle}+\left(\bar{g} \bar{K}_{0}+v^{2} \bar{K}_{2}\right) \boldsymbol{q}_{\mathscr{T} . \#}^{\langle 0\rangle}=\bar{E} \boldsymbol{u}_{\mathscr{T}}
$$

The matrices $\bar{M}, \bar{C}_{1}, \bar{K}_{0}, \bar{K}_{2}$ and $\bar{E}$ are invariants and can be expressed as functions of the physical parameters of the subsystems of $\mathscr{T}$.

It is worth noting that $\dot{x}, x, y, \theta_{\mathcal{W}}, \theta_{\mathcal{F}_{\mathrm{R}}}$ and $\theta_{\mathcal{F}_{\mathrm{I}}}$ never appear in any dynamic equation and that $\psi$ will not appear if $\sin \bar{\xi}=0$, i.e. in case of a horizontal ground. Moreover these equations show that, in the linearized model, the longitudinal dynamics is decoupled for the lateral one, once $\ddot{x}$ only appear in a single equation of motion in which no other variable among $\boldsymbol{q}_{\mathscr{T} \text {.\# }}^{\langle 0\rangle}, \boldsymbol{q}_{\mathscr{T}_{\text {.\# }}}^{\langle 1\rangle}$ and $(\ddot{\delta}, \ddot{\phi})$ does. Supposing that $\sin \bar{\xi}=0$, the following state vector can be adopted for describing the lateral dynamics of $\mathscr{T}$ :

$$
x_{\mathscr{T} . L}=(\delta, \phi, \dot{\delta}, \dot{\phi})
$$

Defining $\boldsymbol{u}_{\mathscr{T} . \mathrm{L}}=\left(u_{\delta}, u_{\phi}, u_{\mathcal{F}_{\mathrm{R}}}, u_{\mathcal{F}_{\mathrm{L}}}\right)$, it can be stated that the dynamic equations that describe the lateral motion of $\mathscr{T}$ can be expressed in the following matrix form:

$$
\dot{\boldsymbol{x}}_{\mathscr{T} . \mathrm{L}}=\left(\overline{\boldsymbol{A}}_{0}+v \overline{\boldsymbol{A}}_{1}+v^{2} \overline{\boldsymbol{A}}_{2}\right) \boldsymbol{x}_{\mathscr{T} . \mathrm{L}}+\overline{\boldsymbol{B}} \boldsymbol{u}_{\mathscr{T} . \mathrm{L}}
$$

The matrices $\bar{A}_{0}, \bar{A}_{1}, \bar{A}_{2}$ and $\bar{B}$ are invariants and can also be expressed as functions of the physical parameters of the subsystems of $\mathscr{T}$ only.

\subsubsection{Stability analysis}

Aiming to a better understanding of the dynamics of the tadpole tricycle and to compare it to the behavior of a typical bicycle, a stability analysis is performed using some reference parameters. The corresponding geometrical 
parameters were measured in the Feetz ${ }^{\circledR}$ tricycle (Figure 5.3) available in the bicycle laboratory of TU Delft. The main inertial parameters were scaled from the benchmark Whipple model (MEIJAARD et al., 2007) using the ratios among some of the most relevant dimensions in both models while the remaining ones were simply estimated. The reference parameters used for the presented stability analysis are listed in Table 5.1. Using these reference parameters three charts are presented:

- Eigenvalues versus speed chart (Figure 5.4): shows the real and imaginary parts (both divided by $2 \pi$, i.e., given in $1 / \mathrm{s}$ and $\mathrm{Hz}$ instead of rad/s) of the eigenvalues of the tadpole tricycle model as functions of the corresponding translation speed.

- Steer-to-lean ratio versus speed chart (Figure 5.5): shows, for each eigenvector, the ratio between the components corresponding to the steer angle and the lean angle as functions of the translation speed; these ratios are represented in the polar form (absolute value and phase angle); in the background there is a gray scale version of the eigenvalues versus speed chart (without dividing the eigenvalues components by $2 \pi$ ).

- Eigenvectors tables (Figure 5.6): for some selected speeds, the normalized eigenvectors are plotted in tables; each column is labeled by the real and imaginary parts, in $1 / \mathrm{s}$ and $\mathrm{Hz}$, respectively, of the corresponding eigenvalue and each component is represented by a colored circle with an arrow inside; the color represents the absolute value of the component, with 0 corresponding to blue and 1 to red; the directions of the arrows represent the phase angles.

In order to inspect the sensitivity of the linearized tadpole tricycle model with respect to its geometrical parameters, eight new scenarios are proposed in which these parameters suffer random increases or decreases up to $4 \%$ of the reference values (except from the trail, which is multiplied by a random number between -2 and 11). For all these scenarios, eigenvalues versus speed and steer-to-lean ratio versus speed plots are presented in Figures 5.7 and 5.8, respectively.

It can be observed that, although being very sensitive to the parameters, the modes of the linearized tadpole tricycle model resemble the modes of the benchmark Whipple bicycle model proposed by Meijaard et al. (2007). Particularly, the so called "castering" and "weave" modes are totally analogous to the 


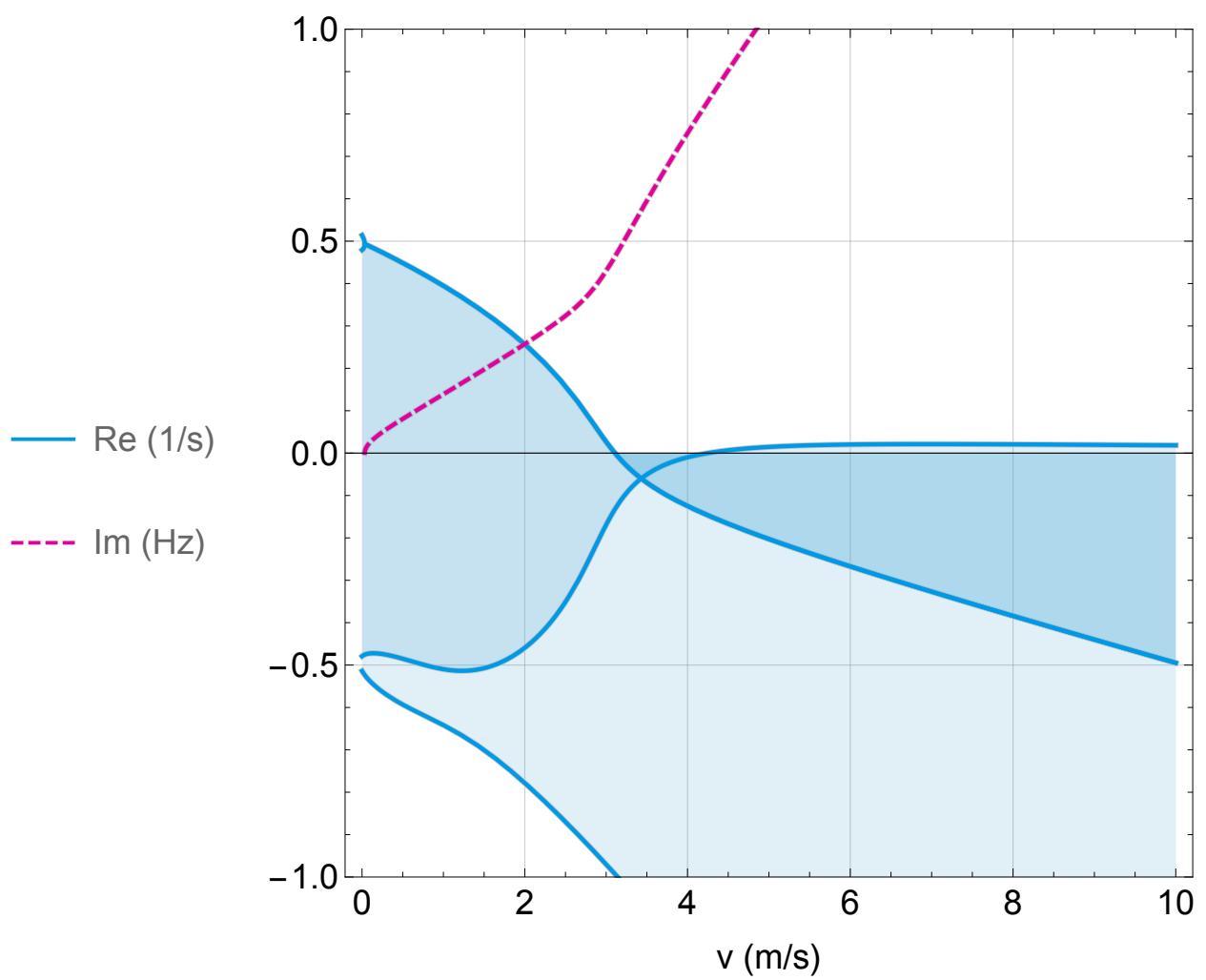

Figure 5.4: Eigenvalues versus speed chart for the linearized tadpole tricycle model using reference parameters.

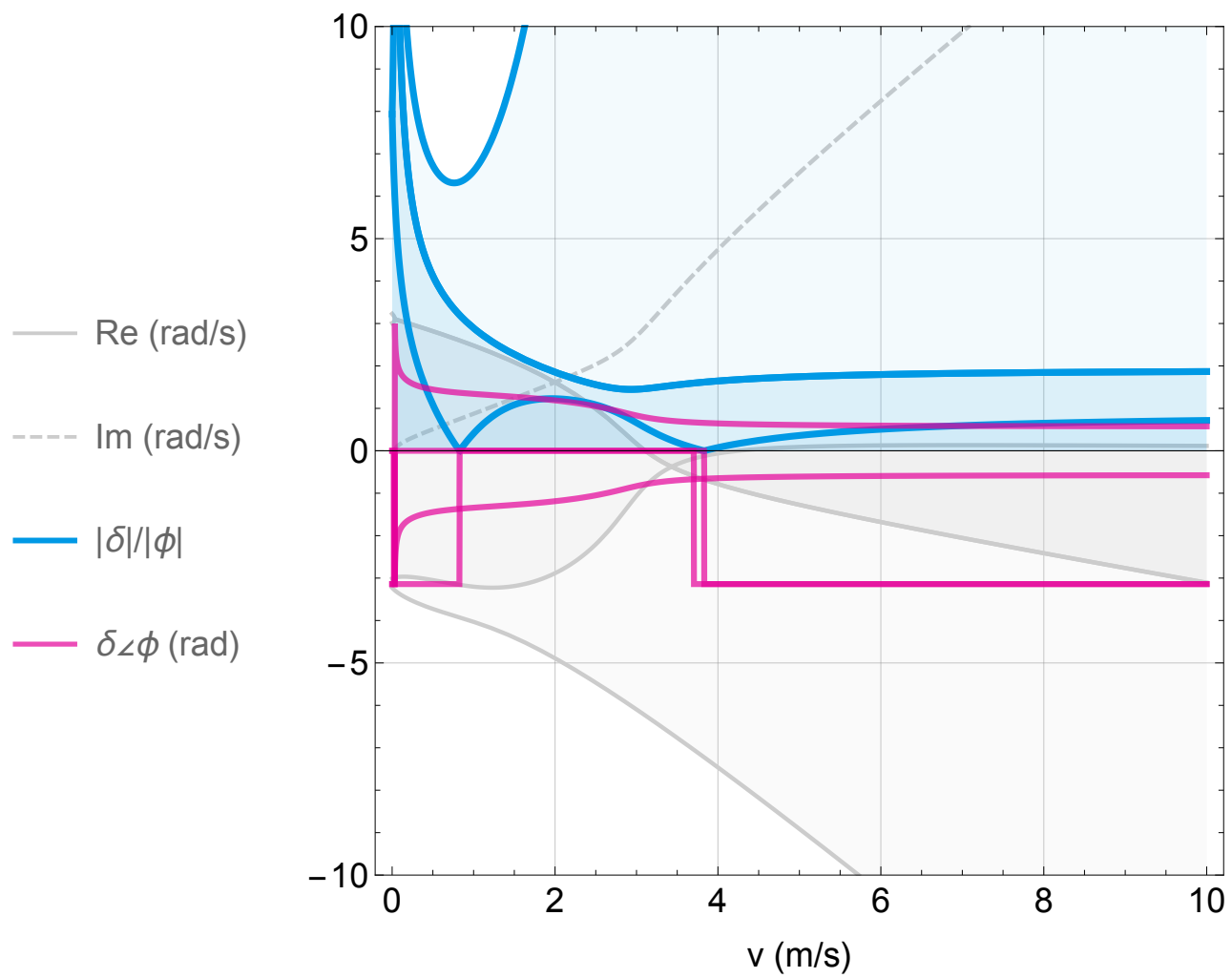

Figure 5.5: Steer-to-lean ratio versus speed chart for the linearized tadpole tricycle model using reference parameters. 

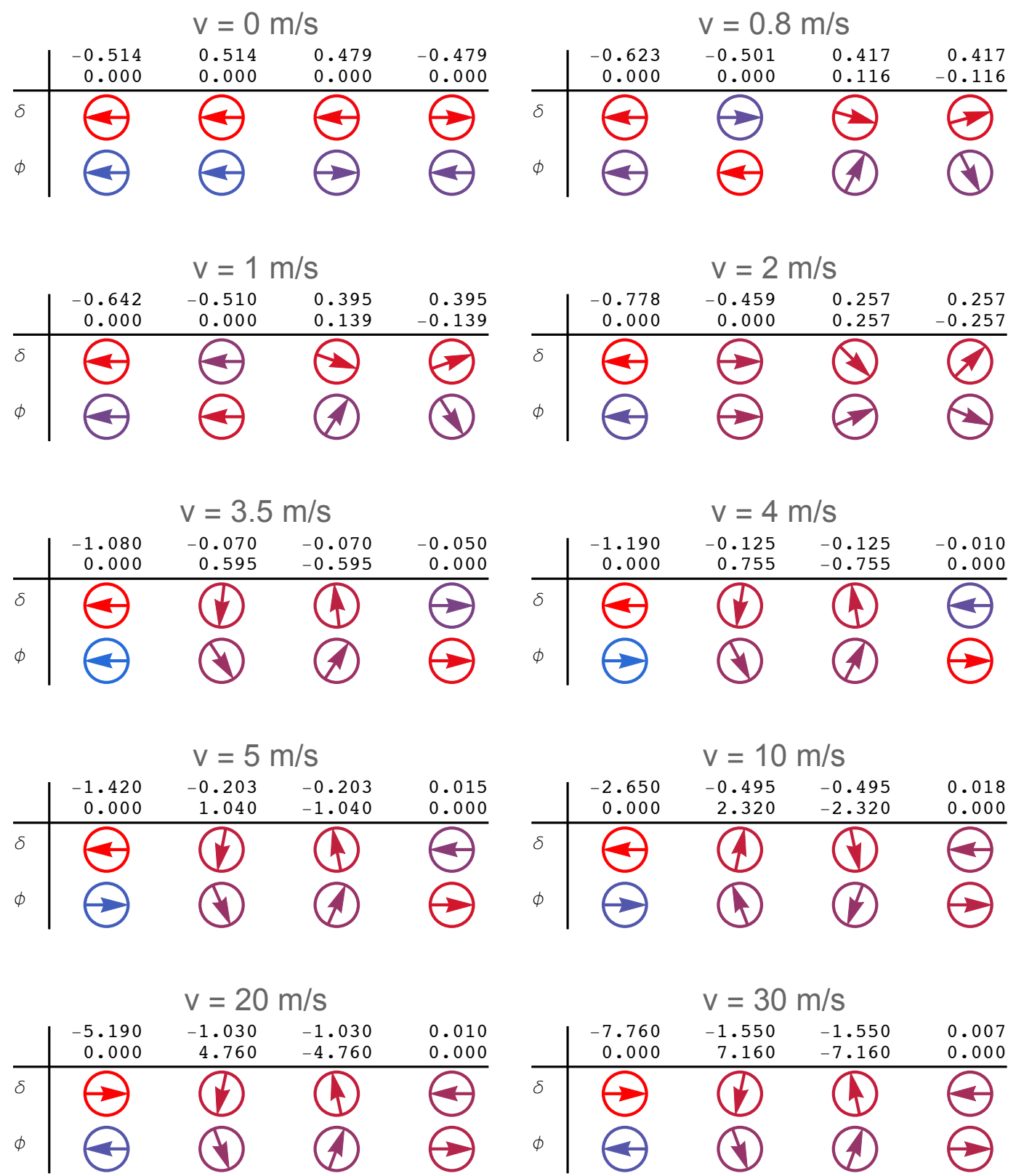

Figure 5.6: Eigenvectors tables for different speeds of the tadpole tricycle using reference parameters. 

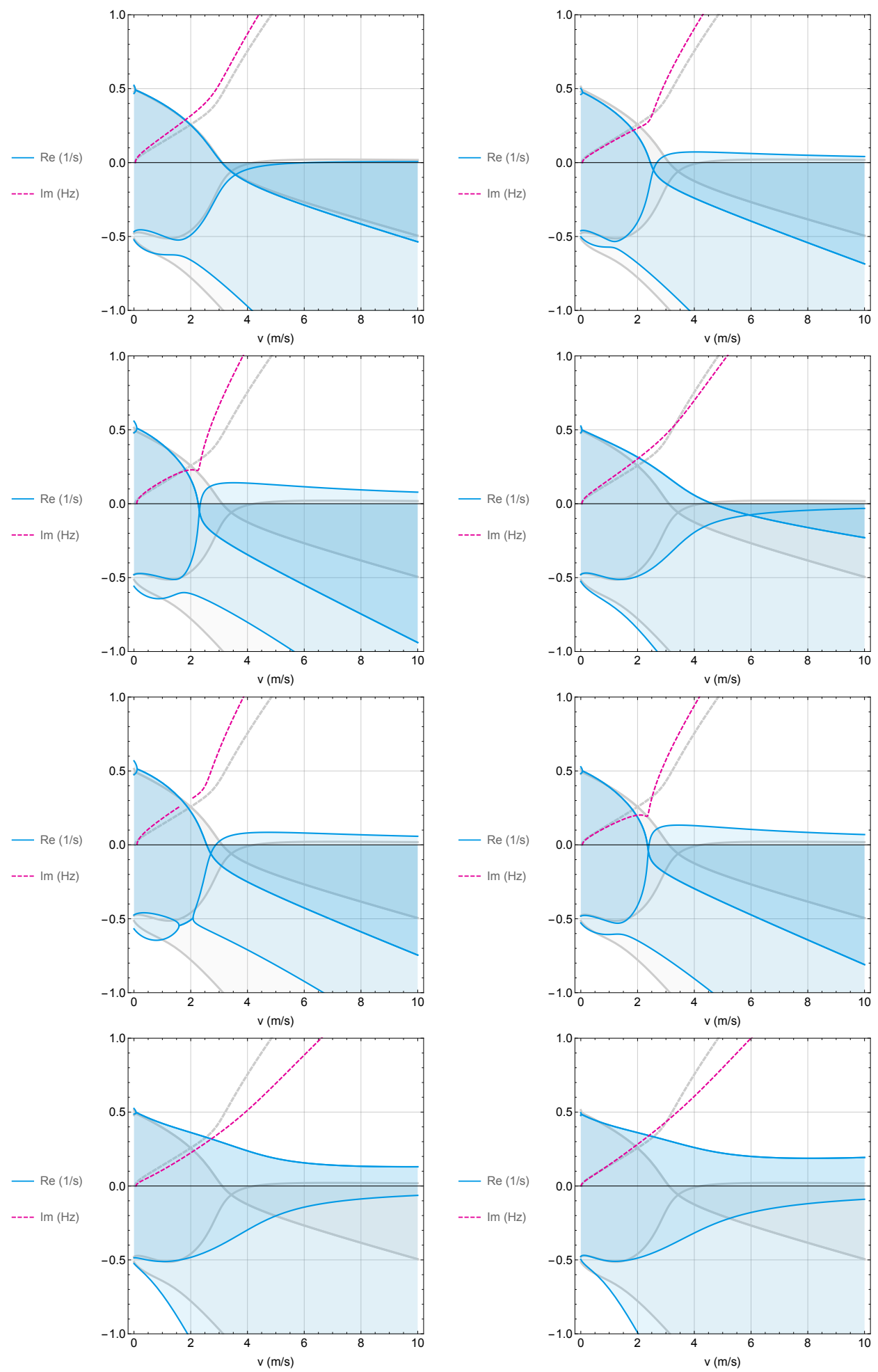

Figure 5.7: Eigenvalues versus speed charts for variant scenarios 

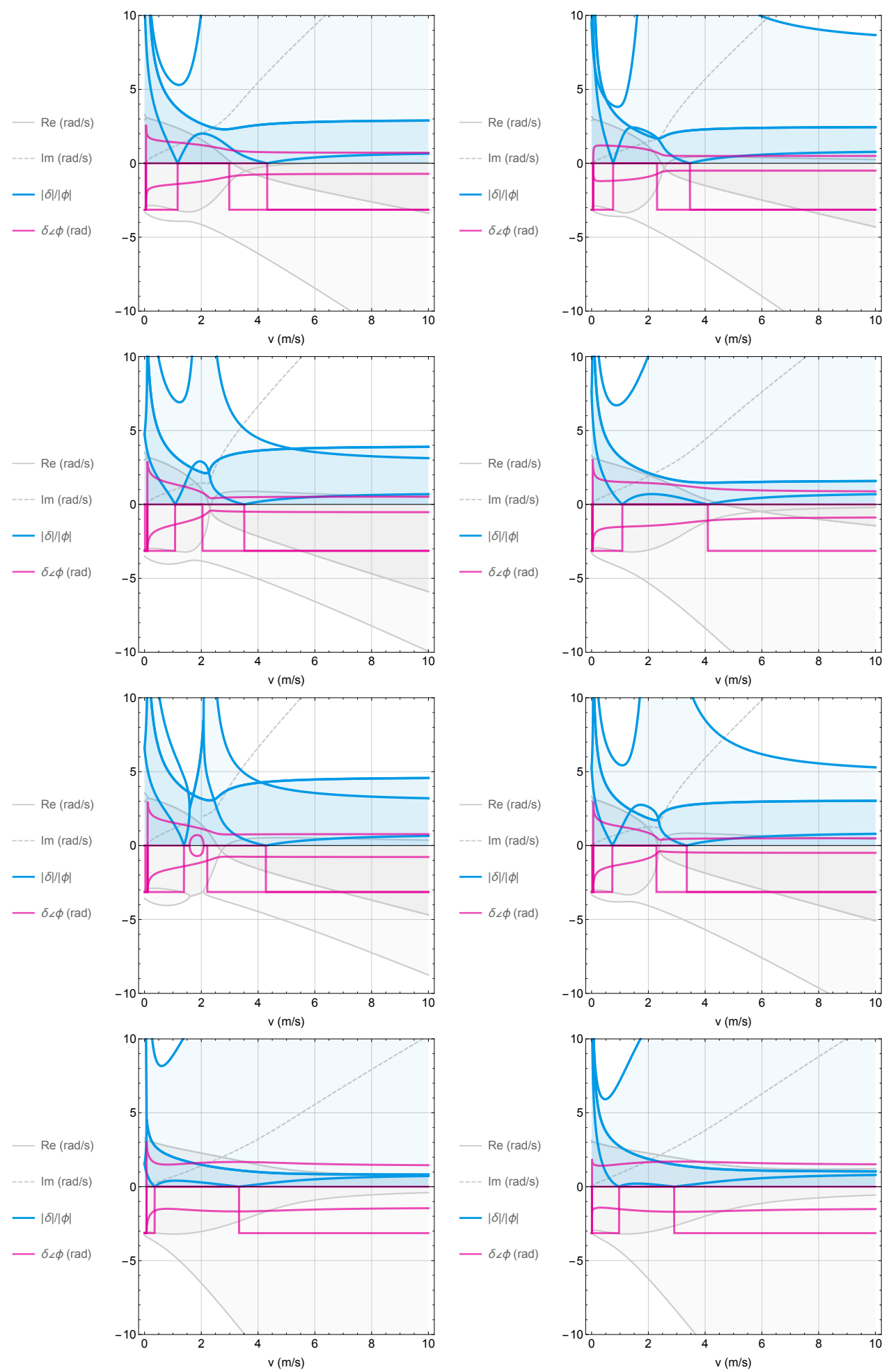

Figure 5.8: Steer-to-lean ratio versus speed charts for variant scenarios 
Table 5.1: Physical parameters for the tadpole tricycle model

\begin{tabular}{|c|c|c|c|}
\hline General & Wheels & Steering bars & Knuckles \\
\hline $\begin{array}{l}\bar{w}=1.075 \mathrm{~m} \\
\bar{c}=0.08 \mathrm{~m} \\
\bar{\lambda}=15.25 \mathrm{deg} \\
\bar{g}=9.81 \mathrm{~m} / \mathrm{s}^{2} \\
\bar{\xi}=0 \mathrm{rad}\end{array}$ & $\begin{array}{l}\bar{r}_{\mathcal{W}}=0.253 \mathrm{~m} \\
\bar{r}_{\mathcal{F}}=0.255 \mathrm{~m} \\
\bar{m}_{\mathcal{W}}=2.50 \mathrm{~m} \\
\bar{I}_{\mathcal{W}, \mathrm{a}}=0.180 \mathrm{~kg} \cdot \mathrm{m}^{2} \\
\bar{l}_{\mathcal{W}, \mathrm{r}}=0.094 \mathrm{~kg} \cdot \mathrm{m}^{2} \\
\bar{m}_{\mathcal{F}}=2.00 \mathrm{~m} \\
\bar{l}_{\mathcal{F}, \mathrm{a}}=0.140 \mathrm{~kg} \cdot \mathrm{m}^{2} \\
\bar{l}_{\mathcal{F}, \mathrm{r}}=0.075 \mathrm{~kg} \cdot \mathrm{m}^{2}\end{array}$ & $\begin{array}{l}\bar{l}_{s}=0.220 \mathrm{~m} \\
\hat{\gamma}_{s}=0.50 \\
\bar{\phi}_{S}=0 \mathrm{deg} \\
\bar{\psi}_{s}=70 \mathrm{deg} \\
\bar{m}_{\mathcal{S}}=0.30 \mathrm{~kg} \\
\bar{l}_{S, \mathrm{r}}=0.001 \mathrm{~kg} \cdot \mathrm{m}^{2} \\
\bar{x}_{\mathrm{s} \mid \mathrm{K}}=-0.080 \mathrm{~m} \\
\bar{y}_{\mathrm{s} \mid \mathrm{K}}=-0.015 \mathrm{~m} \\
\bar{z}_{\mathrm{S} \mid \mathrm{K}}=0.012 \mathrm{~m}\end{array}$ & 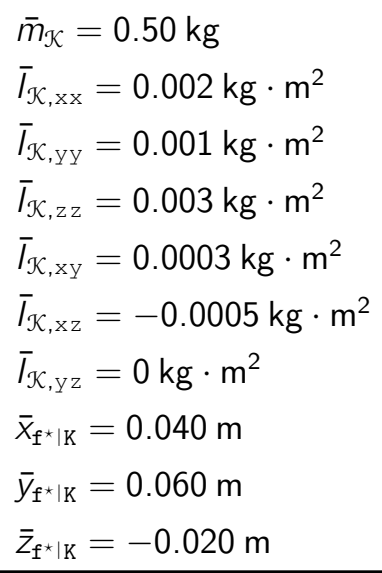 \\
\hline $\mathscr{B}$ & $\mathscr{H}$ & $\mathscr{P}$ & Q \\
\hline $\begin{array}{l}\bar{m}_{\mathcal{B}}=85 \mathrm{~kg} \\
\bar{I}_{\mathcal{B}, \mathrm{xx}}=9.70 \mathrm{~kg} \cdot \mathrm{m}^{2} \\
\bar{I}_{\mathcal{B}, \mathrm{zz}}=3.10 \mathrm{~kg} \cdot \mathrm{m}^{2} \\
\bar{I}_{\mathcal{B}, \mathrm{xz}}=2.70 \mathrm{~kg} \cdot \mathrm{m}^{2} \\
\bar{x}_{\mathrm{b}^{\star} \mid \mathrm{B}}=0.375 \mathrm{~m} \\
{\overline{\mathrm{z}_{\mathrm{b}} \mid \mathrm{B}}}=-0.950 \mathrm{~m}\end{array}$ & $\begin{array}{l}\bar{m}_{\mathcal{H}}=3.0 \mathrm{~kg} \\
\bar{l}_{\mathcal{H}, \mathrm{xx}}=0.097 \mathrm{~kg} \cdot \mathrm{m}^{2} \\
\bar{l}_{\mathcal{H}, \mathrm{zz}}=0.015 \mathrm{~kg} \cdot \mathrm{m}^{2} \\
\bar{l}_{\mathcal{H}, \mathrm{xz}}=-0.009 \mathrm{~kg} \cdot \mathrm{m}^{2} \\
\bar{x}_{\mathrm{h}^{\star} \mid \mathrm{H}}=0.020 \mathrm{~m} \\
{\overline{\mathrm{z}_{\mathrm{h}} \mid \mathrm{H}}}_{\bar{y}^{\prime}}=-0.820 \mathrm{~m} \\
\bar{x}_{\mathrm{h} \mid \mathrm{H}}=-0.120 \mathrm{~m} \\
\bar{y}_{\mathrm{h} \mid \mathrm{H}}=0.015 \mathrm{~m} \\
\bar{z}_{\mathrm{h} \mid \mathrm{H}}=-0.165 \mathrm{~m}\end{array}$ & $\begin{array}{l}\bar{m}_{\mathcal{P}}=0.75 \mathrm{~kg} \\
{\overline{l_{\mathcal{P}, \mathrm{x}}}}=0.010 \mathrm{~kg} \cdot \mathrm{m}^{2} \\
{\overline{I_{\mathcal{P}, \mathrm{Z}}}}=0.009 \mathrm{~kg} \cdot \mathrm{m}^{2} \\
\bar{\lambda}_{\mathcal{P}}=-2.5 \mathrm{deg} \\
\bar{x}_{\mathrm{p}_{\mathrm{o}} \mid \mathrm{B}}=1.040 \mathrm{~m} \\
\bar{z}_{\mathrm{p} \mid \mathrm{B}}=-0.310 \mathrm{~m} \\
\bar{y}_{\mathrm{p} \mid \mathrm{P}}=0.195 \mathrm{~m} \\
\bar{z}_{\mathrm{p} \mid \mathrm{P}}=-0.040 \mathrm{~m} \\
\bar{x}_{\mathrm{p} \mid \mathrm{K}}=0.030 \mathrm{~m} \\
\bar{y}_{\mathrm{p} \mid \mathrm{K}}=0.025 \mathrm{~m} \\
\bar{z}_{\mathrm{p} \mid \mathrm{K}}=-0.060 \mathrm{~m} \\
\bar{x}_{\mathrm{p}^{\star} \mid \mathrm{K}}=0 \mathrm{~m} \\
\bar{z}_{\mathrm{p}^{\star} \mid \mathrm{P}}=-0.015 \mathrm{~m}\end{array}$ & $\begin{array}{l}\bar{m}_{Q}=0.75 \mathrm{~kg} \\
\bar{l}_{Q, \mathrm{x}}=0.012 \mathrm{~kg} \cdot \mathrm{m}^{2} \\
{\overline{l_{Q}, \mathrm{z}}}=0.011 \mathrm{~kg} \cdot \mathrm{m}^{2} \\
\bar{\lambda}_{Q}=-2.5 \mathrm{deg} \\
\bar{x}_{\mathrm{q}_{\bullet} \mid \mathrm{B}}=1.070 \mathrm{~m} \\
\bar{z}_{\mathrm{q}_{\circ} \mid \mathrm{B}}=-0.209 \mathrm{~m} \\
\bar{y}_{\mathrm{q} \mid \mathrm{Q}}=0.220 \mathrm{~m} \\
\bar{z}_{\mathrm{q} \mid \mathrm{Q}}=-0.040 \mathrm{~m} \\
\bar{x}_{\mathrm{q} \mid \mathrm{K}}=0.050 \mathrm{~m} \\
\bar{y}_{\mathrm{q} \mid \mathrm{K}}=0 \mathrm{~m} \\
\bar{z}_{\mathrm{q} \mid \mathrm{K}}=0.041 \mathrm{~m} \\
\bar{x}_{\mathrm{q}^{\star} \mid \mathrm{K}}=0 \mathrm{~m} \\
\bar{z}_{\mathrm{q}^{\star} \mid \mathrm{Q}}=-0.015 \mathrm{~m}\end{array}$ \\
\hline
\end{tabular}

ones observed in the bicycle model. In the former, the eigenvalues are always negative and decrease with any increase in the translation speed; moreover, the steer-to-lean ratio is always high (the greatest among the all the modes, generally above 5 in magnitude), approaching a local maximum when $v \rightarrow 0$ and approaching infinity at the speed corresponding to a phase shift transition from 0 to $\pi$ (i.e. steer and lean are in-phase at low speeds and are completely out-of-phase after the transition). In the latter, a pair of positive real eigenvalues at very low speeds become a pair of conjugate complex eigenvalues whose real parts keep decreasing with the increase of speed so that, apart from some scenarios, they become negative at some transition speed; the absolute value of the imaginary parts (corresponding to the natural frequencies of "weave"), however, keep increasing with the increase of the speed; the steer-to-lean ratio, which approaches infinity at a speed close to, but slightly greater than $v=0$ 
(about $0.02 \mathrm{~m} / \mathrm{s}$ ) decreases both in magnitude and phase shift with the increase of the speed, stabilizing at some non-zero value.

The only mode that shows major differences when compared to the bicycle model is the "capsize". As in the bicycle model, the corresponding eigenvalues are negative real numbers at low speeds, almost not varying at these low speeds, then increasing with the increase of the translation speed, becoming positive at some transition speed, reaching a peak at some higher speed and finally decreasing asymptotically to zero. The steer-to-lean ratio, however, behaves in quite a different way. It approaches a local maximum as $v \rightarrow 0$, as in the bicycle model, but it has two phase shifts, instead of just one: the first from $\pi$ (opposite phase) to 0 (in-phase) and the second, from 0 to $\pi$. This means that, in some scenarios (including the reference one), within the self-stable speed range, the "capsize" mode, which is (in general) the dominant one, is characterized by steering towards the fall at lower self-stable speeds and steering oppositely to the fall at higher self-stable speeds. Furthermore, instead of going asymptotically to zero as in the bicycle model, the steer-to-lean ratio also stabilizes at a constant value, typically lower in magnitude than the the value of the corresponding ratio for the "weave" mode. Thus, it can be stated that apart from the "capsize" mode behavior, the tadpole tricycle dynamics is quite similar to the typical dynamics of a conventional bicycle.

Finally, it is worth noting that although the range of the self-stable speeds is extremely sensitive to the parameters, in some scenarios it can be a really long range. Some ceteris paribus analyses (i.e. comparisons between the reference scenario alternative scenarios in which a single physical parameter is varied) can be performed in order to figure out which parameters can be varied to increase the range of self-stable speeds. This is out of the scope of this text, however.

\subsection{Mo2DPack - modeling and simulation of pla- nar mechanisms}

Let $\delta$ be a planar mechanism, i.e. a multi-rigid-body system in which all the bodies perform plane motions in parallel planes. Denote by $\mathcal{B}_{n}$ a generic rigid body in $\delta$ and let $\mathcal{B}_{0}$ be the base body whose motion with respect to an inertial reference frame $\mathcal{N}$ is already known. Let $N=\left(n_{\circ}, \hat{\mathbf{x}}, \hat{\mathbf{y}}, \hat{\mathbf{z}}\right)$ be a coordinate system rigidly attached to $\mathcal{N}$ in which $(\hat{\mathbf{x}}, \hat{\mathbf{y}}, \hat{\mathbf{z}})$ is an orthonormal basis and the 
unit vector $\hat{\mathbf{z}}$ is orthogonal to the planes of motion.

As described in Example 2.5, tracking the motion of at least two points of each rigid body, which are called nodes, is enough to fully describe any possible configuration of $\delta$. Actually, once the bodies perform planar motions, descriptions based on the $\mathrm{x}$ and $\mathrm{y}$ coordinates of the nodes only are enough to derive the corresponding mathematical models. Let $b_{n, k}$ denote the $k$-th node of body $\mathcal{B}_{n}$ and $\left(p_{\mathcal{B}_{n}, k, x}, p_{\mathcal{B}_{n}, k, y}\right)$ denote its $x$ and y coordinates in $\mathrm{N}$. Define the nodal generalized coordinates and the nodal quasi-velocities of $\delta$ according to the following conventions:

$$
\begin{array}{ll}
q_{\mathrm{N}, n, 2 k-1}^{\langle 0\rangle}=p_{\mathcal{B}_{n}, k, \mathrm{x}} & q_{\mathrm{N}, n, 2 k-1}^{\langle 1\rangle}=\dot{p}_{\mathcal{B}_{n}, k, \mathrm{x}} \\
q_{\mathrm{N}, n, 2 k}^{\langle 0\rangle}=p_{\mathcal{B}_{n}, k, \mathrm{y}} & q_{\mathrm{N}, n, 2 k}^{\langle 1\rangle}=\dot{p}_{\mathcal{B}_{n}, k, \mathrm{y}}
\end{array}
$$

Note that all the identities presented in Example 2.5 for a $2 \mathrm{D}$ rigid body element, also apply for the nodal generalized coordinates and quasi-velocities introduced.

Assume that the active force system applied to $\mathcal{B}_{n}$ can be reduced to a resultant force ${ }^{\mathrm{A}} f_{n, \mathrm{x}}^{\prime} \hat{\mathbf{x}}+{ }^{\mathrm{A}} f_{n, y}^{\prime} \hat{\mathbf{y}}$ and to a torque with respect to its own center of mass ${ }^{A} f_{n, z}^{\prime} \hat{\mathbf{z}}$. Consider also that the position of the center of mass of $\mathcal{B}_{n}$ is specified in terms of the positions of the nodes $\mathrm{b}_{n, i}$ and $\mathrm{b}_{n, j}$ by the adimensional parameters $\hat{a}_{n, 1}=\hat{\gamma}_{\mathcal{B}_{n}}$ and $\hat{a}_{n, 2}=\hat{\rho}_{\mathcal{B}_{n}}$ (see Example 2.5). Denoting by ${ }^{\mathrm{A}} f_{-}^{\{n\}}$ the parcel of ${ }^{\mathrm{A}} f_{-}$due to the active force system applied to $\mathcal{B}_{n}$, it can be stated that:

$$
\left[\begin{array}{l}
{ }^{\mathrm{A}} f_{\mathrm{N}, n, 2 i-1}^{\{n\}} \\
{ }^{\mathrm{A}} f_{\mathrm{N}, n, 2 i}^{\{n\}} \\
{ }^{\mathrm{A}} f_{\mathrm{N}, n, 2 j-1}^{\{n\}} \\
{ }^{\mathrm{A}} f_{\mathrm{N}, n, 2 j}^{\{n\}} \\
{ }^{\mathrm{A}} f_{\mathrm{B}, n, 3}^{\{n\}}
\end{array}\right]=\left[\begin{array}{ccc}
1-\hat{a}_{n, 1} & -\hat{a}_{n, 2} & 0 \\
\hat{a}_{n, 2} & 1-\hat{a}_{n, 1} & 0 \\
\hat{a}_{n, 1} & \hat{a}_{n, 2} & 0 \\
-\hat{a}_{n, 2} & \hat{a}_{n, 1} & 0 \\
0 & 0 & 1
\end{array}\right]\left[\begin{array}{c}
{ }^{\mathrm{A}} f_{n, \mathrm{x}}^{\prime} \\
{ }^{\mathrm{A}} f_{n, \mathrm{Y}}^{\prime} \\
{ }^{\mathrm{A}} f_{n, \mathrm{z}}^{\prime}
\end{array}\right]
$$

Moreover, defining $\hat{a}_{n, 3}=\left(\hat{a}_{n, 1}-1\right)^{2}+\hat{a}_{n, 2}^{2}, \hat{a}_{n, 4}=\hat{a}_{n, 1}^{2}+\hat{a}_{n, 2}^{2}$ and $\hat{a}_{n, 5}=\hat{a}_{n, 2}^{2}+$ $\left(\hat{a}_{n, 1}-1\right) \hat{a}_{n, 1}$, and denoting by $\bar{m}_{n}$ the mass of $\mathcal{B}_{n}$, by $\bar{I}_{n}$ the moment of inertia of $\mathcal{B}_{n}$ with respect to an axis parallel to $\hat{z}$ passing through the its center of mass and by ${ }^{\mathrm{I}} f_{-}^{\{n\}}$ the parcel of ${ }^{\mathrm{I}} f_{-}$due to the inertia forces of $\mathcal{B}_{n}$, it can be stated 
that:

$$
\left[\begin{array}{c}
{ }^{\mathrm{I}} \mathrm{I}_{\mathrm{N}, n, 2 i-1}^{\{n\}} \\
{ }^{\mathrm{I}} f_{\mathrm{N}, n, 2 i}^{\{n\}} \\
{ }^{\mathrm{I}} f_{\mathrm{N}, n, 2 j-1}^{\{n\}} \\
{ }^{\mathrm{I}} \mathrm{I}_{\mathrm{N}, n, 2 j}^{\{n\}} \\
{ }^{\mathrm{I}} f_{\mathrm{B}, n, 3}^{\{n\}}
\end{array}\right]=\left[\begin{array}{ccccc}
-\bar{m}_{n} \hat{a}_{n, 3} & 0 & \bar{m}_{n} \hat{a}_{n, 5} & \bar{m}_{n} \hat{a}_{n, 2} & 0 \\
0 & -\bar{m}_{n} \hat{a}_{n, 3} & -\bar{m}_{n} \hat{a}_{n, 2} & \bar{m}_{n} \hat{a}_{n, 5} & 0 \\
\bar{m}_{n} \hat{a}_{n, 5} & -\bar{m}_{n} \hat{a}_{n, 2} & -\bar{m}_{n} \hat{a}_{n, 4} & 0 & 0 \\
\bar{m}_{n} \hat{a}_{n, 2} & \bar{m}_{n} \hat{a}_{n, 5} & 0 & -\bar{m}_{n} \hat{a}_{n, 4} & 0 \\
0 & 0 & 0 & 0 & -\bar{l}_{n}
\end{array}\right]\left[\begin{array}{l}
q_{\mathrm{N}, n, 2 i-1}^{\langle 1\rangle} \\
q_{\mathrm{N}, n, 2 i}^{\langle 1\rangle} \\
q_{\mathrm{N}, n, 2 j-1}^{\langle 1\rangle} \\
q_{\mathrm{N}, n, 2 j}^{\langle 1\rangle} \\
q_{\mathrm{B}, n, 3}^{\langle 1\rangle}
\end{array}\right]
$$

with $q_{\mathrm{B}, n, 3}^{\langle 1\rangle}=\omega_{\mathcal{B}_{n}, z}$.

Suppose that two bodies $\mathcal{B}_{n_{1}}$ and $\mathcal{B}_{n_{2}}, n_{1}<n_{2}$, constitute a revolute joint. Once $\delta$ is a planar mechanism, the revolute joint axis must be parallel to $\hat{z}$. Thus, assuming that there is a node $b_{n_{1}, i_{1}}$ in $\mathcal{B}_{1}$ and $b_{n_{2}, i_{2}}$ in $\mathcal{B}_{2}$ such that the revolute joint axis is defined by the line $b_{n_{1}, i_{1}} b_{n_{2}, i_{2}}$, it can be stated that: $q_{\mathrm{N}, n_{2}, 2 i_{2}-1}^{\langle 0\rangle}=q_{\mathrm{N}, n_{1}, 2 i_{1}-1}^{\langle 0\rangle}, q_{\mathrm{N}, n_{2}, 2 i_{2}}^{\langle 0\rangle}=q_{\mathrm{N}, n_{1}, 2 i_{1}}^{\langle 0\rangle}, q_{\mathrm{N}, n_{2}, 2 i_{2}-1}^{\langle 1\rangle}=q_{\mathrm{N}, n_{1}, 2 i_{1}-1}^{\langle 1\rangle}$ and $q_{\mathrm{N}, n_{2}, 2 i_{2}}^{\langle 1\rangle}=$ $q_{\mathrm{N}, n_{1}, 2 i_{1}}^{\langle 1\rangle}$. Considering that $n_{1}<n_{2}$, the generalized variables associated to the point $b_{n_{2}, i_{2}}$ can be deleted from the model using these trivial identities. Moreover, defining ${ }^{\mathrm{A}} f_{\mathrm{N}, n_{1}, 2 i_{1}-1}^{\left\{n_{2}\right\}}={ }^{\mathrm{A}} f_{\mathrm{N}, n_{2}, 2 i_{2}-1}^{\left\{n_{2}\right\}},{ }^{\mathrm{A}} f_{\mathrm{N}, n_{1}, 2 i_{1}}^{\left\{n_{2}\right\}}={ }^{\mathrm{A}} f_{\mathrm{N}, n_{2}, 2 i_{2}}^{\left\{n_{2}\right\}},{ }^{\mathrm{I}} f_{\mathrm{N}, n_{1}, 2 i_{1}-1}^{\left\{n_{2}\right\}}={ }^{\mathrm{I}} f_{\mathrm{N}, n_{2}, 2 i_{2}-1}^{\left\{n_{2}\right\}}$ and ${ }^{\mathrm{I}} f_{\mathrm{N}, n_{1}, 2 i_{1}}\left\{{ }^{\mathrm{I}} f_{\mathrm{N}, n_{2}, 2 i_{2}}\left\{n_{2}\right\}\right.$, after all variable eliminations due to revolute joint constraints, it can be stated that:

$$
\left\{\begin{array}{l}
{ }^{\mathrm{A}} f_{\mathrm{N}, n, r}=\sum_{k \geq n}{ }^{\mathrm{A}} f_{\mathrm{N}, n, r}^{\{k\}} \\
{ }^{\mathrm{I}} f_{\mathrm{N}, n, r}=\sum_{k \geq n}{ }^{\mathrm{I}} f_{\mathrm{N}, n, r}^{\{k\}}
\end{array}\right.
$$

Moreover, if the revolute joint constituted by $\mathcal{B}_{n_{1}}$ and $\mathcal{B}_{n_{2}}$ is active, i.e., if there is an actuator providing a torque $u_{R, n_{1}, n_{2}}$ to control the relative revolute motion between them, it is convenient to define a new quasi-velocity $q_{\mathrm{R}, n_{1}, n_{2}}^{\langle 1\rangle}$ satisfying the following constraint equation:

$$
q_{\mathrm{R}, n_{1}, n_{2}}^{\langle 1\rangle}-\left(q_{\mathrm{B}, n_{2}, 3}^{\langle 1\rangle}-q_{\mathrm{B}, n_{1}, 3}^{\langle 1\rangle}\right)=0
$$

The corresponding generalized forces are the following:

$$
\left\{\begin{array}{l}
{ }^{\mathrm{A}} f_{\mathrm{R}, n_{1}, n_{2}}=u_{\mathrm{R}, n_{1}, n_{2}} \\
{ }^{\mathrm{I}} f_{\mathrm{R}, n_{1}, n_{2}}=0
\end{array}\right.
$$

Suppose now that the a prismatic joint constituted by $\mathcal{B}_{n_{1}}$ and $\mathcal{B}_{n_{2}}\left(n_{1}<n_{2}\right)$. In this case no relative revolute motion is allowed between these bodies, being possible to delete from the model the quasi-velocity $q_{\mathrm{B}, n_{2}, 3}^{\langle 1\rangle}$ once $q_{\mathrm{B}, n_{2}, 3}^{\langle 1\rangle}=q_{\mathrm{B}, n_{1}, 3}^{\langle 1\rangle}$. 
In this case define ${ }^{\mathrm{A}} f_{\mathrm{B}, n_{1}, 3}^{\left\{n_{2}\right\}}={ }^{\mathrm{A}} f_{\mathrm{B}, n_{2}, 3}^{\left\{n_{2}\right\}}$ and ${ }^{\mathrm{I}} f_{\mathrm{B}, n_{1}, 3}^{\left\{n_{2}\right\}}={ }^{\mathrm{I}} f_{\mathrm{B}, n_{2}, 3}^{\left\{n_{2}\right\}}$. After all the eliminations of generalized variables due to prismatic joint constraints, it can be stated that:

$$
\left\{\begin{array}{l}
{ }^{\mathrm{A}} f_{\mathrm{B}, n, 3}=\sum_{k \geq n}{ }^{\mathrm{A}}{ }_{\mathrm{B}, n, 3}^{\{k\}} \\
{ }^{\mathrm{I}} f_{\mathrm{B}, n, 3}=\sum_{k \geq n}{ }^{\mathrm{I}} f_{\mathrm{B}, n, 3}^{\{k\}}
\end{array}\right.
$$

Moreover, it can be stated that two nodes $\mathrm{b}_{n_{1}, i_{1}}$ and $\mathrm{b}_{n_{1}, j_{1}}\left(i_{1}<j_{1}\right)$ of $\mathcal{B}_{n_{1}}$ and one node $b_{n_{2}, i_{2}}$ of $\mathcal{B}_{n_{2}}$, will always be aligned ${ }^{2}$. In order to describe the constraint imposed by this joint, it is convenient to define an extra generalized coordinate $q_{\mathrm{P}, n_{1}, n_{2}}^{\langle 0\rangle}$ and an associated quasi-velocity $q_{\mathrm{P}, n_{1}, n_{2}}^{\langle 1\rangle}=\dot{q}_{\mathrm{P}, n_{1}, n_{2}}^{\langle 0\rangle}$, such that the following invariants can be stated:

$$
\left\{\begin{array}{l}
\bar{\ell}_{n_{1}, i_{1}, j_{1}} q_{\mathrm{N}, n_{2}, 2 i_{2}-1}^{\langle 0\rangle}-\left(\bar{\ell}_{n_{1}, i_{1}, j_{1}}-q_{\mathrm{P}, n_{1}, n_{2}}^{\langle 0\rangle}\right) q_{\mathrm{N}, n_{1}, 2 i_{1}-1}^{\langle 0\rangle}-q_{\mathrm{P}, n_{1}, n_{2}}^{\langle 0\rangle} q_{\mathrm{N}, n_{1}, 2 j_{1}-1}^{\langle 0\rangle}=0 \\
\bar{\ell}_{n_{1}, i_{1}, j_{1}} q_{\mathrm{N}, n_{2}, 2 i_{2}}^{\langle 0\rangle}-\left(\bar{\ell}_{n_{1}, i_{1}, j_{1}}-q_{\mathrm{P}, n_{1}, n_{2}}^{\langle 0\rangle}\right) q_{\mathrm{N}, n_{1}, 2 i_{1}}^{\langle 0\rangle}-q_{\mathrm{P}, n_{1}, n_{2}}^{\langle 0\rangle} q_{\mathrm{N}, n_{1}, 2 j_{1}}^{\langle 0\rangle}=0
\end{array}\right.
$$

with $\bar{\ell}_{n_{1}, i_{1}, j_{1}}$ representing the distance between the nodes $\mathrm{b}_{n_{1}, i_{1}}$ and $\mathrm{b}_{n_{1}, j_{1}}$. Finally, if there is an actuator in this prismatic joint, providing an input force $u_{\mathrm{p}, n_{1}, n_{2}}$, it can be stated ${ }^{3}$ :

$$
\left\{\begin{array}{l}
{ }^{\mathrm{A}} f_{\mathrm{P}, n_{1}, n_{2}}=u_{\mathrm{P}, n_{1}, n_{2}} \\
{ }^{\mathrm{I}} f_{\mathrm{P}, n_{1}, n_{2}}=0
\end{array}\right.
$$

The application of these specific techniques for selecting variables and computing the corresponding generalized forces, along with the modular modeling methodology presented in Sections 4.1 and 4.2, leads to a specialized algorithm for modeling planar mechanisms. This algorithm was implemented as a package of functions for Wolfram Mathematica 10.2 called Mo2DPack, which can be downloaded, along with its complete documentation, at https: //github.com/renatoorsino/Mo2DPack. Mo2DPack not only implements the modeling algorithm described above, but also presents functions for obtaining numerical solutions for the forward and inverse dynamics problems associated to the model of a particular planar mechanism.

Example 5.1. This example shows the application of Mo2DPack in the modeling and simulation of the system $\delta$, discussed in Examples 4.2 and 4.7. Basically two rigid bodies are defined, $\mathcal{B}_{1}$ representing an homogeneous ring whose center is $b_{1}^{\star} \equiv b_{1,1}$ and $\mathcal{B}_{2}$ representing an homogeneous disc whose center is

\footnotetext{
2 Without loss in generality, the description of the prismatic joint can also be done by choosing one node in $\mathcal{B}_{n_{1}}$ and two nodes in $\mathcal{B}_{n_{2}}$.

${ }^{3}$ In case of a passive prismatic joint, consider $u_{\mathrm{p}, n_{1}, n_{2}}$ identically zero.
} 
$b_{2}^{\star} \equiv b_{2,1}$. The constraints among these systems are imposed according to the no-slip conditions, given by the invariants e of Example 4.2.

The piece of code for obtaining the mathematical model of $\delta$ using Mo2DPack is shown in Figure 5.9a. To show the expression of matrix $C_{\delta}$ used in the modeling and to obtain the explicit form of the equations of motion of $\mathcal{S}$ along with the relation $\bar{m}_{1} / \bar{m}_{2}$ in terms of $\bar{\theta}$ and $\bar{\phi}$ for a motion in which $q_{1}^{\langle 0\rangle}(t)=\theta, \dot{q}_{1}^{\langle 0\rangle}(t)=0$ and $\ddot{q}_{1}^{\langle 0\rangle}(t)=0$, the piece of code shown in Figure $5.9 \mathrm{~b}$ can be applied. Finally, the piece of code shown in Figure 5.9c can be used to perform the simulations of the (non-linear) model shown in Example 4.7.

The following subsections present the application of Mo2DPack to the modeling and inverse dynamics simulations of a Whitworth quick-return mechanism and a 3 RRR parallel mechanism. 


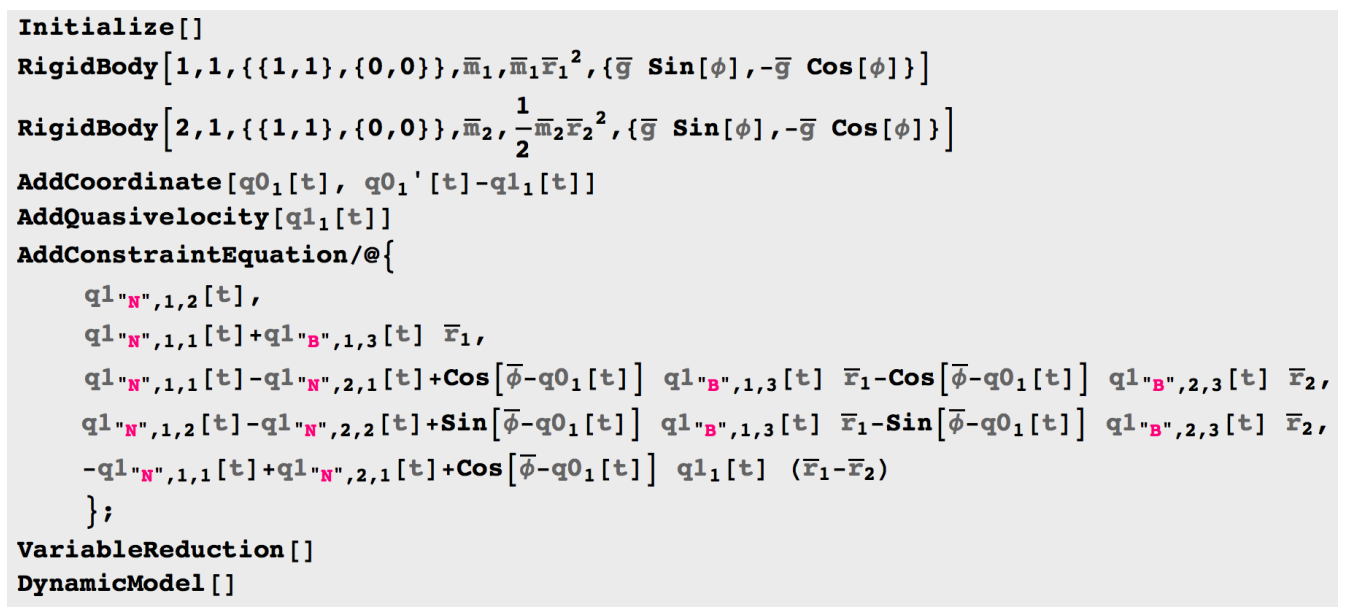

(a) Derivation of the model

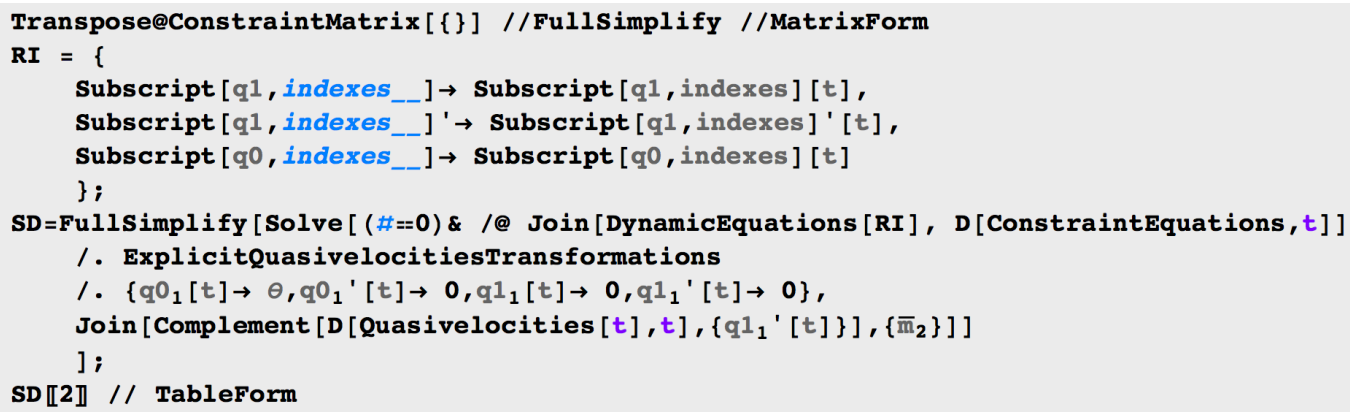

(b) Finding the explicit form of the equations of motion (see Example 4.2)

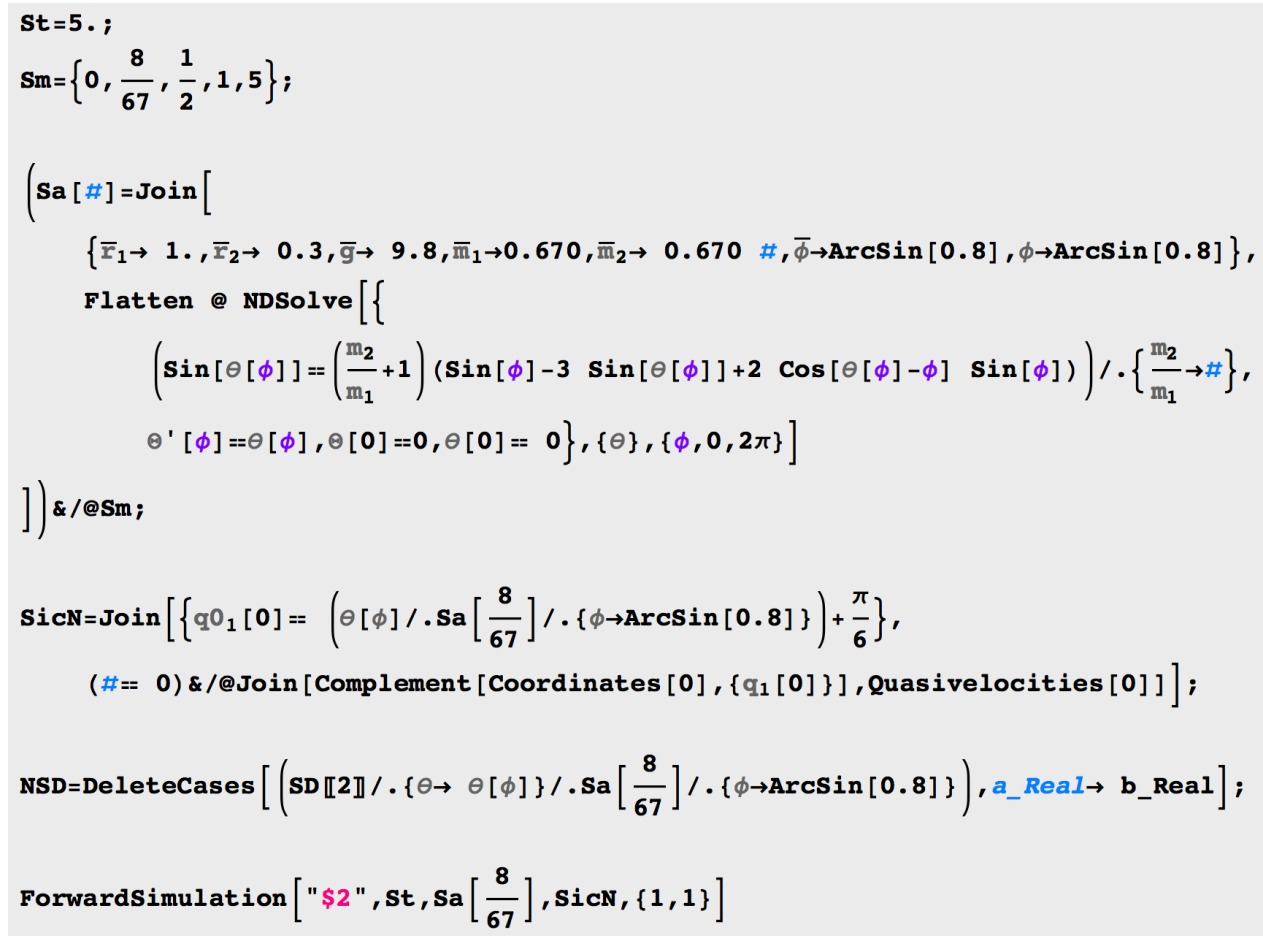

(c) Forward simulation of the non-linear model (see Example 4.7)

Figure 5.9: Algorithm for modeling and simulation of system $\mathcal{S}$. 


\subsubsection{Modeling and inverse dynamics simulation of a Whit- worth quick-return mechanism}

Consider the planar mechanism represented in Figure 5.10, which is a Whitworth quick-return mechanism. The following piece of code can be used to model this system using Mo2DPack:

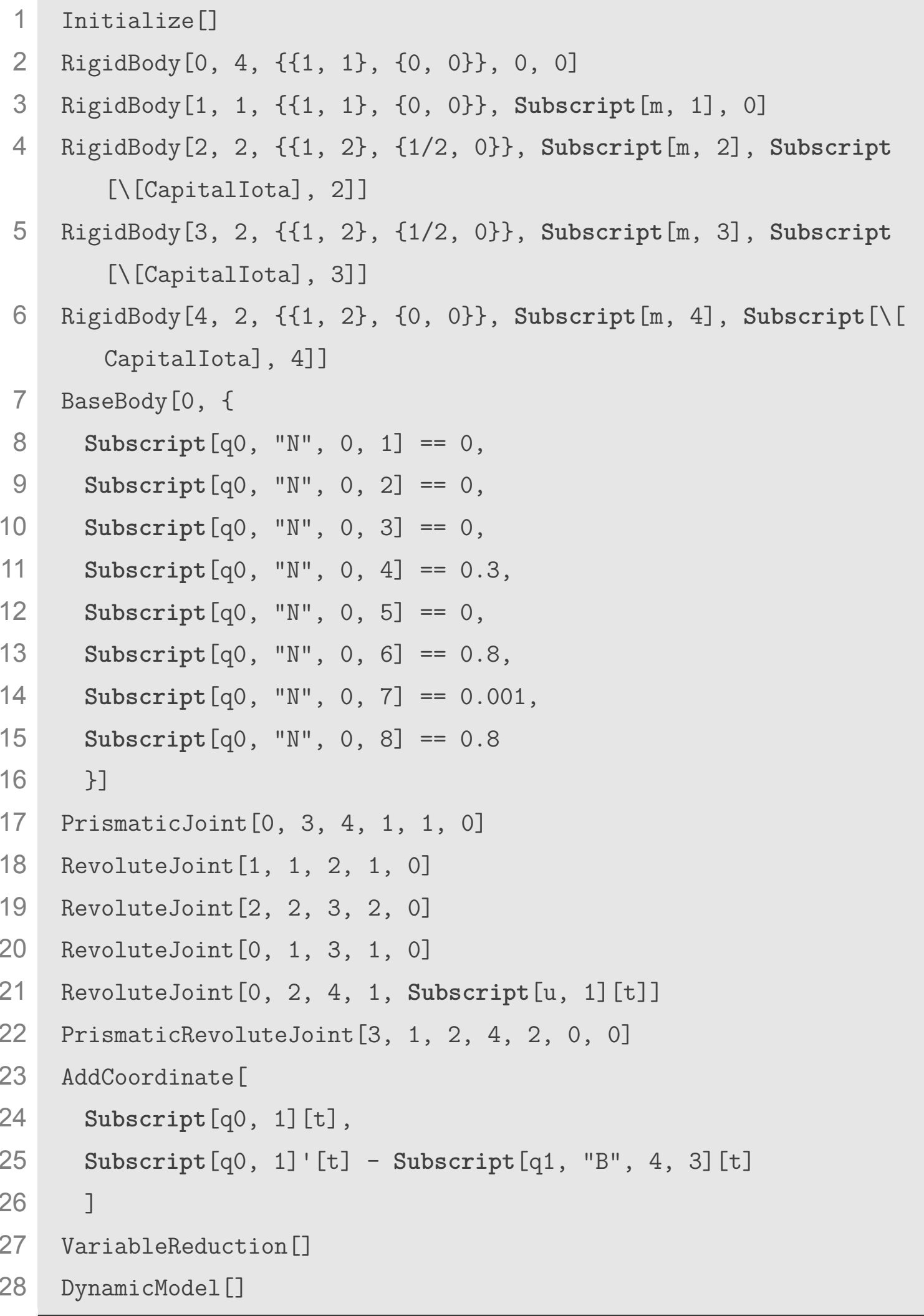


In this code, $\mathcal{B}_{0}$ is defined as being the base body of this mechanism (rigidly attached to an inertial reference frame $\mathcal{N}$ ). Four nodes are chosen to describe $\mathcal{B}_{0}, b_{0,1}, b_{0,2}, b_{0,3}$ and $b_{0,4}$, whose coordinates are, respectvely, $(0,0),(0,0.3 \mathrm{~m})$, $(0,0.8 \mathrm{~m})$ and $(0.001 \mathrm{~m}, 0.3 \mathrm{~m})$. Also, it is supposed that the centers of mass of $\mathcal{B}_{2}$ and $\mathcal{B}_{3}$ are in the midpoints of the line segments defined by their extreme nodes, and that the center of mass of $\mathcal{B}_{4}$ is the node $b_{4,1}$. Revolute joints are defined by the kinematic pairs $\left(\mathcal{B}_{0}, \mathcal{B}_{3}\right),\left(\mathcal{B}_{0}, \mathcal{B}_{4}\right),\left(\mathcal{B}_{1}, \mathcal{B}_{2}\right)$ and $\left(\mathcal{B}_{2}, \mathcal{B}_{3}\right)$, a prismatic joint is defined by the pair $\left(\mathcal{B}_{0}, \mathcal{B}_{1}\right)$ and a superposition between a prismatic and a revolute joint is defined by $\left(\mathcal{B}_{3}, \mathcal{B}_{4}\right)$. The only active joint is the revolute one defined by the pair $\left(\mathcal{B}_{0}, \mathcal{B}_{4}\right)$. in which there is an input torque $u_{1}$. Moreover, it is supposed that the mechanism is assembled in the horizontal, so that the gravitational effects are not considered. By convenience, define the generalized coordinate $q_{1}^{\langle 0\rangle}$ such that $\dot{q}_{1}^{\langle 0\rangle}=q_{\mathrm{B}, 4,3}^{\langle 1\rangle}$

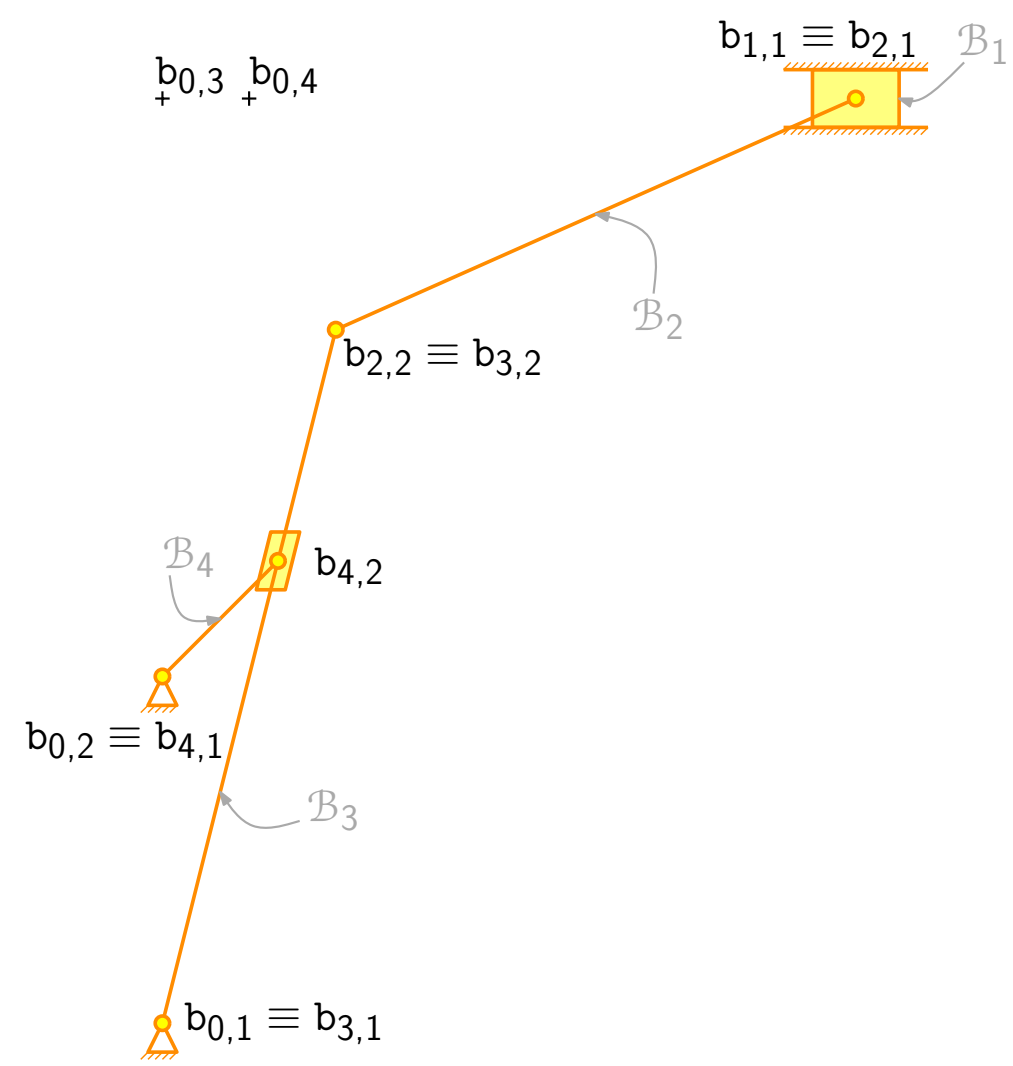

Figure 5.10: Representation of a Whitworth quick-return mechanism.

The following piece of code can be used to set and run an inverse dynamics simulation, labeled as "Sl", in which $q_{\mathrm{B}, 4,3}^{\langle 1\rangle}$ is constant and equal to $\pi / 2 \mathrm{rad} / \mathrm{s}$ :

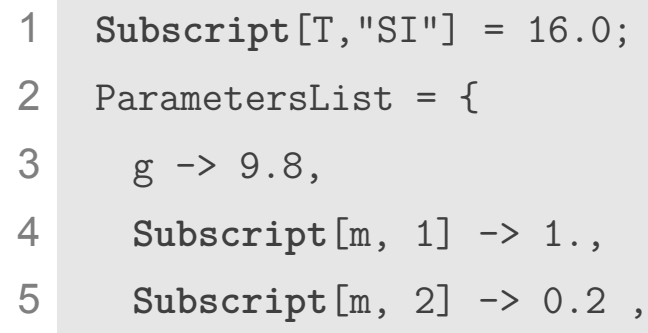




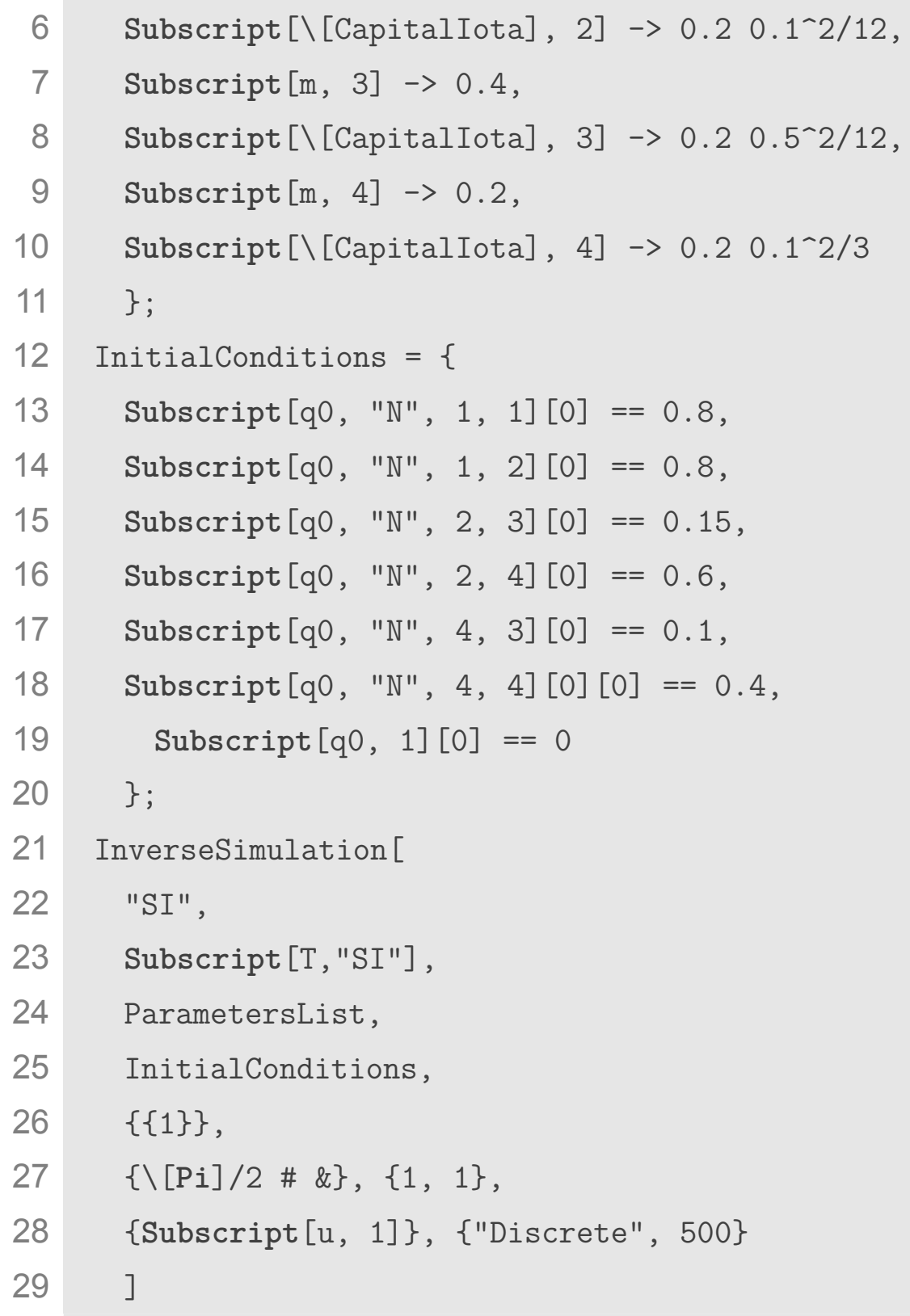

Some of the results of this simulation are shown in Figures $5.11-5.14$. Particularly, it is relevant to mention that the quick-return behavior (noticed, for instance, in the time history associated to the quasi-velocity $\left.q_{\mathrm{P}, 0,1}^{\langle\langle\rangle}\right)$is associated to peaks of torque provided by the actuator. 


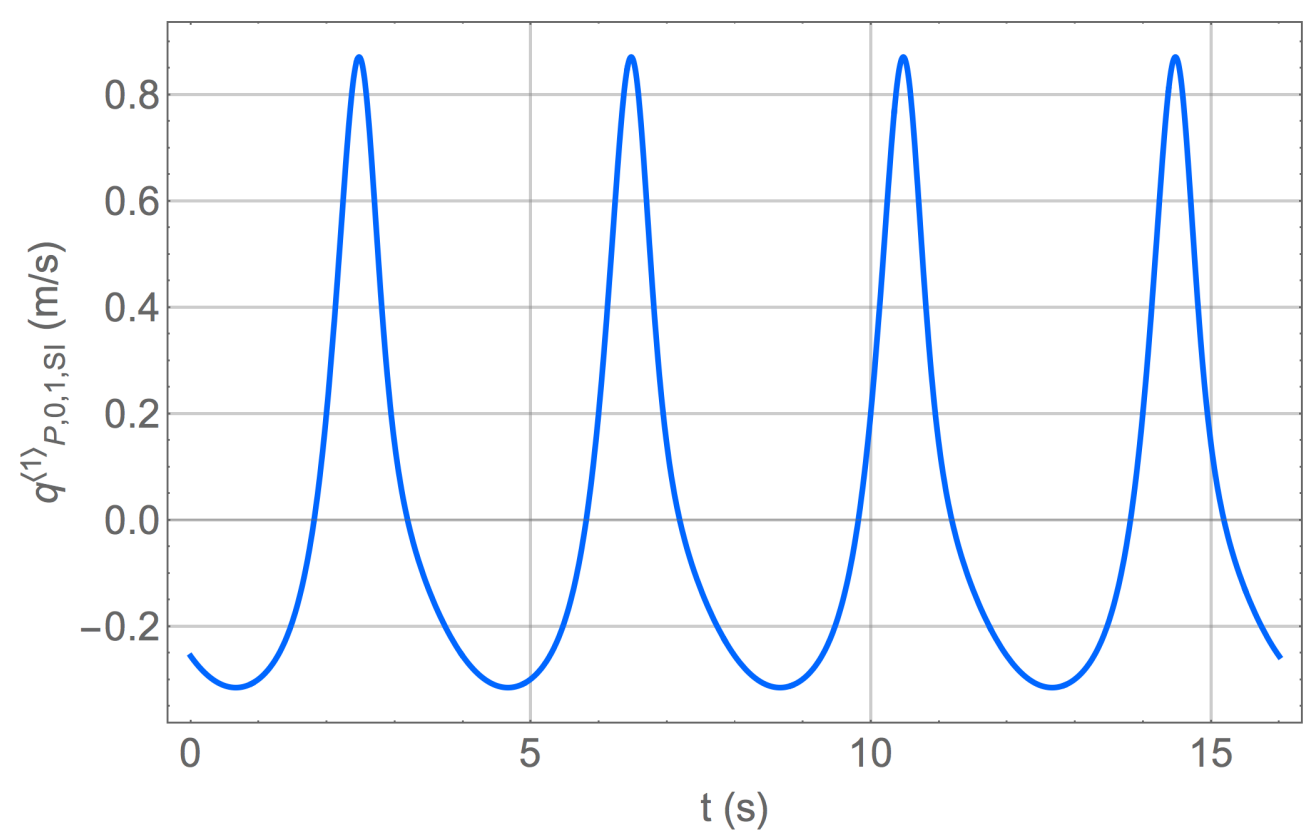

Figure 5.11: Time history of the quasi-velocity $q_{\mathrm{P}, 0,1}^{\langle 1\rangle}$ in the simulation "SI".

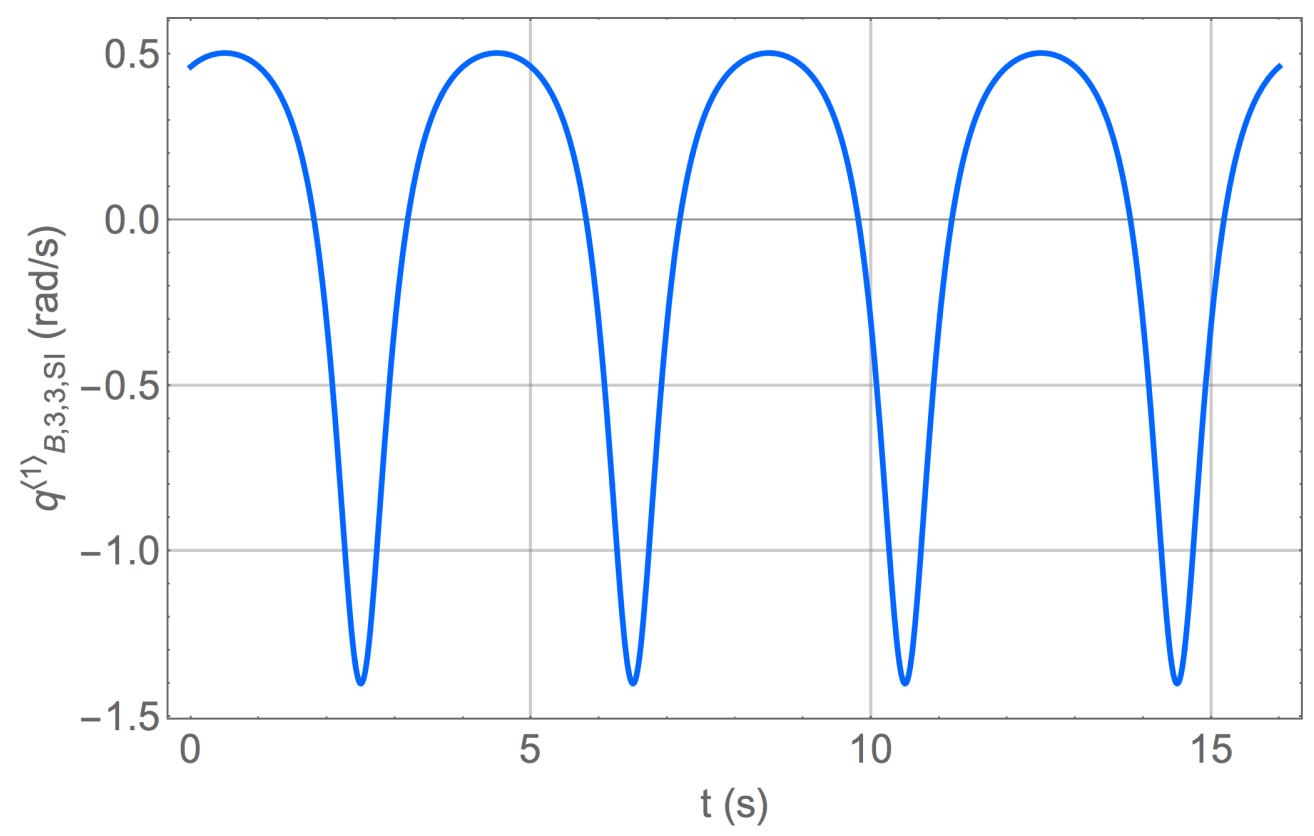

Figure 5.12: Time history of the quasi-velocity $q_{P, 3,3}^{\langle 1\rangle}$ in the simulation "SI". 


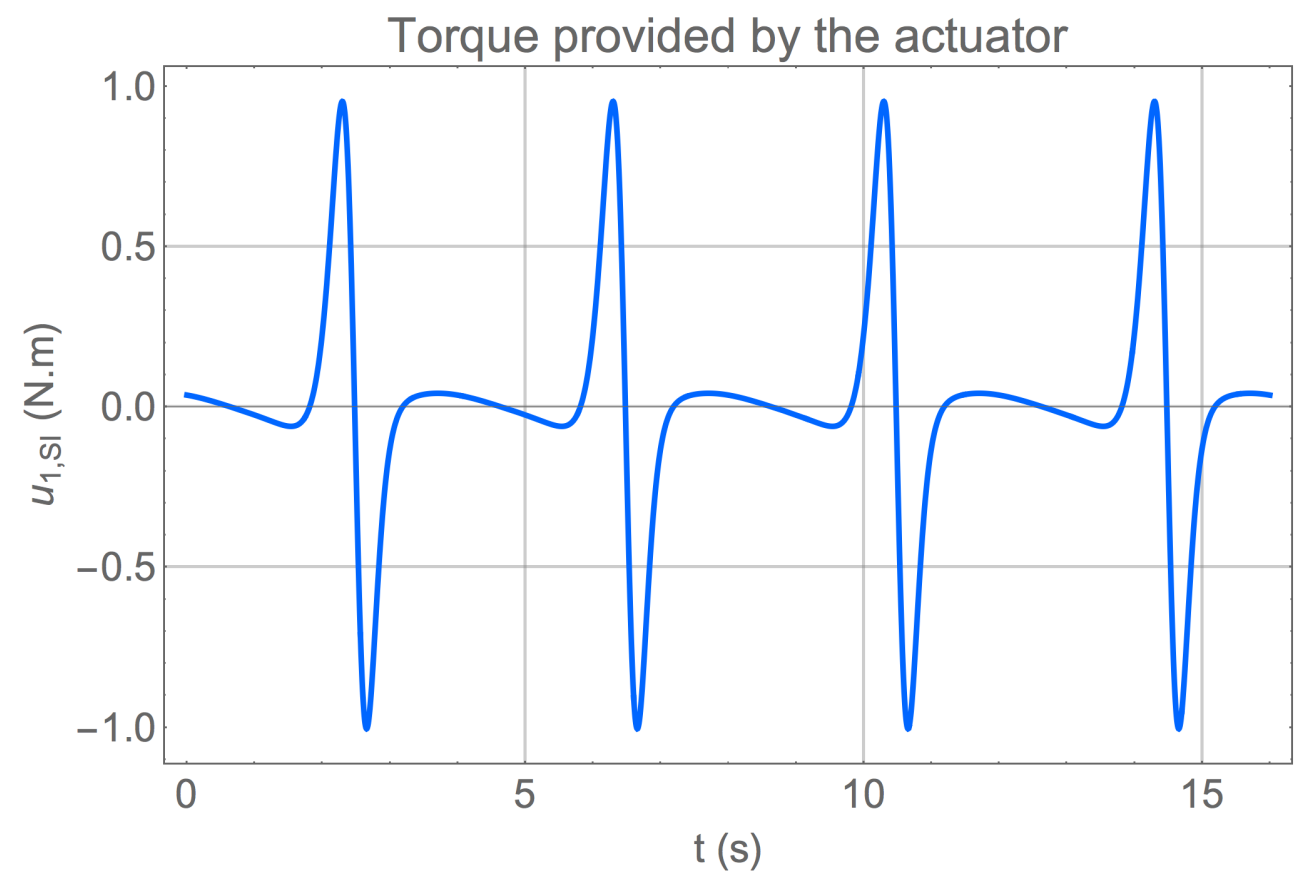

Figure 5.13: Time history of the input torque $u_{1}$ in the simulation "SI".

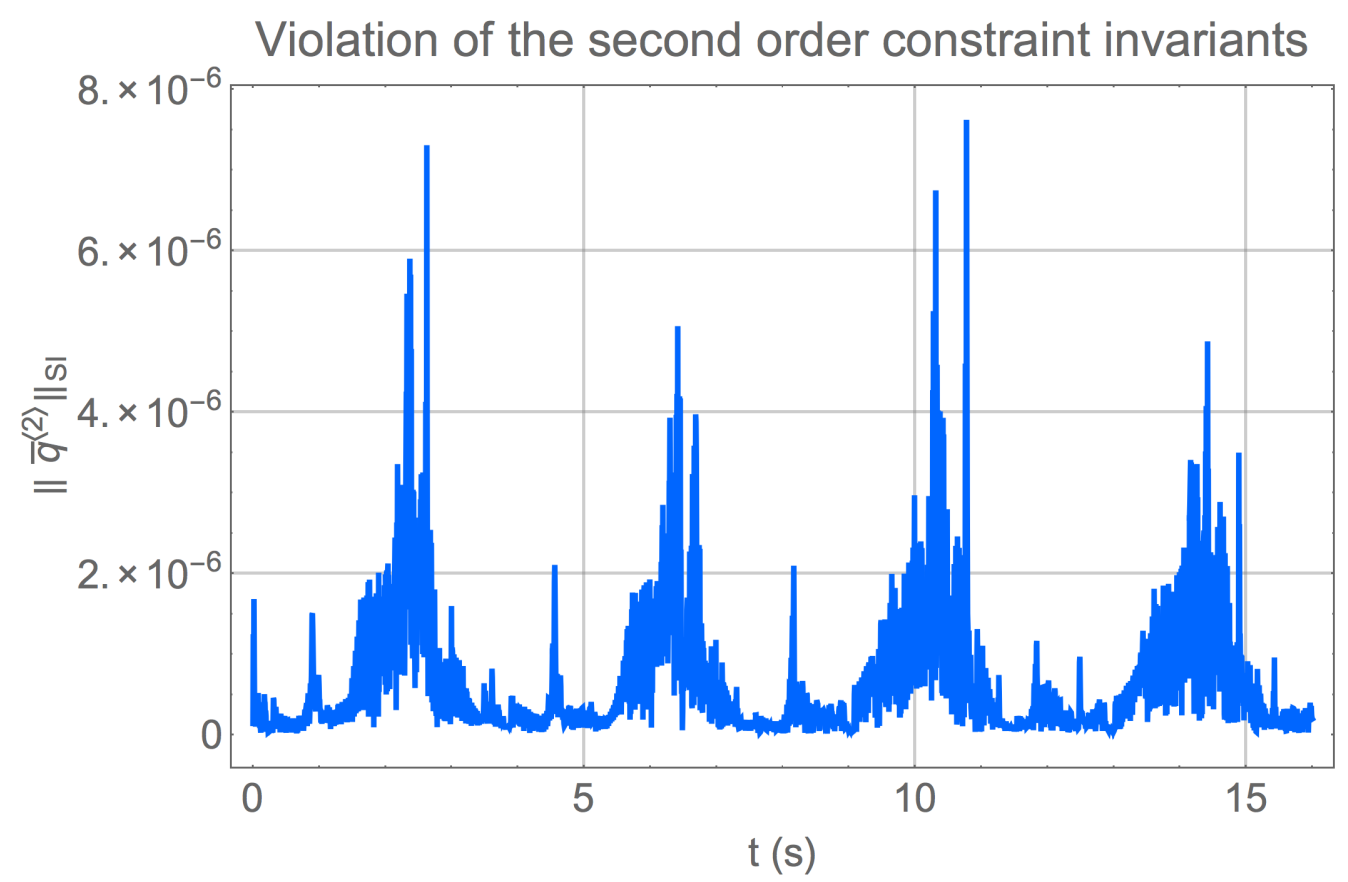

Figure 5.14: Inspection of the error in the numerical solution of the constraint equations in the simulation "SI". 


\subsubsection{Modeling and inverse dynamics simulation of a 3 RRR mechanism}

Consider the $3 \underline{R R R}$ parallel mechanism represented in Figure 5.15. The following piece of code can be used for the modeling of this system ${ }^{4}$ :

Initialize []

$2 \operatorname{RigidBody}[0,3,\{\{1,1\},\{0,0\}\}, 0,0]$

3 RigidBody $[1,4,\{\{1,2\},\{0,0\}\}$, Subscript $[m, 1]$, Subscript $[\backslash[$

Capitallota], 1]]

RigidBody $[2,2,\{\{1,2\},\{1 / 2,0\}\}$, Subscript $[m, 2]$, Subscript

[\[CapitalIota $], 2]]$

RigidBody $[3,2,\{\{1,2\},\{1 / 2,0\}\}$, Subscript $[m, 2]$, Subscript

[\[CapitalIota], 2]]

RigidBody $[4,2,\{\{1,2\},\{1 / 2,0\}\}$, Subscript $[m, 2]$, Subscript

[\[CapitalIota], 2]]

RigidBody $[5,2,\{\{1,2\},\{0,0\}\}$, Subscript $[m, 3]$, Subscript $[\backslash[$

Capitallota], 3] ]

RigidBody $[6,2,\{\{1,2\},\{0,0\}\}$, Subscript $[m, 3]$, Subscript $[\backslash[$

Capitallota], 3] ]

RigidBody $[7,2,\{\{1,2\},\{0,0\}\}$, Subscript $[m, 3]$, Subscript $[\backslash[$

Capitallota], 3] ]

BaseBody [0, \{

Subscript $[q 0, " N ", 0,1]==3$,

Subscript $[q 0, " N ", 0,2]==0$,

Subscript $[q 0, " N ", 0,3]==-(3 / 2)$,

Subscript $[q 0, " N ", 0,4]==(3 \operatorname{Sqrt}[3]) / 2$,

Subscript [q0, "N", 0,5$]==-(3 / 2)$,

Subscript $[q 0, " N ", 0,6]==-((3 \operatorname{Sqrt}[3]) / 2)$

18 RevoluteJoint [0, 1, 5, 1, Subscript [u, 1] [t]]

19 RevoluteJoint [0, 2, 6, 1, Subscript [u, 2] [t]]

20 RevoluteJoint [0, 3, 7, 1, Subscript [u, 3] [t]]

21 RevoluteJoint $[1,2,2,1,0]$

22 RevoluteJoint [1, 3, 3, 1, 0]

23 RevoluteJoint $[1,4,4,1,0]$

\footnotetext{
${ }^{4}$ It is worth noting that Mo2DPack automatically derives the mathematical model of a planar mechanism using a particular version of the modular modeling algorithm that does not allow, for example, to conceive the model of this mechanism as shown in the tree structure presented in Figure 2.8.
} 
RevoluteJoint [2, 2, 5, 2, 0]

RevoluteJoint [3, 2, 6, 2, 0]

RevoluteJoint $[4,2,7,2,0]$

\section{AddCoordinate [}

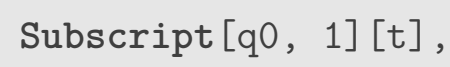

All the kinematic pairs in this mechanism constitute revolute joints, being $\left(\mathcal{B}_{0}, \mathcal{B}_{5}\right),\left(\mathcal{B}_{0}, \mathcal{B}_{6}\right)$ and $\left(\mathcal{B}_{0}, \mathcal{B}_{7}\right)$ the active ones. The torques provided by the actuators in these joints are denoted respectively by $u_{1}, u_{2}$ and $u_{3}$. It is supposed that the mechanism is assembled in the horizontal, so that the gravitational effects are not considered. Moreover, by convenience define the angular generalized coordinate $q_{1}^{\langle 0\rangle}$ such that $\dot{q}_{1}^{\langle 0\rangle}=\dot{q}_{\mathrm{B}, 1,3}^{\langle 1\rangle}$.

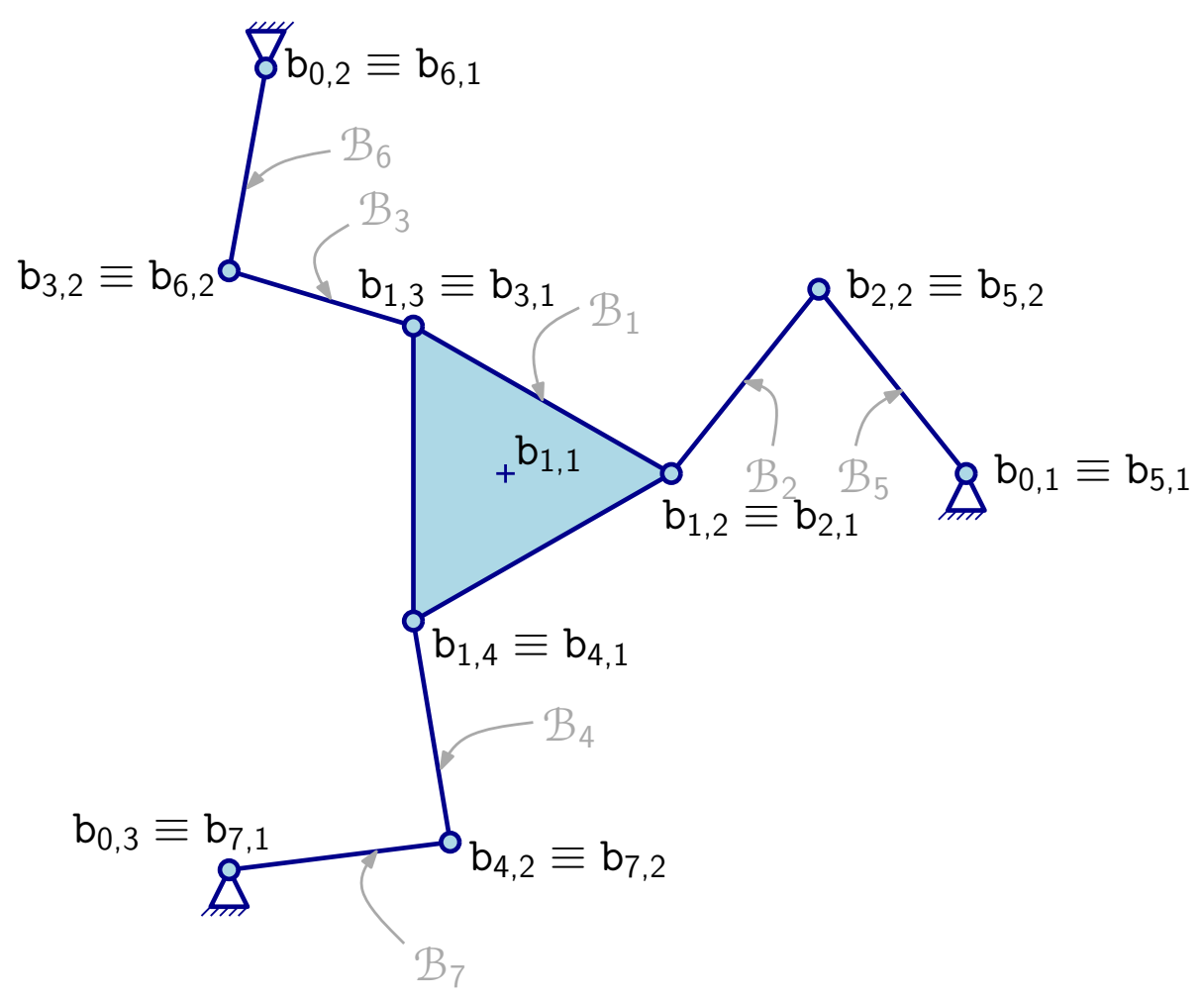

Figure 5.15: Representation of a 3 RRR parallel mechanism.

The following piece of code can be used to set and run an inverse dynamics simulation "SI" in which the following generalized coordinates are prescribed: $q_{\mathrm{N}, 1,1}^{\langle 0\rangle}(t)=-\sin (\pi t / 2), q_{\mathrm{N}, 1,1}^{\langle 0\rangle}(t)=\sin (\pi t / 4)$ and $q_{1}^{\langle 0\rangle}(t)=(\pi / 6) \sin (\pi t / 4)$ : 


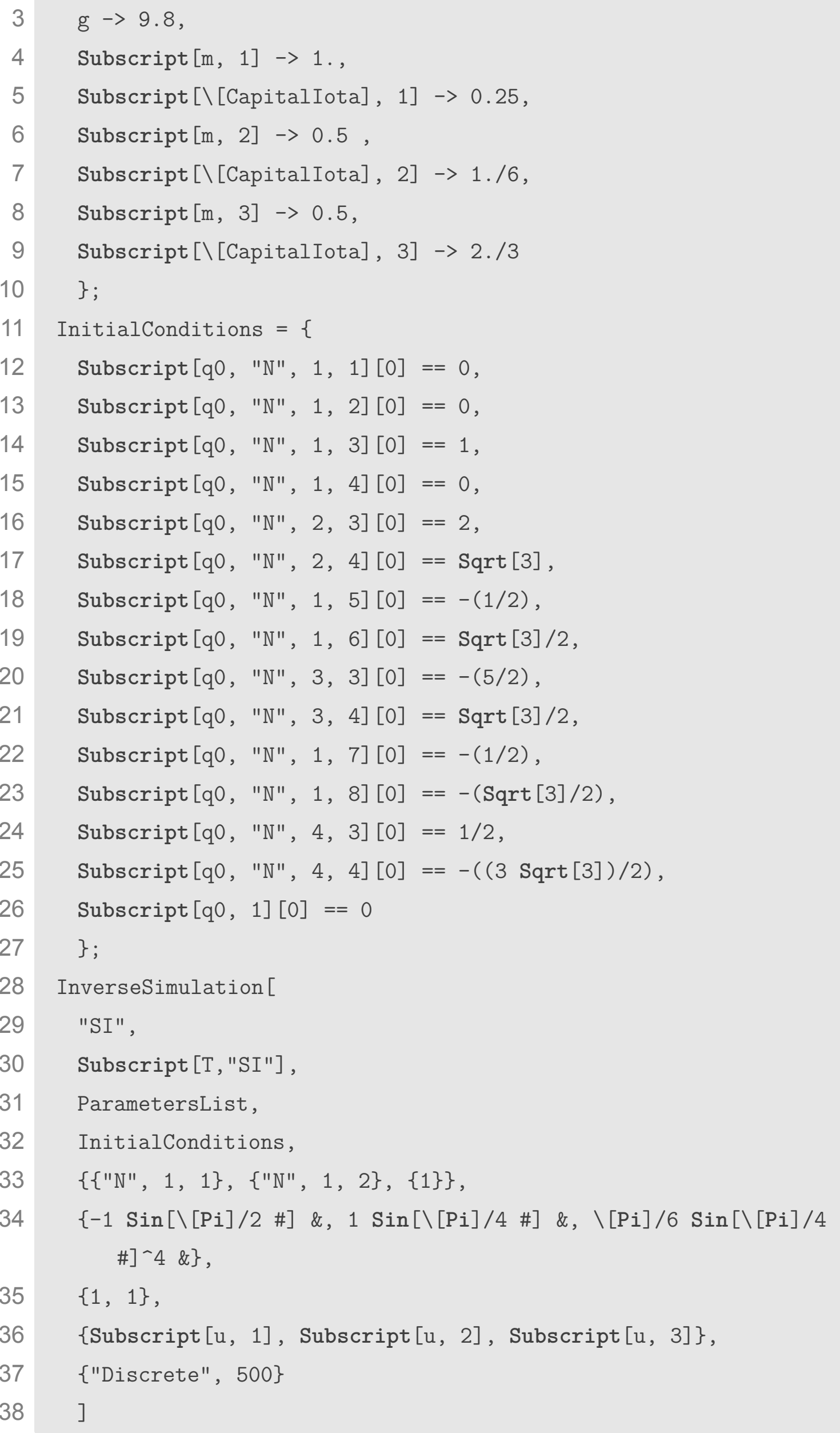


Some of the results obtained in this simulation are shown in Figures 5.165.22. Moreover, Figure 5.23 shows a representation of the initial configuration of the mechanism generated by the command Animation [0] .

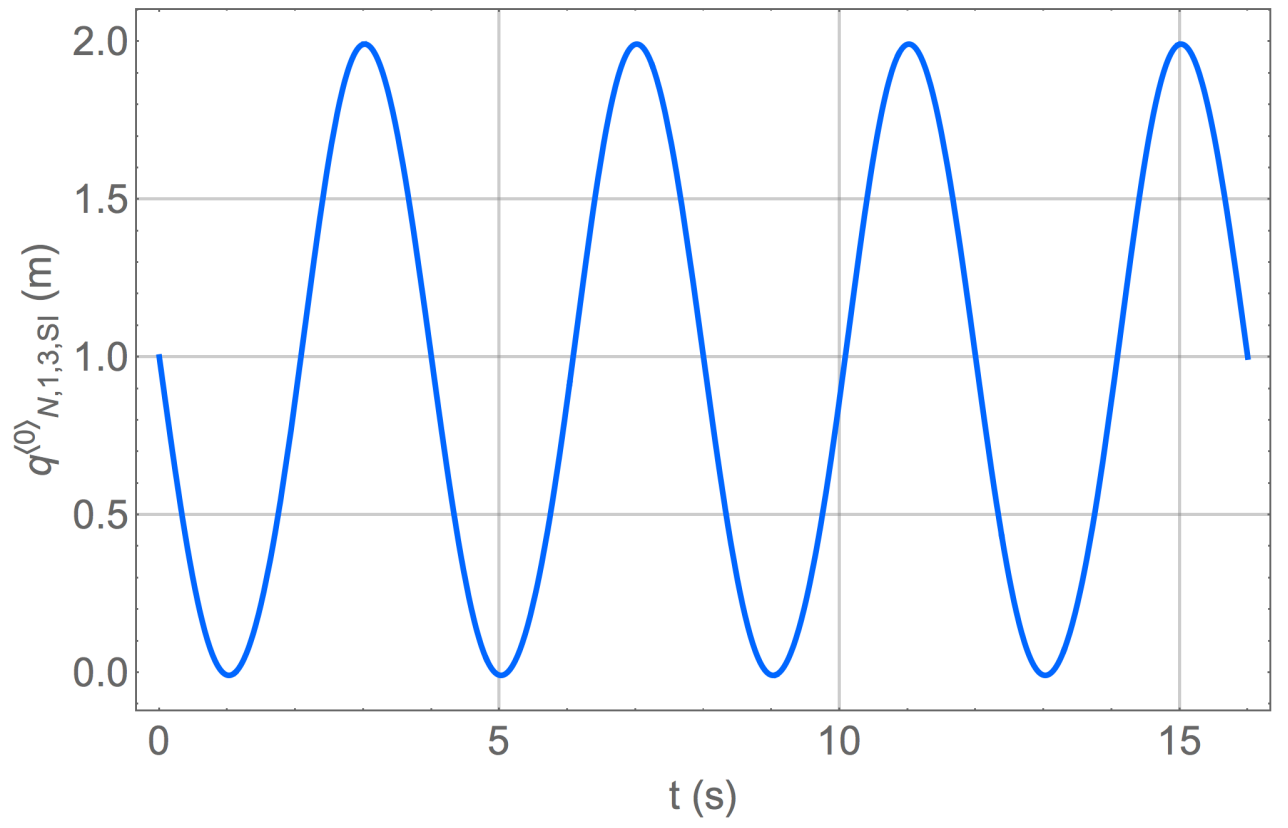

Figure 5.16: Time history of the generalized coordinate $q_{\mathbb{N}, 1,3}^{\langle 0\rangle}$ in the simulation "Sl".

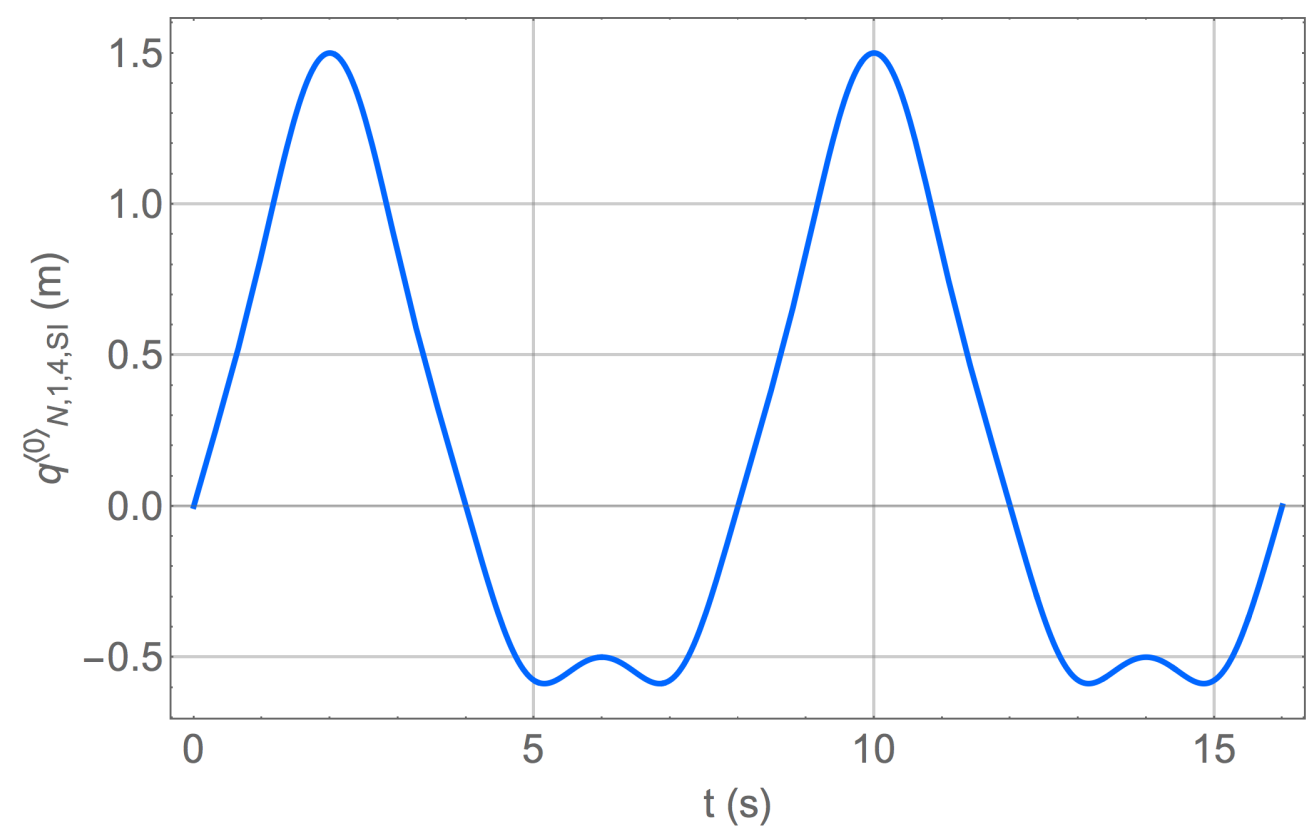

Figure 5.17: Time history of the generalized coordinate $q_{\mathrm{N}, 1,4}^{\langle 0\rangle}$ in the simulation "SI". 


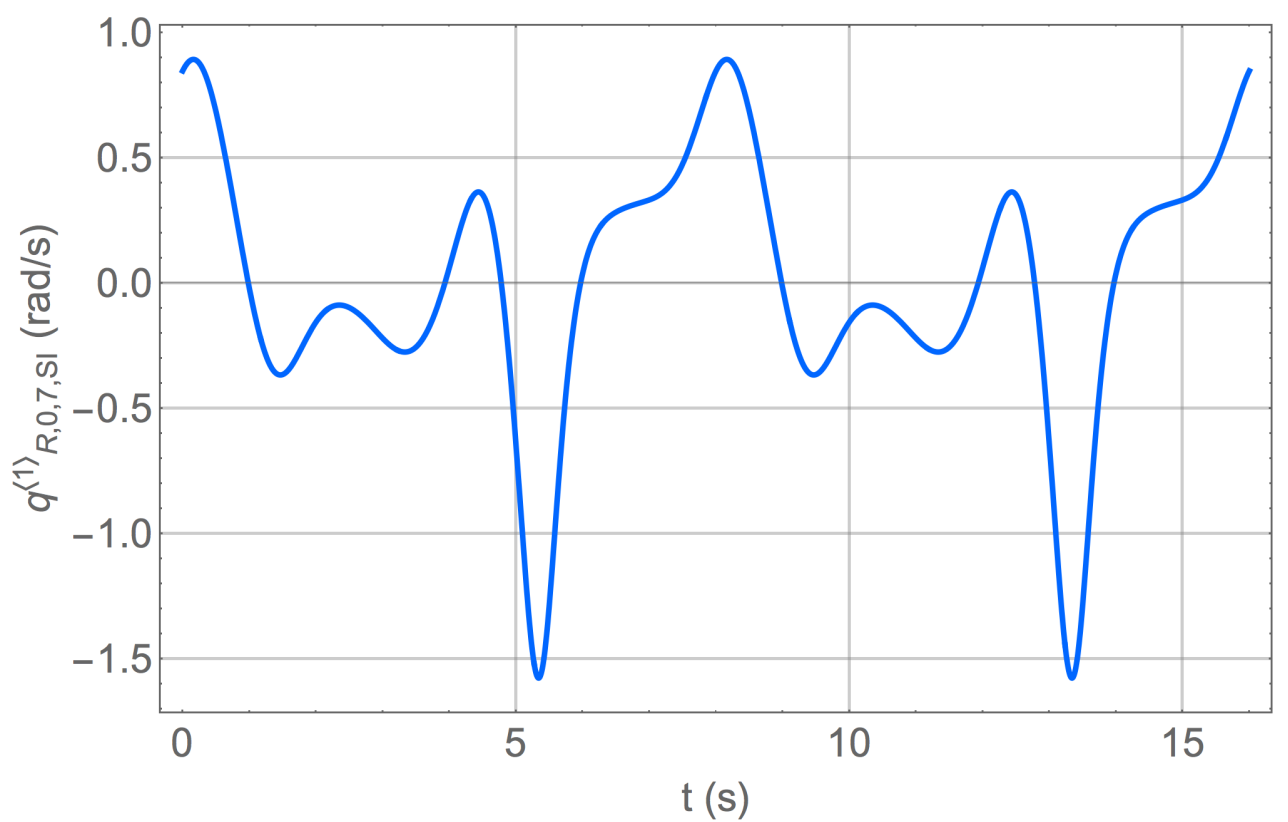

Figure 5.18: Time history of the quasi-velocity $q_{R, 0,7}^{\langle 1\rangle}$ in the simulation "SI".

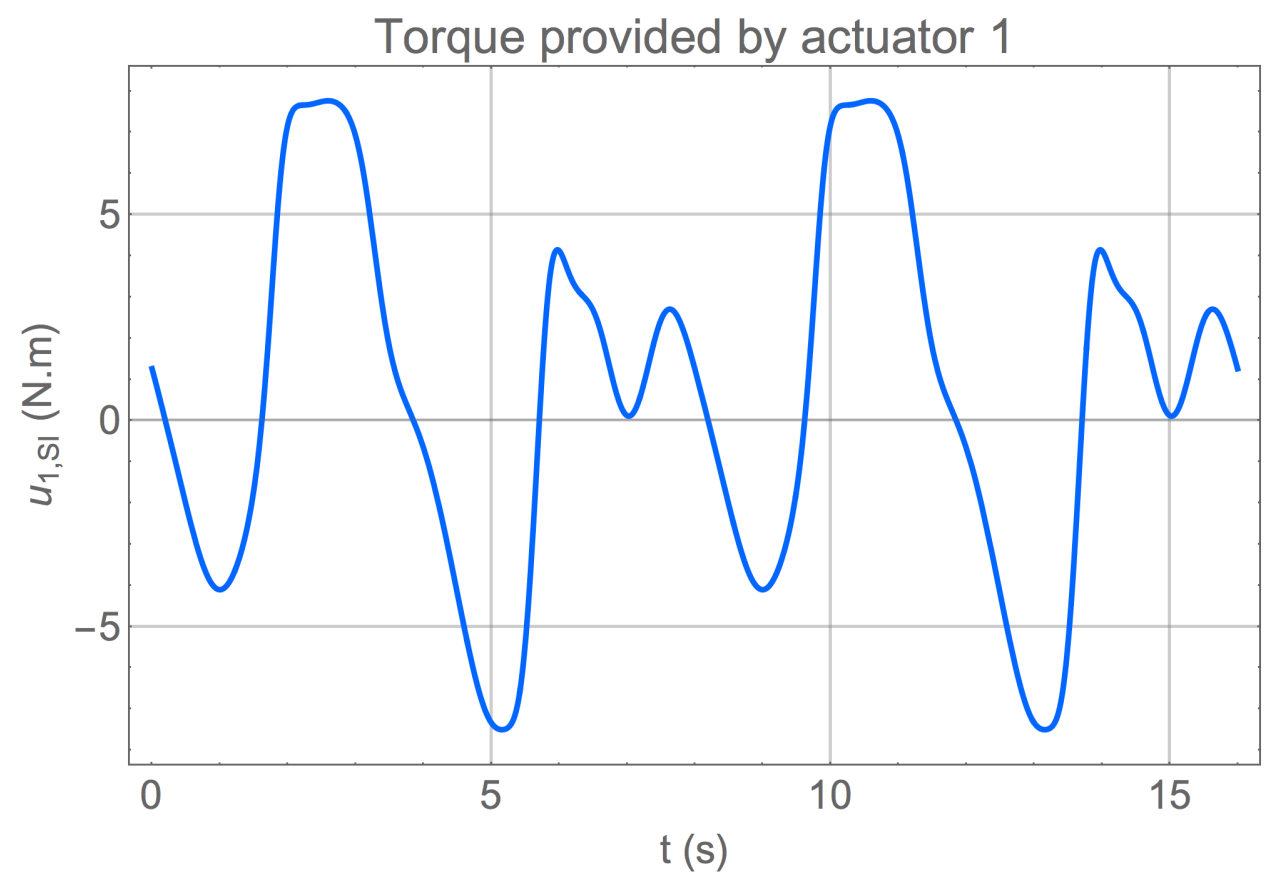

Figure 5.19: Time history of the input torque $u_{1}$ in the simulation "SI". 


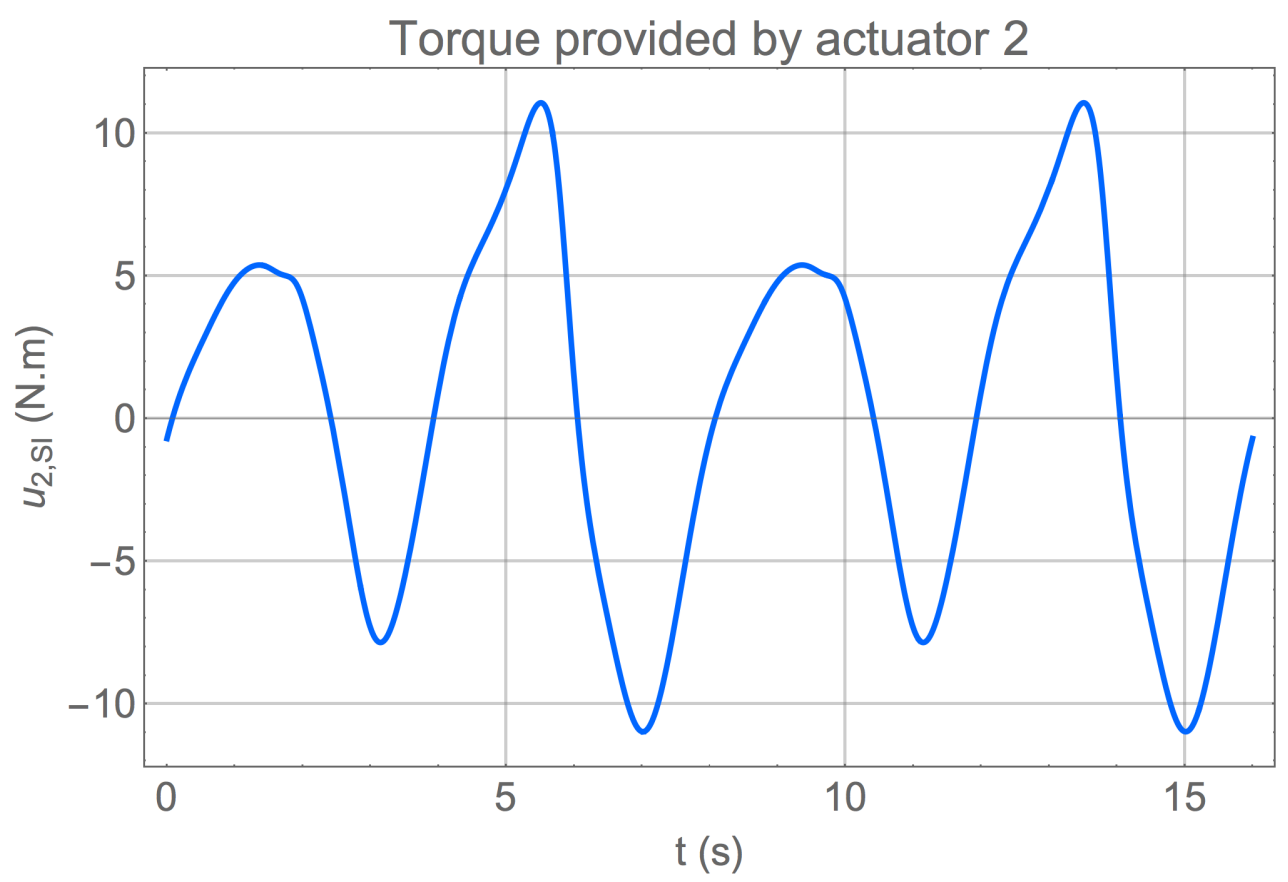

Figure 5.20: Time history of the input torque $u_{2}$ in the simulation "SI".

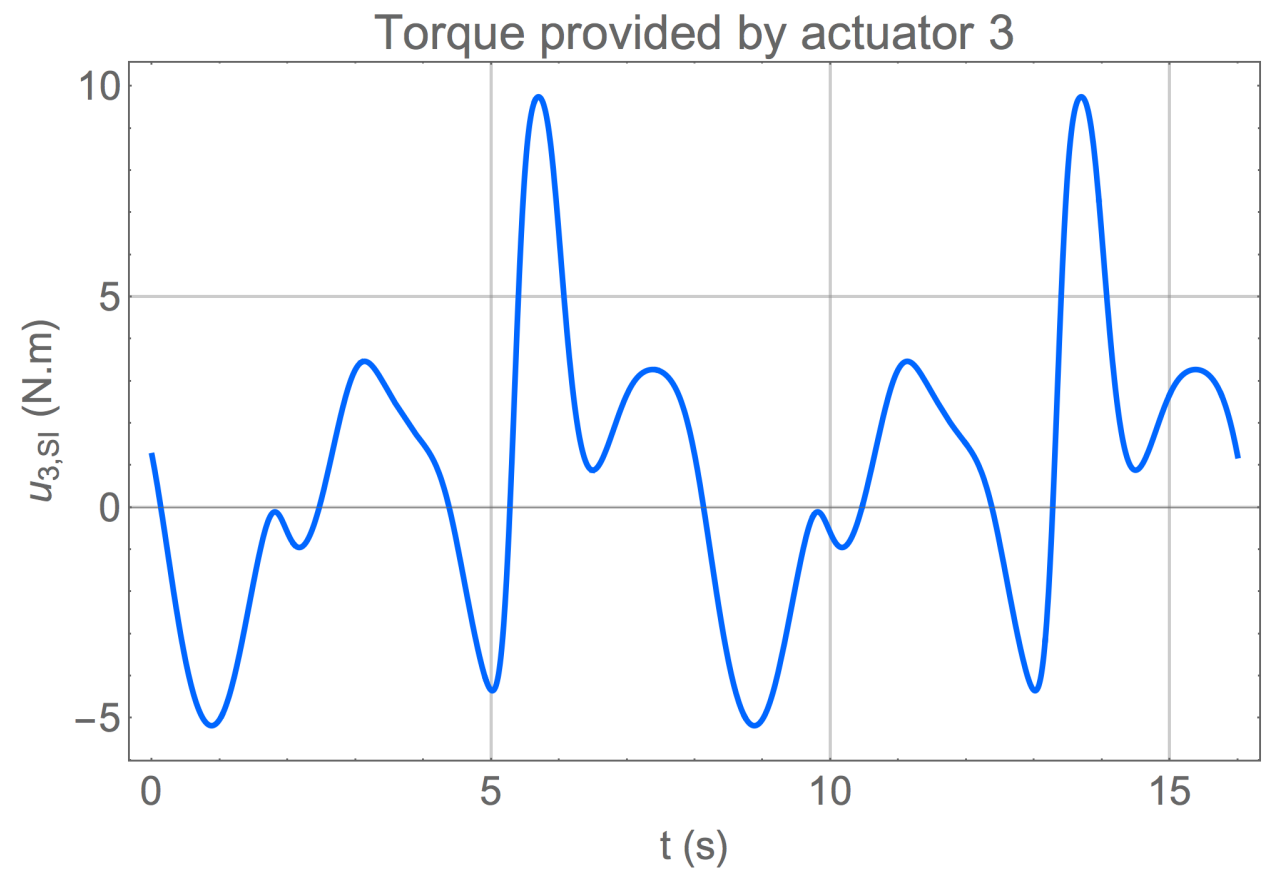

Figure 5.21: Time history of the input torque $u_{3}$ in the simulation "Sl". 


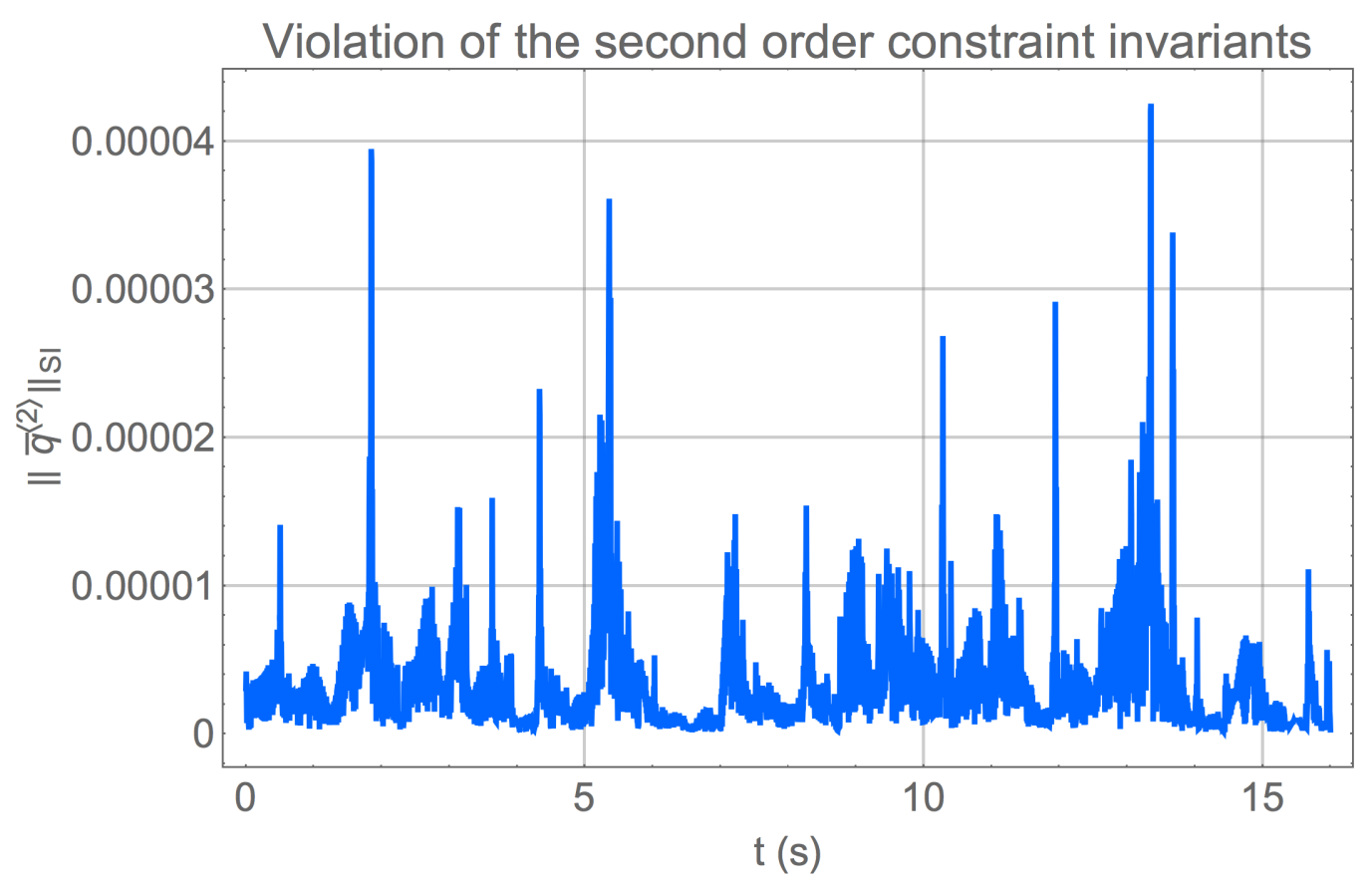

Figure 5.22: Inspection of the error in the numerical solution of the constraint equations in the simulation "SI".

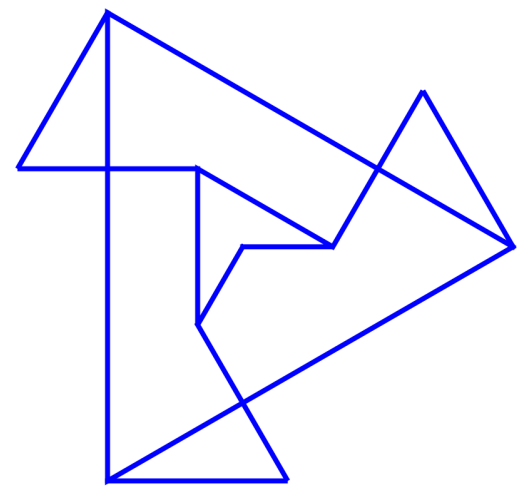

Figure 5.23: Representation of the initial configuration of the mechanism in the simulation "SI". 


\section{Conclusion}

This thesis accomplishes its mission of developing a novel recursive modular modeling methodology, based on a new theorem, Theorem 2.10, that unifies the main advantages of the conventional formalisms of Multibody System Dynamics. In this new perspective, it is enough to conceive a multibody system as a hierarchical structure of subsystems that can be represented by a tree diagram, in which the root represents the system itself and the leaves are indivisible subsystems whose models can be derived by any conventional approach, not being necessary to use the same methodology for all the leaves.

The novel methodology presented in this work does not restrict the parametric description of the motion of a system to the use of coordinates, quasivelocities and its time derivatives only as generalized variables. Nor there is other restriction than the need of being representable by an invariant, to the admissible motion constraints of a system. These properties, not only ensure a great versatility and generality to the methodology, but also enable to extend its scope of application to other areas within the Engineering (e.g. applications to the design of control systems based on the use of non-material programmed constraints, cited in Section 1.3) and eventually to other types of dynamical systems from other areas of knowledge that somehow preserve some similarity to multibody systems.

This work also illustrates with case studies and two examples of computational packages, the potential for using the novel methodology in the development of both generic and specialized algorithms for the modeling of multibody systems. It is evident that apart from the advantages brought by the use of Wolfram Mathematica 10.2 (due to its wide range of built in functions and its suitability for both symbolic and numerical computations), there is no real need of using any proprietary software to implement packages based on the novel methodology. Considering that one of the motivations of the development of the novel methodology was to reduce the dependency on proprietary software for applications to Multibody System Dynamics, it can be stated that this goal 
has also been accomplished. The effective implementation of generic and specialized open source packages based on the novel methodology, however, is still an open issue. The candidate intends to implement an open source version of MoSsPack in Python soon.

It is also worth mentioning the discussions on linearization of equations of motion performed in Section 4.5, which led to a new algorithm for obtaining linearized least squares approximations for orthogonal complement matrices (Proposition 4.4).

As future perspectives, some of the following topics can be explored, based on the contribution presented in this work:

- Development of several specialized algorithms based on the novel modular modeling methodology, for different areas in Mechanical Engineering.

- Design of new model-based control strategies inspired by the hierarchical modular conception of multibody systems.

- Extension of the methodology for problems involving contact and impact in which there are impulsive forces and constraints expressed by inequalities.

- Extension of the methodology to Continuum Mechanics, i.e. to infinite DOF systems, for applications in problems of Fluid Mechanics, FluidStructure Interaction, Elasticity, Plasticity, etc.

- Improved and optimized algorithms based on the novel modular modeling methodology for applications in Finite Elements Analysis (the candidate has already started some studies in this area). 


\section{References}

ANGELES, J. Rational Kinematics. [S.I.]: Springer New York, 1988. (Springer Tracts in Natural Philosophy).

APPELL, P. Sur une forme générale des équations de la dynamique. Journal für die reine und angewandte Mathematik, v. 121, p. 310-319, 1900.

APPELL, P. Sur une forme générale des équations de la dynamique. Paris: Gauthier-Villars, 1925. (Mémorial des Sciences Mathématiques).

BALL, R. A Treatise on the Theory of Screws. Cambridge: Cambridge University Press, 1998. (Cambridge Mathematical Library).

BARUH, H. Analytical Dynamics. Boston: McGraw-Hill Higher Education, 1999. (Engineering Mechanics Series).

$\mathrm{BARUH}, \mathrm{H}$. Another Look at the Describing Equations of Dynamics. The Journal of the Chinese Society of Mechanical Engineers, v. 21, n. 1, p. 15-24, 2000.

BAUCHAU, O. A.; BOTTASSO, C. L.; TRAINELLI, L. Robust integration schemes for flexible multibody systems. Computer Methods in Applied Mechanics and Engineering, v. 192, n. 3-4, p. 395-420, 2003.

BAUMGARTE, J. Stabilization of constraints and integrals of motion in dynamical systems. Computer Methods in Applied Mechanics and Engineering, v. 1, n. 1, p. 1-16, 1972.

BAYO, E.; JALON, J. G. de; SERNA, M. A. A modified lagrangian formulation for the dynamic analysis of constrained mechanical systems. Computer Methods in Applied Mechanics and Engineering, v. 71, n. 2, p. 183-195, 1988.

BLOCH, A. Nonholonomic Mechanics and Control. New York: Springer, 2003. (Interdisciplinary Applied Mathematics).

BLOCH, A. M.; MARSDEN, J. E.; ZENKOV, D. V. Quasivelocities and symmetries in non-holonomic systems. Dynamical Systems, v. 24, n. 2, p. 187-222, 2009.

BOHM, D. Wholeness and the Implicate Order. New York: Routledge, 2005. (Routledge Classics). ISBN 9781134438723.

BOLENDER, M. Rigid Multi-Body Equations-of-Motion for Flapping Wing MAVs Using Kane's Equations. In: AIAA Guidance, Navigation, and Control Conference. Chicago, Estados Unidos: American Institute of Aeronautics and Astronautics, 2009. (Guidance, Navigation, and Control and Co-located Conferences), p. 1-23. 
BORRI, M.; BOTTASSO, C.; MANTEGAZZA, P. Equivalence of Kane's and Maggi's equations. Meccanica, Kluwer Academic Publishers, v. 25, n. 4, p. 272-274, 1990.

BOTTEMA, O.; ROTH, B. Theoretical kinematics. New York: Dover Publications, 1979. (Dover books on engineering).

BREMER, H. Heun and Hamel - Representatives of Mechanics around 1900. In: STEIN, E. (Ed.). The History of Theoretical, Material and Computational Mechanics - Mathematics Meets Mechanics and Engineering SE - 5. Berlin: Springer Berlin Heidelberg, 2014, (Lecture Notes in Applied Mathematics and Mechanics, v. 1). p. 73-80.

BUFFINTON, K. W. Kane's Method in Robotics. In: Robotics and automation handbook. Boca Raton: CRC Press, 2005. p. 90 - 120.

BYACHKOV, A. B.; SUSLONOV, V. M. Maggi's equations in terms of quasi-coordinates. Regular and Chaotic Dynamics, v. 7, n. 3, p. 269-279, 2002.

CAMERON, J. M.; BOOK, W. J. Modeling mechanisms with nonholonomic joints using the Boltzmann-Hamel equations. The International Journal of Robotics Research, v. 16, n. 1, p. 47-59, 1997.

CRAIG, J. Introduction to robotics: mechanics and control. Reading: Pearson/Prentice Hall, 2005. (Addison-Wesley series in electrical and computer engineering: control engineering).

DESLOGE, E. A. Relationship between Kane's equations and the Gibbs-Appell equations. Journal of Guidance, Control, and Dynamics, American Institute of Aeronautics and Astronautics, v. 10, n. 1, p. 120-122, 1987.

DOPICO, D.; GONZÁLEZ, F.; CUADRADO, J.; KÖVECSES, J. Determination of Holonomic and Nonholonomic Constraint Reactions in an Index-3 Augmented Lagrangian Formulation With Velocity and Acceleration Projections. Journal of Computational and Nonlinear Dynamics, v. 9, n. October, p. 041006, 2014.

FALCO, D. de; PENNESTRÌ, E.; VITA, L. The Udwadia-Kalaba formulation: a report on its numerical efficiency in multibody dynamics simulations and on its teaching effectiveness. Multibody Dynamics, v. 1, n. June, p. 21-24, 2005.

FEATHERSTONE, R. A Divide-and-Conquer Articulated-Body Algorithm for Parallel $O(\log (n))$ Calculation of Rigid-Body Dynamics. Part 1: Basic Algorithm. The International Journal of Robotics Research, v. 18, n. 9, p. 867-875, 1999.

FEATHERSTONE, R. A Divide-and-Conquer Articulated-Body Algorithm for Parallel $\mathrm{O}(\log (\mathrm{n}))$ Calculation of Rigid-Body Dynamics. Part 2: Trees, Loops, and Accuracy. The International Journal of Robotics Research, v. 18, n. 9, p. 876-892, 1999.

FEATHERSTONE, R. Rigid Body Dynamics Algorithms. New York: Springer, 2008. (Kluwer international series in engineering and computer science: Robotics). 
FEATHERSTONE, R.; ORIN, D. Robot dynamics: equations and algorithms. In: Proceedings 2000 ICRA. Millennium Conference. IEEE International Conference on Robotics and Automation. Symposia Proceedings (Cat. No.00CH37065). San Francisco: IEEE, 2000. v. 1.

FIJANY, A.; FEATHERSTONE, R. A new factorization of the mass matrix for optimal serial and parallel calculation of multibody dynamics. Multibody System Dynamics, v. 29, n. 2, p. 169-187, 2012.

GELFAND, I.; FOMIN, S.; SILVERMAN, R. Calculus of Variations. New York: Dover Publications, 2000. (Dover Books on Mathematics).

GIBBS, J. W. On the Fundamental Formulae of Dynamics. American Journal of Mathematics, v. 2, n. 1, p. 49-64, 1879.

GILLESPIE, R. B.; ARBOR, A. Kane's Equations for Haptics Display of Multibody Systems. The Electronic Journal of Haptics Research, v. 3, n. 2, p. $1-20,2003$.

GOGU, G. Mobility of mechanisms: A critical review. Mechanism and Machine Theory, v. 40, n. 9, p. 1068-1097, 2005.

GOLDSTEIN, H.; POOLE JR., C. P.; SAFKO, J. L. Classical Mechanics. San Francisco: Pearson Education, 2002.

HAASER, N.; SULLIVAN, J. Real Analysis. New York: Dover Publications, 1991. (Dover Books on Mathematics Series).

HE, J.-H. Variational iteration method - a kind of non-linear analytical technique: some examples. International Journal of Non-Linear Mechanics, v. 34, n. 4, p. 699-708, 1999.

HOLICS, L. 300 Creative Physics Problems with Solutions. London: Anthem Press, 2011.

HU, Q.; JIA, Y.; XU, S. A new computer-oriented approach with efficient variables for multibody dynamics with motion constraints. Acta Astronautica, Elsevier, v. 81, n. 1, p. 380-389, 2012.

JAMMER, M. Concepts of Force. New York: Dover Publications, 2012. (Dover Books on Physics).

JARZEBOWSKA, E. M. On Derivation of Motion Equations for Systems with Non-Holonomic High-Order Program Constraints. Multibody System Dynamics, v. 7, p. 307-329, 2002.

JARZEBOWSKA, E. M. Advanced Programmed Motion Tracking Control of Nonholonomic Mechanical Systems. IEEE Transactions on Robotics, v. 24, n. 6, p. 1315-1328, 2008.

JARZEBOWSKA, E. M. Quasi-coordinates based dynamics modeling and control design for nonholonomic systems. Nonlinear Analysis: Theory, Methods \& Applications, v. 71, n. 12, p. e118-e131, 2009.

JARZEBOWSKA, E. M. Model-Based Tracking Control of Nonlinear Systems. Boca Raton: Taylor \& Francis, 2012. (Modern Mechanics and Mathematics). 
KANE, T.; LEVINSON, D. Dynamics, theory and applications. New York: McGraw-Hill, 1985. (McGraw-Hill series in mechanical engineering).

KANE, T. R. Dynamics of Nonholonomic Systems. Journal of Applied Mechanics, v. 28, n. 4, p. 574-578, 1961.

KANE, T. R.; LEVINSON, D. A. Multibody Dynamics. Journal of Applied Mechanics, v. 50, n. 4b, p. 1071-1078, 1983.

KANE, T. R.; LEVINSON, D. A. The Use of Kane's Dynamical Equations in Robotics. The International Journal of Robotics Research, v. 2, n. 3, p. 3-21, 1983.

KANE, T. R.; WANG, C. On the Derivation of Equations of Motion. Journal of the Society for Industrial and Applied Mathematics, Society for Industrial and Applied Mathematics, v. 13, n. 2, p. 487-492, 1965.

KARNOPP, D. Understanding multibody dynamics using bond graph representations. Journal of the Franklin Institute, v. 334, n. 4, p. 631-642, 1997.

KHAN, W. A.; KROVI, V. N.; SAHA, S. K.; ANGELES, J. Modular and recursive kinematics and dynamics for parallel manipulators. Multibody System Dynamics, v. 14, n. 3-4, p. 419-455, 2005.

KHAN, W. A.; KROVI, V. N.; SAHA, S. K.; ANGELES, J. Modular and Recursive Kinematics and Dynamics for Parallel Manipulators. Multibody System Dynamics, Kluwer Academic Publishers, v. 14, n. 3-4, p. 419-455, 2005.

KIRGETOV, V. I. On the equations of motion of controlled mechanical systems. Journal of Applied Mathematics and Mechanics, v. 28, n. 2, p. 285-296, 1964.

KORAYEM, M. H.; SHAFEI, A. M. Motion equations proper for forward dynamics od robotic manipulator with flexible links by using recursive GibbsAppell formulation. Scientia Iranica Transaction B: Mechanical Engineering, v. 16, n. 6 , p. $479-495,2009$.

LANCZOS, C. The variational principles of mechanics. New York: Dover Publications, 1986. (Mathematical expositions).

LAULUSA, A.; BAUCHAU, O. A. Review of Contemporary Approaches for Constraint Enforcement in Multibody Systems. Journal of Computational and Nonlinear Dynamics, v. 3, n. 1, p. 011005, 2008.

LEECH, J. W. Mecânica Analítica. Rio de Janeiro: Ao Livro Técnico S. A. e Editora da Universidade de São Paulo, 1971.

LEVINSON, D. A. Comment on 'Relationship between Kane's equations and the Gibbs-Appell equations'. Journal of Guidance, Control, and Dynamics, American Institute of Aeronautics and Astronautics, v. 10, n. 6, p. 593, 1987.

LI, Y.; STAICU, S. Inverse dynamics of a 3-PRC parallel kinematic machine. Nonlinear Dynamics, v. 67, n. 2, p. 1031-1041, 2011. 
MANGERON, D.; DELEANU, S. Sur une classe d'équations de la mécanique analytique au sens de Tzenoff. Comptes Rendus de l'Académie Bulgare des Sciences: Sciences Mathématiques et Naturelles, v. 15, n. 1, p. 1-9, 1962.

MARUSKIN, J. M.; BLOCH, A. M. The Boltzmann-Hamel equations for optimal control. In: Proceedings of the 46th IEEE Conference on Decision and Control. New Orleans, Estados Unidos: leee, 2007. p. 554-559. ISBN 978-1-4244-1497-0.

MATA, V.; PROVENZANO, S.; CUADRADO, J.; VALERO, F. Inverse dynamic problem in robots using Gibbs-Appell equations. Robotica, v. 20, n. 01, p. 59-67, 2002.

MATA, V.; PROVENZANO, S.; VALERO, F.; CUADRADO, J. Serial-robot dynamics algorithms for moderately large numbers of joints. Mechanism and Machine Theory, v. 37, n. 8, p. 739-755, 2002.

MAUGIN, G. A. The principle of virtual power: from eliminating metaphysical forces to providing an efficient modelling tool. Continuum Mechanics and Thermodynamics, Springer-Verlag, v. 25, n. 2-4, p. 127-146, 2013.

MCPHEE, J. J.; REDMOND, S. M. Modelling multibody systems with indirect coordinates. Computer Methods in Applied Mechanics and Engineering, v. 195, n. 50-51, p. 6942-6957, 2006.

MCPHEE, J. J.; SCHMITKE, C.; REDMOND, S. M. Dynamic Modelling of Mechatronic Multibody Systems With Symbolic Computing and Linear Graph Theory. Mathematical and Computer Modelling of Dynamical Systems, v. 10, n. 1, p. 1-23, 2004.

MEIJAARD, J. P.; PAPADOPOULOS, J. M.; RUINA, A.; SCHWAB, A. L. Linearized dynamics equations for the balance and steer of a bicycle: a benchmark and review. Proceedings of the Royal Society A: Mathematical, Physical and Engineering Sciences, v. 463, n. 2084, p. 1955-1982, 2007.

MITIGUY, P. C.; BANERJEE, A. K. A constraint force algorithm for formulating equations of motion. In: Proceedings of the Asian Conference on Multibody Dynamics. Fukushima, Japão: JSME, 2002. p. 606 - 608.

NEJMARK, J. I.; FUFAEV, N. A. Dynamics of nonholonomic systems. Providence, Rhode Island: American Mathematical Society, 1972.

ORSINO, R. M. M.; COUTINHO, A. G.; HESS-COELHO, T. A. Dynamic Modelling and Control of Balanced Parallel Mechanisms. In: ZHANG, D.; WEI, B. (Ed.). Dynamic Balancing of Mechanisms and Synthesizing of Parallel Robots SE - 16. [S.I.]: Springer International Publishing, 2016. p. 403-445. ISBN 978-3-319-17682-6.

ORSINO, R. M. M.; HESS-COELHO, T. A. A contribution for developing more efficient dynamic modelling algorithms of parallel robots. International Journal of Mechanisms and Robotic Systems, v. 1, n. 1, p. 15-34, 2013.

ORSINO, R. M. M.; HESS-COELHO, T. A. A contribution on the modular modelling of multibody systems. Proceedings of the Royal Society A:

Mathematical, Physical and Engineering Sciences, v. 471, n. 2183, p. 1-20, 2015. 
ORSINO, R. M. M.; HESS-COELHO, T. A.; PESCE, C. P. Analytical mechanics approaches in the dynamic modelling of Delta mechanism. Robotica, v. 33, n. 04, p. 953-973, 2015.

PAPASTAVRIDIS, J. G. The variational principles of mechanics, and a reply to C. D. Bailey. Journal of Sound and Vibration, v. 118, n. 2, p. 378-393, 1987.

PAPASTAVRIDIS, J. G. On the nonlinear Appell's equations and the determination of generalized reaction forces. International Journal of Engineering Science, v. 26, n. 6, p. 609-625, 1988.

PAPASTAVRIDIS, J. G. On Jourdain's principle. International Journal of Engineering Science, v. 30, n. 2, p. 135-140, 1992.

PAPASTAVRIDIS, J. G. A Panoramic Overview of the Principles and Equations of Motion of Advanced Engineering Dynamics. Applied Mechanics Reviews, v. 51, n. 4, p. 239-265, 1998.

PAUL, B. Analytical Dynamics of Mechanisms - A Computer Oriented Overview. Mechanism and Machine Theory, v. 10, p. 481-507, 1975.

PENNESTRÌ, E.; VALENTINI, P. P. Coordinate reduction strategies in multibody dynamics: a review. In: Conference on Multibody System Dynamics. Pitesti, Romênia: [s.n.], 2004. p. 1-17.

PENNESTRÌ, E.; VALENTINI, P. P.; FALCO, D. de. An application of the Udwadia-Kalaba dynamic formulation to flexible multibody systems. Journal of the Franklin Institute, v. 347, n. 1, p. 173-194, 2010.

PESCE, C. P. The Application of Lagrange Equations to Mechanical Systems With Mass Explicitly Dependent on Position. Journal of Applied Mechanics, v. 70, n. 5, p. 751-756, 2003.

PIEDBOEUF, J.-C. Kane's equations or Jourdain's principle? In: Proceedings of the 36th Midwest Symposium on Circuits and Systems. [S.I.: s.n.], 1993. v. 2, p. 1471-1474. ISBN VO -.

POINCARE, H. Sur une forme nouvelle des équations de la méchanique. Comptes Rendus de l'Académie des Sciences, v. 132, n. 3, p. 369-371, 1901.

RICHARD, M. J.; BOUAZARA, M. Graph-Theoretic Approach for the Dynamic Simulation of Flexible Multibody Systems. Advances in Mechanical Engineering, v. 2012, p. 1-12, 2012.

RODRIGUEZ, G. Kalman filtering, smoothing, and recursive robot arm forward and inverse dynamics. Robotics and Automation, IEEE Journal of, v. 3, n. 6 , p. 624-639, 1987.

SAHA, S. K.; ANGELES, J. Dynamics of Nonholonomic Mechanical Systems Using a Natural Orthogonal Complement. Journal of Applied Mechanics, v. 58, n. 1, p. 238-243, 1991.

SANDINO, L. A.; BEJAR, M.; OLLERO, A. Tutorial for the application of Kane's Method to model a small-size helicopter. In: Proceedings of the 1st Workshop on Research, Development and Education on Unmanned Aerial Systems. Seville, Spain: [s.n.], 2011. p. 162-173. ISBN 9788461562695. 
SCHIEHLEN, W. Multibody System Dynamics: Roots and Perspectives. Multibody System Dynamics, v. 1, n. 2, p. 149-188, 1997.

SCHIEHLEN, W. Research trends in multibody system dynamics. Multibody System Dynamics, Kluwer Academic Publishers, v. 18, n. 1, p. 3-13, 2007.

SCHIEHLEN, W.; GUSE, N.; SEIFRIED, R. Multibody dynamics in computational mechanics and engineering applications. Computer Methods in Applied Mechanics and Engineering, v. 195, n. 41-43, p. 5509-5522, 2006.

SCHMITKE, C.; MCPHEE, J. J. Forming Equivalent Subsystem Components to Facilitate the Modelling of Mechatronic Multibody Systems. Multibody System Dynamics, Kluwer Academic Publishers, v. 14, n. 1, p. 81-110, 2005.

SCHWAB, A. L.; MEIJAARD, J. P. Dynamics of Flexible Multibody Systems with Non-Holonomic Constraints : A Finite Element Approach. Multibody System Dynamics, v. 10, n. 1, p. 107-123, 2003.

SELIG, J. Geometric Fundamentals of Robotics. New York: Springer, 2005. (Monographs in Computer Science).

SELIGER, R. L.; WHITHAM, G. B. Variational Principles in Continuum Mechanics. Proceedings of the Royal Society A: Mathematical, Physical and Engineering Sciences, v. 305, n. 1480, p. 1-25, 1968.

SHAH, S. V.; SAHA, S. K.; DUTT, J. K. Modular framework for dynamic modeling and analyses of legged robots. Mechanism and Machine Theory, Elsevier Ltd, v. 49, p. 234-255, 2012.

SHI, P.; MCPHEE, J. J. Dynamics of Flexible Multibody Systems Using Virtual Work and Linear Graph Theory. Multibody System Dynamics, Kluwer Academic Publishers, v. 4, n. 4, p. 355-381, 2000.

SOTOMAYOR-TELLO, J. Equações Diferenciais Ordinárias. São Paulo: Editora Livraria da Física, 2011.

STAICU, S. Calculus of Joint Forces in Dynamics of a Planar Parallel Robot. UPB Scientific Bulletin, Series D, v. 75, n. 1, p. 3-12, 2013.

STAICU, S.; ZHANG, D. A novel dynamic modelling approach for parallel mechanisms analysis. Robotics and Computer-Integrated Manufacturing, v. 24 , n. 1, p. 167-172, 2008.

TENENBAUM, R. Fundamentals of Applied Dynamics. New York: Springer, 2004. (Advanced texts in physics).

TEODORESCU, P. Mechanical Systems, Classical Models - Volume 3: Analytical Mechanics. Dordrecht: Springer, 2009. (Mathematical and Analytical Techniques with Applications to Engineering).

TSAI, L. Robot analysis: the mechanics of serial and parallel manipulators. New York: John Wiley \& Sons, 1999. (Wiley-Interscience publication).

UDWADIA, F. E. A new perspective on the tracking control of nonlinear structural and mechanical systems. Proceedings of the Royal Society of London. Series A: Mathematical, Physical and Engineering Sciences, v. 459, n. 2035, p. 1783-1800, 2003. 
UDWADIA, F. E. Recent Advances in Multi-body Dynamics and Nonlinear Control. Journal of the Brazilian Society of Mechanical Science and Engineering, XXVIII, n. 3, p. 311-315, 2006.

UDWADIA, F. E.; KALABA, R. E. A new perspective on constrained motion. Proceedings: Mathematical and Physical Sciences, v. 439, n. 1906, p. 407-410, 1992.

UDWADIA, F. E.; KALABA, R. E. On the foundations of analytical dynamics. International Journal of Non-Linear Mechanics, v. 37, n. 6, p. 1079-1090, 2002.

UDWADIA, F. E.; PHOHOMSIRI, P. Explicit equations of motion for constrained mechanical systems with singular mass matrices and applications to multi-body dynamics. Proceedings of the Royal Society A: Mathematical, Physical and Engineering Sciences, v. 462, n. 2071, p. 2097-2117, 2006.

UDWADIA, F. E.; PHOHOMSIRI, P. Explicit Poincaré equations of motion for general constrained systems. Part I. Analytical results. Proceedings of the Royal Society A: Mathematical, Physical and Engineering Sciences, v. 463, n. 2082, p. 1421-1434, 2007.

UDWADIA, F. E.; PHOHOMSIRI, P. Explicit Poincaré equations of motion for general constrained systems. Part II. Applications to multi-body dynamics and nonlinear control. Proceedings of the Royal Society A: Mathematical, Physical and Engineering Sciences, v. 463, n. 2082, p. 1435-1446, 2007.

UDWADIA, F. E.; SCHUTTE, A. D. Equations of motion for general constrained systems in Lagrangian mechanics. Acta Mechanica, v. 213, n. 1-2, p. 111-129, 2010.

UDWADIA, F. E.; WANICHANON, T. Hamel's paradox and the foundations of analytical dynamics. Applied Mathematics and Computation, v. 217, n. 3, p. 1253-1265, 2010. 


\section{Appendix A - Use of energy-like functions in the modeling of multibody systems}

\section{A.1 Generalized inertia forces and energy-like functions}

Let $\mathscr{M}$ de a $\nu^{\#-D O F}$ multibody system whose constraint order is equal to $\nu^{\circ}$. Assume that the generalized variables of $\mathscr{M}$ are trivial above first order and that it is possible to express the corresponding Gibbs-Appell function (see Section 3.3.1) as follows: $S=\underline{S}\left(t, \boldsymbol{q}^{\langle 0\rangle}, \boldsymbol{q}^{\langle 1\rangle}, \dot{\boldsymbol{q}}^{\langle 1\rangle}\right)$. Following a derivation similar to the one that led to equation (3.112), it can be stated that the unconstrained generalized inertia forces associated to $\mathscr{M}$ are given by the following expression:

$$
{ }^{\mathrm{I}} \boldsymbol{f}=-\frac{\partial \underline{S}}{\partial \dot{\boldsymbol{q}}^{\langle 1\rangle}}
$$

Now consider that expression of the kinetic energy of $\mathscr{M}$ is known. The following proposition can be established.

Proposition A.1. Assume that the expression of the kinetic energy of $\mathscr{M}$ is expressed as a function of time $t$ and of the generalized coordinates of the model of $\mathscr{M}$ along with its time derivatives, i.e. $T=\underline{T}^{\bullet}\left(t, \boldsymbol{q}^{\langle 0\rangle}, \dot{\boldsymbol{q}}^{\langle 0\rangle}\right)$. The unconstrained generalized inertia forces associated to $\mathscr{M}$ are given the following expression:

$$
{ }^{\mathrm{I}} \boldsymbol{f}=-\left(\frac{\partial \dot{\dot{\boldsymbol{q}}}^{\langle 0\rangle}}{\partial \boldsymbol{q}^{\langle 1\rangle}}\right)^{\top}\left(\frac{\mathrm{d}}{\mathrm{d} t} \frac{\partial \underline{T}^{\bullet}}{\partial \dot{\boldsymbol{q}}^{\langle 0\rangle}}-\frac{\partial \underline{T}^{\bullet}}{\partial \boldsymbol{q}^{\langle 0\rangle}}\right)
$$

Proof.Assume, without any loss in generality that the kinetic energy of $\mathscr{M}$ is given by equation (3.58). Suppose that $\mathbf{r}_{k}$ represents the position vector of a point $\mathrm{p}_{k}$ of $\mathscr{M}$ with respect to a coordinate system $\mathrm{N}$ rigidly attached to an inertial reference frame $\mathcal{N}$. It can be stated that:

$$
\mathbf{v}_{k}=\frac{\mathrm{d} \mathbf{r}_{k}}{\mathrm{~d} t}=\frac{\mathrm{d}}{\mathrm{d} t}\left(\underline{\mathbf{r}}_{k}\left(t, \boldsymbol{q}^{\langle 0\rangle}\right)\right)=\frac{\partial \underline{\mathbf{r}}_{k}}{\partial \boldsymbol{q}^{\langle 0\rangle}} \dot{\boldsymbol{q}}^{\langle 0\rangle}+\frac{\partial \underline{\mathbf{r}}_{k}}{\partial t}=\underline{\mathbf{v}}_{k}^{\bullet}\left(t, \boldsymbol{q}^{\langle 0\rangle}, \dot{\boldsymbol{q}}^{\langle 0\rangle}\right)
$$


Thus:

$$
\frac{\mathrm{d}}{\mathrm{d} t}\left(\frac{\partial \underline{\mathbf{v}}_{k}}{\partial \dot{\boldsymbol{q}}^{\langle 0\rangle}}\right)=\frac{\mathrm{d}}{\mathrm{d} t}\left(\frac{\partial \underline{\mathbf{r}}_{k}}{\partial \boldsymbol{q}^{\langle 0\rangle}}\right)=\frac{\partial}{\partial \boldsymbol{q}^{\langle 0\rangle}}\left(\frac{\partial \underline{\mathbf{r}}_{k}}{\partial \boldsymbol{q}^{\langle 0\rangle}} \dot{\boldsymbol{q}}^{\langle 0\rangle}+\frac{\partial \underline{\mathbf{r}}_{k}}{\partial t}\right)=\frac{\partial \underline{\mathbf{v}}_{k}}{\partial \boldsymbol{q}^{\langle 0\rangle}}
$$

Moreover:

$$
\begin{aligned}
\frac{\mathrm{d}}{\mathrm{d} t} \frac{\partial \underline{T}^{\bullet}}{\partial \dot{\boldsymbol{q}}^{\langle 0\rangle}}-\frac{\partial T^{\bullet}}{\partial \boldsymbol{q}^{\langle 0\rangle}} & =\sum_{k}\left(\frac{\mathrm{d}}{\mathrm{d} t}\left(m_{k} \frac{\partial \underline{\mathbf{v}}_{k}^{\bullet}}{\partial \dot{\boldsymbol{q}}^{\langle 0\rangle}} \cdot \mathbf{v}_{k}\right)-m_{k} \frac{\partial \underline{\mathbf{v}}_{k}^{\bullet}}{\partial \boldsymbol{q}^{\langle 0\rangle}} \cdot \mathbf{v}_{k}\right) \\
& =\sum_{k} m_{k} \frac{\partial \underline{\mathbf{v}}_{k}^{\bullet}}{\partial \dot{\boldsymbol{q}}^{\langle 0\rangle}} \cdot \dot{\mathbf{v}}_{k}=\sum_{k} m_{k} \frac{\partial \underline{\mathbf{r}}_{k}}{\partial \boldsymbol{q}^{\langle 0\rangle}} \cdot \dot{\mathbf{v}}_{k}
\end{aligned}
$$

Finally:

$$
\begin{aligned}
\left(\frac{\partial \dot{\boldsymbol{q}}^{\langle 0\rangle}}{\partial \boldsymbol{q}^{\langle 1\rangle}}\right)^{\top}\left(\frac{\mathrm{d}}{\mathrm{d} t} \frac{\partial \underline{\underline{T}}^{\bullet}}{\partial \dot{\boldsymbol{q}}^{\langle 0\rangle}}-\frac{\partial \underline{T}^{\bullet}}{\partial \boldsymbol{q}^{\langle 0\rangle}}\right) & =\sum_{k} m_{k} \dot{\mathbf{v}}_{k} \cdot\left(\frac{\partial \underline{\mathbf{r}}_{k}}{\partial \boldsymbol{q}^{\langle 0\rangle}} \frac{\partial \underline{\dot{\boldsymbol{q}}}^{\langle 0\rangle}}{\partial \boldsymbol{q}^{\langle 1\rangle}}\right) \\
& =\sum_{k} m_{k} \dot{\mathbf{v}}_{k} \cdot \mathbf{V}_{k}=-{ }^{\mathrm{I}} \boldsymbol{f}
\end{aligned}
$$

This completes the proof.

Assume now that the kinetic energy is expressed as a function of time $t$, of the generalized coordinates $\boldsymbol{q}^{\langle 0\rangle}$ and of the quasi-velocities $\boldsymbol{q}^{\langle 1\rangle}$ of the model of $\mathscr{M}$, i.e. $T=\underline{T}\left(t, \boldsymbol{q}^{\langle 0\rangle}, \boldsymbol{q}^{\langle 1\rangle}\right)$. It can be stated that:

$$
\underline{T}^{\bullet}\left(t, \boldsymbol{q}^{\langle 0\rangle}, \dot{\boldsymbol{q}}^{\langle 0\rangle}\right)=\underline{T}\left(t, \boldsymbol{q}^{\langle 0\rangle}, \underline{\boldsymbol{q}}^{\langle 1\rangle}\left(t, \boldsymbol{q}^{\langle 0\rangle}, \dot{\boldsymbol{q}}^{\langle 0\rangle}\right)\right)
$$

Thus:

$$
\begin{aligned}
& \frac{\partial \underline{T}^{\bullet}}{\partial \dot{\boldsymbol{q}}^{\langle 0\rangle}}=\left(\frac{\partial \underline{\boldsymbol{q}}^{\langle 1\rangle}}{\partial \dot{\boldsymbol{q}}^{\langle 0\rangle}}\right)^{\top} \frac{\partial \underline{T}}{\partial \boldsymbol{q}^{\langle 1\rangle}} \\
& \frac{\partial \underline{T}^{\bullet}}{\partial \boldsymbol{q}^{\langle 0\rangle}}=\frac{\partial \underline{T}}{\partial \boldsymbol{q}^{\langle 0\rangle}}+\left(\frac{\partial \underline{\boldsymbol{q}}^{\langle 1\rangle}}{\partial \boldsymbol{q}^{\langle 0\rangle}}\right)^{\top} \frac{\partial \underline{T}}{\partial \boldsymbol{q}^{\langle 1\rangle}}
\end{aligned}
$$

Define:

$$
\left\{\begin{array}{l}
\boldsymbol{N}_{0,0}=\left(\frac{\partial \dot{\dot{q}}^{\langle 0\rangle}}{\partial \boldsymbol{q}^{\langle 1\rangle}}\right)^{\top} \\
\boldsymbol{N}_{0,1}=\left(\left(\frac{\partial \underline{\boldsymbol{q}}^{\langle 1\rangle}}{\partial \boldsymbol{q}^{\langle 0\rangle}}-\frac{\mathrm{d}}{\mathrm{d} t} \frac{\partial \underline{\boldsymbol{q}}^{\langle 1\rangle}}{\partial \dot{\boldsymbol{q}}^{\langle 0\rangle}}\right) \frac{\partial \underline{\dot{\boldsymbol{q}}}^{\langle 0\rangle}}{\partial \boldsymbol{q}^{\langle 1\rangle}}\right)^{\top} \\
\boldsymbol{N}_{1,1}=-\left(\frac{\partial \underline{\boldsymbol{q}}^{\langle 1\rangle}}{\partial \dot{\boldsymbol{q}}^{\langle 0\rangle}} \frac{\partial \dot{\boldsymbol{q}}^{\langle 0\rangle}}{\partial \boldsymbol{q}^{\langle 1\rangle}}\right)^{\top}
\end{array}\right.
$$

Replacing equations (A.4, A.5) in the expression (A.2), it can be stated that:

$$
{ }^{\mathrm{I}} \boldsymbol{f}=\boldsymbol{N}_{0,0} \frac{\partial \underline{T}}{\partial \boldsymbol{q}^{\langle 0\rangle}}+N_{0,1} \frac{\partial \underline{T}}{\partial \boldsymbol{q}^{\langle 1\rangle}}+\boldsymbol{N}_{1,1} \frac{\mathrm{d}}{\mathrm{d} t} \frac{\partial \underline{T}}{\partial \boldsymbol{q}^{\langle 1\rangle}}
$$

Example A.1 (Modeling of a spherical pendulum). Back to Example 4.1, con- 
sider the second version of the model in Cartesian coordinates, in which $\boldsymbol{q}^{\langle 0\rangle}=(x, y, z)$ and $\boldsymbol{q}^{\langle 1\rangle}=(\dot{x}, \dot{y}, \dot{z}, v)$ with $v=\dot{x}^{2}+\dot{y}^{2}+\dot{z}^{2}$. The kinetic energy of this system can be expressed as follows:

$$
T=\frac{m v}{2}
$$

It can be stated that:

$$
\frac{\partial \underline{T}}{\partial \boldsymbol{q}}=0 \quad \frac{\partial \underline{T}}{\partial \boldsymbol{q}^{\langle 1\rangle}}=\frac{1}{2}\left[\begin{array}{c}
0 \\
0 \\
0 \\
m
\end{array}\right] \quad \frac{\mathrm{d}}{\mathrm{d} t} \frac{\partial \underline{T}}{\partial \boldsymbol{q}^{\langle 1\rangle}}=0
$$

Moreover, from equation (A.6):

$$
N_{0,1}=\left(-\frac{d}{d t}\left[\begin{array}{ccc}
1 & 0 & 0 \\
0 & 1 & 0 \\
0 & 0 & 1 \\
2 \dot{x} & 2 \dot{y} & 2 \dot{z}
\end{array}\right]\left[\begin{array}{cccc}
1 & 0 & 0 & 0 \\
0 & 1 & 0 & 0 \\
0 & 0 & 1 & 0
\end{array}\right]\right)^{\top}=\left[\begin{array}{cccc}
0 & 0 & 0 & -2 \ddot{x} \\
0 & 0 & 0 & -2 \ddot{y} \\
0 & 0 & 0 & -2 \ddot{z} \\
0 & 0 & 0 & 0
\end{array}\right]
$$

Finally, using equation (A.7):

$$
{ }^{\mathrm{I}} \boldsymbol{f}=\frac{1}{2}\left[\begin{array}{cccc}
0 & 0 & 0 & -2 \ddot{x} \\
0 & 0 & 0 & -2 \ddot{y} \\
0 & 0 & 0 & -2 \ddot{z} \\
0 & 0 & 0 & 0
\end{array}\right]\left[\begin{array}{c}
0 \\
0 \\
0 \\
m
\end{array}\right]=\left[\begin{array}{c}
-m \ddot{x} \\
-m \ddot{y} \\
-m \ddot{z} \\
0
\end{array}\right]
$$

Comparing to Example 4.1, it can be noticed that this last expression corresponds to the unconstrained generalized inertia forces associated to the model of $\mathscr{M}$.

Proposition A.2. Let $F$ be a motion variable defined by a class $\mathscr{C}^{\rho}$ function $F=\underline{F}\left(t, \boldsymbol{q}^{\langle 0\rangle}, \boldsymbol{q}^{\langle 1\rangle}\right)$, with $\rho \geq 1$. Let:

$$
F^{(\rho)}=\underline{F}^{(\rho)}\left(t, \boldsymbol{q}^{\langle\rho \mid 1\rangle}\right)=\frac{\mathrm{d}^{\rho}}{\mathrm{d} t^{\rho}}\left(\underline{F}\left(t, \boldsymbol{q}^{\langle 1\rangle}\right)\right)
$$

It can be stated that (JARZEBOWSKA, 2009):

$$
\frac{\mathrm{d}}{\mathrm{d} t}\left(\frac{\partial \underline{F}}{\partial \boldsymbol{q}^{\langle 1\rangle}}\right)=\frac{1}{\rho}\left(\frac{\partial \underline{F}^{(\rho)}}{\partial \boldsymbol{q}^{\langle\rho \mid 1\rangle}}-\left(\frac{\partial \dot{\dot{q}}^{\langle 0\rangle}}{\partial \boldsymbol{q}^{|1\rangle}}\right)^{\top} \frac{\partial \underline{F}}{\partial \boldsymbol{q}^{\langle 0\rangle}}\right)
$$


Proof.First of all, note that:

$$
\frac{\mathrm{d}}{\mathrm{d} t}\left(\frac{\partial \underline{F}}{\partial q_{. j}^{\langle 1\rangle}}\right)=\frac{\partial^{2} \underline{F}}{\partial t \partial q_{. j}^{\langle 1\rangle}}+\sum_{i} \frac{\partial^{2} \underline{F}}{\partial q_{. i}^{\langle 0\rangle} \partial q_{. j}^{\langle 1\rangle}} \dot{q}_{. i}^{\langle 0\rangle}+\sum_{k} \frac{\partial^{2} \underline{F}}{\partial q_{. k}^{\langle 1\rangle} \partial q_{. j}^{\langle 1\rangle}} \dot{q}_{. k}^{\langle 1\rangle}
$$

Assuming that $\dot{\boldsymbol{q}}^{\langle 0\rangle}=\dot{\boldsymbol{q}}^{\langle 0\rangle}\left(t, \boldsymbol{q}^{\langle 1\rangle}\right)$, this expression can be rewritten as a function of $\left(t, \boldsymbol{q}^{\langle 1\rangle}\right)$. Moreover, for $\rho=1$ :

$$
\begin{aligned}
F^{(1)} & =\frac{\partial \underline{F}}{\partial t}+\sum_{i} \frac{\partial \underline{F}}{\partial q_{. i}^{\langle 0\rangle}} \dot{q}_{. i}^{\langle 0\rangle}+\sum_{k} \frac{\partial \underline{F}}{\partial q_{. k}^{\langle 1\rangle}} \dot{q}_{. k}^{\langle 1\rangle} \\
\frac{\partial \underline{F}^{(1)}}{\partial q_{. j}^{\langle 1\rangle}} & =\frac{\partial^{2} \underline{F}}{\partial t \partial q_{. j}^{\langle 1\rangle}}+\sum_{i} \frac{\partial^{2} \underline{F}}{\partial q_{. i}^{\langle 0\rangle} \partial q_{. j}^{\langle 1\rangle}} \dot{q}_{. i}^{\langle 0\rangle}+\sum_{k} \frac{\partial^{2} \underline{F}}{\partial q_{. k}^{\langle 1\rangle} \partial q_{. j}^{\langle 1\rangle}} \dot{q}_{. k}^{\langle 1\rangle} \\
& +\sum_{i} \frac{\partial \underline{F}_{i}^{\langle 0\rangle}}{\partial q_{. i}^{\langle 0}} \frac{\partial \dot{q}_{. i}^{\langle 0\rangle}}{\partial q_{. j}^{\langle 1\rangle}}
\end{aligned}
$$

Thus:

$$
\frac{\mathrm{d}}{\mathrm{d} t}\left(\frac{\partial \underline{F}}{\partial q_{. j}^{\langle 1\rangle}}\right)=\left(\frac{\partial \underline{F}^{(1)}}{\partial q_{. j}^{\langle 1\rangle}}-\sum_{i} \frac{\partial \underline{F}^{\langle 0\rangle}}{\partial q_{. i}^{\langle 0}} \frac{\partial \dot{q}_{. i}^{\langle 0\rangle}}{\partial q_{. j}^{\langle 1\rangle}}\right)
$$

Also, for $\rho=2$, it can be stated that:

$$
\begin{gathered}
F^{(2)}=\sum_{j} \dot{q}_{. j}^{\langle 1\rangle}\left(2 \frac{\partial^{2} \underline{\underline{F}}}{\partial t \partial q_{. j}^{\langle 1\rangle}}+\sum_{i} 2 \frac{\partial^{2} \underline{F}}{\partial q_{. i}^{\langle 0\rangle} \partial q_{. j}^{\langle 1\rangle}} \dot{q}_{. i}^{\langle 0\rangle}+\sum_{k \neq j} 2 \frac{\partial^{2} \underline{F}}{\partial q_{. k}^{\langle 1\rangle} \partial q_{. j}^{\langle 1\rangle}} \dot{q}_{. k}^{\langle 1\rangle}\right. \\
\left.+\frac{\partial^{2} \underline{F}}{\partial q_{. j}^{\langle 1\rangle} \partial q_{. j}^{\langle 1\rangle}} \dot{q}_{. j}^{\langle 1\rangle}\right)+\sum_{j} \dot{q}_{. j}^{\langle 1\rangle}\left(\sum_{i} \frac{\partial \underline{F}}{\partial q_{. i}^{\langle 0\rangle}} \frac{\partial \dot{q}_{. i}^{\langle 0\rangle}}{\partial q_{. j}^{\langle 1\rangle}}\right)+\tilde{F}^{(2)}
\end{gathered}
$$

with $\tilde{F}^{(2)}$ representing all the terms of $F^{(2)}$ that are independent of $\dot{q}_{. j}^{\langle 1\rangle}$. Thus:

$$
\begin{aligned}
\frac{\partial \underline{F}^{(2)}}{\partial q_{. j}^{\langle 2 \mid 1\rangle}}=2( & \left.\frac{\partial^{2} \underline{F}}{\partial t \partial q_{. j}^{\langle 1\rangle}}+\sum_{i} \frac{\partial^{2} \underline{F}}{\partial q_{. i}^{\langle 0\rangle} \partial q_{. j}^{\langle 1\rangle}} \dot{q}_{. i}^{\langle 0\rangle}+\sum_{k} \frac{\partial^{2} \underline{F}}{\partial q_{. k}^{\langle 1\rangle} \partial q_{. j}^{\langle 1\rangle}} \dot{q}_{. k}^{\langle 1\rangle}\right) \\
& +\sum_{i} \frac{\partial \underline{F}}{\partial q_{. i}^{\langle 0\rangle}} \frac{\partial \underline{\dot{q}}_{. i}^{\langle 0\rangle}}{\partial q_{. j}^{\langle 1\rangle}}
\end{aligned}
$$

Therefore:

$$
\frac{\mathrm{d}}{\mathrm{d} t}\left(\frac{\partial \underline{F}^{\langle 1\rangle}}{\partial q_{. j}^{(1)}}\right)=\frac{1}{2}\left(\frac{\partial \underline{F}^{(2)}}{\partial q_{. j}^{\langle 2 \mid 1\rangle}}-\sum_{i} \frac{\partial \underline{F}}{\partial q_{. i}^{\langle 0\rangle}} \frac{\partial \underline{\dot{q}}_{. i}^{\langle 0\rangle}}{\partial q_{. j}^{\langle 1\rangle}}\right)
$$


For $\rho>2$, it can be stated that:

$$
\begin{aligned}
F^{(\rho)}=\sum_{j} \rho q_{. j}^{\langle\rho \mid 1\rangle}\left(\frac{\partial^{2} \underline{F}}{\partial t \partial q_{. j}^{\langle 1\rangle}}+\sum_{i} \frac{\partial^{2} \underline{F}}{\partial q_{. i}^{\langle 0\rangle} \partial q_{. j}^{\langle 1\rangle}} \dot{q}_{. i}^{\langle 0\rangle}+\sum_{k} \frac{\partial^{2} \underline{F}}{\partial q_{. k}^{\langle 1\rangle} \partial q_{. j}^{\langle 1\rangle}} \dot{q}_{. k}^{\langle 1\rangle}\right) \\
+\sum_{j} q_{. j}^{\langle\rho \mid 1\rangle}\left(\sum_{i} \frac{\partial \underline{F}}{\partial q_{. i}^{\langle 0\rangle}} \frac{\partial \dot{q}_{. i}^{\langle 0\rangle}}{\partial q_{. j}^{\langle 1\rangle}}\right)+\tilde{F}^{(\rho)} \\
\frac{\partial \underline{F}^{(\rho)}}{\partial q_{. j}^{\langle\rho \mid 1\rangle}}=\rho\left(\frac{\partial^{2} \underline{F}}{\partial t \partial q_{. j}^{\langle 1\rangle}}+\sum_{i} \frac{\partial^{2} \underline{F}}{\partial q_{. i}^{\langle 0\rangle} \partial q_{. j}^{\langle 1\rangle}} \dot{q}_{. i}^{\langle 0\rangle}+\sum_{k} \frac{\partial^{2} \underline{F}}{\partial q_{. k}^{\langle 1\rangle} \partial q_{. j}^{\langle 1\rangle}} \dot{q}_{. k}^{\langle 1\rangle}\right) \\
+\sum_{i} \frac{\partial \underline{F}}{\partial q_{. i}^{\langle 0\rangle}} \frac{\partial \underline{\dot{q}}_{. i}^{\langle 0\rangle}}{\partial q_{. j}^{\langle 1\rangle}}
\end{aligned}
$$

Finally:

$$
\frac{\mathrm{d}}{\mathrm{d} t}\left(\frac{\partial \underline{F}^{\langle 1\rangle}}{\partial q_{. j}^{(1\rangle}}\right)=\frac{1}{\rho}\left(\frac{\partial \underline{F}^{(\rho)}}{\partial q_{. j}^{\langle\rho \mid 1\rangle}}-\sum_{i} \frac{\partial \underline{F}^{\langle 0}}{\partial q_{. i} \frac{\partial \underline{\dot{q}}_{. i}^{\langle 0\rangle}}{\partial q_{. j}^{\langle 1\rangle}}}\right)
$$

This completes the proof.

Define:

$$
\left\{\begin{array}{l}
\boldsymbol{N}_{0,0}^{*}=\left(\frac{\partial \underline{\dot{q}}}{\partial \boldsymbol{q}^{\langle 1\rangle}}+\frac{1}{\rho} \frac{\partial \underline{\dot{q}}}{\partial \boldsymbol{q}^{\langle 1\rangle}} \frac{\partial \underline{\boldsymbol{q}^{\langle 1\rangle}}}{\partial \dot{\boldsymbol{q}}} \frac{\partial \underline{\dot{\boldsymbol{q}}}}{\partial \boldsymbol{q}^{\langle 1\rangle}}\right)^{\top} \\
\boldsymbol{N}_{0,1}^{*}=\left(\left(\frac{\partial \underline{\boldsymbol{q}}^{\langle 1\rangle}}{\partial \boldsymbol{q}}-\frac{\mathrm{d}}{\mathrm{d} t} \frac{\partial \underline{\boldsymbol{q}}^{\langle 1\rangle}}{\partial \dot{\boldsymbol{q}}}\right) \frac{\partial \underline{\dot{\boldsymbol{q}}}}{\partial \boldsymbol{q}^{\langle 1\rangle}}\right)^{\top} \\
\boldsymbol{N}_{\rho, \rho}^{*}=-\frac{1}{\rho}\left(\frac{\partial \underline{\boldsymbol{q}}^{\langle 1\rangle}}{\partial \dot{\boldsymbol{q}}} \frac{\partial \underline{\dot{\boldsymbol{q}}}}{\partial \boldsymbol{q}^{\langle 1\rangle}}\right)^{\top}
\end{array}\right.
$$

According to the result presented in Proposition A.2 equation (A.7) can be rewritten as follows:

$$
{ }^{\mathrm{I}} \boldsymbol{f}=\boldsymbol{N}_{0,0}^{*} \frac{\partial \underline{T}}{\partial \boldsymbol{q}}+\boldsymbol{N}_{0,1}^{*} \frac{\partial \underline{T}}{\partial \boldsymbol{q}^{\langle 1\rangle}}+\boldsymbol{N}_{\rho, \rho}^{*} \frac{\partial \underline{T}^{(\rho)}}{\partial \boldsymbol{q}^{\langle\rho \mid 1\rangle}}
$$

Particularly, for $\rho=1$, it can be stated that:

$$
{ }^{\mathrm{I}} \boldsymbol{f}=\boldsymbol{N}_{0,0}^{*} \frac{\partial \underline{T}}{\partial \boldsymbol{q}}+\boldsymbol{N}_{0,1}^{*} \frac{\partial \underline{T}}{\partial \boldsymbol{q}^{\langle 1\rangle}}+\boldsymbol{N}_{1,1}^{*} \frac{\partial \underline{T}^{(1)}}{\partial \boldsymbol{q}^{\langle 1\rangle}}
$$

Proposition A.3. There is a square matrix $M$, called unconstrained generalized inertia matrix, and a column-matrix ${ }^{G} \boldsymbol{f}$, whose elements are called unconstrained generalized gyroscopic inertia forces, such that:

$$
{ }^{\mathrm{I}} \boldsymbol{f}=-\underline{\boldsymbol{M}}\left(t, \boldsymbol{q}, \boldsymbol{q}^{\langle 1\rangle}\right) \dot{\boldsymbol{q}}^{\langle 1\rangle}+{ }^{\mathrm{G}} \boldsymbol{f}\left(t, \boldsymbol{q}, \boldsymbol{q}^{\langle 1\rangle}\right)
$$

Moreover it can be stated that matrix $M$ is symmetric and its elements can be 
obtained as follows:

$$
M_{. i . j}=-\frac{\partial{ }^{\mathrm{I}} f_{. i}}{\partial \dot{q}_{. j}^{\langle 1\rangle}}=\frac{\partial S}{\partial \dot{q}_{. i}^{\langle 1\rangle} \partial \dot{q}_{. j}^{\langle 1\rangle}}
$$

Proof.Noting that $\left(\partial \underline{T} / \partial \boldsymbol{q}^{\langle 1\rangle}\right)$ and $\left(\partial \underline{\boldsymbol{q}}^{\langle 1\rangle} / \partial \dot{\boldsymbol{q}}\right)$ can be expressed as functions of $\left(t, \boldsymbol{q}, \boldsymbol{q}^{\langle 1\rangle}\right)$, it can be stated that, in equation (A.7), there can only be terms dependent of $\dot{\boldsymbol{q}}^{\langle 1\rangle}$ when the following time derivatives are obtained:

$$
\frac{\mathrm{d}}{\mathrm{d} t}\left(\frac{\partial \underline{T}}{\partial \boldsymbol{q}^{\langle 1\rangle}}\right) \quad \text { e } \quad \frac{\mathrm{d}}{\mathrm{d} t}\left(\frac{\partial \underline{\boldsymbol{q}}^{\langle 1\rangle}}{\partial \dot{\boldsymbol{q}}}\right)
$$

Once the terms in which the variables $\dot{\boldsymbol{q}}^{\langle 1\rangle}$ appear are associated with time derivatives of terms expressed as functions of $\left(t, \boldsymbol{q}, \boldsymbol{q}^{\langle 1\rangle}\right)$, it can be stated that ${ }^{\mathrm{I}} \boldsymbol{f}$ is an affine function of $\dot{\boldsymbol{q}}^{\langle 1\rangle}$. Particularly, $M$ can be obtained as follows:

$$
M=\left(\frac{\partial \dot{\dot{q}}}{\partial \boldsymbol{q}^{\langle 1\rangle}}\right)^{\top} \frac{\partial}{\partial \dot{\boldsymbol{q}}^{\langle 1\rangle}}\left(\frac{\mathrm{d}}{\mathrm{d} t}\left(\left(\frac{\partial \underline{\boldsymbol{q}}^{\langle 1\rangle}}{\partial \dot{\boldsymbol{q}}}\right)^{\top} \frac{\partial \underline{T}}{\partial \boldsymbol{q}^{\langle 1\rangle}}\right)\right)
$$

Moreover, from equation (A.7), it can be noted that the column-matrix:

$$
{ }^{\mathrm{G}} \boldsymbol{f}={ }^{\mathrm{I}} \boldsymbol{f}+\boldsymbol{M} \dot{\boldsymbol{q}}\langle 1\rangle
$$

can be expressed as a function of $t$, of the generalized coordinates $\boldsymbol{q}$ and of the quasi-velocities $\boldsymbol{q}^{\langle 1\rangle}$, only. Finally, once ${ }^{\mathrm{I}} \boldsymbol{f}$ is an affine function with respect to $\dot{\boldsymbol{q}}^{\langle 1\rangle}$ it can be stated that:

$$
M_{. i . j}=-\frac{\partial{ }^{\mathrm{I}} f_{. i}}{\partial \dot{q}_{. j}^{\langle 1\rangle}}=\frac{\partial S}{\partial \dot{q}_{. j}^{\langle 1\rangle} \partial \dot{q}_{. i}^{\langle 1\rangle}}=\frac{\partial S}{\partial \dot{q}_{. i}^{\langle 1\rangle} \partial \dot{q}_{. j}^{\langle 1\rangle}}=-\frac{\partial{ }^{\mathrm{I}} f_{. j}}{\partial \dot{q}_{. i}^{\langle 1\rangle}}=M_{. j . i}
$$

This completes the proof.

\section{A.2 Kinetic energy and Gibbs-Appell function of a rigid body}

In order to develop algorithms to derive the equations of motion of a multirigid-body system, it is convenient to be able to obtain directly the expressions of the kinetic energy and Gibbs-Appell function of a rigid body from a description of its instantaneous motion, given by the velocity of its center of mass and by the angular velocity of the body with respect to an inertial reference frame, along with its inertia properties, given by its mass and inertia tensor with respect to its own center of mass.

Let $\mathcal{B}$ denote a tridimensional rigid body which will be modeled as a contin- 
uous system of finite mass $m$. Denote by $v$ the velocity of an arbitrary point $p$ of $\mathcal{B}$ with respect to an inertial reference frame $\mathcal{N}$ and let $\mathbf{v}^{\star}$ denote the velocity of its center of mass $b^{\star}$ with respect to $\mathcal{N}, \omega$ denote the angular velocity of $\mathcal{B}$ with respect to $\mathcal{N}$ and $r$ denote the position vector of $\mathrm{p}$ with respect to $\mathrm{b}^{\star}$. It can be stated that (TENENBAUM, 2004):

$$
\begin{aligned}
& \mathbf{v}=\mathbf{v}^{\star}+\omega \times \mathbf{r} \\
& \dot{\mathbf{v}}=\dot{\mathbf{v}}^{\star}+\dot{\omega} \times \mathbf{r}+\omega \times(\omega \times \mathbf{r})
\end{aligned}
$$

Denote by $\mu$ the density function of $\mathcal{B}$ which will be supposed to be an integrable function defined for each point of $\mathcal{B}$. Assume, by hypothesis, that $\mu$ is not dependent of time $t$. Once $\mathcal{B}$ is a rigid body, its volume is also independent of $t$, and the identities in equation (3.4) are valid. The kinetic energy of $\mathcal{B}$ can be computed as follows:

$$
T=\int_{\mathcal{B}} \frac{1}{2}(\mathbf{v} \cdot \mathbf{v}) \mu \mathrm{dB}
$$

Replacing the expression of $\mathbf{v}$ given by equation (A.15), it can be stated that:

$$
T=\int_{\mathcal{B}} \frac{1}{2}\left(\mathbf{v}^{\star} \cdot \mathbf{v}^{\star}+2 \mathbf{v}^{\star} \cdot(\omega \times \mathbf{r})+(\omega \times \mathbf{r}) \cdot(\omega \times \mathbf{r})\right) \mu \mathrm{d} \mathcal{B}
$$

Using the properties of dot and cross product, this expression can be simplified as follows:

$$
\begin{aligned}
T= & \frac{1}{2}\left(\mathbf{v}^{\star} \cdot \mathbf{v}^{\star}\right) \int_{\mathcal{B}} \mu \mathrm{d} \mathcal{B}+\left(\mathbf{v}^{\star} \times \omega\right) \cdot \int_{\mathcal{B}} \mathbf{r} \mu \mathrm{d} \mathcal{B} \\
& -\frac{1}{2} \int_{\mathcal{B}} \mathbf{r} \cdot \omega \times(\omega \times \mathbf{r}) \mu \mathrm{d} \mathcal{B}
\end{aligned}
$$

And, applying the identities in equation (3.4):

$$
T=\frac{1}{2} m\left(\mathbf{v}^{\star} \cdot \mathbf{v}^{\star}\right)-\frac{1}{2} \int_{\mathcal{B}} \mathbf{r} \cdot \omega \times(\omega \times \mathbf{r}) \mu \mathrm{d} \mathcal{B}
$$

For further simplification, it must be considered that for any pair of vectors $\mathrm{x}$ and $\mathbf{y}$ :

$$
\mathbf{x} \times(\mathbf{x} \times \mathbf{y})=(\mathbf{x} \cdot \mathrm{y}) \mathbf{x}-(\mathrm{x} \cdot \mathrm{x}) \mathrm{y}
$$

Thus, the expression of the kinetic energy of $\mathcal{B}$ can be rewritten as follows:

$$
T=\frac{1}{2} m\left(\mathbf{v}^{\star} \cdot \mathbf{v}^{\star}\right)+\frac{1}{2} \int_{\mathcal{B}}\left((\omega \cdot \omega)(\mathbf{r} \cdot \mathbf{r})-(\omega \cdot \mathbf{r})^{2}\right) \mu \mathrm{d} \mathcal{B}
$$

Once the vector $\omega$ does not vary within the domain of $\mathcal{B}$, the inertia tensor I of $\mathcal{B}$ with respect with its center of mass can be defined as being the second 
order tensor for which given any pair of vectors ${ }^{1} \mathrm{x}$ and $\mathrm{y}$ :

$$
\begin{aligned}
& \mathbf{I} \cdot \mathbf{x}=\int_{\mathcal{B}} \mathbf{r} \times(\mathbf{x} \times \mathbf{r}) \mu \mathrm{d} \mathcal{B}=\int_{\mathcal{B}}((\mathbf{r} \cdot \mathbf{r}) \mathbf{x}-(\mathbf{x} \cdot \mathbf{r}) \mathbf{r}) \mu \mathrm{d} \mathcal{B} \\
& \mathbf{x} \cdot(\mathbf{I} \cdot \mathbf{y})=\int_{\mathcal{B}}((\mathbf{x} \cdot \mathbf{y})(\mathbf{r} \cdot \mathbf{r})-(\mathbf{x} \cdot \mathbf{r})(\mathbf{y} \cdot \mathbf{r})) \mu \mathrm{d} \mathcal{B}=\mathbf{y} \cdot(\mathbf{I} \cdot \mathbf{x})
\end{aligned}
$$

Due to the commutative property of the dot product, it can e stated that I tensor is a symmetric second order tensor, being possible to compute the kinetic energy of $\mathcal{B}$ as follows:

$$
T=\frac{1}{2} m \mathbf{v}^{\star} \cdot \mathbf{v}^{\star}+\frac{1}{2} \omega \cdot \mathbf{I} \cdot \omega
$$

Analogously, the Gibbs-Appell function associated to the generic rigid body $\mathcal{B}$ is given by:

$$
S=\int_{\mathcal{B}} \frac{1}{2}(\dot{\mathbf{v}} \cdot \dot{\mathbf{v}}) \mu \mathrm{d} \mathcal{B}
$$

Considering that $\dot{\mathbf{v}}=\dot{\mathbf{v}}^{\star}+\ddot{\mathbf{r}}$, with $\ddot{\mathbf{r}}=\dot{\omega} \times \mathbf{r}+\omega \times(\omega \times \mathbf{r})$ :

$$
S=\frac{1}{2}\left(\dot{\mathbf{v}}^{\star} \cdot \dot{\mathbf{v}}^{\star}\right) \int_{\mathcal{B}} \mu \mathrm{d} \mathcal{B}+\dot{\mathbf{v}}^{\star} \cdot \int_{\mathcal{B}} \ddot{\mathbf{r}} \mu \mathrm{d} \mathcal{B}+\frac{1}{2} \int_{\mathcal{B}}(\ddot{\mathbf{r}} \cdot \ddot{\mathbf{r}}) \mu \mathrm{d} \mathcal{B}
$$

Applying the identities in equation (3.4):

$$
S=\frac{1}{2} m\left(\dot{\mathbf{v}}^{\star} \cdot \dot{\mathbf{v}}^{\star}\right)+\frac{1}{2} \int_{\mathcal{B}}(\ddot{\mathbf{r}} \cdot \ddot{\mathbf{r}}) \mu \mathrm{d} \mathcal{B}
$$

Note that:

$$
\ddot{\mathbf{r}} \cdot \ddot{\mathbf{r}}=(\dot{\omega} \times \mathbf{r})^{2}+2(\dot{\omega} \times \mathbf{r}) \cdot(\omega \times(\omega \times \mathbf{r}))+(\omega \times(\omega \times \mathbf{r}))^{2}
$$

Thus, it can be stated that:

$$
\int_{\mathcal{B}}(\dot{\omega} \times \mathbf{r}) \cdot(\dot{\omega} \times \mathbf{r}) \mu \mathrm{d} \mathcal{B}=-\int_{\mathcal{B}} \mathbf{r} \cdot \dot{\omega} \times(\dot{\omega} \times \mathbf{r}) \mu \mathrm{d} \mathcal{B}=\dot{\omega} \cdot(\mathbf{I} \cdot \dot{\omega})
$$

Also using equation (A.21):

$$
\begin{aligned}
\int_{\mathcal{B}}(\dot{\omega} \times \mathbf{r}) \cdot(\omega \times(\omega \times \mathbf{r})) \mu \mathrm{d} \mathcal{B} \\
=\int_{\mathcal{B}}(\dot{\omega} \times \mathbf{r}) \cdot((\omega \cdot \mathbf{r}) \omega-(\omega \cdot \omega) \mathbf{r}) \mu \mathrm{d} \mathcal{B} \\
=\dot{\omega} \cdot \int_{\mathcal{B}}(\mathbf{r} \times \omega)(\omega \cdot \mathbf{r}) \mu \mathrm{dB}
\end{aligned}
$$

\footnotetext{
${ }^{1}$ It is important to highlight that $\mathbf{r}$ is a function defined for each point of $\mathcal{B}$ that gives the position of the point with respect to $\mathrm{b}^{\star}$, while $\mathrm{x}$ and $\mathrm{y}$ are treated as arbitrary vectors that do not vary within the domain of $\mathcal{B}$.
} 
It can be proved that (KANE; LEVINSON, 1985):

$$
\int_{\mathcal{B}}(\mathbf{r} \times \omega)(\omega \cdot \mathbf{r}) \mu \mathrm{dB}=\omega \times(\mathbf{I} \cdot \omega)
$$

For the last term, the identity of equation (A.21) can be applied leading to:

$$
\begin{aligned}
\int_{\mathcal{B}}( & \times(\omega \times \mathbf{r})) \cdot(\omega \times(\omega \times \mathbf{r})) \mu \mathrm{d} \mathcal{B} \\
& =\int_{\mathcal{B}}\left((\omega \cdot \omega)^{2}(\mathbf{r} \cdot \mathbf{r})-(\omega \cdot \mathbf{r})^{2}(\omega \cdot \omega)\right) \mu \mathrm{d} \mathcal{B} \\
& =(\omega \cdot \omega) \int_{\mathcal{B}}\left((\omega \cdot \omega)(\mathbf{r} \cdot \mathbf{r})-(\omega \cdot \mathbf{r})^{2}\right) \mu \mathrm{dB}=(\omega \cdot \omega)(\omega \cdot \mathbf{I} \cdot \omega)
\end{aligned}
$$

Therefore, the Gibbs-Appell function for a generic rigid body $\mathcal{B}$ can be computed by the following expression:

$$
S=\frac{1}{2} m \dot{\mathbf{v}}^{\star} \cdot \dot{\mathbf{v}}^{\star}+\frac{1}{2}(\dot{\omega} \cdot \mathbf{I} \cdot \dot{\omega}+2 \dot{\omega} \cdot(\omega \times \mathbf{I} \cdot \omega)+(\omega \cdot \omega)(\omega \cdot \mathbf{I} \cdot \omega))
$$

\section{A.3 Generalized forces in a multi-rigid-body sys- tem}

Let $\mathcal{B}$ be a rigid body, which is conceived as a continuous system of finite and constant mass. Let $\mathcal{N}$ denote an inertial reference frame and consider that $\mathrm{N}$ is a coordinate system rigidly attached to $\mathcal{N}$. Denote by $\mathrm{v}$ the velocity of a generic point $\mathrm{p}$ of $\mathcal{B}$ and by $\dot{\mathrm{v}}$ its acceleration, both measured with respect to $\mathcal{N}$. Define $\mathscr{B}$ as a mechanical system constituted by $\mathcal{B}$ only. Eventually, $\mathscr{B}$ might have other constraints than the ones imposed by the rigid body condition, being a $\nu^{\#-D O F ~ s y s t e m ~ w h o s e ~ c o n s t r a i n t ~ o r d e r ~ i s ~ e q u a l ~ t o ~} \nu^{\circ}$.

Using a notation similar to the one adopted in Section 3.1, it can be stated that the Principle of Virtual Power for $\mathscr{B}$ can be written as follows:

$$
{ }^{\mathrm{A}} \tilde{P}+{ }^{\mathrm{I}} \tilde{P} \leq 0
$$

with the virtual power associated to the active and inertia forces defined respectively by:

$$
\begin{aligned}
& { }^{\mathrm{A}} \tilde{P}=\int_{\mathcal{B}} \delta \mathbf{v} \cdot{ }^{\mathrm{A}} \boldsymbol{\gamma} \mathrm{d} \mathcal{B}+\int_{\widetilde{\partial}_{\mathcal{B}}} \delta \mathbf{v} \cdot{ }^{\mathrm{A}} \boldsymbol{\sigma} \mathrm{d} \widetilde{\partial}_{\mathcal{B}}+\sum_{k} \delta \mathbf{v}_{k} \cdot{ }^{\mathrm{A}} \mathbf{f}_{k} \\
& { }^{\mathrm{I}} \tilde{P}=-\int_{\mathcal{B}} \delta \mathbf{v} \cdot \dot{\mathbf{v}} \mu \mathrm{d} \mathcal{B}
\end{aligned}
$$

Note that in these equations ${ }^{\mathrm{A}} \boldsymbol{\gamma},{ }^{\mathrm{A}} \boldsymbol{\sigma}$ and ${ }^{\mathrm{A}} \mathbf{f}_{k}$ refer to the active force system acting on $\mathcal{B}$ only, i.e. there are no parcels in these terms associated to constraint 
forces.

Denoting by $\mathbf{v}^{\star}$ the velocity of the center of mass $b^{\star}$ of $\mathcal{B}$ and by $\omega$ the angular velocity of $\mathcal{B}$ with respect to $\mathcal{N}$, it can be stated that whenever it can be assumed that $\delta \mathbf{r}=\mathbf{0}$, then:

$$
\delta \mathbf{v}=\delta \mathbf{v}^{\star}+\delta \omega \times \mathbf{r}
$$

Take an integer $\sigma \geq \max \left\{1, \nu^{\circ}\right\}$, assume that it is possible to express $\mathbf{v}^{\star}$ and $\omega$ as explicit functions of the generalized variables defined for $\mathscr{B}$ up to first order, i.e. $\mathbf{v}^{\star}=\underline{\mathbf{v}}^{\star}\left(t, \boldsymbol{q}^{\langle 0\rangle}, \boldsymbol{q}^{\langle 1\rangle}\right)$ and $\omega=\underline{\omega}\left(t, \boldsymbol{q}^{\langle 0\rangle}, \boldsymbol{q}^{\langle 1\rangle}\right)$, and define, in analogy to equations (3.108) and (3.137):

$$
\mathbf{V}^{\star}=\frac{\partial \underline{\mathbf{v}}^{\star}}{\partial \boldsymbol{q}^{\langle 1\rangle}} \frac{\partial \underline{\boldsymbol{q}}^{\langle\sigma \mid 1\rangle}}{\partial \boldsymbol{q}^{\langle\sigma\rangle}} \quad \Omega=\frac{\partial \underline{\omega}}{\partial \boldsymbol{q}^{\langle 1\rangle}} \frac{\partial \underline{\boldsymbol{q}}^{\langle\sigma \mid 1\rangle}}{\partial \boldsymbol{q}^{\langle\sigma\rangle}}
$$

Thus, the unconstrained generalized active forces of $\mathscr{B}$ are defined as follows:

$$
\begin{aligned}
{ }^{\mathrm{A}} & =\mathbf{V}^{\star} \cdot{ }^{\mathrm{A}} \mathbf{f}+\boldsymbol{\Omega} \cdot{ }^{\mathrm{A}} \mathbf{t}^{\star} \\
{ }^{\mathrm{A}} \mathbf{f} & =\int_{\mathcal{B}}{ }^{\mathrm{A}} \boldsymbol{\gamma} \mathrm{d} \mathcal{B}+\int_{\widetilde{\partial}_{\mathcal{B}}}{ }^{\mathrm{A}} \boldsymbol{\sigma} \mathrm{d} \widetilde{\partial}_{\mathcal{B}}+\sum_{k}{ }^{\mathrm{A}} \mathbf{f}_{k} \\
{ }^{\mathrm{A}} \mathbf{t}^{\star} & =\int_{\mathcal{B}} \mathbf{r} \times{ }^{\mathrm{A}} \boldsymbol{\gamma} \mathrm{d} \mathcal{B}+\int_{\widetilde{\partial}_{\mathcal{B}}} \mathbf{r} \times{ }^{\mathrm{A}} \boldsymbol{\sigma} \mathrm{d} \widetilde{\partial}_{\mathcal{B}}+\sum_{k} \mathbf{r}_{k} \times{ }^{\mathrm{A}} \mathbf{f}_{k}
\end{aligned}
$$

Analogously, the unconstrained generalized inertia forces of $\mathscr{B}$ are computed by the following expressions, see equations (3.7) and (3.8):

$$
\begin{aligned}
& { }^{\mathrm{I}} \boldsymbol{f}=\mathbf{V}^{\star} \cdot{ }^{\mathrm{I}} \mathbf{f}+\boldsymbol{\Omega} \cdot{ }^{\mathrm{I}} \mathbf{t}^{\star} \\
& { }^{\mathrm{I}} \mathbf{f}=-\int_{\mathcal{B}} \mu \dot{\mathbf{v}} \mathrm{d} \mathcal{B}=-m \dot{\mathbf{v}}^{\star} \\
& { }^{\mathrm{I}} \mathbf{t}^{\star}=-\int_{\mathcal{B}} \mu(\mathbf{r} \times \dot{\mathbf{v}}) \mathrm{d} \mathcal{B}=-\left(\mathbf{I}^{\star} \cdot \dot{\boldsymbol{\omega}}+\boldsymbol{\omega} \times\left(\mathbf{I}^{\star} \cdot \boldsymbol{\omega}\right)\right)
\end{aligned}
$$

with $m$ denoting the mass of $\mathcal{B}$, and $\mathbf{I}^{\star}$ representing the inertia tensor of $\mathcal{B}$ with respect to its center of mass $b^{\star}$. Equation (A.43) can alternatively be obtained from (A.1) using the expression of $S$ in (A.34) or from (A.7) or (A.11) using the expression of $T$ in (A.25).

Let $\mathscr{M}$ be a multibody system constituted by a finite number of rigid bodies, generically denoted by $\mathcal{B}_{r}$. Use the subscript $r$ to denote the physical quantities 
associated to $\mathcal{B}_{r}$. It can be stated that for $\mathscr{M}$ :

$$
\begin{aligned}
& { }^{\mathrm{A}} \boldsymbol{f}=\sum_{r}\left(\mathbf{V}_{r}^{\star} \cdot{ }^{\mathrm{A}} \mathbf{f}_{r}+\boldsymbol{\Omega}_{r} \cdot{ }^{\mathrm{A}} \mathbf{t}_{r}^{\star}\right) \\
& { }^{\mathrm{I}} \boldsymbol{f}=-\sum_{r}\left(\mathbf{V}_{r}^{\star} \cdot\left(m_{r} \dot{\mathbf{V}}_{r}^{\star}\right)+\boldsymbol{\Omega}_{r} \cdot\left(\mathbf{I}_{r}^{\star} \cdot \dot{\boldsymbol{\omega}}_{r}+\boldsymbol{\omega}_{r} \times\left(\mathbf{I}_{r}^{\star} \cdot \boldsymbol{\omega}_{r}\right)\right)\right)
\end{aligned}
$$

Therefore, the modeling a multi-rigid-body system involves the following steps:

(1) Define a set of generalized variables which must be sufficient and adequate for describing the motions performed by this system.

(2) Obtain invariants associated to constraints which are not automatically imposed by the chosen variables, if any.

(3) Describe positions, velocities, angular velocities, accelerations and angular accelerations in terms of the defined variables and geometric parameters of the model.

(4) Provide, for each rigid body, the mass and the inertia tensor with respect to its own center of mass (which is typically represented in matrix form, in terms of its components associated to a coordinate system rigidly attached to the body).

(5) Describe, in terms of the generalized variables and physical parameters of the model, the active force systems acting in the system.

After these steps, the unconstrained active and inertia forces of $\mathscr{M}$ can be obtained from equations (A.46) and (A.47), begin possible to complete the derivation of the equations of motion of $\mathscr{M}$ applying the modular modeling algorithm presented in Section 4.2.

Finally, it is important to highlight that all the results presented in Section A.1 are applicable for $\mathscr{M}$ once the corresponding Gibbs-Appell function and kinetic enery are computed as follows ${ }^{2}$ :

$$
\begin{gathered}
S=\sum_{r} \int_{\mathcal{B}_{r}} \frac{1}{2}(\dot{\mathbf{v}} \cdot \dot{\mathbf{v}}) \mu \mathrm{d} \mathcal{B}_{r}=\sum_{r}\left(\frac{1}{2} m_{r}\left(\dot{\mathbf{v}}_{r}^{\star} \cdot \dot{\mathbf{v}}_{r}^{\star}\right)+\frac{1}{2} \dot{\omega}_{r} \cdot\left(\mathbf{I}_{r}^{\star} \cdot \dot{\boldsymbol{\omega}}\right)\right. \\
\left.+\dot{\omega}_{r} \cdot\left(\omega_{r} \times\left(\mathbf{I}_{r}^{\star} \cdot \omega_{r}\right)\right)+\frac{1}{2}\left(\omega_{r} \cdot \omega_{r}\right)\left(\omega_{r} \cdot\left(\mathbf{I}_{r}^{\star} \cdot \omega_{r}\right)\right)\right) \\
T=\sum_{r} \int_{\mathcal{B}_{r}} \frac{1}{2}(\mathbf{v} \cdot \mathbf{v}) \mu \mathrm{d} \mathcal{B}_{r}=\sum_{r}\left(\frac{1}{2} m_{r}\left(\mathbf{v}_{r}^{\star} \cdot \mathbf{v}_{r}^{\star}\right)+\frac{1}{2} \omega_{r} \cdot\left(\mathbf{I}_{r}^{\star} \cdot \boldsymbol{\omega}_{r}\right)\right)
\end{gathered}
$$

2 The corresponding demonstrations are totally analogous to the ones presented in Section A.1 and will be omitted from this text. 